\title{
Parents in prevention: A meta-analysis of randomized controlled trials of parenting interventions to prevent internalizing problems in children from birth to age 18
}

Marie B. H. Yap ${ }^{\mathrm{a}, \mathrm{b}}$, Amy J. Morgan ${ }^{\mathrm{b}, \mathrm{c}}$, Kathryn Cairns ${ }^{\mathrm{b}}$, Anthony F. Jorm ${ }^{\mathrm{b}}$, SarahE. Hetrick $^{\mathrm{d}}$, Sally Merry

aSchool of Psychological Sciences, Monash Institute of Cognitive and Clinical Neurosciences, Monash University, Melbourne, Australia

bMelbourne School of Population and Global Health, University of Melbourne, Melbourne, Australia

'School of Psychology and Public Health, La Trobe University, Melbourne, Australia ${ }^{\mathrm{d} C e n t r e ~ o f ~ E x c e l l e n c e ~ i n ~ Y o u t h ~ M e n t a l ~ H e a l t h, ~ O r y g e n ~ Y o u t h ~ H e a l t h ~ R e s e a r c h ~ C e n t r e, ~}$ Centre for Youth Mental Health, University of Melbourne, Melbourne, Australia

eSchool of Medicine, University of Auckland.

Corresponding author: Dr Marie Yap

Address: Bld 17, 18 Innovation Walk, Monash University, Clayton, Victoria 3800, Australia.

Telephone: + +61399050723

Fax: $\quad$ +61399053948

E-mail: marie.yap@monash.edu (Marie Yap). 


\begin{abstract}
Purpose of the research:

Burgeoning evidence that modifiable parental factors can influence children's and adolescents' risk for depression and anxiety indicates that parents can play a crucial role in prevention of these disorders in their children. However, it remains unclear whether preventive interventions that are directed primarily at the parent (i.e. where the parent receives more than half of the intervention) are effective in reducing child internalizing (including both depression and anxiety) problems in the longer term.
\end{abstract}

\title{
Principal results:
}

Compared to a range of comparison conditions, parenting interventions reduced child internalizing problems, at a minimum of six months after the intervention was delivered. Mean effects were very small for measures of internalizing and depressive symptoms, and small for measures of anxiety symptoms. Pooled effects for anxiety diagnoses were significant and indicated a number needed to treat (NNT) of 10. Pooled effects for depression diagnoses approached significance but suggested a NNT of 11. These results were based on effects reported at the longest follow-up interval for each included study, which ranged from six months up to 15 years for internalizing measures, 5.5 years for depressive measures, and 11 years for anxietymeasures.

\section{Major conclusions:}

Our findings underscore the likely benefits of increasing parental involvement in preventing internalizing problems, particularly anxiety problems, in young people.

Keywords: Mental disorders; emotional problems; family; systematic review; young people 


\section{Introduction}

Mental disorders are the largest contributors to disability in young people, with one in four youths affected during their lifetime (Patel, Flisher, Hetrick, \& McGorry, 2007). Anxiety and mood disorders are amongst the most common disorders, with lifetime prevalence rates of $38 \%$ and $18 \%$ respectively amongst young people aged 13-17 years (Kessler, Petukhova, Sampson, Zaslavsky, \& Wittchen, 2012). Given the deleterious long-term sequelae resulting from early onset disorders (Last et al., 1997; Rao et al., 1995), there is a strong impetus for an effective, integrated approach to prevent these disorders in young people.

There is substantial overlap between depression and anxiety symptoms especially in childhood (Hankin, Fraley, Lahey, \& Waldman, 2005; Lahey, Hulle, D'Onofrio, Rodgers, \& Waldman, 2008), and increasing evidence indicates that anxiety and depression comprise an 'internalizing cluster' (Schleider \& Weisz, 2016; Weersing, Rozenman, Maher-Bridge, \& Campo, 2012). In addition to their phenomenological and diagnostic similarities, depression and anxiety disorders share some risk factors, including parental factors (Dozois, Seeds, \& Collins, 2009; Schleider \& Weisz, 2016; Wilamowska et al., 2010). Of note, extant evidence indicates that many parental factors examined in depression and anxiety research are not specific to either disorder (e.g., Bögels \& BrechmanToussaint, 2006; Restifo \& Bögels, 2009; Yap \& Jorm, 2015; Yap, Pilkington, Ryan, \& Jorm, 2014). Consequently, an increasing number of researchers have called for transdiagnostic approachesto prevention of these disorders (Dozois et al., 2009; Schleider \& Weisz, 2016; Yap \& Jorm, 2015; Yap et al., 2014), because of their potential to enhance the efficacy, generalizability, and cost-effectiveness of such interventions, as well as to facilitate their implementation. Moreover, studies examining internalizing outcomes across childhood and adolescence tend to use a range of measures assessing anxiety symptoms, depressive symptoms, or a combination of both, otherwise termed 'internalizing' symptoms. Hence, this review takes a transdiagnostic approach by including both depression and anxiety as outcomes of interest, along with measures of internalizing symptoms. Henceforth, the 
broad term 'internalizing cluster or problems' will be used to refer to measures of internalizing, anxiety or depressive symptoms or disorders.

\section{Parental influence in the etiology of child internalizing problems}

Within the developmental psychopathological framework, interpersonal theories posit that psychopathology both results from, and contributes to, disruptions in developmentally-salient interpersonal processes (starting with early parent-infant attachment), which in turn interfere with young people's need for relatedness (Rudolph, Lansford, \& Rodkin, 2016). From an etiological perspective, parents clearly have an important influence on young people's risk for internalizing disorders, in terms of both nature and nurture. Evidence from genetic studies suggests that the heritability of liability to internalizing disorders ranges from 30\%-80\% (e.g. Boomsma, Van Beijsterveldt, \& Hudziak, 2005; Eley et al., 2003). In contrast, meta-analytic reviews have found that single parenting behaviors account for only small amounts of variance in child internalizing problems (between 1\% to 18\%; McLeod, Weisz, \& Wood, 2007; McLeod, Wood, \& Weisz, 2007; Yap \& Jorm, 2015; Yap et al., 2014). Nonetheless, based on a large twin study in the Netherlands, Boomsma and colleagues (2005) concluded that as children grow up, the variation in internalizing problems is due in equal parts to genetic and environmental (namely the shared family environment) influences, and may partly account for the findings that some family-based approaches for child internalizing problems are effective. Furthermore, from a prevention and intervention perspective (Mrazek\& Haggerty, 1994; O'Connell, Boat, \& Warner, 2009), the focus needs to be on modifiable factors that can be targeted in intervention.

\section{Involving parents in prevention}

Given the burgeoning evidence underscoring a range of modifiable parental factors that can increase or decrease the risk for depression and anxiety in children (Yap \& Jorm, 2015) and adolescents(Yap et al., 2014), it is clear that parents play a crucial role in prevention of these disorders in their children. To that end, in the past several decades, parenting programs have been developed to capitalise on parents' influence on their child's development and mental health, based on the 
underlying assumption that changing parenting (mediators) will in turn change a child's riskfor depression and anxiety (Sandler, Schoenfelder, Wolchik, \& MacKinnon, 2011). Specifically, one of the theoretical pathways proposed by Sandler and colleagues (2011) posits that parenting programs can have long-term benefits for child outcomes through their effects on parenting skills, perceptions of parental efficacy, and reduction in barriers to effective parenting (e.g. parental psychopathology). These parental changes in turn lead to changes in their child (e.g. emotion regulation), and the transactions that their child has with their environment (e.g. peer relations).

\section{How long do preventive effects last?}

Inherent in the evaluation of preventive parenting programs for child internalizing problems, is whether they have long-term effects. That is: does the program prevent child internalizing problems in the long term, well after the intervention is over? The absence of evidence for long-term effects is one of the key limitations bemoaned in previous reviews of preventive programs (Barlow, Smailagic, Ferriter, Bennett, \& Jones, 2010; Fisak, Richard, \& Mann, 2011; Greenberg, Domitrovich, \& Bumbarger, 2001; Merry et al., 2011), which have mostly observed effects lasting less than 2 years. Based on developmental theories, some researchers have argued that preventive parenting interventions could have long-lasting effects, and that in fact there might be sleeper effects that increase over time (Greenberg et al., 2001; Rapee, 2013). In particular, following Bell's(1968) seminal writings and Sameroff and Chandler's (1975) transactional model of development, developmental researchers have long recognised that children are not passive recipients of parenting, but actively evoke and respond to parental behaviours based on their early temperamental dispositions (Dwyer, Nicholson, \& Battistutta, 2006), giving rise to dynamic interactive processes between parent and child over the course of the child's development (Sameroff, 2009). These bidirectional processes are likely to influence and build on one another to shape subsequent interactions and experiences, altering the child's developmental trajectoryand their risk of internalizing problems (Yap, Allen, \& Sheeber, 2007). Hence, it is possible that by intervening in these processes (e.g. by modifying parental risk or protective factors; Yap \& Jorm, 
2015; Yap et al., 2014), parenting interventions may also result in magnified effects on child internalizing outcomes over time; or at least produce effects that are sustained even after the intervention has ended. Investigation of the long-term effects of parenting programs testedin randomized controlled trials (RCTs) is required to address the question of whether such interventions have lasting effects, and whether these effects vary with the duration of the follow-up interval (post-intervention).

\section{Factors that may influence preventive effects}

Various program-level factors can influence the effects of preventive parenting programs, including: (1) at which stage of child development is the program delivered; (2) who the target population of parents is; (3) the focus of intervention in the program; and (4) whether the child receives direct intervention (in addition to what the parentreceives).

\section{Does the timing of preventive intervention matter?}

When considering the effects of preventive programs, one question is whether there is an 'optimal time' to intervene with parents. Within the developmental psychopathology framework, different aspects of parenting are salient at different stages of the child's development (Rudolph et al., 2016). The emergence of internalizing symptoms and rates of onset of disorders also vary across childhood (Kessler et al., 2012), and inform the need for preventive intervention at different developmental stages. Consistent with this, existing parenting interventions vary widely in their timing of delivery, from pre-birth programs for expectant parents to programs for parents of adolescents. Classic attachment theory (Bowlby, 1971) underscores the importance of early caregiver-infant attachment for optimal child development. Based on this, various programs target the very early days of 'parenthood', beginning during pregnancy, recognizing the challenges of parenting for first-time parents, especially parents with existing risk factors (e.g. age, poverty, trauma status; Ordway et al., 2014),. For instance, perinatal programs have been designed to equip couples with communication and co-parenting skills (Feinberg, Jones, Kan, \& Goslin, 2010; Feinberg \& Kan, 2008), promotion of maternal feelings of competence in infant caretaking as well as improvement of maternal sensitivity 
and the quality of mother-infant interactions (Cheng et al., 2007) and attachment (Ordway etal., 2014). Similar programs target parents in the first few months post-birth, specifically to address the risks associated with prematurity/low birth weight (e.g. parental distress; Singer et al., 1999), which in turn predict poorer child outcomes including internalizing problems (Spittle et al., 2010). Given that internalizing problems have their modal onset in middle to late childhood (Kessler et al., 2012), programs that target the early-childhood, preschool years attempt to address the risk and protective factors for internalizing problems, including behavioural inhibition and overprotective parenting (Dadds \& Roth, 2008; Rapee, Kennedy, Ingram, Edwards, \& Sweeney, 2005), before these factors become entrenched or start to impact on the child's functioning. Programs that are delivered during the mid-late childhood phase (primary school age) target parents of children at increased risk for internalizing problems, for example, due to parental psychopathology (Lam, Fals-Stewart, \& Kelley, 2008; Solantaus, Toikka, Alasuutari, Beardslee, \& Paavonen, 2009) or parental separation (Wolchik et al., 1993). Others target parents whose child has existing difficulties such as sleep problems (Quach, Hiscock, \& Wake, 2010) or childhood phobias (Santacruz, Mendez, \& Sanchez-Meca, 2006); or aim to prepare parents for the challenges of the adolescent years (Mason et al., 2007). Parenting interventions targeted at parents of adolescents are rare, but the few notable ones include a program targeting parents with the Human Immunodeficiency Virus, given the increased risk it imposes on their adolescents (e.g. prematurely assuming more responsibility, threat of loss of parent; Rotheram-Borus, Lee, Gwadz, \& Draimin, 2001); and another that aims to increase parents' emotion socialization skills (Kehoe, Havighurst, \& Harley, 2014) in the face of increased negative emotionality in parent-child interactions during adolescence (Yap, Schwartz, Byrne, Simmons, \& Allen, 2010). In this review, we will examine whether preventive effects differ as a function of the developmental stage during which the intervention is delivered.

\section{Who is the target population of parents?}

Related to the question of timing, is one about the target population: do preventive effects vary depending on the target population of parents the interventions were designed for? Within the 
original Institute of Medicine's proposed classification system delineating the mental health intervention spectrum (Mrazek \& Haggerty, 1994), preventive parenting programs can be universal, i.e. delivered to all parents regardless of their child's risk (e.g. Cheng et al., 2007; Dadds \& Roth, 2008; Mason et al., 2007); selective, focussing on parents of children with known risk factors (e.g. Ginsburg, 2009; Rapee et al., 2005; Spittle et al., 2010); or indicated, focussing on parents whose child has signs or symptoms of incipient disorder (e.g. Ruffolo, Kuhn, \& Evans, 2005; Simon, Bogels, \& Voncken, 2011). Other parenting interventions have been designed to treat early childhood problems (e.g. childhood trauma, J. A. Cohen \& Mannarino, 1998; darkness phobia, Santacruz et al., 2006) with the aim to prevent subsequent internalizing problems (i.e. secondary prevention). Given our whole-of-childhood focus in this review, we adopted a broader definition of prevention which includes such treatment or secondary prevention interventions (O'Connell et al., 2009).

Universal preventive parenting programs are advantageous because they can reduce the stigma parents fear would be attached to them as 'bad parents' or their child as having problems that require intervention, if they participate in parenting programs (Yap, Fowler, Reavley, \& Jorm, 2015). Universal interventions can be cost-effective if they are effective and acceptable to individuals, incur a low cost per person, and carry a low risk of harm (Mrazek \& Haggerty, 1994). In line with the classic, empirically-supported Health Belief Model (Becker, 1974), when parents are aware of their child's increased risk or otherwise concerned about their child, they are more likely to engage in preventive parenting programs. Consequently, parents tend to self-select into prevention programs based on their child's increased risk (Dadds \& Roth, 2008), which seems to suggest that selective and indicated programs may have better uptake. Another potential advantage of selective and indicated programs is that they may yield larger effect sizes relative to universal programs (Teubert \& Pinquart, 2011), although meta-analyses of preventive programs for internalizing problems (targeting the individual directly) have not consistently demonstrated this difference (e.g. Fisak et al., 2011; Merry et al., 2011; van Zoonen et al., 2014). This review of preventive parenting 
interventions will examine whether the type of prevention or target population moderates the preventive effects.

\section{Does the focus of the parenting program matter?}

As a reflection of the multifaceted nature of a parent's role in prevention of child internalizing problems, preventive parenting programs have incorporated various sets of intervention foci, including parenting skills, parent-child interaction/relationship, parents' own mental health, and training parents to be a co-therapist or coach for the child. Most parenting interventions have been designed to modify specific familial or parental factors that are theoretically and empirically believed to influence risk for child anxiety or depression. For example, many focus on building specific parenting skills, such as minimizing family conflict (e.g. Ginsburg, 2009; Wolchik et al., 2000), given its impact on the child's emotional security and subsequent risk for internalizing problems (Cummings \& Davies, 2002). Parental over-involvement has also been a target of parenting programs (e.g. Ginsburg, 2009; Rapee et al., 2005), given its well-established link especially with child anxiety, through reinforcement of the child's perceptions of the world as a dangerous place (Rapee, 1991). Notably, these parenting skills have been identified in recent meta-analyses as havingsound evidence for their association with depression, anxiety, and/or internalizing symptoms in children (Yap \& Jorm, 2015) and adolescents (Yap et al., 2014).

Fundamentally influenced by classic attachment theory (Bowlby, 1971) highlighting the importance of the parent-child bond throughout development, other programs have targeted the parent-child relationship. These focus on developing healthy parent-child communication and supportive parenting (e.g. Beardslee, Gladstone, Wright, \& Cooper, 2003), which comprise the general positive parenting construct of warmth or acceptance, another modifiable parental factor identified as having sound evidence for its association with internalizing symptoms in children (Yap \& Jorm, 2015), and reduced depression and anxiety in adolescents (Yap et al., 2014). In a similar vein, programs for parents of pre-term infants (e.g. Spittle et al., 2010) or first-time parents (Cheng et al., 
2007) focus on improving parent responsiveness and sensitivity in their interactions with their infants.

Given that children of parents with mood or anxiety disorders are at higher risk for developing such problems themselves, due to both genetic heritability (Boomsma et al., 2005) and environmental factors including the impact of parental psychopathology on parenting, parent-child attachment, and interparental conflict (Siegenthaler, Munder, \& Egger, 2012); various programs also specifically focus on improving parental mental health (e.g. Solantaus et al., 2009). Finally, some programs, especially those targeting anxiety in younger children within a cognitive behavior therapy framework, are designed to train parents to be their child's therapist or coach in overcoming the child's fears and anxieties (e.g. Rapee et al., 2005; Santacruz et al., 2006). While parenting programs may include more than one intervention focus, this review will examine whether preventive effects vary depending on whether any of the above-mentioned foci is included in the intervention.

\section{Does additional direct intervention with the child make a difference?}

Extant evidence indicates that internalizing problems in young people can be prevented. Some recent reviews and meta-analyses of randomized controlled trials (RCTs) have demonstrated that preventive interventions primarily directed at young people themselves have the potential to reduce depressive symptoms and disorder (Merry et al., 2011; van Zoonen et al., 2014), and anxiety symptoms and disorder (Fisak et al., 2011; Teubert \& Pinquart, 2011). Other reviews have included RCTs of interventions that focused on the family, parent, couple, child or variouscombinations (Siegenthaler et al., 2012; Stathakos \& Roehrle, 2003) or group parenting programs only (Barlow et al., 2010) and have also found small but significant mean effects on child internalizing symptoms. However, it is unclear whether interventions that are directed primarily at the parent (i.e where the parent receives most of the intervention), regardless of whether the child receives any direct intervention, or whether the program is individual- or group-based, are effective in reducing child internalizing problems. In order to address the question of whether it is fruitful to primarily target parents in preventive efforts for child internalizing problems, a review of preventive parenting 
programs where the parent receives most of the intervention is required. In addition, within such a review, moderator analyses can examine whether adding direct intervention with the childcan enhance the preventive effects.

\section{Aim of this review}

The main aim of this review was to examine the long-term effects of preventive parenting interventions for child internalizing problems. We also sought to examine whether the size of preventive effects changes over time. In addition, we examined whether the type of prevention (target population), the focus of the parenting intervention, the timing of the intervention, and whether the child receives any direct intervention modifies the effects of parenting interventions.

\section{Method}

\section{Literature search and study selection}

PubMed, PsycINFO, Embase and the Cochrane Central Register of Controlled Trials were searched from inception up until 2 July 2015 using controlled vocabulary and free text words. The search terms used for PubMed were: (depressive disorder[mh] OR depressi*[tw] OR anxiety[tw] OR anxiety disorders[mh] OR internalizing[tw] OR externalising[tw] OR externalizing[tw]) AND (program[tw] OR training[tw] OR education[tw] OR "Early Intervention (Education)"[mh]) AND ("clinical trial"[tw] OR randomized controlled trial[pt] OR controlled clinical trial[pt]) AND parent*[tw]. The search terms used for the other databases are presented in Appendix A. Titles and abstracts were screened by a single author, and the full-text of any potentially eligible papers was then examined independently by two authors. Disagreements about eligibility were settled by consensus or by a third author. References in included studies and relevant reviews were also examined for potential inclusion. See Figure 1 for the flow of selection of eligible papers, and Appendix B for the list of excluded papers, along with the reason(s) for exclusion.

Studies were included in the current review if they: 
a) were reported as randomized controlled trials with a no-treatment control group, an attention control group (including minimal interventions not intended to be active), or treatment as usual or normal service provision;

b) targeted parents or caregivers of children from birth to 18 years;

c) involved interventions that aimed to improve child outcomes indirectly through parents as a mediator (e.g., modification of parenting practices, improving parent/child relationships, improving parental functioning, coaching parents to provide an intervention for their children's own difficulties);

d) aimed to improve child internalizing problems through universal, selective or indicated prevention, or secondary prevention (e.g., treatment of darkness phobia in early childhood to prevent later internalizing problems). Whilst the inclusion of secondary prevention programs deviates from the more conventional purist definition of prevention (Mrazek\& Haggerty, 1994), it is aligned with the more recent and broader definition (O'Connell etal., 2009). Secondary prevention studies included participants with diagnosable difficulties identified through rigorous assessment (e.g. structured clinical interviews), and thus could be considered a form of treatment. However, most other studies fail to use similarly rigorous assessment to ascertain that their participants did not in fact have current or previous clinically diagnosable disorders; hence making it less logical to exclude secondary prevention studies. Moreover, intervening with early difficulties such as specific phobia has the potential benefit of preventing more generalised anxiety and depression outcomes later in life (Kessler et al., 2005; Mathew, Pettit, Lewinsohn, Seeley, \& Roberts, 2011);

e) evaluated interventions that focused on improving internalizing problems, including internalising measures, anxiety measures, and depression measures, as a major goal and not merely as a by-product. Interventions that were designed to prevent other outcomes (e.g. substance use or externalising disorders) were excluded, even if they retrospectively 
examined the effects of their interventions on internalizing problems (e.g. Trudeau, Spoth, Randall, \& Azevedo, 2007);

f) spent the majority of the intervention time with the parents (i.e., more than $50 \%$ ), although children could have received some intervention directly from the interventionist. Therefore studies were excluded if they evaluated a dual-component intervention delivered equally to parents and children (e.g. Compas, 2009; Sandler et al., 2003), or the parenting component was a small add-on to a child-focused intervention (e.g. Lowry-Webster, Barrett, \& Lock, 2003);

g) used validated internalising measures, anxiety measures and depression measures at least 6 months after the intervention ended ${ }^{1}$. A follow-up point of at least 6 months postintervention was chosen as this was thought to be the minimum length of time required for any true preventive effects to be demonstrated (whilst being sufficiently distant to differentiate these from any immediate effects post-intervention), and is a typical follow-up point in the literature (e.g. Cairns, Yap, Pilkington, \& Jorm, 2014; Ryan, Jorm, \& Lubman, 2010);

h) were reported in English.

Studies that evaluated interventions in children with developmental disorders, physical disabilities, medical conditions, or distress about facing medical interventions were excluded.

\section{Data extraction}

Two authors independently extracted key study characteristics and outcomes using a data extraction form that was piloted on three studies. Study characteristics included: the targetprevention population, inclusion and exclusion criteria, intervention/recruitment setting, country, age and gender of children, sample size and dropouts, description of the intervention and comparison

\footnotetext{
${ }^{1}$ Only two studies offered booster sessions after the intervention had ended (Etter, 2014; Ginsburg, 2009); but neither reported the uptake of these sessions. These booster sessions were not considered as part of the intervention when calculating the follow-up interval.
} 
condition (program name, focus of intervention, content outline, delivery format, number/length of sessions scheduled and delivered, length of intervention, whether child received direct intervention), risk of bias (see below), and assessment time points and measures used. The focus of the intervention was coded as parenting skills (e.g. modelling, child management strategies), parentchild relationship (e.g. reducing family conflict, improving interactions), parental mental health (e.g. managing cancer-related emotions), parent as coach of child's problem (e.g. applying anxiety exposure hierarchies with their child), or other, with multiple foci possible. Comparison conditions were coded as usual care (defined by the study), no treatment, minimal intervention (minimal versions of the intervention not expected to be as effective, e.g., fact sheets, parenting lecture), attention control, or extended waitlist (where the intervention was offered to the control group once the long-term follow-up assessments were completed). In studies that included more than one eligible intervention arm, data were extracted from the condition that was the main focus of the study, or was randomly selected by a coin toss (for 2 eligible arms) or a web-based randomizer (www.randomizer.org; for more than 2 eligible arms) if this was unclear. Where studies reported analyses that combined multiple eligible intervention arms and data for the intervention arm of interest could not be obtained from study authors, the combined data was extracted if it was meaningful to include the combined data. This decision was reached by consensus amongst the authors and was based on whether the intervention arms were considered similar enough to each other in terms of intervention foci (e.g. the same intervention but in segregated gender groups), and to other included studies in general.

Information needed to compute effect sizes (e.g., means and standard deviations) was extracted from measures of internalizing symptoms, anxiety symptoms, and depressive symptoms for each informant, where available, and at each follow-up time-point 6 months or more after the end of the intervention. Where means and standard deviations were not available, we extracted the effect size as reported (e.g., means and sample size of each group, and the $p$ value) if it was accepted by Comprehensive Meta-Analysis (CMA) Version 3 (Comprehensive Meta Analysis, 2013). When 
adjusted and unadjusted data were reported, unadjusted data were extracted unless the adjusted data controlled for baseline differences in the dependent variable or variables that were closely associated with the dependent variable (e.g., externalizing symptoms). CMA then converted all extracted continuous effect size measures into Cohen's standardized difference in means, $d$ (Borenstein, Hedges, Higgins, \& Rothstein, 2009). For anxiety and depressive disorder diagnoses, we extracted the number of participants meeting criteria for clinically significant disorder on standardised instruments yielding diagnoses, such as the Schedule for Affective Disorders for Children (K-SADS). Following Merry and colleagues' Cochrane review (Merry et al., 2011), these data were pooled using a Risk Difference (RD). Data from studies which used a pre-determined cut-off to identify participants who were likely to have, or did have clinically significant depressive or anxiety symptoms, were included in the meta-analysis of continuous measures (to derive Cohen's $d$ ) only. Disagreements were settled by consensus or by a third author. Attempts were made to contact study authors to provide missing information.

\section{Risk ofbias}

Risk of bias of each study was independently assessed by two authors using a descriptiveapproach. Information about potential sources of bias was extracted for each of the following criteria according to the Cochrane Collaboration's risk of bias tool: random sequence generation, allocation concealment, blinding of outcome assessment, incomplete outcome data, and selective reporting of internalizing problems (Higgins \& Green, 2011). These criteria were judged as high-risk, low-risk, or unclear, with supportingjustification.

\section{Data synthesis}

A meta-analysis was conducted separately for internalizing symptoms, depressive symptoms, depressive diagnoses, anxiety symptoms, and anxiety diagnoses using CMA. To examine intervention effects for internalizing problems as a broad construct, a meta-analysis was also conducted that included data from all included studies regardless of the type of outcome measure they used 
(measures of internalizing, anxiety, or depressive symptoms). Where studies used more than one of these outcome measures, we applied the following hierarchy for selecting which outcome from that study to include in the meta-analysis: (1) internalizing measure, given that the majority of studies used this type of measure; (2) anxiety measure, given that anxiety symptoms are more prevalent than depressive symptoms across childhood, and are believed to be a precursor of subsequent depression (Kessler et al., 2005; Mathew et al., 2011); and (3) depression measure. A random effects model was used in all meta-analyses because we expected considerable heterogeneity. We calculated the $Q$ and $I^{2}$ statistics as indicators of heterogeneity. An $I^{2}$ value of $0 \%$ indicates no observed heterogeneity and larger values indicate greater heterogeneity $(25 \%=10 w, 50 \%=$ moderate, 75\%=high; Higgins, Thompson, Deeks, \& Altman, 2003). When the meta-analysis involved at least 3 studies, we tested for publication bias by inspecting the funnel plot of the meta-analysis and by using Egger's test (Egger, Smith, \& Phillips, 1997). To obtain an estimate of the effect sizeafter publication bias, we used the Duval \& Tweedie (2000) 'trim-and-fill' procedure, which allows the imputation of potentially missing studies. A guideline of four or more studies imputed is taken as indicative of publication bias (Borenstein et al., 2009).

For each outcome of interest, we conducted one overall meta-analysis which included one effect size from each eligible study. When studies reported data from more than one follow-up, we used the effect size from the longest follow-up interval. When a study reported an effect size from more than one informant (e.g., child-report and mother-report), we applied the following hierarchy, which was adapted from a recent meta-analytic review (Yap \& Jorm, 2015), in order of preference for data extraction: (1) composite of parent and child computed through models which take into account the correlation between measures, based on the argument that this reduces the risk of bias associated with reliance on a sole informant (Podsakoff, MacKenzie, Lee, \& Podsakoff, 2003); (2) child-report, given that in most studies the child did not receive direct intervention and is hence less subject to outcome assessment bias compared to parents; (3) parent-report, which is less preferred compared to child-report because parents are more subject to bias than are children; but preferable to 
teacher-report because parent-child agreement on child internalizing symptoms has been found to be higher than teacher-child agreement (Phares, Compas, \& Howell, 1989; Stanger \& Lewis, 1993). If both mother-report and father-report were obtained, mother-report data was selected over fatherreport, because most other included studies with parent-reported outcomes involved a mother informant; and (4) teacher. However, since the mean effect could differ at varying follow-up periods and depending on the informant, we conducted a series of supplementary analyses for each of these factors. Firstly, we examined Cohen's $d$ for each follow-up period separately (6-10 months, $>10-12$ months, $>12-24$ months, $>24-48$ months, and $>48$ months). Secondly, where subsets of studies with data from each informant (e.g., father, mother, child, teacher, combinations) had more than two comparisons each, we also examined Cohen's $d$ for each subset. Given that some studies had data at more than one follow-up interval or from more than one informant (e.g., one study with 7-month, 13-month, and 36-month follow-up data would be included in three analyses), the subsets of studies were not mutually exclusive and hence differences between subsets were not examined.

If a study reported an effect size for more than one independent subgroup in their sample (e.g., older versus younger mothers, single- versus dual-family, female versus male adolescents), we included each subgroup as a separate 'study'.

Where there were more than two comparisons in each meta-analysis and heterogeneity was not zero, we conducted subgroup analyses for dichotomous moderators and meta-regression analyses for continuous variables, according to the procedures implemented in CMA. These analyseswere conducted using mixed-effects analyses that pooled studies within subgroups with the random effects model, but tested for significant differences between subgroups with the fixed effects model. We examined whether the mean effect size (Cohen's $d$ ) was modified by type of prevention (universal, selective, indicated, secondary); whether the intervention included a focus on parentchild relationship, parenting skills, parental mental health, or training parents to coach their child (e.g., using exposure techniques); timing of intervention (birth: 0-2.99 months, preschool : 3 months- 
4 years, primary-school: >5-12 years, adolescence: $12+$ years); type of comparison (notreatment, attention control, extended waitlist, minimal intervention, usual care); and whether there was direct intervention with the child. We also conducted meta-regression analyses in which we used only the longest follow-up period reported in each study, to explore whether the effect size varied depending on the duration of the follow-up period. When interpreting mean effect sizes, we followed Cohen's (1992) guidelines whereby $d$ of at least $0.2=$ small, $0.5=$ =medium, and $0.8=$ large.

\section{Results}

\section{Study characteristics}

A total of 66 reports of 51 studies were eligible for inclusion. Of these, six studies were excluded from meta-analyses because they did not provide sufficient data to compute effect sizes. Key characteristics are summarised in Table 1 and characteristics of each study are available in Appendix C (Tables C1 and C2). Most studies were classed as selective prevention, based on a variety of risk factors including pre-term infants, children of a parent with a mental disorder, children at risk of maltreatment, and children of divorced parents. Interventions were usually conducted with parents of children from birth to primary school age, with very few studies targeting parents of adolescents. The majority of studies ( $k=34)$ had multiple foci of intervention. A dual focus on improving both parenting skills and the parent-child relationship was the most common combination $(k=18)$. Interventions were delivered in a variety of formats, including parent groups, individual sessions, and home visits, and in most studies the child had no direct involvement in the intervention. When reported, the scheduled intervention dose varied greatly from 1 to 114 hours, but about half lasted 10 hours or less. Most were delivered in 3 months or less, but a few lasted more than one year, with one intervention lasting 4 years on average (Fergusson, Boden, \& Horwood, 2013). The level of adherence to the scheduled intervention length (i.e., number of sessions delivered) was poorly reported and is available in Appendix Table C2. Most parenting interventions were compared to a 
'usual care' or no treatment condition, with few studies using an attention control condition. About a third of studies followed up participants more than once, with the longest follow-up occurring 15 years after the end of the intervention. However, half followed participants for 12 months or less after the end of the intervention.

\section{Risk of bias in included studies (see Appendix C3)}

\section{Selection bias}

Of the 51 included studies reports, the majority $(k=33)$ described an adequate method to generate the allocation sequence (e.g., computer-generated random number sequence), and were judged as low risk of bias on the random sequence generation criterion. One study was judged as high-risk (Dadds \& Roth, 2008), and the remaining did not report sufficient detail to be confident that the allocation sequence was genuinely randomized.

In many studies ( $k=28)$, it was unclear what techniques (if any) had been used to conceal the allocation sequence from participants and those involved in the enrolment and assignment of participants. Two studies were judged to be at high risk of bias, and 20 studies were judged to have a low risk of bias from allocation concealment.

\section{Detection bias}

The risk of bias associated with blinding of outcome assessment varied within studies depending on the outcome measure used. Measures that were completed by parents were usually judged to be at high risk of bias, as parents were usually aware of which condition they had been allocated to. These were typically more common than instruments completed by others, such as clinicians or teachers, which were more likely to be judged as low risk of bias. Child self-report measures were assumed to be low risk unless the child was directly involved in the intervention, in which case they were judged to be at high risk of bias. 
There were 57 reports (from 44 studies) that used internalizing measures, and only nine of these included a measure rated low-risk of detection bias due to adequate blinding of outcome assessment. Five reports measured depression diagnoses, and two of these reported adequate blinding of outcome assessors, with the remaining rated high risk of bias $(k=2)$ or unclear $(k=1)$. Eleven reports assessed depression symptoms, and six of these relied on an informant whowas adequately blinded and at low risk of bias. For anxiety diagnoses, 8 of 11 reports were judged as low risk of bias due to adequate blinding of outcome assessment. Sixteen studies measured anxiety symptoms, with seven of these judged as low risk of bias due to adequate blinding of assessment.

\section{Attrition bias}

Given the lengthy follow-up periods involved in included studies, it is unsurprising that many reports $(k=34)$ were judged to be at high risk of bias from incomplete outcome data. Most of thesehad dropout rates greater than $15 \%$ and analysed data from complete cases only. Thirty reports were judged to have a low risk of bias due to low levels of incomplete outcome data and/or the use of adequate statistical methods to account for missing data (e.g. multiple imputation, maximumlikelihood based methods; Salim \& et al., 2008), and two reports did not provide enough information from which to make a validjudgement.

\section{Reporting bias}

The risk of bias from selective reporting of outcome measures was unclear in 44 reports (33 studies) due to the unavailability of a study protocol. Seven reports were judged as low risk of bias due to the reporting of all relevant outcomes included in their study protocol, and 15 reports were judged as high-risk. High-risk selective reporting was typically because outcomes were reported incompletely and couldn't be used in the meta-analyses (e.g. reporting 'no significant differences' and providing no data), or results from a key outcome of interest were not reported.

Overall, for the meta-analysis involving measures of internalizing symptoms, there were only 2 out of 35 studies that did not have a potential high risk of bias in any category. This was mainly due to a 
high risk of detection bias from the use of parent self-report outcome measures $(k=31)$ or incomplete outcome data $(\mathrm{k}=16)$. Similarly, most studies in the meta-analyses involving measures of anxiety symptoms (10 out of 13) and depression symptoms (6 out of 9) were at high risk of bias from parents completing outcome assessments and/or incomplete outcome data. For the metaanalysis involving measures of anxiety diagnoses, 3 of 5 studies were at high risk of bias, mainly due to incomplete outcome data, and 2 of the 3 studies in the meta-analysis involving measures of depression diagnoses were judged to have a high risk of bias in at least one category.

\section{Overallstandardized difference in meansand riskdifference}

\section{Measures of internalizing symptoms}

As shown in Table 2 and Figure 2, the mean Cohen's $d$ of the 37 comparisons was very small but significant $(d=-0.123)$, with low-to-moderate heterogeneity. The funnel plot showed marginally significant asymmetry in the studies (Egger's test, two-tailed $p=0.08$ ). The Duval \& Tweedie trim-andfill approach suggested that seven studies were potentially missing and, if imputed, the mean effect size would drop to $d=-0.065$, and would no longer be significant $(95 \% \mathrm{Cl}:-0.125,0.011)$.

Supplementary analyses revealed a significant mean effect size only from studies reporting effects at 6-10 months $(\mathrm{k}=16 ; d=-0.148)$ and at $>24-48$ months $(\mathrm{k}=6 ; d=-0.124)$ post-intervention. The mean effect sizes for all intervals were very small, especially at 1-2 years post-intervention $(d=$ -0.050). Regarding informants, supplementary analyses revealed significant mean effects for the mother $(\mathrm{k}=17 ; d=-0.139)$ and parent $(\mathrm{k}=13 ; d=-0.170)$ informant groups, which were also the most common informants across studies.

Subgroup analyses revealed that the mean Cohen's $d$ differed according to the type of prevention ( $p$ $=0.033 ;$ see Table 2 ). However, only two subgroups (universal and selective) could be included in this analysis, because the other subgroups had $<3$ comparisons each. Selective prevention programs $(k=26 ; d=-0.134)$ yielded significant effects, whereas universal programs $(k=7 ; d=0.110)$ did not. 
Findings from the remaining subgroup analyses, involving the focus and timing of intervention, type of comparison, and whether there was direct intervention with the child, were all non-significant.

We also conducted a meta-regression analysis to see whether there was any decay of effect over time, using the effect size from the longest follow-up interval reported in each study. There was no significant association between Cohen's $d$ and follow-up duration (the point estimate of the slope was $0.0008 ; 95 \% \mathrm{Cl}:-0.002$ to $0.003 ; p=0.494 ;$ median $=14 ;$ range $=6-180$ months).

\section{Measures of depressive symptoms and diagnoses}

As shown in Table 3 and Figure 3, the mean Cohen's $d$ of the 10 comparisons reporting depressive symptoms was very small but significant $(d=-0.156)$, with low heterogeneity and noapparent publication bias (Egger's test, two-tailed $p=0.55$ ). Supplementary analyses revealed a significant mean effect size from studies relying on child ( $\mathrm{k}=8$ comparisons; $d=-0.169)$ or parent $(\mathrm{k}=3 ; d=-$ 0.459) informants. Mean effects for each follow-up interval were all non-significant.

Subgroup analyses were not conducted for the type of prevention or comparison, whether parenting skills or parent as coach was an intervention focus, and whether the child received direct intervention, because only one subgroup in each case had $>2$ comparisons. No subgroup differences were found for whether parent-child relationship or parental mental health was an intervention focus, or for timing of intervention (see Table 3).

We also conducted a meta-regression analysis to see whether there was any decay of effect over time, using the effect size from the longest follow-up interval reported in each study. We found no significant association between Cohen's $d$ and follow-up duration (the point estimate of the slope was $<-0.0001 ; 95 \% \mathrm{Cl}:-0.003$ to $0.003 ; p=0.998 ;$ median $=21 ;$ range $=6-129$ months $)$. There were four comparisons reporting depression diagnoses (see Table 3 and Figure 4), and these yielded a pooled risk difference of -0.095 which approached significance $(p=0.071)$, with moderate heterogeneity but no apparent publication bias (Egger's test, two-tailed $p=0.85$ ). 


\section{Measures of anxiety symptoms and diagnoses}

As shown in Table 4 and Figure 5, the 14 comparisons reporting anxiety symptoms yielded a significant small effect $(d=-0.273)$, but with high heterogeneity. There was no apparent publication bias, Egger's test two-tailed $p=0.89$. Supplementary analyses revealed a significant mean effect size from studies relying on child $(k=9 ; d=-0.192)$, parent $(k=5 ; d=-0.535)$, or the combination of parent and child informants $(k=1 ; d=-1.831)$. Mean effects for each follow-up interval were all nonsignificant, except for studies reporting outcomes at $>10-12$ month post-intervention $(k=5 ; d=$ $-0.851)$.

Subgroup analyses revealed that the mean Cohen's $d$ differed according to whether the intervention included a focus on parental mental health ( $p=0.031$; see Table 4). Specifically, the 8 comparisons that did not include this focus yielded an almost-medium effect size $(d=-0.458)$; in contrast to the 6 comparisons that did $(d=-0.047)$. A subgroup analysis for type of comparison condition was not run because only one subgroup had $>2$ comparison conditions, but RCTs that involved a no-treatment $(k$ $=7 ; d=-0.381)$ or extended waitlist $(k=1 ; d=-1.831)$ yielded significant mean effect sizes, in contrast to RCTs that involved active control interventions. Subgroup analyses were also not run for type of prevention and whether parenting skills was an intervention focus, because only one subgroup in each case had $>2$ comparisons. Findings from the remaining subgroup analyses involving the focus or timing of intervention, and whether there was direct intervention with the child, were all non-significant.

We also conducted a meta-regression analysis to see whether there was any decay of effect over time, using the effect size from the longest follow-up interval reported in each study. We found no significant association between Cohen's $d$ and follow-up duration ( $p=0.842$; the point estimate of the slope was $0.0005 ; 95 \% \mathrm{Cl}:-0.005$ to $0.006 ;$ median $=12 ;$ range $=6-129$ months). 
There were six comparisons reporting anxiety diagnoses (see Table 4 and Figure 6), and these yielded a significant pooled risk difference of $-0.109(p=0.020)$. There was moderate-to-high heterogeneity but no apparent publication bias (Egger's test, two-tailed $p=0.22$ ).

\section{Internalizing cluster}

As shown in Table 5 and Figure 6 , the 45 comparisons yielded a very small but significant effect $(d=-$ 0.177) on internalizing problems (based on data from internalizing, depressive or anxiety symptom measures), with moderate-to-high heterogeneity. There was no apparent publication bias, Egger's test two-tailed $p=0.38$.

Supplementary analyses revealed a significant mean effect size from studies reporting effects at 6-10 months $(\mathrm{k}=20 ; d=-0.151)$, between $10-12$ months $(\mathrm{k}=14 ; d=-0.288)$, between $24-48$ months $(\mathrm{k}=$ 7; $d=-0.138)$, and $>48$ months $(\mathrm{k}=9 ; d=-0.138)$ post-intervention. The mean effect sizes for all intervals were very small, especially at $1-2$ years post-intervention $(d=-0.078)$. Regarding informants, supplementary analyses revealed significant mean effects for the mother $(\mathrm{k}=17 ; d=-$ $0.139)$ and parent $(\mathrm{k}=13 ; d=-0.170)$ informant groups, which were also the most common informants across studies; as well as the child informant group ( $\mathrm{k}=9 ; d=-0.175)$.

Subgroup analyses revealed that the mean Cohen's $d$ differed according to the type of prevention ( $p$ $=0.011 ;$ see Table 5). However, only 3 subgroups (universal, selective, and secondary prevention) could be included in this analysis, because the other subgroups had $<3$ comparisons each. Selective $(k=30 ; d=-0.186)$ and secondary $(k=3 ; d=-0.770)$ prevention programs yielded significant effects, whereas universal programs $(k=9 ; d=0.006)$ did not. Findings from the remaining subgroup analyses, involving the focus and timing of intervention, type of comparison, and whether there was direct intervention with the child, were all non-significant.

We also conducted a meta-regression analysis to see whether there was any decay of effect over time, using the effect size from the longest follow-up interval reported in each study. There was no 
significant association between Cohen's $d$ and follow-up duration (the point estimate of the slope was $0.0005 ; 95 \% \mathrm{Cl}:-0.002$ to $0.003 ; p=0.679 ;$ median $=14 ;$ range $=6-180$ months).

\section{Discussion}

\section{Main findings}

We examined whether parenting interventions directed primarily at parents are effective in preventing child internalizing problems in the longer-term. Results showed that compared to controls, parenting interventions reduced child internalizing, depressive, and anxiety symptoms, at a minimum of six months after the intervention was delivered. The mean effects were very small for internalizing and depressive symptoms, and small for anxiety symptoms. The mean effect for the internalizing cluster was also very small. The sizes of these mean effects are similar to those reported in recent reviews of effects of family-based interventions on child internalizing outcomes (Barlow et al., 2010; Siegenthaler et al., 2012). Few studies were judged to have a low risk of bias overall, and this must qualify the review's findings. Furthermore, there may be publication bias amongst studies using internalizing symptom measures, hence those findings may not be as robust and need to be interpreted with caution.

Despite the small effect sizes, the effects on anxiety diagnoses translate into a number needed to treat (NNT) of 10, which means that relative to children whose parents do not receive a preventive parenting intervention, for every 10 children whose parents do receive it, one additional child could be free of an anxiety diagnosis. The effects on depression diagnoses fell just short of statistical significance but are nonetheless promising, because they translate into an NNT of less than 11 , which is similar to the average NNT for the prevention of cases of depression using programs targeting young people directly (NNT=11; Merry et al., 2011). Although there is no formally recognized system of classification for the size of NNT, the NNTs for preventive parenting programs compare very favorably with programs directed towards individuals which are delivered later in life 
(NNT=20; van Zoonen et al., 2014). Moreover, drawing from the broader health literature, a recent review of low-dose aspirin reported a NNT=284 to prevent 1 major cardiovascular event, anda NNT=614 to prevent 1 ischaemic stroke, over a mean follow-up of 6.8 years (Xie et al., 2014). Similarly, a Cochrane review of the benefits of statins the primary prevention of cardiovascular disease reported NNT values of 167 and 67 for individuals at $<5 \%$ five-year risk and 5-10\% five-year risk, respectively (Taylor et al., 2013). Another review of antihypertensive therapy forindividuals with pre-hypertensive blood pressure levels reported a NNT=169 over an average of 4.3 years to prevent one stroke (Sipahi et al., 2012). The conclusion drawn in the respective reviews was that aspirin, statins, and antihypertensive therapy were of benefit. Furthermore, it is a known phenomenon that a small change in mean scores across a population can have a beneficial effect for those in a tail of a population distribution - hence the NNTs found in our review are promising despite the small population effect sizes. Given the burden of internalizing problems especially in terms of their early onset and long-term negative sequelae, we argue that preventive parenting programs would still be a worthwhile investment in public health terms.

Findings in this review were based on effects reported at the longest follow-up interval for each included study (from a minimum of 6 months post-intervention), and significant effects were maintained up to 3 years for internalizing symptoms (Spencer-Smith et al., 2012), 5.5 years for depressive symptoms (Mason et al., 2007), and 11 years for anxiety symptoms and diagnoses (Rapee, 2013). Although the intensity and duration of interventions varied widely, it is remarkable that preventive interventions directed primarily at parents can have such long-term benefits for children.

\section{Findings with implications for future prevention policy and practice}

\section{Duration of preventive effects}

One of the key questions in this review is: for how long do the preventive effects of parenting interventions last, and do effects vary with the duration of the follow-up interval? We did not find 
the duration of follow-up interval to be associated with mean effects on internalizing, depressive or anxiety symptoms, when examined separately and as an internalizing cluster (i.e. any of the 3 measures). When we examined the mean effects of parenting interventions at different follow-up intervals (6-10 months, $>10-12$ months, $>12-24$ months, $>24-48$ months, and $>48$ months), we found very small but significant mean effects on internalizing symptoms at 6-10 months and at $>24-48$ months, and a large mean effect on anxiety symptoms only at $>10-12$ months. The mean effects on depressive symptoms were non-significant for each follow-up interval, although there werefew studies with data at each interval. Mean effects on any internalizing measure were significant for all time intervals except between 12-24 months, and were all very small except between 10-12 months, where the mean effect was small. Overall, the current findings suggest that the small but persistent effects that preventive parenting interventions have on internalizing problems are notassociated with the time that has lapsed after the intervention. Based on developmental theories, the longevity of intervention effects may be due to the bidirectional and interactive effects of parenting and child behaviours on child outcomes over time (Branje, Hale, Frijns, \& Meeus, 2010; Lengua \& Kovacs, 2005; Restifo \& Bögels, 2009; Yap et al., 2007). For instance, it is possible that parenting interventions may improve parenting skills (e.g. reducing overprotection), which may in turn increase child exploration, and in turn increase parental encouragement of child independence. Over time, these interactive, bidirectional processes may build on one another to maintain positive intervention effects in decreasing the child's risk for internalizing problems, for years after the intervention had ended.

\section{Timing of intervention}

An important question for implementation of preventive parenting interventions is when in the child's development should interventions be delivered. Subgroup analyses revealed no significant moderation of intervention effects by the timing of delivery of preventive parenting interventions, on any of the three sets of measures, or on the internalizing cluster. Nonetheless, some patterns of findings are noteworthy. Firstly, children seem to benefit if their parents received parenting 
interventions during the primary-school years, with a small mean effect based on internalizing measures and for the internalizing cluster, and a medium mean effect based on measures of anxiety symptoms. Secondly, programs delivered to parents very early in their child's life, including the antenatal period, may yield long-term (albeit small) preventive effects on measures of child internalizing symptoms, and on internalizing problems as a cluster. Thirdly, timing of intervention did not modify the effects of parenting interventions on depressive symptoms, possibly due to inadequate power to detect group differences. Nonetheless it is notable that the two studies where the parenting intervention was delivered during adolescence yielded a significant mean effect (albeit very small), whereas the other studies delivered in preschool and primary-school phases did not. It is possible that the effects of parenting programs on child depression may not be apparent until later in adolescence, given that the rates of depression increase more rapidly during mid-adolescence (Kessler et al., 2007).

Overall, very few parenting interventions were delivered during adolescence. This is unsurprising given that most interventions for youth mental health are directed at young people themselves, with parents either not receiving any direct intervention or receiving only a brief psychoeducation supplement focusing on informing parents about what their child is receiving, and/or how parents can support their child's intervention (Brunwasser \& Garber, 2015; Hetrick, Cox, \& Merry, 2015). However, this approach seems amiss given the sound evidence that some modifiable parental factors are associated with adolescent depression and anxiety (Yap et al., 2014). Such evidence highlights the potential of parenting interventions that target such modifiable parental factors for reducing adolescent risk for depression and anxiety. Taken together, findings to date underscore an important gap in preventive parenting interventions targeted at parents of adolescents, as a complement to adolescent-directed interventions, some of which have shown promising benefits for adolescent depression (Merry et al., 2011). 


\section{Target population}

When implementing preventive interventions, a key question is who should receive the intervention. We sought to examine whether the mean effects of parenting interventions varied depending on the target population or the type of prevention the interventions were designed for: universal, selective, indicated, or secondary/treatment. Unfortunately, due to the small number of studies which used measures of depressive and anxiety symptoms and diagnoses, a subgroup analysis could only be conducted for internalizing symptoms and the internalizing cluster. However, in the former analysis, only two groups could be compared (universal versus selective), and a significant group difference was found, favoring selective (very small but significant mean effect) over universal (non-significant mean effect) programs. Nonetheless, a large effect was found for the single indicated prevention program and a medium mean effect for two secondary prevention programs. Findings for the internalizing cluster were similar: a significant difference emerged in the subgroup analysis involving 3 subgroups: universal, selective, and secondary. Mean effects were significant for selective and secondary prevention but not universal prevention; however an examination of confidence intervals suggests that the difference is significant only between secondary prevention and universal prevention programs. Although subgroup analyses were not conducted for measures of depressive or anxiety symptoms, it is interesting to note that for both outcomes, universal programs yielded significant mean effects, albeit in the very small to small range; whereas all other targeted programs yielded non-significant mean effects.

In sum, evidence to-date is inconclusive about whether the type of prevention modifies the longterm effects of parenting interventions. However, consistent with findings from other preventive programs (e.g. Merry et al., 2011), they suggest the promise of selective and secondary prevention parenting programs for internalizing problems, at least relative to universal programs. This is unsurprising given that universal programs are subject to 'floor effects', whereby participants have minimal problems at pre-intervention, making it more difficult to demonstrate improvement. Further research is required to address this question more definitively, given the disproportionately 
larger number of RCTs of selective programs, and the exploratory and observational nature of the subgroup analyses conducted. Nonetheless, it has also been argued that although universal programs may have a smaller effect than selective or indicated programs at the level of the individual, they may have a great public health impact because they reach a larger proportion of the population (Phares et al., 1989; Rose, 1992), and have the potential to shift the population mean (Rose, 1992).

\section{Focus ofintervention}

Parenting interventions have been developed from a wide range of approaches and theoretical frameworks, and hence vary widely in their focus and targets. To inform future development and implementation of parenting interventions, we were interested in whether the focus of interventions modified their effects. Most of the interventions identified in this review included a focus on improving parenting skills and/or the parent-child relationship, and most included one or two foci. Subgroup analyses revealed no significant differences between interventions that included each of the four foci and interventions that did not, for all three measures of child internalizing problems. The only exception was for parental mental health, whereby interventions that did not include a focus on parents' own mental health had an almost-medium mean effect on child anxiety symptoms, whereas interventions that did include that focus yielded a non-significant mean effect. This concurs with evidence that parenting behaviors (e.g. parental over-involvement orovercontrolling behaviors) may have a stronger association with child anxiety than with parent anxiety (Van Der Bruggen, Stams, \& Bögels, 2008), as well as findings from a recent Cochrane review that group parenting programs improved parental depression and anxiety only at post-intervention, but had no lasting effects on parental mental health (Barlow, Smailagic, Huband, Roloff, \& Bennett, 2014). These findings suggest that parental mental health may not be a fruitful target for parenting interventions to prevent child anxiety. However, other possible explanations for thesefindings should be considered. Firstly, all 6 studies which included a focus on parental mental health also included one (in 3 studies) or two (in 3 other studies) other intervention foci. It is possible that a 
focus on parental mental health was included at the expense of other foci, or simply reduced the amount of intervention time that could be focused on modifying other risk or protective factors for child anxiety, resulting in the dilution of effects from each component. Secondly, it could be argued that programs which included a focus on parental mental health may have targeted parents with mental health problems or who may be at elevated risk for such difficulties, and this may have limited the degree of change in parenting and hence in child benefits. Indeed, of the 6 studies that included a focus on parental mental health, 1 targeted parental risk factors, 2 targeted parents with psychopathology (mood disorder or alcohol abuse), 2 targeted parents with terminal illness (HIV/AIDS or breast cancer), and 1 targeted young mothers from an ethnic minority group. However, Sieganthaler and colleagues' (2012) review found that parenting programs for parents with mental illness yielded promising effects on child internalizing problems, suggesting that parental mental illness does not hinder the effectiveness of parenting programs. An important caveat to note is that this review did not identify any program that focused solely on parental mental health; hence it remains possible that such programs may have preventive effects on child anxiety. It is also important to note that the main meta-analyses examining effects on measures of internalizing symptoms and the internalizing cluster yielded significant mean effects in programs that did include a focus on parental mental health. More research is clearly required before any meaningful conclusions can be drawn.

All other subgroup analyses for all three sets of measures and the internalizing cluster revealed no significant differences between interventions that included a particular focus and those that did not. There was high between-trial heterogeneity in many subgroups, suggesting that interventions categorized as sharing a similar focus differed in meaningful ways that may have influenced their effects on child internalizing problems. 


\section{Direct intervention with child}

This review only included interventions where parents received the majority of the intervention, were the primary targets of intervention, and were assumed to be key agents of change in child outcomes. However, some interventions included direct intervention with the child either in family sessions or individually with the interventionist. Of interest, we found that including a component of direct intervention with the child in such programs did not yield significantly different mean effects on measures of internalizing and anxiety symptoms, or the internalizing cluster. A subgroup analysis could not be conducted for measures of depressive symptoms, but the pattern of findings was similar to that for internalizing and anxiety symptoms: the 8 comparisons involving no direct child intervention yielded a significant small mean effect, whereas the other 2 did not. Taken together, our findings suggest that in parenting interventions primarily directed at the parents, adding a child intervention component may not yield significant differences in benefits for child internalizing problems. Although some parents may prefer to involve their child in an intervention, and it may be logical to expect that intervening directly with the child in addition to the parent would produce synergistic effects, the current findings suggest that this may not be necessary. This is promising in terms of intervention delivery, because it is likely to be more efficient and easier logistically to deliver the intervention to parents only, especially on a larger scale. It also underscores the potential of interventions directed primarily at parents to indirectly benefit the child, presumably through modifying parental behaviors, even into the longer term.

It is important to note however, that substantive evidence has already demonstrated that direct intervention with young people can prevent depression (Merry et al., 2011) and anxiety (Fisak et al., 2011; Teubert \& Pinquart, 2011); hence our findings should not be interpreted as suggesting that child intervention is ineffective. Indeed, the subgroup analysis for the internalizing cluster yielded significant mean effects regardless of whether the program included direct intervention with the child (i.e. mean effects were significant in both subgroups). Instead, it is likely that the child intervention component of some of the parenting programs included in our review may have been 
too small to have yielded significant additional benefits. Nonetheless, our findings are consistent with those reported in a review of the effects of preventive interventions in mentally-ill parents on children's internalizing symptoms, which included some interventions which were directed solely at adolescents, some which involved both parents and children (equally), and some-like the current review - which were directly solely or primarily at parents (Siegenthaler et al., 2012). On the other hand, there is also evidence suggesting that in intervention programs primarily directed at young people, adding a parent intervention component may not yield significant additive benefits for child depression (David-Ferdon \& Kaslow, 2008) or anxiety (Hudson et al., 2015). Whilst it remains to be ascertained why dual-component programs do not seem to yield robust synergistic benefits, what is clear is that each type of program - those directed at young people, and those directed at parentshas preventive effects on child internalizing problems.

\section{Training and qualifications of interventionists}

Although not central to the focus of this review, it is noteworthy that of the 51 included studies, the vast majority involved intervention programs that were delivered by trained specialist professionals (e.g. clinical psychologists; $k=30$ ) or non-specialist professionals or paraprofessionals (e.g. trained nurses or research assistants; $k=15$ ). This has important implications for the cost and feasibility of disseminating such interventions in the larger scale, and needs to be taken into account when assessing the cost-effectiveness of the programs. Unfortunately, the training, experience and qualification of interventionists are not easy to quantify objectively and consistently, and is inadequately described in manystudies.

\section{Implications for future research}

\section{Informant}

Given existing evidence of modest cross-informant agreements (Phares et al., 1989; Stanger \& Lewis, 1993), we examined the mean effects of parenting interventions for each group of informants of child outcomes. For internalizing measures, significant mean effects were found only for studies that 
relied on mother or parent informants; mean effects were non-significant for all other informant groups. Despite increasing interest in the effects of fathering on child mental health and discrepancies between father- and mother-reports (e.g. Bögels, Bamelis, \& van der Bruggen, 2008; Teubert \& Pinquart, 2011), there were only three studies that included both father and mother as independent reporters of their child's symptoms, all utilizing measures of internalizing symptoms, and these yielded a non-significant mean effect.

For depressive and anxiety measures, significant small mean effects were found for child informants and medium effects for parent informants; but mean effects were non-significant for other informant groups. For the internalizing cluster, mean effects were significant for 3 groups of informants: child, mother, and parent. Notably, when evaluating the effects of parenting interventions directed primarily at parents, it is likely that child informants would be less subject to outcome assessment bias than parent informants, given that children are not direct recipients of the intervention in most cases. Moreover, as argued in a recent review (Yap \& Jorm, 2015), widely-used child self-report depressive and anxiety symptom measures have been validated with childrenas young as 7 (e.g. the Children's Depression Inventory; Craighead, Smucker, Craighead, \& Ilardi, 1998; Saylor, Finch, Spirito, \& Bennett, 1984; Smucker, Craighead, Craighead, \& Green, 1986; Timbremont, Braet, \& Dreessen, 2004) or 8 (e.g. the Spence Children's Anxiety Scale; Spence, 1998;Spence, Barrett, \& Turner, 2003) years. Furthermore, given that children are privy to their own internal experiences, and internalizing problems are less observable to independent observers (De Los Reyes \& Kazdin, 2005), evidence to date suggests that when it is age appropriate, child informants should be relied upon as the primary source of child outcomes in trials of parenting interventions.

\section{Type of comparisoncondition}

Although our main meta-analyses included all eligible studies regardless of the comparison or control conditions employed in the trials, we expected mean effects to vary depending on the comparison condition. However, due to small numbers of comparisons in most subgroups, a 
subgroup analysis could only be conducted for measures of internalizing symptoms, comparing four subgroups (excluding extended waitlist comparisons), and this revealed no significant group differences. A subgroup analysis was also conducted for the internalizing cluster, which included all 5 subgroups, but this also yielded no significant group differences. Nonetheless, only studies that compared their interventions to usual-care (the most common comparison condition) yieldeda significant mean effect on internalizing measures. For the internalizing cluster, significantmean effects emerged for interventions that were compared to either no-treatment or usual-care comparisons. It is noteworthy that the usual-care comparison employed in many of these studies involved standard medical care, case management or maternal and child health nursing support. Although some of these included referrals for psychotherapeutic or counselling support for the parent if required, most of them did not provide any active intervention specifically aimed at reducing risk for child internalizing problems. Hence these findings suggest that parenting interventions may yield significant benefits for child internalizing problems compared to usual care. However, further research comparing these parenting interventions to active comparisons (especially alternative parenting interventions) is required in order to most strongly demonstrate the effectiveness of these interventions (Stathakos \& Roehrle, 2003).

For measures of depressive and anxiety symptoms, we found that studies employing non-active (i.e. no treatment, extended waitlist) or less active (e.g. minimal intervention) comparisons yielded significant mean effects, whereas studies employing more active comparisons (i.e. attention-control and usual-care) did not. This is consistent with findings from Merry and colleagues' (2011) review. These are important caveats to note when considering the effectiveness of parenting interventions to date on child anxiety and depressive symptoms, and underscore the importance of future trials employing more active comparison conditions, in order to establish whether these parenting interventions offer preventive benefits for the child beyond simply involving parents in a parenting intervention (Stathakos \& Roehrle, 2003). Nonetheless, the persistence of intervention effects makes it less likely that the effects are merely the results of attention. 


\section{Symptom versus diagnostic outcomes}

Although it would have been preferable to evaluate the long-term effects of parenting interventions on reducing the incidence of child depressive and anxiety disorders, this review identified only 3 studies reporting depressive diagnoses and 5 reporting anxiety diagnoses, which could be included in meta-analyses. Whilst the evidence on diagnostic outcomes are promising and are consistent with evidence on symptom-based measures, the small samples providing diagnostic data precludeany firm conclusions to be drawn. More studies are required that include a diagnostic measure of depression and anxiety outcomes, especially at longer-term follow-up during the second decade of life, when the incidence of case-level disorders starts to increase (Kessler et al., 2012).

\section{Differences in effects on measures of internalizing, depression and anxiety outcomes}

Findings from this review revealed that the effects of parenting interventions may be weaker for measures of internalizing and depressive symptoms and depressive diagnoses, compared to anxiety symptoms and diagnoses. We discuss briefly a few possible reasons for this pattern of findings. Firstly, although we did not find significant moderation of intervention effects by the timing of intervention, we did find that most of the interventions which examined internalizing symptoms as outcomes (68\%) were delivered earlier in life (before the schooling years) whereas most of the interventions which used measures of depression (70\%) and anxiety (71\%) were delivered in primary and high school years. Moreover, most studies using measures of internalizing symptoms only had follow-up assessments up to two years post intervention; which means that in many cases the children may still be pre-school aged when rates of internalizing problems are low. Hence, even when group differences in intervention effects on internalizing symptoms are found, the size of those effects are likely to be very small. It is also possible that the high comorbidity between anxiety and depression symptoms earlier in life (commonly measured as 'internalizing symptoms') reduces over the course of development as the symptoms start to differentiate more clearly into anxietyspecific or depression-specific symptoms. This may also account for the smaller sizes of mean effects on internalizing symptoms. 
Intervention effects on depression outcomes may be less marked than those on anxiety outcomes because the rates of depression increase more rapidly during mid-adolescence (Kessler et al., 2007), but only $22 \%$ of the studies which examined depressive symptoms involved parents of adolescents; and only 33\% included follow-up assessments longer than 2 years post intervention, which means that depression outcomes were assessed prior to mid-adolescence for most studies.

Another possible reason for the apparent difference in effect sizes across the three outcomes is the relevance of parental risk and protective factors targeted in parenting interventions to each outcome. For example, interventions delivered earlier in the child's life tend to have a broader aim of improving general adaptation and functioning, and included internalizing as one of multiple indicators. Hence the parental factors targeted may also be more general, or may be more relevant in infancy (e.g. parental sensitivity to infant cues) but fail to be adapted for older children with more complex social interaction demands. In contrast, interventions specifically developed to prevent child anxiety may more intentionally target evidence-based parental factors associated with children's risk for anxiety (e.g. over-protection; Yap \& Jorm, 2015; Yap et al., 2014). Ironically, recent reviews found that the evidence base for parental factors associated with child (Yap \& Jorm, 2015) and adolescent (Yap et al., 2014) anxiety is more limited than the evidence base for depression and internalizing problems more generally. Nonetheless, it is possible that interventionsspecifically designed to prevent child anxiety are more strongly driven by the albeit-limited evidence base, relative to other programs which examined depression and internalizing problems. It is also possible that programs which target more than one evidence-based parental factor may have larger effects (Yap \& Jorm, 2015; Yap et al., 2014). For example, Ginsburg and colleagues' (2009) Coping and Promoting Strength program (for parents of primary-school aged children) targets over-protection, aversiveness and inter-parental conflict, all of which have sound evidence for increasing riskfor anxiety in adolescents (Yap et al., 2014) and emerging evidence for anxiety in children (Yap \& Jorm, 2015). 


\section{Strengths and limitations}

To our knowledge, this is one of the most comprehensive systematic reviews and meta-analyses of the long-term effects of parenting interventions to prevent child internalizing problems to date, including studies reporting significant intervention effects extending up to 11 years postintervention. Unlike the Cochrane review of group parenting programs which focused on interventions delivered in the first 3 years of the child's life (Barlow et al., 2010), we placedno restrictions on the intervention mode of delivery, and included a wider child age range. Employing a whole-of-childhood focus allowed us to examine whether there are periods of development when parenting interventions may be best targeted. The growing number of randomized controlled trials (RCTs) examining parenting interventions enabled us to include only RCTs in this review, permitting meta-analyses of more rigorously-designed and higher-quality studies. By focusing only on parenting interventions that were directed primarily at parents (i.e. where parents received majority of the direct intervention), we were able to examine the question of whether directly intervening with parents (without any substantial direct intervention with the child) is sufficient to reduce risk for child internalizing outcomes. Unlike Siegenthaler and colleagues' review (2012) which focused on interventions involving mentally-ill parents of children without established mental disorders at the time of randomization into the RCT (selective or indicated prevention), our review had a broader scope in capturing universal, selective and indicated prevention, as well as secondary prevention of internalizing outcomes. We also conducted a series of supplementary and subgroup analyses to examine potential moderators of the effects found.

However, a few limitations to our findings should be noted. Firstly, it was beyond the scope of the current review to examine mediators of the intervention effects found. As cogently argued by Sandler and colleagues (Sandler et al., 2011; Sandler et al., 2014), mediation analyses are required to identify the processes by which interventions have their effects, and the key components that must be preserved when interventions are disseminated (Teubert \& Pinquart, 2011). Like many other 
meta-analyses to date (Sandler et al., 2014), we could not adequately assess the quality of all included trials because of poor reporting, especially of allocation concealment and selective reporting of outcome measures. Overall, there were few studies judged to be at low risk of bias across the domains that were assessed, and therefore results should be interpreted cautiously. We also could not assess for moderation by intensity or duration of intervention due to inadequate descriptions of this aspect of intervention programs in about one-third of included trials. Notably, programs varied widely from a few group sessions scheduled within 1-3 months, to multiple weekly home visits tapering off into less-frequent but ongoing interventions over a few years. The number of sessions actually delivered was even more poorly reported. We believe that it is at least partly due to such variability in program design, delivery and reporting that past meta-analyses have not found consistent associations between intensity of programs and effect sizes (Sandler et al., 2014; van Zoonen et al., 2014). We attempted to estimate intervention intensity by multiplying the number of intervention sessions by the duration of each session, because sessions varied significantly between programs (e.g. 30 minutes to 3 hours), but one or both variables were inadequately reported in some trials. Clearly, such information is crucial for determining the costs and cost-effectiveness of preventive programs, and improved reporting is required in future studies. There also remains a need to clarify the dimensions along which implementation should be measured as predictors of outcome; e.g. dosage, fidelity, and quality of implementation (including training and supervision of those administering interventions). Moreover, while $44 \%$ of included studies involved group sessions, many of these made the assumption that individual members of the group programs could be regarded as independent observations, without adequately controlling for or considering potential clustering effects. Furthermore, even in interventions involving individual sessions with an interventionist, many studies involved only one or very few interventionists, and fail to consider the potential interventionist clustering effects. Hence it is possible that clustering effects may have overestimated the precision of the effect size estimates (by affecting the confidence intervals). This review examined a large number of potential moderators of intervention effects relative to the small 
number of studies included, especially those examining depressive and anxiety outcomes; hence findings from the supplementary analyses should be regarded as primarily observational and hypothesis generating in nature. This review also could not draw firm conclusions about the longterm effects of parenting interventions in preventing incidence of anxiety or depression disorders, because too few studies assessed diagnostic outcomes. Finally, most included trials were conducted in English-speaking developed countries; hence our findings may not generalize to ethnic minorities or developing countries.

\section{Summary and conclusions}

This review of parenting interventions found evidence for very small to small mean change based on measures of internalizing, depressive, and anxiety symptoms but potentially significant long-term effects on clinical presentations of internalizing disorders, with more substantial effects based on measures of anxiety than depression. Although the size of effects found seem small in absolute terms, they are comparable to the effects of other preventive interventions, including those directed at young people themselves (Merry et al., 2011) or later in life (van Zoonen et al., 2014), and could have an important public health impact (Rose, 1992; Sandler et al., 2014). Importantly, the persistence of intervention effects suggests that these are real and not placebo effects. Most noteworthy are the findings involving measures of anxiety symptoms and diagnoses, which compare favourably to interventions targeted directly at young people themselves (Fisak et al., 2011; Teubert \& Pinquart, 2011). Given the central role and involvement that parents have throughout most of young people's development, our findings underscore the likely benefits of increasing parental involvement in preventing and reducing internalizing problems in young people. 


\section{Figure Captions}

Figure $1 \quad$ PRISMA flow chart showing the flow of selection of eligible papers.

Figure 2 The effects of preventive parenting interventions on measures of child internalizing symptoms. Lines represent standardized difference in means (Cohen's $d$ ) and 95\%confidence intervals ( $\mathrm{Cl})$; the size of the box represents the weight of each study. Studies are ordered by the duration of the follow-up interval (shortest to longest).

Figure 3 The effects of preventive parenting interventions on measures of child depressive symptoms. Lines represent standardized difference in means (Cohen's $d$ ) and 95\%confidence intervals (Cl); the size of the box represents the weight of each study. Studies are ordered by the duration of the follow-up interval (shortest to longest).

Figure 4 The effects of preventive parenting interventions on measures of child depression diagnoses. Lines represent risk difference and 95\% confidence intervals ( $\mathrm{Cl})$; the size of the box represents the weight of each study. Studies are ordered by the duration of the follow-up interval (shortest to longest).

Figure 5 The effects of preventive parenting interventions on measures of child anxiety symptoms. Lines represent standardized difference in means (Cohen's $d$ ) and 95\% confidence intervals $(\mathrm{CI})$; the size of the box represents the weight of each study. Studies are ordered by the duration of the follow-up interval (shortest to longest).

Figure 6 The effects of preventive parenting interventions on measures of child anxiety diagnoses. Lines represent risk difference and 95\% confidence intervals ( $\mathrm{Cl}$ ); the size of the box represents the weight of each study. Studies are ordered by the duration of the follow-up interval (shortest to longest).

Figure $7 \quad$ The effects of preventive parenting interventions on the broad cluster of child internalizing problems. Lines represent standardized difference in means (Cohen's $d$ ) and 95\% 
confidence intervals $(\mathrm{Cl})$; the size of the box represents the weight of each study. Studies are ordered by the duration of the follow-up interval (shortest to longest). 


\section{References}

Barlow, J., Smailagic, N., Ferriter, M., Bennett, C., \& Jones, H. (2010). Group-based parent-training programmes for improving emotional and behavioural adjustment in children from birth to three years old. Cochrane Database of Systematic Reviews, (3).

http://www.mrw.interscience.wiley.com/cochrane/clsysrev/articles/CD003680/frame.html doi:10.1002/14651858.CD003680.pub2

Barlow, J., Smailagic, N., Huband, N., Roloff, V., \& Bennett, C. (2014). Group-based parent training programmes for improving parental psychosocial health. Cochrane Database of Systematic Reviews(5). doi: 10.1002/14651858.CD002020.pub4

Beardslee, W. R., Gladstone, T. R. G., Wright, E. J., \& Cooper, A. B. (2003). A Family-Based Approach to the Prevention of Depressive Symptoms in Children at Risk: Evidence of Parental and Child Change. Pediatrics, 112(2), e119-131. doi: 10.1542/peds.112.2.e119

Becker, M. H. (Ed.). (1974). The health belief model and personal health behavior (Vol. 2).

Bell, R. Q. (1968). A reinterpretation of the direction of effects in studies of socialisation. Psychology Review, 75, 81-95.

Bögels, S. M., Bamelis, L., \& van der Bruggen, C. (2008). Parental rearing as a function of parent's own, partner's, and child's anxiety status: Fathers make the difference. Cognition \& Emotion, 22(3), 522-538. doi: 10.1080/02699930801886706

Bögels, S. M., \& Brechman-Toussaint, M. L. (2006). Family issues in child anxiety: Attachment, family functioning, parental rearing and beliefs. Clinical Psychology Review, 26(7), 834-856. doi: 10.1016/j.cpr.2005.08.001

Boomsma, D. I., Van Beijsterveldt, C. E. M., \& Hudziak, J. J. (2005). Genetic and environmental influences on Anxious/Depression during childhood: a study from the Netherlands Twin Register. Genes, Brain and Behavior, 4(8), 466-481. doi: 10.1111/j.1601-183X.2005.00141.x

Borenstein, M., Hedges, L. V., Higgins, J. P. T., \& Rothstein, H. R. (2009). Introduction to metaanalysis. West Sussex, UK: Wiley \& Sons.

Bowlby, J. (1971). Attachment and Loss (Vol. 1). London: Penguin Books.

Branje, S., Hale, W., Frijns, T., \& Meeus, W. (2010). Longitudinal associations between perceived parent-child relationship quality and depressive symptoms in adolescence. Journal of Abnormal Child Psychology, 38(6), 751-763. doi: 10.1007/s10802-010-9401-6

Brunwasser, S. M., \& Garber, J. (2015). Programs for the Prevention of Youth Depression: Evaluation of Efficacy, Effectiveness, and Readiness for Dissemination. Journal of Clinical Child \& Adolescent Psychology, 1-21. doi: 10.1080/15374416.2015.1020541

Cairns, K. E., Yap, M. B., Pilkington, P. D., \& Jorm, A. F. (2014). Risk and protective factors for depression that adolescents can modify: a systematic review and meta-analysis of longitudinal studies. J Affect Disord, 169, 61-75. doi: 10.1016/j.jad.2014.08.006

Cheng, S., Kondo, N., Aoki, Y., Kitamura, Y., Takeda, Y., \& Yamagata, Z. (2007). The effectiveness of early intervention and the factors related to child behavioural problems at age 2:A randomized controlled trial. Early Human Development, 83(10), 683-691. doi: 10.1016/j.earlhumdev.2007.01.008

Cohen, J. (1992). A power primer. Psychological Bulletin, 112, 155-159.

Cohen, J. A., \& Mannarino, A. P. (1998). Factors that mediate treatment outcome of sexually abused preschool children: six- and 12-month follow-up. Journal of the American Academy of Child \& Adolescent Psychiatry, (1), 44-51. http://www.mrw.interscience.wiley.com/cochrane/clcentral/articles/193/CN00367193/frame.html

Compas, B. E. (2009). Randomized controlled trial of a family cognitive-behavioral preventive intervention for children of depressed parents. Journal of Consulting and Clinical Psychology, 77(6), 1007. 
Comprehensive Meta Analysis. (2013). (Version 3) [computer software] (Version 3). Englewood, NJ: Biostat.

Craighead, W. E., Smucker, M. R., Craighead, L. W., \& Ilardi, S. S. (1998). Factor analysis of the Children's Depression Inventory in a community sample. Psychological Assessment, 10(2), 156.

Cummings, E. M., \& Davies, P. T. (2002). Effects of marital conflict on children: recent advances and emerging themes in process-oriented research. Journal of Child Psychology and Psychiatry and Allied Disciplines, 43(1), 31-63.

Dadds, M. R., \& Roth, J. H. (2008). Prevention of anxiety disorders: results of a universal trial with young children. Journal of Child and Family Studies, 17(3), 320-335. doi: 10.1007/s10826007-9144-3

David-Ferdon, C., \& Kaslow, N. J. (2008). Evidence-based psychosocial treatments for child and adolescent depression. J Clin Child Adolesc Psychol, 37(1), 62-104. doi: 10.1080/15374410701817865

De Los Reyes, A., \& Kazdin, A. E. (2005). Informant Discrepancies in the Assessment of Childhood Psychopathology: A Critical Review, Theoretical Framework, and Recommendations for Further Study. Psychological Bulletin, 131(4), 483-509.

Dozois, D. J., Seeds, P. M., \& Collins, K. A. (2009). Transdiagnostic approaches to the prevention of depression and anxiety. J Cogn Psychother, 23(1), 44-59.

Duval, S. J., \& Tweedie, R. L. (2000). A non-parametric trim and fill method of accounting for publication bias in meta-analysis. J. Am. Stat. Assoc, 95, 89-98.

Dwyer, S., Nicholson, J., \& Battistutta, D. (2006). Parent and Teacher Identification of Children at Risk of Developing Internalizing or Externalizing Mental Health Problems: A Comparison of Screening Methods. Prevention Science, 7(4), 343-357. doi: 10.1007/s11121-006-0026-5

Egger, M., Smith, G., \& Phillips, A. (1997). Meta-analysis: principles and procedures. BMJ, 315, 15331537.

Eley, T. C., Bolton, D., O'Connor, T. G., Perrin, S., Smith, P., \& Plomin, R. (2003). A twin study of anxiety-related behaviours in pre-school children. Journal of Child Psychologyand Psychiatry, 44, 945-960.

Etter, M. E. (2014). Longitudinal effects of improving inter-parental relationships in low-income couples: Child outcomes. Dissertation Abstracts International: Section B: The Sciences and Engineering, 75(1-B E).

Feinberg, M. E., Jones, D. E., Kan, M. L., \& Goslin, M. C. (2010). Effects of family foundations on parents and children: 3.5 years after baseline. J Fam Psychol, 24(5), 532-542. doi: 201021484-003 [pii]

10.1037/a0020837 [doi]

Feinberg, M. E., \& Kan, M. L. (2008). Establishing family foundations: intervention effects on coparenting, parent/infant well-being, and parent-child relations. J Fam Psychol, 22(2), 253263. doi: 2008-03770-008 [pii]

10.1037/0893-3200.22.2.253 [doi]

Fergusson, D. M., Boden, J. M., \& Horwood, L. J. (2013). Nine-year follow-up of a home-visitation program: a randomized trial. Pediatrics, 131(2), 297-303. doi: 10.1542/peds.2012-1612

Fisak, B., D. , Richard, D., \& Mann, A. (2011). The prevention of child and adolescent anxiety: A metaanalytic review. Prevention Science. doi:DOI 10.1007/s11121-011-0210-0

Ginsburg, G. S. (2009). The Child Anxiety Prevention Study: Intervention Model and Primary Outcomes. Journal of Consulting and Clinical Psychology, 77(3), 580-587.

Greenberg, M. T., Domitrovich, C., \& Bumbarger, B. (2001). The prevention of mental disorders in school-aged children: Current state of the field. Prevention and Treatment, 4(March).

Hankin, B. L., Fraley, R. C., Lahey, B. B., \& Waldman, I. D. (2005). Is depression best viewed as a continuum or discrete category? A taxometric analysis of childhood and adolescent 
depression in a population-based sample. J Abnorm Psychol, 114(1), 96-110. doi: 10.1037/0021-843x.114.1.96

Hetrick, S., Cox, G., \& Merry, S. (2015). Where to Go from Here? An Exploratory Meta-Analysis of the Most Promising Approaches to Depression Prevention Programs for Children and Adolescents. International Journal of Environmental Research and Public Health, 12(5), 4758.

Higgins, J. P., \& Green, S. (Eds.). (2011). Cochrane Handbook for Systematic Reviews of Interventions. Version 5.1.0 [updated March 2011]: The Cochrane Collaboration.

Higgins, J. P., Thompson, S. G., Deeks, J. J., \& Altman, D. G. (2003). Measuring inconsistency in metaanalyses. BMJ, 327, 557-560.

Hudson, J. L., Rapee, R. M., Lyneham, H. J., McLellan, L. F., Wuthrich, V. M., \& Schniering, C. A. (2015). Comparing outcomes for children with different anxiety disorders following cognitive behavioural therapy. Behaviour research and therapy, 72, 30-37.

Kehoe, C. E., Havighurst, S. S., \& Harley, A. E. (2014). Tuning in to Teens: Improving parent emotion socialization to reduce youth internalizing difficulties. Social Development, 23(2), 413-431.

Kessler, R. C., Amminger, G. P., Aguilar-Gaxiola, S., Alonso, J., Lee, S., \& Ustun, T. B. (2007). Age of onset of mental disorders: a review of recent literature. Current Opinion in Psychiatry, 20(4), 359-364.

Kessler, R. C., Berglund, P., Demler, O., Jin, R., Merikangas, K. R., \& Walters, E. E. (2005). Lifetime prevalence and age-of-onset distributions of DSM-IV disorders in the National Comorbidity Survey Replication. Archives of General Psychiatry, 62(6), 593-602. doi: 62/6/593 [pii]

10.1001/archpsyc.62.6.593 [doi]

Kessler, R. C., Petukhova, M., Sampson, N. A., Zaslavsky, A. M., \& Wittchen, H.-U. (2012). Twelvemonth and lifetime prevalence and lifetime morbid risk of anxiety and mood disorders in the United States. International Journal of Methods in Psychiatric Research, 21(3), 169-184. doi: 10.1002/mpr.1359

Lahey, B., Hulle, C., D'Onofrio, B., Rodgers, J., \& Waldman, I. (2008). Is Parental Knowledge of their Adolescent Offspring's Whereabouts and Peer Associations Spuriously Associated with Offspring Delinquency? Journal of Abnormal Child Psychology, 36(6), 807-823.

Lam, W. K., Fals-Stewart, W., \& Kelley, M. L. (2008). Effects of Parent Skills Training with Behavioral Couples Therapy for alcoholism on children: a randomized clinical pilot trial. Addict Behav, 33(8), 1076-1080. doi: S0306-4603(08)00092-0 [pii]

10.1016/j.addbeh.2008.04.002 [doi]

Lengua, L. J., \& Kovacs, E. A. (2005). Bidirectional associations between temperament and parenting and the prediction of adjustment problems in middle childhood. Journal of Applied Developmental Psychology, 26(1), 21-38. doi: http://dx.doi.org/10.1016/i.appdev.2004.10.001

Lowry-Webster, H. M., Barrett, P. M., \& Lock, S. (2003). A universal prevention trial of anxiety symptomology during childhood: Results at 1-year follow-up. Behaviour Change, 20(1), 2543. doi:10.1375/bech.20.1.25.24843

Mason, W. A., Kosterman, R., Hawkins, J. D., Haggerty, K. P., Spoth, R. L., \& Redmond, C. (2007). Influence of a family-focused substance use preventive intervention on growth in adolescent depressive symptoms. Journal of Research on Adolescence, 17(3), 541-564. doi: 10.1111/j.1532-7795.2007.00534.x

Mathew, A. R., Pettit, J. W., Lewinsohn, P. M., Seeley, J. R., \& Roberts, R. E. (2011). Co-morbidity between major depressive disorder and anxiety disorders: shared etiology or direct causation? Psychological Medicine, FirstView, 1-12. doi: doi:10.1017/S0033291711000407

McLeod, B. D., Weisz, J. R., \& Wood, J. J. (2007). Examining the association between parenting and childhood depression: A meta-analysis. Clinical Psychology Review, 27(8), 986-1003.

McLeod, B. D., Wood, J. J., \& Weisz, J. R. (2007). Examining the association between parenting and childhood anxiety: A meta-analysis. Clin Psychol Rev, 27(2), 155-172. 
Merry, S. N., Hetrick, S. E., Cox, G. R., Brudevold-Iversen, T., Bir, J. J., \& McDowel, H. H. (2011). Prevention of depression in children and adolescents. Cochrane Database of Systematic Reviews, 12.

Mrazek, P. J., \& Haggerty, R. J. (1994). Reducing Risks for Mental Disorders: Frontiers for Preventive Intervention Research.

O'Connell, M. E., Boat, T., \& Warner, K. (2009). Preventing Mental, Emotional, and Behavioral Disorders Among Young People: Progress and Possibilities Committee on Prevention of Mental Disorders and Substance Abuse Among Children, Youth and Young Adults: Research Advances and Promising Interventions, Board on Children, Youth, and Families, Division of Behavioral and Social Sciences and Education. Washington, DC: The National Academies Press.

Ordway, M. R., Sadler, L. S., Dixon, J., Close, N., Mayes, L., \& Slade, A. (2014). Lasting effects of an interdisciplinary home visiting program on child behavior: Preliminary follow-up results of a randomized trial. Journal of pediatric nursing, 29(1), 10.1016/j.pedn.2013.1004.1006. doi: 10.1016/j.pedn.2013.04.006

Patel, V., Flisher, A. J., Hetrick, S., \& McGorry, P. (2007). Mental health of young people: a global public-health challenge. The Lancet, 369(9569), 1302-1313.

Phares, V., Compas, B. E., \& Howell, D. C. (1989). Perspectives on Child Behavior Problems: Comparisons of Children's Self-Reports With Parent and Teacher Reports. Psychological Assessment, 1(1), 68-71.

Podsakoff, P. M., MacKenzie, S. B., Lee, J.-Y., \& Podsakoff, N. P. (2003). Common Method Biases in Behavioral Research: A Critical Review of the Literature and Recommended Remedies. Journal of Applied Psychology, 88(5),879-903.

Quach, J., Hiscock, H., \& Wake, M. (2010). Impact of a brief school-entry sleep intervention on child and parent outcomes: Randomised controlled trial. Journal of Paediatrics and Child Health, $46,11$.

Rapee, R. M. (1991). Generalized anxiety disorder: A review of clinical features and theoretical concepts. Clin Psych Rev, 11, 419-440.

Rapee, R. M. (2013). The preventative effects of a brief, early intervention for preschool-aged children at risk for internalising: follow-up into middle adolescence. Journal of Child Psychology and Psychiatry, 54(7), 780-788. doi: 10.1111/jcpp.12048

Rapee, R. M., Kennedy, S., Ingram, M., Edwards, S. L., \& Sweeney, L. (2005). Prevention and early intervention of anxiety disorders in inhibited preschool children. J Consult Clin Psychol, 73(3), 488-497.

Restifo, K., \& Bögels, S. (2009). Family processes in the development of youth depression: Translating the evidence to treatment. Clinical Psychology Review, 29(4), 294-316. doi: 10.1016/j.cpr.2009.02.005

Rose, G. (1992). The Strategy of Preventive Medicine (Vol. 1). Oxford: Oxford UniversityPress.

Rotheram-Borus, M. J., Lee, M. B., Gwadz, M., \& Draimin, B. (2001). An intervention for parents with AIDS and their adolescent children. American journal of public health, (8), 1294-1302. http://www.mrw.interscience.wiley.com/cochrane/clcentral/articles/994/CN00349994/frame.html

Rudolph, K. D., Lansford, J. E., \& Rodkin, P. C. (2016). Interpersonal theories of developmental psychopathology. In D. Cichetti (Ed.), Developmental Psychopathology: Maladpation and Psychopathology (Third ed.). New Jersey, U.S.A.: John Wiley \& Sons.

Ruffolo, M. C., Kuhn, M. T., \& Evans, M. E. (2005). Support, empowerment, and education: A study of multiple family group psychoeducation. Journal of Emotional and Behavioral Disorders, 13(4), 200-212. doi: 10.1177/10634266050130040201

Ryan, S. M., Jorm, A. F., \& Lubman, D. I. (2010). Parenting factors associated with reduced adolescent alcohol use: a systematic review of longitudinal studies. Aust N Z J Psychiatry, 44(9), 774-783. doi: doi:10.1080/00048674.2010.501759 
Salim, A., \& et al. (2008). Comparison of data analysis strategies for intent-to-treat analysis in pretest-post-test designs with substantial dropout rates. Psychiatry Research, 160, 335-345.

Sameroff, A. J. (2009). The Transactional Model of Development: How Children and Contexts Shape Each Other: American Psychological Association.

Sameroff, A. J., \& Chandler, M. J. (1975). Reproductive risk and the continuum of caretaking casualty. In F. D. Harrowitz, S. Scarr-Salapatek \& G. Siegel (Eds.), Review of Child Development Research (Vol. 4, pp. 187-224). Chicago: University of Chicago Press.

Sandler, I. N., Ayers, T. S., Wolchik, S. A., Tein, J. Y., Kwok, O. M., Haine, R. A., . . Griffin, W. A. (2003). The family bereavement program: efficacy evaluation of a theory-based prevention program for parentally bereaved children and adolescents. J Consult Clin Psychol, 71(3), 587600.

Sandler, I. N., Schoenfelder, E. N., Wolchik, S. A., \& MacKinnon, D. P. (2011). Long-term impact of prevention programs to promote effective parenting: Lasting effects but uncertain processes. Ann Rev Psychol, 62(1), 299-329. doi: doi:10.1146/annurev.psych.121208.131619

Sandler, I. N., Wolchik, S. A., Cruden, G., Mahrer, N. E., Ahn, S., Brincks, A., \& Brown, C. H. (2014). Overview of Meta-Analyses of the Prevention of Mental Health, Substance Use, and Conduct Problems*. Annual Review of Clinical Psychology, 10(1), 243-273. doi: doi:10.1146/annurevclinpsy-050212-185524

Santacruz, I., Mendez, F. J., \& Sanchez-Meca, J. (2006). Play Therapy Applied by Parents for Children with Darkness Phobia: Comparison of Two Programmes. Child \& Family Behavior Therapy, 28(1), 19-35. doi:10.1300/J019v28n01_02

Saylor, C. F., Finch, A., Spirito, A., \& Bennett, B. (1984). The children's depression inventory: a systematic evaluation of psychometric properties. Journal of consulting and clinical psychology, 52(6), 955.

Schleider, J. L., \& Weisz, J. R. (2016). Family process and youth internalizing problems: A triadic model of etiology and intervention. Dev Psychopathol, 1-29. doi: $10.1017 /$ s095457941600016x

Siegenthaler, E., Munder, T., \& Egger, M. (2012). Effect of Preventive Interventions in Mentally III Parents on the Mental Health of the Offspring: Systematic Review and Meta-Analysis. J Am Acad Child Adolesc Psychiatry, 51(1), 8-17.e18. doi: 10.1016/j.jaac.2011.10.018

Simon, E., Bogels, S. M., \& Voncken, J. M. (2011). Efficacy of child-focused and parent-focused interventions in a child anxiety prevention study. J Clin Child Adolesc Psychol, 40(2), 204-219. doi: 10.1080/15374416.2011.546039

Singer, L. T., Salvator, A., Guo, S., Collin, M., Lilien, L., \& Baley, J. (1999). Maternal psychological distress and parenting stress after the birth of a very low-birth-weight infant.JAMA Pediatrics, 281(9), 799-805.

Sipahi, I., Swaminathan, A., Natesan, V., Debanne, S. M., Simon, D. I., \& Fang, J. C. (2012). Effect of antihypertensive therapy on incident stroke in cohorts with prehypertensive blood pressure levels: a meta-analysis of randomized controlled trials. Stroke, 43(2), 432-440. doi: 10.1161/strokeaha.111.636829

Smucker, M. R., Craighead, W. E., Craighead, L. W., \& Green, B. J. (1986). Normative and reliability data for the Children's Depression Inventory. Journal of abnormal child psychology, 14(1), 25-39.

Solantaus, T., Toikka, S., Alasuutari, M., Beardslee, W. R., \& Paavonen, E. J. (2009). Safety, feasibility and family experiences of preventive interventions for children and families with parental depression. The International Journal of Mental Health Promotion, 11(4), 15-24.

Spence, S. H. (1998). A measure of anxiety symptoms among children. Behaviour research and therapy, 36(5), 545-566.

Spence, S. H., Barrett, P. M., \& Turner, C. M. (2003). Psychometric properties of the Spence Children's Anxiety Scale with young adolescents. Journal of anxiety disorders, 17(6), 605-625. 
Spencer-Smith, M. M., Spittle, A. J., Doyle, L. W., Lee, K. J., Lorefice, L. S., A., ... , \& Anderson, P. J. (2012). Long-term benefits of home-based preventive care for preterm infants: a randomized trial. Pediatrics, 130(6), 1094-1101.

Spittle, A. J., Anderson, P. J., Lee, K. J., Ferretti, C., Eeles, A., Orton, J., ... Doyle, L. W. (2010). Preventive care at home for very preterm infants improves infant and caregiver outcomes at 2 years. Pediatrics, 126(1), e171-178. doi: 10.1542/peds.2009-3137

Stanger, C., \& Lewis, M. (1993). Agreement Among Parents, Teachers, and Children on Internalizing and Externalizing Behavior Problems. Journal of Clinical Child Psychology, 22(1), 107-116. doi:10.1207/s15374424jccp2201_11

Stathakos, P., \& Roehrle, B. (2003). The effectiveness of intervention programmes for children of divorce-a metaanalysis. Int. J. Mental Health Promot., 5, 31-37.

Taylor, F., Huffman, M. D., Macedo, A. F., Moore, T. H. M., Burke, M., Davey Smith, G., . . Ebrahim, S. (2013). Statins for the primary prevention of cardiovascular disease. Cochrane Database of Systematic Reviews(1). doi: 10.1002/14651858.CD004816.pub5

Teubert, D., \& Pinquart, M. (2011). A meta-analytic review on the prevention of symptoms of anxiety in children and adolescents. Journal of Anxiety Disorders, 25(8), 1046-1059. doi: http://dx.doi.org/10.1016/i.janxdis.2011.07.001

Timbremont, B., Braet, C., \& Dreessen, L. (2004). Assessing depression in youth: relation between the Children's Depression Inventory and a structured interview. Journal of clinical child and adolescent psychology, 33(1), 149-157.

Trudeau, L., Spoth, R., Randall, G. K., \& Azevedo, K. (2007). Longitudinal effects of a universal familyfocused intervention on growth patterns of adolescent internalizing symptoms and polysubstance use: gender comparisons. J. Youth Adolesc, 36, 725-740.

Van Der Bruggen, C. O., Stams, G. J. J. M., \& Bögels, S. M. (2008). Research Review: The relation between child and parent anxiety and parental control: a meta-analytic review. Journalof Child Psychology and Psychiatry, 49(12), 1257-1269. doi: 10.1111/j.1469-7610.2008.01898.x

van Zoonen, K., Buntrock, C., Ebert, D. D., Smit, F., Reynolds, C. F., 3rd, Beekman, A. T., \& Cuijpers, P. (2014). Preventing the onset of major depressive disorder: a meta-analytic review of psychological interventions. Int J Epidemiol, 43(2), 318-329. doi: 10.1093/ije/dyt175

Weersing, V. R., Rozenman, M. S., Maher-Bridge, M., \& Campo, J. V. (2012). Anxiety, Depression, and Somatic Distress: Developing a Transdiagnostic Internalizing Toolbox for Pediatric Practice. Cogn Behav Pract, 19(1), 68-82. doi: 10.1016/j.cbpra.2011.06.002

Wilamowska, Z. A., Thompson-Hollands, J., Fairholme, C. P., Ellard, K. K., Farchione, T. J., \& Barlow, D. H. (2010). Conceptual background, development, and preliminary data from the unified protocol for transdiagnostic treatment of emotional disorders. Depression and Anxiety, 27(10), 882-890.

Wolchik, S. A., West, S. G., Sandler, I. N., Tein, J.-Y., Coatsworth, D., Lengua, L., . . Griffin, W. A. (2000). An experimental evaluation of theory-based mother and mother-child programs for children of divorce. Journal of Consulting and Clinical Psychology, 68(5), 843-856. doi: 10.1037/0022-006x.68.5.843

Wolchik, S. A., West, S. G., Westover, S., Sandler, I. N., Martin, A., Lustig, J., . . Fisher, J. (1993). The children of divorce parenting intervention: outcome evaluation of an empiricallybased program. American journal of community psychology, (3), 293-231. http://www.mrw.interscience.wiley.com/cochrane/clcentral/articles/024/CN00099024/frame.html

Xie, M., Shan, Z., Zhang, Y., Chen, S., Yang, W., Bao, W., . . Liu, L. (2014). Aspirin for Primary Prevention of Cardiovascular Events: Meta-Analysis of Randomized Controlled Trials and Subgroup Analysis by Sex and Diabetes Status. PloS one, 9(10), e90286. doi: 10.1371/journal.pone.0090286 
Yap, M. B. H., Allen, N. B., \& Sheeber, L. (2007). Using an emotion regulation framework to understand the role of temperament and family processes in risk for adolescent depressive disorders. Clinical Child \& Family Psychology Review, 10(2), 180-196.

Yap, M. B. H., Fowler, M., Reavley, N., \& Jorm, A. F. (2015). Parenting strategies for reducing the risk of childhood depression and anxiety disorders: A Delphi consensus study. J AffectDisord, 183, 330-338. doi:10.1016/j.jad.2015.05.031

Yap, M. B. H., \& Jorm, A. F. (2015). Parental factors associated with childhood anxiety, depression, and internalizing problems: A systematic review and meta-analysis. J Affect Disord, 175C, 424-440. doi:10.1016/j.jad.2015.01.050

Yap, M. B. H., Pilkington, P. D., Ryan, S. M., \& Jorm, A. F. (2014). Parental factors associated with depression and anxiety in young people: A systematic review and meta-analysis. Journal of Affective Disorders, 156, 8-23.

Yap, M. B. H., Schwartz, O. S., Byrne, M. L., Simmons, J. G., \& Allen, N. B. (2010). Maternal positive and negative interaction behaviors and early adolescents' depressive symptoms: Adolescent emotion regulation as a mediator. J Res Adolesc, 20(4), 1014-1043. 
2427 records identified through database searching
256 additional records

identified through other sources

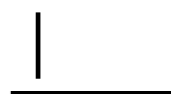

1918 records after duplicates removed

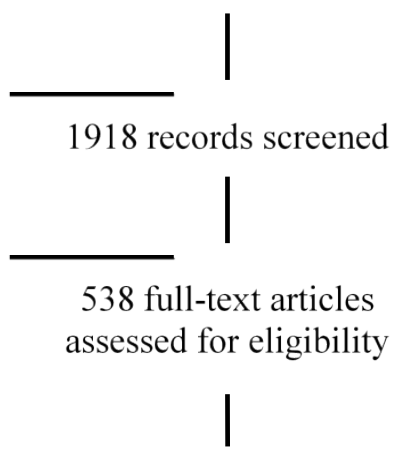

51 studies (66 articles) included in qualitative synthesis

范
尝<smiles>[CH]C</smiles>

45 studies included in quantitative synthesis (meta-analysis)
1380 records excluded
472 full-text articles excluded:

- No child internalising outcomes or not a major goal of study $(n=140)$

- No long term follow-up $(\mathrm{n}=113)$

- Parent component of intervention too small $(n=83)$

- Not an RCT $(n=50)$

- Targets externalising $(\mathrm{n}=59)$

- Follow-up less than 6 months $(n=43)$

- Follow-up data collected only in active group $(\mathrm{n}=34)$

- Comparison condition not a minimal control $(\mathrm{n}=28$

- Not a parenting intervention $(\mathrm{n}=15)$

- Secondary analysis (data not useable) (n 11)

- Data not available/reported (e.g. protocol) $(\mathrm{n}=9)$

- Not in English $(\mathrm{n}=5$,

- Not with children aged 0 to $18(\mathrm{n}=2$

- Intervention for developmental disorders $(n=2)$

Figure 1 PRISMA flow chart showing the flow of selection of eligible papers. 
Study name

Carta 2013

Hoff 2005

Lowell 2011

Oswalt 2013; olderMo

Oswalt 2013 _youngerMo

van Doesum 2008

Walkup 2009

Williamson 2014

Dadds 2008_T

Riuf folo 200.5

Cartwright-Hatton 2011

Cohen 1997 (12mth)

Connell $200 B$

Lam 2008 (12mth)

Lewis 2015

Strayhorn 1991

Wu 2014

Cheng 2007_Mo

Wake 2011

Butz 2001

Etter 2013

Solantaus 2010 (18mth)

Hahlweg 2007_1P (23mth)

Hahlweg 2007 2P (23mth)

Beardslee 2003

Cowan 2005i (24mth)

MoDonald 2006_T

Ordway 2014_Mo

Velderman 2006

Spencer-Smith 2012

Verkerk 201.2

Nondhov 2012

Feinberg 2014_P

Rotheram-Borws 20013 (69mth)

Fergusson 2013 (70mth)

Black 2007

Wolchik 2013
Statistics for each study

Std diff
in means $\begin{gathered}\text { Lower Upper } \\ \text { limit }\end{gathered}$ limit p-Value

$\begin{array}{llll}-0.069 & -0.328 & 0.190 & 0.602\end{array}$

$\begin{array}{llll}-0.783 & -1.615 & 0.050 & 0.086\end{array}$

$\begin{array}{llll}-0.232 & -0.595 & 0.132 & 0.212\end{array}$

$\begin{array}{llll}0.253 & -0.080 & 0.568 & 0.113\end{array}$

$\begin{array}{llll}-0.58 .5 & -1.23 .7 & 0.068 & 0.078\end{array}$

$\begin{array}{llll}0.304 & -0.164 & 0.772 & 0.203\end{array}$

$\begin{array}{llll}-0.358 & -0.850 & 0.133 & 0.153\end{array}$

$\begin{array}{llll}-0.240 & -0.558 & 0.078 & 0.139\end{array}$

$\begin{array}{llll}-0.156 & -0.336 & 0.023 & 0.088\end{array}$

$\begin{array}{llll}-0.84 .2 & -1.479 & -0.205 & 0.010\end{array}$

$\begin{array}{llll}-0.616 & -1.096 & -0.137 & 0.012\end{array}$

$\begin{array}{llll}-0.406 & -1.039 & 0.227 & 0.209\end{array}$

$\begin{array}{llll}-0.210 & -0.583 & 0.143 & 0.243\end{array}$

$\begin{array}{llll}-0.650 & -1.550 & 0.249 & 0.156\end{array}$

$\begin{array}{llll}0.040 & -0.314 & 0.393 & 0.828\end{array}$

$\begin{array}{llll}-0.510 & -1.045 & 0.025 & 0.062\end{array}$

$\begin{array}{lllll}-0.355 & -0.780 & 0.051 & 0.087\end{array}$

$\begin{array}{llll}0.096 & -0.331 & 0.523 & 0.680\end{array}$

$\begin{array}{llll}0.058 & -0.18: 3 & 0.298 & 0.638\end{array}$

$\begin{array}{llll}-0.282 & -0.676 & 0.112 & 0.161\end{array}$

$\begin{array}{llll}-0.320 & -0.751 & 0.111 & 0.146\end{array}$

$\begin{array}{cccc}-0.133 & -0.562 & 0.296 & 0.543\end{array}$

$\begin{array}{llll}0.477 & -0.042 & 0.995 & 0.071\end{array}$

$\begin{array}{llll}0.103 & -0.193 & 0.400 & 0.495\end{array}$

$\begin{array}{llll}-0.103 & -0.568 & 0.359 & 0.681\end{array}$

$\begin{array}{llll}0.760 & 0.201 & 1.317 & 0.008\end{array}$

$\begin{array}{llll}-0.009 & -0.383 & 0.344 & 0.958\end{array}$

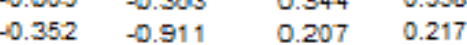

$\begin{array}{llll}0.090 & -0.449 & 0.629 & 0.743\end{array}$

$\begin{array}{llll}-0.505 & -0.930 & -0.080 & 0.020\end{array}$

$\begin{array}{llll}-0.144 & -0.46: 5 & 0.176 & 0.378\end{array}$

$\begin{array}{llll}-0.287 & -0.616 & 0.083 & 0.135\end{array}$

$\begin{array}{llll}-0.021 & -0.468 & 0.423 & 0.925\end{array}$

$\begin{array}{llll}-0.058 & -0.466 & 0.423 & 0.925 \\ 0.058 & -0.162 & 0.278 & 0.606\end{array}$

$\begin{array}{rrrr}-0.150 & -0.355 & 0.055 & 0.151\end{array}$

$\begin{array}{llll}-0.212 & -0.613 & 0.189 & 0.301\end{array}$

$\begin{array}{llll}-0.016 & -0.348 & 0.315 & 0.923\end{array}$

$\begin{array}{llll}-0.123 & -0.20 .5 & -0.042 & 0.003\end{array}$
Std difference in means and $95 \% \mathrm{Cl}$

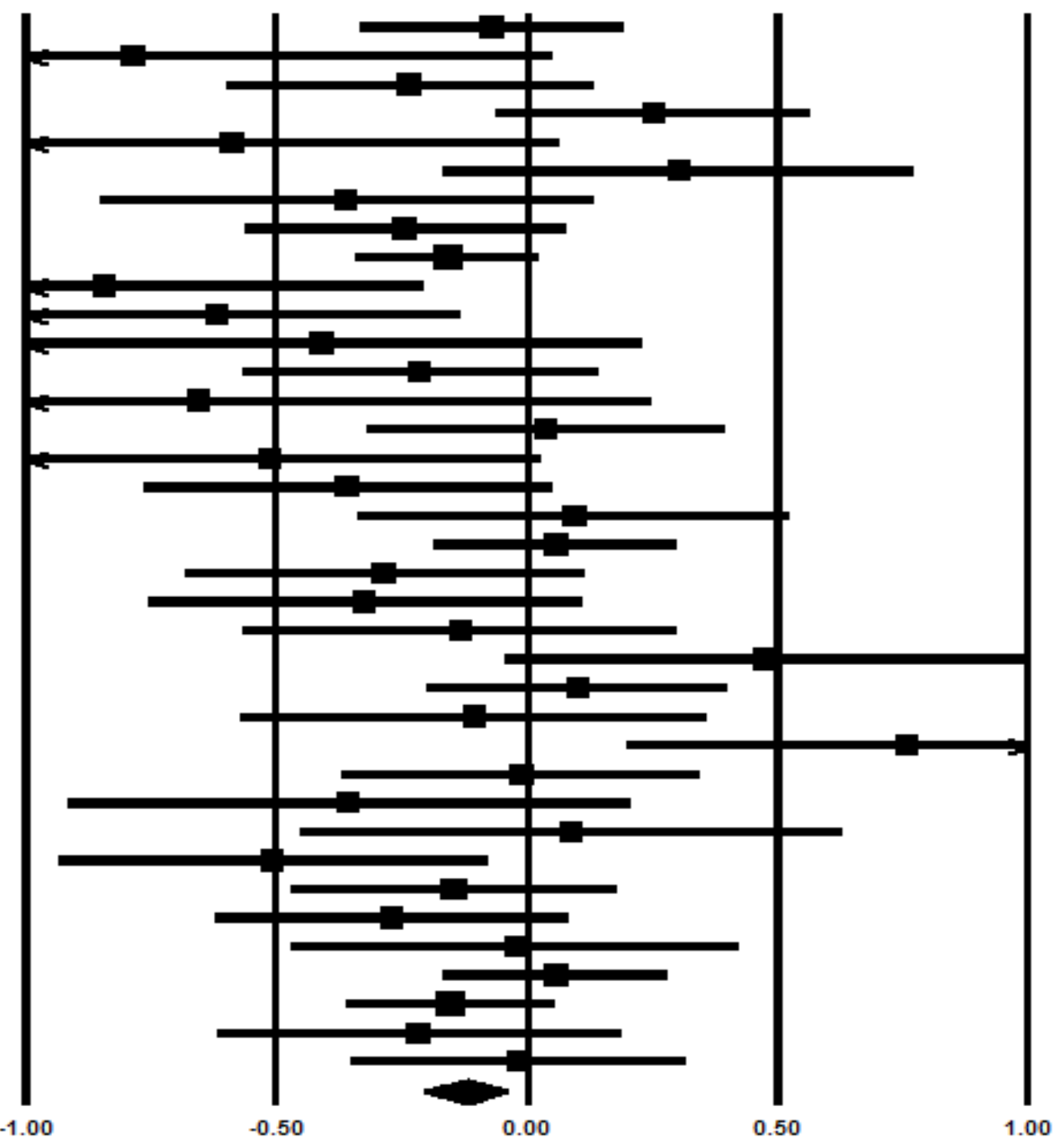

Favours Intervention 
Figure 2

The effects of preventive parenting interventions on measures of child internalizing symptoms. Lines represent standardized difference in means (Cohen's $d$ ) and 95\% confidence intervals $(\mathrm{Cl})$; the size of the box represents the weight of each study. Studies are ordered by the duration of the follow-up interval (shortest tolongest). 


$\begin{array}{lrrrr} & \begin{array}{c}\text { Std diff } \\ \text { in means }\end{array} & \begin{array}{c}\text { Lower } \\ \text { limit }\end{array} & \begin{array}{c}\text { Upper } \\ \text { limit }\end{array} & \text { p-Value } \\ \text { Forgatch 1999 } & 0.085 & -0.240 & 0.411 & 0.608 \\ \text { Walkup 2009 } & -0.136 & -0.624 & 0.352 & 0.586 \\ \text { Lewis 2015 } & -0.535 & -0.895 & -0.175 & 0.004 \\ \text { Kehoe2014_C } & -0.140 & -0.175 & -0.105 & 0.000 \\ \text { Lam 2008 (12mth) } & -0.971 & -1.898 & -0.045 & 0.040 \\ \text { Velderman 2006_Sx } & 0.005 & -0.534 & 0.544 & 0.986 \\ \text { Mason 2007 (68mth) } & -0.209 & -0.399 & -0.019 & 0.031 \\ \text { Rotheram-Borus 2006 (69mth) } & 0.000 & -0.231 & 0.231 & 1.000 \\ \text { Rapee 2013-F } & -0.483 & -0.988 & 0.022 & 0.061 \\ \text { Rapee 2013-M } & 0.069 & -0.548 & 0.686 & 0.827 \\ & -0.156 & -0.287 & -0.046 & 0.005\end{array}$

Std difference in means and $95 \% \mathrm{Cl}$
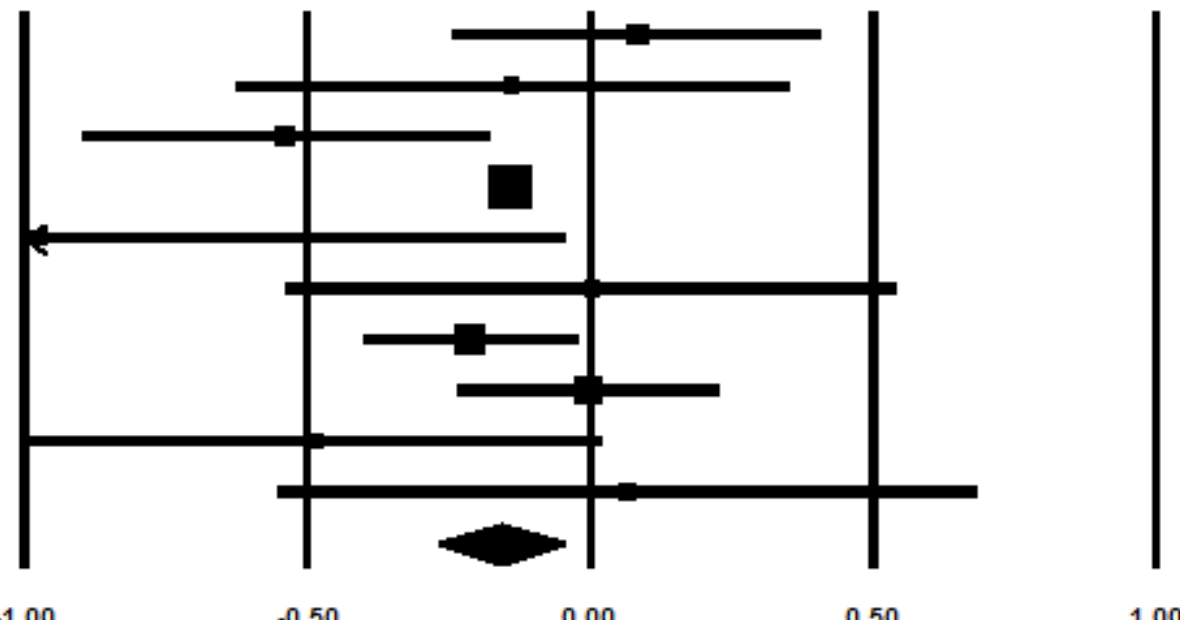

Favours Intervention

Favours Comparison

Figure 3 The effects of preventive parenting interventions on measures of child depressive symptoms. Lines represent standardized difference in means (Cohen's $d$ ) and 95\% confidence intervals (Cl); the size of the box represents the weight of each study. Studies are ordered by the duration of the follow-up interval (shortest tolongest). 


\section{Study name}

\section{Beardslee 1997}

Rapee 2013-F_P\&C_Dx

Rapee 2013-M_P\&C_Dx

Wolchik 2013 (15-year)
Statistics for each study

$$
\text { Risk Lower Upper }
$$

difference limit limit

$\begin{array}{llll}-0.179 & -0.376 & 0.019 & 0.077 \\ -0.161 & -0.299 & -0.024 & 0.022 \\ 0.068 & -0.100 & 0.235 & 0.428 \\ -0.104 & -0.238 & 0.030 & 0.128 \\ -0.095 & -0.198 & 0.008 & 0.071\end{array}$

$-0.40$

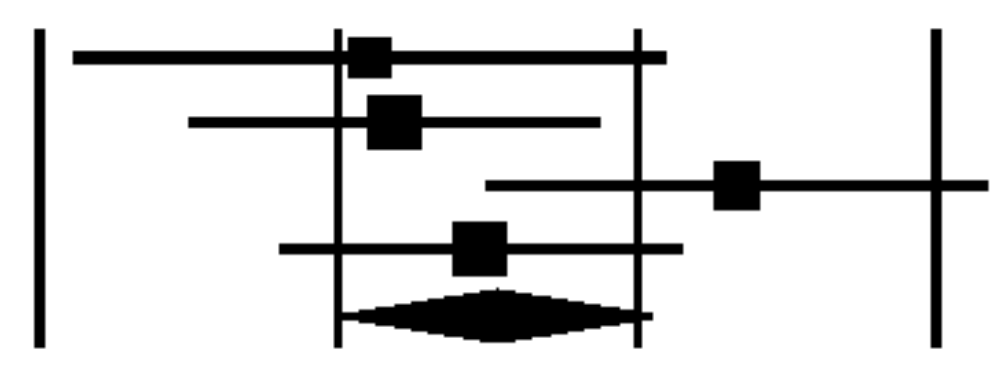

$-0.20$
Risk difference and $95 \% \mathrm{Cl}$

-Value

Favours Intervention

Favours Comparison

Figure $4 \quad$ The effects of preventive parenting interventions on measures of child depression diagnoses. Lines represent risk difference and 95\% confidence intervals $(\mathrm{Cl})$; the size of the box represents the weight of each study. Studies are ordered by the duration of the follow-up interval (shortest to longest). 


$\begin{array}{rrrr}\begin{array}{r}\text { Std diff } \\ \text { in means }\end{array} & \begin{array}{c}\text { Lower } \\ \text { limit }\end{array} & \begin{array}{r}\text { Upper } \\ \text { limit }\end{array} & \text { p-Value } \\ -0.055 & -0.380 & 0.271 & 0.742 \\ -0.045 & -0.532 & 0.443 & 0.858 \\ 0.021 & -0.332 & 0.375 & 0.905 \\ -0.260 & -0.295 & -0.225 & 0.000 \\ -0.169 & -0.743 & 0.410 & 0.567 \\ -1.831 & -2.569 & -1.093 & 0.000 \\ -0.902 & -1.822 & 0.018 & 0.055 \\ -1.354 & -2.018 & -0.690 & 0.000 \\ 0.080 & -0.349 & 0.508 & 0.715 \\ -0.353 & -0.771 & 0.054 & 0.097 \\ 0.136 & -0.403 & 0.675 & 0.622 \\ 0.143 & -0.089 & 0.374 & 0.227 \\ -0.607 & -1.116 & -0.098 & 0.019 \\ -0.188 & -0.805 & 0.430 & 0.551 \\ -0.273 & -0.465 & -0.082 & 0.005\end{array}$

Forgation 1999_P

Walkup 2009_P_s

Lewls 2015

Kenoe 2014_C

Cartwright-Hamon 2011_C_SX

Ginsburg 2009_P+C_Sx (12mm)

Lam 2008 (12men)

Santacruz 2005_P_BTR

Solantaus 2010_P SN (18mtm)

Simon 2011_C (22 mm)

Velderman 2005 _P_S

Fotheram-Bonus 2005 (69mm)

Frapee 2013-F_SK (11yr)

Fapee 2013-M Sx (11yr)
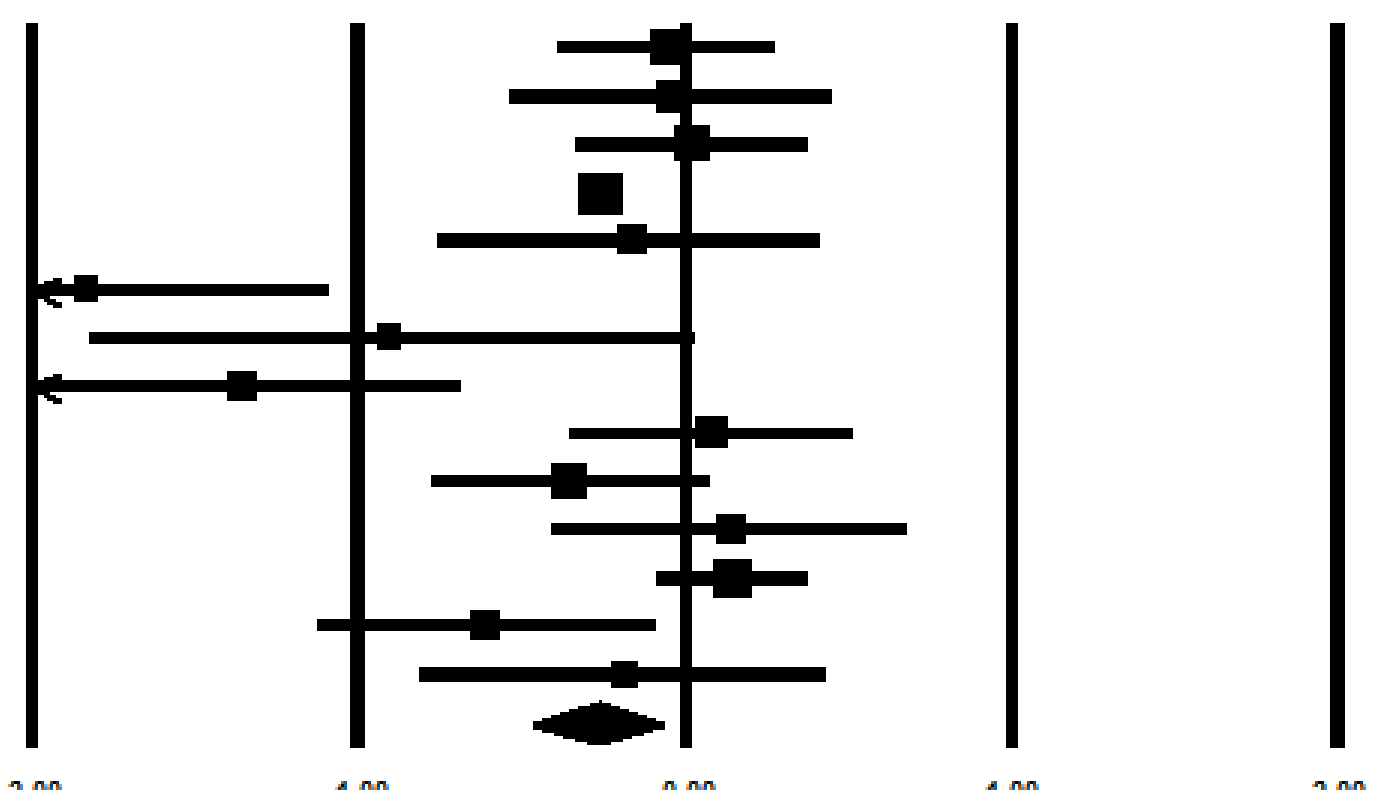

Favours Intervention

Favours Comparison

Figure 5 The effects of preventive parenting interventions on measures of child anxiety symptoms. Lines represent standardized difference in means (Cohen's $d$ ) and $95 \%$ confidence intervals ( $\mathrm{Cl}$ ); the size of the box represents the weight of each study. Studies are ordered by the duration of the follow-up interval (shortest to longest). 
Study name

Statistics for each study

Risk Lower Upper

difference limit limit $p$-Value

Ginsburg 2009 _ P+C_Dx (6mm)

Dados 2008

Cartwright-Hamon 2011_P\&C_Dx

Frapee 2013-F_PSC_DX (11yr)

Frapee 2013-M_PSC_Dx (11yr)

Wolchlk 2013 (15yr)

$\begin{array}{llll}-0.150 & -0.322 & 0.022 & 0.088 \\ -0.025 & -0.087 & 0.036 & 0.421 \\ -0.369 & -0.557 & -0.180 & 0.000 \\ -0.226 & -0.468 & 0.017 & 0.068 \\ 0.077 & -0.230 & 0.384 & 0.622 \\ -0.047 & -0.109 & 0.015 & 0.141 \\ -0.109 & -0.200 & -0.017 & 0.020\end{array}$

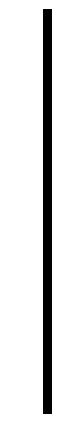

$-0.60$

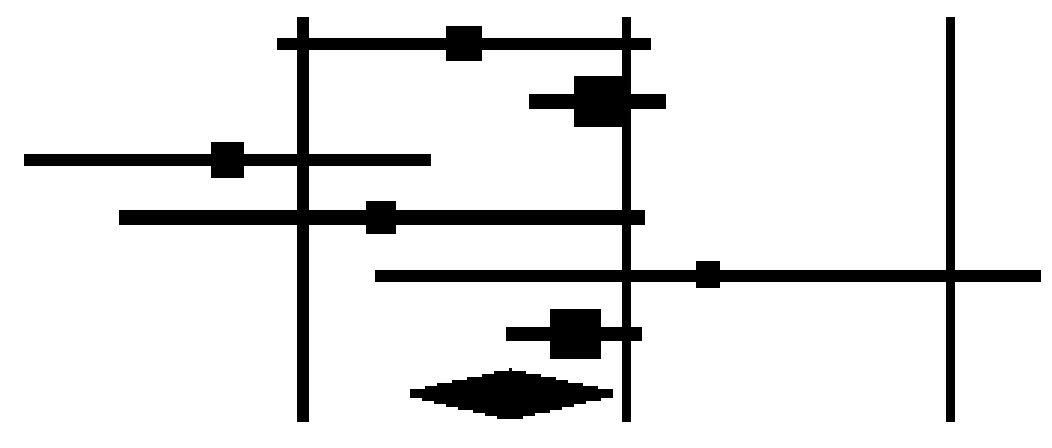

0.00

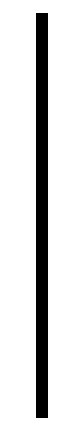

0.30

0.60

Favours Intervention

Favours Comparison

Figure 6 The effects of preventive parenting interventions on measures of child anxiety diagnoses. Lines represent risk difference and 95\% confidence intervals $(\mathrm{Cl})$; the size of the box represents the weight of each study. Studies are ordered by the duration of the follow-up interval (shortest to longest). 


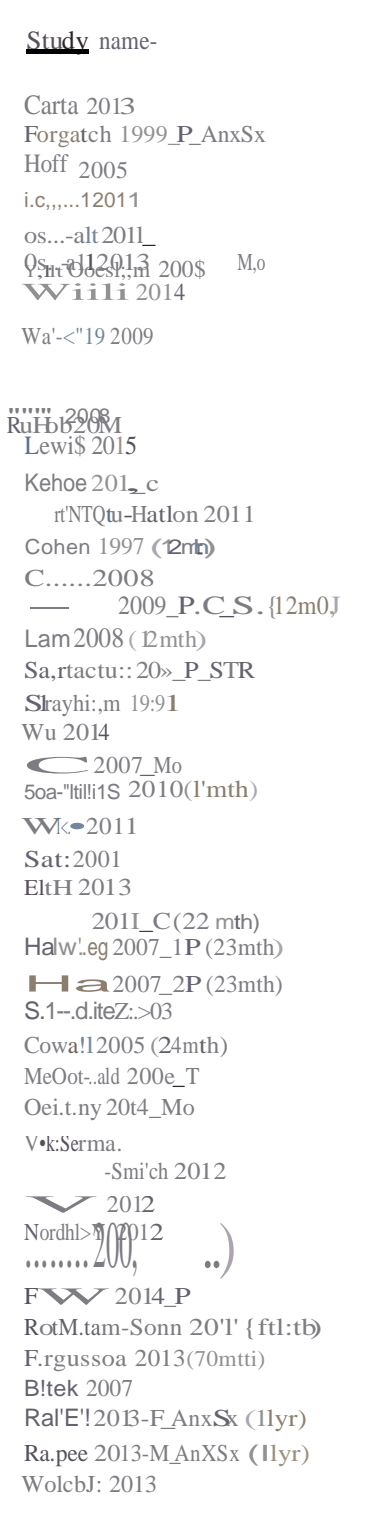

\begin{tabular}{|c|c|c|}
\hline $\begin{array}{l}\text { Std diff } \\
\text { in m9.tns }\end{array}$ & $\begin{array}{l}\text { I.owe, } \\
\text { limit }\end{array}$ & $\begin{array}{c}\text { Ul..,., } \\
\text { limit }\end{array}$ \\
\hline $\begin{array}{r}-0.009 \\
.0 .0 ! 6 \\
-0.783\end{array}$ & $\begin{array}{l}-0.328 \\
.0380 \\
.015\end{array}$ & $\begin{array}{l}\text { O. } 190 \\
\text { O271 } \\
\text { (1) C.0 }\end{array}$ \\
\hline .02233 & linpe & 0.? \\
\hline G. $240{ }^{-O B A S}$ & $\begin{array}{l}1230164 \\
00000\end{array}$ & Q:IYH \\
\hline$-0 ., \$ 8$ & $.0 . S S 0$ & $\begin{array}{l}0: 978 \\
0\end{array}$ \\
\hline $\begin{array}{l}-0.119 \\
-0 S 42\end{array}$ & $\begin{array}{c}.0410 \\
+479\end{array}$ & 0.172 \\
\hline 0. & -0.314 & 0.333 \\
\hline-0.200 & $.0 \mathrm{~m}$ & $.0 . \mathrm{m}$ \\
\hline .0010 & 109\& & -0.137 \\
\hline-0.40 & $703 . !$ & 0.227 \\
\hline 1831 & $z=10 \mathrm{~S}$ & $\mathrm{Sl}{ }^{0.143}$ \\
\hline . P.Sesp & -20 & -82490 \\
\hline-0.510 & 945 & 0025 \\
\hline-0355 & -01eo & 0.061 \\
\hline $\begin{array}{r}0.09 \& \\
-0.133\end{array}$ & $\begin{array}{l}.0 .311 \\
-0502\end{array}$ & $\begin{array}{l}\text { o. } 3 \\
0.250\end{array}$ \\
\hline 0. & J).183 & o.m \\
\hline $\begin{array}{l}.0282 \\
-0320\end{array}$ & $\begin{array}{l}-(\mathbb{R} 18 \\
-0.71\end{array}$ & $\begin{array}{l}0.112 \\
0.111\end{array}$ \\
\hline $\begin{array}{r}-0.53 \\
0.477\end{array}$ & $\begin{array}{l}-0.711 \\
-0042\end{array}$ & $\begin{array}{l}\text { O.ce. } \\
0.995\end{array}$ \\
\hline $\begin{array}{r}0,103 \\
-0.103\end{array}$ & $\begin{array}{l}-0.193 \\
\text { "IIIIIO }\end{array}$ & $\begin{array}{l}0 ., 00 \\
0 . " 9\end{array}$ \\
\hline $0.1 \& 0$ & O202 & 1.317 \\
\hline-0009 & $.03 \& 3$ & 0.344 \\
\hline$-0.31 \cdot \cdot 2$ & -0.911 & 0.207 \\
\hline $\begin{array}{l}\text { uyyu } \\
\text {.a.sos }\end{array}$ & IIIST & intón \\
\hline-0.1 & $-U \leq \mathrm{M}$ & U1: \\
\hline Iopging & $-0 \& 16$ & 0033 \\
\hline .0 .209 & $=83 \$ \$$ & -0.019 \\
\hline .0.021 & & 0.423 \\
\hline 0. & , $0.1 \& 2$ & 0218 \\
\hline-0.150 & -0.356 & $0.0 \mathrm{SS}$ \\
\hline$-0.2 \mathbb{D}$ & $-0 \& 13$ & O. 189 \\
\hline e01 & 7.11\& & .0093 \\
\hline .0.188 & $.0,00$ & 0.430 \\
\hline$-0,01 \&$ & 等. 0.348 & 0315 \\
\hline$-0.1 n$ & -0257 & $-1.0 S 7$ \\
\hline
\end{tabular}

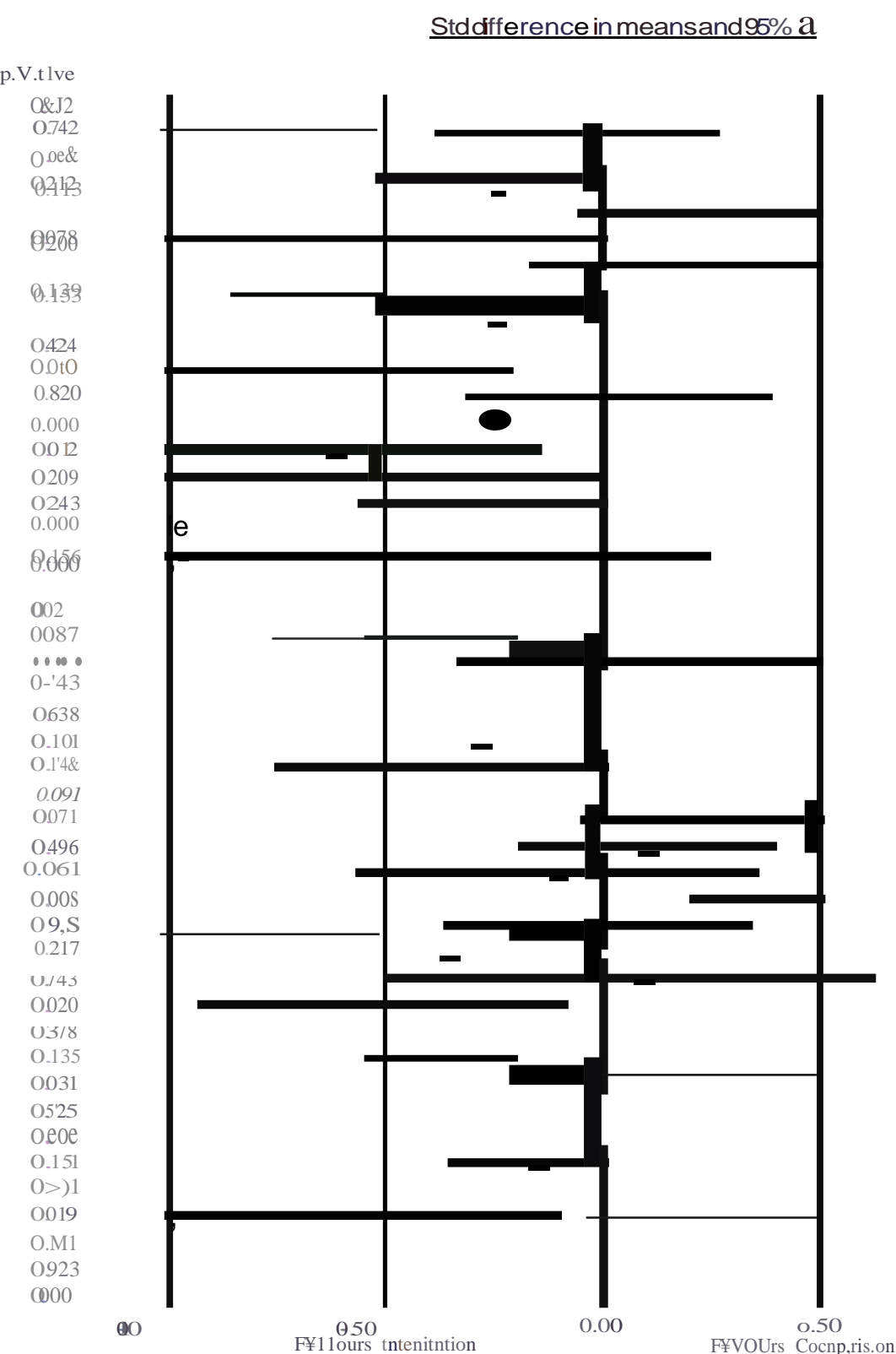


Figure 7

The effects of preventive parenting interventions on the broad cluster of child internalizing problems. Lines represent standardized difference in means (Cohen's $d$ ) and 95\% confidence intervals $(\mathrm{Cl})$; the size of the box represents the weight of each study. Studies are ordered by the duration of the follow-up interval (shortest to longest). 
Table 1

Summary of study characteristics

\begin{tabular}{|c|c|c|c|}
\hline Participant characteristics & Number of comparisons ( $k$ ) & $\%$ & $\%$ after excluding missing data \\
\hline \multicolumn{4}{|l|}{ Type of prevention population } \\
\hline Universal & 9 & 17.6 & \\
\hline Selective & 36 & 70.6 & \\
\hline Indicated & 2 & 3.9 & \\
\hline Selective + Indicated & 1 & 2.0 & \\
\hline Secondary & 3 & 5.9 & \\
\hline \multicolumn{4}{|l|}{ Country } \\
\hline USA & 32 & 62.7 & \\
\hline Australia/New Zealand & 7 & 13.7 & \\
\hline Europe & 10 & 19.6 & \\
\hline Asia & 2 & 3.9 & \\
\hline \multicolumn{4}{|l|}{ Age of children at recruitment } \\
\hline Pre-birth/birth (0-2.99 months) & 13 & 25.5 & \\
\hline Infancy/preschool (3 months-5years) & 16 & 31.4 & \\
\hline Primary school (>5-12 years) & 18 & 35.3 & \\
\hline Adolescence (>12-18 years) & 3 & 5.9 & \\
\hline Not reported & 1 & 2.0 & \\
\hline \multicolumn{4}{|l|}{ Gender mix } \\
\hline Approx. even (40-60\% female) & 37 & 72.5 & 86.0 \\
\hline$>60 \%$ female & 1 & 2.0 & 2.3 \\
\hline$>60 \%$ male & 5 & 9.8 & 11.6 \\
\hline Not reported & 8 & 15.7 & \\
\hline \multicolumn{4}{|l|}{ Program characteristics } \\
\hline \multicolumn{4}{|l|}{ Focus of intervention ${ }^{a}$} \\
\hline Parenting skills & 37 & 72.5 & \\
\hline Parent-child relationship & 31 & 60.8 & \\
\hline
\end{tabular}




\begin{tabular}{|c|c|c|c|}
\hline Participant characteristics & Number of comparisons ( $k$ ) & $\%$ & $\%$ after excluding missing data \\
\hline Parental mental health & 13 & 25.5 & \\
\hline Parent as coach & 9 & 17.6 & \\
\hline Other & 2 & 3.9 & \\
\hline \multicolumn{4}{|l|}{ Delivery format } \\
\hline Group sessions (parent/family) & 20 & 39.2 & \\
\hline Individual sessions (parent/family) & 13 & 25.5 & \\
\hline Mix of group and individual sessions & 2 & 3.9 & \\
\hline Home visits & 11 & 21.6 & \\
\hline Individual sessions and home visits & 3 & 5.9 & \\
\hline Other & 2 & 3.9 & \\
\hline \multicolumn{4}{|l|}{ Total number of intervention hours ${ }^{b}$} \\
\hline 1 to 10 & 16 & 31.4 & 47.1 \\
\hline 11 to 20 & 10 & 19.6 & 29.4 \\
\hline$>20$ & 8 & 15.7 & 23.5 \\
\hline Not reported/Unclear & 17 & 33.3 & \\
\hline \multicolumn{4}{|l|}{ Length of intervention } \\
\hline 0-3 months & 25 & 49.0 & 58.1 \\
\hline$>3-6$ months & 6 & 11.8 & 14.0 \\
\hline$>6-12$ months & 7 & 13.7 & 16.3 \\
\hline$>12$ months & 5 & 9.8 & 11.6 \\
\hline Not reported & 8 & 15.7 & \\
\hline \multicolumn{4}{|l|}{ Direct intervention with child } \\
\hline Yes & 10 & 19.6 & \\
\hline No & 41 & 80.4 & \\
\hline \multicolumn{4}{|l|}{ Method characteristics } \\
\hline \multicolumn{4}{|l|}{ Length of follow-upa } \\
\hline 6-10 months & 25 & 49.0 & \\
\hline$>10-12$ months & 16 & 31.4 & \\
\hline$>12-24$ months & 18 & 35.3 & \\
\hline
\end{tabular}




\begin{tabular}{|c|c|c|c|}
\hline Participant characteristics & Number of comparisons ( $k$ ) & $\%$ & $\%$ after excluding missing data \\
\hline$>24-48$ months & 10 & 19.6 & \\
\hline$>48$ months & 10 & 19.6 & \\
\hline \multicolumn{4}{|l|}{ Comparison condition } \\
\hline Usual care & 19 & 37.3 & \\
\hline No treatment & 14 & 27.5 & \\
\hline Minimal intervention & 9 & 17.6 & \\
\hline Attention control & 6 & 11.8 & \\
\hline Extended waitlist & 3 & 5.9 & \\
\hline
\end{tabular}

aPercentage does not add to 100 because studies could fall into multiple categories.

${ }^{b}$ Calculated from the number of sessions scheduled multiplied by the length of each session. 
Table 2

Meta-analysis and supplementary analyses for internalizing symptoms

\begin{tabular}{|c|c|c|c|c|c|c|c|c|c|c|}
\hline \multirow[t]{2}{*}{ Analysis and Variable } & \multirow[t]{2}{*}{$\begin{array}{c}\text { Number of } \\
\text { Comparisons } \\
\text { (k) }\end{array}$} & \multicolumn{6}{|c|}{ Analysis of Standardised Difference in Means (Cohen's d) } & \multicolumn{3}{|c|}{$\begin{array}{r}\text { Analysis of Group Difference } \\
\text { (Subgroup analyses) }\end{array}$} \\
\hline & & $d$ & $95 \% \mathrm{Cl}$ & z & $p$ & $\begin{array}{c}\mathrm{Q}- \\
\text { value }\end{array}$ & $I^{2}(\%)$ & $\begin{array}{r}\text { Q- } \\
\text { value }\end{array}$ & $\mathrm{df}(\mathrm{Q})$ & $\begin{array}{r}p \text { for } Q- \\
\text { value }\end{array}$ \\
\hline \multicolumn{11}{|l|}{ Overall Analysis } \\
\hline Internalizing symptoms & 37 & -0.123 & $-0.205,-0.042$ & -2.960 & 0.003 & 60.702 & 40.69 & & & \\
\hline \multicolumn{11}{|l|}{ Supplementary Analyses } \\
\hline \multicolumn{11}{|l|}{ Follow-up Interval ${ }^{a}$} \\
\hline 6-10 months post-intervention & 16 & -0.148 & $-0.272,-0.023$ & -2.330 & 0.020 & 22.912 & 34.53 & & & \\
\hline$>10-12$ months post-intervention & 10 & -0.185 & $-0.400,0.030$ & -1.689 & 0.091 & 24.179 & 62.78 & & & \\
\hline$>12-24$ months post-intervention & 15 & -0.050 & $-0.160,0.061$ & -0.877 & 0.380 & 21.249 & 34.11 & & & \\
\hline >24-48 months post-intervention & 6 & -0.124 & $-0.239,-0.009$ & -2.121 & 0.034 & 4.420 & 0.00 & & & \\
\hline$>48$ months post-intervention & 6 & -0.084 & $-0.201,0.033$ & -1.409 & 0.159 & 3.669 & 0.00 & & & \\
\hline \multicolumn{11}{|l|}{ Informant of child outcome $e^{\mathrm{a}}$} \\
\hline $\begin{array}{lll}\text { Child } \\
\end{array}$ & 2 & 0.028 & $-0.171,0.227$ & 0.277 & 0.782 & 0.381 & 0.00 & & & \\
\hline Father & 3 & -0.260 & $-0.742,0.222$ & -1.057 & 0.290 & 3.158 & 36.66 & & & \\
\hline Mother & 17 & -0.139 & $-0.260,-0.018$ & -2.258 & 0.024 & 25.110 & 36.28 & & & \\
\hline Parent or Primary Carer & 13 & -0.170 & $-0.317,-0.023$ & -2.265 & 0.024 & 20.576 & 41.68 & & & \\
\hline Parent and child combined & 1 & -0.016 & $-0.348,0.315$ & -0.097 & 0.923 & 0.000 & 0.00 & & & \\
\hline Teacher & 9 & -0.086 & $-0.289,0.116$ & -0.838 & 0.402 & 17.671 & 54.73 & & & \\
\hline
\end{tabular}




\begin{tabular}{|c|c|c|c|c|c|c|c|c|c|c|}
\hline \multirow[t]{2}{*}{ Analysis and Variable } & \multirow[t]{2}{*}{$\begin{array}{c}\text { Number of } \\
\text { Comparisons } \\
\text { (k) }\end{array}$} & \multicolumn{6}{|c|}{ Analysis of Standardised Difference in Means (Cohen's d) } & \multicolumn{3}{|c|}{$\begin{array}{r}\text { Analysis of Group Difference } \\
\text { (Subgroup analyses) }\end{array}$} \\
\hline & & $d$ & $95 \% \mathrm{Cl}$ & $z$ & $p$ & $\begin{array}{c}\mathrm{Q}- \\
\text { value }\end{array}$ & $I^{2}(\%)$ & $\begin{array}{c}\text { Q- } \\
\text { value }\end{array}$ & $\mathrm{df}(\mathrm{Q})$ & $\begin{array}{r}p \text { for } Q- \\
\text { value }\end{array}$ \\
\hline \multicolumn{11}{|l|}{ Subgroup Analyses } \\
\hline Type of prevention & & & & & & & & 4.539 & 1 & 0.033 \\
\hline Universal & 7 & 0.110 & $-0.099,0.318$ & 1.029 & 0.303 & 13.923 & 56.91 & & & \\
\hline Selective & 26 & -0.134 & $-0.217,-0.052$ & -3.179 & 0.001 & 32.147 & 22.23 & & & \\
\hline Indicated ${ }^{b}$ & 1 & -0.842 & $-1.479,-0.205$ & -2.592 & 0.010 & 0.000 & 0.00 & & & \\
\hline Secondary ${ }^{b}$ & 2 & -0.539 & $-0.922,-0.157$ & -2.765 & 0.006 & 0.270 & 0.00 & & & \\
\hline Selective \& Indicated ${ }^{b}$ & 1 & -0.232 & $-0.595,0.132$ & -1.248 & 0.212 & 0.000 & 0.00 & & & \\
\hline \multicolumn{11}{|l|}{ Focus of intervention } \\
\hline Parenting skills & & & & & & & & 0.445 & 1 & 0.505 \\
\hline Not included & 7 & -0.046 & $-0.295,0.203$ & -0.361 & 0.718 & 12.289 & 51.18 & & & \\
\hline Included & 30 & -0.136 & $-0.222,-0.049$ & -3.073 & 0.002 & 47.930 & 39.50 & & & \\
\hline Parent-child relationship & & & & & & & & 0.183 & 1 & 0.669 \\
\hline Not included & 12 & -0.155 & $-0.320,0.010$ & -1.847 & 0.065 & 23.715 & 53.62 & & & \\
\hline Included & 25 & -0.114 & $-0.209,-0.019$ & -2.349 & 0.019 & 36.986 & 35.11 & & & \\
\hline Parent mental health & & & & & & & & 2.159 & 1 & 0.142 \\
\hline Not included & 26 & -0.089 & $-0.183,0.006$ & -1.844 & 0.065 & 44.187 & 43.42 & & & \\
\hline Included & 11 & -0.233 & $-0.401,-0.065$ & -2.717 & 0.007 & 15.311 & 34.69 & & & \\
\hline Parent as coach & & & & & & & & 0.430 & 1 & 0.512 \\
\hline Not included & 34 & -0.116 & $-0.200,0.033$ & -2.724 & 0.006 & 53.714 & 0.013 & & & \\
\hline Included & 3 & -0.276 & $-0.748,0.195$ & -1.149 & 0.251 & 6.987 & 71.38 & & & \\
\hline Timing of intervention ${ }^{c}$ & & & & & & & & 3.914 & 2 & 0.141 \\
\hline Pre-birth/birth & 12 & -0.178 & $-0.309,-0.048$ & -2.680 & 0.007 & 15.322 & 28.21 & & & \\
\hline Preschool age & 13 & -0.021 & $-0.164,0.122$ & -0.292 & 0.770 & 24.102 & 50.21 & & & \\
\hline Primary-school age & 9 & -0.240 & $-0.439,-0.041$ & -2.363 & 0.018 & 13.920 & 42.53 & & & \\
\hline
\end{tabular}




\begin{tabular}{|c|c|c|c|c|c|c|c|c|c|c|}
\hline \multirow[t]{2}{*}{ Analysis and Variable } & \multirow[t]{2}{*}{$\begin{array}{c}\text { Number of } \\
\text { Comparisons } \\
\text { (k) }\end{array}$} & \multicolumn{6}{|c|}{ Analysis of Standardised Difference in Means (Cohen's d) } & \multicolumn{3}{|c|}{$\begin{array}{r}\text { Analysis of Group Difference } \\
\text { (Subgroup analyses) }\end{array}$} \\
\hline & & $d$ & $95 \% \mathrm{Cl}$ & $\mathrm{z}$ & $p$ & $\begin{array}{c}\mathrm{Q}- \\
\text { value }\end{array}$ & $I^{2}(\%)$ & $\begin{array}{r}\text { Q- } \\
\text { value }\end{array}$ & $\mathrm{df}(\mathrm{Q})$ & $\begin{array}{l}p \text { for } Q- \\
\text { value }\end{array}$ \\
\hline Adolescence $^{b}$ & 2 & 0.018 & $-0.178,0.214$ & 0.180 & 0.857 & 0.603 & 0.00 & & & \\
\hline Type of comparison & & & & & & & & 5.328 & 3 & 0.149 \\
\hline No treatment & 8 & -0.079 & $-0.208,0.049$ & -1.212 & 0.226 & 9.133 & 23.35 & & & \\
\hline Extended waitlist $^{\mathrm{b}}$ & 2 & -0.137 & $-0.338,0.064$ & -1.338 & 0.181 & 0.667 & 0.00 & & & \\
\hline Minimal intervention & 8 & 0.028 & $-0.175,0.230$ & 0.267 & 0.789 & 12.784 & 45.25 & & & \\
\hline Attention control & 5 & -0.271 & $-0.670,0.129$ & -1.329 & 0.184 & 10.426 & 61.64 & & & \\
\hline Usual care & 14 & -0.228 & $-0.367,-0.090$ & -3.237 & 0.001 & 9.133 & 43.74 & & & \\
\hline Direct intervention with child & & & & & & & & 0.386 & 1 & 0.535 \\
\hline No & 31 & -0.127 & $-0.220,-0.034$ & -2.680 & 0.007 & 55.144 & 45.60 & & & \\
\hline Yes & 6 & -0.070 & $-0.224,0.085$ & -0.885 & 0.376 & 5.183 & 3.53 & & & \\
\hline
\end{tabular}

Total number of comparisons exceeds 30 because studies could fall into multiple categories.

${ }^{b}$ Excluded from subgroup analysis because less than 3 comparisons available.

'One study (Etter 2013) was excluded from this subgroup analysis because information about child age at intervention was not reported.

Findings significant at $p<0.05$ are presented in bold. 
Table 3

Meta-analysis and supplementary analyses for depressive symptoms and disorders

\begin{tabular}{|c|c|c|c|c|c|c|c|c|c|c|}
\hline \multirow[t]{2}{*}{ Analysis and Variable } & \multirow[t]{2}{*}{$\begin{array}{c}\text { Number of } \\
\text { Comparisons (k) }\end{array}$} & \multicolumn{6}{|c|}{ Analysis of Standardised Difference in Means (Cohen's $d$ ) } & \multicolumn{3}{|c|}{$\begin{array}{c}\text { Analysis of Group Difference } \\
\text { (Subgroup analyses) }\end{array}$} \\
\hline & & $d$ & $95 \% \mathrm{Cl}$ & $\mathrm{z}$ & $p$ & $\begin{array}{c}\mathrm{Q}- \\
\text { value }\end{array}$ & $I^{2}(\%)$ & Q-value & $\mathrm{df}(\mathrm{Q})$ & $\begin{array}{l}p \text { for } Q- \\
\text { value }\end{array}$ \\
\hline \multicolumn{11}{|l|}{ Overall Analyses } \\
\hline Depressive Symptoms & 10 & -0.156 & $-0.267,-0.046$ & -2.779 & 0.005 & 13.960 & 35.53 & & & \\
\hline Depressive Diagnoses $^{\mathrm{a}}$ & 4 & -0.095 & $-0.198,0.008$ & -1.803 & 0.071 & 5.218 & 42.51 & & & \\
\hline \multicolumn{11}{|c|}{ Supplementary Analyses (Depressive Symptoms only) } \\
\hline \multicolumn{11}{|c|}{ Informant of child outcome ${ }^{\mathrm{b}}$} \\
\hline Child & 8 & -0.169 & $-0.298,-0.040$ & -2.572 & 0.010 & 13.674 & 48.81 & & & \\
\hline Mother & 3 & 0.014 & $-0.228,0.256$ & 0.115 & 0.908 & 0.541 & 0.00 & & & \\
\hline Parent & 3 & -0.459 & $-0.493,-0.424$ & -25.917 & 0.000 & 1.752 & 0.00 & & & \\
\hline \multicolumn{11}{|l|}{ Follow-up Interval ${ }^{b}$} \\
\hline 6-10 months post-intervention & 4 & -0.292 & $-0.689,0.106$ & -1.436 & 0.151 & 8.907 & 66.32 & & & \\
\hline$>10-12$ months post-intervention & 3 & -0.105 & $-0.333,0.122$ & -0.907 & 0.364 & 7.266 & 72.47 & & & \\
\hline$>12-24$ months post-intervention & 1 & -0.139 & $-0.329,0.051$ & -1.437 & 0.151 & 0.000 & 0.00 & & & \\
\hline$>24-48$ months post-intervention & 3 & -0.035 & $-0.176,0.106$ & -0.487 & 0.626 & 0.104 & 0.00 & & & \\
\hline$>48$ months post-intervention & 4 & -0.142 & $-0.322,0.038$ & -1.551 & 0.121 & 4.128 & 27.33 & & & \\
\hline
\end{tabular}




\begin{tabular}{|c|c|c|c|c|c|c|c|c|c|c|}
\hline \multirow[t]{2}{*}{ Analysis and Variable } & \multirow[t]{2}{*}{$\begin{array}{c}\text { Number of } \\
\text { Comparisons (k) }\end{array}$} & \multicolumn{6}{|c|}{ Analysis of Standardised Difference in Means (Cohen's $d$ ) } & \multicolumn{3}{|c|}{$\begin{array}{l}\text { Analysis of Group Difference } \\
\text { (Subgroup analyses) }\end{array}$} \\
\hline & & $d$ & $95 \% \mathrm{Cl}$ & $z$ & $p$ & $\begin{array}{c}\text { Q- } \\
\text { value }\end{array}$ & $1^{2}(\%)$ & Q-value & $\mathrm{df}(\mathrm{Q})$ & $\begin{array}{l}p \text { for Q- } \\
\text { value }\end{array}$ \\
\hline \multicolumn{11}{|c|}{ Subgroup Analyses (Depressive Symptoms only) } \\
\hline Type of prevention & & & & & & & & $\mathrm{n} / \mathrm{a}$ & & \\
\hline Universal & 2 & -0.142 & $-0.176,-0.108$ & -8.145 & 0.000 & 0.491 & 0.00 & & & \\
\hline Selective & 8 & -0.176 & $-0.389,0.038$ & -1.613 & 0.107 & 13.446 & 47.94 & & & \\
\hline Indicated & 0 & $\mathrm{n} / \mathrm{a}$ & $\mathrm{n} / \mathrm{a}$ & $\mathrm{n} / \mathrm{a}$ & $\mathrm{n} / \mathrm{a}$ & $\mathrm{n} / \mathrm{a}$ & $\mathrm{n} / \mathrm{a}$ & & & \\
\hline Secondary & 0 & $\mathrm{n} / \mathrm{a}$ & $\mathrm{n} / \mathrm{a}$ & $\mathrm{n} / \mathrm{a}$ & $\mathrm{n} / \mathrm{a}$ & $\mathrm{n} / \mathrm{a}$ & $\mathrm{n} / \mathrm{a}$ & & & \\
\hline \multicolumn{11}{|l|}{ Focus of intervention } \\
\hline Parenting skills & & & & & & & & $\mathrm{n} / \mathrm{a}$ & & \\
\hline Not included & 0 & $\mathrm{n} / \mathrm{a}$ & $\mathrm{n} / \mathrm{a}$ & $\mathrm{n} / \mathrm{a}$ & $\mathrm{n} / \mathrm{a}$ & $\mathrm{n} / \mathrm{a}$ & $\mathrm{n} / \mathrm{a}$ & & & \\
\hline Included & 10 & -0.156 & $-0.267,-0.046$ & -2.779 & 0.005 & 13.960 & 35.53 & & & \\
\hline Parent-child relationship & & & & & & & & 0.019 & 1 & 0.890 \\
\hline Not included & 5 & -0.182 & $-0.494,0.129$ & -1.147 & 0.251 & 7.115 & 43.78 & & & \\
\hline Included & 5 & -0.159 & $-0.274,-0.044$ & -2.714 & 0.007 & 6.813 & 41.29 & & & \\
\hline Parent mental health & & & & & & & & 0.727 & 1 & 0.394 \\
\hline Not included & 6 & -0.140 & $-0.174,-0.106$ & -8.095 & 0.000 & 4.295 & 0.00 & & & \\
\hline Included & 4 & -0.300 & $-0.665,0.065$ & -1.609 & 0.108 & 8.903 & 66.30 & & & \\
\hline Parent as coach & & & & & & & & $\mathrm{n} / \mathrm{a}$ & & \\
\hline Not included & 8 & -0.149 & $-0.265,-0.033$ & -2.519 & 0.012 & 11.756 & 40.46 & & & \\
\hline Included & 2 & -0.237 & $-0.774,0.301$ & -0.863 & 0.388 & 1.840 & 45.64 & & & \\
\hline Timing of intervention & & & & & & & & 0.139 & 1 & 0.709 \\
\hline Pre-birth/birth & 0 & $\mathrm{n} / \mathrm{a}$ & $\mathrm{n} / \mathrm{a}$ & $\mathrm{n} / \mathrm{a}$ & $\mathrm{n} / \mathrm{a}$ & $\mathrm{n} / \mathrm{a}$ & $\mathrm{n} / \mathrm{a}$ & & & \\
\hline Preschool age & 3 & -0.163 & $-0.515,0.188$ & -0.911 & 0.362 & 2.456 & 18.56 & & & \\
\hline Primary-school age & 5 & -0.246 & $-0.496,0.005$ & -1.924 & 0.054 & 8.971 & 55.41 & & & \\
\hline Adolescence ${ }^{c}$ & 2 & -0.119 & $-0.217,-0.020$ & -2.349 & 0.019 & 1.379 & 27.48 & & & \\
\hline
\end{tabular}




\begin{tabular}{|c|c|c|c|c|c|c|c|c|c|c|}
\hline \multirow[t]{2}{*}{ Analysis and Variable } & \multirow[t]{2}{*}{$\begin{array}{c}\text { Number of } \\
\text { Comparisons (k) }\end{array}$} & \multicolumn{6}{|c|}{ Analysis of Standardised Difference in Means (Cohen's $d$ ) } & \multicolumn{3}{|c|}{$\begin{array}{l}\text { Analysis of Group Difference } \\
\text { (Subgroup analyses) }\end{array}$} \\
\hline & & $d$ & $95 \% \mathrm{Cl}$ & $z$ & $p$ & $\begin{array}{c}\text { Q- } \\
\text { value }\end{array}$ & $\mathrm{I}^{2}(\%)$ & Q-value & $\mathrm{df}(\mathrm{Q})$ & $\begin{array}{l}p \text { for } Q- \\
\text { value }\end{array}$ \\
\hline Type of comparison & & & & & & & & $\mathrm{n} / \mathrm{a}$ & & \\
\hline No treatment & 5 & -0.126 & $-0.216,-0.037$ & -2.769 & 0.006 & 4.313 & 7.27 & & & \\
\hline Extended waitlist & 0 & $\mathrm{n} / \mathrm{a}$ & $\mathrm{n} / \mathrm{a}$ & $\mathrm{n} / \mathrm{a}$ & $\mathrm{n} / \mathrm{a}$ & $\mathrm{n} / \mathrm{a}$ & $\mathrm{n} / \mathrm{a}$ & & & \\
\hline Usual care & 1 & 0.000 & $-0.231,0.231$ & 0.000 & 1.000 & 0.000 & 0.00 & & & \\
\hline Direct intervention with child & & & & & & & & $\mathrm{n} / \mathrm{a}$ & & \\
\hline No & 8 & -0.190 & $-0.359,-0.020$ & -2.192 & 0.028 & 12.030 & 41.81 & & & \\
\hline Yes & 2 & -0.115 & $-0.319,0.088$ & -1.109 & 0.267 & 1.877 & 46.71 & & & \\
\hline
\end{tabular}

aRisk difference rather than Cohen's $d$ statistics are shown in this row.

bTotal number of comparisons exceeds 10 because studies could fall into multiple categories.

${ }^{c}$ Excluded from subgroup analysis because less than 3 comparisons available.

Findings significant at $p<0.05$ are presented in bold. 
Table 4

Meta-analysis and supplementary analyses for anxiety symptoms and disorders

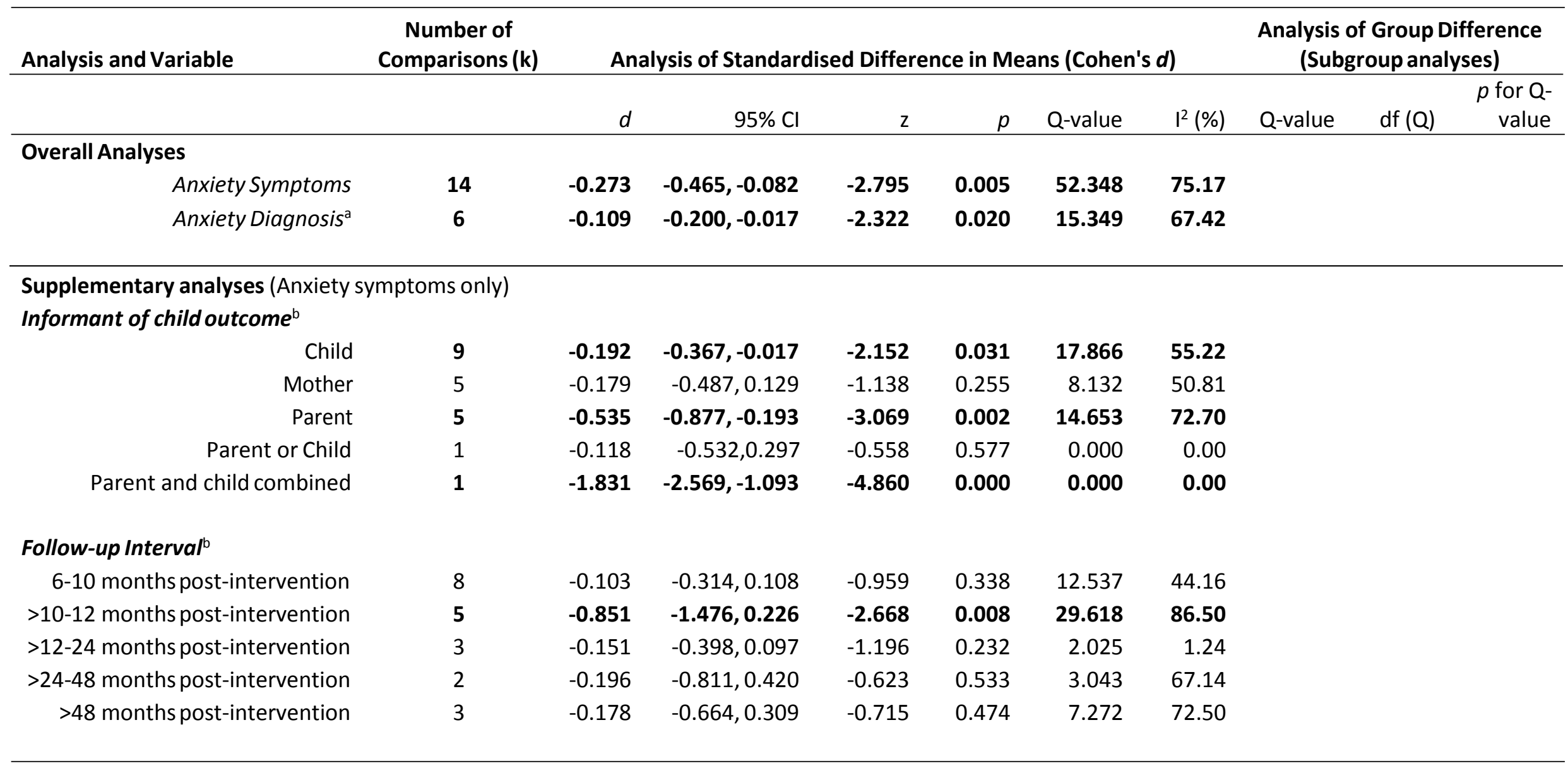




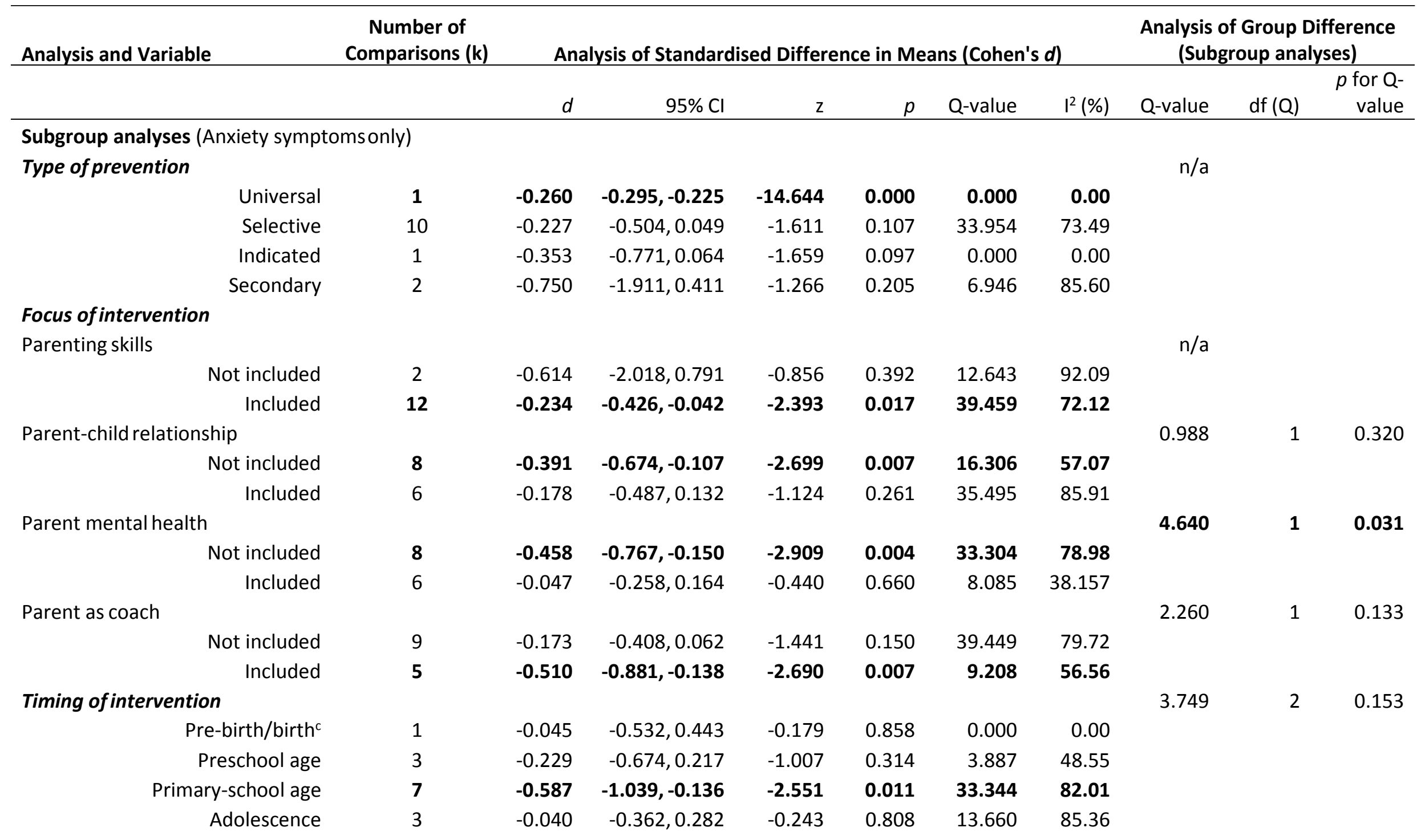




\begin{tabular}{|c|c|c|c|c|c|c|c|c|c|c|}
\hline Analysis and Variable & $\begin{array}{c}\text { Number of } \\
\text { Comparisons (k) }\end{array}$ & \multicolumn{6}{|c|}{ Analysis of Standardised Difference in Means (Cohen's $d$ ) } & \multicolumn{3}{|c|}{$\begin{array}{l}\text { Analysis of Group Difference } \\
\text { (Subgroup analyses) }\end{array}$} \\
\hline Extended waitlist & 1 & -1.831 & $-2.569,-1.093$ & -4.860 & 0.000 & 0.000 & 0.00 & & & \\
\hline Minimal intervention & 2 & 0.045 & $-0.228,0.318$ & 0.323 & 0.746 & 0.043 & 0.00 & & & \\
\hline Attention control & 2 & -0.381 & $-1.201,0.440$ & -0.910 & 0.363 & 2.603 & 61.58 & & & \\
\hline Direct intervention with child & & & & & & & & 0.197 & 1 & 0.657 \\
\hline No & 11 & -0.258 & $-0.431,-0.085$ & -2.926 & 0.003 & 21.181 & 52.79 & & & \\
\hline Yes & 3 & -0.460 & $-1.337,0.416$ & -1.029 & 0.303 & 25.209 & 92.07 & & & \\
\hline
\end{tabular}

aRisk difference rather than Cohen's $d$ statistics are shown in this row.

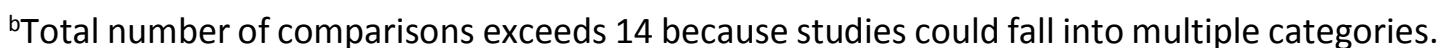

cExcluded from subgroup analysis because less than 3 comparisons available.

Findings significant at $p<0.05$ are presented in bold. 
Table 5

Meta-analysis and supplementary analyses for measures of internalizing, anxiety or depressive symptoms

\begin{tabular}{|c|c|c|c|c|c|c|c|c|c|c|}
\hline \multirow[t]{2}{*}{ Analysis and Variable } & \multirow[t]{2}{*}{$\begin{array}{c}\text { Number of } \\
\text { Comparisons (k) }\end{array}$} & \multicolumn{6}{|c|}{ Analysis of Standardised Difference in Means (Cohen's d) } & \multicolumn{3}{|c|}{$\begin{array}{r}\text { Analysis of Group Difference } \\
\text { (Subgroup analyses) }\end{array}$} \\
\hline & & $d$ & $95 \% \mathrm{Cl}$ & $\mathbf{z}$ & $p$ & Q-value & $I^{2}(\%)$ & $\begin{array}{c}\text { Q- } \\
\text { value }\end{array}$ & df (Q) & $\begin{array}{l}p \text { for } Q- \\
\text { value }\end{array}$ \\
\hline \multicolumn{11}{|l|}{ Overall Analysis } \\
\hline $\begin{array}{r}\text { Internalizing/anxiety/depressive } \\
\text { symptoms }\end{array}$ & 45 & -0.177 & $-0.257,-0.097$ & -4.350 & 0.000 & 112.746 & 60.97 & & & \\
\hline \multicolumn{11}{|l|}{ Supplementary Analyses } \\
\hline \multicolumn{11}{|l|}{ Follow-up Intervala } \\
\hline 6-10 months post-intervention & 20 & -0.151 & $-0.266,-0.035$ & -2.549 & 0.011 & 28.962 & 34.40 & & & \\
\hline$>10-12$ months post-intervention & 14 & -0.288 & $-0.469,-0.107$ & -3.120 & 0.002 & 65.309 & 80.10 & & & \\
\hline$>12-24$ months post-intervention & 18 & -0.078 & $-0.172,0.016$ & -1.635 & 0.102 & 23.676 & 28.20 & & & \\
\hline$>24-48$ months post-intervention & 7 & -0.138 & $-0.253,-0.023$ & -2.347 & 0.019 & 7.247 & 17.20 & & & \\
\hline$>48$ months post-intervention & 9 & -0.138 & $-0.238,-0.039$ & -2.722 & 0.006 & 8.305 & 3.68 & & & \\
\hline \multicolumn{11}{|l|}{ Informant of child outcome $e^{\mathrm{a}}$} \\
\hline Child & 9 & -0.175 & $-0.297,-0.053$ & -2.817 & 0.005 & 14.675 & 45.49 & & & \\
\hline Father & 3 & -0.260 & $-0.742,0.222$ & -1.057 & 0.290 & 3.158 & 36.66 & & & \\
\hline Mother & 22 & -0.139 & $-0.253,-0.026$ & -2.409 & 0.016 & 34.737 & 39.55 & & & \\
\hline Parent or Primary Carer & 18 & -0.254 & $-0.409,-0.098$ & -3.200 & 0.001 & 69.644 & 75.59 & & & \\
\hline Parent and child combined & 2 & -0.892 & $-2.670,0.885$ & -0.984 & 0.325 & 19.308 & 94.82 & & & \\
\hline Teacher & 9 & -0.086 & $-0.289,0.116$ & -0.838 & 0.402 & 17.671 & 54.73 & & & \\
\hline \multicolumn{11}{|l|}{ Subgroup Analyses } \\
\hline \multirow[t]{2}{*}{ Type of prevention } & & & & & & & & 9.087 & 2 & 0.011 \\
\hline & 9 & 0.006 & $-0.169,0.181$ & 0.069 & 0.945 & 31.822 & 74.86 & & & \\
\hline
\end{tabular}




\begin{tabular}{|c|c|c|c|c|c|c|c|c|c|c|}
\hline \multirow[t]{2}{*}{ Analysis and Variable } & \multirow[t]{2}{*}{$\begin{array}{c}\text { Number of } \\
\text { Comparisons (k) }\end{array}$} & \multicolumn{6}{|c|}{ Analysis of Standardised Difference in Means (Cohen's d) } & \multicolumn{3}{|c|}{$\begin{array}{r}\text { Analysis of Group Difference } \\
\text { (Subgroup analyses) }\end{array}$} \\
\hline & & $d$ & $95 \% \mathrm{Cl}$ & $\mathbf{z}$ & $p$ & Q-value & $I^{2}(\%)$ & $\begin{array}{c}\text { Q- } \\
\text { value }\end{array}$ & df (Q) & $\begin{array}{r}p \text { for Q- } \\
\text { value }\end{array}$ \\
\hline Selective & 30 & -0.186 & $-0.285,-0.086$ & -3.654 & 0.000 & 56.143 & 48.35 & & & \\
\hline Indicated $^{b}$ & 2 & -0.536 & $-1.000,-0.073$ & -2.267 & 0.023 & 1.584 & 36.86 & & & \\
\hline Secondary & 3 & -0.770 & $-1.286,-0.255$ & -2.929 & 0.003 & 4.608 & 56.60 & & & \\
\hline Selective $\&$ Indicated ${ }^{b}$ & 1 & -0.232 & $-0.595,0.132$ & -1.248 & 0.212 & 0.000 & 0.00 & & & \\
\hline \multicolumn{11}{|l|}{ Focus of intervention } \\
\hline Parenting skills & & & & & & & & 0.002 & 1 & 0.968 \\
\hline Not included & 8 & -0.185 & $-0.512,0.143$ & -1.106 & 0.269 & 26.172 & 73.25 & & & \\
\hline Included & 37 & -0.178 & $-0.259,-0.096$ & -4.283 & 0.000 & 85.188 & 57.74 & & & \\
\hline Parent-child relationship & & & & & & & & 0.790 & 1 & 0.374 \\
\hline Not included & 17 & -0.240 & $-0.400,-0.081$ & -2.948 & 0.003 & 41.317 & 61.28 & & & \\
\hline Included & 29 & -0.155 & $-0.253,-0.058$ & -3.136 & 0.002 & 68.883 & 60.80 & & & \\
\hline Parent mental health & & & & & & & & 0.616 & 1 & 0.432 \\
\hline Not included & 34 & -0.161 & $-0.253,-0.069$ & -3.417 & 0.001 & 96.443 & 65.78 & & & \\
\hline Included & 11 & -0.238 & $-0.408,-0.069$ & -2.670 & 0.006 & 15.584 & 35.83 & & & \\
\hline Parent as coach & & & & & & & & 2.999 & 1 & 0.083 \\
\hline Not included & 38 & -0.145 & $-0.228,-0.063$ & -3.449 & 0.001 & 90.929 & 59.31 & & & \\
\hline Included & 7 & -0.452 & $-0.789,-0.115$ & -2.628 & 0.009 & 21.454 & 72.03 & & & \\
\hline Timing of intervention ${ }^{c}$ & & & & & & & & 6.845 & 3 & 0.077 \\
\hline Pre-birth/birth & 12 & -0.178 & $-0.309,-0.048$ & -2.680 & 0.007 & 15.322 & 28.21 & & & \\
\hline Preschool age & 15 & -0.050 & $-0.196,0.096$ & -0.670 & 0.503 & 27.754 & 49.56 & & & \\
\hline Primary-school age & 14 & -0.386 & $-0.594,-0.178$ & -3.630 & 0.000 & 44.576 & 70.84 & & & \\
\hline Adolescence & 3 & -0.128 & $-0.367,0.111$ & -1.049 & 0.294 & 8.104 & 75.32 & & & \\
\hline
\end{tabular}




\begin{tabular}{|c|c|c|c|c|c|c|c|c|c|c|}
\hline \multirow[t]{2}{*}{ Analysis and Variable } & \multirow[t]{2}{*}{$\begin{array}{c}\text { Number of } \\
\text { Comparisons (k) }\end{array}$} & \multicolumn{6}{|c|}{ Analysis of Standardised Difference in Means (Cohen's d) } & \multicolumn{3}{|c|}{$\begin{array}{r}\text { Analysis of Group Difference } \\
\text { (Subgroup analyses) }\end{array}$} \\
\hline & & $d$ & $95 \% \mathrm{Cl}$ & $\mathbf{z}$ & $p$ & Q-value & $I^{2}(\%)$ & $\begin{array}{c}\text { Q- } \\
\text { value }\end{array}$ & df (Q) & $\begin{array}{r}p \text { for } Q- \\
\text { value }\end{array}$ \\
\hline Type of comparison & & & & & & & & 5.238 & 4 & 0.264 \\
\hline No treatment & 14 & -0.176 & $-0.307,-0.045$ & -2.634 & 0.008 & 31.863 & 59.20 & & & \\
\hline Extended waitlist & 3 & -0.605 & $-1.292,0.082$ & -1.726 & 0.084 & 19.491 & 89.74 & & & \\
\hline Minimal intervention & 9 & -0.017 & $-0.197,0.164$ & -0.182 & 0.856 & 16.289 & 50.89 & & & \\
\hline Attention control & 5 & -0.271 & $-0.670,0.129$ & -1.329 & 0.184 & 10.426 & 61.64 & & & \\
\hline Usual care & 14 & -0.228 & $-0.367,-0.090$ & -3.237 & 0.001 & 23.105 & 43.74 & & & \\
\hline Direct intervention with child & & & & & & & & 0.751 & 1 & 0.386 \\
\hline No & 37 & -0.164 & $-0.250,-0.078$ & -3.753 & 0.000 & 84.805 & 57.55 & & & \\
\hline Yes & 8 & -0.285 & $-0.544,-0.026$ & -2.154 & 0.031 & 26.682 & 73.77 & & & \\
\hline
\end{tabular}

Total number of comparisons exceeds 45 because studies could fall into multiple categories.

${ }^{b}$ Excluded from subgroup analysis because less than 3 comparisons available.

'One study (Etter 2013) was excluded from this subgroup analysis because information about child age at intervention could not be obtained.

Findings significant at $p<0.05$ are presented in bold. 


\section{Appendix A. Searchterms}

\section{Searches performed in April 2011 and updated in November}

2012 used the following terms:

\section{PubMed}

(depressive disorder[mh] OR depressi*[tw] OR anxiety[tw] OR anxiety disorders [mh] OR internalizing[ $\mathrm{tw}]$ OR externalising[tw] OR externalizing[tw]) AND (program[tw] OR training[tw] OR education[tw] OR "Early I ntervention (Education)"[mh]) AND ("clinical trial"[tw] OR randomized controlled trial[pt] OR controlled clinical trial[pt]) AND parent*[tw]

\section{Psycl NFO (CSAI lumina)}

( $(\mathrm{kw}=$ internali?ing $\mathrm{OR} \mathrm{kw}=$ externali?ing) or $\mathrm{DE}=$ (major depression or seasonal affective disorder or Dysthymic Disorder or Anxiety disorders or "acute stress disorder" or "generalized anxiety disorder" or "obsessive compulsive disorder" or "panic disorder" or "phobias" or "agoraphobia" or "claustrophobia" or "school phobia" or "social phobia" or "posttraumatic stress disorder" or adolescent psychopathology or child psychopathology or Adolescentpsychology or adolescent psychiatry or Child psychology or child psychiatry or Psychiatric symptoms or Abnormal psychology)) and (parent* within 5 (training or program or education or intervention)) AND ("clinical trial" OR "controlled trial" OR random*) AND (ME=(empirical study) or $\mathrm{ME}=$ (followup study) or $\mathrm{ME}=$ (longitudinal study) or $\mathrm{ME}=$ (prospective study) or $\mathrm{ME}=$ (quantitative study) or $\mathrm{ME}=($ treatment outcome/clinicaltrial $))$

\section{CENTRAL}

(parent* near/5 training or parent* near/5 program* or parent* near/ 5 education or parent* near/5 intervention) AND (depressi* OR anxi* OR internali?ing OR externali?ing OR emotional OR mental health)

(in Clinical Trials)

\section{Embase (Elsevier)}

'depression'/exp OR 'depression' OR 'anxiety disorder'/exp OR 'anxiety disorder' OR 'anxiety'/exp OR 'anxiety' OR internali?ing OR externali?ing OR 'emotional disorder'/exp OR 'emotional disorder' AND parent* NEAR/5 (training OR program OR education OR intervention) AND ([controlled clinical trial]/lim OR [ randomized controlled trial]/lim OR 'clinical trial' OR 'controlled trial')

207 hits

An updated search was conducted in April 2014, with slight modification of some search terms for the Embase and Psycl NFO databases, due to a change in database providers: 


\section{Embase (Ovid)}

(exp depression/ or exp 'anxiety disorder'/ or exp 'emotional disorder'/ or exp 'anxiety'/ or 'emotional disorder'.mp. or depression.mp. or anxiety.mp. or 'anxiety disorder'.mp. or internali\#ing.mp. or externali\#ing.mp.) AND (parent* adj5 (training or program or education or intervention)). mp AND (clinical trial' or 'controlled trial).mp.

Limits applied

Clinical trial or randomized controlled trial or controlled clinical trial or multicenter study or phase 1 clinical trial or phase 2 clinical trial or phase 3 clinical trial or phase 4 clinical trial; Publication date from 2012-2014

\section{Psycl NFO (EBSCOhost)}

( ( $\mathrm{KW}$ internali?ing OR KW externali?ing) OR (DE "major depression" or DE "seasonal affective disorder" or DE "Dysthymic Disorder" or DE "Anxiety disorders" or DE "acute stress disorder" or DE "generalized anxiety disorder" or DE "obsessive compulsive disorder" or DE "panic disorder" or DE "phobias" or De "agoraphobia" or DE "claustrophobia" or DE "school phobia" or DE "social phobia" or DE "posttraumatic stress disorder" or DE "adolescent psychopathology" or DE "child psychopathology" or DE "Adolescent psychology" or DE "adolescent psychiatry" or DE "Child psychology" or DE "child psychiatry" or DE "Psychiatric symptoms" or DE "Abnormal psychology")) ) AND ( (parent* W5 (training OR program OR education OR intervention) ) ) AND ( ("controlled trial" OR "clinical trial" OR random*) )

Limits applied

Publication Year: 2012-2014; Methodology: EMPI RI CALSTUDY, -Followup Study, -Longitudinal Study, ---Prospective Study, -Quantitative Study, TREATMENT OUTCOME/CLI NI CAL TRIAL

\section{A further updated search was conducted in J uly 2015 and used the terms from the 2011 search above, dueto database providers changing back.}




\begin{tabular}{|c|c|}
\hline Citation & $\begin{array}{l}\text { Reason for } \\
\text { exclusion }\end{array}$ \\
\hline $\begin{array}{l}\text { 1. Abedi MR and Vostanis P. 2010. Evaluation of quality of life therapy } \\
\text { for parents of children with obsessive-compulsive disorders in Iran. } \\
\text { European child \& adolescent psychiatry, 19(7), 605-613. }\end{array}$ & $\begin{array}{l}\text { No long term } \\
\text { follow-up }\end{array}$ \\
\hline $\begin{array}{l}\text { 2. Abendroth D, Moser DK, Dracup K and Doering LV. 1999. Do apnea } \\
\text { monitors decrease emotional distress in parents of infants at high risk } \\
\text { for cardiopulmonary arrest? J Pediatr Health Care. 13(2):50-7. }\end{array}$ & $\begin{array}{l}\text { No child } \\
\text { internalising } \\
\text { outcomes or not } \\
\text { a major goal }\end{array}$ \\
\hline $\begin{array}{l}\text { 3. Abikoff, H., Hechtman, L., Klein, R. G., Weiss, G., Fleiss, K., Etcovitch, J., } \\
\text { et al. (2004). Symptomatic improvement in children with ADHD } \\
\text { treated with long-term methylphenidate and multimodal psychosocial } \\
\text { treatment. Journal of the American Academy of Child and Adolescent } \\
\text { Psychiatry, (7), 802-811. }\end{array}$ & $\begin{array}{l}\text { No child } \\
\text { internalising } \\
\text { outcomes or not } \\
\text { a major goal }\end{array}$ \\
\hline $\begin{array}{l}\text { 4. Aboud, F. E., Singla, D. R., Nahil, M. I., \& Borisova, I. (2013). } \\
\text { Effectiveness of a parenting program in Bangladesh to address early } \\
\text { childhood health, growth and development. Soc Sci Med, 97, 250-258. } \\
\text { doi: } 10.1016 / \text { j.socscimed.2013.06.020 }\end{array}$ & $\begin{array}{l}\text { No child } \\
\text { internalising } \\
\text { outcomes or not } \\
\text { a major goal }\end{array}$ \\
\hline $\begin{array}{l}\text { 5. Achenbach, T. M., et al. (1990). Seven-year outcome of the Vermont } \\
\text { Intervention Program for Low-Birthweight Infants. Child Development } \\
\text { 61(6): 1672-1681. }\end{array}$ & $\begin{array}{l}\text { No child } \\
\text { internalising } \\
\text { outcomes or not } \\
\text { a major goal }\end{array}$ \\
\hline $\begin{array}{l}\text { 6. Adams, S., Morris, D., Gilmore, G., \& Frampton, I. (2010). A novel } \\
\text { parent-supported emotional literacy programme for children. } \\
\text { Community practitioner : the journal of the Community Practitioners' } \\
\text { \& Health Visitors' Association, (8), 27-30. }\end{array}$ & $\begin{array}{l}\text { No long term } \\
\text { follow-up }\end{array}$ \\
\hline $\begin{array}{l}\text { 7. Adams, W. G., Phillips, B. D., Bacic, J. D., Walsh, K. E., Shanahan, C. W., } \\
\text { \& Paasche-Orlow, M. K. (2014). Automated Conversation System } \\
\text { Before Pediatric Primary Care Visits: A Randomized Trial. Pediatrics, } \\
\text { 134(3), e691-e699. }\end{array}$ & $\begin{array}{l}\text { Not a parenting } \\
\text { intervention }\end{array}$ \\
\hline $\begin{array}{l}\text { 8. Adesso VJ and Lipson JW. 1981. Group training of parents as therapists } \\
\text { for the children. Behavior Therapy, } 12(5), 625-633 \text {. }\end{array}$ & $\begin{array}{l}\text { Follow-up less } \\
\text { than } 6 \text { months }\end{array}$ \\
\hline $\begin{array}{l}\text { 9. Afshar, H., Nakhjavani, Y. B., Mahmoudi-Gharaei, J., Paryab, M., \& } \\
\text { Zadhoosh, S. (2011). The effect of parental presence on the } 5 \text { year-old } \\
\text { children's anxiety and cooperative behavior in the first and second } \\
\text { dental visit. Iranian Journal of Pediatrics, } 21,193-200 .\end{array}$ & $\begin{array}{l}\text { Not a parenting } \\
\text { intervention }\end{array}$ \\
\hline $\begin{array}{l}\text { 10. Albano, A. M., Marten, P. A., Holt, C. S., Heimberg, R. G., \& Barlow, D. } \\
\text { H. (1995). Cognitive-behavioral group treatment for social phobia in } \\
\text { adolescents. A preliminary study. Journal of Nervous and Mental } \\
\text { Disease, 183(10), 649-656. }\end{array}$ & $\begin{array}{l}\text { Not an RCT; } \\
\text { Parent } \\
\text { component too } \\
\text { small }\end{array}$ \\
\hline $\begin{array}{l}\text { 11. Alcázar, A. I. R., Olivares-Olivares, P. J., \& Rodríguez, J. O. (2009). The } \\
\text { role of non-specific effects in the psychological treatment of } \\
\text { adolescents with social phobia. Anuario de Psicología/The UB Journal } \\
\text { of Psychology, 40(1), 43-61. }\end{array}$ & $\begin{array}{l}\text { Not a parenting } \\
\text { intervention }\end{array}$ \\
\hline
\end{tabular}


12. Alcock DS, Feldman W, Goodman JT, McGrath PJ and Park JM. 1985. Evaluation of child life intervention in emergency department suturing. Pediatr Emerg Care. 1(3):111-5.

13. Ale, C. M., \& Krackow, E. (2011). Concurrent treatment of early childhood OCD and ODD: A case illustration. Clinical Case Studies, 10, 312-323.

14. Alexander JF, Robbins MS, Sexton TL. Family-based interventions with older, at-risk youth: from promise to proof to practice. J Prim Prev. 2000;21:185-205

15. Alfano CA, Pina AA, Villalta IK, Beidel DC, Ammerman RT and Crosby LE. 2009. Mediators and moderators of outcome in the behavioral treatment of childhood social phobia. Journal of the American Academy of Child \& Adolescent Psychiatry. 48(9):945-53.

16. Alink LRA, Mesman J, van Zeijl J, Stolk MN, Juffer F, BakermansKranenburg MJ, van ljzendoorn MH and Koot HM. 2009. Maternal sensitivity moderates the relation between negative discipline and aggression in early childhood. Social Development. 18(1):99-120.

17. Al-Mutairi H. 2000. Conduct disorders among Kuwaiti children living in low-income families. Journal of the Social Sciences. 28(3):65-88.

18. Als, L. C., Vickers, B., Nadel, S., Cooper, M., \& Garralda, M. E. (2014). A Brief Intervention To Improve Parent Post-traumatic Stress Symptoms Following Paediatric Critical Illness: A Pilot Randomised Controlled Trial. Archives of Disease in Childhood, 99(Suppl 2), A159-A160.

19. Al-Turkait FA and Ohaeri JU. 2008. Psychopathological status, behavior problems, and family adjustment of Kuwaiti children whose fathers were involved in the first Gulf War. Child and Adolescent Psychiatry and Mental Health. 2(12).

20. Aman, M. G., McDougle, C. J., Scahill, L., Handen, B., Arnold, L. E., Johnson, C., et al. (2009). Medication and Parent Training in Children With Pervasive Developmental Disorders and Serious Behavior Problems: Results From a Randomized Clinical Trial. Journal of the American Academy of Child and Adolescent Psychiatry, 48(12), 11431154.

21. Ambrosino JM, Fennie K, Whittemore R, Jaser S, Dowd MF and Grey M. 2008. Short-term effects of coping skills training in school-age children with type 1 diabetes. Pediatr Diabetes. 9(3 Pt 2):74-82.

22. Aminabadi, N. A., Vafaei, A., Erfanparast, L., Oskouei, S. G., \& Jamali, Z. (2011). Impact of pictorial story on pain perception, situational anxiety and behavior in children: a cognitive-behavioral schema. J Clin Pediatr Dent, 36, 127-132.

Intervention for developmental disorders (autism, autism spectrum disorder), physical disabilities, medical conditions or distress about facing medical interventions

Not an RCT

Review

Not a parenting intervention

Not an RCT

No child internalising outcomes or not a major goal Not an RCT

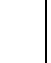

\section{Follow-up less} than 6 months

Follow-up less than 6 months

Not a parenting intervention 
23. Ammerman RT, Putnam FW, Altaye M, Chen L, Holleb LJ, Stevens J, Short JA and Van Ginkel JB. 2009. Changes in depressive symptoms in first time mothers in home visitation. Child Abuse \& Neglect. 33(3):127-38.

24. Amorós, M. O., Carrillo, F. J. M., \& Sanchez, J. P. E. (2005). Contribución de la Economía de Fichas a la Eficacia de las Escenificaciones Emotivas en el Tratamiento de la Fobia a la Oscuridad / Contribution of the token economy to the efficacy of emotive performances for treating phobia of the dark. Ansiedad y estrés, 11(1), 7-16.

25. Anastopoulos, A. D., Shelton, T. L., DuPaul, G. J., \& Guevremont, D. C. (1993). Parent training for attention-deficit hyperactivity disorder: Its impact on parent functioning. Journal of Abnormal Child Psychology, 21(5), 581-596. doi: 10.1007/bf00916320

26. Anderson, B., Katz, L. L., McKay, S., Nadeau, K., Casey, T., Higgins, L., Hirst, K., Izquierdo, R., Lauer, A., Wauters, A. (2014). Study medication adherence and outcomes in the TODAY cohort of youth with type 2 diabetes (T2D). Pediatric Diabetes, 1522.

27. Anderson, K. M., Danis, F. S., \& Havig, K. (2011). Adult daughters of battered women: Recovery and posttraumatic growth following childhood adversity. Families in Society, 92, 154-160.

28. Angold, A., Erkanli, A., Copeland, W., Goodman, R., Fisher, P. W., \& Costello, E. J. (2012). Psychiatric diagnostic interviews for children and adolescents: a comparative study. J Am Acad Child Adolesc Psychiatry, 51, 506-517.

29. Annett RD, Bender BG, Skipper B and Allen C. 2010. Predicting moderate improvement and decline in pediatric asthma quality of life over 24 months. Qual Life Res. 19(10):1517-27.

30. Antshel KM and Remer R. 2003. Social skills training in children with attention deficit hyperactivity disorder: a randomized-controlled clinical trial. J Clin Child Adolesc Psychol. 32(1):153-65.

31. Arch LM, Humphris GM and Lee GT. 2001. Children choosing between general anaesthesia or inhalation sedation for dental extractions: the effect on dental anxiety. International Journal of Paediatric Dentistry, 11(1), 41-48.

32. Armstrong KL, Fraser JA, Dadds MR and Morris J. 2000. Promoting secure attachment, maternal mood and child health in a vulnerable population: a randomized controlled trial. Journal of paediatrics and child health, 36(6), 555-562.
No child

internalising outcomes or not a major goal

Not in English

Follow-up less than 6 months

Not a parenting intervention

Not a parenting intervention

Not a parenting intervention

Not an RCT

Not a parenting intervention

Intervention for developmental disorders (autism, autism spectrum disorder), physical disabilities, medical conditions or distress about facing medical interventions Follow-up less than 6 months 
33. Armstrong, K. L., Fraser, J. A., Dadds, M. R., \& Morris, J. (1999). A randomized, controlled trial of nurse home visiting to vulnerable families with newborns. Journal of Paediatrics and Child Health, 35(3), 237-244.

34. Arnold DH, Brown SA, Meagher S, Baker CN, Dobbs J and DoctoroffGL. 2006. Preschool-Based Programs for Externalizing Problems. Education \& Treatment of Children. 29(2):311-39.

35. Arnold LE, Farmer C, Kraemer HC, Davies M, Witwer A, Chuang S, Disilvestro R, McDougle CJ, McCracken J, Vitiello B, Aman MG, Scahill L, Posey DJ and Swiezy NB. 2010. Moderators, mediators, and other predictors of risperidone response in children with autistic disorder and irritability. Journal of Child and Adolescent Psychopharmacology. 20(2):83-93.

36. Arnold, L. E., Gadow, K. D., Farmer, C. A., Findling, R. L., Bukstein, O., Molina, B. S., ... \& Aman, M. G. (2015). Comorbid anxiety and social avoidance in treatment of severe childhood aggression: response to adding risperidone to stimulant and parent training; mediation of disruptive symptom response. Journal of child and adolescent psychopharmacology, 25(3), 203-212.

37. Aronen ET, Arajärvi T. Effects of early intervention on psychiatric symptoms of young adults in low-risk and high-risk families. American Journal of Orthopsychiatry. 2000 Apr;70(2):223-32.

38. Aronen ET, Kurkela SA. Long-term effects of an early home-based intervention. J Am Acad Child Adolesc Psychiatry. 1996 Dec;35(12):1665-72.

39. Aronen, E., Kuosa, M., Tallila, M., \& Arajärvi, T. (1995). Kotikäynteihin perustuvan perheneuvonnan vaikutus nuoren psyykkiseen terveyteen-15 vuoden seurantatutkimus. [Effect of home-based family counseling on the mental health of youths--15-year follow-up study]. Duodecim, 111(6), 505-509.

40. Asarnow, J. R., Scott, C. V., \& Mintz, J. (2002). A combined cognitivebehavioral family education intervention for depression in children: $A$ treatment development study. Cognitive Therapy and Research, 26(2), 221-229. doi: 10.1023/a:1014573803928

41. Asgeirsdottir, B. B., Sigfusdottir, I. D., Gudjonsson, G. H., \& Sigurdsson, J. F. (2011). Associations between sexual abuse and family conflict/violence, self-injurious behavior, and substance use: The mediating role of depressed mood and anger. Child Abuse \& Neglect, 35, 210-219.

42. Atwine B, Cantor-Graae E and Bajunirwe F. 2005. Psychological distress among AIDS orphans in rural Uganda. Social Science \& Medicine. 61(3):555-64.

43. Auerbach, V., Nixon, R., Forrest, K., Gooley, S., \& Gemke, G. (1999). Group intervention program for oppositional, noncompliant and aggressive preschoolers. Clinical Psychologist, 3, 30-34.

44. August GJ, Lee SS, Bloomquist ML, Realmuto GM and Hektner JM. 2004. Maintenance Effects of an Evidence-Based Prevention Innovation for Aggressive Children Living in Culturally Diverse Urban Neighborhoods: The Early Risers Effectiveness Study. Journal of Emotional and Behavioral Disorders. 12(4):194-205. 


\begin{tabular}{|c|c|}
\hline $\begin{array}{l}\text { 45. Aune, T., \& Stiles, T. C. (2009). Universal-based prevention of } \\
\text { syndromal and subsyndromal social anxiety: A randomized controlled } \\
\text { study. J Consult Clin Psychol, 77(5), 867-879. doi:2009-17643-008 }\end{array}$ & $\begin{array}{l}\text { Parent } \\
\text { component too } \\
\text { small }\end{array}$ \\
\hline $\begin{array}{l}\text { 46. Auslander BA, Short MB, Succop PA and Rosenthal SL. } 2009 \text {. } \\
\text { Associations between parenting behaviors and adolescent romantic } \\
\text { relationships. Journal of Adolescent Health. 45(1):98-101. }\end{array}$ & Not an RCT \\
\hline $\begin{array}{l}\text { 47. Babinski, D. E., Pelham, W. E., Jr., Molina, B. S. G., Waschbusch, D. A., } \\
\text { Gnagy, E. M., Yu, J., Sibley, M. H., \& Biswas, A. (2011). Women with } \\
\text { childhood ADHD: Comparisons by diagnostic group and gender. } \\
\text { Journal of Psychopathology and Behavioral Assessment, 33, 420-429. }\end{array}$ & $\begin{array}{l}\text { Not a parenting } \\
\text { intervention }\end{array}$ \\
\hline $\begin{array}{l}\text { 48. Baer S and Garland EJ. 2005. Pilot Study of Community-Based } \\
\text { Cognitive Behavioral Group Therapy for Adolescents With Social } \\
\text { Phobia. Journal of the American Academy of Child \& Adolescent } \\
\text { Psychiatry. 44(3):258-64. }\end{array}$ & $\begin{array}{l}\text { Not a parenting } \\
\text { intervention }\end{array}$ \\
\hline $\begin{array}{l}\text { 49. Bagner DM and Eyberg SM. 2003. Father involvement in parent } \\
\text { training: when does it matter? J Clin Child Adolesc Psychol. 32(4):599- } \\
605 .\end{array}$ & $\begin{array}{l}\text { Follow-up less } \\
\text { than } 6 \text { months }\end{array}$ \\
\hline $\begin{array}{l}\text { 50. Bagner DM, Sheinkopf SJ, Miller-Loncar C, LaGasse LL, Lester BM, Liu J, } \\
\text { Bauer CR, Shankaran S, Bada H and Das A. 2009. The effect of } \\
\text { parenting stress on child behavior problems in high-risk children with } \\
\text { prenatal drug exposure. Child Psychiatry and Human Development. } \\
\text { 40(1):73-84. }\end{array}$ & Not an RCT \\
\hline $\begin{array}{l}\text { 51. Bagner DM, Sheinkopf SJ, Miller-Loncar CL, Vohr BR, HinckleyM, } \\
\text { Eyberg SM and Lester BM. 2009. Parent-child interaction therapy for } \\
\text { children born premature: A case study and illustration of vagal tone as } \\
\text { a physiological measure of treatment outcome. Cognitive and } \\
\text { Behavioral Practice. 16(4):468-77. }\end{array}$ & Not an RCT \\
\hline $\begin{array}{l}\text { 52. Bagner DM, Sheinkopf SJ, Vohr BR and Lester BM. 2010. Parenting } \\
\text { intervention for externalizing behavior problems in children born } \\
\text { premature: An initial examination. Journal of Developmental and } \\
\text { Behavioral Pediatrics. 31(3):209-16. }\end{array}$ & $\begin{array}{l}\text { Follow-up less } \\
\text { than } 6 \text { months }\end{array}$ \\
\hline $\begin{array}{l}\text { 53. Bagner, D. M., \& Graziano, P. A. (2013). Barriers to success in parent } \\
\text { training for young children with developmental delay: the role of } \\
\text { cumulative risk. Behav Modif, 37(3), 356-377. doi: } \\
10.1177 / 0145445512465307\end{array}$ & $\begin{array}{l}\text { Secondary } \\
\text { analysis (data } \\
\text { not usable) }\end{array}$ \\
\hline $\begin{array}{l}\text { 54. Bagner, D. M., Graziano, P. A., Jaccard, J., Sheinkopf, S. J., Vohr, B. R., } \\
\text { \& Lester, B. M. (2012). An Initial Investigation of Baseline Respiratory } \\
\text { Sinus Arrhythmia as a Moderator of Treatment Outcome for Young } \\
\text { Children Born Premature With Externalizing Behavior Problems. } \\
\text { Behavior Therapy, 43(3), 652-665. doi: } \\
\text { http://dx.doi.org/10.1016/j.beth.2011.12.002 }\end{array}$ & $\begin{array}{l}\text { No child } \\
\text { internalising } \\
\text { outcomes or not } \\
\text { a major goal }\end{array}$ \\
\hline $\begin{array}{l}\text { 55. Bagner, D. M., Rodriguez, G. M., Blake, C. A., \& Rosa-Olivares, J. } \\
\text { (2013). Home-Based Preventive Parenting Intervention for at-Risk } \\
\text { Infants and Their Families: An Open Trial. Cognitive and Behavioral } \\
\text { Practice, 20(3), 334-348. doi: } \\
\text { http://dx.doi.org/10.1016/j.cbpra.2012.08.001 }\end{array}$ & $\begin{array}{l}\text { Follow-up less } \\
\text { than } 6 \text { months }\end{array}$ \\
\hline $\begin{array}{l}\text { 56. Bailey KA, Chavira DA, Stein MT and Stein MB. 2006. Brief Measures to } \\
\text { Screen for Social Phobia in Primary Care Pediatrics. Journal of Pediatric } \\
\text { Psychology. 31(5):513-21. }\end{array}$ & $\begin{array}{l}\text { Not a parenting } \\
\text { intervention }\end{array}$ \\
\hline
\end{tabular}


57. Baker, C. N. (2010). Relationships between contextual characteristics, parent implementation, and child outcome within an academic preventive intervention for preschoolers (Order No. 3427496). Available from ProQuest Dissertations \& Theses Global. (814731426).

58. Baker, C. N., Arnold, D. H., \& Meagher, S. (2011). Enrollment and attendance in a parent training prevention program for conduct problems. Prevention Science, 12, 126-138.

59. Baker-Henningham H, Walker SP, Powell C and Gardner JM. 2009. Preventing behaviour problems through a universal intervention in Jamaican basic schools: a pilot study. West Indian Med J. 58(5):460-4.

60. Bakermans-Kranenburg MJ and van ljzendoorn MH. 2008. Oxytocin receptor (OXTR) and serotonin transporter (5-HTT) genes associated with observed parenting. Social Cognitive and Affective Neuroscience. $3(2): 128-34$.

61. Bakermans-Kranenburg MJ and van ljzendoorn MH. 2009. The first 10,000 adult attachment interviews: Distributions of adult attachment representations in clinical and non-clinical groups. Attachment \& Human Development. 11(3):223-63.

62. Bakermans-Kranenburg, M. J., Van ljzendoorn, M. H., Mesman, J., Alink, L. R., \& Juffer, F. (2008). Effects of an attachment-based intervention on daily cortisol moderated by dopamine receptor D4: a randomized control trial on 1- to 3-year-olds screened for externalizing behavior. Dev Psychopathol, 20(3), 805-820. doi: S0954579408000382

63. Bakermans-Kranenburg, M. J., Van ljzendoorn, M. H., Pijlman, F. T. A., Mesman, J., \& Juffer, F. (2008). Experimental evidence for differential susceptibility: Dopamine D4 receptor polymorphism (DRD4 VNTR) moderates intervention effects on toddlers' externalizing behavior in a randomized controlled trial. Developmental Psychology, 44(1), 293300. doi: 10.1037/0012-1649.44.1.293

64. Bang KS. 2009. [Effects of an early nursing intervention program for infants' development and mother's child rearing in poverty]. J Korean Acad Nurs. 39(6):796-804.

65. Bank, L., Marlowe, J. H., Reid, J. B., Patterson, G. R., \& Weinrott, M. R. (1991). A comparative evaluation of parent-training interventions for families of chronic delinquents. Journal of abnormal child psychology, (1), 15-33.

66. Bannon, W. M., Jr., \& McKay, M. M. (2007). Addressing Urban African American Youth Externalizing and Social Problem Behavioral Difficulties in a Family Oriented Prevention Project. Social Work in Mental Health, 5(1-2), 221-240. doi:10.1300/J200v05n01_11

67. Bao X, Sun S, Wei S. Early intervention promotes intellectual development of premature infants: a preliminary report. Chin Med J 1999;116:520-3.

Targets externalising

No long term follow-up Not a parenting intervention Not a parenting intervention

Not a parenting intervention follow-up . No long term follow-up No child internalising outcomes or not a major goal No long term follow-up

No child internalising outcomes or not a major goal 
68. Barbieri, L., Cicconetti, A., Serveli, S., Blanchi, I., D'Ulivo, B., Mezzano, P., \& Da Rin Della Mora, R. (2011). Assessment of an educationalbehavioral intervention program for premature infants' mothers in nicu. Acta Paediatrica, International Journal of Paediatrics, 100, 111.

69. Barker ED, Vitaro F, Lacourse E, Fontaine NM, Carbonneau R, Tremblay RE. Testing the developmental distinctiveness of male proactive and reactive aggression with a nested longitudinal experimental intervention. Aggressive behavior [serial on the Internet]. 2010; (2): Available from: http://www.mrw.interscience.wiley.com/cochrane/clcentral/articles/ 616/CN-00743616/frame.html.

70. Barkley RA, Guevremont DC, Anastopoulos AD and Fletcher KE.1992. A comparison of three family therapy programs for treating family conflicts in adolescents with attention-deficit hyperactivity disorder. J Consult Clin Psychol. 60(3):450-62.

71. Barkley RA, Shelton TL, Crosswait C, Moorehouse M, Fletcher K, Barrett S, Jenkins L and Metevia L. 2002. Preschool children with disruptive behavior: three-year outcome as a function of adaptive disability. Dev Psychopathol. 14(1):45-67.

72. Barkley, R. A., Shelton, T. L., Crosswait, C., Moorehouse, M., Fletcher, K., Barrett, S., et al. (2000). Multi-method psycho-educational intervention for preschool children with disruptive behavior: preliminary results at post-treatment. J Child Psychol Psychiatry, 41(3), 319-332.

73. Barlow A, Varipatis-Baker E, Speakman K et al. Home-visiting intervention to improve child care among American Indian adolescent mothers. Arch Pediatr Adolesc Med. 2006;160:1101-1107.

74. Barlow J, Coren E and Stewart-Brown S. 2002. Meta-analysis of the effectiveness of parenting programmes in improving maternal psychosocial health. British Journal of General Practice. 52(476):22333.

75. Barlow J, Davis $\mathrm{H}$, Mclntosh $\mathrm{E}$, et al. Role of home visiting in improving parenting and health in families at risk of abuse and neglect: results of a multicentre randomised controlled trial and economic evaluation. Arch Dis Child 2007;92:229-33.

76. Barlow J, Powell L and Gilchrist M. 2006. The influence of the training and support programme on the self-efficacy and psychological wellbeing of parents of children with disabilities: a controlled trial. Complement Ther Clin Pract. 12(1):55-63.

No child

internalising outcomes or not a major goal; Follow-up less than 6 months No child internalising outcomes or not a major goal

Follow-up less than 6 months

Not an RCT

No long term follow-up

No child internalising outcomes or not a major goal

Review

No long term follow-up

Intervention for developmental disorders (autism, autism spectrum disorder), physical disabilities, medical conditions or distress about facing medical interventions 


\begin{tabular}{|c|c|}
\hline $\begin{array}{l}\text { 77. Barlow JH, Powell LA, Gilchrist M and Fotiadou M. 2008. The } \\
\text { effectiveness of the Training and Support Program for parents of } \\
\text { children with disabilities: a randomized controlled trial. J Psychosom } \\
\text { Res. } 64(1): 55-62 \text {. }\end{array}$ & $\begin{array}{l}\text { Intervention for } \\
\text { developmental } \\
\text { disorders } \\
\text { (autism, autism } \\
\text { spectrum } \\
\text { disorder), } \\
\text { physical } \\
\text { disabilities, } \\
\text { medical } \\
\text { conditions or } \\
\text { distress about } \\
\text { facing medical } \\
\text { interventions }\end{array}$ \\
\hline $\begin{array}{l}\text { 78. Barlow, A., Mullany, B., Neault, N., Compton, S., Carter, A., Hastings, } \\
\text { R., ,.. \& Walkup, J. T. (2013). Effect of a Paraprofessional Home-Visiting } \\
\text { Intervention on American Indian Teen Mothers' and Infants' } \\
\text { Behavioral Risks: A Randomized Controlled Trial. American Journal of } \\
\text { Psychiatry, 170(1), 83-93. }\end{array}$ & $\begin{array}{l}\text { Follow-up less } \\
\text { than } 6 \text { months }\end{array}$ \\
\hline $\begin{array}{l}\text { 79. Barlow, A., Mullany, B., Neault, N., Goklish, N., Billy, T., Hastings, } \\
\text { R., ... Walkup, J. T. (2015). Paraprofessional-delivered home-visiting } \\
\text { intervention for American Indian teen mothers and children: 3-year } \\
\text { outcomes from a randomized controlled trial. The American Journal of } \\
\text { Psychiatry, 172(2), 154-162. }\end{array}$ & $\begin{array}{l}\text { No long term } \\
\text { follow-up }\end{array}$ \\
\hline $\begin{array}{l}\text { 80. Barlow, J., \& Stewart-Brown, S. (2000). Behavior problems and group- } \\
\text { based parent education programs. Journal of Developmental and } \\
\text { Behavioral Pediatrics, 21(5), 356-370. }\end{array}$ & Review \\
\hline $\begin{array}{l}\text { 81. Barlow, J., N. Smailagic, et al. (2011). Individual and group based } \\
\text { parenting programmes for improving psychosocial outcomes for } \\
\text { teenage parents and their children. Cochrane Database of Systematic } \\
\text { Reviews(3), 1-97. }\end{array}$ & Review \\
\hline $\begin{array}{l}\text { 82. Barlow, J., Sembi, S., Gardner, F., Macdonald, G., Petrou, S., Parsons, } \\
\text { H., . . Dawe, S. (2013). An evaluation of the parents under pressure } \\
\text { programme: a study protocol for an RCT into its clinical and cost } \\
\text { effectiveness. Trials, 14, 210. doi:10.1186/1745-6215-14-210 }\end{array}$ & $\begin{array}{l}\text { Data not } \\
\text { available/report } \\
\text { ed }\end{array}$ \\
\hline $\begin{array}{l}\text { 83. Barnet B, Liu J, DeVoe M, Alperovitz-Bichell K and Duggan AK. } 2007 . \\
\text { Home visiting for adolescent mothers: effects on parenting, maternal } \\
\text { life course, and primary care linkage. Ann Fam Med.5(3):224-32. }\end{array}$ & $\begin{array}{l}\text { No child } \\
\text { internalising } \\
\text { outcomes or not } \\
\text { a major goal }\end{array}$ \\
\hline $\begin{array}{l}\text { 84. Barnet, B., Duggan, A. K., Devoe, M., \& Burrell, L. (2002). The effect of } \\
\text { volunteer home visitation for adolescent mothers on parenting and } \\
\text { mental health outcomes: a randomized trial. Archives of pediatrics \& } \\
\text { adolescent medicine, (12), 1216-1222. }\end{array}$ & $\begin{array}{l}\text { No child } \\
\text { internalising } \\
\text { outcomes or not } \\
\text { a major goal }\end{array}$ \\
\hline $\begin{array}{l}\text { 85. Barrera M and Schulte F. 2009. A group social skills intervention } \\
\text { program for survivors of childhood brain tumors. J Pediatr Psychol. } \\
\text { 34(10):1108-18. }\end{array}$ & $\begin{array}{l}\text { Not a parenting } \\
\text { intervention }\end{array}$ \\
\hline $\begin{array}{l}\text { 86. Barrera M, Chung JYY, Greenberg M and Fleming C. 2002. Preliminary } \\
\text { investigation of a group intervention for siblings of pediatric cancer } \\
\text { patients. Children's Health Care. } 31(2): 131-42 \text {. }\end{array}$ & Not an RCT \\
\hline
\end{tabular}


87. Barrera, M. E. (1986). Low birth weight and homeintervention strategies: preterm infants. Journal of developmental and behavioral pediatrics 7(6): 361 .

88. Barrera, M. E. (1987). Stability of early home intervention effects with preterm infants: one-year follow-up. Early Child Development and Care 27(4): 635-649.

89. Barrera, M. E., et al. (1991). A 3-Year Early Home Intervention Followup Study with Low Birthweight Infants and Their Parents. Topics in Early Childhood Special Education 10(4): 14-28.

90. Barrera, M. E., Rosenbaum, P. L., \& Cunningham, C. E. (1986). Early home intervention with low-birth-weight infants and their parents. Child development, (1), 20-33.

91. Barrera, M., Jr., Biglan, A., Taylor, T. K., Gunn, B. K., Smolkowski, K., Black, C., et al. (2002). Early elementary school intervention to reduce conduct problems: a randomized trial with Hispanic and non-Hispanic children. Prev Sci, 3(2), 83-94.

92. Barrera, M., Rokeach, A., Hancock, K., Cataudella, D., Schulte, F., Chung, J., ... \& Atenafu, E. (2014). A randomized controlled trial to evaluate the efficacy of a group social skills intervention for children with brain tumours. Neuro-oncology, 16 i111.

93. Barrera, M., Rokeach, A., Hancock, K., Schulte, F., Atenafu, E., \& Nathan, P. (2014). Reduction of anxiety levels in parents and siblings of children with cancer after sibling participation in a psychosocial group intervention: A randomized controlled trial. Pediatric blood \& cancer, $61 \mathrm{~S} 161$.

94. Barrett PM, Dadds MR and Rapee RM. 1996. Family treatment of childhood anxiety: A controlled trial. Journal of Consulting and Clinical Psychology. 64(2):333-42.

95. Barrett PM, Duffy AL, Dadds MR and Rapee RM. 2001. Cognitivebehavioral treatment of anxiety disorders in children: Long-term (6year) follow-up. Journal of Consulting and Clinical Psychology. 69(1):135-41.

96. Barrett PM, Shortt AL and Wescombe K. 2001. Examining the social validity of the FRIENDS treatment program for anxious children. Behaviour Change. 18(2):63-77.

97. Barrett, P. M., Farrell, L. J., Ollendick, T. H., \& Dadds, M. (2006). Longterm outcomes of an Australian universal prevention trial of anxiety and depression symptoms in children and youth: an evaluation of the friends program. Journal Of Clinical Child And Adolescent Psychology: The Official Journal For The Society Of Clinical Child And Adolescent Psychology, American Psychological Association, Division 53, 35, 403411.
Follow-up data collected only in active group; Parent component too small

No child

internalising

outcomes or not a major goal

No child

internalising

outcomes or not

a major goal

No child

internalising

outcomes or not

a major goal

No child

internalising

outcomes or not

a major goal

Parent

component too

small

Not a parenting

intervention

Not a parenting

intervention

Follow-up data collected only in active group;

Parent

component too

small

Not an RCT

Not a parenting intervention 


\begin{tabular}{|c|c|}
\hline $\begin{array}{l}\text { 98. Barrett, P. M., Lock, S., \& Farrell, L. J. (2005). Developmental } \\
\text { Differences in Universal Preventive Intervention for Child Anxiety. } \\
\text { Clinical Child Psychology and Psychiatry, 10, 539-555. }\end{array}$ & $\begin{array}{l}\text { Not a parenting } \\
\text { intervention }\end{array}$ \\
\hline $\begin{array}{l}\text { 99. Barrett, P. M., Moore, A. F., \& Sonderegger, R. (2000). The FRIENDS } \\
\text { Program for young former-Yugoslavian refugees in Australia: A pilot } \\
\text { study. Behaviour Change. Special Issue: Adolescent health, 17(3), 124- } \\
\text { 133. doi: } 10.1375 / \text { bech.17.3.124 }\end{array}$ & $\begin{array}{l}\text { Not a parenting } \\
\text { intervention; No } \\
\text { long term } \\
\text { follow-up; Not } \\
\text { an RCT }\end{array}$ \\
\hline $\begin{array}{l}\text { 100. Barrett, P. M., Sonderegger, R., \& Sonderegger, N. L. (2001). } \\
\text { Evaluation of an Anxiety-prevention and Positive-coping Program } \\
\text { (FRIENDS) for Children and Adolescents of Non-English-speaking } \\
\text { Background. Behaviour Change, 18, 78-91. }\end{array}$ & $\begin{array}{l}\text { Not a parenting } \\
\text { intervention }\end{array}$ \\
\hline $\begin{array}{l}\text { Barrett, P., \& Turner, C. (2001). Prevention of anxiety } \\
\text { symptoms in primary school children: preliminary results from a } \\
\text { universal school-based trial. The British Journal Of Clinical Psychology, } \\
40,399-410 .\end{array}$ & $\begin{array}{l}\text { Not a parenting } \\
\text { intervention }\end{array}$ \\
\hline $\begin{array}{l}102 . \quad \text { Barrett, P., Healy, L., \& March, J. S. (2003). Behavioral } \\
\text { avoidance test for childhood obsessive-compulsive disorder: A home- } \\
\text { based observation. American Journal of Psychotherapy, 57(1), 80-100. }\end{array}$ & $\begin{array}{l}\text { No long term } \\
\text { follow-up }\end{array}$ \\
\hline $\begin{array}{l}\text { 103. Barrett, P., Turner, C., Rombouts, S., \& Duffy, A. (2000). } \\
\text { Reciprocal skills training in the treatment of externalising behaviour } \\
\text { disorders in childhood: A preliminary investigation. Behaviour Change, } \\
\text { 17(4), 221-234. doi: } 10.1375 / \text { bech.17.4.221 }\end{array}$ & $\begin{array}{l}\text { Not a parenting } \\
\text { intervention; No } \\
\text { long term } \\
\text { follow-up }\end{array}$ \\
\hline $\begin{array}{l}\text { 104. Barrington J, Prior M, Richardson M and Allen K. } 2005 . \\
\text { Effectiveness of CBT Versus Standard Treatment for Childhood Anxiety } \\
\text { Disorders in a Community Clinic Setting. Behaviour Change. 22(1):29- } \\
\text { 43. }\end{array}$ & $\begin{array}{l}\text { Not a parenting } \\
\text { intervention }\end{array}$ \\
\hline $\begin{array}{l}\text { 105. Baruch G, Vrouva I and Wells C. 2011. Outcome findings from } \\
\text { a parent training programme for young people with conduct } \\
\text { problems. Child and Adolescent Mental Health. 16(1):47-54. }\end{array}$ & Not an RCT \\
\hline $\begin{array}{l}\text { 106. Baskin, T. W., Rhody, M., Schoolmeesters, S., \& Ellingson, C. } \\
\text { (2011). Supporting special-needs adoptive couples: Assessing an } \\
\text { intervention to enhance forgiveness, increase marital satisfaction, and } \\
\text { prevent depression ?. The Counseling Psychologist, 39, 933-955. }\end{array}$ & $\begin{array}{l}\text { Follow-up less } \\
\text { than } 6 \text { months }\end{array}$ \\
\hline $\begin{array}{l}\text { 107. Bastani, F., Ali Abadi, T., \& Haghani, H. (2012). The } \\
\text { effectiveness of participatory care program in neonatal intensive care } \\
\text { unit on state anxiety of mothers of preterm newborns. Journal of } \\
\text { Babol University of Medical Sciences, 14, 59-65. }\end{array}$ & $\begin{array}{l}\text { No child } \\
\text { internalising } \\
\text { outcomes or not } \\
\text { a major goal; No } \\
\text { long term } \\
\text { follow-up }\end{array}$ \\
\hline $\begin{array}{l}\text { 108. Bateson, K., Delaney, J., \& Pybus, R. (2008). Meeting } \\
\text { expectations: the pilot evaluation of the Solihull Approach Parenting } \\
\text { Group. Community Pract, } 81(5), 28-31 .\end{array}$ & Not an RCT \\
\hline
\end{tabular}




\begin{tabular}{|c|c|}
\hline $\begin{array}{l}\text { 109. Bauchner H, Vinci R, Bak S, Pearson C and Corwin MJ. } 1996 . \\
\text { Parents and procedures: a randomized controlled trial. Pediatrics, } \\
\text { 98(5), 861-867. }\end{array}$ & $\begin{array}{l}\text { Intervention for } \\
\text { developmental } \\
\text { disorders } \\
\text { (autism, autism } \\
\text { spectrum } \\
\text { disorder), } \\
\text { physical } \\
\text { disabilities, } \\
\text { medical } \\
\text { conditions or } \\
\text { distress about } \\
\text { facing medical } \\
\text { interventions }\end{array}$ \\
\hline $\begin{array}{l}\text { 110. Baum \& Forehand } 1981 \text { Long term follow-up assessment of } \\
\text { parent training by multiple outcome measures. Behavior Therapy. }\end{array}$ & $\begin{array}{l}\text { Not an RCT; No } \\
\text { child } \\
\text { internalising } \\
\text { outcomes or not } \\
\text { a major goal }\end{array}$ \\
\hline $\begin{array}{l}\text { Baydar N, Reid MJ and Webster-Stratton C. 2003. The role of } \\
\text { mental health factors and program engagement in the effectiveness of } \\
\text { a preventive parenting program for Head Start mothers. Child Dev. } \\
74(5): 1433-53 \text {. }\end{array}$ & $\begin{array}{l}\text { No child } \\
\text { internalising } \\
\text { outcomes or not } \\
\text { a major goal }\end{array}$ \\
\hline $\begin{array}{l}\text { 112. Bayer J, Hiscock H, Scalzo K, Mathers M, McDonald M, Morris } \\
\text { A, Birdseye J and Wake M. 2009. Systematic review of preventive } \\
\text { interventions for children's mental health: What would work in } \\
\text { Australian contexts? Australian and New Zealand Journal of Psychiatry. } \\
\text { 43(8):695-710. }\end{array}$ & Review \\
\hline $\begin{array}{l}\text { 113. Bayer J, Sanson AV and Hemphill SA. 2009. Early childhood } \\
\text { aetiology of internalising difficulties: A longitudinal community study. } \\
\text { The International Journal of Mental Health Promotion. 11(1):4-14. }\end{array}$ & $\begin{array}{l}\text { Not a parenting } \\
\text { intervention }\end{array}$ \\
\hline $\begin{array}{l}\text { Bayer JK, Hiscock H, Ukoumunne OC, Price A and Wake M. } \\
\text { 2008. Early childhood aetiology of mental health problems: A } \\
\text { longitudinal population-based study. Journal of Child Psychology and } \\
\text { Psychiatry. 49(11):1166-74. }\end{array}$ & $\begin{array}{l}\text { Not a parenting } \\
\text { intervention }\end{array}$ \\
\hline $\begin{array}{l}\text { 115. Bayer JK, Rapee RM, Hiscock H, Ukoumunne OC, Mihalopoulos } \\
\text { C and Wake M. 2011. Translational research to prevent internalizing } \\
\text { problems early in childhood. Depression and Anxiety. 28(1):50-7. }\end{array}$ & Review \\
\hline $\begin{array}{l}\text { 116. Bayer JK, Rapee RM, Hiscock H, Ukoumunne OC, Mihalopoulos } \\
\text { C, Clifford S and Wake M. 2011. The Cool Little Kids randomised } \\
\text { controlled trial: population-level early prevention for anxiety } \\
\text { disorders. BMC Public Health. 11:11. }\end{array}$ & $\begin{array}{l}\text { Data not } \\
\text { available/report } \\
\text { ed (protocol) }\end{array}$ \\
\hline $\begin{array}{l}\text { 117. Bayer JK, Sanson AV and Hemphill SA. 2006. Parent influences } \\
\text { on early childhood internalizing difficulties. Journal of Applied } \\
\text { Developmental Psychology. 27(6):542-59. }\end{array}$ & Not an RCT \\
\hline $\begin{array}{l}\text { 118. Bayer, J. K., Hiscock, H., Ukoumunne, O. C., Scalzo, K., \& Wake, } \\
\text { M. (2010). Three-year-old outcomes of a brief universal parenting } \\
\text { intervention to prevent behaviour problems: randomised controlled } \\
\text { trial. Arch Dis Child, 95(3), 187-192. doi: adc.2009.168302 }\end{array}$ & $\begin{array}{l}\text { Targets } \\
\text { externalising }\end{array}$ \\
\hline $\begin{array}{l}\text { 119. Bayer. (2003). Preventing the Development of Emotional } \\
\text { Mental Health Problems from Early Childhood: Recent Advances in the } \\
\text { Field. The International Journal of Mental Health Promotion, } 5,4-16 \text {. }\end{array}$ & Review \\
\hline
\end{tabular}




\begin{tabular}{|c|c|}
\hline $\begin{array}{l}\text { 120. Beach S. 2003. Affective disorders. Journal of Marital and } \\
\text { Family Therapy. 29(2):247-61. }\end{array}$ & Review \\
\hline $\begin{array}{l}\text { 121. Beach SRH, Brody GH, Kogan SM, Philibert RA, Chen Y-f and Lei } \\
\text { MK. 2009. Change in caregiver depression in response to parent } \\
\text { training: Genetic moderation of intervention effects. Journal of Family } \\
\text { Psychology. 23(1):112-7. }\end{array}$ & $\begin{array}{l}\text { No child } \\
\text { internalising } \\
\text { outcomes or not } \\
\text { a major goal }\end{array}$ \\
\hline $\begin{array}{l}\text { 122. Beach, S. R., Kogan, S. M., Brody, G. H., Chen, Y. F., Lei, M. K., } \\
\text { \& Murry, V. M. (2008). Change in caregiver depression as a function of } \\
\text { the Strong African American Families Program. J Fam Psychol, 22(2), } \\
\text { 241-252. doi: 2008-03770-007 }\end{array}$ & $\begin{array}{l}\text { No child } \\
\text { internalising } \\
\text { outcomes or not } \\
\text { a major goal }\end{array}$ \\
\hline $\begin{array}{l}\text { Beardslee W, Hoke L, Wheelock I, Rothberg P, Van De Velde P } \\
\text { and Swatling S. 1992. Initial findings on preventive intervention for } \\
\text { families with parental affective disorders. Am J Psychiatry, 149, } 1335 \text {. }\end{array}$ & $\begin{array}{l}\text { No child } \\
\text { internalising } \\
\text { outcomes or not } \\
\text { a major goal }\end{array}$ \\
\hline $\begin{array}{l}\text { 124. Beardslee WR, Salt P, Porterfield K, Rothberg PC, van de Velde } \\
\text { P, Swatling S, Hoke L, Moilanen DL and Wheelock I. 1993. Comparison } \\
\text { of preventive interventions for families with parental affective } \\
\text { disorder. Journal of the American Academy of Child \& Adolescent } \\
\text { Psychiatry, 32(2), 254-263. }\end{array}$ & $\begin{array}{l}\text { Follow-up less } \\
\text { than } 6 \text { months }\end{array}$ \\
\hline $\begin{array}{l}125 . \quad \text { Beardslee, W. R. (1998). Prevention and the clinical encounter. } \\
\text { American Journal of Orthopsychiatry, } 68,521-533 \text {. }\end{array}$ & Review \\
\hline $\begin{array}{l}\text { 126. Beardslee, W. R., Brent, D. A., Weersing, V. R., Clarke, G. N., } \\
\text { Porta, G., Hollon, S. D., . . Garber, J. (2013). Prevention of depression } \\
\text { in at-risk adolescents: longer-term effects. JAMA Psychiatry, 70(11), } \\
\text { 1161-1170. doi: 10.1001/jamapsychiatry.2013.295 }\end{array}$ & $\begin{array}{l}\text { Parent } \\
\text { component too } \\
\text { small }\end{array}$ \\
\hline $\begin{array}{l}\text { 127. Beardslee, W. R., Salt, P., Versage, E. M., Gladstone, T. R., } \\
\text { Wright, E. J., \& Rothberg, P. C. (1997). Sustained change in parents } \\
\text { receiving preventive interventions for families with depression. Am J } \\
\text { Psychiatry, 154(4), 510-515. }\end{array}$ & $\begin{array}{l}\text { No child } \\
\text { internalising } \\
\text { outcomes or not } \\
\text { a major goal }\end{array}$ \\
\hline $\begin{array}{l}\text { 128. Beardslee, W. R., Wright, E., Rothberg, P. C., Salt, P., \& } \\
\text { Versage, E. (1996). Response of families to two preventive } \\
\text { intervention strategies: long-term differences in behavior and attitude } \\
\text { change. J Am Acad Child Adolesc Psychiatry, 35(6), 774-782. doi: } \\
\text { S0890-8567(09)63912-5 }\end{array}$ & $\begin{array}{l}\text { No child } \\
\text { internalising } \\
\text { outcomes or not } \\
\text { a major goal }\end{array}$ \\
\hline $\begin{array}{l}\text { 129. Beatson, R. M., Bayer, J. K., Perry, A., Mathers, M., Hiscock, H., } \\
\text { Wake, M., Beesley, K., Rapee, R. M. (2014). Community screening for } \\
\text { preschool child inhibition to offer the 'Cool Little Kids' anxiety } \\
\text { prevention programme. Infant and Child Development, } 23 \text { (6), 650- } \\
661 .\end{array}$ & $\begin{array}{l}\text { Data not } \\
\text { available/report } \\
\text { ed }\end{array}$ \\
\hline $\begin{array}{l}\text { 130. Beauchaine, T. P., Webster-Stratton, C., \& Reid, M. J. (2005). } \\
\text { Mediators, moderators, and predictors of 1-year outcomes among } \\
\text { children treated for early-onset conduct problems: a latent growth } \\
\text { curve analysis. J Consult Clin Psychol, 73(3), 371-388. doi: 2005-06517- } \\
001\end{array}$ & $\begin{array}{l}\text { No long term } \\
\text { follow-up }\end{array}$ \\
\hline
\end{tabular}




\begin{tabular}{|c|c|}
\hline $\begin{array}{l}\text { 131. Beaumont R and Sofronoff K. 2008. A multi-component social } \\
\text { skills intervention for children with Asperger syndrome: the Junior } \\
\text { Detective Training Program. Journal of Child Psychology and } \\
\text { Psychiatry, 49(7), 743-753. }\end{array}$ & $\begin{array}{l}\text { Intervention for } \\
\text { developmental } \\
\text { disorders } \\
\text { (autism, autism } \\
\text { spectrum } \\
\text { disorder), } \\
\text { physical } \\
\text { disabilities, } \\
\text { medical } \\
\text { conditions or } \\
\text { distress about } \\
\text { facing medical } \\
\text { interventions } \\
\end{array}$ \\
\hline $\begin{array}{l}\text { 132. Becker KD, Mathis G, Mueller CW, Issari K and Atta SS. } 2009 . \\
\text { Community-based treatment outcomes for parents and children } \\
\text { exposed to domestic violence. In: Children exposed to violence: } \\
\text { Current issues, interventions and research. (pp. 179-95). New York, } \\
\text { NY, US: Routledge/Taylor \& Francis Group. xiii. }\end{array}$ & Review \\
\hline $\begin{array}{l}\text { 133. Becker, E. M., Becker, K. D., \& Ginsburg, G. S. (2012). Modular } \\
\text { cognitive behavioral therapy for youth with anxiety disorders: A closer } \\
\text { look at the use of specific modules and their relation to treatment } \\
\text { process and response. School Mental Health, } 4(4), 243-253 . \text { doi: } \\
10.1007 / \text { s12310-012-9080-2 }\end{array}$ & $\begin{array}{l}\text { Not a parenting } \\
\text { intervention }\end{array}$ \\
\hline $\begin{array}{l}\text { 134. Beeber LS, Cooper C, Van Noy BE, Schwartz TA, Blanchard HC, } \\
\text { Canuso R, Robb K, Laudenbacher C and Emory SL. 2007. Flying under } \\
\text { the radar: Engagement and retention of depressed low-income } \\
\text { mothers in a mental health intervention. Advances in Nursing Science. } \\
\text { 30(3):221-34. }\end{array}$ & $\begin{array}{l}\text { No child } \\
\text { internalising } \\
\text { outcomes or not } \\
\text { a major goal }\end{array}$ \\
\hline $\begin{array}{l}\text { 135. Beeber LS, Holditch-Davis D, Belyea MJ, Funk SG and Canuso } \\
\text { R. 2004. In-home intervention for depressive symptoms with low- } \\
\text { income mothers of infants and toddlers in the United States. Health } \\
\text { Care Women Int. 25(6):561-80. }\end{array}$ & $\begin{array}{l}\text { Follow-up less } \\
\text { than } 6 \text { months }\end{array}$ \\
\hline $\begin{array}{l}\text { 136. Beeber, L. S., Holditch-Davis, D., Perreira, K., Schwartz, T. A., } \\
\text { Lewis, V., Blanchard, H., et al. (2010). Short-term in-home intervention } \\
\text { reduces depressive symptoms in Early Head Start Latina mothers of } \\
\text { infants and toddlers. Res Nurs Health, 33(1), 60-76. doi: } \\
\text { 10.1002/nur.20363 }\end{array}$ & $\begin{array}{l}\text { Follow-up less } \\
\text { than } 6 \text { months }\end{array}$ \\
\hline $\begin{array}{l}137 . \quad \text { Beeber, L. S., Schwartz, T. A., Holditch-Davis, D., Canuso, R., } \\
\text { Lewis, V., \& Hall, H. W. (2013). Parenting enhancement, interpersonal } \\
\text { psychotherapy to reduce depression in low-income mothers of infants } \\
\text { and toddlers: A randomized trial. Nursing Research, 62(2), 82-90. doi: } \\
\text { 10.1097/NNR.0b013e31828324c2 }\end{array}$ & $\begin{array}{l}\text { No child } \\
\text { internalising } \\
\text { outcomes or not } \\
\text { a major goal }\end{array}$ \\
\hline $\begin{array}{l}\text { 138. Beeber, L. S., Schwartz, T. A., Martinez, M. I., Holditch-Davis, } \\
\text { D., Bledsoe, S. E., Canuso, R., Lewis, V. S. (2014). Depressive } \\
\text { symptoms and compromised parenting in low-income mothers of } \\
\text { infants and toddlers: Distal and proximal risks. Research in Nursing \& } \\
\text { Health, } 37 \text { (4), 276-291. }\end{array}$ & $\begin{array}{l}\text { Not a parenting } \\
\text { intervention }\end{array}$ \\
\hline $\begin{array}{l}\text { 139. Beelmann A. 2003. Effectiveness of a social problem-solving } \\
\text { program in preschool children with developmental deficits. Zeitschrift } \\
\text { Fur Padagogische Psychologie/ German Journal of Educational } \\
\text { Psychology. 17(1):27-41. }\end{array}$ & $\begin{array}{l}\text { Not a parenting } \\
\text { intervention }\end{array}$ \\
\hline
\end{tabular}




\begin{tabular}{|c|c|}
\hline $\begin{array}{l}\text { 140. Beeson M, Davison I, Vostanis P and Window S. } 2006 . \\
\text { Parenting Programmes for Behavioural Problems: Where Do Tertiary } \\
\text { Units Fit in a Comprehensive Service? Clinical Child Psychology and } \\
\text { Psychiatry. 11(3):335-48. }\end{array}$ & Not an RCT \\
\hline $\begin{array}{l}\text { 141. Behan, J. (2001). Evaluation of the parenting plus programme. } \\
\text { Irish Journal of Psychology, 22(3-4), 238-256. }\end{array}$ & $\begin{array}{l}\text { Follow-up less } \\
\text { than } 6 \text { months }\end{array}$ \\
\hline $\begin{array}{l}\text { 142. Beidel DC, Turner SM and Young BJ. 2006. Social Effectiveness } \\
\text { Therapy for Children: Five Years Later. Behavior Therapy. 37(4):416- } \\
25 .\end{array}$ & $\begin{array}{l}\text { Not a parenting } \\
\text { intervention }\end{array}$ \\
\hline $\begin{array}{l}\text { 143. Beidel DC, Turner SM, Sallee FR, Ammerman RT, Crosby LA } \\
\text { and Pathak S. 2007. SET-C versus fluoxetine in the treatment of } \\
\text { childhood social phobia. Journal of the American Academy of Child \& } \\
\text { Adolescent Psychiatry. 46(12):1622-32. }\end{array}$ & $\begin{array}{l}\text { Not a parenting } \\
\text { intervention }\end{array}$ \\
\hline $\begin{array}{l}\text { 144. Bender BG, Annett RD, Ikle D, DuHamel TR, Rand C and Strunk } \\
\text { RC. 2000. Relationship between disease and psychological adaptation } \\
\text { in children in the Childhood Asthma Management Program and their } \\
\text { families. CAMP Research Group. Arch Pediatr Adolesc Med. } \\
\text { 154(7):706-13. }\end{array}$ & $\begin{array}{l}\text { Not a parenting } \\
\text { intervention }\end{array}$ \\
\hline $\begin{array}{l}\text { 145. Benn, R., Akiva, T., Arel, S., \& Roeser, R. W. (2012). } \\
\text { Mindfulness training effects for parents and educators of children with } \\
\text { special needs. Developmental psychology, 48(5),1476-1487. }\end{array}$ & $\begin{array}{l}\text { Not a parenting } \\
\text { intervention }\end{array}$ \\
\hline $\begin{array}{l}\text { 146. Bennett, Tess, Algozzine and Bob. 1983. Effects of Family- } \\
\text { Oriented Intervention with Young Handicapped Children on Indicators } \\
\text { of Parental Stress. Special Education Programs, Handicapped } \\
\text { Children's Early Education Program, Washington, DC. }\end{array}$ & $\begin{array}{l}\text { Intervention for } \\
\text { developmental } \\
\text { disorders } \\
\text { (autism, autism } \\
\text { spectrum } \\
\text { disorder), } \\
\text { physical } \\
\text { disabilities, } \\
\text { medical } \\
\text { conditions or } \\
\text { distress about } \\
\text { facing medical } \\
\text { interventions }\end{array}$ \\
\hline $\begin{array}{l}\text { 147. Berger R and Gelkopf M. 2009. School-based intervention for } \\
\text { the treatment of tsunami-related distress in children: A quasi- } \\
\text { randomized controlled trial. Psychotherapy and Psychosomatics. } \\
78(6): 364-71 .\end{array}$ & $\begin{array}{l}\text { Not a parenting } \\
\text { intervention }\end{array}$ \\
\hline $\begin{array}{l}\text { 148. Berghmans, J., Weber, F., Van Akoleyen, C., Utens, E., } \\
\text { Adriaenssens, P., Klein, J., \& Himpe, D. (2012). Audiovisual aid viewing } \\
\text { immediately before pediatric induction moderates the accompanying } \\
\text { parents' anxiety. Paediatric Anaesthesia, 22, 386-392. }\end{array}$ & $\begin{array}{l}\text { No long term } \\
\text { follow-up }\end{array}$ \\
\hline $\begin{array}{l}\text { 149. Bergner A, Beyer R, Klapp BF and Rauchfuvü M. } 2009 . \\
\text { Mourning, coping and subjective attribution after early miscarriage. } \\
\text { Psychotherapie Psychosomatik Medizinische Psychologie. 59(2):57-67. }\end{array}$ & $\begin{array}{l}\text { Not a parenting } \\
\text { intervention }\end{array}$ \\
\hline $\begin{array}{l}\text { 150. Bergström, M., Kieler, H., \& Waldenström, U. (2011). A } \\
\text { randomised controlled multicentre trial of women's and men's } \\
\text { satisfaction with two models of antenatal education. In Midwifery } \\
\text { (pp. e195-200). }\end{array}$ & $\begin{array}{l}\text { No child } \\
\text { internalising } \\
\text { outcomes or not } \\
\text { a major goal }\end{array}$ \\
\hline
\end{tabular}




\begin{tabular}{|c|c|}
\hline $\begin{array}{l}\text { 151. Bergstrom, M., Rudman, A., Waldenstrom, U., \& Kieler, H. } \\
\text { (2013). Fear of childbirth in expectant fathers, subsequent childbirth } \\
\text { experience and impact of antenatal education: Subanalysis of results } \\
\text { from a randomized controlled trial. Acta Obstetricia et Gynecologica } \\
\text { Scandinavica, 92(8), 967-973. doi: } \\
\text { http://dx.doi.org/10.1111/aogs.12147 }\end{array}$ & $\begin{array}{l}\text { No child } \\
\text { internalising } \\
\text { outcomes or not } \\
\text { a major goal }\end{array}$ \\
\hline $\begin{array}{l}152 . \quad \text { Berkule, S. B., Cates, C. B., Dreyer, B. P., Huberman, H. S., } \\
\text { Arevalo, J., Burtchen, N., ... \& Mendelsohn, A. L. (2014). Reducing } \\
\text { Maternal Depressive Symptoms Through Promotion of Parenting in } \\
\text { Pediatric Primary Care. Clinical pediatrics, 53(5), 460-469. }\end{array}$ & $\begin{array}{l}\text { No child } \\
\text { internalising } \\
\text { outcomes or not } \\
\text { a major goal }\end{array}$ \\
\hline $\begin{array}{l}\text { 153. Berlin, L. J., Shanahan, M., \& Appleyard Carmody, K. (2014). } \\
\text { Promoting supportive parenting in new mothers with substance-use } \\
\text { problems: A pilot randomized trial of residential treatment plus an } \\
\text { attachment-based parenting program. Infant mental health journal, } \\
\text { 35(1), 81-85. }\end{array}$ & $\begin{array}{l}\text { No long term } \\
\text { follow-up }\end{array}$ \\
\hline $\begin{array}{l}\text { 154. Berliner, L., \& Saunders, B. E. (1996). Treating Fear and Anxiety } \\
\text { in Sexually Abused Children: Results of a Controlled 2-Year Follow-Up } \\
\text { Study. Child Maltreatment, 1, 294-309. }\end{array}$ & $\begin{array}{l}\text { Not a parenting } \\
\text { intervention }\end{array}$ \\
\hline $\begin{array}{l}\text { 155. Bernard, K., Dozier, M., \& Zwerling, J. (2014). Blunted diurnal } \\
\text { cortisol mediates the association between maltreatment risk and } \\
\text { externalizing behavior: Results from an early parenting intervention. } \\
\text { Psychosomatic Medicine, } 76 \text { (3) A-57. }\end{array}$ & $\begin{array}{l}\text { No child } \\
\text { internalising } \\
\text { outcomes or not } \\
\text { a major goal }\end{array}$ \\
\hline $\begin{array}{l}\text { 156. Bernstein, G. A., Bernat, D. H., Victor, A. M., \& Layne, A. E. } \\
\text { (2008). School-based interventions for anxious children: 3-, 6-, and 12- } \\
\text { month follow-ups. J Am Acad Child Adolesc Psychiatry, 47(9), 1039- } \\
\text { 1047. doi: 10.1097/CHI.ob013e31817eecco }\end{array}$ & $\begin{array}{l}\text { Parent } \\
\text { component too } \\
\text { small }\end{array}$ \\
\hline $\begin{array}{l}\text { 157. Bernstein, G. A., Layne, A. E., Egan, E. A., \& Tennison, D. M. } \\
\text { (2005). School-based interventions for anxious children. J Am Acad } \\
\text { Child Adolesc Psychiatry, 44(11), 1118-1127. doi: S0890- } \\
8567(09) 62214-0\end{array}$ & $\begin{array}{l}\text { Parent } \\
\text { component too } \\
\text { small }\end{array}$ \\
\hline $\begin{array}{l}\text { 158. Berry, D. C., Schwartz, T. A., McMurray, R. G., Skelly, A. H., } \\
\text { Neal, M., Hall, E. G., ... \& Melkus, G. (2014). The family partners for } \\
\text { health study: a cluster randomized controlled trial for child and parent } \\
\text { weight management. Nutrition \& diabetes, 4(1), e101. }\end{array}$ & $\begin{array}{l}\text { No child } \\
\text { internalising } \\
\text { outcomes or not } \\
\text { a major goal }\end{array}$ \\
\hline $\begin{array}{l}\text { 159. Bert, S. C., Farris, J. R., \& Borkowski, J. G. (2008). Parent } \\
\text { training: implementation strategies for adventures in parenting. The } \\
\text { journal of primary prevention, (3), 243-261. }\end{array}$ & $\begin{array}{l}\text { No long term } \\
\text { follow-up }\end{array}$ \\
\hline $\begin{array}{l}\text { 160. Bierman KL, Coie JD, Dodge KA, Greenberg MT, Lochman JE, } \\
\text { McMahon RJ and Pinderhughes EE. 2002. Evaluation of the first } 3 \\
\text { years of the Fast Track prevention trial with children at high risk for } \\
\text { adolescent conduct problems. Journal of Abnormal Child Psychology. } \\
\text { 30(1):19-35. }\end{array}$ & $\begin{array}{l}\text { No child } \\
\text { internalising } \\
\text { outcomes or not } \\
\text { a major goal; No } \\
\text { long term } \\
\text { follow-up }\end{array}$ \\
\hline $\begin{array}{l}\text { 161. Bilszta, J. L., Buist, A. E., Wang, F., \& Zulkefli, N. R. (2012). Use } \\
\text { of video feedback intervention in an inpatient perinatal psychiatric } \\
\text { setting to improve maternal parenting. Archives of women's mental } \\
\text { health, 15(4), 249-257. }\end{array}$ & $\begin{array}{l}\text { No child } \\
\text { internalising } \\
\text { outcomes or not } \\
\text { a major goal }\end{array}$ \\
\hline $\begin{array}{l}\text { 162. Bjørknes, R., Jakobsen, R., \& Nærde, A. (2011). Recruiting } \\
\text { ethnic minority groups to evidence-based parent training. Who will } \\
\text { come and how? Children and Youth Services Review, 33, 351-357. }\end{array}$ & $\begin{array}{l}\text { No child } \\
\text { internalising } \\
\text { outcomes or not } \\
\text { a major goal }\end{array}$ \\
\hline
\end{tabular}




\begin{tabular}{|c|c|}
\hline $\begin{array}{l}\text { 163. Black MM, Nair P, Kight C, Wachtel R, Roby P, Schuler M. } \\
\text { Parenting and early development among children of drug-abusing } \\
\text { women: effects of home intervention. Pediatrics.1994;94:440-448. }\end{array}$ & $\begin{array}{l}\text { No child } \\
\text { internalising } \\
\text { outcomes or not } \\
\text { a major goal; No } \\
\text { long term } \\
\text { follow-up }\end{array}$ \\
\hline $\begin{array}{l}\text { 164. Black MM, Sazawal S, Black RE, Khosla S, Kumar J and Menon } \\
\text { V. 2004. Cognitive and motor development among small-for- } \\
\text { gestational-age infants: impact of zinc supplementation, birth weight, } \\
\text { and caregiving practices. Pediatrics, 113(5), 1297-1305. }\end{array}$ & $\begin{array}{l}\text { Not a parenting } \\
\text { intervention }\end{array}$ \\
\hline $\begin{array}{l}\text { 165. Blader JC. 2006. Which family factors predict children's } \\
\text { externalizing behaviors following discharge from psychiatric inpatient } \\
\text { treatment? Journal of Child Psychology and Psychiatry. 47(11):1133- } \\
42 .\end{array}$ & Not an RCT \\
\hline $\begin{array}{l}\text { 166. Blanchard A, Hodgson J, Gunn W, Jesse E and White M. } 2009 . \\
\text { Understanding social support and the couple's relationship among } \\
\text { women with depressive symptoms in pregnancy. Issues in Mental } \\
\text { Health Nursing. 30(12):764-76. }\end{array}$ & $\begin{array}{l}\text { Not a parenting } \\
\text { intervention }\end{array}$ \\
\hline $\begin{array}{l}\text { 167. Blatt-Eisengart I, Drabick DAG, Monahan KC and Steinberg L. } \\
\text { 2009. Sex differences in the longitudinal relations among family risk } \\
\text { factors and childhood externalizing symptoms. Developmental } \\
\text { Psychology. 45(2):491-502. }\end{array}$ & Not an RCT \\
\hline $\begin{array}{l}\text { 168. Blitstein JL, Murray DM, Lytle LA, Birnbaum AS and Perry CL. } \\
\text { 2005. Predictors of Violent Behavior in an Early Adolescent Cohort: } \\
\text { Similarities and Differences Across Genders. Health Education \& } \\
\text { Behavior. 32(2):175-94. }\end{array}$ & $\begin{array}{l}\text { Not a parenting } \\
\text { intervention }\end{array}$ \\
\hline $\begin{array}{l}\text { 169. Bocca, G., Kuitert, M. W. B., Sauer, P. J. J., Stolk, R. P., Flapper, } \\
\text { B. C., \& Corpeleijn, E. (2014). A multidisciplinary intervention } \\
\text { programme has positive effects on quality of life in overweight and } \\
\text { obese preschool children. Acta Paediatrica, 103(9), 962-967. }\end{array}$ & $\begin{array}{l}\text { Not a parenting } \\
\text { intervention }\end{array}$ \\
\hline $\begin{array}{l}\text { 170. Bodden DHM, Bogels SM, Nauta MH, De Haan E, Riingrose J, } \\
\text { Appelboom C, Brinkman AG and Appelboom-Geerts KCMMJ. } 2008 . \\
\text { Child versus family cognitive-behavioral therapy in clinically anxious } \\
\text { youth: An efficacy and partial effectiveness study. Journal of the } \\
\text { American Academy of Child \& Adolescent Psychiatry. 47(12):1384-94. }\end{array}$ & $\begin{array}{l}\text { Follow-up less } \\
\text { than } 6 \text { months }\end{array}$ \\
\hline $\begin{array}{l}\text { 171. Bodden DHM, Dirksen CD, Bogels SM, Nauta MH, De Haan E, } \\
\text { Ringrose J, et al. Costs and cost-effectiveness of family CBT versus } \\
\text { individual CBT in clinically anxious children. Clinical Child Psychology } \\
\text { and Psychiatry. } 2008 \text { Oct;13(4):543-64. }\end{array}$ & $\begin{array}{l}\text { Comparison } \\
\text { condition not a } \\
\text { minimal control }\end{array}$ \\
\hline $\begin{array}{l}\text { 172. Bodenmann, G., Cina, A., Ledermann, T., \& Sanders, M. R. } \\
\text { (2008). The efficacy of the Triple P-Positive Parenting Program in } \\
\text { improving parenting and child behavior: A comparison with two other } \\
\text { treatment conditions. Behaviour Research and Therapy, } 46(4), 411- \\
427 .\end{array}$ & $\begin{array}{l}\text { No child } \\
\text { internalising } \\
\text { outcomes or not } \\
\text { a major goal }\end{array}$ \\
\hline $\begin{array}{l}\text { 173. Bøe, T., Øverland, S., Lundervold, A. J., \& Hysing, M. (2012). } \\
\text { Socioeconomic status and children's mental health: Results from the } \\
\text { Bergen child study. Social Psychiatry and Psychiatric Epidemiology, 47, } \\
\text { 1557-1566. }\end{array}$ & $\begin{array}{l}\text { Not a parenting } \\
\text { intervention }\end{array}$ \\
\hline
\end{tabular}




\begin{tabular}{|c|c|}
\hline $\begin{array}{l}\text { 174. Bogels SM, Siqueland L. Family cognitive behavioral therapy } \\
\text { for children and adolescents with clinical anxiety disorders. Journal of } \\
\text { the American Academy of Child \& Adolescent Psychiatry. } 2006 \\
\text { Feb;45(2):134-41. }\end{array}$ & Not an RCT \\
\hline $\begin{array}{l}\text { Bohlin G and Janols LO. 2004. Behavioural problems and } \\
\text { psychiatric symptoms in 5-13 year-old Swedish children - A } \\
\text { comparison of parent ratings on the FTF (Five to Fifteen) with the } \\
\text { ratings on CBCL (Child Behavior Checklist). European Child \& } \\
\text { Adolescent Psychiatry. 13(Suppl3):14-22. }\end{array}$ & $\begin{array}{l}\text { Not a parenting } \\
\text { intervention }\end{array}$ \\
\hline $\begin{array}{l}\text { 176. Bohnert, K. M., Anthony, J. C., \& Breslau, N. (2012). Paternal } \\
\text { monitoring at age } 11 \text { and subsequent onset of cannabis use up to age } \\
\text { 17: Results from a prospective study. Journal of Studies on Alcohol and } \\
\text { Drugs, } 73,173-177 \text {. }\end{array}$ & $\begin{array}{l}\text { Not a parenting } \\
\text { intervention }\end{array}$ \\
\hline $\begin{array}{l}\text { 177. Bonanno GA, Galea S, Bucciarelli A and Vlahov D. 2007. What } \\
\text { predicts psychological resilience after disaster? The role of } \\
\text { demographics, resources, and life stress. Journal of Consulting and } \\
\text { Clinical Psychology. } 75(5): 671-82 \text {. }\end{array}$ & $\begin{array}{l}\text { Not a parenting } \\
\text { intervention }\end{array}$ \\
\hline $\begin{array}{l}\text { 178. Bonds DD, Wolchik SA, Winslow E, Tein JY, Sandler IN, Millsap } \\
\text { RE. Developmental cascade effects of the New Beginnings Program on } \\
\text { adolescent adaptation outcomes. Dev Psychopathol. } 2010 \\
\text { Nov;22(4):771-84. }\end{array}$ & $\begin{array}{l}\text { Secondary } \\
\text { analysis } \\
\text { (mediation } \\
\text { analysis of data } \\
\text { in Wolchik et al } \\
\text { 2002) }\end{array}$ \\
\hline $\begin{array}{l}\text { 179. Bor, W., Sanders, M. R., \& Markie-Dadds, C. (2002). The } \\
\text { effects of the Triple P-Positive Parenting Program on preschool } \\
\text { children with co-occurring disruptive behavior and } \\
\text { attentional/hyperactive difficulties. Journal of abnormal child } \\
\text { psychology, (6), 571-587. }\end{array}$ & $\begin{array}{l}\text { No child } \\
\text { internalising } \\
\text { outcomes or not } \\
\text { a major goal }\end{array}$ \\
\hline $\begin{array}{l}\text { 180. Borelli JL, Luthar SS and Suchman NE. 2010. Discrepancies in } \\
\text { perceptions of maternal aggression: implications for children of } \\
\text { methadone-maintained mothers. American journal of orthopsychiatry, } \\
80(3), 412-21 \text {. }\end{array}$ & Not an RCT \\
\hline $\begin{array}{l}\text { 181. Borghini, A., Habersaat, S., Forcada-Guex, M., Nessi, J., } \\
\text { Pierrehumbert, B., Ansermet, F., \& Müller-Nix, C. (2014). Effects of an } \\
\text { early intervention on maternal post-traumatic stress symptoms and } \\
\text { the quality of mother-infant interaction: The case of preterm birth. } \\
\text { Infant Behavior and Development, 37(4), 624-631. }\end{array}$ & $\begin{array}{l}\text { No child } \\
\text { internalising } \\
\text { outcomes or not } \\
\text { a major goal }\end{array}$ \\
\hline $\begin{array}{l}\text { 182. Borowsky, I. W., Mozayeny, S., Stuenkel, K., \& Ireland, M. } \\
\text { (2004). Effects of a primary care-based intervention on violent } \\
\text { behavior and injury in children. Pediatrics, (4), e392-399. }\end{array}$ & $\begin{array}{l}\text { Targets } \\
\text { externalising } \\
\text { (violence) }\end{array}$ \\
\hline $\begin{array}{l}\text { 183. Borrego J, Jr., Anhalt K, Terao SY, Vargas EC and Urquiza AJ. } \\
\text { 2006. Parent-Child Interaction Therapy With a Spanish-Speaking } \\
\text { Family. Cognitive and Behavioral Practice. 13(2):121-33. }\end{array}$ & Not an RCT \\
\hline $\begin{array}{l}\text { 184. Borrego, J., Gutow, M. R., Reicher, S., \& Barker, C. H. (2008). } \\
\text { Parent-child interaction therapy with domestic violence populations. } \\
\text { Journal of Family Violence, 23(6), 495-505. }\end{array}$ & Not an RCT \\
\hline $\begin{array}{l}\text { 185. Bosmans, G., Braet, C., Beyers, W., Van Leeuwen, K., \& Van } \\
\text { Vlierberghe, L. (2011). Parents' power assertive discipline and } \\
\text { internalizing problems in adolescents: The role of attachment. } \\
\text { Parenting: Science and Practice, } 11,34-55 .\end{array}$ & $\begin{array}{l}\text { Not a parenting } \\
\text { intervention }\end{array}$ \\
\hline
\end{tabular}




\begin{tabular}{|c|c|}
\hline $\begin{array}{l}\text { Bosworth K, Espelage D, DuBay T, Dahlberg LL and Daytner G. } \\
\text { 1996. Using multimedia to teach conflict-resolution skills to young } \\
\text { adolescents. Am J Prev Med. } 12 \text { ( } 5 \text { Suppl):65-74. }\end{array}$ & $\begin{array}{l}\text { Not a parenting } \\
\text { intervention }\end{array}$ \\
\hline $\begin{array}{l}\text { B87. Botngard, A., Skranes, L. P., Skranes, J., \& Dollner, H. (2013). } \\
\text { Multimedia based health information to parents in a pediatric acute } \\
\text { ward: A randomized controlled trial. Patient Education and } \\
\text { Counseling, 93(3), 389-393. doi: } \\
\text { http://dx.doi.org/10.1016/j.pec.2013.04.017 }\end{array}$ & $\begin{array}{l}\text { No child } \\
\text { internalising } \\
\text { outcomes or not } \\
\text { a major goal }\end{array}$ \\
\hline $\begin{array}{l}\text { 188. Bouve LR, Rozmus CL and Giordano P. 1999. Preparing parents } \\
\text { for their child's transfer from the PICU to the pediatric floor. Appl Nurs } \\
\text { Res. 12(3):114-20. }\end{array}$ & $\begin{array}{l}\text { No child } \\
\text { internalising } \\
\text { outcomes or not } \\
\text { a major goal }\end{array}$ \\
\hline $\begin{array}{l}\text { 189. Bowers JW. 2002. A preliminary investigation of the additive } \\
\text { effect of a parent training protocol in the treatment of phobic } \\
\text { children. Dissertation Abstracts International: Section B: The Sciences } \\
\text { and Engineering. 63(2-B). }\end{array}$ & $\begin{array}{l}\text { Follow-up less } \\
\text { than } 6 \text { months }\end{array}$ \\
\hline $\begin{array}{l}\text { 190. Boyd RC, Zayas LH and McKee MD. 2006. Mother-Infant } \\
\text { Interaction, Life Events and Prenatal and Postpartum Depressive } \\
\text { Symptoms Among Urban Minority Women in Primary Care. Maternal } \\
\text { and Child Health Journal. 10(2):139-48. }\end{array}$ & $\begin{array}{l}\text { Not a parenting } \\
\text { intervention }\end{array}$ \\
\hline $\begin{array}{l}\text { 191. Bradley, S. J., Jadaa, D. A., Brody, J., Landy, S., Tallett, S. E., } \\
\text { Watson, W., . . Stephens, D. (2003). Brief psychoeducational } \\
\text { parenting program: An evaluation and 1-year follow-up. Journal of the } \\
\text { American Academy of Child and Adolescent Psychiatry, 42(10), 1171- } \\
\text { 1178. }\end{array}$ & $\begin{array}{l}\text { Follow-up data } \\
\text { collected only in } \\
\text { active group }\end{array}$ \\
\hline $\begin{array}{l}\text { 192. Bradway KP. 1935. Paternal occupational intelligence and } \\
\text { mental deficiency. Journal of Applied Psychology. 19(5):527-42. }\end{array}$ & $\begin{array}{l}\text { Not a parenting } \\
\text { intervention }\end{array}$ \\
\hline $\begin{array}{l}\text { 193. Brann P, Coleman G and Luk E. 2001. Routine outcome } \\
\text { measurement in a child and adolescent mental health service: An } \\
\text { evaluation of HoNOSCA. Australian and New Zealand Journal of } \\
\text { Psychiatry. 35(3):370-6. }\end{array}$ & $\begin{array}{l}\text { Not a parenting } \\
\text { intervention }\end{array}$ \\
\hline $\begin{array}{l}\text { 194. Braswell L, August GJ, Bloomquist ML, Realmuto GM, Skare SS } \\
\text { and Crosby RD. 1997. School-based secondary prevention for children } \\
\text { with disruptive behavior: initial outcomes. J Abnorm Child Psychol. } \\
\text { 25(3):197-208. }\end{array}$ & $\begin{array}{l}\text { Not a parenting } \\
\text { intervention }\end{array}$ \\
\hline $\begin{array}{l}\text { 195. Brechman-Toussaint, M., \& Anderson, J. (2004). Modifying } \\
\text { Cognitive Behavioural Therapy to train parents as therapists of young } \\
\text { children at risk of anxiety. 32nd Congress of the British Association for } \\
\text { Behavioural and Cognitive Psychotherapies (jointly with the European } \\
\text { Association of Behavioural and Cognitive Therapies); } 2004 \text { September } \\
7 \text { - 11; Manchester, 106. Retrieved from } \\
\text { http://www.mrw.interscience.wiley.com/cochrane/clcentral/articles/ } \\
\text { 837/CN-00712837/frame.html }\end{array}$ & $\begin{array}{l}\text { Data not } \\
\text { available/report } \\
\text { ed }\end{array}$ \\
\hline $\begin{array}{l}\text { 196. Brent DA, Baugher M, Birmaher B, Kolko DJ and Bridge J. } 2000 . \\
\text { Compliance with recommendations to remove firearms in families } \\
\text { participating in a clinical trial for adolescent depression. J Am Acad } \\
\text { Child Adolesc Psychiatry. 39(10):1220-6. }\end{array}$ & $\begin{array}{l}\text { No child } \\
\text { internalising } \\
\text { outcomes or not } \\
\text { a major goal }\end{array}$ \\
\hline $\begin{array}{l}197 . \quad \text { Brent, D. A., Holder, D., Kolko, D., Birmaher, B., Baugher, M., } \\
\text { Roth, C., . . Johnson, B. A. (1997). A clinical psychotherapy trial for } \\
\text { adolescent depression comparing cognitive, family, and supportive } \\
\text { therapy. Archives of General Psychiatry, 54(9), 877-885. }\end{array}$ & $\begin{array}{l}\text { No long term } \\
\text { follow-up }\end{array}$ \\
\hline
\end{tabular}




\begin{tabular}{|c|c|}
\hline $\begin{array}{l}\text { 198. Brightman, R. P., Baker, B. L., Clark, D. B. \& Ambrose, S. A. } \\
\text { (1982). Effectiveness of alternative parent training formats. Behaviour } \\
\text { Therapy and Experimental Psychiatry, 13, } 113\end{array}$ & $\begin{array}{l}\text { No child } \\
\text { internalising } \\
\text { outcomes or not } \\
\text { a major goal }\end{array}$ \\
\hline $\begin{array}{l}\text { 199. Brody, G. H., Murry, V. M., Chen, Y. F., Kogan, S. M., \& Brown, } \\
\text { A. C. (2006). Effects of family risk factors on dosage and efficacy of a } \\
\text { family-centered preventive intervention for rural African Americans. } \\
\text { Prevention science : the official journal of the Society for Prevention } \\
\text { Research, (3), 281-291. }\end{array}$ & $\begin{array}{l}\text { No long term } \\
\text { follow-up }\end{array}$ \\
\hline $\begin{array}{l}200 . \quad \text { Brody, G. H., Yu, T., Chen, E., \& Miller, G. E. (2014). Prevention } \\
\text { moderates associations between family risks and youth catecholamine } \\
\text { levels. Health Psychology, 33(11), 1435. }\end{array}$ & $\begin{array}{l}\text { No child } \\
\text { internalising } \\
\text { outcomes or not } \\
\text { a major goal }\end{array}$ \\
\hline $\begin{array}{l}\text { 201. Bröning, S., Sack, P. M., Thomsen, M., Stolle, M., Wendell, A., } \\
\text { Stappenbeck, J., \& Thomasius, R. (2014). Implementing and evaluating } \\
\text { the German adaptation of the "Strengthening Families Program 10-14 } \\
\text { "-a randomized-controlled multicentre study. BMC public health, } \\
\text { 14(1), 83. }\end{array}$ & $\begin{array}{l}\text { Data not } \\
\text { available/report } \\
\text { ed }\end{array}$ \\
\hline $\begin{array}{l}202 . \quad \text { Brooks-Gunn, J., McCarton, C. M., Casey, P. H., McCormick, M. } \\
\text { C., Bauer, C. R., Bernbaum, J. C., Tyson, J., Swanson, M., Bennett, F. C., } \\
\text { Scott, D. T., \& et al. (1994). Early intervention in low-birth-weight } \\
\text { premature infants. Results through age } 5 \text { years from the Infant Health } \\
\text { and Development Program. JAMA, 272, 1257-1262. }\end{array}$ & $\begin{array}{l}\text { Parent } \\
\text { component too } \\
\text { small }\end{array}$ \\
\hline $\begin{array}{l}\text { 203. Brotman LM, Gouley KK and Chesir-Teran D. 2005. Assessing } \\
\text { peer entry and play in preschoolers at risk for maladjustment. Journal } \\
\text { of Clinical Child and Adolescent Psychology. 34(4):671-80. }\end{array}$ & $\begin{array}{l}\text { Not a parenting } \\
\text { intervention }\end{array}$ \\
\hline $\begin{array}{l}204 . \quad \text { Brotman, L. M., Calzada, E., Huang, K. Y., Kingston, S., Dawson- } \\
\text { McClure, S., Kamboukos, D., ... \& Petkova, E. (2011). Promoting } \\
\text { effective parenting practices and preventing child behavior problems } \\
\text { in school among ethnically diverse families from underserved, urban } \\
\text { communities. Child Development, 82(1), 258-276. doi:10.1111/j.1467- } \\
8624.2010 .01554 . x\end{array}$ & $\begin{array}{l}\text { No long term } \\
\text { follow-up }\end{array}$ \\
\hline $\begin{array}{l}\text { 205. Brown C-SE. 1997. The aftermath of Hurricane Iniki: } \\
\text { Development of a school-based intervention. Dissertation Abstracts } \\
\text { International: Section B: The Sciences and Engineering. 57(10-B). }\end{array}$ & $\begin{array}{l}\text { Not a parenting } \\
\text { intervention }\end{array}$ \\
\hline $\begin{array}{l}\text { 206. Brown, F. L., Whittingham, K., McKinlay, L., Boyd, R. N., } \\
\text { Sofronoff, K. (2014). The efficacy of a parenting programme for } \\
\text { improving child and parenting outcomes following paediatric acquired } \\
\text { brain injury. Developmental medicine and child neurology, } 5641 .\end{array}$ & $\begin{array}{l}\text { No child } \\
\text { internalising } \\
\text { outcomes or not } \\
\text { a major goal }\end{array}$ \\
\hline $\begin{array}{l}207 . \quad \text { Brown, F. L., Whittingham, K., McKinlay, L., Boyd, R. N., } \\
\text { Sofronoff, K. (2014). An RCT of a parenting intervention for improving } \\
\text { parent and family outcomes following paediatric acquired brain injury. } \\
\text { Developmental medicine and child neurology, } 5658 \text {. }\end{array}$ & $\begin{array}{l}\text { Follow-up data } \\
\text { collected only in } \\
\text { active group }\end{array}$ \\
\hline $\begin{array}{l}\text { 208. Brown, F. L., Whittingham, K., McKinlay, L., Boyd, R., \& } \\
\text { Sofronoff, K. (2013). Efficacy of stepping stones triple p plus a stress } \\
\text { management adjunct for parents of children with an acquired brain } \\
\text { injury: The protocol of a Randomised controlled trial. Brain } \\
\text { Impairment, 14(2), 253-269. doi: } \\
\text { http://dx.doi.org/10.1017/Brlmp.2013.18 }\end{array}$ & $\begin{array}{l}\text { Data not } \\
\text { available/report } \\
\text { ed }\end{array}$ \\
\hline
\end{tabular}




\begin{tabular}{|c|c|}
\hline $\begin{array}{l}\text { 209. Brown, L. D., Feinberg, M., \& Kan, M. L. (2012). Predicting } \\
\text { engagement in a transition to parenthood program for couples. } \\
\text { Evaluation and program planning, 35(1), 1-8. }\end{array}$ & $\begin{array}{l}\text { No child } \\
\text { internalising } \\
\text { outcomes or not } \\
\text { a major goal }\end{array}$ \\
\hline $\begin{array}{l}210 . \quad \text { Brown, L. K., Hadley, W., Donenberg, G. R., DiClemente, R. J., } \\
\text { Lescano, C., Lang, D. M., ... \& Oster, D. (2014). Project STYLE: A } \\
\text { multisite RCT for HIV prevention among youths in mental health } \\
\text { treatment. Psychiatric Services, 65(3), 338-344. }\end{array}$ & $\begin{array}{l}\text { Not a parenting } \\
\text { intervention }\end{array}$ \\
\hline $\begin{array}{l}\text { 211. Brugha TS, Wheatley S, Taub NA, Culverwell A, Friedman T, } \\
\text { Kirwan P, Jones DR and Shapiro DA. 2000. Pragmatic randomized trial } \\
\text { of antenatal intervention to prevent post-natal depression by reducing } \\
\text { psychosocial risk factors. Psychol Med. 30(6):1273-81. }\end{array}$ & $\begin{array}{l}\text { No child } \\
\text { internalising } \\
\text { outcomes or not } \\
\text { a major goal }\end{array}$ \\
\hline $\begin{array}{l}\text { 212. Bry BH and George FE. 1980. The preventive effects of early } \\
\text { intervention on the attendance and grades of urban adolescents. } \\
\text { Professional Psychology, 11(2), 252-60. }\end{array}$ & $\begin{array}{l}\text { Not a parenting } \\
\text { intervention }\end{array}$ \\
\hline $\begin{array}{l}\text { 213. Bryan AA. 2000. Enhancing parent-child interaction with a } \\
\text { prenatal couple intervention. MCN: The American Journal of } \\
\text { Maternal/Child Nursing, 25(3), 139-145. }\end{array}$ & $\begin{array}{l}\text { No child } \\
\text { internalising } \\
\text { outcomes or not } \\
\text { a major goal }\end{array}$ \\
\hline $\begin{array}{l}\text { 214. Buckworth J, Dishman RK and Cureton KJ. 1994. Autonomic } \\
\text { responses of women with parental hypertension. Effects of physical } \\
\text { activity and fitness. Hypertension. 24(5):576-84. }\end{array}$ & $\begin{array}{l}\text { Not a parenting } \\
\text { intervention }\end{array}$ \\
\hline $\begin{array}{l}215 . \quad \text { Buelow, J. M., Johnson, C. S., Perkins, S. M., Austin, J. K., \& } \\
\text { Dunn, D. W. (2013). Creating Avenues for Parent Partnership (CAPP): } \\
\text { An intervention for parents of children with epilepsy and learning } \\
\text { problems. Epilepsy and Behavior, 27(1), 64-69. } \\
\text { doi:http://dx.doi.org/10.1016/j.yebeh.2012.12.013 }\end{array}$ & $\begin{array}{l}\text { No long term } \\
\text { follow-up }\end{array}$ \\
\hline $\begin{array}{l}\text { 216. Bühler, A., Kötter, C., Jaursch, S., \& Lösel, F. (2011). Prevention } \\
\text { of familial transmission of depression: EFFEKT-E, a selective program } \\
\text { for emotionally burdened families. Journal of Public Health, 19, 321- } \\
\text { 327. }\end{array}$ & $\begin{array}{l}\text { Not an RCT; } \\
\text { Parent } \\
\text { component too } \\
\text { small }\end{array}$ \\
\hline $\begin{array}{l}\text { 217. Buist A, Speelman C, Hayes B, Reay R, Milgrom J, Meyer D and } \\
\text { Condon J. 2007. Impact of education on women with perinatal } \\
\text { depression. Journal of Psychosomatic Obstetrics \& Gynecology. } \\
\text { 28(1):49-54. }\end{array}$ & $\begin{array}{l}\text { Not a parenting } \\
\text { intervention }\end{array}$ \\
\hline $\begin{array}{l}\text { 218. Bullock LF, Wells JE, Duff GB and Hornblow AR. } 1995 . \\
\text { Telephone support for pregnant women: outcome in late pregnancy. } \\
\text { N Z Med J. 108(1012):476-8. }\end{array}$ & $\begin{array}{l}\text { No child } \\
\text { internalising } \\
\text { outcomes or not } \\
\text { a major goal }\end{array}$ \\
\hline $\begin{array}{l}\text { 219. Bunaciu, L., Leen-Feldner, E. W., Blumenthal, H., Knapp, A. A., } \\
\text { Badour, C. L., \& Feldner, M. T. (2014). An Experimental Test of the } \\
\text { Effects of Parental Modeling on Panic-Relevant Escape and Avoidance } \\
\text { Among Early Adolescents. Behavior therapy, 45(4), 517-529. }\end{array}$ & $\begin{array}{l}\text { No long term } \\
\text { follow-up }\end{array}$ \\
\hline $\begin{array}{l}\text { 220. Bunton, P., Hindley, P., Woods, M., Grose, C., \& Hedderly, T. } \\
\text { (2013). Innovations in psychological treatment for tic disorders: } \\
\text { Feasibility, acceptability and effectiveness data from two pilot studies. } \\
\text { [Conference Abstract]. Developmental Medicine and Child Neurology, } \\
\text { 55, 67. doi: http://dx.doi.org/10.1111/dmcn.12070 }\end{array}$ & Not an RCT \\
\hline
\end{tabular}




\begin{tabular}{|c|c|}
\hline $\begin{array}{l}\text { 221. Burke, K., Brennan, L., \& Cann, W. (2012). Promoting } \\
\text { protective factors for young adolescents: ABCD Parenting Young } \\
\text { Adolescents Program randomized controlled trial. Journal of } \\
\text { Adolescence, } 35,1315-1328 .\end{array}$ & $\begin{array}{l}\text { No long term } \\
\text { follow-up }\end{array}$ \\
\hline $\begin{array}{l}\text { 222. Burke, S. O., Handley-Derry, M. H., Costello, E. A., Kauffmann, } \\
\text { E., \& Dillon, M. C. (1997). Stress-point intervention for parents of } \\
\text { repeatedly hospitalized children with chronic conditions. Res Nurs } \\
\text { Health, 20(6), 475-485. doi: } 10.1002 /(\mathrm{SICl}) 1098- \\
240 X(199712) 20: 6<475:: \text { AID-NUR2>3.0.CO;2-J }\end{array}$ & $\begin{array}{l}\text { Follow-up less } \\
\text { than } 6 \text { months }\end{array}$ \\
\hline $\begin{array}{l}\text { 223. Burns CD, Cortell R and Wagner BM. 2008. Treatment } \\
\text { compliance in adolescents after attempted suicide: A 2-year follow-up } \\
\text { study. Journal of the American Academy of Child \& Adolescent } \\
\text { Psychiatry. 47(8):948-57. }\end{array}$ & $\begin{array}{l}\text { Not a parenting } \\
\text { intervention }\end{array}$ \\
\hline $\begin{array}{l}\text { 224. Burstein M, Ginsburg GS, Petras H and lalongo N. 2010. Parent } \\
\text { psychopathology and youth internalizing symptoms in an urban } \\
\text { community: A latent growth model analysis. Child Psychiatry and } \\
\text { Human Development. 41(1):61-87. }\end{array}$ & $\begin{array}{l}\text { Not a parenting } \\
\text { intervention }\end{array}$ \\
\hline $\begin{array}{l}\text { 225. Butler LD, Symons BK, Henderson SL, Shortliffe LD and Spiegel } \\
\text { D. 2005. Hypnosis reduces distress and duration of an invasive medical } \\
\text { procedure for children. Pediatrics. 115(1):e77-85. }\end{array}$ & $\begin{array}{l}\text { Intervention for } \\
\text { developmental } \\
\text { disorders } \\
\text { (autism, autism } \\
\text { spectrum } \\
\text { disorder), } \\
\text { physical } \\
\text { disabilities, } \\
\text { medical } \\
\text { conditions or } \\
\text { distress about } \\
\text { facing medical } \\
\text { interventions }\end{array}$ \\
\hline $\begin{array}{l}\text { 226. Butler SF, Budman SH and Beardslee W. 2000. Risk reduction } \\
\text { in children from families with parental depression: A videotape } \\
\text { psychoeducation program. National Academies of Practice Forum: } \\
\text { Issues in Interdisciplinary Care (Vol. 2, No. 4, pp. 267-276). }\end{array}$ & $\begin{array}{l}\text { Follow-up less } \\
\text { than } 6 \text { months }\end{array}$ \\
\hline $\begin{array}{l}\text { 227. Butler, A. M. (2006). Parent-child interaction therapy and } \\
\text { ethnic minority children. Vulnerable children and youth studies, 1(3), } \\
246 .\end{array}$ & Review \\
\hline $\begin{array}{l}\text { 228. Byers JF, Yovaish W, Lowman LB and Francis JD. 2003. Co- } \\
\text { bedding versus single-bedding premature multiple-gestation infants in } \\
\text { incubators. Journal of Obstetric, Gynecologic, \& Neonatal Nursing, } \\
\text { 32(3), 340-347. }\end{array}$ & $\begin{array}{l}\text { Not a parenting } \\
\text { intervention }\end{array}$ \\
\hline $\begin{array}{l}229 . \quad \text { Bywater, T., Hutchings, J., Daley, D., Whitaker, C., Yeo, S. T., } \\
\text { Jones, K., et al. (2009). Long-term effectiveness of a parenting } \\
\text { intervention for children at risk of developing conduct disorder. Br J } \\
\text { Psychiatry, 195(4), 318-324. doi: 195/4/318 }\end{array}$ & $\begin{array}{l}\text { Follow-up data } \\
\text { collected only in } \\
\text { active group }\end{array}$ \\
\hline $\begin{array}{l}\text { 230. Bywater, T., Hutchings, J., Linck, P., Whitaker, C., Daley, D., } \\
\text { Yeo, S. T., \& Edwards, R. T. (2011). Incredible Years parent training } \\
\text { support for foster carers in Wales: a multi-centre feasibility study. } \\
\text { Child Care Health Dev, 37, 233-243. }\end{array}$ & $\begin{array}{l}\text { Targets } \\
\text { externalising }\end{array}$ \\
\hline
\end{tabular}




\begin{tabular}{|c|c|}
\hline $\begin{array}{l}\text { 231. Cadman, D., Chambers, L. W., Walter, S. D., Ferguson, R., } \\
\text { Johnston, N., \& McNamee, J. (1987). Evaluation of public health } \\
\text { preschool child developmental screening: the process and outcomes } \\
\text { of a community program. American journal of public health, (1), 45-51. }\end{array}$ & $\begin{array}{l}\text { Not a parenting } \\
\text { intervention }\end{array}$ \\
\hline $\begin{array}{l}\text { 232. Cadzow SP, Armstrong KL and Fraser JA. 1999. Stressed } \\
\text { parents with infants: reassessing physical abuse risk factors. Child } \\
\text { Abuse Negl. 23(9):845-53. }\end{array}$ & $\begin{array}{l}\text { Not a parenting } \\
\text { intervention }\end{array}$ \\
\hline $\begin{array}{l}\text { 233. Calam R and Peters S. 2006. Assessing expressed emotion: } \\
\text { Comparing Camberwell Family Interview and Five-minute Speech } \\
\text { Sample ratings for mothers of children with behaviour problems. } \\
\text { International Journal of Methods in Psychiatric Research. 15(3):107- } \\
\text { 15. }\end{array}$ & $\begin{array}{l}\text { No child } \\
\text { internalising } \\
\text { outcomes or not } \\
\text { a major goal }\end{array}$ \\
\hline $\begin{array}{l}\text { 234. Calam, R., Sanders, M. R., Miller, C., Sadhnani, V., \& Carmont, } \\
\text { S. A. (2008). Can technology and the media help reduce dysfunctional } \\
\text { parenting and increase engagement with preventative parenting } \\
\text { interventions? Child Maltreat, 13(4), 347-361. doi: } 1077559508321272\end{array}$ & $\begin{array}{l}\text { No child } \\
\text { internalising } \\
\text { outcomes or not } \\
\text { a major goal }\end{array}$ \\
\hline $\begin{array}{l}235 . \quad \text { Camp JM, Finkelstein N. Parenting training for women in } \\
\text { residential substance abuse treatment. Journal of Substance Abuse } \\
\text { Treatment 1997;14:311-422. }\end{array}$ & Not an RCT \\
\hline $\begin{array}{l}\text { 236. Campbell, L, Clark, M, and K and S E. 1986. Stress } \\
\text { management training for parents and their children undergoing } \\
\text { cardiac catheterization. American Journal of Orthopsychiatry, 56(2), } \\
\text { 234-43. }\end{array}$ & $\begin{array}{l}\text { Intervention for } \\
\text { developmental } \\
\text { disorders } \\
\text { (autism, autism } \\
\text { spectrum } \\
\text { disorder), } \\
\text { physical } \\
\text { disabilities, } \\
\text { medical } \\
\text { conditions or } \\
\text { distress about } \\
\text { facing medical } \\
\text { interventions }\end{array}$ \\
\hline $\begin{array}{l}\text { 237. Campbell, S. B. \& Ewing, L.J. (1990). Follow-up of hard to } \\
\text { manage preschoolers: adjustment at age } 9 \text { and predictors of } \\
\text { continuing symptoms. Joumai of Child Psychology and Psychiatry, } \\
\text { 31,871- }\end{array}$ & $\begin{array}{l}\text { Not an RCT; Not } \\
\text { a parenting } \\
\text { intervention }\end{array}$ \\
\hline $\begin{array}{l}\text { 238. Campbell, S. B., Ewing, L. J., Breaux, A. M., \& Szumowski, E. K. } \\
\text { (1986). Parent-referred problem three-year olds: Follow-up at school } \\
\text { entry. Journal of Child Psychology and Psychiatry, 27, 473-488 }\end{array}$ & $\begin{array}{l}\text { Not an RCT; Not } \\
\text { a parenting } \\
\text { intervention }\end{array}$ \\
\hline $\begin{array}{l}\text { 239. Capage, L. C., Bennett, G. M., \& McNeil, C. B. (2001). A } \\
\text { comparison between African American and Caucasian children } \\
\text { referred for treatment of disruptive behavior disorders. Child \& Family } \\
\text { Behavior Therapy, 23(1), 1-14. }\end{array}$ & Not an RCT \\
\hline
\end{tabular}




\begin{tabular}{|c|c|}
\hline $\begin{array}{l}\text { 240. Caprilli S, Anastasi F, Grotto RP, Scollo Abeti M and Messeri A. } \\
\text { 2007. Interactive music as a treatment for pain and stress in children } \\
\text { during venipuncture: a randomized prospective study. J Dev Behav } \\
\text { Pediatr. 28(5):399-403. }\end{array}$ & $\begin{array}{l}\text { Intervention for } \\
\text { developmental } \\
\text { disorders } \\
\text { (autism, autism } \\
\text { spectrum } \\
\text { disorder), } \\
\text { physical } \\
\text { disabilities, } \\
\text { medical } \\
\text { conditions or } \\
\text { distress about } \\
\text { facing medical } \\
\text { interventions }\end{array}$ \\
\hline $\begin{array}{l}241 . \quad \text { Caramlau, I., Barlow, J., Sembi, S., McKenzie-McHarg, K., \& } \\
\text { McCabe, C. (2011). Mums } 4 \text { Mums: Structured telephone peer- } \\
\text { support for women experiencing postnatal depression. Pilot and } \\
\text { exploratory RCT of its clinical and cost effectiveness. Trials, } 12 \text {. }\end{array}$ & $\begin{array}{l}\text { No child } \\
\text { internalising } \\
\text { outcomes or not } \\
\text { a major goal; } \\
\text { Data not } \\
\text { reported/availab } \\
\text { le (protocol) }\end{array}$ \\
\hline $\begin{array}{l}242 . \quad \text { Carlo, W. A., Goudar, S. S., Pasha, O., Chomba, E., Wallander, J. } \\
\text { L., Biasini, F. J., ... Wright, L. L. (2013). Randomized trial of early } \\
\text { developmental intervention on outcomes in children after birth } \\
\text { asphyxia in developing countries. Journal of pediatrics, } 162(4), 705- \\
\text { 712.e703. doi:10.1016/j.jpeds.2012.09.052 }\end{array}$ & $\begin{array}{l}\text { No child } \\
\text { internalising } \\
\text { outcomes or not } \\
\text { a major goal }\end{array}$ \\
\hline $\begin{array}{l}\text { 243. Carrasco, J. M. (2011). The impact of treatment intensity on a } \\
\text { parent and child therapy program (Order No. 3419562). Available from } \\
\text { ProQuest Dissertations \& Theses Global. (755486026). }\end{array}$ & $\begin{array}{l}\text { Targets } \\
\text { externalising }\end{array}$ \\
\hline $\begin{array}{l}\text { 244. Carrick-Sen, D. M., Steen, N., \& Robson, S. C. (2014). Twin } \\
\text { parenthood: the midwife's role-a randomised controlled trial. BJOG: } \\
\text { An International Journal of Obstetrics \& Gynaecology, 121(10), 1302- } \\
1310 .\end{array}$ & $\begin{array}{l}\text { No child } \\
\text { internalising } \\
\text { outcomes or not } \\
\text { a major goal }\end{array}$ \\
\hline $\begin{array}{l}\text { 245. Carrillo X.M., Amoros, M. O., \& Sanchez. Emotive } \\
\text { performances for the phobia of the dark: A controlled essay. } \\
\text { International Journal of Clinical and Health Psychology. } 2004 \\
\text { Sep;4(3):505-20. }\end{array}$ & Not in English \\
\hline $\begin{array}{l}\text { 246. Cartwright-Hatton, S., McNally, D., White, C., \& Verduyn, C. } \\
\text { (2005). Parenting skills training: an effective intervention for } \\
\text { internalizing symptoms in younger children? J Child Adolesc Psychiatr } \\
\text { Nurs, 18(2), 45-52. doi: JCAP014 }\end{array}$ & Not an RCT \\
\hline $\begin{array}{l}\text { 247. Cartwright-Hatton, S., Roberts, C., Chitsabesan, P., Fothergill, } \\
\text { C., \& Harrington, R. (2004). Systematic review of the efficacy of } \\
\text { cognitive behaviour therapies for childhood and adolescent anxiety } \\
\text { disorders. British Journal of Clinical Psychology, 43(4), 421-436. doi: } \\
\text { 10.1348/0144665042388928 }\end{array}$ & Review \\
\hline $\begin{array}{l}\text { 248. Casey PH, Kelleher KJ, Bradley RH, Kellogg KW, Kirby RS, } \\
\text { Whiteside L. A multifaceted intervention for infants with failure to } \\
\text { thrive: a prospective study. Arch Pediatr Adoles Med. 1994;148:1071- } \\
1077\end{array}$ & $\begin{array}{l}\text { No long term } \\
\text { follow-up }\end{array}$ \\
\hline
\end{tabular}




\begin{tabular}{|c|c|}
\hline $\begin{array}{l}\text { 249. Casey R, Ludwig S and McCormick MC. } 1987 \text {. Minor head } \\
\text { trauma in children: an intervention to decrease functional morbidity. } \\
\text { Pediatrics, } 80(2), 159-164 \text {. }\end{array}$ & $\begin{array}{l}\text { Intervention for } \\
\text { developmental } \\
\text { disorders } \\
\text { (autism, autism } \\
\text { spectrum } \\
\text { disorder), } \\
\text { physical } \\
\text { disabilities, } \\
\text { medical } \\
\text { conditions or } \\
\text { distress about } \\
\text { facing medical } \\
\text { interventions }\end{array}$ \\
\hline $\begin{array}{l}\text { 250. Cassady JF, Jr., Wysocki TT, Miller KM, Cancel DD and Izenberg } \\
\text { N. 1999. Use of a preanesthetic video for facilitation of parental } \\
\text { education and anxiolysis before pediatric ambulatory surgery. Anesth } \\
\text { Analg. } 88(2): 246-50 \text {. }\end{array}$ & $\begin{array}{l}\text { Intervention for } \\
\text { developmental } \\
\text { disorders } \\
\text { (autism, autism } \\
\text { spectrum } \\
\text { disorder), } \\
\text { physical } \\
\text { disabilities, } \\
\text { medical } \\
\text { conditions or } \\
\text { distress about } \\
\text { facing medical } \\
\text { interventions }\end{array}$ \\
\hline $\begin{array}{l}\text { 251. Cassidy KL, Reid GJ, McGrath PJ, Finley GA, Smith DJ, Morley C, } \\
\text { Szudek EA and Morton B. 2002. Watch needle, watch TV: Audiovisual } \\
\text { distraction in preschool immunization. Pain Medicine, 3(2), 108-118. }\end{array}$ & $\begin{array}{l}\text { Intervention for } \\
\text { developmental } \\
\text { disorders } \\
\text { (autism, autism } \\
\text { spectrum } \\
\text { disorder), } \\
\text { physical } \\
\text { disabilities, } \\
\text { medical } \\
\text { conditions or } \\
\text { distress about } \\
\text { facing medical } \\
\text { interventions }\end{array}$ \\
\hline $\begin{array}{l}\text { 252. Catalano RR, Gainey RR, Fleming CB, Haggerty KP, Johnson } \\
\text { NO. An experimental intervention with families of substance abusers: } \\
\text { One-year follow-up of the focus on families project. Addiction } \\
\text { 1999;94:241-254 }\end{array}$ & $\begin{array}{l}\text { No child } \\
\text { internalising } \\
\text { outcomes or not } \\
\text { a major goal }\end{array}$ \\
\hline $\begin{array}{l}\text { 253. Caughy, M. O. B., Miller, T. L., Genevro, J. L., Huang, K.-Y., \& } \\
\text { Nautiyal, C. (2003). The effects of Healthy Steps on discipline } \\
\text { strategies of parents of young children. Journal of Applied } \\
\text { Developmental Psychology, 24, 517-534. }\end{array}$ & $\begin{array}{l}\text { No child } \\
\text { internalising } \\
\text { outcomes or not } \\
\text { a major goal }\end{array}$ \\
\hline $\begin{array}{l}\text { 254. Cavell, T. A., Elledge, L. C., Malcolm, K. T., Faith, M. A., \& } \\
\text { Hughes, J. N. (2009). Relationship quality and the mentoring of } \\
\text { aggressive, high-risk children. J Clin Child Adolesc Psychol, 38(2), 185- } \\
\text { 198. doi: } 909504563\end{array}$ & $\begin{array}{l}\text { Parent } \\
\text { component too } \\
\text { small }\end{array}$ \\
\hline
\end{tabular}




\begin{tabular}{|c|c|}
\hline $\begin{array}{l}255 . \quad \text { Celano, M., Hazzard, A., Webb, C., \& McCall, C. (1996). } \\
\text { Treatment of traumagenic beliefs among sexually abused girls and } \\
\text { their mothers: an evaluation study. J Abnorm Child Psychol, 24(1), 1- } \\
\text { 17. }\end{array}$ & $\begin{array}{l}\text { No long term } \\
\text { follow-up }\end{array}$ \\
\hline $\begin{array}{l}256 . \quad \text { Cernvall, M., Carlbring, P., Ljungman, G., \& Essen, L. (2013). } \\
\text { Guided self-help as intervention for traumatic stress in parents of } \\
\text { children with cancer: conceptualization, intervention strategies, and a } \\
\text { case study. Journal of psychosocial oncology, 31(1), 13-29. } \\
\text { doi:10.1080/07347332.2012.741095 }\end{array}$ & Not an RCT \\
\hline $\begin{array}{l}257 . \quad \text { Cernvall, M., Carlbring, P., Ljungman, L., Ljungman, G., \& } \\
\text { Essen, L. V. (2014). Guided Self-help via the Internet for Parents of } \\
\text { Children Recently Diagnosed with Cancer: A Randomized Controlled } \\
\text { Trial. In Psycho-Oncology (Vol. 23, No. Suppl. 3, pp. 102-102). }\end{array}$ & $\begin{array}{l}\text { No child } \\
\text { internalising } \\
\text { outcomes or not } \\
\text { a major goal }\end{array}$ \\
\hline $\begin{array}{l}258 . \quad \text { Chaffin, M., Bard, D., Bigfoot, D. S., \& Maher, E. J. (2012). Is a } \\
\text { structured, manualized, evidence-based treatment protocol culturally } \\
\text { competent and equivalently effective among American Indian parents } \\
\text { in child welfare? Child maltreatment, 17(3), 242-252. } \\
\text { doi:10.1177/1077559512457239 }\end{array}$ & $\begin{array}{l}\text { No child } \\
\text { internalising } \\
\text { outcomes or not } \\
\text { a major goal }\end{array}$ \\
\hline $\begin{array}{l}\text { 259. Chan CSM and Molassiotis A. 2002. The effects of an } \\
\text { educational programme on the anxiety and satisfaction level of } \\
\text { parents having parent present induction and visitation in a } \\
\text { postanaesthesia care unit. Pediatric Anesthesia, 12(2), 131-139. }\end{array}$ & $\begin{array}{l}\text { Intervention for } \\
\text { developmental } \\
\text { disorders } \\
\text { (autism, autism } \\
\text { spectrum } \\
\text { disorder), } \\
\text { physical } \\
\text { disabilities, } \\
\text { medical } \\
\text { conditions or } \\
\text { distress about } \\
\text { facing medical } \\
\text { interventions }\end{array}$ \\
\hline $\begin{array}{l}\text { 260. Chaplin TM, Gillham JE, Reivich K, Elkon AGL, Samuels B, } \\
\text { Freres DR, Winder B and Seligman MEP. 2006. Depression prevention } \\
\text { for early adolescent girls: A pilot study of all girls versus co-ed groups. } \\
\text { The Journal of Early Adolescence. 26(1):110-26. }\end{array}$ & $\begin{array}{l}\text { Not a parenting } \\
\text { intervention }\end{array}$ \\
\hline $\begin{array}{l}\text { 261. Chau, K., Kabuth, B., \& Chau, N. (2014). Gender and family } \\
\text { disparities in suicide attempt and role of socioeconomic, school, and } \\
\text { health-related difficulties in early adolescence. BioMed research } \\
\text { international, } 2014 .\end{array}$ & $\begin{array}{l}\text { Not a parenting } \\
\text { intervention }\end{array}$ \\
\hline $\begin{array}{l}\text { 262. Chavira DA and Stein MB. 2002. Combined psychoeducation } \\
\text { and treatment with selective serotonin reuptake inhibitors for youth } \\
\text { with generalized social anxiety disorder. J Child Adolesc } \\
\text { Psychopharmacol. 12(1):47-54. }\end{array}$ & Not an RCT \\
\hline $\begin{array}{l}\text { 263. Chavira DA, Stein MB, Bailey K and Stein MT. 2003. Parental } \\
\text { Opinions Regarding Treatment for Social Anxiety Disorder in Youth. } \\
\text { Journal of Developmental and Behavioral Pediatrics. 24(5):315-22. }\end{array}$ & $\begin{array}{l}\text { Not a parenting } \\
\text { intervention }\end{array}$ \\
\hline $\begin{array}{l}264 . \quad \text { Chazan-Cohen, R., Ayoub, C., Pan, B. A., Roggman, L., Raikes, } \\
\text { H., McKelvey, L., Whiteside-Mansell, L., \& Hart, A. (2007). It takes } \\
\text { time: Impacts on Early Head Start that lead to reductions in maternal } \\
\text { depression two years later. Infant Mental Health Journal. Special } \\
\text { Issue: Infant Mental Health in Early Head Start, } 28,151-170 .\end{array}$ & $\begin{array}{l}\text { No child } \\
\text { internalising } \\
\text { outcomes or not } \\
\text { a major goal }\end{array}$ \\
\hline
\end{tabular}




\begin{tabular}{|c|c|}
\hline $\begin{array}{l}265 . \quad \text { Chen, J. J.-L., \& Liu, X. (2012). The mediating role of perceived } \\
\text { parental warmth and parental punishment in the psychological well- } \\
\text { being of children in rural China. Social Indicators Research, 107, 483- } \\
508 .\end{array}$ & $\begin{array}{l}\text { Not a parenting } \\
\text { intervention }\end{array}$ \\
\hline $\begin{array}{l}\text { 266. Chen, J. L., Weiss, S. J., Heyman, M. B., Cooper, B., \& Lustig, R. } \\
\text { H. (2010). The Active Balance Childhood program for improving coping } \\
\text { and quality of life in Chinese American children. Nursing research, (4), } \\
\text { 270-279. }\end{array}$ & $\begin{array}{l}\text { Parent } \\
\text { component too } \\
\text { small }\end{array}$ \\
\hline $\begin{array}{l}267 . \quad \text { Chen, Y., Shen, W. W., Gao, K., Lam, C. S., Chang, W. C., \& } \\
\text { Deng, H. (2014). Effectiveness RCT of a CBT intervention for } \\
\text { youthswho lost parents in the Sichuan, China, earthquake. Psychiatric } \\
\text { Services, 65(2), 259-262. doi: } \\
\text { http://dx.doi.org/10.1176/appi.ps.201200470 }\end{array}$ & $\begin{array}{l}\text { Not a parenting } \\
\text { intervention }\end{array}$ \\
\hline $\begin{array}{l}\text { 268. Cheng, T. L., Wright, J. L., Markakis, D., Copeland-Linder, N., \& } \\
\text { Menvielle, E. (2008). Randomized trial of a case management program } \\
\text { for assault-injured youth: impact on service utilization and risk for } \\
\text { reinjury. Pediatric emergency care, (3), 130-136. }\end{array}$ & $\begin{array}{l}\text { Follow-up less } \\
\text { than } 6 \text { months }\end{array}$ \\
\hline $\begin{array}{l}\text { 269. Chernoff, R. G., Ireys, H. T., DeVet, K. A., \& Kim, Y. J. (2002). A } \\
\text { randomized, controlled trial of a community-based support program } \\
\text { for families of children with chronic illness: pediatric outcomes. Arch } \\
\text { Pediatr Adolesc Med, 156(6), 533-539. doi: poa10379 }\end{array}$ & $\begin{array}{l}\text { No long term } \\
\text { follow-up }\end{array}$ \\
\hline $\begin{array}{l}\text { 270. Cherry, V. R., Belgrave, F. Z., Jones, W., Kennon, D. K., Gray, F. } \\
\text { S., \& Phillips, F. (1998). NTU: An africentric approach to substance } \\
\text { abuse prevention among African American youth. Journal of Primary } \\
\text { Prevention, (3), 319-339. }\end{array}$ & $\begin{array}{l}\text { Parent } \\
\text { component too } \\
\text { small; Not an } \\
\text { RCT; Follow-up } \\
\text { less than } 6 \\
\text { months }\end{array}$ \\
\hline $\begin{array}{l}\text { 271. Chhangur, R. R., Weeland, J., Overbeek, G., Matthys, W., \& } \\
\text { Orobio de Castro, B. (2012). ORCHIDS: an observational randomized } \\
\text { controlled trial on childhood differential susceptibility. BMC public } \\
\text { health, } 12,917 . \text { doi:10.1186/1471-2458-12-917 }\end{array}$ & $\begin{array}{l}\text { Data not } \\
\text { available/report } \\
\text { ed (protocol) }\end{array}$ \\
\hline $\begin{array}{l}\text { 272. Chiang LC, Ma WF, Huang JL, Tseng LF and Hsueh KC. } 2009 . \\
\text { Effect of relaxation-breathing training on anxiety and asthma } \\
\text { signs/symptoms of children with moderate-to-severe asthma: a } \\
\text { randomized controlled trial. Int J Nurs Stud. 46(8):1061-70. }\end{array}$ & $\begin{array}{l}\text { Not a parenting } \\
\text { intervention }\end{array}$ \\
\hline $\begin{array}{l}\text { 273. Chisholm V, Atkinson L, Donaldson C, Noyes K, Payne A and } \\
\text { Kelnar C. 2007. Predictors of treatment adherence in young children } \\
\text { with type } 1 \text { diabetes. Journal of Advanced Nursing. 57(5):482-93. }\end{array}$ & $\begin{array}{l}\text { Not a parenting } \\
\text { intervention }\end{array}$ \\
\hline $\begin{array}{l}\text { 274. Choate, M. L., Pincus, D. B., Eyberg, S. M., \& Barlow, D. H. } \\
\text { (2005). Parent-child interaction therapy for treatment of separation } \\
\text { anxiety disorder in young children: A pilot study. Cognitive and } \\
\text { Behavioral Practice, 12(1), 126-135. }\end{array}$ & Not an RCT \\
\hline $\begin{array}{l}\text { 275. Chorpita BF, Taylor AA, Francis SE, Moffitt C and Austin AA. } \\
\text { 2004. Efficacy of Modular Cognitive Behavior Therapy for Childhood } \\
\text { Anxiety Disorders. Behavior Therapy. 35(2):263-87. }\end{array}$ & $\begin{array}{l}\text { Not a parenting } \\
\text { intervention }\end{array}$ \\
\hline $\begin{array}{l}\text { 276. Chorpita, B. F., Bernstein, A., \& Daleiden, E. L. (2011). } \\
\text { Empirically guided coordination of multiple evidence-based } \\
\text { treatments: An illustration of relevance mapping in children's mental } \\
\text { health services. J Consult Clin Psychol, 79, 470-480. }\end{array}$ & $\begin{array}{l}\text { Not a parenting } \\
\text { intervention }\end{array}$ \\
\hline
\end{tabular}




\begin{tabular}{|c|c|}
\hline $\begin{array}{l}\text { 277. Christakis, D. A., Garrison, M. M., Herrenkohl, T., Haggerty, K., } \\
\text { Rivara, F. P., Zhou, C., \& Liekweg, K. (2013). Modifying media content } \\
\text { for preschool children: A randomized controlled trial. Pediatrics, } \\
\text { 131(3), 431-438. }\end{array}$ & $\begin{array}{l}\text { Follow-up less } \\
\text { than } 6 \text { months }\end{array}$ \\
\hline $\begin{array}{l}\text { 278. Christiano B and Russ SW. 1998. Matching preparatory } \\
\text { intervention to coping style: The effects on children's distress in the } \\
\text { dental setting. J Pediatr Psychol. 23(1):17-27; discussion 9-32. }\end{array}$ & $\begin{array}{l}\text { Not a parenting } \\
\text { intervention }\end{array}$ \\
\hline $\begin{array}{l}\text { 279. Christiansen H, Oades RD, Psychogiou L, Hauffa BP and } \\
\text { Sonuga-Barke EJ. 2010. Does the cortisol response to stress mediate } \\
\text { the link between expressed emotion and oppositional behavior in } \\
\text { Attention-Deficit/Hyperactivity-Disorder (ADHD)? Behavioral and Brain } \\
\text { Functions. 6(15). }\end{array}$ & $\begin{array}{l}\text { Not a parenting } \\
\text { intervention }\end{array}$ \\
\hline $\begin{array}{l}280 . \quad \text { Christie, D. (2014). Effectiveness of a Structured Educational } \\
\text { Intervention Using Psychological Delivery Methods in Children and } \\
\text { Adolescents with Poorly Controlled Type } 1 \text { Diabetes: A Cluster } \\
\text { Randomized Controlled Trial of the CASCADE Intervention. Journal of } \\
\text { Adolescent Health, 2(54), S41. }\end{array}$ & $\begin{array}{l}\text { Not a parenting } \\
\text { intervention }\end{array}$ \\
\hline $\begin{array}{l}\text { 281. Christie, D., Thompson, R., Sawtell, M., Allen, E., Cairns, J., } \\
\text { Smith, F., ... \& Viner, R. (2014). Structured, intensive education } \\
\text { maximising engagement, motivation and long-term change for } \\
\text { children and young people with diabetes: a cluster randomised } \\
\text { controlled trial with integral process and economic evaluation-the } \\
\text { CASCADE study.Health technology assessment, } 18(20), 1-202 .\end{array}$ & $\begin{array}{l}\text { Not a parenting } \\
\text { intervention }\end{array}$ \\
\hline $\begin{array}{l}\text { 282. Chronis AM, Fabiano GA, Gnagy EM, Wymbs BT, Burrows- } \\
\text { MacLean L and Pelham WE, Jr. 2001. Comprehensive, sustained } \\
\text { behavioral and pharmacological treatment for attention- } \\
\text { deficit/hyperactivity disorder: A case study. Cognitive and Behavioral } \\
\text { Practice. 8(4):346-58. }\end{array}$ & Not an RCT \\
\hline $\begin{array}{l}\text { 283. Chronis AM, Lahey BB, Pelham WE, Jr., Williams SH, Baumann } \\
\text { BL, Kipp H, Jones HA and Rathouz PJ. 2007. Maternal depression and } \\
\text { early positive parenting predict future conduct problems in young } \\
\text { children with attention-deficit/hyperactivity disorder. Developmental } \\
\text { Psychology. 43(1):70-82. }\end{array}$ & $\begin{array}{l}\text { Not a parenting } \\
\text { intervention }\end{array}$ \\
\hline $\begin{array}{l}\text { 284. Chronis, A. M. (2003). The addition of the coping with } \\
\text { depression course to behavioral parent training for mothers of } \\
\text { children with attention deficit hyperactivity disorder. [Dissertation } \\
\text { Abstract; Dissertation]. Dissertation Abstracts International: Section B: } \\
\text { The Sciences and Engineering, 63(8-B). }\end{array}$ & $\begin{array}{l}\text { Follow-up less } \\
\text { than } 6 \text { months; } \\
\text { No child } \\
\text { internalising } \\
\text { outcomes }\end{array}$ \\
\hline $\begin{array}{l}\text { 285. Chronis, A. M., Gamble, S. A., Roberts, J. E., \& Pelham, W. E., } \\
\text { Jr. (2006). Cognitive-behavioral depression treatment for mothers of } \\
\text { children with attention-deficit/hyperactivity disorder. Behav Ther, } \\
\text { 37(2), 143-158. doi:S0005-7894(06)00016-5 }\end{array}$ & $\begin{array}{l}\text { Follow-up less } \\
\text { than } 6 \text { months; } \\
\text { No child } \\
\text { internalising } \\
\text { outcomes }\end{array}$ \\
\hline $\begin{array}{l}\text { 286. Chronis, A. M., Jones, H. A., \& Raggi, V. L. (2006). Evidence- } \\
\text { based psychosocial treatments for children and adolescents with } \\
\text { attention-deficit/hyperactivity disorder. Clinical Psychology Review, } \\
\text { 26(4), 486-502. }\end{array}$ & Review \\
\hline
\end{tabular}




\begin{tabular}{|c|c|}
\hline $\begin{array}{l}\text { 287. Chronis-Tuscano, A., \& Clarke, T. L. (2008). Behavioral skills } \\
\text { training for depressed mothers of children with ADHD L'Abate, } \\
\text { Lucciano (2008) (pp. Toward a science of clinical psychology: } \\
\text { Laboratory evaluations and interventions. (pp. 57-77). Hauppauge, NY, } \\
\text { US: Nova Science Publishers. xxi, 414). }\end{array}$ & $\begin{array}{l}\text { Not an RCT; } \\
\text { Follow-up less } \\
\text { than } 6 \text { months }\end{array}$ \\
\hline $\begin{array}{l}\text { 288. Chronis-Tuscano, A., Clarke, T. L., O'Brien, K. A., Raggi, V. L., } \\
\text { Diaz, Y., Mintz, A. D., .. Lewinsohn, P. (2013). Development and } \\
\text { preliminary evaluation of an integrated treatment targeting parenting } \\
\text { and depressive symptoms in mothers of children with attention- } \\
\text { deficit/hyperactivity disorder. Journal of Consulting and Clinical } \\
\text { Psychology, } 81(5), 918-925 . \text { doi: http://dx.doi.org/10.1037/a0032112 }\end{array}$ & $\begin{array}{l}\text { Follow-up less } \\
\text { than } 6 \text { months }\end{array}$ \\
\hline $\begin{array}{l}\text { 289. Chronis-Tuscano, A., Rubin, K. H., O'Brien, K. A., Coplan, R. J., } \\
\text { Thomas, S. R., Dougherty, L. R., ... \& Wimsatt, M. (2015). Preliminary } \\
\text { evaluation of a multimodal early intervention program for behaviorally } \\
\text { inhibited preschoolers. Journal of consulting and clinical psychology, } \\
\text { 83(3), 534. }\end{array}$ & $\begin{array}{l}\text { No long term } \\
\text { follow-up }\end{array}$ \\
\hline $\begin{array}{l}\text { 290. Chu BC, Colognori D, Weissman AS and Bannon K. 2009. An } \\
\text { initial description and pilot of group behavioral activation therapy for } \\
\text { anxious and depressed youth. Cognitive and Behavioral Practice. } \\
\text { 16(4):408-19. }\end{array}$ & $\begin{array}{l}\text { Not a parenting } \\
\text { intervention }\end{array}$ \\
\hline $\begin{array}{l}\text { 291. Chung B, Suzuki AR and McGough JJ. 2002. New drugs for } \\
\text { treatment of attention-deficit/hyperactivity disorder. Expert Opinion } \\
\text { on Emerging Drugs. 7(2):269-76. }\end{array}$ & $\begin{array}{l}\text { Not a parenting } \\
\text { intervention }\end{array}$ \\
\hline $\begin{array}{l}\text { 292. Cicchetti, D., Rogosch, F. A., \& Toth, S. L. (2000). The efficacy } \\
\text { of toddler-parent psychotherapy for fostering cognitive development } \\
\text { in offspring of depressed mothers. Journal of Abnormal Child } \\
\text { Psychology, 28, 135-148. }\end{array}$ & $\begin{array}{l}\text { No long term } \\
\text { follow-up; No } \\
\text { child } \\
\text { internalising } \\
\text { outcomes or not } \\
\text { a major goal }\end{array}$ \\
\hline $\begin{array}{l}\text { 293. Cicchetti, D., Toth, S. L., \& Rogosch, F. A. (1999). The efficacy } \\
\text { of toddler-parent psychotherapy to increase attachment security in } \\
\text { offspring of depressed mothers. Attachment \& Human Development, } \\
\text { 1, 34-66. }\end{array}$ & $\begin{array}{l}\text { No child } \\
\text { internalising } \\
\text { outcomes or not } \\
\text { a major goal }\end{array}$ \\
\hline $\begin{array}{l}\text { 294. Ciechomski, L. D., Jackson, K. L., Tonge, B., King, N. J., \& Heyne, } \\
\text { D. A. (2001). Intellectual disability and anxiety in children: A group- } \\
\text { based parent skills-training intervention. Behaviour Change, (4), 204- } \\
212 .\end{array}$ & $\begin{array}{l}\text { Data not } \\
\text { available/report } \\
\text { ed }\end{array}$ \\
\hline $\begin{array}{l}\text { 295. Clarke GN, Hornbrook M, Lynch F, Polen M, Gale J, Beardslee } \\
\text { W, O'Connor E and Seeley J. 2001. A randomized trial of a group } \\
\text { cognitive intervention for preventing depression in adolescent } \\
\text { offspring of depressed parents. Arch Gen Psychiatry.58(12):1127-34. }\end{array}$ & $\begin{array}{l}\text { Not a parenting } \\
\text { intervention }\end{array}$ \\
\hline $\begin{array}{l}\text { 296. Clarke GN, Hornbrook M, Lynch F, Polen M, Gale J, O'Connor } \\
\text { E, Seeley JR and Debar L. 2002. Group cognitive-behavioral treatment } \\
\text { for depressed adolescent offspring of depressed parents in a health } \\
\text { maintenance organization. J Am Acad Child Adolesc Psychiatry. } \\
\text { 41(3):305-13. }\end{array}$ & $\begin{array}{l}\text { Not a parenting } \\
\text { intervention }\end{array}$ \\
\hline $\begin{array}{l}\text { 297. Clarke-Pounder, J. P., Boss, R. D., Roter, D. L., Hutton, N., } \\
\text { Larson, S., \& Donohue, P. K. (2015). Communication intervention in } \\
\text { the neonatal intensive care unit: Can it backfire?. Journal of palliative } \\
\text { medicine, 18(2), 157-161. }\end{array}$ & $\begin{array}{l}\text { No child } \\
\text { internalising } \\
\text { outcomes or not } \\
\text { a major goal }\end{array}$ \\
\hline
\end{tabular}




\begin{tabular}{|c|c|}
\hline $\begin{array}{l}\text { 298. Clayton DM. 2007. Parenting education for low-income } \\
\text { parents of preschoolers: What is the most effective approach? } \\
\text { Dissertation Abstracts International: Section B: The Sciences and } \\
\text { Engineering. 68(5-B). }\end{array}$ & Not an RCT \\
\hline $\begin{array}{l}\text { 299. Coard, S. I., Wallace, S. A., Stevenson Jr, H. C., \& Brotman, L. } \\
\text { M. (2004). Towards Culturally Relevant Preventive Interventions: The } \\
\text { Consideration of Racial Socialization in Parent Training with African } \\
\text { American Families. Journal of Child \& Family Studies, 13, 277-293. }\end{array}$ & Not an RCT \\
\hline $\begin{array}{l}\text { 300. Coates AO, Schaefer CA and Alexander JL. 2004. Detection of } \\
\text { Postpartum Depression and Anxiety in a Large Health Plan. The Journal } \\
\text { of Behavioral Health Services \& Research. 31(2):117-33. }\end{array}$ & $\begin{array}{l}\text { Not a parenting } \\
\text { intervention }\end{array}$ \\
\hline $\begin{array}{l}\text { Coates S and Schechter D. 2004. Preschoolers' traumatic stress } \\
\text { post-9/11: Relational and developmental perspectives. Psychiatric } \\
\text { Clinics of North America. Special Issue: Disaster psychiatry, a closer } \\
\text { look. 27(3):473-89. }\end{array}$ & $\begin{array}{l}\text { Not a parenting } \\
\text { intervention }\end{array}$ \\
\hline $\begin{array}{l}\text { 302. Cobham VE, Dadds MR and Spence SH. 1998. The role of } \\
\text { parental anxiety in the treatment of childhood anxiety. Journal of } \\
\text { Consulting and Clinical Psychology.66(6):893-905. }\end{array}$ & $\begin{array}{l}\text { Comparison } \\
\text { condition not a } \\
\text { minimal control }\end{array}$ \\
\hline $\begin{array}{l}\text { 303. Cobham VE. 2003. Evaluation of a Brief Child-focused Group- } \\
\text { based Intervention for Anxiety-disordered Children. Behaviour } \\
\text { Change. 20(2):109-16. }\end{array}$ & $\begin{array}{l}\text { Not a parenting } \\
\text { intervention }\end{array}$ \\
\hline $\begin{array}{l}\text { 304. Cobham, V. E. (2012). Do anxiety-disordered children need to } \\
\text { come into the clinic for efficacious treatment? J Consult Clin Psychol, } \\
80,465-476 .\end{array}$ & $\begin{array}{l}\text { Follow-up data } \\
\text { collected only in } \\
\text { active group }\end{array}$ \\
\hline $\begin{array}{l}\text { 305. Cobham, V. E., Dadds, M. R., Spence, S. H., \& McDermott, B. } \\
\text { (2010). Parental anxiety in the treatment of childhood anxiety: A } \\
\text { different story three years later. Journal of Clinical Child and } \\
\text { Adolescent Psychology, 39(3), 410-420. doi: } \\
\text { 10.1080/15374411003691719 }\end{array}$ & $\begin{array}{l}\text { Comparison } \\
\text { condition not a } \\
\text { minimal control }\end{array}$ \\
\hline $\begin{array}{l}\text { 306. Coelho KF. 1999. Attachment Guided Multisystemic Therapy: } \\
\text { A multicomponent intervention for children who are displaying } \\
\text { externalizing behaviors. Dissertation Abstracts International: Section } \\
\text { B: The Sciences and Engineering. 59(9-B). }\end{array}$ & Not an RCT \\
\hline $\begin{array}{l}\text { 307. Coffman S, Levitt MJ and Brown L. 1994. Effects of clarification } \\
\text { of support expectations in prenatal couples. Nursing Research, 43(2), } \\
\text { 111-116. }\end{array}$ & $\begin{array}{l}\text { No child } \\
\text { internalising } \\
\text { outcomes or not } \\
\text { a major goal }\end{array}$ \\
\hline $\begin{array}{l}\text { 308. Cohen JA, Mannarino AP (1996a), A treatment outcome study } \\
\text { for sexually abused preschool children: initial findings. J Am Acad Child } \\
\text { Adolesc Psychiatry 35:42-50. }\end{array}$ & $\begin{array}{l}\text { No long term } \\
\text { follow-up }\end{array}$ \\
\hline
\end{tabular}




\begin{tabular}{|c|c|}
\hline $\begin{array}{l}\text { 309. Cohen LL, Blount RL and Panopoulos G. 1997. Nurse coaching } \\
\text { and cartoon distraction: An effective and practical intervention to } \\
\text { reduce child, parent, and nurse distress during immunizations. Journal } \\
\text { of Pediatric Psychology. 22(3):355-70. }\end{array}$ & $\begin{array}{l}\text { Intervention for } \\
\text { developmental } \\
\text { disorders } \\
\text { (autism, autism } \\
\text { spectrum } \\
\text { disorder), } \\
\text { physical } \\
\text { disabilities, } \\
\text { medical } \\
\text { conditions or } \\
\text { distress about } \\
\text { facing medical } \\
\text { interventions }\end{array}$ \\
\hline $\begin{array}{l}\text { 310. Cohen, J. A., \& Mannarino, A. P. (1998). Factors that mediate } \\
\text { treatment outcome of sexually abused preschool children: six- and 12- } \\
\text { month follow-up. Journal of the American Academy of Child \& } \\
\text { Adolescent Psychiatry, (1), 44-51. }\end{array}$ & $\begin{array}{l}\text { Secondary } \\
\text { analysis of } \\
\text { Cohen } 1997 \text { - } \\
\text { predictors only, } \\
\text { not primary } \\
\text { outcomes }\end{array}$ \\
\hline $\begin{array}{l}\text { 311. Cohen, N. J., Sullivan, J., Minde, K., Novak, C., \& Helwig, C. } \\
\text { (1981). Evaluation of the Relative Effectiveness of Methylphenidate } \\
\text { and Cognitive Behavior-Modification in the Treatment of } \\
\text { Kindergarten-Aged Hyperactive-Children. Journal of Abnormal Child } \\
\text { Psychology, 9(1), 43-54. }\end{array}$ & $\begin{array}{l}\text { Not a parenting } \\
\text { intervention }\end{array}$ \\
\hline $\begin{array}{l}\text { 312. Colditz, P., Sanders, M. R., Boyd, R., Pritchard, M., Gray, P., } \\
\text { O'Callaghan, M. J., ... \& Jardine, L. (2015). Prem Baby Triple P: a } \\
\text { randomised controlled trial of enhanced parenting capacity to } \\
\text { improve developmental outcomes in preterm infants. BMC pediatrics, } \\
\text { 15(1), } 15 .\end{array}$ & $\begin{array}{l}\text { Data not } \\
\text { available/report } \\
\text { ed }\end{array}$ \\
\hline $\begin{array}{l}\text { 313. Colletti CJM, Forehand R, Garai E, McKee L, Potts J, Haker K, } \\
\text { Champion J and Compas BE. 2010. Associations of parent-child anxious } \\
\text { and depressive symptoms when a caregiver has a history of } \\
\text { depression. Journal of Child and Family Studies. 19(6):762-70. }\end{array}$ & $\begin{array}{l}\text { Not a parenting } \\
\text { intervention }\end{array}$ \\
\hline $\begin{array}{l}\text { 314. Colville G, Cream P and Kerry S. 2010. High stress during } \\
\text { admission predicts the impact of a PICU follow-up clinic on parents' } \\
\text { psychological outcome. Critical Care. 14:S200. }\end{array}$ & $\begin{array}{l}\text { No child } \\
\text { internalising } \\
\text { outcomes or not } \\
\text { a major goal }\end{array}$ \\
\hline $\begin{array}{l}\text { 315. Colville GA, Cream PR and Kerry SM. 2010. Do parents benefit } \\
\text { from the offer of a follow-up appointment after their child's admission } \\
\text { to intensive care?: an exploratory randomised controlled trial. } \\
\text { Intensive and Critical Care Nursing, 26(3), 146-153. }\end{array}$ & $\begin{array}{l}\text { No child } \\
\text { internalising } \\
\text { outcomes or not } \\
\text { a major goal }\end{array}$ \\
\hline $\begin{array}{l}\text { 316. Comer, J. S., Furr, J. M., Beidas, R. S., Weiner, C. L., \& Kendall, } \\
\text { P. C. (2008). Children and terrorism-related news: training parents in } \\
\text { Coping and Media Literacy. J Consult Clin Psychol, 76(4), 568-578. doi: } \\
\text { 2008-09736-004 }\end{array}$ & $\begin{array}{l}\text { No long term } \\
\text { follow-up }\end{array}$ \\
\hline $\begin{array}{l}\text { 317. Comer, J. S., Puliafico, A. C., Aschenbrand, S. G., McKnight, K., } \\
\text { Robin, J. A., Goldfine, M. E., \& Albano, A. M. (2012). A pilot feasibility } \\
\text { evaluation of the CALM Program for anxiety disorders in early } \\
\text { childhood. J Anxiety Disord, 26, 40-49. }\end{array}$ & Not an RCT \\
\hline
\end{tabular}




\begin{tabular}{|c|c|}
\hline $\begin{array}{l}\text { 318. Compas BE, Champion JE, Forehand R, Cole DA, Reeslund KL, } \\
\text { Fear J, et al. Coping and parenting: Mediators of 12-month outcomes } \\
\text { of a family group cognitive-behavioral preventive intervention with } \\
\text { families of depressed parents. J Consult Clin Psychol. } 2010 \\
\text { Oct; } 78(5): 623-34 \text {. }\end{array}$ & $\begin{array}{l}\text { Parent } \\
\text { component too } \\
\text { small }\end{array}$ \\
\hline $\begin{array}{l}\text { 319. Compas BE, Forehand R, Keller G, Champion JE, Rakow A, } \\
\text { Reeslund KL, et al. Randomized controlled trial of a family cognitive- } \\
\text { behavioral preventive intervention for children of depressed parents. } \\
\text { Journal of Consulting and Clinical Psychology. 2009 Dec;77(6):1007-20. }\end{array}$ & $\begin{array}{l}\text { Parent } \\
\text { component too } \\
\text { small }\end{array}$ \\
\hline $\begin{array}{l}\text { 320. Compas, B. E., Forehand, R., Thigpen, J. C., Keller, G., } \\
\text { Hardcastle, E. J., Cole, D. A., Potts, J., H. Watson, K., Rakow, A., Colletti, } \\
\text { C., Reeslund, K., Fear, J., Garai, E., McKee, L., Merchant, M. J., \& } \\
\text { Roberts, L. (2011). Family group cognitive-behavioral preventive } \\
\text { intervention for families of depressed parents: } 18 \text { - and 24-month } \\
\text { outcomes. J Consult Clin Psychol, 79, 488-499. }\end{array}$ & $\begin{array}{l}\text { Secondary } \\
\text { analysis of an } \\
\text { excluded study } \\
\text { (Compas 2009) } \\
\text { which was } \\
\text { excluded } \\
\text { because parent } \\
\text { component too } \\
\text { small. }\end{array}$ \\
\hline $\begin{array}{l}\text { 321. Compas, B. E., Forehand, R., Thigpen, J., Hardcastle, E., Garai, } \\
\text { E., McKee, L., ... \& Sterba, S. (2015). Efficacy and moderators of a } \\
\text { family group cognitive-behavioral preventive intervention for children } \\
\text { of parents with depression. Journal of consulting and clinical } \\
\text { psychology, } 83(3), 541 .\end{array}$ & $\begin{array}{l}\text { Parent } \\
\text { component too } \\
\text { small }\end{array}$ \\
\hline $\begin{array}{l}\text { 322. Compas, B. E., Keller, G., \& Forehand, R. (2011). Preventive } \\
\text { intervention in families of depressed parents: A family cognitive- } \\
\text { behavioral intervention. In T. J. Strauman, P. R. Costanzo \& J. Garber } \\
\text { (Eds.), Depression in adolescent girls: Science and prevention. (pp. } \\
\text { 318-339). New York, NY US: Guilford Press. }\end{array}$ & $\begin{array}{l}\text { Secondary } \\
\text { analysis of an } \\
\text { excluded study } \\
\text { (Compas 2009) } \\
\text { which was } \\
\text { excluded } \\
\text { because parent } \\
\text { component too } \\
\text { small. }\end{array}$ \\
\hline $\begin{array}{l}\text { 323. Conant KD, Morgan AK, Muzykewicz D, Clark DC and Thiele EA. } \\
\text { 2008. A karate program for improving self-concept and quality of life } \\
\text { in childhood epilepsy: results of a pilot study. Epilepsy Behav. } \\
\text { 12(1):61-5. }\end{array}$ & $\begin{array}{l}\text { Not a parenting } \\
\text { intervention }\end{array}$ \\
\hline $\begin{array}{l}\text { 324. Conduct Problems Prevention Research Group. (1999). Initial } \\
\text { impact of the Fast Track prevention trial for conduct problems: I. The } \\
\text { high-risk sample. Journal of consulting and clinical psychology, (5), } \\
\text { 631-647. }\end{array}$ & $\begin{array}{l}\text { No child } \\
\text { internalising } \\
\text { outcomes or not } \\
\text { a major goal }\end{array}$ \\
\hline $\begin{array}{l}\text { 325. Conduct Problems Prevention Research Group. (2007). Fast } \\
\text { track randomized controlled trial to prevent externalizing psychiatric } \\
\text { disorders: findings from grades } 3 \text { to } 9 \text {. J Am Acad Child Adolesc } \\
\text { Psychiatry, } 46(10), 1250-1262 \text {. doi: } 10.1097 / \text { chi.0b013e31813e5d39 }\end{array}$ & $\begin{array}{l}\text { No child } \\
\text { internalising } \\
\text { outcomes or not } \\
\text { a major goal }\end{array}$ \\
\hline $\begin{array}{l}\text { 326. Conduct Problems Prevention Research Group. (2011). The } \\
\text { effects of the Fast Track preventive intervention on the development } \\
\text { of conduct disorder across childhood. Child Development, 82(1), 331- } \\
\text { 345. doi: } 10.1111 / \text { j.1467-8624.2010.01558.x }\end{array}$ & $\begin{array}{l}\text { No child } \\
\text { internalising } \\
\text { outcomes or not } \\
\text { a major goal }\end{array}$ \\
\hline
\end{tabular}




\begin{tabular}{|c|c|}
\hline $\begin{array}{l}\text { 327. Connell AM, Dishion TJ. Reducing depression among at-risk } \\
\text { early adolescents: Three-year effects of a family-centered intervention } \\
\text { embedded within schools. Journal of Family Psychology Special Issue: } \\
\text { Public health perspectives on family interventions. } 2008 \\
\text { Aug;22(4):574-85. }\end{array}$ & $\begin{array}{l}\text { No long term } \\
\text { follow-up }\end{array}$ \\
\hline $\begin{array}{l}\text { 328. Connell S, Sanders MR and Markie-Dadds C. 1997. Self- } \\
\text { directed behavioral family intervention for parents of oppositional } \\
\text { children in rural and remote areas. Behav Modif. 21(4):379-408. }\end{array}$ & $\begin{array}{l}\text { Follow-up less } \\
\text { than } 6 \text { months; } \\
\text { no child } \\
\text { internalising } \\
\text { outcomes or not } \\
\text { a major goal }\end{array}$ \\
\hline $\begin{array}{l}\text { 329. Connell, A., Dishion, T., Yasui, M., \& Kavanagh, K. (2007). An } \\
\text { adaptive approach to family intervention: Linking engagement in } \\
\text { family-centered intervention to reductions in adolescent problem } \\
\text { behavior. Journal of Consulting and Clinical Psychology, 75, 568-579. }\end{array}$ & $\begin{array}{l}\text { No child } \\
\text { internalising } \\
\text { outcomes or not } \\
\text { a major goal }\end{array}$ \\
\hline $\begin{array}{l}\text { 330. Connolly L, Sharry J, Fitzpatrick C (2001) Evaluation of a group } \\
\text { treatment programme for parents of children with behavioural } \\
\text { disorders. Child Psychol Psychiatry Rev 6:159-165 }\end{array}$ & $\begin{array}{l}\text { Follow-up data } \\
\text { collected only in } \\
\text { active group }\end{array}$ \\
\hline $\begin{array}{l}\text { 331. Connor-Smith JK and Weisz JR. 2003. Applying treatment } \\
\text { outcome research in clinical practice: Techniques for adapting } \\
\text { interventions to the real world. Child and Adolescent Mental Health. } \\
\text { 8(1):3-10. }\end{array}$ & $\begin{array}{l}\text { Not a parenting } \\
\text { intervention }\end{array}$ \\
\hline $\begin{array}{l}\text { 332. Cook, F., Bayer, J., Le, H. N. D., Mensah, F., Cann, W., \& } \\
\text { Hiscock, H. (2012). Baby Business: A randomised controlled trial of a } \\
\text { universal parenting program that aims to prevent early infant sleep } \\
\text { and cry problems and associated parental depression. BMC Pediatr, } \\
\text { 12. }\end{array}$ & $\begin{array}{l}\text { Data not } \\
\text { available/report } \\
\text { ed (protocol) }\end{array}$ \\
\hline $\begin{array}{l}\text { 333. Cookston, J. T., \& Fung, W. W. (2011). The kids' turn program } \\
\text { evaluation: Probing change within a community?based intervention } \\
\text { for separating families. Family Court Review, } 49,348-363 \text {. }\end{array}$ & $\begin{array}{l}\text { No long term } \\
\text { follow-up }\end{array}$ \\
\hline $\begin{array}{l}\text { 334. Cooper PJ, Landman M, Tomlinson M, Molteno C, Swartz L and } \\
\text { Murray L. 2002. Impact of a mother-infant intervention in an indigent } \\
\text { peri-urban South African context: pilot study. Br J Psychiatry. 180:76- } \\
81 .\end{array}$ & Not an RCT \\
\hline $\begin{array}{l}\text { 335. Cooper, P. J., Tomlinson, M., Swartz, L., Landman, M., } \\
\text { Molteno, C., Stein, A., et al. (2009). Improving quality of mother-infant } \\
\text { relationship and infant attachment in socioeconomically deprived } \\
\text { community in South Africa: randomised controlled trial. BMJ (Clinical } \\
\text { research ed.), b974. }\end{array}$ & $\begin{array}{l}\text { No child } \\
\text { internalising } \\
\text { outcomes or not } \\
\text { a major goal }\end{array}$ \\
\hline $\begin{array}{l}\text { 336. Copping VE, Warling DL, Benner DG and Woodside DW. } 2001 . \\
\text { A child trauma treatment pilot study. Journal of Child and Family } \\
\text { Studies. 10(4):467-75. }\end{array}$ & Not an RCT \\
\hline $\begin{array}{l}\text { 337. Corrin, E. G. (2004). Child group training versus parent and } \\
\text { child group training for young children with ADHD. [Dissertation } \\
\text { Abstract; Dissertation]. Dissertation Abstracts International: Section B: } \\
\text { The Sciences and Engineering, 64(7-B). }\end{array}$ & $\begin{array}{l}\text { No long term } \\
\text { follow-up }\end{array}$ \\
\hline $\begin{array}{l}\text { 338. Costin J, Vance A, Barnett R, O'Shea M and Luk ESL. } 2002 . \\
\text { Attention deficit hyperactivity disorder and comorbid anxiety: } \\
\text { Practitioner problems in treatment planning. Child and Adolescent } \\
\text { Mental Health. 7(1):16-24. }\end{array}$ & Not an RCT \\
\hline
\end{tabular}




\begin{tabular}{|c|c|}
\hline $\begin{array}{l}\text { 339. Costin, J., \& Chambers, S. (2007). Parent Management } \\
\text { Training as a treatment for children with Oppositional Defiant Disorder } \\
\text { referred to a mental health clinic. Clinical Child Psychology and } \\
\text { Psychiatry, 12, 511-524.of Child Psychology and Psychiatry, 40,1185- } \\
1196 .\end{array}$ & $\begin{array}{l}\text { Follow-up less } \\
\text { than } 6 \text { months }\end{array}$ \\
\hline $\begin{array}{l}\text { 340. Coughlin, M., Sharry, J., Fitzpatrick, C., Guerin, S., \& Drumm, } \\
\text { M. (2009). A controlled clinical evaluation of the parents plus } \\
\text { children's programme: a video-based programme for parents of } \\
\text { children aged } 6 \text { to } 11 \text { with behavioural and developmental problems. } \\
\text { Clinical child psychology and psychiatry, (4), 541-558. }\end{array}$ & $\begin{array}{l}\text { Follow-up less } \\
\text { than } 6 \text { months }\end{array}$ \\
\hline $\begin{array}{l}\text { 341. Cox, C. M., Kenardy, J. A., \& Hendrikz, J. K. (2010). A } \\
\text { randomized controlled trial of a web-based early intervention for } \\
\text { children and their parents following unintentional injury. J Pediatr } \\
\text { Psychol, 35(6), 581-592. doi: jsp095 }\end{array}$ & $\begin{array}{l}\text { Follow-up less } \\
\text { than } 6 \text { months }\end{array}$ \\
\hline $\begin{array}{l}\text { 342. Coyne CA, Xu R, Raich P, Plomer K, Dignan M, Wenzel LB, } \\
\text { Fairclough D, Habermann T, Schnell L, Quella S, Cella D and Eastern } \\
\text { Cooperative Oncology G. 2003. Randomized, controlled trial of an } \\
\text { easy-to-read informed consent statement for clinical trial } \\
\text { participation: a study of the Eastern Cooperative Oncology Group. } \\
\text { Journal of Clinical Oncology, 21(5), 836-842. }\end{array}$ & $\begin{array}{l}\text { Not a parenting } \\
\text { intervention }\end{array}$ \\
\hline $\begin{array}{l}\text { 343. Crawford AM and Manassis K. 2001. Familial predictors of } \\
\text { treatment outcome in childhood anxiety disorders. Journal of the } \\
\text { American Academy of Child \& Adolescent Psychiatry. 40(10):1182-9. }\end{array}$ & $\begin{array}{l}\text { Not a parenting } \\
\text { intervention }\end{array}$ \\
\hline $\begin{array}{l}\text { 344. Crawley SA, Beidas RS, Benjamin CL, Martin E and Kendall PC. } \\
\text { 2008. Treating socially phobic youth with CBT: Differential outcomes } \\
\text { and treatment considerations. Behavioural and Cognitive } \\
\text { Psychotherapy. 36(4):379-89. }\end{array}$ & $\begin{array}{l}\text { Not a parenting } \\
\text { intervention }\end{array}$ \\
\hline $\begin{array}{l}\text { 345. Crisante, L. (2003). Implementation and Process Issues in } \\
\text { Using Group Triple P with Chinese Parents: Preliminary Findings. } \\
\text { Australian eJournal for the advancement of mental health, 2, n3. }\end{array}$ & $\begin{array}{l}\text { No long term } \\
\text { follow-up }\end{array}$ \\
\hline $\begin{array}{l}\text { 346. Crockett K, Zlotnick C, Davis M, Payne N and Washington R. } \\
\text { 2008. A depression preventive intervention for rural low-income } \\
\text { African-American pregnant women at risk for postpartum depression. } \\
\text { Arch Womens Ment Health. 11(5-6):319-25. }\end{array}$ & $\begin{array}{l}\text { No child } \\
\text { internalising } \\
\text { outcomes or not } \\
\text { a major goal }\end{array}$ \\
\hline $\begin{array}{l}\text { 347. Crosby DA, Dowsett CJ, Gennetian LA and Huston AC. 2010. A } \\
\text { tale of two methods: Comparing regression and instrumental variables } \\
\text { estimates of the effects of preschool child care type on the } \\
\text { subsequent externalizing behavior of children in low-income families. } \\
\text { Developmental Psychology. 46(5):1030-48. }\end{array}$ & $\begin{array}{l}\text { Not a parenting } \\
\text { intervention }\end{array}$ \\
\hline $\begin{array}{l}\text { 348. Cross, W., West, J., Wyman, P. A., Schmeelk-Cone, K., Xia, Y., } \\
\text { Tu, X., ... \& Forgatch, M. (2015). Observational measures of } \\
\text { implementer fidelity for a school-based preventive intervention: } \\
\text { development, reliability, and validity. Prevention Science, 16(1), 122- } \\
\text { 132. }\end{array}$ & $\begin{array}{l}\text { Not a parenting } \\
\text { intervention }\end{array}$ \\
\hline $\begin{array}{l}\text { 349. Crowe } M \text { and Luty S. 2005. Interpersonal psychotherapy: An } \\
\text { effective psychotherapeutic intervention for mental health nursing } \\
\text { practice. International Journal of Mental Health Nursing. 14(2):126-33. }\end{array}$ & $\begin{array}{l}\text { Not a parenting } \\
\text { intervention }\end{array}$ \\
\hline $\begin{array}{l}\text { 350. Crowe M and Luty S. 2005. The Process of Change in } \\
\text { Interpersonal Psychotherapy (IPT) for Depression: A Case Study for the } \\
\text { New IPT Therapist. Psychiatry: Interpersonal and Biological Processes. } \\
\text { 68(1):43-54. }\end{array}$ & $\begin{array}{l}\text { Not a parenting } \\
\text { intervention }\end{array}$ \\
\hline
\end{tabular}




\begin{tabular}{|c|c|}
\hline $\begin{array}{l}\text { 351. Cuesta-Barriuso, R., Torres-Ortuño, A., López-García, M., \& } \\
\text { Nieto-Munuera, J. (2014). Effectiveness of an educational intervention } \\
\text { of Physiotherapy in parents of children with haemophilia. } \\
\text { Haemophilia, 20(6), 866-872. }\end{array}$ & Not an RCT \\
\hline $\begin{array}{l}\text { 352. Cukrowicz KC, Smith PN, Hohmeister HC and Joiner TE, Jr. } \\
\text { 2009. The moderation of an early intervention program for anxiety } \\
\text { and depression by specific psychological symptoms. Journal of Clinical } \\
\text { Psychology. 65(4):337-51. }\end{array}$ & $\begin{array}{l}\text { Not a parenting } \\
\text { intervention }\end{array}$ \\
\hline $\begin{array}{l}\text { 353. Cummings CM and Fristad MA. 2007. Medications prescribed } \\
\text { for children with mood disorders: effects of a family-based } \\
\text { psychoeducation program. Exp Clin Psychopharmacol. 15(6):555-62. }\end{array}$ & $\begin{array}{l}\text { No child } \\
\text { internalising } \\
\text { outcomes or not } \\
\text { a major goal }\end{array}$ \\
\hline $\begin{array}{l}\text { 354. Cummings, J. G., \& Wittenberg, J.-V. (2008). Supportive } \\
\text { expressive therapy--Parent child version: An exploratory study. } \\
\text { Psychotherapy: Theory, Research, Practice, Training. Special Issue: } \\
\text { New treatments in psychotherapy, 45(2), 148-164. doi: 10.1037/0033- } \\
\text { 3204.45.2.148 }\end{array}$ & $\begin{array}{l}\text { Targets } \\
\text { externalising }\end{array}$ \\
\hline $\begin{array}{l}\text { 355. Cunningham CE, Boyle M, Offord D, Racine Y, Hundert J, } \\
\text { Secord M and McDonald J. 2000. Tri-ministry study: Correlates of } \\
\text { school-based parenting course utilization. Journal of Consulting and } \\
\text { Clinical Psychology. 68(5):928-33. }\end{array}$ & Not an RCT \\
\hline $\begin{array}{l}\text { 356. Cunningham S, Deere S, Symon A, Elton RA and McIntosh N. } \\
\text { 1998. A randomized, controlled trial of computerized physiologic } \\
\text { trend monitoring in an intensive care unit. Crit Care Med. 26(12):2053- } \\
60 .\end{array}$ & $\begin{array}{l}\text { Not a parenting } \\
\text { intervention }\end{array}$ \\
\hline $\begin{array}{l}\text { 357. Cunningham, C. E., Bremner, R., \& Boyle, M. (1995). Large } \\
\text { group community-based parenting programs for families of } \\
\text { preschoolers at risk for disruptive behaviour disorders: utilization, cost } \\
\text { effectiveness, and outcome. Journal of child psychology and } \\
\text { psychiatry, and allied disciplines, (7), 1141-1159. }\end{array}$ & $\begin{array}{l}\text { Targets } \\
\text { externalising } \\
\text { (preschoolers at } \\
\text { risk for } \\
\text { disruptive } \\
\text { behaviour } \\
\text { disorders) } \\
\end{array}$ \\
\hline $\begin{array}{l}\text { 358. Curry, J., Rohde, P., Simons, A., Silva, S., Vitiello, B., Kratochvil, } \\
\text { C., ... March, J. (2006). Predictors and Moderators of Acute Outcome } \\
\text { in the Treatment for Adolescents With Depression Study (TADS). } \\
\text { Journal of the American Academy of Child \& Adolescent Psychiatry, } \\
\text { 45(12), 1427-1439. }\end{array}$ & $\begin{array}{l}\text { Not a parenting } \\
\text { intervention }\end{array}$ \\
\hline $\begin{array}{l}\text { 359. Cutuli, J. J., Gillham, J. E., Chaplin, T. M., Reivich, K. J., } \\
\text { Seligman, M.E.P., Gallop, R.J., Abenavoli, R. M., Freres, D. R. (2013). } \\
\text { Preventing adolescents' externalizing and internalizing symptoms: } \\
\text { Effects of the Penn Resiliency Program. The International Journal of } \\
\text { Emotional Education, } 5 \text { (2), 67-79. }\end{array}$ & $\begin{array}{l}\text { Not a parenting } \\
\text { intervention }\end{array}$ \\
\hline $\begin{array}{l}\text { 360. Dadds MR, Holland DE, Laurens KR, Mullins M, Barrett PM, } \\
\text { Spence SH. Early intervention and prevention of anxiety disorders in } \\
\text { children: results at 2-year follow-up. J Consult Clin Psychol. } 1999 \\
\text { Feb;67(1):145-50. }\end{array}$ & $\begin{array}{l}\text { Parent } \\
\text { component too } \\
\text { small }\end{array}$ \\
\hline
\end{tabular}




\begin{tabular}{|c|c|}
\hline $\begin{array}{l}\text { 361. Dadds MR, Schwartz S and Sanders MR. 1987. Marital Discord } \\
\text { and Treatment Outcome in Behavioral Treatment of Child Conduct } \\
\text { Disorders. Journal of Consulting and Clinical Psychology. 55(3):396- } \\
\text { 403. }\end{array}$ & $\begin{array}{l}\text { Comparison } \\
\text { condition not a } \\
\text { minimal control; } \\
\text { No child } \\
\text { internalising } \\
\text { outcomes or not } \\
\text { a major goal }\end{array}$ \\
\hline $\begin{array}{l}\text { 362. Dadds MR, Spence SH, Holland DE, Barrett PM, Laurens KR. } \\
\text { Prevention and early intervention for anxiety disorders: a controlled } \\
\text { trial. J Consult Clin Psychol. } 1997 \text { Aug;65(4):627-35. }\end{array}$ & $\begin{array}{l}\text { Parent } \\
\text { component too } \\
\text { small }\end{array}$ \\
\hline $\begin{array}{l}\text { 363. Dadds, M. R., \& McHugh, T. A. (1992). Social support and } \\
\text { treatment outcome in behavioral family therapy for child conduct } \\
\text { problems. J Consult Clin Psychol, 60(2), 252-259. }\end{array}$ & $\begin{array}{l}\text { Targets } \\
\text { externalising }\end{array}$ \\
\hline $\begin{array}{l}\text { 364. Dadds, M. R., MacDonald, E., Cauchi, A., Williams, K., Levy, F., } \\
\text { \& Brennan, J. (2014). Nasal oxytocin for social deficits in childhood } \\
\text { autism: A randomized controlled trial. Journal of Autism and } \\
\text { Developmental Disorders, 44(3), 521-531. doi: } \\
\text { http://dx.doi.org/10.1007/s10803-013-1899-3 }\end{array}$ & $\begin{array}{l}\text { Not a parenting } \\
\text { intervention }\end{array}$ \\
\hline $\begin{array}{l}\text { 365. Dadsetan, P., Ghanbari, S., Heydari, M. (2014). The } \\
\text { effectiveness of behavioral parent training on reducing externalizing } \\
\text { problems in } 7 \text { to } 9 \text { years old children. Journal of Psychology, } 17 \text { (4), } \\
401-419 .\end{array}$ & $\begin{array}{l}\text { Targets } \\
\text { externalising }\end{array}$ \\
\hline $\begin{array}{l}\text { 366. Dahlquist LM, Pendley JS, Landthrip DS, Jones CL and Steuber } \\
\text { CP. 2002. Distraction intervention for preschoolers undergoing } \\
\text { intramuscular injections and subcutaneous port access. Health } \\
\text { Psychol. 21(1):94-9. }\end{array}$ & $\begin{array}{l}\text { Intervention for } \\
\text { developmental } \\
\text { disorders } \\
\text { (autism, autism } \\
\text { spectrum } \\
\text { disorder), } \\
\text { physical } \\
\text { disabilities, } \\
\text { medical } \\
\text { conditions or } \\
\text { distress about } \\
\text { facing medical } \\
\text { interventions }\end{array}$ \\
\hline $\begin{array}{l}\text { 367. Daley D, Jones K, Hutchings J and Thompson M. } 2009 . \\
\text { Attention deficit hyperactivity disorder in pre-school children: Current } \\
\text { findings, recommended interventions and future directions. Child: } \\
\text { Care, Health and Development. 35(6):754-66. }\end{array}$ & Review \\
\hline $\begin{array}{l}368 . \quad \text { Daley, D., \& O'Brien, M. (2013). A small-scale randomized } \\
\text { controlled trial of the self-help version of the New Forest Parent } \\
\text { Training Programme for children with ADHD symptoms. European } \\
\text { child \& adolescent psychiatry, 22(9), 543-552. doi:10.1007/s00787- } \\
013-0396-8\end{array}$ & $\begin{array}{l}\text { No child } \\
\text { internalising } \\
\text { outcomes or not } \\
\text { a major goal }\end{array}$ \\
\hline $\begin{array}{l}\text { 369. Dallaire DH, Cole DA, Smith TM, Ciesla JA, LaGrange B, Jacquez } \\
\text { FM, Pineda AQ, Truss AE and Folmer AS. 2008. Predicting children's } \\
\text { depressive symptoms from community and individual risk factors. } \\
\text { Journal of Youth and Adolescence. } 37(7): 830-46 .\end{array}$ & $\begin{array}{l}\text { Not a parenting } \\
\text { intervention }\end{array}$ \\
\hline
\end{tabular}




\begin{tabular}{|c|c|}
\hline $\begin{array}{l}\text { 370. Daly, R. M., Holland, C. J., Forrest, P. A., \& Fellbaum, G. A. } \\
\text { (1985). Temporal generalization of treatment effects over a three-year } \\
\text { period for a parent training program: Directive Parental Counseling } \\
\text { (DPC). Canadian Journal of Behavioural Science/Revue canadienne des } \\
\text { sciences du comportement, 17(4), 379-388. doi:10.1037/h0080049 }\end{array}$ & $\begin{array}{l}\text { Follow-up data } \\
\text { collected only in } \\
\text { active group }\end{array}$ \\
\hline $\begin{array}{l}\text { D71. Angelo EJ, Llerena-Quinn R, Shapiro R, Colon F, Rodriguez P, } \\
\text { Gallagher K and Beardslee WR. 2009. Adaptation of the Preventive } \\
\text { Intervention Program for Depression for use with predominantly low- } \\
\text { income Latino families. Family Process. 48(2):269-91. }\end{array}$ & Not an RCT \\
\hline $\begin{array}{l}\text { 372. Dashtbozorgi B, Ghadirian F, Khajeddin N and Karatni K. } 2009 . \\
\text { Effect of family psychoeducation on the level of adaptation and } \\
\text { improvement of patients with mood disorders. Iranian Journal of } \\
\text { Psychiatry and Clinical Psychology. Special Issue: On bipolar disorder. } \\
\text { 15(2):193-200. }\end{array}$ & $\begin{array}{l}\text { Follow-up less } \\
\text { than } 6 \text { months }\end{array}$ \\
\hline $\begin{array}{l}\text { 373. Davey, M. P., Kissil, K., Lynch, L., Harmon, L. R., \& Hodgson, N. } \\
\text { (2013). A culturally adapted family intervention for African American } \\
\text { families coping with parental cancer: Outcomes of a pilot study. } \\
\text { Psycho-Oncology, 22(7), 1572-1580. doi: } \\
\text { http://dx.doi.org/10.1002/pon.3172 }\end{array}$ & $\begin{array}{l}\text { No long term } \\
\text { follow-up }\end{array}$ \\
\hline $\begin{array}{l}\text { 374. David, D. H., McMahon, T. J., Luthar, S. L., \& Suchman, N. E. } \\
\text { (2012). Sensation Seeking, Coping With Stress, and Readiness to } \\
\text { Engage in Therapy: Does Ego Development Influence the Psychosocial } \\
\text { Functioning of Substance-Abusing Mothers? American Journal of } \\
\text { Orthopsychiatry, } 82,231-240 .\end{array}$ & $\begin{array}{l}\text { Secondary } \\
\text { analysis } \\
\text { (moderators) of } \\
\text { Luthar } 2007\end{array}$ \\
\hline $\begin{array}{l}\text { 375. David, O. A. (2014). The Rational Positive Parenting program } \\
\text { for child externalizing behavior: Mechanisms of change analysis. } \\
\text { Journal of Evidence Based Psychotherapies, } 14 \text { (1), 21-38. }\end{array}$ & $\begin{array}{l}\text { Targets } \\
\text { externalising }\end{array}$ \\
\hline $\begin{array}{l}\text { 376. David, O. A., David, D., Dobrean, A. (2014). Efficacy of the } \\
\text { Rational Positive Parenting Program for child externalizing behavior: } \\
\text { Can an emotion-regulation enhanced cognitive-behavioral parent } \\
\text { program be more effective than a standard one? Journal of Evidence } \\
\text { Based Psychotherapies, } 14 \text { (2), 159-178. }\end{array}$ & $\begin{array}{l}\text { Targets } \\
\text { externalising }\end{array}$ \\
\hline $\begin{array}{l}\text { D77. Davies SL, Horton TV, Williams AG, Martin MY and Stewart KE. } \\
\text { 2009. MOMS: formative evaluation and subsequent intervention for } \\
\text { mothers living with HIV. AIDS Care. 21(5):552-60. }\end{array}$ & $\begin{array}{l}\text { No child } \\
\text { internalising } \\
\text { outcomes or not } \\
\text { a major goal } \\
\text { (focus study) }\end{array}$ \\
\hline $\begin{array}{l}\text { 378. Dawson, G., \& Burner, K. (2011). Behavioral interventions in } \\
\text { children and adolescents with autism spectrum disorder: A review of } \\
\text { recent findings. Current Opinion in Pediatrics, 23, 616-620. }\end{array}$ & Review \\
\hline $\begin{array}{l}\text { 379. Dawson, W. W. (2003). Symptomatology of bereaved parents } \\
\text { who have lost a child to cancer: A 6-week group therapy intervention } \\
\text { (Order No. 3085520). Available from ProQuest Dissertations \& Theses } \\
\text { Global. (305284152). }\end{array}$ & $\begin{array}{l}\text { No child } \\
\text { internalising } \\
\text { outcomes or not } \\
\text { a major goal }\end{array}$ \\
\hline $\begin{array}{l}\text { 380. Dawson-McClure, S. R., Sandler, I. N., Wolchik, S. A., \& Millsap, } \\
\text { R. E. (2004). Risk as a Moderator of the Effects of Prevention Programs } \\
\text { for Children From Divorced Families: A Six-Year Longitudinal Study. } \\
\text { Journal of Abnormal Child Psychology: An official publication of the } \\
\text { International Society for Research in Child and Adolescent } \\
\text { Psychopathology, 32(2), 175-190. doi: } \\
\text { 10.1023/b:jacp.0000019769.75578.79 }\end{array}$ & $\begin{array}{l}\text { Secondary } \\
\text { analysis of } \\
\text { Wolchik West et } \\
\text { al } 2000 \\
\text { (moderator } \\
\text { analysis only, no } \\
\text { useable data) }\end{array}$ \\
\hline
\end{tabular}




\begin{tabular}{|c|c|}
\hline $\begin{array}{l}\text { 381. de Brouwer, S. J., Kraaimaat, F. W., Sweep, F. C., Donders, R. } \\
\text { T., Eijsbouts, A., van Koulil, S., van Riel, P. L., \& Evers, A. W. (2011). } \\
\text { Psychophysiological responses to stress after stress management } \\
\text { training in patients with rheumatoid arthritis. PLoS One, 6, e27432. }\end{array}$ & $\begin{array}{l}\text { Not a parenting } \\
\text { intervention }\end{array}$ \\
\hline $\begin{array}{l}\text { 382. De Cuyper S, Timbremont B, Braet C, De Backer V and } \\
\text { Wullaert T. 2004. Treating depressive symptoms in schoolchildren: a } \\
\text { pilot study. Eur Child Adolesc Psychiatry. 13(2):105-14. }\end{array}$ & $\begin{array}{l}\text { Not a parenting } \\
\text { intervention }\end{array}$ \\
\hline $\begin{array}{l}\text { 383. de Groot J, Cobham V, Leong J, McDermott B. Individual } \\
\text { versus group family-focused cognitive-behaviour therapy for } \\
\text { childhood anxiety: Pilot randomized controlled trial. Australian and } \\
\text { New Zealand Journal of Psychiatry. 2007 Dec;41(12):990-7. }\end{array}$ & $\begin{array}{l}\text { Comparison } \\
\text { condition not a } \\
\text { minimal control }\end{array}$ \\
\hline $\begin{array}{l}\text { 384. De Los Reyes, A., Alfano, C. A., \& Beidel, D. C. (2010). The } \\
\text { relations among measurements of informant discrepancies within a } \\
\text { multisite trial of treatments for childhood social phobia. Journal of } \\
\text { Abnormal Child Psychology: An official publication of the International } \\
\text { Society for Research in Child and Adolescent Psychopathology, 38(3), } \\
\text { 395-404. doi: } 10.1007 / \text { s10802-009-9373-6 }\end{array}$ & $\begin{array}{l}\text { No long term } \\
\text { follow-up }\end{array}$ \\
\hline $\begin{array}{l}\text { 385. de Mello MF, de Jesus Mari J, Bacaltchuk J, Verdeli H and } \\
\text { Neugebauer R. 2005. A systematic review of research findings on the } \\
\text { efficacy of interpersonal therapy for depressive disorders. European } \\
\text { Archives of Psychiatry and Clinical Neuroscience. 255(2):75-82. }\end{array}$ & Review \\
\hline $\begin{array}{l}\text { 386. De Rubeis, S., \& Granic, I. (2012). Understanding treatment } \\
\text { effectiveness for aggressive youth: The importance of regulation in } \\
\text { mother-child interactions. Journal of Family Psychology, 26, 66-75. }\end{array}$ & $\begin{array}{l}\text { Targets } \\
\text { externalising }\end{array}$ \\
\hline $\begin{array}{l}\text { 387. de Souza, M. A., Salum, G. A., Jarros, R. B., Isolan, L., Davis, R., } \\
\text { Knijnik, D., . . Heldt, E. (2013). Cognitive-behavioral group therapy for } \\
\text { youths with anxiety disorders in the community: effectiveness in low } \\
\text { and middle income countries. Behav Cogn Psychother, 41(3), 255-264. } \\
\text { doi: } 10.1017 / \text { s1352465813000015 }\end{array}$ & $\begin{array}{l}\text { Parent } \\
\text { component too } \\
\text { small }\end{array}$ \\
\hline $\begin{array}{l}\text { 388. Dean KL, Stein BD, Jaycox LH, Kataoka SH, Wong M, Pincus HA } \\
\text { and Tanielian TL. 2004. Acceptability of Asking Parents About Their } \\
\text { Children's Traumatic Symptoms. Psychiatric Services. 55(8):866. }\end{array}$ & $\begin{array}{l}\text { Not a parenting } \\
\text { intervention }\end{array}$ \\
\hline $\begin{array}{l}\text { D89. Deault LC. 2010. A systematic review of parenting in relation } \\
\text { to the development of comorbidities and functional impairments in } \\
\text { children with attention-deficit/hyperactivity disorder (ADHD). Child } \\
\text { Psychiatry and Human Development. 41(2):168-92. }\end{array}$ & Review \\
\hline $\begin{array}{l}\text { 390. Deblinger, E., Mannarino, A. P., Cohen, J. A., Runyon, M. K., \& } \\
\text { Steer, R. A. (2011). Trauma-focused cognitive behavioral therapy for } \\
\text { children: impact of the trauma narrative and treatment length. } \\
\text { Depress Anxiety, } 28,67-75 .\end{array}$ & $\begin{array}{l}\text { Not a parenting } \\
\text { intervention }\end{array}$ \\
\hline $\begin{array}{l}\text { 391. DeGarmo DS, Patterson GR, Forgatch MS. How do outcomes in } \\
\text { a specified parent training intervention maintain or wane over time? } \\
\text { Prev Sci. } 2004 \text { Jun;5(2):73-89. }\end{array}$ & $\begin{array}{l}\text { Secondary } \\
\text { analysis } \\
\text { (mediation) of } \\
\text { study already } \\
\text { included } \\
\text { (Forgatch \& } \\
\text { DeGarmo 1999) }\end{array}$ \\
\hline $\begin{array}{l}\text { 392. DeGarmo, D. S., \& Forgatch, M. S. (2005). Early development } \\
\text { of delinquency within divorced families: evaluating a randomized } \\
\text { preventive intervention trial. Developmental Science, 8(3), 229-239. } \\
\text { doi: 10.1111/j.1467-7687.2005.00412.x }\end{array}$ & $\begin{array}{l}\text { No child } \\
\text { internalising } \\
\text { outcomes or not } \\
\text { a major goal }\end{array}$ \\
\hline
\end{tabular}




\begin{tabular}{|c|c|}
\hline $\begin{array}{l}\text { 393. Dégi, C. L., Kállay, É., \& Vincze, A. E. 2007. Differences in } \\
\text { illness-related distress in ethnically different cancer patients: } \\
\text { Romanians, Romanian Hungarians and Hungarians.Cognitie, Creier, } \\
\text { Comportament/Cognition, Brain, Behavior, 11(1): } 143 \text {. }\end{array}$ & $\begin{array}{l}\text { Not a parenting } \\
\text { intervention }\end{array}$ \\
\hline $\begin{array}{l}\text { 394. Deitz DK, Cook RF, Billings DW and Hendrickson A. 2009. A } \\
\text { web-based mental health program: reaching parents at work. Journal } \\
\text { of pediatric psychology, 34(5), 488-494. }\end{array}$ & $\begin{array}{l}\text { No child } \\
\text { internalising } \\
\text { outcomes or not } \\
\text { a major goal }\end{array}$ \\
\hline $\begin{array}{l}\text { 395. Dekovic, M., Asscher, J. J., Manders, W. A., Prins, P. J. M, van } \\
\text { der Laan, P. (2012). Within-intervention change: Mediators of } \\
\text { intervention effects during multisystemic therapy. Journal of } \\
\text { Consulting and Clinical Psychology, } 80 \text { (4), 574-587. }\end{array}$ & $\begin{array}{l}\text { Targets } \\
\text { externalising }\end{array}$ \\
\hline $\begin{array}{l}\text { 396. Dell'Agnello G, Maschietto D, Bravaccio C, Calamoneri F, Masi } \\
\text { G, Curatolo P, Besana D, Mancini F, Rossi A, Poole L, Escobar R and } \\
\text { Zuddas A. 2009. Atomoxetine hydrochloride in the treatment of } \\
\text { children and adolescents with attention-deficit/hyperactivity disorder } \\
\text { and comorbid oppositional defiant disorder: A placebo-controlled } \\
\text { Italian study. European Neuropsychopharmacology. 19(11):822-34. }\end{array}$ & $\begin{array}{l}\text { Not a parenting } \\
\text { intervention }\end{array}$ \\
\hline $\begin{array}{l}\text { 397. Dennis CL, Ross LE and Grigoriadis S. 2007. Psychosocial and } \\
\text { psychological interventions for treating antenatal depression. } \\
\text { Cochrane Database of Systematic Reviews. (3), CD006309. }\end{array}$ & $\begin{array}{l}\text { No child } \\
\text { internalising } \\
\text { outcomes or not } \\
\text { a major goal }\end{array}$ \\
\hline $\begin{array}{l}\text { 398. Dent A. 1999. Evaluation of community interventions for } \\
\text { bereaved parents using a randomised controlled trial. National } \\
\text { Research Register, Retrieved from } \\
\text { http://onlinelibrary.wiley.com/o/cochrane/clcentral/articles/738/CN- } \\
\text { 00315738/frame.html. }\end{array}$ & $\begin{array}{l}\text { No child } \\
\text { internalising } \\
\text { outcomes or not } \\
\text { a major goal }\end{array}$ \\
\hline $\begin{array}{l}\text { 399. DePanfilis, D., \& Dubowitz, H. (2005). Family Connections: A } \\
\text { Program for Preventing Child Neglect. Child Maltreatment, 10(2), 108- } \\
\text { 123. doi: } 10.1177 / 1077559505275252\end{array}$ & $\begin{array}{l}\text { Comparison } \\
\text { condition not a } \\
\text { minimal control }\end{array}$ \\
\hline $\begin{array}{l}\text { 400. Descheemaeker MJ, Swillen A, Plissart L, Borghgraef M, } \\
\text { Rasenberg S, Curfs LMG and Fryns JP. 1994. The Prader-Willi } \\
\text { syndrome: A self supporting program for children, youngsters and } \\
\text { adults. Genetic Counseling. 5(2):199-205. }\end{array}$ & Not an RCT \\
\hline $\begin{array}{l}\text { 401. Diamond, G., Reis, B., Diamond, G., Siqueland, L., \& Isaacs, L. } \\
\text { (2002). Attachment-based family therapy for depressed adolescents: A } \\
\text { treatment development study. Journal of the American Academy of } \\
\text { Child and Adolescent Psychiatry, 41, 1190-1196. }\end{array}$ & $\begin{array}{l}\text { Follow-up data } \\
\text { collected only in } \\
\text { active group }\end{array}$ \\
\hline $\begin{array}{l}\text { 402. Diaz MA, Le H-N, Cooper BA and Munoz RF. } 2007 . \\
\text { Interpersonal factors and perinatal depressive symptomatology in a } \\
\text { low-income Latina sample. Cultural Diversity and Ethnic Minority } \\
\text { Psychology. 13(4):328-36. }\end{array}$ & $\begin{array}{l}\text { Not a parenting } \\
\text { intervention }\end{array}$ \\
\hline $\begin{array}{l}\text { 403. Dieter JNI, Emory EK, Johnson KC and Raynor BD. } 2008 . \\
\text { Maternal depression and anxiety effects on the human fetus: } \\
\text { Preliminary findings and clinical implications. Infant Mental Health } \\
\text { Journal. Special Issue: Perinatal mood and anxiety disorders and } \\
\text { mother-infant relationships. 29(5):420-41. }\end{array}$ & $\begin{array}{l}\text { Not a parenting } \\
\text { intervention }\end{array}$ \\
\hline $\begin{array}{l}\text { 404. Dietz LJ, Jennings KD, Kelley SA and Marshal M. } 2009 . \\
\text { Maternal depression, paternal psychopathology, and toddlers' } \\
\text { behavior problems. Journal of Clinical Child and Adolescent } \\
\text { Psychology. 38(1):48-61. }\end{array}$ & $\begin{array}{l}\text { Not a parenting } \\
\text { intervention }\end{array}$ \\
\hline
\end{tabular}




\begin{tabular}{|c|c|}
\hline $\begin{array}{l}\text { 405. Díez-Juan, M., Schneider, A., Phillips, T., Lozano, R., Tassone, } \\
\text { F., Solomon, M., \& Hagerman, R. J. (2014). Parent-delivered } \\
\text { touchscreen intervention for children with fragile X syndrome. } \\
\text { Intractable \& rare diseases research, 3(4), } 166 .\end{array}$ & $\begin{array}{l}\text { Intervention for } \\
\text { developmental } \\
\text { disorders } \\
\text { (autism, autism } \\
\text { spectrum } \\
\text { disorder), } \\
\text { physical } \\
\text { disabilities, } \\
\text { medical } \\
\text { conditions or } \\
\text { distress about } \\
\text { facing medical } \\
\text { interventions }\end{array}$ \\
\hline $\begin{array}{l}\text { 406. Dillman Carpentier FR, Mauricio AM, Gonzales NA, Millsap RE, } \\
\text { Meza CM, Dumka LE, Germ·n M and Genalo MT. 2007. Engaging } \\
\text { Mexican origin families in a school-based preventive intervention. The } \\
\text { journal of primary prevention, } 28(6), 521-546 \text {. }\end{array}$ & $\begin{array}{l}\text { No child } \\
\text { internalising } \\
\text { outcomes or not } \\
\text { a major goal }\end{array}$ \\
\hline $\begin{array}{l}\text { 407. Dillon FR, Pantin H, Robbins MS and Szapocznik J. } 2008 \text {. } \\
\text { Exploring the role of parental monitoring of peers on the relationship } \\
\text { between family functioning and delinquency in the lives of African } \\
\text { American and Hispanic adolescents. Crime \& Delinquency. 54(1):65- } \\
94 .\end{array}$ & Not an RCT \\
\hline $\begin{array}{l}\text { 408. Dishion TJ and Andrews DW. 1995. Preventing Escalation in } \\
\text { Problem Behaviors with High-Risk Young Adolescents - Immediate and } \\
\text { 1-Year Outcomes. Journal of Consulting and Clinical Psychology. } \\
\text { 63(4):538-48. }\end{array}$ & $\begin{array}{l}\text { Targets } \\
\text { externalising }\end{array}$ \\
\hline $\begin{array}{l}\text { 409. Dishion TJ, Kavanagh K, Schneiger A, Nelson SE, Kaufman N. } \\
\text { Preventing early adolescent substance use: A family-centered strategy } \\
\text { for the public middle-school ecology. Prevention Science 2002;3:191- } \\
\text { 201. [PubMed: } 12387554 \text { ] }\end{array}$ & $\begin{array}{l}\text { No child } \\
\text { internalising } \\
\text { outcomes or not } \\
\text { a major goal }\end{array}$ \\
\hline $\begin{array}{l}\text { 410. Dishion TJ, Shaw D, Connell A, Gardner F, Weaver C and } \\
\text { Wilson M. 2008. The Family Check-Up with high-risk indigent families: } \\
\text { Preventing problem behavior by increasing parents' positive behavior } \\
\text { support in early childhood. Child Development. } 79(5): 1395-414 .\end{array}$ & $\begin{array}{l}\text { Targets } \\
\text { externalising }\end{array}$ \\
\hline $\begin{array}{l}\text { 411. Dishion, T. J., Brennan, L. M., Shaw, D. S., McEachern, A. D., } \\
\text { Wilson, M. N., \& Jo, B. (2014). Prevention of problem behavior } \\
\text { through annual family check-ups in early childhood: intervention } \\
\text { effects from home to early elementary school. Journal of abnormal } \\
\text { child psychology, 42(3), 343-354. }\end{array}$ & $\begin{array}{l}\text { No child } \\
\text { internalising } \\
\text { outcomes or not } \\
\text { a major goal }\end{array}$ \\
\hline $\begin{array}{l}\text { 412. Dobson KS, Hopkins JA, Fata L, Scherrer M and Allan LC. } 2010 . \\
\text { The prevention of depression and anxiety in a sample of high-risk } \\
\text { adolescents: A randomized controlled trial. Canadian Journal of School } \\
\text { Psychology. 25(4):291-310. }\end{array}$ & $\begin{array}{l}\text { Not a parenting } \\
\text { intervention }\end{array}$ \\
\hline $\begin{array}{l}\text { 413. Dodge, K. A., Bierman, K. L., Coie, J. D., Greenberg, M. T., } \\
\text { Lochman, J. E., McMahon, R. J., \& Pinderhughes, E. E. (2014). Impact } \\
\text { of early intervention on psychopathology, crime, and well-being at age } \\
\text { 25. American journal of psychiatry, } 172(1), 59-70 .\end{array}$ & $\begin{array}{l}\text { Not a parenting } \\
\text { intervention }\end{array}$ \\
\hline
\end{tabular}




\begin{tabular}{|c|c|}
\hline $\begin{array}{l}\text { 414. Dodge, K. A., Goodman, W. B., Murphy, R. A., O'Donnell, K., } \\
\text { Sato, J., \& Guptill, S. (2014). Implementation and randomized } \\
\text { controlled trial evaluation of universal postnatal nurse home visiting. } \\
\text { Am J Public Health, } 104 \text { Suppl 1, S136-143. doi: } \\
\text { 10.2105/ajph.2013.301361 }\end{array}$ & $\begin{array}{l}\text { No child } \\
\text { internalising } \\
\text { outcomes or not } \\
\text { a major goal }\end{array}$ \\
\hline $\begin{array}{l}\text { 415. Doepfner, M., \& Rothenberger, A. (2011). Progress in real } \\
\text { world interventions for externalizing disorders using innovative } \\
\text { designs and data analyses. European Child and Adolescent Psychiatry, } \\
\text { 20, S66-S67. }\end{array}$ & Not an RCT \\
\hline $\begin{array}{l}\text { 416. Doering JJ, Morin K and Stetzer FC. 2009. Severe fatigue and } \\
\text { depressive symptoms in lower-income urban postpartum women. } \\
\text { Western Journal of Nursing Research. 31(5):599-612. }\end{array}$ & $\begin{array}{l}\text { Not a parenting } \\
\text { intervention }\end{array}$ \\
\hline $\begin{array}{l}\text { 417. Doherty, W. J., Erickson, M. F., \& LaRossa, R. (2006). An } \\
\text { intervention to increase father involvement and skills with infants } \\
\text { during the transition to parenthood. Journal of Family Psychology, } \\
\text { 20(3), 438-447. }\end{array}$ & $\begin{array}{l}\text { No long term } \\
\text { follow-up; No } \\
\text { child } \\
\text { internalising } \\
\text { outcomes or not } \\
\text { a major goal }\end{array}$ \\
\hline $\begin{array}{l}\text { 418. Domitrovich, C. E., Cortes, R. C., \& Greenberg, M. T. (2007). } \\
\text { Improving young children's social and emotional competence: A } \\
\text { randomized trial of the preschool “PATHS" curriculum. The Journal of } \\
\text { Primary Prevention, } 28,67-91 .\end{array}$ & $\begin{array}{l}\text { Not a parenting } \\
\text { intervention; No } \\
\text { long term } \\
\text { follow-up }\end{array}$ \\
\hline $\begin{array}{l}\text { 419. Donovan, C. L., \& March, S. (2014). Online CBT for preschool } \\
\text { anxiety disorders: a randomised control trial. Behav Res Ther, 58, 24- } \\
\text { 35. doi: } 10.1016 / \text { j.brat.2014.05.001 }\end{array}$ & $\begin{array}{l}\text { Follow-up data } \\
\text { collected only in } \\
\text { active group }\end{array}$ \\
\hline $\begin{array}{l}\text { 420. Donovick, M. R. (2010). Parenting practices and child mental } \\
\text { health among spanish speaking latino families: Examining the role of } \\
\text { parental cultural values (Order No. 3423994). Available from ProQuest } \\
\text { Dissertations \& Theses Global. (759968090). }\end{array}$ & $\begin{array}{l}\text { Data not } \\
\text { available/report } \\
\text { ed (reports on } \\
\text { baseline data } \\
\text { from larger RCT - } \\
\text { no intervention } \\
\text { here) }\end{array}$ \\
\hline $\begin{array}{l}\text { 421. Dooley, B., Fitzgerald, A., \& Giollabhui, N. M. (2015). The risk } \\
\text { and protective factors associated with depression and anxiety in a } \\
\text { national sample of Irish adolescents. Irish Journal of Psychological } \\
\text { Medicine, 32(01), 93-105. }\end{array}$ & $\begin{array}{l}\text { Not a parenting } \\
\text { intervention }\end{array}$ \\
\hline $\begin{array}{l}\text { 422. Dornelas EA, Ferrand J, Stepnowski R, Barbagallo J and } \\
\text { McCullough L. 2010. A pilot study of affect-focused psychotherapy for } \\
\text { antepartum depression. Journal of Psychotherapy Integration. } \\
\text { 20(4):364-82. }\end{array}$ & $\begin{array}{l}\text { No child } \\
\text { internalising } \\
\text { outcomes or not } \\
\text { a major goal }\end{array}$ \\
\hline $\begin{array}{l}\text { 423. Douglas, P. S., Miller, Y., Bucetti, A., Hill, P. S., \& Creedy, D. K. } \\
\text { (2013). Preliminary evaluation of a primary care intervention for cry- } \\
\text { fuss behaviours in the first 3-4 months of life ('The Possums } \\
\text { Approach'): effects on cry-fuss behaviours and maternal mood. } \\
\text { Australian journal of primary health. }\end{array}$ & $\begin{array}{l}\text { No child } \\
\text { internalising } \\
\text { outcomes or not } \\
\text { a major goal }\end{array}$ \\
\hline $\begin{array}{l}\text { D24. Dowd, T. (2007). An Australian Pilot Study of a Parent-Child } \\
\text { Interaction Program You Make the Difference. Neonatal, paediatric, } \\
\text { and child health nursing, 10,13. }\end{array}$ & Not an RCT \\
\hline
\end{tabular}




\begin{tabular}{|c|c|}
\hline $\begin{array}{l}\text { 425. Doyle AC, McLean C, Washington BN, Hoste RR and le Grange } \\
\text { D. 2009. Are single-parent families different from two-parent families } \\
\text { in the treatment of adolescent bulimia nervosa using family-based } \\
\text { treatment? International Journal of Eating Disorders, 42(2), 153-157. }\end{array}$ & $\begin{array}{l}\text { Not a parenting } \\
\text { intervention }\end{array}$ \\
\hline $\begin{array}{l}\text { 426. Dracup K, Moser DK, Doering LV, Guzy PM and Juarbe T. } 2000 . \\
\text { A controlled trial of cardiopulmonary resuscitation training for } \\
\text { ethnically diverse parents of infants at high risk for cardiopulmonary } \\
\text { arrest. Crit Care Med. 28(9):3289-95. }\end{array}$ & $\begin{array}{l}\text { No child } \\
\text { internalising } \\
\text { outcomes or not } \\
\text { a major goal }\end{array}$ \\
\hline $\begin{array}{l}\text { D27. Drahota A, Wood JJ, Sze KM and Van Dyke M. 2011. Effects of } \\
\text { cognitive behavioral therapy on daily living skills in children with high- } \\
\text { functioning autism and concurrent anxiety disorders. Journal of } \\
\text { Autism and Developmental Disorders. 41(3):257-65. }\end{array}$ & $\begin{array}{l}\text { Intervention for } \\
\text { developmental } \\
\text { disorders } \\
\text { (autism, autism } \\
\text { spectrum } \\
\text { disorder), } \\
\text { physical } \\
\text { disabilities, } \\
\text { medical } \\
\text { conditions or } \\
\text { distress about } \\
\text { facing medical } \\
\text { interventions }\end{array}$ \\
\hline $\begin{array}{l}\text { 428. Draper, K., Siegel, C., White, J., Solis, C. M., \& Mishna, F. } \\
\text { (2009). Preschoolers, parents, and teachers (PPT): a preventive } \\
\text { intervention with an at risk population. International journal of group } \\
\text { psychotherapy, (2), 221-242. }\end{array}$ & $\begin{array}{l}\text { No long term } \\
\text { follow-up }\end{array}$ \\
\hline $\begin{array}{l}\text { 429. Dreifuss FE, Rosman NP, Cloyd JC, Pellock JM, Kuzniecky RI, Lo } \\
\text { WD, Matsuo F, Sharp GB, Conry JA, Bergen DC and Bell WE. 1998. A } \\
\text { comparison of rectal diazepam gel and placebo for acute repetitive } \\
\text { seizures. N Engl J Med. 338(26):1869-75. }\end{array}$ & $\begin{array}{l}\text { Not a parenting } \\
\text { intervention }\end{array}$ \\
\hline $\begin{array}{l}\text { 430. Drendel, A. L. (2013). High anxiety in the Emergency } \\
\text { Department. [Conference Abstract]. Pediatric Emergency Care, } 29 \\
\text { (10), 1147. doi:http://dx.doi.org/10.1097/PEC.0b013e3182a684be }\end{array}$ & $\begin{array}{l}\text { No long term } \\
\text { follow-up }\end{array}$ \\
\hline $\begin{array}{l}\text { 431. Dretzke J, Davenport C, Frew E, Barlow J, Stewart-Brown S, } \\
\text { Bayliss S, Taylor RS, Sandercock J and Hyde C. 2009. The clinical } \\
\text { effectiveness of different parenting programmes for children with } \\
\text { conduct problems: A systematic review of randomised controlled } \\
\text { trials. Child and Adolescent Psychiatry and Mental Health. 3:7. }\end{array}$ & Review \\
\hline $\begin{array}{l}\text { 432. Drew A, Baird G, Baron-Cohen S, Cox A, Slonims V, } \\
\text { Wheelwright S, Swettenham J, Berry B and Charman T. 2002. A pilot } \\
\text { randomised control trial of a parent training intervention for pre- } \\
\text { school children with autism. Preliminary findings and methodological } \\
\text { challenges. European child \& adolescent psychiatry, 11(6), 266-272. }\end{array}$ & $\begin{array}{l}\text { Intervention for } \\
\text { developmental } \\
\text { disorders } \\
\text { (autism, autism } \\
\text { spectrum } \\
\text { disorder), } \\
\text { physical } \\
\text { disabilities, } \\
\text { medical } \\
\text { conditions or } \\
\text { distress about } \\
\text { facing medical } \\
\text { interventions }\end{array}$ \\
\hline
\end{tabular}




\begin{tabular}{|c|c|}
\hline $\begin{array}{l}\text { 433. Dreyer BP. 2006. The diagnosis and management of Attention- } \\
\text { Deficit/Hyperactivity Disorder in preschool children: The state of our } \\
\text { knowledge and practice. Current Problems in Pediatric and Adolescent } \\
\text { Health Care. } 36(1): 6-30 \text {. }\end{array}$ & Review \\
\hline $\begin{array}{l}\text { 434. Drotar D. 2002. Enhancing reviews of psychological } \\
\text { treatments with pediatric populations: Thoughts on next steps. } \\
\text { Journal of Pediatric Psychology. 27(2):167-76. }\end{array}$ & $\begin{array}{l}\text { Not a parenting } \\
\text { intervention }\end{array}$ \\
\hline $\begin{array}{l}\text { D35. Drugli MB, Fossum S, Larsson B, Morch WT. Characteristics of } \\
\text { young children with persistent conduct problems } 1 \text { year after } \\
\text { treatment with the Incredible Years program. Eur Child Adolesc } \\
\text { Psychiatry. 2010 Jul;19(7):559-65. }\end{array}$ & $\begin{array}{l}\text { Comparison } \\
\text { condition not a } \\
\text { minimal control }\end{array}$ \\
\hline $\begin{array}{l}\text { 436. Drugli, M. B., \& Larsson, B. (2006). Children aged 4-8 years } \\
\text { treated with parent training and child therapy because of conduct } \\
\text { problems: generalisation effects to day-care and school settings. } \\
\text { European child \& adolescent psychiatry, (7), 392-399. }\end{array}$ & $\begin{array}{l}\text { Follow-up data } \\
\text { collected only in } \\
\text { active group; } \\
\text { Targets } \\
\text { externalising }\end{array}$ \\
\hline $\begin{array}{l}\text { 437. Drugli, M. B., \& Larsson, B. (2009). When young children have } \\
\text { conduct problems - Who are the nonresponders after parent training? } \\
\text { European Psychiatry, 24, S1042. }\end{array}$ & $\begin{array}{l}\text { Targets } \\
\text { externalising }\end{array}$ \\
\hline $\begin{array}{l}\text { 438. Drugli, M. B., Larsson, B., Fossum, S., \& Morch, W. T. (2010). } \\
\text { Five- to six-year outcome and its prediction for children with ODD/CD } \\
\text { treated with parent training. J Child Psychol Psychiatry, 51(5), 559-566. } \\
\text { doi: JCPP2178 }\end{array}$ & $\begin{array}{l}\text { Follow-up data } \\
\text { collected only in } \\
\text { active group; } \\
\text { Targets } \\
\text { externalising }\end{array}$ \\
\hline $\begin{array}{l}\text { 439. Duarte CS, Sourander A, Nikolakaros G, Pihlajamaki H, } \\
\text { Helenius H, Piha J, Kumpulainen K, Moilanen I, Tamminen T, Almqvist } \\
\text { F and Must A. 2010. Child mental health problems and obesity in early } \\
\text { adulthood. The Journal of Pediatrics. 156(1):93-7. }\end{array}$ & $\begin{array}{l}\text { Not a parenting } \\
\text { intervention }\end{array}$ \\
\hline $\begin{array}{l}\text { 440. Dubey, D. R., O'Leary, S. 8c Kaufman, K. F. (1983). Training } \\
\text { parents of hyperactive children in child management: a comparative } \\
\text { outcome study. Joumai of Abnormal Child Psychology, 11, 229-2 }\end{array}$ & $\begin{array}{l}\text { No child } \\
\text { internalising } \\
\text { outcomes or not } \\
\text { a major goal; } \\
\text { Comparison } \\
\text { condition not a } \\
\text { minimal control }\end{array}$ \\
\hline $\begin{array}{l}\text { 441. Dubow, E. F., Huesmann, L. R., Boxer, P., Landau, S., Dvir, S., } \\
\text { Shikaki, K., \& Ginges, J. (2012). Exposure to political conflict and } \\
\text { violence and posttraumatic stress in Middle East youth: Protective } \\
\text { factors. Journal of Clinical Child and Adolescent Psychology, 41, 402- } \\
416 .\end{array}$ & $\begin{array}{l}\text { Not a parenting } \\
\text { intervention }\end{array}$ \\
\hline $\begin{array}{l}\text { 442. Dubowitz, H., Lane, W. G., Semiatin, J. N., Magder, L. S., } \\
\text { Venepally, M., \& Jans, M. (2011). The safe environment for every kid } \\
\text { model: impact on pediatric primary care professionals. Pediatrics, 127, } \\
\text { e962-970. }\end{array}$ & $\begin{array}{l}\text { Not a parenting } \\
\text { intervention }\end{array}$ \\
\hline $\begin{array}{l}\text { 443. Duch, H., \& Rodriguez, C. (2011). Strengthening families in } \\
\text { Head Start: The impact of a parent education programme on the } \\
\text { emotional well-being of Latino families. Early Child Development and } \\
\text { Care, 181, 733-748. }\end{array}$ & $\begin{array}{l}\text { Not an RCT; No } \\
\text { long term } \\
\text { follow-up }\end{array}$ \\
\hline
\end{tabular}




\begin{tabular}{|c|c|}
\hline $\begin{array}{l}\text { 444. Dudley AL, Melvin GA, Williams NJ, Tonge BJ and King NJ. } \\
\text { 2005. Investigation of consumer satisfaction with cognitive-behaviour } \\
\text { therapy and sertraline in the treatment of adolescent depression. Aust } \\
\text { N Z J Psychiatry. } 39(6): 500-6 \text {. }\end{array}$ & $\begin{array}{l}\text { Not a parenting } \\
\text { intervention }\end{array}$ \\
\hline $\begin{array}{l}\text { 445. Duggan AK, McFarlane EC, Windham AM, et al. Evaluation of } \\
\text { Hawaii's Healthy Start Program. FutureChild.1999;9:66-90. }\end{array}$ & $\begin{array}{l}\text { Not an RCT; No } \\
\text { long term } \\
\text { follow-up }\end{array}$ \\
\hline $\begin{array}{l}\text { 446. Duggan, A. K., Berlin, L. J., Cassidy, J., Burrell, L., \& Tandon, S. } \\
\text { D. (2009). Examining maternal depression and attachment insecurity } \\
\text { as moderators of the impacts of home visiting for at-risk mothers and } \\
\text { infants. Journal of Consulting and Clinical Psychology, } 77(4), 788-799 . \\
\text { doi: } 10.1037 / \text { a0015709 }\end{array}$ & $\begin{array}{l}\text { No child } \\
\text { internalising } \\
\text { outcomes or not } \\
\text { a major goal }\end{array}$ \\
\hline $\begin{array}{l}\text { 447. Duggan, A., Caldera, D., Rodriguez, K., Burrell, L., Rohde, C., \& } \\
\text { Crowne, S. S. (2007). Impact of a statewide home visiting program to } \\
\text { prevent child abuse. Child Abuse \& Neglect, 31(8), 801-827. doi: } \\
\text { 10.1016/j.chiabu.2006.06.011 }\end{array}$ & $\begin{array}{l}\text { No child } \\
\text { internalising } \\
\text { outcomes or not } \\
\text { a major goal }\end{array}$ \\
\hline $\begin{array}{l}\text { 448. Duggan, A., Fuddy, L., Burrell, L., Higman, S. M., McFarlane, E., } \\
\text { Windham, A., et al. (2004). Randomized trial of a statewide home } \\
\text { visiting program to prevent child abuse: impact in reducing parental } \\
\text { risk factors. Child abuse \& neglect, (6), 623-643. }\end{array}$ & $\begin{array}{l}\text { No child } \\
\text { internalising } \\
\text { outcomes or not } \\
\text { a major goal }\end{array}$ \\
\hline $\begin{array}{l}\text { 449. Dumas, J. E. \& Wahler, R. G. (1983). Predictors of treatment } \\
\text { outcome in parent training: mother insularity and socioeconomic } \\
\text { disadvantage. Behavioral Assessment, 5, 301-31 }\end{array}$ & Not an RCT \\
\hline $\begin{array}{l}\text { 450. Dunning, M., Seymour, M., Cooklin, A., \& Giallo, R. (2013). } \\
\text { Wide Awake Parenting: study protocol for a randomised controlled } \\
\text { trial of a parenting program for the management of post-partum } \\
\text { fatigue. BMC public health, 13, 26. doi:10.1186/1471-2458-13-26 }\end{array}$ & $\begin{array}{l}\text { Data not } \\
\text { available/report } \\
\text { ed }\end{array}$ \\
\hline $\begin{array}{l}\text { 451. Dusak, A., Sen, V., Yildirim, Z. B., Porzig-Drummond, R., } \\
\text { Stevenson, R. J., Stevenson, C. (2014). The 1-2-3 Magic parenting } \\
\text { program and its effect on child problem behaviors and dysfunctional } \\
\text { parenting: a randomized controlled trial. Biomed Res Int, 58 52-64. }\end{array}$ & $\begin{array}{l}\text { Targets } \\
\text { externalising; No } \\
\text { internalising } \\
\text { outcomes } \\
\text { measured or not } \\
\text { a major goal; } \\
\text { Follow-up data } \\
\text { collected only in } \\
\text { active group }\end{array}$ \\
\hline $\begin{array}{l}\text { 452. Dybdahl, R. (2001). Children and mothers in war: an outcome } \\
\text { study of a psychosocial intervention program. Child Dev, } 72(4), 1214- \\
1230 .\end{array}$ & $\begin{array}{l}\text { No long term } \\
\text { follow-up }\end{array}$ \\
\hline $\begin{array}{l}\text { 453. Dykman RA and Ackerman PT. 1991. Attention deficit disorder } \\
\text { and specific reading disability: separate but often overlapping } \\
\text { disorders. J Learn Disabil. 24(2):96-103. }\end{array}$ & Not an RCT \\
\hline $\begin{array}{l}\text { 454. Eather, N., Morgan, P. J., \& Lubans, D. R. (2011). Improving } \\
\text { health-related fitness in children: the Fit-4-Fun randomized controlled } \\
\text { trial study protocol. BMC public health, 11(1), } 902 .\end{array}$ & $\begin{array}{l}\text { Not a parenting } \\
\text { intervention; } \\
\text { Data not } \\
\text { available/report } \\
\text { ed (protocol } \\
\text { only) }\end{array}$ \\
\hline $\begin{array}{l}\text { 455. Eber, L., Nelson, M., \& Miles, P. (1997). School-based } \\
\text { wraparound for students with emotional and behavioral challenges. } \\
\text { Exceptional Children, 63, 539-555. }\end{array}$ & Not an RCT \\
\hline
\end{tabular}




\begin{tabular}{|c|c|}
\hline $\begin{array}{l}\text { 456. Eckshtain D and Gaynor ST. 2009. Assessing outcome in } \\
\text { cognitive behavior therapy for child depression: An illustrative case } \\
\text { series. Child \& Family Behavior Therapy. 31(2):94-116. }\end{array}$ & Not an RCT \\
\hline $\begin{array}{l}\text { E57. Eckshtain, D., \& Gaynor, S. T. (2013). Combined individual } \\
\text { cognitive behavior therapy and parent training for childhood } \\
\text { depression: } 2 \text { - to 3-year follow-up. Child \& Family Behavior Therapy, } \\
\text { 35(2), 132-143. doi: } 10.1080 / 07317107.2013 .789362\end{array}$ & Not an RCT \\
\hline $\begin{array}{l}\text { 458. Edwards, N. M. (2010). The maternal role in promoting } \\
\text { emotional competence: Predicting head start mothers' } \\
\text { expressiveness, perceived role, and receptivity to support (Order No. } \\
\text { 3409571). Available from ProQuest Dissertations \& Theses Global. } \\
\text { (717681372). }\end{array}$ & $\begin{array}{l}\text { Not a parenting } \\
\text { intervention }\end{array}$ \\
\hline $\begin{array}{l}\text { 459. Eimecke S, Pauschardt J and Mattejat F. 2010. Prevention of } \\
\text { childhood anxiety and depression: Efficacy of an additional parent } \\
\text { training program. Verhaltenstherapie. 20(3):193-200. }\end{array}$ & $\begin{array}{l}\text { No long term } \\
\text { follow-up }\end{array}$ \\
\hline $\begin{array}{l}\text { 460. Eisen AR, Raleigh } \mathrm{H} \text { and Neuhoff CC. 2008. The unique impact } \\
\text { of parent training for separation anxiety disorder in children. Behavior } \\
\text { Therapy. } 39(2): 195-206 \text {. }\end{array}$ & Not an RCT \\
\hline $\begin{array}{l}\text { 461. Eisenhower AS. 2009. Improving student-teacher relationships } \\
\text { and school adjustment for children with disruptive behavior problems } \\
\text { during the transition to kindergarten. Dissertation Abstracts } \\
\text { International: Section B: The Sciences and Engineering. 69(10-B). }\end{array}$ & $\begin{array}{l}\text { Follow-up less } \\
\text { than } 6 \text { months }\end{array}$ \\
\hline $\begin{array}{l}\text { 462. Eisner, M., \& Meidert, U. (2011). Stages of parental } \\
\text { engagement in a universal parent training program. The Journal of } \\
\text { Primary Prevention, 32, 83-93. }\end{array}$ & $\begin{array}{l}\text { No child } \\
\text { internalising } \\
\text { outcomes or not } \\
\text { a major goal }\end{array}$ \\
\hline $\begin{array}{l}\text { 463. Elgan, T. H., Hansson, H., Zetterlind, U., Kartengren, N., \& } \\
\text { Leifman, H. (2012). Design of a Web-based individual coping and } \\
\text { alcohol-intervention program (web-ICAIP) for children of parents with } \\
\text { alcohol problems: study protocol for a randomized controlled trial. } \\
\text { BMC Public Health, 12, } 35 \text {. }\end{array}$ & $\begin{array}{l}\text { Not a parenting } \\
\text { intervention; } \\
\text { Data not } \\
\text { available/report } \\
\text { ed (protocol } \\
\text { only) }\end{array}$ \\
\hline $\begin{array}{l}\text { 464. Elgar FJ, Curtis LL, McGrath PJ, Waschbusch DA and Stewart } \\
\text { SH. 2003. Antecedent-Consequence Conditions in Maternal Mood and } \\
\text { Child Adjustment: A Four-Year Cross-Lagged Study. Journal of Clinical } \\
\text { Child and Adolescent Psychology. 32(3):362-74. }\end{array}$ & $\begin{array}{l}\text { Not a parenting } \\
\text { intervention }\end{array}$ \\
\hline $\begin{array}{l}465 . \quad \text { El-Kamary, S. S., Higman, S. M., Fuddy, L., McFarlane, E., Sia, } \\
\text { C., \& Duggan, A. K. (2004). Hawaii's healthy start home visiting } \\
\text { program: determinants and impact of rapid repeat birth. Pediatrics, } \\
\text { 114(3), e317-326. doi: } 10.1542 / \text { peds. } 2004-0618\end{array}$ & $\begin{array}{l}\text { No child } \\
\text { internalising } \\
\text { outcomes or not } \\
\text { a major goal } \\
\text { (Targets } \\
\text { reduction in } \\
\text { rapid repeat } \\
\text { births) }\end{array}$ \\
\hline
\end{tabular}




\begin{tabular}{|c|c|}
\hline $\begin{array}{l}\text { 466. Ellerton ML and Merriam C. 1994. Preparing children and } \\
\text { families psychologically for day surgery: an evaluation. J Adv Nurs. } \\
\text { 19(6):1057-62. }\end{array}$ & $\begin{array}{l}\text { Intervention for } \\
\text { developmental } \\
\text { disorders } \\
\text { (autism, autism } \\
\text { spectrum } \\
\text { disorder), } \\
\text { physical } \\
\text { disabilities, } \\
\text { medical } \\
\text { conditions or } \\
\text { distress about } \\
\text { facing medical } \\
\text { interventions }\end{array}$ \\
\hline $\begin{array}{l}\text { 467. Elliot, J., Prior, M., Merrigan, C., \& Ballinger, K. (2002). } \\
\text { Evaluation of a community intervention programme for preschool } \\
\text { behavior problems. Journal of paediatrics and child health, (1), 41-50. }\end{array}$ & $\begin{array}{l}\text { Targets } \\
\text { externalising } \\
\text { (selected for } \\
\text { high on } \\
\text { externalising) }\end{array}$ \\
\hline $\begin{array}{l}\text { 468. Elliott SA, Sanjack M and Leverton TJ. 1988. Parents groups in } \\
\text { pregnancy A preventive intervention for postnatal depression? } \\
\text { Gottlieb B: Marshalling Social Support: Formats, Processes and Effects. } \\
\text { London: Sage, 87-110. }\end{array}$ & $\begin{array}{l}\text { No child } \\
\text { internalising } \\
\text { outcomes or not } \\
\text { a major goal }\end{array}$ \\
\hline $\begin{array}{l}\text { 469. Ellis J, McCarthy P, Gosselin P and Splinter W. } 2000 . \\
\text { Intravenous sedation for control of distress during lumbar punctures } \\
\text { for pediatric cancer patients. Pain Research \& Management, 5(2), 141- } \\
\text { 147. }\end{array}$ & $\begin{array}{l}\text { Intervention for } \\
\text { developmental } \\
\text { disorders } \\
\text { (autism, autism } \\
\text { spectrum } \\
\text { disorder), } \\
\text { physical } \\
\text { disabilities, } \\
\text { medical } \\
\text { conditions or } \\
\text { distress about } \\
\text { facing medical } \\
\text { interventions }\end{array}$ \\
\hline $\begin{array}{l}\text { 470. Embry DD. 2011. Behavioral Vaccines and Evidence-Based } \\
\text { Kernels: Nonpharmaceutical Approaches for the Prevention of Mental, } \\
\text { Emotional, and Behavioral Disorders. Psychiatric Clinics of North } \\
\text { America. 34(1):1-34. }\end{array}$ & Not an RCT \\
\hline $\begin{array}{l}\text { 471. Emslie GJ, Hughes CW, Crismon ML, Lopez M, Pliszka S, Toprac } \\
\text { MG and Boemer C. 2004. A feasibility study of the childhood } \\
\text { depression medication algorithm: the Texas Children's Medication } \\
\text { Algorithm Project (CMAP). J Am Acad Child Adolesc Psychiatry. } \\
\text { 43(5):519-27. }\end{array}$ & $\begin{array}{l}\text { Not a parenting } \\
\text { intervention }\end{array}$ \\
\hline $\begin{array}{l}\text { 472. Enebrink, P., Högström, J., Forster, M., \& Ghaderi, A. (2012). } \\
\text { Internet-based parent management training: A randomized controlled } \\
\text { study. Behav Res Ther, 50, 240-249. }\end{array}$ & $\begin{array}{l}\text { Targets } \\
\text { externalising }\end{array}$ \\
\hline
\end{tabular}




\begin{tabular}{|c|c|}
\hline $\begin{array}{l}\text { 473. Epstein, L. H., Paluch, R. A., Saelens, B. E., Ernst, M. M., \& } \\
\text { Wilfley, D. E. (2001). Changes in eating disorder symptoms with } \\
\text { pediatric obesity treatment. J Pediatr, 139(1), 58-65. doi: S0022- } \\
3476(01) 61305-2\end{array}$ & $\begin{array}{l}\text { No child } \\
\text { internalising } \\
\text { outcomes or not } \\
\text { a major goal } \\
\text { (Targets weight } \\
\text { reduction in } \\
\text { overweight } \\
\text { children); Parent } \\
\text { component too } \\
\text { small }\end{array}$ \\
\hline $\begin{array}{l}74 . \quad \text { Erhardt, D., \& Baker, B. L. (1990). The Effects of Behavioral } \\
\text { Parent Training on Families with Young Hyperactive-Children. Journal } \\
\text { of Behavior Therapy and Experimental Psychiatry, 21(2), 121-132. }\end{array}$ & Not an RCT \\
\hline $\begin{array}{l}\text { 475. Ericksen, J., Milgrom, J., Schembri, C., \& Gemmill, A. (2013). } \\
\text { Towards parenthood. A public health intervention to prepare for the } \\
\text { transition to parenthood. [Conference Abstract]. Archives of Women's } \\
\text { Mental Health, 16, S32. doi: http://dx.doi.org/10.1007/s00737-013- } \\
\text { 0355-x }\end{array}$ & $\begin{array}{l}\text { No child } \\
\text { internalising } \\
\text { outcomes or not } \\
\text { a major goal }\end{array}$ \\
\hline $\begin{array}{l}\text { 476. Ernst, E. (1999). Massage therapy is promising for childhood } \\
\text { asthma. Focus Alternat Complement Ther, } 4(1), 30-31 \text {. }\end{array}$ & $\begin{array}{l}\text { No long term } \\
\text { follow-up }\end{array}$ \\
\hline $\begin{array}{l}\text { 477. Esbjørn, B. H., Reinholdt-Dunne, M. L., Nielsen, S. K., Smith, A. } \\
\text { C., Breinholst, S., \& Leth, I. (2015). Exploring the effect of case } \\
\text { formulation driven CBT for children with anxiety disorders: a feasibility } \\
\text { study. Behavioural and cognitive psychotherapy, } 43(01), 20-30 \text {. }\end{array}$ & $\begin{array}{l}\text { Not a parenting } \\
\text { intervention }\end{array}$ \\
\hline $\begin{array}{l}\text { 478. Esfahanizadeh, N. (2011). Dental health education programme } \\
\text { for 6-year-olds: a cluster randomised controlled trial. In European } \\
\text { journal of paediatric dentistry : official journal of European Academy } \\
\text { of Paediatric Dentistry (pp. 167-170). }\end{array}$ & $\begin{array}{l}\text { No child } \\
\text { internalising } \\
\text { outcomes or not } \\
\text { a major goal }\end{array}$ \\
\hline $\begin{array}{l}\text { 479. Esposito-Smythers C, Birmaher B, Valeri S, Chiappetta L, Hunt } \\
\text { J, Ryan N, Axelson D, Strober M, Leonard H, Sindelar H and Keller M. } \\
\text { 2006. Child Comorbidity, Maternal Mood Disorder, and Perceptions of } \\
\text { Family Functioning Among Bipolar Youth. Journal of the American } \\
\text { Academy of Child \& Adolescent Psychiatry. 45(8):955-64. }\end{array}$ & $\begin{array}{l}\text { Not a parenting } \\
\text { intervention }\end{array}$ \\
\hline $\begin{array}{l}\text { 480. Eyberg, S. M., \& Robinson, E. A. (1982). Parent-Child } \\
\text { Interaction Training - Effects on Family Functioning. Journal of Clinical } \\
\text { Child Psychology, 11(2), 130-137. }\end{array}$ & Not an RCT \\
\hline $\begin{array}{l}\text { 481. Fabiano, G. A. (2006). Behavioral parent training for fathers of } \\
\text { children with attention-deficit/hyperactivity disorder: Effectiveness of } \\
\text { the intervention and a comparison of two formats. [Dissertation } \\
\text { Abstract; Dissertation]. Dissertation Abstracts International: Section B: } \\
\text { The Sciences and Engineering, 66(8-B). }\end{array}$ & $\begin{array}{l}\text { No child } \\
\text { internalising } \\
\text { outcomes or not } \\
\text { a major goal }\end{array}$ \\
\hline $\begin{array}{l}\text { 482. Falkin, S. M. (1989). Relaxation/stress management training to } \\
\text { reduce cardiovascular reactivity in offspring of essential hypertensive } \\
\text { parents (Order No. 9007749). Available from ProQuest Dissertations \& } \\
\text { Theses Global. (303779753). }\end{array}$ & $\begin{array}{l}\text { Not a parenting } \\
\text { intervention }\end{array}$ \\
\hline $\begin{array}{l}\text { 483. Fann JR, Fan MY and Unutzer J. 2009. Improving primary care } \\
\text { for older adults with cancer and depression. J Gen Intern Med. } 24 \\
\text { Suppl 2:S417-24. }\end{array}$ & $\begin{array}{l}\text { Not a parenting } \\
\text { intervention }\end{array}$ \\
\hline
\end{tabular}




\begin{tabular}{|c|c|}
\hline $\begin{array}{l}\text { 484. Faramarzi, M., Pasha, H., Esmailzadeh, S., Kheirkhah, F., } \\
\text { Heidary, S., \& Afshar, Z. (2013). The effect of the cognitive behavioral } \\
\text { therapy and pharmacotherapy on infertility stress: a randomized } \\
\text { controlled trial. Int J Fertil Steril, } 7(3), 199-206 .\end{array}$ & $\begin{array}{l}\text { Not a parenting } \\
\text { intervention }\end{array}$ \\
\hline $\begin{array}{l}\text { 485. Farris, J. R., Bert, S. S., Nicholson, J. S., Glass, K., \& Borkowski, } \\
\text { J. G. (2013). Effective intervention programming: improving maternal } \\
\text { adjustment through parent education. Administration and policy in } \\
\text { mental health, } 40(3), 211-223 . \text { doi:10.1007/s10488-011-0397-1 }\end{array}$ & $\begin{array}{l}\text { No child } \\
\text { internalising } \\
\text { outcomes or not } \\
\text { a major goal }\end{array}$ \\
\hline $\begin{array}{l}\text { 486. Favara-Scacco C, Smirne G, Schilirú G and Di Cataldo A. } 2001 . \\
\text { Art therapy as support for children with leukemia during painful } \\
\text { procedures. Medical and pediatric oncology, 36(4), 474-480. }\end{array}$ & $\begin{array}{l}\text { Not a parenting } \\
\text { intervention }\end{array}$ \\
\hline $\begin{array}{l}\text { 487. Feather JS and Ronan KR. 2009. Trauma-focused CBT with } \\
\text { maltreated children: A clinic-based evaluation of a new treatment } \\
\text { manual. Australian Psychologist. 44(3):174-94. }\end{array}$ & Not an RCT \\
\hline $\begin{array}{l}\text { 488. Fedele, D. A., Hullmann, S. E., Chaffin, M., Kenner, C., Fisher, } \\
\text { M. J., Kirk, K., ... \& Mullins, L. L. (2013). Impact of a parent-based } \\
\text { interdisciplinary intervention for mothers on adjustment in children } \\
\text { newly diagnosed with cancer. Journal of pediatric psychology, 38(5), } \\
\text { 531-540. }\end{array}$ & $\begin{array}{l}\text { Follow-up less } \\
\text { than } 6 \text { months }\end{array}$ \\
\hline $\begin{array}{l}\text { 489. Federico B, Falese L and Capelli G. 2009. Socio-economic } \\
\text { inequalities in physical activity practice among Italian children and } \\
\text { adolescents: a cross-sectional study. Journal of Public Health. } \\
\text { 17(6):377-84. }\end{array}$ & $\begin{array}{l}\text { Not a parenting } \\
\text { intervention }\end{array}$ \\
\hline $\begin{array}{l}\text { 490. Feehan CJ and Vostanis P. 1996. Cognitive-behavioural } \\
\text { therapy for depressed children: Children's and therapists' impressions. } \\
\text { Behavioural and Cognitive Psychotherapy, 24(02), 171-183. }\end{array}$ & $\begin{array}{l}\text { Not a parenting } \\
\text { intervention }\end{array}$ \\
\hline $\begin{array}{l}\text { Feeny NC, Silva SG, Reinecke MA, McNulty S, Findling RL, } \\
\text { Rohde P, Curry JF, Ginsburg GS, Kratochvil CJ, Pathak SM, May DE, } \\
\text { Kennard BD, Simons AD, Wells KC, Robins M, Rosenberg D and March } \\
\text { JS. 2009. An exploratory analysis of the impact of family functioning on } \\
\text { treatment for depression in adolescents. Journal of Clinical Child and } \\
\text { Adolescent Psychology. 38(6):814-25. }\end{array}$ & $\begin{array}{l}\text { Not a parenting } \\
\text { intervention }\end{array}$ \\
\hline $\begin{array}{l}\text { 492. Feigelman, S., Dubowitz, H., Lane, W., Grube, L., \& Kim, J. } \\
\text { (2011). Training pediatric residents in a primary care clinic to help } \\
\text { address psychosocial problems and prevent child maltreatment. Acad } \\
\text { Pediatr, } 11,474-480 .\end{array}$ & $\begin{array}{l}\text { Not a parenting } \\
\text { intervention }\end{array}$ \\
\hline $\begin{array}{l}\text { 493. Feinberg ME, Button TMM, Neiderhiser JM, Reiss D and } \\
\text { Hetherington EM. 2007. Parenting and adolescent antisocial behavior } \\
\text { and depression: Evidence of genotype Vó parenting environment } \\
\text { interaction. Archives of General Psychiatry. 64(4):457-65. }\end{array}$ & $\begin{array}{l}\text { Not a parenting } \\
\text { intervention }\end{array}$ \\
\hline
\end{tabular}




\begin{tabular}{|c|c|}
\hline $\begin{array}{l}\text { 494. Feinberg, E., Augustyn, M., Fitzgerald, E., Sandler, J., Ferreira- } \\
\text { Cesar Suarez, Z., Chen, N., . . Silverstein, M. (2014). Improving } \\
\text { maternal mental health after a child's diagnosis of autism spectrum } \\
\text { disorder: results from a randomized clinical trial. JAMA Pediatr, 168(1), } \\
\text { 40-46. doi: 10.1001/jamapediatrics.2013.3445 }\end{array}$ & $\begin{array}{l}\text { Intervention for } \\
\text { developmental } \\
\text { disorders } \\
\text { (autism, autism } \\
\text { spectrum } \\
\text { disorder), } \\
\text { physical } \\
\text { disabilities, } \\
\text { medical } \\
\text { conditions or } \\
\text { distress about } \\
\text { facing medical } \\
\text { interventions }\end{array}$ \\
\hline $\begin{array}{l}495 . \quad \text { Feinberg, E., Stein, R., Diaz-Linhart, Y., Egbert, L., Beardslee, } \\
\text { W., Hegel, M. T., \& Silverstein, M. (2012). Adaptation of problem- } \\
\text { solving treatment for prevention of depression among low-income, } \\
\text { culturally diverse mothers. Family \& Community Health: The Journal of } \\
\text { Health Promotion \& Maintenance, 35, 57-67. }\end{array}$ & $\begin{array}{l}\text { No child } \\
\text { internalising } \\
\text { outcomes or not } \\
\text { a major goal }\end{array}$ \\
\hline $\begin{array}{l}\text { 496. Feinberg, M. E., \& Kan, M. L. (2008). Establishing family } \\
\text { foundations: intervention effects on coparenting, parent/infant well- } \\
\text { being, and parent-child relations. J Fam Psychol, 22(2), 253-263. doi: } \\
\text { 2008-03770-008 }\end{array}$ & $\begin{array}{l}\text { No long term } \\
\text { follow-up; No } \\
\text { child } \\
\text { internalising } \\
\text { outcomes or not } \\
\text { a major goal }\end{array}$ \\
\hline $\begin{array}{l}\text { 497. Feinberg, M. E., Kan, M. L., \& Goslin, M. (2009). Enhancing } \\
\text { coparenting, parenting, and child self-regulation at the transition to } \\
\text { parenthood: Effects of family foundations one year after birth. } \\
\text { Prevention Science, 10, } 276-285 \text {. }\end{array}$ & $\begin{array}{l}\text { No child } \\
\text { internalising } \\
\text { outcomes or not } \\
\text { a major goal }\end{array}$ \\
\hline $\begin{array}{l}\text { 498. Feinberg, M. E., Roettger, M. E., Jones, D. E., Paul, I. M., \& Kan, } \\
\text { M. L. (2015). Effects of a psychosocial couple-based prevention } \\
\text { program on adverse birth outcomes. Maternal and child health } \\
\text { journal, 19(1), 102-111. }\end{array}$ & $\begin{array}{l}\text { No child } \\
\text { internalising } \\
\text { outcomes or not } \\
\text { a major goal }\end{array}$ \\
\hline $\begin{array}{l}\text { 499. Feinberg, M. E., Solmeyer, A. R., Hostetler, M. L., Sakuma, K. } \\
\text { L., Jones, D., \& McHale, S. M. (2013). Siblings are special: initial test of } \\
\text { a new approach for preventing youth behavior problems. J Adolesc } \\
\text { Health, 53(2), 166-173. doi: } 10.1016 / \text { j.jadohealth.2012.10.004 }\end{array}$ & $\begin{array}{l}\text { Parent } \\
\text { component too } \\
\text { small }\end{array}$ \\
\hline $\begin{array}{l}\text { 500. Feinfield, K. A., \& Baker, B. L. (2004). Empirical support for a } \\
\text { treatment program for families of young children with externalizing } \\
\text { problems. J Clin Child Adolesc Psychol, 33(1), 182-195. doi: } \\
\text { 10.1207/S15374424JCCP3301_17 }\end{array}$ & $\begin{array}{l}\text { Follow-up less } \\
\text { than } 6 \text { months }\end{array}$ \\
\hline
\end{tabular}




\begin{tabular}{|c|c|}
\hline $\begin{array}{l}\text { 501. Felder-Puig R, Maksys A, Noestlinger C, Gadner H, Stark H, } \\
\text { Pfluegler A and Topf R. 2003. Using a children's book to prepare } \\
\text { children and parents for elective ENT surgery: results of a randomized } \\
\text { clinical trial. Int J Pediatr Otorhinolaryngol. 67(1):35-41. }\end{array}$ & $\begin{array}{l}\text { Intervention for } \\
\text { developmental } \\
\text { disorders } \\
\text { (autism, autism } \\
\text { spectrum } \\
\text { disorder), } \\
\text { physical } \\
\text { disabilities, } \\
\text { medical } \\
\text { conditions or } \\
\text { distress about } \\
\text { facing medical } \\
\text { interventions }\end{array}$ \\
\hline $\begin{array}{l}\text { 502. Feldman, M. A., Sparks, B., \& Case, L. (1993). Effectiveness of } \\
\text { home-based early intervention on the language development of } \\
\text { children of mothers with mental retardation. Research in } \\
\text { developmental disabilities, (5), 387-408. }\end{array}$ & $\begin{array}{l}\text { No child } \\
\text { internalising } \\
\text { outcomes or not } \\
\text { a major goal }\end{array}$ \\
\hline $\begin{array}{l}\text { 503. Fergusson DM, Grant H, Horwood } \mathrm{L} \text { and Ridder EM. } 2005 . \\
\text { Randomized trial of the early start program of home visitation. } \\
\text { Pediatrics. 116(6):E803-E9. }\end{array}$ & $\begin{array}{l}\text { No long term } \\
\text { follow-up } \\
\text { (intervention } \\
\text { lasted for } 36 \\
\text { months) }\end{array}$ \\
\hline $\begin{array}{l}\text { 504. Fergusson, D. M., Grant, H., Horwood, L. J., \& Ridder, E. M. } \\
\text { (2006). Randomized trial of the early start program of home visitation: } \\
\text { Parent and family outcomes. Pediatrics, } 117(3), 781-786 .\end{array}$ & $\begin{array}{l}\text { No child } \\
\text { internalising } \\
\text { outcomes or not } \\
\text { a major goal }\end{array}$ \\
\hline $\begin{array}{l}\text { 505. Ferrin, M., Moreno-Granados, J. M., Salcedo-Marin, M. D., } \\
\text { Ruiz-Veguilla, M., Perez-Ayala, V., Taylor, E. (2014). Evaluation of a } \\
\text { psychoeducation programme for parents of children and adolescents } \\
\text { with ADHD: Immediate and long-term effects using a blind } \\
\text { randomized controlled trial. European child \& adolescent psychiatry, } \\
23 \text { (8), 637-47. }\end{array}$ & $\begin{array}{l}\text { Intervention for } \\
\text { developmental } \\
\text { disorders } \\
\text { (autism, autism } \\
\text { spectrum } \\
\text { disorder), } \\
\text { physical } \\
\text { disabilities, } \\
\text { medical } \\
\text { conditions or } \\
\text { distress about } \\
\text { facing medical } \\
\text { interventions }\end{array}$ \\
\hline $\begin{array}{l}\text { 506. Field, T. M., et al. (1980). Teenage, Lower-Class, Black Mothers } \\
\text { and Their Preterm Infants: An Intervention and Developmental Follow- } \\
\text { up. Child Development 51(2): 426-436. }\end{array}$ & $\begin{array}{l}\text { No child } \\
\text { internalising } \\
\text { outcomes or not } \\
\text { a major goal }\end{array}$ \\
\hline $\begin{array}{l}\text { 507. Fincher, W., Shaw, J., \& Ramelet, A. S. (2012). The } \\
\text { effectiveness of a standardised preoperative preparation in reducing } \\
\text { child and parent anxiety: a single-blind randomised controlled trial. J } \\
\text { Clin Nurs, 21, 946-955. }\end{array}$ & $\begin{array}{l}\text { Not a parenting } \\
\text { intervention }\end{array}$ \\
\hline $\begin{array}{l}\text { 508. Flament MF, Cohen D, Choquet M, Jeammet P and Ledoux S. } \\
\text { 2001. Phenomenology, psychosocial correlates, and treatment seeking } \\
\text { in major depression and dysthymia of adolescence. Journal of the } \\
\text { American Academy of Child \& Adolescent Psychiatry. 40(9):1070-8. }\end{array}$ & $\begin{array}{l}\text { Not a parenting } \\
\text { intervention }\end{array}$ \\
\hline
\end{tabular}




\begin{tabular}{|c|c|}
\hline $\begin{array}{l}\text { 509. Flay, B. R., Graumlich, S., Segawa, E., Burns, J. L., Holliday, M. } \\
\text { Y., \& Aban Aya, I. (2004). Effects of } 2 \text { prevention programs on high-risk } \\
\text { behaviors among African American youth: a randomized trial. Archives } \\
\text { of pediatrics \& adolescent medicine, (4), 377-384. }\end{array}$ & $\begin{array}{l}\text { No child } \\
\text { internalising } \\
\text { outcomes or not } \\
\text { a major goal }\end{array}$ \\
\hline $\begin{array}{l}\text { 510. Fleischman, M. J. (1981). A replication of Patterson's } \\
\text { Intervention for boys with conduct problems. Journal of Consulting } \\
\text { and Clinical Psychology, 49(3), 342-351. doi: 10.1037/0022- } \\
\text { 006x.49.3.342 }\end{array}$ & $\begin{array}{l}\text { No long term } \\
\text { follow-up }\end{array}$ \\
\hline $\begin{array}{l}\text { 511. Fleming CB, Mason WA, Mazza JJ, Abbott RD and Catalano RF. } \\
\text { 2008. Latent growth modeling of the relationship between depressive } \\
\text { symptoms and substance use during adolescence. Psychology of } \\
\text { Addictive Behaviors. 22(2):186-97. }\end{array}$ & $\begin{array}{l}\text { Not a parenting } \\
\text { intervention }\end{array}$ \\
\hline $\begin{array}{l}\text { 512. Flory V. 2004. A Novel Clinical Intervention for Severe } \\
\text { Childhood Depression and Anxiety. Clinical Child Psychology and } \\
\text { Psychiatry. 9(1):9-23. }\end{array}$ & Not an RCT \\
\hline $\begin{array}{l}\text { 513. Flouri, E., \& Kallis, C. (2011). Adverse life events and mental } \\
\text { health in middle adolescence. Journal of Adolescence, 34, 371-377. }\end{array}$ & $\begin{array}{l}\text { Not a parenting } \\
\text { intervention }\end{array}$ \\
\hline $\begin{array}{l}\text { 514. Fonagy P. 1998. Prevention, the appropriate target of infant } \\
\text { psychotherapy. Infant Mental Health Journal, 19(2), 124-150. }\end{array}$ & Review \\
\hline $\begin{array}{l}\text { 515. Forbes F, Duffy JC, Mok J and Lemvig J. 2003. Early } \\
\text { intervention service for non-abusing parents of victims of child sexual } \\
\text { abuse: Pilot study. British Journal of Psychiatry. 183(1):66-72. }\end{array}$ & Not an RCT \\
\hline $\begin{array}{l}\text { 516. Ford, T., Edwards, V., Sharkey, S., Ukoumunne, O. C., Byford, } \\
\text { S., Norwich, B., \& Logan, S. (2012). Supporting teachers and children in } \\
\text { schools: the effectiveness and cost-effectiveness of the Incredible } \\
\text { Years teacher classroom management programme in primary school } \\
\text { children: a cluster randomised controlled trial, with parallel economic } \\
\text { and process evaluations. BMC public health, 12, 719. } \\
\text { doi:10.1186/1471-2458-12-719 }\end{array}$ & $\begin{array}{l}\text { Not a parenting } \\
\text { intervention }\end{array}$ \\
\hline $\begin{array}{l}\text { 517. Forehand R and Long N. 1988. Outpatient Treatment of the } \\
\text { Acting-out Child - Procedures, Long-Term Follow-up Data, and Clinical } \\
\text { Problems. Advances in Behaviour Research and Therapy. 10(3):129-77. }\end{array}$ & Not an RCT \\
\hline $\begin{array}{l}\text { 518. Forehand, R., Thigpen, J. C., Parent, J., Hardcastle, E. J., Bettis, } \\
\text { A., \& Compas, B. E. (2012). The role of parent depressive symptoms in } \\
\text { positive and negative parenting in a preventive intervention. Journal } \\
\text { of Family Psychology, 26, 532-541. }\end{array}$ & $\begin{array}{l}\text { Secondary } \\
\text { analysis of } \\
\text { Compas et al } \\
\text { 2009, which was } \\
\text { excluded } \\
\text { because parent } \\
\text { component too } \\
\text { small. Also no } \\
\text { child } \\
\text { internalising } \\
\text { outcomes }\end{array}$ \\
\hline $\begin{array}{l}\text { 519. Forgatch MS and Patterson GR. 2010. Parent Management } \\
\text { Training-Oregon Model: An intervention for antisocial behavior in } \\
\text { children and adolescents. Evidence-based psychotherapies for } \\
\text { children and adolescents (2nd ed.). (pp. 159-177). New York, NY, US: } \\
\text { Guilford Press. xx, } 602 \text {. }\end{array}$ & Review \\
\hline
\end{tabular}




\begin{tabular}{|c|c|}
\hline $\begin{array}{l}\text { 520. Forgatch, M. S., Patterson, G. R., Degarmo, D. S., \& Beldavs, Z. } \\
\text { G. (2009). Testing the Oregon delinquency model with 9-year follow- } \\
\text { up of the Oregon Divorce Study. Development and Psychopathology, } \\
\text { 21(02), 637-660. doi: doi:10.1017/S0954579409000340 }\end{array}$ & $\begin{array}{l}\text { No child } \\
\text { internalising } \\
\text { outcomes or not } \\
\text { a major goal }\end{array}$ \\
\hline $\begin{array}{l}\text { 521. FORMAN, D. R., et al. (2007). Effective treatment for } \\
\text { postpartum depression is not sufficient to improve the developing } \\
\text { mother-child relationship. Development and Psychopathology 19(02): } \\
585-602 \text {. }\end{array}$ & $\begin{array}{l}\text { Follow-up data } \\
\text { collected only in } \\
\text { active group }\end{array}$ \\
\hline $\begin{array}{l}\text { 522. Forman, S. G., Linney, J. A., \& Brondino, M. J. (1990). Effects of } \\
\text { coping skills training on adolescents at risk for substance use. } \\
\text { Psychology of Addictive Behaviors, (2), 67-76. }\end{array}$ & $\begin{array}{l}\text { Parent } \\
\text { component too } \\
\text { small }\end{array}$ \\
\hline $\begin{array}{l}\text { 523. Fortier, M. A., Blount, R. L., Wang, S. M., Mayes, L. C., \& Kain, } \\
\text { Z. N. (2011). Analysing a family-centred preoperative intervention } \\
\text { programme: A dismantling approach. Br J Anaesth, 106, 713-718. }\end{array}$ & $\begin{array}{l}\text { Intervention for } \\
\text { developmental } \\
\text { disorders } \\
\text { (autism, autism } \\
\text { spectrum } \\
\text { disorder), } \\
\text { physical } \\
\text { disabilities, } \\
\text { medical } \\
\text { conditions or } \\
\text { distress about } \\
\text { facing medical } \\
\text { interventions }\end{array}$ \\
\hline $\begin{array}{l}\text { 524. Fortier, M. A., Bunzli, E., Walthall, J., Olshansky, E., Saadat, H., } \\
\text { Santistevan, R., ... \& Kain, Z. N. (2015). Web-based tailored } \\
\text { intervention for preparation of parents and children for outpatient } \\
\text { surgery (WebTIPS): formative evaluation and randomized controlled } \\
\text { trial. Anesthesia \& Analgesia, 120(4), 915-922. }\end{array}$ & $\begin{array}{l}\text { Intervention for } \\
\text { developmental } \\
\text { disorders } \\
\text { (autism, autism } \\
\text { spectrum } \\
\text { disorder), } \\
\text { physical } \\
\text { disabilities, } \\
\text { medical } \\
\text { conditions or } \\
\text { distress about } \\
\text { facing medical } \\
\text { interventions }\end{array}$ \\
\hline $\begin{array}{l}\text { 525. Fossum, S., Handegård, B. H., Drugli, M. B., \& Mørch, W. T. } \\
\text { (2010). Utviklingen i barns utagerende atferd og mødres } \\
\text { oppdragelsespraksis etter foreldretrening. / The development of } \\
\text { disruptive behaviour and harsh parenting following participation in a } \\
\text { parent training: Do risk factors influence treatment outcome? } \\
\text { Tidsskrift for Norsk Psykologforening, 47(7), 601-607. }\end{array}$ & $\begin{array}{l}\text { Secondary } \\
\text { analysis } \\
\text { (excludes } \\
\text { controls); } \\
\text { Targets } \\
\text { externalising }\end{array}$ \\
\hline $\begin{array}{l}\text { 526. Fossum, S., Mørch, W. T., Handegård, B. H., \& Drugli, M. B. } \\
\text { 2007. Childhood disruptive behaviors and family functioning in } \\
\text { clinically referred children: Are girls different from boys? Scandinavian } \\
\text { Journal of Psychology. 48(5):375-82. }\end{array}$ & $\begin{array}{l}\text { Not a parenting } \\
\text { intervention }\end{array}$ \\
\hline $\begin{array}{l}\text { 527. Fossum, S., Morch, W. T., Handegard, B. H., Drugli, M. B., \& } \\
\text { Larsson, B. (2009). Parent training for young Norwegian children with } \\
\text { ODD and CD problems: predictors and mediators of treatment } \\
\text { outcome. Scandinavian Journal of Psychology, (2), 173-181. }\end{array}$ & $\begin{array}{l}\text { No long term } \\
\text { follow-up }\end{array}$ \\
\hline
\end{tabular}




\begin{tabular}{|c|c|}
\hline $\begin{array}{l}\text { 528. Foster RP. 2007. Treating depression in vulnerable urban } \\
\text { women: A feasibility study of clinical outcomes in community service } \\
\text { settings. American Journal of Orthopsychiatry. } 77(3): 443-53 \text {. }\end{array}$ & $\begin{array}{l}\text { Not a parenting } \\
\text { intervention }\end{array}$ \\
\hline $\begin{array}{l}\text { 529. Fox, C. K., Eisenberg, M. E., McMorris, B. J., Pettingell, S. L., \& } \\
\text { Borowsky, I. W. (2013). Survey of Minnesota parent attitudes } \\
\text { regarding school-based depression and suicide screening and } \\
\text { education. Maternal and Child Health Journal, 17(3), 456-462. doi: } \\
\text { 10.1007/s10995-012-1017-8 }\end{array}$ & $\begin{array}{l}\text { Not a parenting } \\
\text { intervention }\end{array}$ \\
\hline $\begin{array}{l}\text { 530. Fox, J. K., Warner, C. M., Lerner, A. B., Ludwig, K., Ryan, J. L., } \\
\text { Colognori, D., Lucas, C. P., \& Brotman, L. M. (2012). Preventive } \\
\text { intervention for anxious preschoolers and their parents: Strengthening } \\
\text { early emotional development. Child Psychiatry and Human } \\
\text { Development, 43, 544-559. }\end{array}$ & $\begin{array}{l}\text { Not an RCT; } \\
\text { Follow-up less } \\
\text { than } 6 \text { months; } \\
\text { Parent } \\
\text { component too } \\
\text { small }\end{array}$ \\
\hline $\begin{array}{l}\text { 531. Frampton, I., McArthur, C., Crowe, B., Linn, J., \& Lovering, K. } \\
\text { (2008). Beyond parent training: Predictors of clinical status and service } \\
\text { use two to three years after Scallywags. Clinical Child Psychology and } \\
\text { Psychiatry, 13, 593-608 }\end{array}$ & Not an RCT \\
\hline $\begin{array}{l}\text { 532. Franck, L. S., Oulton, K., \& Bruce, E. (2012). Parental } \\
\text { involvement in neonatal pain management: an empirical and } \\
\text { conceptual update. Journal of nursing scholarship, 44(1), 45-54. } \\
\text { doi:10.1111/j.1547-5069.2011.01434.x }\end{array}$ & $\begin{array}{l}\text { Intervention for } \\
\text { developmental } \\
\text { disorders } \\
\text { (autism, autism } \\
\text { spectrum } \\
\text { disorder), } \\
\text { physical } \\
\text { disabilities, } \\
\text { medical } \\
\text { conditions or } \\
\text { distress about } \\
\text { facing medical } \\
\text { interventions }\end{array}$ \\
\hline $\begin{array}{l}\text { 533. Franck, L. S., Oulton, K., Nderitu, S., Lim, M., Fang, S., \& Kaiser, } \\
\text { A. (2011). Parent involvement in pain management for NICU infants: A } \\
\text { randomized controlled trial. Pediatrics, 128, 510-518. }\end{array}$ & $\begin{array}{l}\text { Intervention for } \\
\text { developmental } \\
\text { disorders } \\
\text { (autism, autism } \\
\text { spectrum } \\
\text { disorder), } \\
\text { physical } \\
\text { disabilities, } \\
\text { medical } \\
\text { conditions or } \\
\text { distress about } \\
\text { facing medical } \\
\text { interventions }\end{array}$ \\
\hline $\begin{array}{l}\text { 534. Frankel, F., \& Simmons, J. Q. (1992). Parent behavioral } \\
\text { training: Why and when some parents drop out. Journal of Clinical } \\
\text { Child Psychology, 21(4), 322-330. doi:10.1207/s15374424jccp2104_1 }\end{array}$ & $\begin{array}{l}\text { No child } \\
\text { internalising } \\
\text { outcomes or not } \\
\text { a major goal }\end{array}$ \\
\hline $\begin{array}{l}\text { 535. Franz, M., Weihrauch, L., \& Schafer, R. (2011). PALME: A } \\
\text { preventive parental training program for single mothers with } \\
\text { preschool aged children. Journal of Public Health, 19, 305-319. }\end{array}$ & $\begin{array}{l}\text { No long term } \\
\text { follow-up }\end{array}$ \\
\hline
\end{tabular}




\begin{tabular}{|c|c|}
\hline $\begin{array}{l}\text { 536. Franz, M., Weihrauch, L., Buddenberg, T., Güttgemanns, J., } \\
\text { Haubold, S., \& Schäfer1, R. (2010). Effekte eines bindungstheoretisch } \\
\text { fundierten Gruppenprogramms für alleinerziehende Mütter und ihre } \\
\text { Kinder: PALME. / Effectiveness of an attachment-oriented parental } \\
\text { training program for single mothers and their children: PALME. } \\
\text { Kindheit und Entwicklung, 19(2), 90-101. doi: 10.1026/0942- } \\
\text { 5403/a000013 }\end{array}$ & $\begin{array}{l}\text { No long term } \\
\text { follow-up }\end{array}$ \\
\hline $\begin{array}{l}\text { 537. Fraser E and Pakenham KI. 2008. Evaluation of a resilience- } \\
\text { based intervention for children of parents with mental illness. Aust N Z } \\
\text { J Psychiatry. } 42(12): 1041-50 .\end{array}$ & $\begin{array}{l}\text { Not a parenting } \\
\text { intervention }\end{array}$ \\
\hline $\begin{array}{l}\text { 538. Fraser JA, Armstrong KL, Morris JP and Dadds MR. 2000. Home } \\
\text { visiting intervention for vulnerable families with newborns: follow-up } \\
\text { results of a randomized controlled trial. Child Abuse Negl. } \\
\text { 24(11):1399-429. }\end{array}$ & $\begin{array}{l}\text { No child } \\
\text { internalising } \\
\text { outcomes or not } \\
\text { a major goal }\end{array}$ \\
\hline $\begin{array}{l}\text { 539. Freedman SR and Enright RD. 1996. Forgiveness as an } \\
\text { intervention goal with incest survivors. Journal of Consulting and } \\
\text { Clinical Psychology. 64(5):983-92. }\end{array}$ & $\begin{array}{l}\text { Not a parenting } \\
\text { intervention }\end{array}$ \\
\hline $\begin{array}{l}\text { 540. Freeman MP and Davis MF. 2010. Supportive psychotherapy } \\
\text { for perinatal depression: Preliminary data for adherence and } \\
\text { response. Depression and Anxiety. 27(1):39-45. }\end{array}$ & $\begin{array}{l}\text { No child } \\
\text { internalising } \\
\text { outcomes or not } \\
\text { a major goal }\end{array}$ \\
\hline $\begin{array}{l}\text { 541. Freitag, C. M., Cholemkery, H., Elsuni, L., Kroeger, A. K., } \\
\text { Bender, S., Kunz, C. U., \& Kieser, M. (2013). The group-based social } \\
\text { skills training SOSTA-FRA in children and adolescents with high } \\
\text { functioning autism spectrum disorder--study protocol of the } \\
\text { randomised, multi-centre controlled SOSTA--net trial. Trials, 14, 6. doi: } \\
\text { 10.1186/1745-6215-14-6 }\end{array}$ & $\begin{array}{l}\text { Intervention for } \\
\text { developmental } \\
\text { disorders } \\
\text { (autism, autism } \\
\text { spectrum } \\
\text { disorder), } \\
\text { physical } \\
\text { disabilities, } \\
\text { medical } \\
\text { conditions or } \\
\text { distress about } \\
\text { facing medical } \\
\text { interventions }\end{array}$ \\
\hline $\begin{array}{l}\text { 542. Fricker-Elhai, A. E., Ruggiero, K. J., \& Smith, D. W. (2005). } \\
\text { Parent-Child Interaction Therapy With Two Maltreated Siblings in } \\
\text { Foster Care. Clinical Case Studies, 4(1), 13-39. doi: } \\
\text { 10.1177/1534650103259671 }\end{array}$ & Not an RCT \\
\hline $\begin{array}{l}\text { 543. Fristad MA, Goldberg-Arnold JS and Gavazzi SM. } 2002 . \\
\text { Multifamily psychoeducation groups (MFPG) for families of children } \\
\text { with bipolar disorder. Bipolar Disord. 4(4):254-62. }\end{array}$ & $\begin{array}{l}\text { Follow-up less } \\
\text { than } 6 \text { months }\end{array}$ \\
\hline $\begin{array}{l}\text { 544. Fristad, M. A., Goldberg-Arnold, J. S., \& Gavazzi, S. M. (2003). } \\
\text { Multifamily psychoeducation groups in the treatment of children with } \\
\text { mood disorders. Journal of Marital \& Family Therapy, 29, 491-504. }\end{array}$ & $\begin{array}{l}\text { No child } \\
\text { internalising } \\
\text { outcomes or not } \\
\text { a major goal }\end{array}$ \\
\hline $\begin{array}{l}\text { 545. Fristad, M. A., Verducci, J. S., Walters, K., \& Young, M. E. } \\
\text { (2009). Impact of multifamily psychoeducational psychotherapy in } \\
\text { treating children aged } 8 \text { to } 12 \text { years with mood disorders. Arch Gen } \\
\text { Psychiatry, } 66(9), 1013-1021 \text {. doi: } 66 / 9 / 1013\end{array}$ & $\begin{array}{l}\text { Parent } \\
\text { component too } \\
\text { small }\end{array}$ \\
\hline
\end{tabular}




\begin{tabular}{|c|c|}
\hline $\begin{array}{l}\text { 546. Frohlich, G., Pott, W., Albayrak, O., Hebebrand, J., \& Pauli- } \\
\text { Pott, U. (2011). Conditions of long-term success in a lifestyle } \\
\text { intervention for overweight and obese youths. Pediatrics, 128, e779- } \\
785 .\end{array}$ & $\begin{array}{l}\text { No child } \\
\text { internalising } \\
\text { outcomes or not } \\
\text { a major goal }\end{array}$ \\
\hline $\begin{array}{l}\text { 547. Fukushima-Flores, M., \& Miller, L. (2011). FRIENDS Parent } \\
\text { Project: Effectiveness of parent training in reducing parent anxiety in a } \\
\text { universal prevention program for anxiety symptoms in school children. } \\
\text { Behaviour Change, } 28,57-74 .\end{array}$ & $\begin{array}{l}\text { Not an RCT; No } \\
\text { long term } \\
\text { follow-up; } \\
\text { Parent } \\
\text { component too } \\
\text { small }\end{array}$ \\
\hline $\begin{array}{l}\text { 548. Funk W, Jakob W, Riedl T and Taeger K. 2000. Oral } \\
\text { preanaesthetic medication for children: double-blind randomized } \\
\text { study of a combination of midazolam and ketamine vs midazolam or } \\
\text { ketamine alone. Br J Anaesth. } 84(3): 335-40 \text {. }\end{array}$ & $\begin{array}{l}\text { Not a parenting } \\
\text { intervention }\end{array}$ \\
\hline $\begin{array}{l}\text { 549. Futamura, M., Masuko, I., Hayashi, K., Ohya, Y., \& Ito, K. } \\
\text { (2013). Effects of a short-term parental education program on } \\
\text { childhood atopic dermatitis: a randomized controlled trial. Pediatr } \\
\text { Dermatol, 30(4), 438-443. doi: } 10.1111 / \text { pde.12105 }\end{array}$ & $\begin{array}{l}\text { No child } \\
\text { internalising } \\
\text { outcomes or not } \\
\text { a major goal }\end{array}$ \\
\hline $\begin{array}{l}\text { 550. Gagnon AJ and Sandall J. 2007. Individual or group antenatal } \\
\text { education for childbirth or parenthood, or both. Cochrane Database of } \\
\text { Systematic Reviews. (3), CD002869. }\end{array}$ & $\begin{array}{l}\text { No child } \\
\text { internalising } \\
\text { outcomes or not } \\
\text { a major goal }\end{array}$ \\
\hline $\begin{array}{l}\text { 551. Gainey, R. R., Catalano, R. F., Haggerty, K. P., \& Hoppe, M. J. } \\
\text { (1995). Participation in a parent training program for methadone } \\
\text { clients. Addictive Behaviors, 20, 117-125. }\end{array}$ & $\begin{array}{l}\text { No child } \\
\text { internalising } \\
\text { outcomes or not } \\
\text { a major goal }\end{array}$ \\
\hline $\begin{array}{l}\text { 552. Gallagher HM, Rabian BA and McCloskey MS. 2004. A brief } \\
\text { group cognitive-behavioral intervention for social phobia in childhood. } \\
\text { Journal of Anxiety Disorders. 18(4):459-79. }\end{array}$ & $\begin{array}{l}\text { Not a parenting } \\
\text { intervention }\end{array}$ \\
\hline $\begin{array}{l}\text { 553. Gallart SC and Matthey S. 2005. The effectiveness of Group } \\
\text { Triple P and the impact of the four telephone contacts. Behaviour } \\
\text { Change, 22(02), 71-80. }\end{array}$ & $\begin{array}{l}\text { No long term } \\
\text { follow-up }\end{array}$ \\
\hline $\begin{array}{l}\text { 554. Gamba Szijarto, S., Forcada-Guex, M., Borghini, A., } \\
\text { Pierrehumbert, B., Ansermet, F., \& Muller Nix, C. (2009). Prematurity } \\
\text { and parental post-traumatic stress disorder: Similarities and } \\
\text { differences. Neuropsychiatr Enfance Adolesc, 57(5), 385-391. }\end{array}$ & $\begin{array}{l}\text { Not a parenting } \\
\text { intervention }\end{array}$ \\
\hline $\begin{array}{l}\text { 555. Gao, L. L., Chan, S. W., \& Sun, K. (2012). Effects of an } \\
\text { interpersonal-psychotherapy-oriented childbirth education } \\
\text { programme for Chinese first-time childbearing women at 3-month } \\
\text { follow up: randomised controlled trial. Int J Nurs Stud, 49, 274-281. }\end{array}$ & $\begin{array}{l}\text { No child } \\
\text { internalising } \\
\text { outcomes or not } \\
\text { a major goal }\end{array}$ \\
\hline $\begin{array}{l}\text { 556. Garber J and Cole DA. 2010. Intergenerational transmission of } \\
\text { depression: A launch and grow model of change across adolescence. } \\
\text { Development and Psychopathology. Special Issue: Developmental } \\
\text { cascades: Part 2. 22(4):819-30. }\end{array}$ & $\begin{array}{l}\text { Not a parenting } \\
\text { intervention }\end{array}$ \\
\hline $\begin{array}{l}\text { 557. Garber J, Ciesla JA, McCauley E, Diamond G and Schloredt KA. } \\
\text { 2011. Remission of depression in parents: Links to healthy functioning } \\
\text { in their children. Child Development. 82(1):226-43. }\end{array}$ & Not an RCT \\
\hline
\end{tabular}




\begin{tabular}{|c|c|}
\hline $\begin{array}{l}\text { 558. Garber J, Clarke GN, Weersing VR, Beardslee WR, Brent DA, } \\
\text { Gladstone TR, DeBar LL, Lynch FL, D'Angelo E, Hollon SD, } \\
\text { Shamseddeen W and lyengar S. 2009. Prevention of depression in at- } \\
\text { risk adolescents: a randomized controlled trial. JAMA. 301(21):2215- } \\
24 .\end{array}$ & $\begin{array}{l}\text { Not a parenting } \\
\text { intervention }\end{array}$ \\
\hline $\begin{array}{l}\text { 559. Garber J. 2006. Depression in Children and Adolescents. } \\
\text { Linking Risk Research and Prevention. American Journal of Preventive } \\
\text { Medicine. 31(6 SUPPL. 1):104-25. }\end{array}$ & Review \\
\hline $\begin{array}{l}\text { 560. Garcia-Lopez, L. J., del Mar Díaz-Castela, M., Muela-Martinez, } \\
\text { J. A., \& Espinosa-Fernandez, L. (2014). Can parent training for parents } \\
\text { with high levels of expressed emotion have a positive effect on their } \\
\text { child's social anxiety improvement?. Journal of anxiety disorders, } \\
\text { 28(8), 812-822. }\end{array}$ & $\begin{array}{l}\text { Parent } \\
\text { component too } \\
\text { small; } \\
\text { Comparison } \\
\text { condition not a } \\
\text { minimal control }\end{array}$ \\
\hline $\begin{array}{l}\text { G61. Gardner F, Dishion TJ, Shaw DS, Burton J and Supplee L. } 2007 . \\
\text { Randomized prevention trial for early conduct problems: Effects on } \\
\text { proactive parenting and links to toddler disruptive behavior. Journal of } \\
\text { Family Psychology. 21(3):398-406. }\end{array}$ & $\begin{array}{l}\text { Targets } \\
\text { externalising }\end{array}$ \\
\hline $\begin{array}{l}\text { 562. Gardner, F., Burton, J., \& Klimes, I. (2006). Randomised } \\
\text { controlled trial of a parenting intervention in the voluntary sector for } \\
\text { reducing child conduct problems: outcomes and mechanisms of } \\
\text { change. J Child Psychol Psychiatry, 47(11), 1123-1132. doi: JCPP1668 }\end{array}$ & $\begin{array}{l}\text { No child } \\
\text { internalising } \\
\text { outcomes or not } \\
\text { a major goal }\end{array}$ \\
\hline $\begin{array}{l}\text { 563. Garfin, D. R., Silver, R. C., Gil-Rivas, V., Guzman, J., Murphy, J. } \\
\text { M., Cova, F., Rincon, P. P., Squicciarini, A. M., George, M., Guzman, M. } \\
\text { P. (2014). Children's reactions to the } 2010 \text { Chilean earthquake: The } \\
\text { role of trauma exposure, family context, and school-based mental } \\
\text { health programming. Psychological Trauma: Theory, Research, } \\
\text { Practice, and Policy, } 6 \text { (5), 563-573. }\end{array}$ & $\begin{array}{l}\text { Not a parenting } \\
\text { intervention }\end{array}$ \\
\hline $\begin{array}{l}\text { 564. Garland AF, Hough RL, McCabe KM, Yeh M, Wood PA and } \\
\text { Aarons GA. 2001. Prevalence of psychiatric disorders in youths across } \\
\text { five sectors of care. Journal of the American Academy of Child \& } \\
\text { Adolescent Psychiatry. 40(4):409-18. }\end{array}$ & $\begin{array}{l}\text { Not a parenting } \\
\text { intervention }\end{array}$ \\
\hline $\begin{array}{l}\text { 565. Garnier S, De Tychey C, Lighezzolo J, Claudon P, Rebourg- } \\
\text { Roesler C and Flach I. 2008. Perinatal depression, prevention } \\
\text { strategies, personality, and the importance of therapy acceptance. } \\
\text { Rorschachiana. 29(2):108-47. }\end{array}$ & $\begin{array}{l}\text { No child } \\
\text { internalising } \\
\text { outcomes or not } \\
\text { a major goal }\end{array}$ \\
\hline $\begin{array}{l}\text { 566. Garrison, M. M. (2014). Predictors of treatment success in } \\
\text { behavioral sleep intervention among preschool children. Sleep, } 37 \\
\text { A304. }\end{array}$ & $\begin{array}{l}\text { Follow-up less } \\
\text { than } 6 \text { months }\end{array}$ \\
\hline $\begin{array}{l}\text { 567. Gathron, M. K. (1990). Pregnant African American } \\
\text { adolescents: overcoming negative outcomes associated with early } \\
\text { childbearing. Urban League Review, (1), 91-97. }\end{array}$ & $\begin{array}{l}\text { No child } \\
\text { internalising } \\
\text { outcomes or not } \\
\text { a major goal; No } \\
\text { long term } \\
\text { follow-up }\end{array}$ \\
\hline $\begin{array}{l}\text { 568. Gau SS, Lin YJ, Cheng AT, Chiu YN, Tsai WC and Soong WT. } \\
\text { 2010. Psychopathology and symptom remission at adolescence among } \\
\text { children with attention-deficit-hyperactivity disorder. Australian and } \\
\text { New Zealand Journal of Psychiatry, 44(4), 323-332. }\end{array}$ & $\begin{array}{l}\text { Not a parenting } \\
\text { intervention }\end{array}$ \\
\hline
\end{tabular}




\begin{tabular}{|c|c|}
\hline $\begin{array}{l}\text { 569. Gavi?a, O. A., David, D., Bujoreanu, S., Tiba, A., \& lonu?iu, D. R. } \\
\text { (2012). The efficacy of a short cognitive-behavioral parent program in } \\
\text { the treatment of externalizing behavior disorders in Romanian foster } \\
\text { care children: Building parental emotion-regulation through } \\
\text { unconditional self- and child-acceptance strategies. Children and } \\
\text { Youth Services Review, 34, 1290-1297. }\end{array}$ & $\begin{array}{l}\text { Targets } \\
\text { externalising }\end{array}$ \\
\hline $\begin{array}{l}\text { 570. Gaylord-Harden NK, Cunningham JA, Holmbeck GN and Grant } \\
\text { KE. 2010. Suppressor effects in coping research with African American } \\
\text { adolescents from low-income communities. Journal of Consulting and } \\
\text { Clinical Psychology. 78(6):843-55. }\end{array}$ & $\begin{array}{l}\text { Not a parenting } \\
\text { intervention }\end{array}$ \\
\hline $\begin{array}{l}\text { 571. Gaynor ST. 2001. Complementing cbt with learning through in } \\
\text { vivo experience (live): An open clinic trial with depressed adolescents. } \\
\text { Dissertation Abstracts International: Section B: The Sciences and } \\
\text { Engineering. 61(7-B). }\end{array}$ & $\begin{array}{l}\text { Not a parenting } \\
\text { intervention }\end{array}$ \\
\hline $\begin{array}{l}\text { 572. Gebert, N., Hummelink, R., Konning, J., Staab, D., Schmidt, S., } \\
\text { Szczepanski, R., et al. (1998). Efficacy of a self-management program } \\
\text { for childhood asthma-A prospective controlled study. Patient } \\
\text { Education and Counseling, 35(3), 213-220. }\end{array}$ & $\begin{array}{l}\text { Intervention for } \\
\text { developmental } \\
\text { disorders } \\
\text { (autism, autism } \\
\text { spectrum } \\
\text { disorder), } \\
\text { physical } \\
\text { disabilities, } \\
\text { medical } \\
\text { conditions or } \\
\text { distress about } \\
\text { facing medical } \\
\text { interventions }\end{array}$ \\
\hline $\begin{array}{l}\text { 573. Gelfand DM, Teti DM, Seiner SA and Jameson PB. } 1996 . \\
\text { Helping mothers fight depression: Evaluation of a home-based } \\
\text { intervention program for depressed mothers and their infants. Journal } \\
\text { of Clinical Child Psychology. 25(4):406-22. }\end{array}$ & $\begin{array}{l}\text { Not an RCT; No } \\
\text { long term } \\
\text { follow-up }\end{array}$ \\
\hline $\begin{array}{l}\text { 574. Gerdes AC, Hoza B, Arnold LE, Pelham WE, Swanson JM, Wigal } \\
\text { T and Jensen PS. 2007. Maternal depressive symptomatology and } \\
\text { parenting behavior: Exploration of possible mediators. Journal of } \\
\text { Abnormal Child Psychology: An official publication of the International } \\
\text { Society for Research in Child and Adolescent Psychopathology. } \\
\text { 35(5):705-14. }\end{array}$ & Not an RCT \\
\hline $\begin{array}{l}\text { 575. Gerkensmeyer JE, Perkins SM, Scott EL and Wu J. } 2008 . \\
\text { Depressive symptoms among primary caregivers of children with } \\
\text { mental health needs: Mediating and moderating variables. Archives of } \\
\text { Psychiatric Nursing. 22(3):135-46. }\end{array}$ & Not an RCT \\
\hline $\begin{array}{l}\text { 576. Gershy, G. (2015). Mentalization, mindfulness and emotion } \\
\text { regulation do parents need to mind themselves in order to mind their } \\
\text { children. Dissertation Abstracts International: Section B: The Sciences } \\
\text { and Engineering, } 75 \text { (7-B E). }\end{array}$ & $\begin{array}{l}\text { Targets } \\
\text { externalising }\end{array}$ \\
\hline $\begin{array}{l}\text { G77. Gewirtz, A. H., Pinna, K. L., Hanson, S. K., \& Brockberg, D. } \\
\text { (2014). Promoting parenting to support reintegrating military families: } \\
\text { after deployment, adaptive parenting tools. Psychological services, } \\
\text { 11(1), } 31 \text {. }\end{array}$ & $\begin{array}{l}\text { No child } \\
\text { internalising } \\
\text { outcomes or not } \\
\text { a major goal }\end{array}$ \\
\hline
\end{tabular}




\begin{tabular}{|c|c|}
\hline $\begin{array}{l}\text { 578. Gholamnia Shirvani, Z., Amin Shokravi, F., \& Ardestani, M. S. } \\
\text { (2013). Evaluation of a health education program for head lice } \\
\text { infestation in female primary school students in Chabahar City, Iran. } \\
\text { Archives of Iranian medicine, } 16(1), 42-45 .\end{array}$ & $\begin{array}{l}\text { No child } \\
\text { internalising } \\
\text { outcomes or not } \\
\text { a major goal }\end{array}$ \\
\hline $\begin{array}{l}\text { 579. Ghuman JK, Arnold LE and Anthony BJ. } 2008 . \\
\text { Psychopharmacological and other treatments in preschool children } \\
\text { with attention-deficit/hyperactivity disorder: Current evidence and } \\
\text { practice. Journal of Child and Adolescent Psychopharmacology. } \\
\text { 18(5):413-47. }\end{array}$ & Review \\
\hline $\begin{array}{l}\text { 580. Giallo R, G.-P. S. (2008). Evaluation of a family-based } \\
\text { intervention for siblings of children with a disability or chronic illness. } \\
\text { Aust E J Adv Mental Health, (2), } 179 .\end{array}$ & $\begin{array}{l}\text { No long term } \\
\text { follow-up }\end{array}$ \\
\hline $\begin{array}{l}\text { 581. Giallo, R., Cooklin, A., Dunning, M., \& Seymour, M. (2014). The } \\
\text { efficacy of an intervention for the management of postpartum fatigue. } \\
\text { Journal of Obstetric, Gynecologic, \& Neonatal Nursing, 43(5), 598-613. }\end{array}$ & $\begin{array}{l}\text { No child } \\
\text { internalising } \\
\text { outcomes or not } \\
\text { a major goal }\end{array}$ \\
\hline $\begin{array}{l}\text { 582. Giannakopoulos, G., Tzavara, C. \& Kolaitis, G. (2013). Mental } \\
\text { health promotion interventions in families with depressed parents: } \\
\text { What makes the difference. Paper presented at the 15th International } \\
\text { Congress of European Society for Child and Adolescent Psychiatry, Jul } \\
\text { 6-10, S279. Dublin, Ireland. }\end{array}$ & $\begin{array}{l}\text { Follow-up less } \\
\text { than } 6 \text { months; } \\
\text { Comparison } \\
\text { condition not a } \\
\text { minimal control }\end{array}$ \\
\hline $\begin{array}{l}\text { 583. Giebenhain, J. E. y O'Dell, S. L. (1984). Evaluation of a parent- } \\
\text { training manual for reducing children's fear of the dark. Journal of } \\
\text { Applied Behavior Analysis, 17, 121-125. }\end{array}$ & Not an RCT \\
\hline $\begin{array}{l}\text { 584. Gielen AC, McKenzie LB, McDonald EM, Shields WC, Wang MC, } \\
\text { Cheng YJ, Weaver NL and Walker AR. 2007. Using a computer kiosk to } \\
\text { promote child safety: results of a randomized, controlled trial in an } \\
\text { urban pediatric emergency department. Pediatrics. } 120(2): 330-9 .\end{array}$ & $\begin{array}{l}\text { No child } \\
\text { internalising } \\
\text { outcomes or not } \\
\text { a major goal }\end{array}$ \\
\hline $\begin{array}{l}\text { 585. Gil-Bernal, F., \& Hernández-Guzmán, L. (2009). Cognitive- } \\
\text { behavioural treatment in Mexican children with social phobia. } \\
\text { Anuario de Psicología/The UB Journal of Psychology, 40(1), 89- } \\
\text { 104. }\end{array}$ & $\begin{array}{l}\text { Not a parenting } \\
\text { intervention }\end{array}$ \\
\hline $\begin{array}{l}\text { 586. Gillham JE, Hamilton J, Freres DR, Patton K and Gallop R. } 2006 . \\
\text { Preventing Depression Among Early Adolescents in the Primary Care } \\
\text { Setting: A Randomized Controlled Study of the Penn Resiliency } \\
\text { Program. Journal of Abnormal Child Psychology: An official publication } \\
\text { of the International Society for Research in Child and Adolescent } \\
\text { Psychopathology. } 34(2): 203-19 .\end{array}$ & $\begin{array}{l}\text { Not a parenting } \\
\text { intervention }\end{array}$ \\
\hline $\begin{array}{l}\text { 587. Gillham, J. E., \& Reivich, K. J. (1999). Prevention of depressive } \\
\text { symptoms in schoolchildren: A research update. Psychological Science, } \\
\text { 10, 461-462. }\end{array}$ & $\begin{array}{l}\text { Not a parenting } \\
\text { intervention }\end{array}$ \\
\hline $\begin{array}{l}\text { 588. Gillham, J. E., Reivich, K. J., Brunwasser, S. M., Freres, D. R., } \\
\text { Chajon, N. D., Kash-Macdonald, V. M., . . Seligman, M. E. (2012). } \\
\text { Evaluation of a group cognitive-behavioral depression prevention } \\
\text { program for young adolescents: a randomized effectiveness trial. } \\
\text { Journal of clinical child and adolescent psychology, 41(5), 621-639. } \\
\text { doi:10.1080/15374416.2012.706517 }\end{array}$ & $\begin{array}{l}\text { Parent } \\
\text { component too } \\
\text { small }\end{array}$ \\
\hline
\end{tabular}




\begin{tabular}{|c|c|}
\hline $\begin{array}{l}\text { 589. Gillham, J. E., Reivich, K. J., Freres, D. R., Lascher, M., Litzinger, } \\
\text { S., Shatté, A., et al. (2006). School-based prevention of depression and } \\
\text { anxiety symptoms in early adolescence: A pilot of a parent } \\
\text { intervention component. School Psychology Quarterly, 21(3), 323-348. } \\
\text { doi: 10.1521/scpq.2006.21.3.323 }\end{array}$ & $\begin{array}{l}\text { Parent } \\
\text { component too } \\
\text { small }\end{array}$ \\
\hline $\begin{array}{l}\text { 590. Giltay EJ, Tishova Y, Mskhalaya G, Gooren LJG, Saad F and } \\
\text { Kalinchenko S. 2010. Effects of testosterone supplementation on } \\
\text { depressive symptoms and sexual dysfunction in hypogonadal men } \\
\text { with the metabolic syndrome: The Moscow Study. Endocrine } \\
\text { Abstracts. 22:P524. }\end{array}$ & $\begin{array}{l}\text { Not a parenting } \\
\text { intervention }\end{array}$ \\
\hline $\begin{array}{l}\text { 591. Ginsburg GS, Baker EV, Mullany BC, Barlow A, Goklish N, } \\
\text { Hastings R, Thurm AE, Speakman K, Reid R and Walkup J. } 2008 . \\
\text { Depressive symptoms among reservation-based pregnant American } \\
\text { Indian adolescents. Maternal and Child Health Journal. } \\
\text { 12(Suppl1):S110-S8. }\end{array}$ & $\begin{array}{l}\text { Not a parenting } \\
\text { intervention }\end{array}$ \\
\hline $\begin{array}{l}\text { 592. Ginsburg, G. S., Becker, K. D., Drazdowski, T. K., \& Tein, J.-Y. } \\
\text { (2012). Treating anxiety disorders in inner city schools: Results from a } \\
\text { pilot randomized controlled trial comparing CBT and usual care. Child } \\
\text { \& Youth Care Forum, 41, 1-19. }\end{array}$ & $\begin{array}{l}\text { Not a parenting } \\
\text { intervention }\end{array}$ \\
\hline $\begin{array}{l}\text { 593. Girling-Butcher RD and Ronan KR. 2009. Brief cognitive- } \\
\text { behavioural therapy for children with anxiety disorders: Initial } \\
\text { evaluation of a program designed for clinic settings. Behaviour } \\
\text { Change. 26(1):27-53. }\end{array}$ & Not an RCT \\
\hline $\begin{array}{l}\text { 594. Gjerdingen DK and Center B. 2002. A randomized controlled } \\
\text { trial testing the impact of a support/work-planning intervention on } \\
\text { first-time parents' health, partner relationship, and work } \\
\text { responsibilities. Behavioral Medicine, 28(3), 84-91. }\end{array}$ & $\begin{array}{l}\text { No child } \\
\text { internalising } \\
\text { outcomes or not } \\
\text { a major goal }\end{array}$ \\
\hline $\begin{array}{l}\text { 595. Gladstone, T. G., Marko-Holguin, M., Rothberg, P., Nidetz, J., } \\
\text { Diehl, A., DeFrino, D. T., ... \& Van Voorhees, B. W. (2015). An internet- } \\
\text { based adolescent depression preventive intervention: study protocol } \\
\text { for a randomized control trial. Trials, } 16(1), 203 .\end{array}$ & $\begin{array}{l}\text { Data not } \\
\text { available/report } \\
\text { ed }\end{array}$ \\
\hline $\begin{array}{l}596 . \quad \text { Gladstone, T. R., \& Beardslee, W. R. (2009). The } \\
\text { prevention of depression in children and adolescents: a review. } \\
\text { Canadian journal of psychiatry. Revue canadienne de psychiatrie, } \\
54(4), 212-221 \text {. }\end{array}$ & Review \\
\hline $\begin{array}{l}\text { 597. Gol D and Jarus T. 2005. Effect of a social skills training group } \\
\text { on everyday activities of children with attention deficit hyperactivity } \\
\text { disorder. Developmental Medicine \& Child Neurology. 47(8):539-45. }\end{array}$ & $\begin{array}{l}\text { Not a parenting } \\
\text { intervention }\end{array}$ \\
\hline $\begin{array}{l}\text { 598. Goldbeck L and Schmid K. 2003. Effectiveness of autogenic } \\
\text { relaxation training on children and adolescents with behavioral and } \\
\text { emotional problems. J Am Acad Child Adolesc Psychiatry. 42(9):1046- } \\
54 .\end{array}$ & $\begin{array}{l}\text { Not a parenting } \\
\text { intervention }\end{array}$ \\
\hline
\end{tabular}




\begin{tabular}{|c|c|}
\hline $\begin{array}{l}\text { 599. Goldbeck, L., Hölling, I., Schlack, R., West, C., \& Besier, T. } \\
\text { (2011). The impact of an inpatient family-oriented rehabilitation } \\
\text { program on parent-reported psychological symptoms of } \\
\text { chronically ill children. Klinische Pädiatrie, 223(2), } 79 .\end{array}$ & $\begin{array}{l}\text { Intervention for } \\
\text { developmental } \\
\text { disorders } \\
\text { (autism, autism } \\
\text { spectrum } \\
\text { disorder), } \\
\text { physical } \\
\text { disabilities, } \\
\text { medical } \\
\text { conditions or } \\
\text { distress about } \\
\text { facing medical } \\
\text { interventions }\end{array}$ \\
\hline $\begin{array}{l}\text { 600. Goldberg-Lillehoj CJ, Spoth R and Trudeau L. } 2005 . \\
\text { Assertiveness Among Young Rural Adolescents: Relationship to } \\
\text { Alcohol Use. Journal of Child \& Adolescent Substance Abuse. 14(3):39- } \\
68 .\end{array}$ & $\begin{array}{l}\text { Not a parenting } \\
\text { intervention }\end{array}$ \\
\hline $\begin{array}{l}\text { 601. Gonsalves L and Schuermeyer I. 2006. Treating depression in } \\
\text { pregnancy: Practical suggestions. Cleveland Clinic Journal of Medicine. } \\
73(12): 1098-104 \text {. }\end{array}$ & Not an RCT \\
\hline $\begin{array}{l}\text { 602. Gonzales NA, GermV }{ }^{\circ} n \text { M, Kim SY, George P, Fabrett FC, } \\
\text { Millsap R and Dumka LE. 2008. Mexican American adolescents' } \\
\text { cultural orientation, externalizing behavior and academic } \\
\text { engagement: The role of traditional cultural values. American Journal } \\
\text { of Community Psychology. 41(1-2):151-64. }\end{array}$ & $\begin{array}{l}\text { Not a parenting } \\
\text { intervention }\end{array}$ \\
\hline $\begin{array}{l}\text { 603. Gonzales, N. A., Dumka, L. E., Millsap, R. E., Gottschall, A., } \\
\text { McClain, D. B., Wong, J. J., Germán, M., Mauricio, A. M., Wheeler, L., } \\
\text { Carpentier, F. D., \& Kim, S. Y. (2012). Randomized trial of a broad } \\
\text { preventive intervention for Mexican American adolescents. J Consult } \\
\text { Clin Psychol, 80, 1-16. }\end{array}$ & $\begin{array}{l}\text { Parent } \\
\text { component too } \\
\text { small }\end{array}$ \\
\hline $\begin{array}{l}\text { 604. Gonzalves M, Pinto } \mathrm{H} \text { and Araújo MdS. 1998. Anxiety disorders } \\
\text { in children: An experience with a parents' group. Psicologia: Teoria, } \\
\text { Investigacao e Practica. 3(2):327-52. }\end{array}$ & Not an RCT \\
\hline $\begin{array}{l}\text { G05. Goodman E and Whitaker RC. 2002. A prospective study of the } \\
\text { role of depression in the development and persistence of adolescent } \\
\text { obesity. Pediatrics. 110(3):497-504. }\end{array}$ & Not an RCT \\
\hline $\begin{array}{l}\text { 606. Goodnight, J. A. (2010). Youths' motivated attention and } \\
\text { reinforcement matching characteristics as moderators of the effects of } \\
\text { parenting on adolescent communication and externalizing behaviors: } \\
\text { A social learning, matching law approach (Order No. 3423599). } \\
\text { Available from ProQuest Dissertations \& Theses Global. (759823017). }\end{array}$ & Not an RCT \\
\hline $\begin{array}{l}\text { 607. Gopalan G, Dean-Assael K, Klingenstein K, Chacko A and } \\
\text { McKay MM. 2011. Caregiver depression and youth disruptive behavior } \\
\text { difficulties. Social Work in Mental Health. 9(1):56-70. }\end{array}$ & $\begin{array}{l}\text { No child } \\
\text { internalising } \\
\text { outcomes or not } \\
\text { a major goal }\end{array}$ \\
\hline $\begin{array}{l}\text { 608. Gordon, R. A., Usdansky, M. L., Wang, X., \& Gluzman, A. } \\
\text { (2011). Child care and mothers' mental health: Is high-quality care } \\
\text { associated with fewer depressive symptoms? Family Relations: An } \\
\text { Interdisciplinary Journal of Applied Family Studies, 60, 446-460. }\end{array}$ & $\begin{array}{l}\text { Not a parenting } \\
\text { intervention }\end{array}$ \\
\hline
\end{tabular}




\begin{tabular}{|c|c|}
\hline $\begin{array}{l}\text { 609. Graham AL and Papandonatos GD. 2008. Reliability of } \\
\text { internet- versus telephone-administered questionnaires in a diverse } \\
\text { sample of smokers. J Med Internet Res. 10(1):e8. }\end{array}$ & $\begin{array}{l}\text { Not a parenting } \\
\text { intervention }\end{array}$ \\
\hline $\begin{array}{l}\text { 610. Graham-Bermann, S. A., Lynch, S., Banyard, V., DeVoe, E. R., \& } \\
\text { Halabu, H. (2007). Community-based intervention for children } \\
\text { exposed to intimate partner violence: An efficacy trial. Journal of } \\
\text { Consulting and Clinical Psychology, 75(2), 199-209. doi:10.1037/0022- } \\
\text { 006x.75.2.199 }\end{array}$ & $\begin{array}{l}\text { Parent } \\
\text { component too } \\
\text { small }\end{array}$ \\
\hline $\begin{array}{l}\text { 611. Grahame, V., Brett, D., Dixon, L., McConachie, H., Lowry, J., } \\
\text { Rodgers, J., ... \& Le Couteur, A. (2015). Managing Repetitive } \\
\text { Behaviours in Young Children with Autism Spectrum Disorder (ASD): } \\
\text { Pilot Randomised Controlled Trial of a New Parent Group Intervention. } \\
\text { Journal of autism and developmental disorders, 1-15. }\end{array}$ & $\begin{array}{l}\text { Intervention for } \\
\text { developmental } \\
\text { disorders } \\
\text { (autism, autism } \\
\text { spectrum } \\
\text { disorder), } \\
\text { physical } \\
\text { disabilities, } \\
\text { medical } \\
\text { conditions or } \\
\text { distress about } \\
\text { facing medical } \\
\text { interventions }\end{array}$ \\
\hline $\begin{array}{l}\text { 612. Graziano, A. M., \& Mooney, K. C. (1982). Behavioral treatment } \\
\text { of nightfears in children: Maintenance of improvement at 21/2- to 3- } \\
\text { year follow-up. Journal of Consulting and Clinical Psychology, 50, 598- } \\
599 .\end{array}$ & Not an RCT \\
\hline $\begin{array}{l}\text { 613. Green J, Stanley C and Peters S. 2007. Disorganized } \\
\text { attachment representation and atypical parenting in young school age } \\
\text { children with externalizing disorder. Attachment \& Human } \\
\text { Development. 9(3):207-22. }\end{array}$ & $\begin{array}{l}\text { Not a parenting } \\
\text { intervention }\end{array}$ \\
\hline $\begin{array}{l}\text { 614. Greenblatt, D. J., Harmatz, J. S., Walsh, J. K., Luthringer, R., } \\
\text { Staner, L., Otmani, S., Nedelec, J. F., Francart, C., Parent, S. J., \& } \\
\text { Staner, C. (2011). Pharmacokinetic profile of SKP-1041, a modified } \\
\text { release formulation of zaleplon. Biopharmaceutics and Drug } \\
\text { Disposition, 32, 489-497. }\end{array}$ & $\begin{array}{l}\text { Not a parenting } \\
\text { intervention }\end{array}$ \\
\hline $\begin{array}{l}\text { 615. Grembowski D, Spiekerman C, Del Aguila MA, Anderson M, } \\
\text { Reynolds D, Ellersick A, Foster J and Choate L. 2006. Randomized pilot } \\
\text { study to disseminate caries-control services in dentist offices. BMC } \\
\text { Oral Health. } 6 \text {. }\end{array}$ & $\begin{array}{l}\text { Not a parenting } \\
\text { intervention }\end{array}$ \\
\hline $\begin{array}{l}\text { 616. Gren-Landell M, Björklind A, Tillfors M, Furmark T, Svedin CGr } \\
\text { and Andersson G. 2009. Evaluation of the psychometric properties of a } \\
\text { modified version of the Social Phobia Screening Questionnaire for use } \\
\text { in adolescents. Child and Adolescent Psychiatry and Mental Health. } \\
\text { 3(11). }\end{array}$ & $\begin{array}{l}\text { Not a parenting } \\
\text { intervention }\end{array}$ \\
\hline $\begin{array}{l}\text { 617. Grey M, Whittemore R, Jaser S, Ambrosino J, Lindemann E, } \\
\text { Liberti L, Northrup V and Dziura J. 2009. Effects of coping skills training } \\
\text { in school-age children with type } 1 \text { diabetes. Res Nurs Health. } \\
\text { 32(4):405-18. }\end{array}$ & $\begin{array}{l}\text { Not a parenting } \\
\text { intervention }\end{array}$ \\
\hline
\end{tabular}




\begin{tabular}{|c|c|}
\hline $\begin{array}{l}\text { 618. Griggs, M. S., \& Mikami, A. Y. (2011). Parental attention- } \\
\text { deficit/hyperactivity disorder predicts child and parent outcomes of } \\
\text { parental friendship coaching treatment. In J Am Acad Child Adolesc } \\
\text { Psychiatry (pp. 1236-1246). }\end{array}$ & $\begin{array}{l}\text { No long term } \\
\text { follow-up; No } \\
\text { child } \\
\text { internalising } \\
\text { outcomes or not } \\
\text { a major goal }\end{array}$ \\
\hline $\begin{array}{l}\text { 619. Grimbos T and Granic I. 2009. Changes in maternal depression } \\
\text { are associated with MST outcomes for adolescents with co-occurring } \\
\text { externalizing and internalizing problems. Journal of Adolescence. } \\
\text { 32(6):1415-23. }\end{array}$ & Not an RCT \\
\hline $\begin{array}{l}\text { 620. Groeben M, Perren S, Stadelmann S and von Klitzing K. } 2011 . \\
\text { Emotional symptoms from kindergarten to middle childhood: } \\
\text { Associations with self- and other-oriented social skills. European Child } \\
\text { \& Adolescent Psychiatry. 20(1):3-15. }\end{array}$ & $\begin{array}{l}\text { Not a parenting } \\
\text { intervention }\end{array}$ \\
\hline $\begin{array}{l}\text { 621. Gross D, Fogg L and Tucker S. 1995. The efficacy of parent } \\
\text { training for promoting positive parent-toddler relationships. Res Nurs } \\
\text { Health. 18(6):489-99. }\end{array}$ & $\begin{array}{l}\text { Follow-up less } \\
\text { than } 6 \text { months }\end{array}$ \\
\hline $\begin{array}{l}\text { 622. Gross HE, Shaw DS, Moilanen KL, Dishion TJ and Wilson MN. } \\
\text { 2008. Reciprocal models of child behavior and depressive symptoms in } \\
\text { mothers and fathers in a sample of children at risk for early conduct } \\
\text { problems. J Fam Psychol. 22(5):742-51. }\end{array}$ & Not an RCT \\
\hline $\begin{array}{l}\text { 623. Gross, D. A., Belcher, H. M. E., Ofonedu, M. E., Breitenstein, S., } \\
\text { Frick, K. D., \& Chakra, B. (2014). Study protocol for a comparative } \\
\text { effectiveness trial of two parent training programs in a fee-for-service } \\
\text { mental health clinic: Can we improve mental health services to low- } \\
\text { income families? Trials, 15(1). doi: http://dx.doi.org/10.1186/1745- } \\
6215-15-70\end{array}$ & $\begin{array}{l}\text { Data not } \\
\text { available/report } \\
\text { ed }\end{array}$ \\
\hline $\begin{array}{l}\text { 624. Gross, D., Fogg, L., Webster-Stratton, C., Garvey, C., Julion, W., } \\
\text { \& Grady, J. (2003). Parent training of toddlers in day care in low- } \\
\text { income urban communities. Journal of consulting and clinical } \\
\text { psychology, (2), 261-278. }\end{array}$ & $\begin{array}{l}\text { No child } \\
\text { internalising } \\
\text { outcomes or not } \\
\text { a major goal }\end{array}$ \\
\hline $\begin{array}{l}\text { 625. Grossman JB and Rhodes JE. 2002. The test of time: predictors } \\
\text { and effects of duration in youth mentoring relationships. American } \\
\text { journal of community psychology, 30(2), 199-219. }\end{array}$ & $\begin{array}{l}\text { Not a parenting } \\
\text { intervention }\end{array}$ \\
\hline $\begin{array}{l}\text { 626. Grote NK, Bledsoe SE, Swartz HA and Frank E. 2004. Feasibility } \\
\text { of Providing Culturally Relevant, Brief Interpersonal Psychotherapy for } \\
\text { Antenatal Depression in an Obstetrics Clinic: A Pilot Study. Research } \\
\text { on Social Work Practice. 14(6):397-407. }\end{array}$ & $\begin{array}{l}\text { No child } \\
\text { internalising } \\
\text { outcomes or not } \\
\text { a major goal }\end{array}$ \\
\hline $\begin{array}{l}\text { 627. Grunewaldt, K. H., Lohaugen, G. C., Austeng, D., Brubakk, A. } \\
\text { M., \& Skranes, J. (2013). Working memory training improves cognitive } \\
\text { function in VLBW preschoolers. Pediatrics, 131(3), e747-754. doi: } \\
\text { 10.1542/peds.2012-1965 }\end{array}$ & $\begin{array}{l}\text { Not a parenting } \\
\text { intervention }\end{array}$ \\
\hline $\begin{array}{l}\text { 628. Gryczkowski, M. R. (2010). An examination of potential } \\
\text { moderators in the relations between mothers' and fathers' parenting } \\
\text { practices and children's behavior (Order No. 3437893). Available from } \\
\text { ProQuest Dissertations \& Theses Global. (822234006). }\end{array}$ & $\begin{array}{l}\text { Not a parenting } \\
\text { intervention }\end{array}$ \\
\hline
\end{tabular}




\begin{tabular}{|c|c|}
\hline $\begin{array}{l}\text { 629. Guillois, B., Castel, S., Blaizot, X., Beunard, A., Creveuil, C., \& } \\
\text { Proia-Lelouey, N. (2013). Early intervention program after discharge } \\
\text { improves parents-infant relationship and behavioral and cognitive } \\
\text { outcomes for preterm infants. [Conference Abstract]. Archives of } \\
\text { Women's Mental Health, 16, S92. doi: } \\
\text { http://dx.doi.org/10.1007/s00737-013-0355-x }\end{array}$ & $\begin{array}{l}\text { No long term } \\
\text { follow-up }\end{array}$ \\
\hline $\begin{array}{l}\text { 630. Guo G and Tillman KH. 2009. Trajectories of depressive } \\
\text { symptoms, dopamine D2 and D4 receptors, family socioeconomic } \\
\text { status and social support in adolescence and young adulthood. } \\
\text { Psychiatric Genetics. 19(1):14-26. }\end{array}$ & $\begin{array}{l}\text { Not a parenting } \\
\text { intervention }\end{array}$ \\
\hline $\begin{array}{l}\text { 631. Gustafsson, H. C., \& Cox, M. J. (2012). Relations among } \\
\text { intimate partner violence, maternal depressive symptoms, and } \\
\text { maternal parenting behaviors. Journal of Marriage and Family, 74, } \\
\text { 1005-1020. }\end{array}$ & $\begin{array}{l}\text { Not a parenting } \\
\text { intervention }\end{array}$ \\
\hline $\begin{array}{l}\text { 632. Gutelius MF, Kirsch AD, MacDonald S, Brooks MR, McErlean T. } \\
\text { Controlled study of child health supervision: behavioral results. } \\
\text { Pediatrics.1977;60:294-304. }\end{array}$ & Not an RCT \\
\hline $\begin{array}{l}\text { 633. Gutiérrez Casares JR, et al. 2002. Adolescents at risk for major } \\
\text { depressive disorder. I. Variables related to the detection of a sample. } \\
\text { Revista de psiquiatría infanto-juvenil. 19(3):122-31. }\end{array}$ & $\begin{array}{l}\text { Not a parenting } \\
\text { intervention }\end{array}$ \\
\hline $\begin{array}{l}\text { 634. Guyll, M., Spoth, R. L., Chao, W., Wickrama, K. A., \& Russell, D. } \\
\text { (2004). Family-focused preventive interventions: evaluating parental } \\
\text { risk moderation of substance use trajectories. Journal of family } \\
\text { psychology : JFP : journal of the Division of Family Psychology of the } \\
\text { American Psychological Association (Division 43), (2), 293-301. }\end{array}$ & $\begin{array}{l}\text { No child } \\
\text { internalising } \\
\text { outcomes or not } \\
\text { a major goal }\end{array}$ \\
\hline $\begin{array}{l}\text { 635. Güzel, A., Atlı, A., Doğan, E., Çelik, F., Tüfek, A., Dusak, A., ... \& } \\
\text { Baysal Yıldırım, Z. (2014). Magnetic Resonance Imaging in children } \\
\text { under anesthesia: The relationship between the degree of information } \\
\text { provided to parents and parents' anxiety scores. BioMed research } \\
\text { international, 2014. }\end{array}$ & $\begin{array}{l}\text { No child } \\
\text { internalising } \\
\text { outcomes or not } \\
\text { a major goal }\end{array}$ \\
\hline $\begin{array}{l}\text { 636. Gwynne K, Blick BA and Duffy GM. 2009. Pilot evaluation of an } \\
\text { early intervention programme for children at risk. Journal of } \\
\text { Paediatrics and Child Health. 45(3):118-24. }\end{array}$ & Not an RCT \\
\hline $\begin{array}{l}\text { 637. Hagan, M. J., Tein, J. Y., Sandler, I. N., Wolchik, S. A., Ayers, T. } \\
\text { S., \& Luecken, L. J. (2012). Strengthening effective parenting practices } \\
\text { over the long term: Effects of a preventive intervention for parentally } \\
\text { bereaved families. Journal of Clinical Child \& Adolescent Psychology, } \\
\text { 41(2), 177-188. }\end{array}$ & $\begin{array}{l}\text { Parent } \\
\text { component too } \\
\text { small; No } \\
\text { internalising } \\
\text { outcomes or not } \\
\text { a major goal }\end{array}$ \\
\hline $\begin{array}{l}\text { 638. Hahlweg, K., Heinrichs, N., Kuschel, A., \& Feldmann, M. (2008). } \\
\text { Therapist-assisted, self-administered bibliotherapy to enhance } \\
\text { parental competence: short- and long-term effects. Behav Modif, } \\
\text { 32(5), 659-681. doi: } 0145445508317131\end{array}$ & $\begin{array}{l}\text { No long term } \\
\text { follow-up }\end{array}$ \\
\hline $\begin{array}{l}\text { 639. Hahn, E. J., Hall, L. A., \& Simpson, M. R. (1998). Drug } \\
\text { prevention with high risk families and young children. J Drug Educ, } \\
28(4), 327-345 .\end{array}$ & Not an RCT \\
\hline $\begin{array}{l}\text { 640. Haldre K, Rahu K, Rahu M and Karro H. 2009. Individual and } \\
\text { familial factors associated with teenage pregnancy: An interview } \\
\text { study. European Journal of Public Health. 19(3):266-70. }\end{array}$ & $\begin{array}{l}\text { Not a parenting } \\
\text { intervention }\end{array}$ \\
\hline
\end{tabular}




\begin{tabular}{|c|c|}
\hline $\begin{array}{l}\text { 641. Hall JA, Smith DC, Easton SD, An H, Williams JK, Godley SH and } \\
\text { Jang M. 2008. Substance abuse treatment with rural adolescents: } \\
\text { Issues and outcomes. Journal of Psychoactive Drugs. } 40(1): 109-20 \text {. }\end{array}$ & $\begin{array}{l}\text { Not a parenting } \\
\text { intervention }\end{array}$ \\
\hline $\begin{array}{l}\text { 642. Hall TF. 2003. Early intervention multimodal treatment } \\
\text { program for children with attention deficit hyperactivity disorder: An } \\
\text { outcome study. Dissertation Abstracts International: Section B: The } \\
\text { Sciences and Engineering. 63(7-B). }\end{array}$ & $\begin{array}{l}\text { Follow-up less } \\
\text { than } 6 \text { months }\end{array}$ \\
\hline $\begin{array}{l}\text { 643. Hall, J. (2012). Women's and men's satisfaction with two } \\
\text { models of antenatal education. Pract Midwife, } 15,35-37 \text {. }\end{array}$ & $\begin{array}{l}\text { No child } \\
\text { internalising } \\
\text { outcomes or not } \\
\text { a major goal }\end{array}$ \\
\hline $\begin{array}{l}\text { 644. Hallfors DD, Waller MW, Ford CA, Halpern CT, Brodish PH and } \\
\text { Iritani B. 2004. Adolescent Depression and Suicide Risk: Association } \\
\text { with Sex and Drug Behavior. American Journal of Preventive Medicine. } \\
\text { 27(3):224-30. }\end{array}$ & $\begin{array}{l}\text { Not a parenting } \\
\text { intervention }\end{array}$ \\
\hline $\begin{array}{l}\text { 645. Han SS, Catron T, Weiss B and Marciel KK. 2005. A teacher- } \\
\text { consultation approach to social skills training for pre-kindergarten } \\
\text { children: treatment model and short-term outcome effects. J Abnorm } \\
\text { Child Psychol. 33(6):681-93. }\end{array}$ & $\begin{array}{l}\text { Not a parenting } \\
\text { intervention }\end{array}$ \\
\hline $\begin{array}{l}\text { 646. Hanas R, Adolfsson P, Elfvin-Akesson K, Hammaren L, Ilvered } \\
\text { R, Jansson I, Johansson C, Kroon M, Lindgren J, Lindh A, Ludvigsson J, } \\
\text { Sigstrom L, Wiik A and Aman J. 2002. Indwelling catheters used from } \\
\text { the onset of diabetes decrease injection pain and pre-injection } \\
\text { anxiety. J Pediatr. 140(3):315-20. }\end{array}$ & $\begin{array}{l}\text { Not a parenting } \\
\text { intervention }\end{array}$ \\
\hline $\begin{array}{l}\text { 647. Hand, A., McDonnell, E., Honari, B., Sharry, J. (2013). A } \\
\text { community led approach to delivery of the Parents Plus Children's } \\
\text { Programme for the parents of children aged 6-11. International } \\
\text { Journal of Clinical and Health Psychology, } 13 \text { (2), 81-90. }\end{array}$ & $\begin{array}{l}\text { Follow-up data } \\
\text { collected only in } \\
\text { active group; } \\
\text { Targets } \\
\text { externalising } \\
\end{array}$ \\
\hline $\begin{array}{l}\text { 648. Hanisch, C., Freund-Braier, I., Hautmann, C., Janen, N., Pluck, } \\
\text { J., Brix, G., et al. (2010). Detecting effects of the indicated prevention } \\
\text { Programme for Externalizing Problem behaviour (PEP) on child } \\
\text { symptoms, parenting, and parental quality of life in a randomized } \\
\text { controlled trial. Behav Cogn Psychother, 38(1), 95-112. doi: } \\
\text { S1352465809990440 }\end{array}$ & $\begin{array}{l}\text { Follow-up less } \\
\text { than } 6 \text { months }\end{array}$ \\
\hline $\begin{array}{l}\text { 649. Hanisch, C., Hautmann, C., Pluck, J., Eichelberger, l., Dopfner, } \\
\text { M. (2014). The prevention program for externalizing problem behavior } \\
\text { (PEP) improves child behavior by reducing negative parenting: Analysis } \\
\text { of mediating processes in a randomized controlled trial. Journal of } \\
\text { Child Psychology and Psychiatry, } 55 \text { (5), 473-484. }\end{array}$ & $\begin{array}{l}\text { Targets } \\
\text { externalising }\end{array}$ \\
\hline $\begin{array}{l}\text { 650. Hanisch, C., Pluck, J., Hautmann, C., \& Doepfner, M. (2011). } \\
\text { Effectiveness of indicated prevention of disruptive behaviour } \\
\text { problems in children: A multilevel modelling analysis. European Child } \\
\text { and Adolescent Psychiatry, 20, S65. }\end{array}$ & Not an RCT \\
\hline $\begin{array}{l}\text { 651. Hanisch, C., Pluck, J., Meyer, N., Brix, G., Freund-Braier, I., } \\
\text { Hautmann, C., \& Dopfner, M. (2006). Short-term effects of the } \\
\text { indicated Prevention Programme for externalizing Problem Behaviour } \\
\text { (PEP). Zeitschrift Fur Klinische Psychologie Und Psychotherapie, 35(2), } \\
\text { 117-126. }\end{array}$ & $\begin{array}{l}\text { No long term } \\
\text { follow-up }\end{array}$ \\
\hline
\end{tabular}




\begin{tabular}{|c|c|}
\hline $\begin{array}{l}652 . \quad \text { Hans, S. L., Thullen, M., Henson, L. G., Lee, H., Edwards, R. C., } \\
\text { \& Bernstein, V. J. (2013). Promoting Positive Mother-Infant } \\
\text { Relationships: A Randomized Trial of Community Doula Support For } \\
\text { Young Mothers. Infant Mental Health Journal, 34(5), 446-457. doi: } \\
\text { http://dx.doi.org/10.1002/imhj.21400 }\end{array}$ & $\begin{array}{l}\text { No child } \\
\text { internalising } \\
\text { outcomes or not } \\
\text { a major goal }\end{array}$ \\
\hline $\begin{array}{l}\text { 653. Hansen, B. L. (1995). A health education booklet for young } \\
\text { families - Its need, use and effect. Patient Education and Counseling, } \\
25(2), 137-142 .\end{array}$ & $\begin{array}{l}\text { No child } \\
\text { internalising } \\
\text { outcomes or not } \\
\text { a major goal }\end{array}$ \\
\hline $\begin{array}{l}\text { 654. Hanson MD, Gauld M, Wathen CN and MacMillan HL. } 2008 . \\
\text { Nonpharmacological interventions for acute wound care distress in } \\
\text { pediatric patients with burn injury: A systematic review. Journal of } \\
\text { Burn Care and Research. 29(5):730-41. }\end{array}$ & Review \\
\hline $\begin{array}{l}\text { 655. Harari D, Bakermans-Kranenburg MJ, de Kloet CS, Geuze E, } \\
\text { Vermetten E, Westenberg HGM and van ljzendoorn MH. } 2009 . \\
\text { Attachment representations in Dutch veterans with and without } \\
\text { deployment-related PTSD. Attachment \& Human Development. } \\
\text { 11(6):515-36. }\end{array}$ & $\begin{array}{l}\text { Not a parenting } \\
\text { intervention }\end{array}$ \\
\hline $\begin{array}{l}\text { 656. Hardy JB, Streett R. Family support and parenting education in } \\
\text { the home: an effective extension of clinic-based preventive health } \\
\text { care services for poor children. J Pediatr. 1989;115:927-931. }\end{array}$ & $\begin{array}{l}\text { no child } \\
\text { internalising } \\
\text { outcomes or not } \\
\text { a major goal }\end{array}$ \\
\hline $\begin{array}{l}\text { 657. Harrington R. 1996. Controlled trial of a brief cognitive- } \\
\text { behavioural intervention in adolescents parents with depressive } \\
\text { disorders. European Psychiatry, 11, 158s. }\end{array}$ & $\begin{array}{l}\text { Not a parenting } \\
\text { intervention }\end{array}$ \\
\hline $\begin{array}{l}\text { 658. Harrington, R., Kerfoot, M., Dyer, E., McNiven, F., Gill, J., } \\
\text { Harrington, V., et al. (1998). Randomized trial of a home-based family } \\
\text { intervention for children who have deliberately poisoned themselves. } \\
\text { Journal of the American Academy of Child and Adolescent Psychiatry, } \\
\text { (5), 512-518. }\end{array}$ & $\begin{array}{l}\text { Parent } \\
\text { component too } \\
\text { small }\end{array}$ \\
\hline $\begin{array}{l}\text { 659. Harrington, R., Peters, S., Green, J., Byford, S., Woods, J., \& } \\
\text { McGowan, R. (2000). Randomised comparison of the effectiveness and } \\
\text { costs of community and hospital based mental health services for } \\
\text { children with behavioural disorders. BMJ, } 321(7268), 1047-1050 .\end{array}$ & $\begin{array}{l}\text { No child } \\
\text { internalising } \\
\text { outcomes or not } \\
\text { a major goal }\end{array}$ \\
\hline $\begin{array}{l}\text { 660. Hart, C., Love, A., Gibson, N., Morgan, M., \& Paton, J. (2002). } \\
\text { Effects of an educational intervention directed at parental beliefs and } \\
\text { anxieties about inhaled steroid medication on adherence [abstract]. } \\
\text { American Journal of Respiratory and Critical Care Medicine, (8 Suppl), } \\
\text { A420. }\end{array}$ & $\begin{array}{l}\text { No child } \\
\text { internalising } \\
\text { outcomes or not } \\
\text { a major goal }\end{array}$ \\
\hline $\begin{array}{l}\text { 661. Hart, K.C. (2013). Promoting successful transitions to } \\
\text { kindergarten: An early intervention for behaviorally at-risk children } \\
\text { from Head Start preschools. Dissertation Abstracts International: } \\
\text { Section B: The Sciences and Engineering, } 74 \text { (2-B E). }\end{array}$ & $\begin{array}{l}\text { Targets } \\
\text { externalising }\end{array}$ \\
\hline $\begin{array}{l}\text { 662. Hart, L., Nedadur, R., Reardon, J., Sirizzotti, N., Speechley, K., } \\
\text { Loftus, J., Miller, M., Salvadori, M., Poonai, N. (2015). An interactive } \\
\text { web-based module versus website and standard of care for parental } \\
\text { fever education: A randomized controlled trial. Academic Emergency } \\
\text { Medicine, 1) S221. }\end{array}$ & $\begin{array}{l}\text { No child } \\
\text { internalising } \\
\text { outcomes or not } \\
\text { a major goal }\end{array}$ \\
\hline
\end{tabular}




\begin{tabular}{|c|c|}
\hline $\begin{array}{l}\text { 663. Hartman JH, Bena J, McIntyre S and Albert NM. 2009. Does a } \\
\text { Photo Diary Decrease Stress and Anxiety in Children Undergoing } \\
\text { Magnetic Resonance Imaging? A Randomized, Controlled Study. } \\
\text { Journal of Radiology Nursing. 28(4):122-8. }\end{array}$ & $\begin{array}{l}\text { Not a parenting } \\
\text { intervention }\end{array}$ \\
\hline $\begin{array}{l}\text { 664. Hartman RR, Stage SA and Webster-Stratton C. 2003. A growth } \\
\text { curve analysis of parent training outcomes: Examining the influence of } \\
\text { child risk factors (inattention, impulsivity, and hyperactivity problems), } \\
\text { parental and family risk factors. Journal of Child Psychology and } \\
\text { Psychiatry and Allied Disciplines. 44(3):388-98. }\end{array}$ & Not an RCT \\
\hline $\begin{array}{l}\text { 665. Hasmann, R., Blank, R., Hampel, O. A., Jahnel, D., Holl, R., } \\
\text { Karpinski, N., \& Petermann, F. (2012). Group Stepping Stones Triple P: } \\
\text { Evaluation of a preventative parenting intervention for parents of a } \\
\text { child with a disability. Developmental Medicine and Child Neurology, } \\
54,42 .\end{array}$ & $\begin{array}{l}\text { Intervention for } \\
\text { developmental } \\
\text { disorders } \\
\text { (autism, autism } \\
\text { spectrum } \\
\text { disorder), } \\
\text { physical } \\
\text { disabilities, } \\
\text { medical } \\
\text { conditions or } \\
\text { distress about } \\
\text { facing medical } \\
\text { interventions }\end{array}$ \\
\hline $\begin{array}{l}\text { 666. Hauch, Y. (2005). A multimodal treatment program for } \\
\text { children with ADHD: A 16-month follow-up. [Dissertation Abstract; } \\
\text { Dissertation]. Dissertation Abstracts International: Section B: The } \\
\text { Sciences and Engineering, 66(3-B). }\end{array}$ & $\begin{array}{l}\text { follow-up less } \\
\text { than } 6 \text { months; } \\
\text { Comparison } \\
\text { condition not a } \\
\text { minimal control }\end{array}$ \\
\hline $\begin{array}{l}\text { 667. Hauck, Y. L., Hall, W. A., Dhaliwal, S. S., Bennett, E., \& Wells, G. } \\
\text { (2012). The effectiveness of an early parenting intervention for } \\
\text { mothers with infants with sleep and settling concerns: A prospective } \\
\text { non-equivalent before-after design. J Clin Nurs, 21,52-62. }\end{array}$ & Not an RCT \\
\hline $\begin{array}{l}\text { 668. Haus BF and Thompson S. 1976. The effect of nursing } \\
\text { intervention on a program of behavior modification by parents in the } \\
\text { home. Journal of psychiatric nursing and mental health services } 14.8 \\
\text { (1976): 9-16. }\end{array}$ & $\begin{array}{l}\text { Intervention for } \\
\text { developmental } \\
\text { disorders } \\
\text { (autism, autism } \\
\text { spectrum } \\
\text { disorder), } \\
\text { physical } \\
\text { disabilities, } \\
\text { medical } \\
\text { conditions or } \\
\text { distress about } \\
\text { facing medical } \\
\text { interventions }\end{array}$ \\
\hline $\begin{array}{l}\text { 669. Hautmann, C., Eichelberger, I., Hanisch, C., Plück, J., Walter, } \\
\text { D., \& Döpfner, M. (2010). The severely impaired do profit most: Short- } \\
\text { term and long-term predictors of therapeutic change for a parent } \\
\text { management training under routine care conditions for children with } \\
\text { externalizing problem behavior. European Child \& Adolescent } \\
\text { Psychiatry, 19(5), 419-430. doi: } 10.1007 / \text { s00787-009-0072-1 }\end{array}$ & Not an RCT \\
\hline
\end{tabular}




\begin{tabular}{|c|c|}
\hline $\begin{array}{l}\text { 670. Hautmann, C., Greimel, L., Imort, S., Katzmann, J., Pinior, J., } \\
\text { Scholz, K., \& Dopfner, M. (2013). Efficacy of guided self-help for } \\
\text { parents of children with externalizing behaviour problems-a } \\
\text { randomized controlled trial with an active control group. [Conference } \\
\text { Abstract]. European Child and Adolescent Psychiatry, 1), S173. doi: } \\
\text { http://dx.doi.org/10.1007/s00787-013-0423-9 }\end{array}$ & $\begin{array}{l}\text { Comparison } \\
\text { condition not a } \\
\text { minimal control }\end{array}$ \\
\hline $\begin{array}{l}\text { 671. Hautmann, C., Hanisch, C., Mayer, I., Pluck, J., \& Dopfner, M. } \\
\text { (2008). Effectiveness of the prevention program for externalizing } \\
\text { problem behaviour (PEP) in children with symptoms of attention- } \\
\text { deficit/hyperactivity disorder and oppositional defiant disorder-- } \\
\text { generalization to the real world. J Neural Transm, 115(2), 363-370. doi: } \\
\text { 10.1007/s00702-007-0866-6 }\end{array}$ & $\begin{array}{l}\text { Not an RCT; No } \\
\text { long term } \\
\text { follow-up }\end{array}$ \\
\hline $\begin{array}{l}\text { 672. Hautmann, C., Hoijtink, H., Eichelberger, I., Hanisch, C., Plück, } \\
\text { J., Walter, D., et al. (2009). One-year follow-up of a parent } \\
\text { management training for children with externalizing behaviour } \\
\text { problems in the real world. Behavioural and Cognitive Psychotherapy, } \\
\text { 37(4), 379-396. doi: } 10.1017 / \text { s135246580999021x }\end{array}$ & Not an RCT \\
\hline $\begin{array}{l}\text { 673. Hautmann, C., Stein, P., Eichelberger, I., Hanisch, C., Plück, J., } \\
\text { Walter, D., \& Döpfner, M. (2011). The severely impaired do profit } \\
\text { most: Differential effectiveness of a parent management training for } \\
\text { children with externalizing behavior problems in a natural setting. } \\
\text { Journal of Child and Family Studies, 20, 424-435. }\end{array}$ & Not an RCT \\
\hline $\begin{array}{l}\text { 674. Hautmann, C., Stein, P., Hanisch, C., Eichelberger, I., Plück, J., } \\
\text { Walter, D., et al. (2009). Does parent management training for } \\
\text { children with externalizing problem behavior in routine care result in } \\
\text { clinically significant changes? Psychotherapy Research, 19(2), 224-233. } \\
\text { doi: } 10.1080 / 10503300902777148\end{array}$ & $\begin{array}{l}\text { Not an RCT; } \\
\text { Follow-up less } \\
\text { than } 6 \text { months }\end{array}$ \\
\hline $\begin{array}{l}\text { 675. Havighurst, S. S., Kehoe, C. E., \& Harley, A. E. (2015). Tuning in } \\
\text { to teens: Improving parental responses to anger and reducing youth } \\
\text { externalizing behavior problems. Journal of adolescence, } 42,148-158 .\end{array}$ & $\begin{array}{l}\text { Targets } \\
\text { externalising }\end{array}$ \\
\hline $\begin{array}{l}\text { 676. Havighurst, S. S., Wilson, K. R., Harley, A. E., Kehoe, C., Efron, } \\
\text { D., \& Prior, M. R. (2013). "Tuning into Kids": Reducing Young Children's } \\
\text { Behavior Problems Using an Emotion Coaching Parenting Program. } \\
\text { Child Psychiatry \& Human Development, 44(2), 247-264. }\end{array}$ & $\begin{array}{l}\text { No child } \\
\text { internalising } \\
\text { outcomes or not } \\
\text { a major goal }\end{array}$ \\
\hline $\begin{array}{l}\text { 677. Hawkins, J. D., Kosterman, R., Catalano, R. F., Hill, K. G., \& } \\
\text { Abbott, R. D. (2005). Promoting positive adult functioning through } \\
\text { social development intervention in childhood: long-term effects from } \\
\text { the Seattle Social Development Project. Archives of pediatrics \& } \\
\text { adolescent medicine, (1), 25-31. }\end{array}$ & Not an RCT \\
\hline $\begin{array}{l}\text { 678. Hawkins, J. D., Kosterman, R., Catalano, R. F., Hill, K. G., \& } \\
\text { Abbott, R. D. (2008). Effects of social development intervention in } \\
\text { childhood } 15 \text { years later. Archives of pediatrics \& adolescent medicine, } \\
\text { (12), 1133-1141. }\end{array}$ & Not an RCT \\
\hline $\begin{array}{l}\text { 679. Hayes L, Matthews J, Copley A and Welsh D. 2008. A } \\
\text { randomized controlled trial of a mother-infant or toddler parenting } \\
\text { program: demonstrating effectiveness in practice. J Pediatr Psychol. } \\
\text { 33(5):473-86. }\end{array}$ & $\begin{array}{l}\text { Follow-up less } \\
\text { than } 6 \text { months }\end{array}$ \\
\hline $\begin{array}{l}\text { 680. Hazen RA, Eder M, Drotar D, Zyzanski S, Reynolds AE, Patrick } \\
\text { Reynolds C, Kodish E and Noll RB. 2010. A feasibility trial of a video } \\
\text { intervention to improve informed consent for parents of children with } \\
\text { leukemia. Pediatric Blood and Cancer. 55(1):113-8. }\end{array}$ & $\begin{array}{l}\text { No child } \\
\text { internalising } \\
\text { outcomes or not } \\
\text { a major goal }\end{array}$ \\
\hline
\end{tabular}




\begin{tabular}{|c|c|}
\hline $\begin{array}{l}\text { 681. Healy, K. L., \& Sanders, M. R. (2014). Randomized controlled } \\
\text { trial of a family intervention for children bullied by peers. Behavior } \\
\text { therapy, } 45(6), 760-777 \text {. }\end{array}$ & $\begin{array}{l}\text { Parent } \\
\text { component too } \\
\text { small }\end{array}$ \\
\hline $\begin{array}{l}\text { 682. Heaman, M. I., Martens, P. J., Brownell, M. D., Chartier, M. J., } \\
\text { Helewa, M. E., Derksen, S. A., \& Thiessen, K. (2012). Predictors of } \\
\text { inadequate prenatal care: A population-based study in Manitoba, } \\
\text { Canada. [Conference Abstract]. Journal of Paediatrics and Child } \\
\text { Health, 48, 31. doi: http://dx.doi.org/10.1111/j.1440- } \\
\text { 1754.2012.02411.x }\end{array}$ & $\begin{array}{l}\text { Not a parenting } \\
\text { intervention }\end{array}$ \\
\hline $\begin{array}{l}\text { 683. Hechtman, L., Abikoff, H., Klein, R. G., Weiss, G., Respitz, C., } \\
\text { Kouri, J., et al. (2004). Academic achievement and emotional status of } \\
\text { children with ADHD treated with long-term methylphenidate and } \\
\text { multimodal psychosocial treatment. J Am Acad Child Adolesc } \\
\text { Psychiatry, 43(7), 812-819. doi:S0890-8567(09)61399-X }\end{array}$ & $\begin{array}{l}\text { Parent } \\
\text { component too } \\
\text { small }\end{array}$ \\
\hline $\begin{array}{l}\text { 684. Hederos CA, Janson S and Hedlin G. 2007. A gender } \\
\text { perspective on parents' answers to a questionnaire on children's } \\
\text { asthma. Respir Med. 101(3):554-60. }\end{array}$ & $\begin{array}{l}\text { No child } \\
\text { internalising } \\
\text { outcomes or not } \\
\text { a major goal }\end{array}$ \\
\hline $\begin{array}{l}\text { 685. Heinicke, C. M., Fineman, N. R., Ponce, V. A., \& Guthrie, D. } \\
\text { (2001). Relation-based intervention with at-risk mothers: Outcome in } \\
\text { the 2nd year of life. Infant Mental Health Journal, 22, 431-462. }\end{array}$ & $\begin{array}{l}\text { No child } \\
\text { internalising } \\
\text { outcomes or not } \\
\text { a major goal }\end{array}$ \\
\hline $\begin{array}{l}\text { 686. Heinrichs } \mathrm{N} \text {, Bertram H, Kuschel A and Hahlweg K. } 2005 . \\
\text { Parent recruitment and retention in a universal prevention program } \\
\text { for child behavior and emotional problems: barriers to research and } \\
\text { program participation. Prevention Science, 6(4), 275-286. }\end{array}$ & $\begin{array}{l}\text { No child } \\
\text { internalising } \\
\text { outcomes or not } \\
\text { a major goal }\end{array}$ \\
\hline $\begin{array}{l}\text { 687. Heinrichs, N., Hahlweg, K., Naumann, S., Kuschel, A., Bertram, } \\
\text { H., \& Staender, D. (2009). Universal prevention of child behavior } \\
\text { problems with a parent training. Zeitschrift fur Klinische Psychologie } \\
\text { Und Psychotherapie, 38(2), 79-88. doi:10.1026/1616-3443.38.2.79 }\end{array}$ & Not in English \\
\hline $\begin{array}{l}\text { 688. Hektner, J. M., August, G. J., Bloomquist, M. L., Lee, S., \& } \\
\text { Klimes-Dougan, B. (2014). A 10-Year Randomized Controlled Trial of } \\
\text { the Early Risers Conduct Problems Preventive Intervention: Effects on } \\
\text { Externalizing and Internalizing in Late High School. Journal of } \\
\text { consulting and clinical psychology, 82(2), } 355 .\end{array}$ & $\begin{array}{l}\text { Targets } \\
\text { externalising; } \\
\text { Parenting } \\
\text { component too } \\
\text { small }\end{array}$ \\
\hline $\begin{array}{l}\text { 689. Helfenbaum-Kun, E. D., \& Ortiz, C. (2007). Parent-training } \\
\text { groups for fathers of head start children: A pilot study of their } \\
\text { feasibility and impact on child behavior and intra-familial } \\
\text { relationships. Child \& Family Behavior Therapy, 29(2), 47-64. doi: } \\
\text { 10.1300/J019v29n02_04 }\end{array}$ & $\begin{array}{l}\text { No child } \\
\text { internalising } \\
\text { outcomes or not } \\
\text { a major goal }\end{array}$ \\
\hline $\begin{array}{l}\text { 690. Hendrie, G., Sohonpal, G., Lange, K., \& Golley, R. (2013). } \\
\text { Change in the family food environment is associated with positive } \\
\text { dietary change in children. International journal of behavioral nutrition } \\
\text { and physical activity, } 10,4 \text {. doi:10.1186/1479-5868-10-4 }\end{array}$ & $\begin{array}{l}\text { No child } \\
\text { internalising } \\
\text { outcomes or not } \\
\text { a major goal }\end{array}$ \\
\hline $\begin{array}{l}\text { 691. Henggeler, S. W., Letourneau, E. J., Chapman, J. E., Borduin, C. } \\
\text { M., Schewe, P. A., \& McCart, M. R. (2009). Mediators of change for } \\
\text { multisystemic therapy with juvenile sexual offenders. J Consult Clin } \\
\text { Psychol, 77(3), 451-462. doi: 2009-08093-008 }\end{array}$ & $\begin{array}{l}\text { Follow-up less } \\
\text { than } 6 \text { months }\end{array}$ \\
\hline
\end{tabular}




\begin{tabular}{|c|c|}
\hline $\begin{array}{l}\text { 692. Henin A, Biederman J, Mick E, Sachs GS, Hirshfeld-Becker DR, } \\
\text { Siegel RS, McMurrich S, Grandin L and Nierenberg AA. } 2005 \text {. } \\
\text { Psychopathology in the offspring of parents with bipolar disorder: a } \\
\text { controlled study. Biol Psychiatry. 58(7):554-61. }\end{array}$ & $\begin{array}{l}\text { Not a parenting } \\
\text { intervention }\end{array}$ \\
\hline $\begin{array}{l}\text { 693. Hensel T. 2006. Effectiveness of EMDR with psychologically } \\
\text { traumatized children and adolescents. Kindheit und Entwicklung. } \\
\text { 15(2):107-17. }\end{array}$ & Review \\
\hline $\begin{array}{l}\text { 694. Herbert JD, Gaudiano BA, Rheingold AA, Moitra E, Myers VH, } \\
\text { Dalrymple KL and Brandsma LL. 2009. Cognitive behavior therapy for } \\
\text { generalized social anxiety disorder in adolescents: A randomized } \\
\text { controlled trial. Journal of Anxiety Disorders. 23(2):167-77. }\end{array}$ & $\begin{array}{l}\text { Not a parenting } \\
\text { intervention }\end{array}$ \\
\hline $\begin{array}{l}\text { 695. Herbert, S. D. (2014). Parent training for families of } \\
\text { hyperactive preschool-aged children. Dissertation Abstracts } \\
\text { International: Section B: The Sciences and Engineering, } 75 \text { (3-B E), }\end{array}$ & $\begin{array}{l}\text { Targets } \\
\text { externalising; No } \\
\text { long term } \\
\text { follow-up }\end{array}$ \\
\hline $\begin{array}{l}\text { 696. Herbert, S. D., Harvey, E. A., Roberts, J. L., Wichowski, K., \& } \\
\text { Lugo-Candelas, C. I. (2013). A randomized controlled trial of a parent } \\
\text { training and emotion socialization program for families of hyperactive } \\
\text { preschool-aged children. Behavior therapy, } 44(2), 302-316 . \\
\text { doi:10.1016/j.beth.2012.10.004 }\end{array}$ & $\begin{array}{l}\text { Targets } \\
\text { externalising }\end{array}$ \\
\hline $\begin{array}{l}\text { 697. Heriot, S. A., Evans, I. M., \& Foster, T. M. (2008). Critical } \\
\text { influences affecting response to various treatments in young children } \\
\text { with ADHD: A case series. Child: Care, Health and Development, 34(1), } \\
\text { 121-133. }\end{array}$ & $\begin{array}{l}\text { No child } \\
\text { internalising } \\
\text { outcomes or not } \\
\text { a major goal }\end{array}$ \\
\hline $\begin{array}{l}\text { 698. Herman, K. C., Borden, L. A., Reinke, W. M., \& Webster- } \\
\text { Stratton, C. (2011). The impact of the Incredible Years parent, child, } \\
\text { and teacher training programs on children's co-occurring internalizing } \\
\text { symptoms. School Psychology Quarterly, 26, 189-201. }\end{array}$ & $\begin{array}{l}\text { Follow-up data } \\
\text { collected only in } \\
\text { active group }\end{array}$ \\
\hline $\begin{array}{l}\text { 699. Hermanns, J. M. A., Asscher, J. J., Zijlstra, B.J.H., Hoffenaar, } \\
\text { P.J., Dekovic, M. (2013). Long-term changes in parenting and child } \\
\text { behavior after the Home-Start family support program. Children and } \\
\text { Youth Services Review, } 35 \text { (4), 678-684. }\end{array}$ & Not an RCT \\
\hline $\begin{array}{l}\text { 700. Herman-Smith R, Pearson B, Cordiano TS and Aguirre- } \\
\text { McLaughlin A. 2008. Addressing individual client needs in manualized } \\
\text { treatment: Case comparisons. Clinical Case Studies. 7(5):377-96. }\end{array}$ & Not an RCT \\
\hline $\begin{array}{l}\text { 701. Hernandez A, Ruble C, Rockmore L, McKay M, Messam T, } \\
\text { Harris M and Hope S. 2009. An integrated approach to treating non- } \\
\text { offending parents affected by sexual abuse. Social Work in Mental } \\
\text { Health. 7(6):533-55. }\end{array}$ & Not an RCT \\
\hline $\begin{array}{l}\text { 702. Hernes, S., Haugen, M., \& Overby, N. (2013). First food for } \\
\text { infants. [Conference Abstract]. Annals of Nutrition and Metabolism, } \\
\text { 63, 326-327. doi: http://dx.doi.org/10.1159/000354245 }\end{array}$ & $\begin{array}{l}\text { No child } \\
\text { internalising } \\
\text { outcomes or not } \\
\text { a major goal }\end{array}$ \\
\hline $\begin{array}{l}\text { 703. Heyne, D., King, N. J., Tonge, B. J., Rollings, S., Young, D., } \\
\text { Pritchard, M., et al. (2002). Evaluation of child therapy and caregiver } \\
\text { training in the treatment of school refusal. J Am Acad Child Adolesc } \\
\text { Psychiatry, 41(6), 687-695. doi: S0890-8567(09)61023-6 }\end{array}$ & $\begin{array}{l}\text { Follow-up less } \\
\text { than } 6 \text { months }\end{array}$ \\
\hline
\end{tabular}




\begin{tabular}{|c|c|}
\hline $\begin{array}{l}704 . \quad \text { Hiebert-Murphy, D., Williams, E. A., Mills, R. S. L., Walker, J. R., } \\
\text { Feldgaier, S., Warren, M., Freeman, W., McIntyre, M., \& Cox, B. J. } \\
\text { (2012). Listening to parents: The challenges of parenting kindergarten- } \\
\text { aged children who are anxious. Clinical Child Psychology and } \\
\text { Psychiatry, 17, 384-399. }\end{array}$ & $\begin{array}{l}\text { Not a parenting } \\
\text { intervention }\end{array}$ \\
\hline $\begin{array}{l}\text { 705. Hilt LM, McLaughlin KA and Nolen-Hoeksema S. } 2010 . \\
\text { Examination of the Response Styles Theory in a community sample of } \\
\text { young adolescents. Journal of Abnormal Child Psychology: An official } \\
\text { publication of the International Society for Research in Child and } \\
\text { Adolescent Psychopathology. 38(4):545-56. }\end{array}$ & $\begin{array}{l}\text { Not a parenting } \\
\text { intervention }\end{array}$ \\
\hline $\begin{array}{l}\text { 706. Hinshaw, S. P., Owens, E. B., Wells, K. C., Kraemer, H. C., } \\
\text { Abikoff, H. B., Arnold, L. E., et al. (2000). Family processes and } \\
\text { treatment outcome in the MTA: negative/ineffective parenting } \\
\text { practices in relation to multimodal treatment. J Abnorm Child Psychol, } \\
\text { 28(6), 555-568. }\end{array}$ & $\begin{array}{l}\text { No long term } \\
\text { follow-up }\end{array}$ \\
\hline $\begin{array}{l}\text { 707. Hintikka U, Viinamäki H, Pelkonen M, Hintikka J, Laukkanen E, } \\
\text { Korhonen V and Lehtonen J. 2003. Clinical recovery in cognitive } \\
\text { functioning and self-image among adolescents with major depressive } \\
\text { disorder and conduct disorder during psychiatric inpatient care. } \\
\text { American Journal of Orthopsychiatry. } 73(2): 212-22 \text {. }\end{array}$ & $\begin{array}{l}\text { Not a parenting } \\
\text { intervention }\end{array}$ \\
\hline $\begin{array}{l}\text { 708. Hipke, K., Wolchik, S. A., Sandler, I. N., \& Braver, S. L. (2002). } \\
\text { Predictors of children's intervention-induced resilience in a parenting } \\
\text { program for divorced mothers. Family Relations: An Interdisciplinary } \\
\text { Journal of Applied Family Studies, 51(2), 121-129. doi: 10.1111/j.1741- } \\
\text { 3729.2002.00121.x }\end{array}$ & $\begin{array}{l}\text { Secondary } \\
\text { analysis (re- } \\
\text { analysis of data } \\
\text { from Wolchik et } \\
\text { al 2000, } \\
\text { predictors of } \\
\text { improvement, } \\
\text { data not } \\
\text { useable) }\end{array}$ \\
\hline $\begin{array}{l}\text { 709. Hirshfeld-Becker DR, Masek B, Henin A, Blakely LR, Rettew DC, } \\
\text { Dufton L, Segool N and Biederman J. 2008. Cognitive-behavioral } \\
\text { intervention with young anxious children. Harvard Review of } \\
\text { Psychiatry. 16(2):113-25. }\end{array}$ & Not an RCT \\
\hline $\begin{array}{l}\text { 710. Hirshfeld-Becker, D. R., Masek, B., Henin, A., Blakely, L. R., } \\
\text { Pollock-Wurman, R. A., McQuade, J., et al. (2010). Cognitive behavioral } \\
\text { therapy for 4- to 7-year-old children with anxiety disorders: A } \\
\text { randomized clinical trial. Journal of Consulting and Clinical Psychology, } \\
\text { 78(4), 498-510. doi: 10.1037/a0019055 }\end{array}$ & $\begin{array}{l}\text { Follow-up data } \\
\text { collected only in } \\
\text { active group }\end{array}$ \\
\hline $\begin{array}{l}\text { 711. Hiscock H, Bayer JK, Hampton A, Ukoumunne OC, Wake M. } \\
\text { Long-term mother and child mental health effects of a population- } \\
\text { based infant sleep intervention: cluster-randomized, controlled trial. } \\
\text { Pediatrics. 2008 Sep;122(3):e621-7. }\end{array}$ & $\begin{array}{l}\text { No child } \\
\text { internalising } \\
\text { outcomes or not } \\
\text { a major goal }\end{array}$ \\
\hline $\begin{array}{l}\text { 712. Hiscock, H., \& Wake, M. (2002). Randomised controlled trial of } \\
\text { behavioural infant sleep intervention to improve infant sleep and } \\
\text { maternal mood. BMJ, 324, 1062. }\end{array}$ & $\begin{array}{l}\text { No child } \\
\text { internalising } \\
\text { outcomes or not } \\
\text { a major goal }\end{array}$ \\
\hline $\begin{array}{l}\text { 713. Hiscock, H., Bayer, J. K., Lycett, K., Ukoumunne, O. C., Shaw, } \\
\text { D., Gold, L., ... Wake, M. (2012). Preventing mental health problems } \\
\text { in children: the Families in Mind population-based cluster randomised } \\
\text { controlled trial. BMC public health, } 12,420 .\end{array}$ & $\begin{array}{l}\text { Data not } \\
\text { available/report } \\
\text { ed (protocol) }\end{array}$ \\
\hline
\end{tabular}




\begin{tabular}{|c|c|}
\hline $\begin{array}{l}\text { 714. Hiscock, H., Bayer, J. K., Price, A., Ukoumunne, O. C., Rogers, } \\
\text { S., \& Wake, M. (2008). Universal parenting programme to prevent } \\
\text { early childhood behavioural problems: Cluster randomised trial. BMJ: } \\
\text { British Medical Journal, 336(7639), 318-321. doi: } \\
\text { 10.1136/bmj.39451.609676.AE }\end{array}$ & $\begin{array}{l}\text { Targets } \\
\text { externalising }\end{array}$ \\
\hline $\begin{array}{l}\text { 715. Hiscock, H., Cook, F., Bayer, J., Le, H. N., Mensah, F., Cann, } \\
\text { W., ... St James-Roberts, I. (2014). Preventing early infant sleep and } \\
\text { crying problems and postnatal depression: a randomized trial. } \\
\text { Pediatrics, 133(2), e346-354. doi: 10.1542/peds.2013-1886 }\end{array}$ & $\begin{array}{l}\text { No child } \\
\text { internalising } \\
\text { outcomes or not } \\
\text { a major goal }\end{array}$ \\
\hline $\begin{array}{l}\text { 716. Ho TP, Chow V, Fung C, Leung K, Chiu KY, Yu G, Au YW and } \\
\text { Lieh-Mak F. 1999. Parent management training in a Chinese } \\
\text { population: application and outcome. J Am Acad Child Adolesc } \\
\text { Psychiatry. 38(9):1165-72. }\end{array}$ & Not an RCT \\
\hline $\begin{array}{l}\text { 717. Ho, J., Yeh, M., McCabe, K., \& Lau, A. (2012). Perceptions of } \\
\text { the acceptability of parent training among Chinese immigrant parents: } \\
\text { Contributions of cultural factors and clinical need. Behav Ther, 43, } \\
436-449 \text {. }\end{array}$ & $\begin{array}{l}\text { Not a parenting } \\
\text { intervention }\end{array}$ \\
\hline $\begin{array}{l}\text { 718. Hoath FE and Sanders MR. 2002. A feasibility study of } \\
\text { Enhanced Group Triple P - Positive parenting program for parents of } \\
\text { children with attention-deficit/hyperactivity disorder. Behaviour } \\
\text { Change. 19(4):191-206. }\end{array}$ & $\begin{array}{l}\text { Follow-up less } \\
\text { than } 6 \text { months }\end{array}$ \\
\hline $\begin{array}{l}\text { 719. Hodes, M. W., Kef, S., Meppelder, H. M., \& Schuengel, C. } \\
\text { (2012). The effect of VIPP-LD intervention with parents with } \\
\text { intellectual disabilities on their children's behaviour. Journal of } \\
\text { Intellectual Disability Research, 56, 743. }\end{array}$ & $\begin{array}{l}\text { No long term } \\
\text { follow-up }\end{array}$ \\
\hline $\begin{array}{l}720 . \quad \text { Hodgetts, S., Savage, A., \& McConnell, D. (2013). Experience } \\
\text { and outcomes of stepping stones triple P for families of children with } \\
\text { autism. Res Dev Disabil, 34(9), 2572-2585. doi: } \\
\text { 10.1016/j.ridd.2013.05.005 }\end{array}$ & Not an RCT \\
\hline $\begin{array}{l}\text { 721. Hodson KJ, McManus FV, Clark DM and Doll H. 2008. Can Clark } \\
\text { and Wells' (1995) cognitive model of social phobia be applied to young } \\
\text { people. Behavioural and Cognitive Psychotherapy. 36(4):449-61. }\end{array}$ & $\begin{array}{l}\text { Not a parenting } \\
\text { intervention }\end{array}$ \\
\hline $\begin{array}{l}\text { 722. Hoekstra-Weebers JEHM, Heuvel F, Jaspers JPC, Kamps WA } \\
\text { and Klip EC. 1998. Brief report: An intervention program for parents of } \\
\text { pediatric cancer patients: A randomized controlled trial. Journal of } \\
\text { Pediatric Psychology. 23(3):207-14. }\end{array}$ & $\begin{array}{l}\text { No child } \\
\text { internalising } \\
\text { outcomes or not } \\
\text { a major goal }\end{array}$ \\
\hline $\begin{array}{l}\text { 723. Hogue A, Liddle HA, Dauber S and Samuolis J. 2004. Linking } \\
\text { Session Focus to Treatment Outcome in Evidence-Based Treatments } \\
\text { for Adolescent Substance Abuse. Psychotherapy: Theory, Research, } \\
\text { Practice, Training. 41(2):83-96. }\end{array}$ & Not an RCT \\
\hline $\begin{array}{l}\text { 724. Hoivik, M. S., Lydersen, S., Drugli, M. B., Onsoien, R., Hansen, } \\
\text { M. B., Nielsen,T. S. B. (2015). Video feedback compared to treatment } \\
\text { as usual in families with parent-child interactions problems: A } \\
\text { randomized controlled trial. Child and adolescent psychiatry and } \\
\text { mental health, } 9 \text { (1), }\end{array}$ & $\begin{array}{l}\text { No child } \\
\text { internalising } \\
\text { outcomes or not } \\
\text { a major goal }\end{array}$ \\
\hline $\begin{array}{l}\text { 725. Holden, G. W., Lavigne, V. V., \& Cameron, A. M. (1990). } \\
\text { Probing the Continuum of Effectiveness in Parent Training: } \\
\text { Characteristics of Parents and Preschoolers. Journal of Clinical Child } \\
\text { Psychology, 19, } 2 \text {. }\end{array}$ & Not an RCT \\
\hline
\end{tabular}




\begin{tabular}{|c|c|}
\hline $\begin{array}{l}\text { 726. Holditch-Davis, D., White-Traut, R. C., Levy, J. A., O'Shea, T. M., } \\
\text { Geraldo, V., \& David, R. J. (2014). Maternally administered } \\
\text { interventions for preterm infants in the NICU: Effects on maternal } \\
\text { psychological distress and mother-infant relationship. Infant Behavior } \\
\text { and Development, 37(4), 695-710. }\end{array}$ & $\begin{array}{l}\text { No child } \\
\text { internalising } \\
\text { outcomes or not } \\
\text { a major goal }\end{array}$ \\
\hline $\begin{array}{l}\text { 727. Hood, K. K., \& Eyberg, S. M. (2003). Outcomes of Parent-Child } \\
\text { Interaction Therapy: Mothers' Reports of Maintenance Three to Six } \\
\text { Years After Treatment. [Article]. Journal of Clinical Child \& Adolescent } \\
\text { Psychology, 32(3), 419. }\end{array}$ & $\begin{array}{l}\text { Follow-up data } \\
\text { collected only in } \\
\text { active group }\end{array}$ \\
\hline $\begin{array}{l}\text { 728. Hoover HVA. Teachers' ratings of children's behavior } \\
\text { problems: A method for potentially adjusting for rater effects. } \\
\text { Dissertation Abstracts International: Section B: The Sciences and } \\
\text { Engineering. [Dissertation Abstract; Dissertation]. 2001;62(5-B). }\end{array}$ & $\begin{array}{l}\text { Follow-up less } \\
\text { than } 6 \text { months }\end{array}$ \\
\hline $\begin{array}{l}\text { 729. Horn KA, Branstetter SA, Dino GA, Jarrett TD, Tworek C and } \\
\text { Zhang J. 2009. Potential effects of active parental consent: Enrolling } \\
\text { teen smokers into a school-based cessation program. Nicotine \& } \\
\text { Tobacco Research. 11(11):1359-67. }\end{array}$ & $\begin{array}{l}\text { Not a parenting } \\
\text { intervention }\end{array}$ \\
\hline $\begin{array}{l}730 . \quad \text { Horn, W. F., lalongo, N. S., Pascoe, J. M., Greenberg, G., } \\
\text { Packard, T., Lopez, M., . . Puttler, L. (1991). Additive Effects of } \\
\text { Psychostimulants, Parent Training, and Self-Control Therapy with } \\
\text { ADHD Children. [doi: 10.1097/00004583-199103000-00011]. Journal } \\
\text { of the American Academy of Child \& Adolescent Psychiatry, 30(2), 233- } \\
240 .\end{array}$ & $\begin{array}{l}\text { No long term } \\
\text { follow-up }\end{array}$ \\
\hline $\begin{array}{l}\text { 731. Horne, A. M., \& Vandyke, B. (1983). Treatment and } \\
\text { Maintenance of Social-Learning Family-Therapy. Behavior Therapy, } \\
\text { 14(5), 606-613. }\end{array}$ & Not an RCT \\
\hline $\begin{array}{l}\text { 732. Horowitz JL, Garber J, Ciesla JA, Young JF and Mufson L. } 2007 . \\
\text { Prevention of depressive symptoms in adolescents: A randomized trial } \\
\text { of cognitive-behavioral and interpersonal prevention programs. } \\
\text { Journal of Consulting and Clinical Psychology. 75(5):693-706. }\end{array}$ & $\begin{array}{l}\text { Not a parenting } \\
\text { intervention }\end{array}$ \\
\hline $\begin{array}{l}\text { 733. Horowitz, J. A., Bell, M., Trybulski, J., Munro, B. H., Moser, D., } \\
\text { Hartz, S. A., et al. (2001). Promoting responsiveness between mothers } \\
\text { with depressive symptoms and their infants. J Nurs Scholarsh, 33(4), } \\
\text { 323-329. }\end{array}$ & $\begin{array}{l}\text { No long term } \\
\text { follow-up }\end{array}$ \\
\hline $\begin{array}{l}\text { 734. Hourihan F and Hoban D. 2004. Learning, enjoying, growing, } \\
\text { support model: An innovative collaborative approach to the } \\
\text { prevention of conduct disorder in preschoolers in hard to reach rural } \\
\text { families. Australian Journal of Rural Health. } 12(6): 269-76 \text {. }\end{array}$ & Not an RCT \\
\hline $\begin{array}{l}\text { 735. Houtzager BA, Hogendoorn SM, Papatsonis DN, Samsom JF, } \\
\text { van Geijn HP, Bleker OP and van Wassenaer AG. 2006. Long-term } \\
\text { follow up of children exposed in utero to nifedipine or ritodrine for the } \\
\text { management of preterm labour. BJOG: An International Journal of } \\
\text { Obstetrics \& Gynaecology, 113(3), 324-331. }\end{array}$ & $\begin{array}{l}\text { Not a parenting } \\
\text { intervention }\end{array}$ \\
\hline $\begin{array}{l}\text { 736. Howard, A. J., Mallan, K. M., Byrne, R., Magarey, A., \& Daniels, } \\
\text { L. A. (2012). Toddlers' food preferences. The impact of novel food } \\
\text { exposure, maternal preferences and food neophobia. Appetite, 59(3), } \\
\text { 818-825. doi: } 10.1016 / \text { j.appet.2012.08.022 }\end{array}$ & $\begin{array}{l}\text { No child } \\
\text { internalising } \\
\text { outcomes or not } \\
\text { a major goal }\end{array}$ \\
\hline
\end{tabular}




\begin{tabular}{|c|c|}
\hline $\begin{array}{l}\text { 737. Huang MC, Liu CC and Huang CC. 1998. Effects of an } \\
\text { educational program on parents with febrile convulsive children. } \\
\text { Pediatr Neurol. 18(2):150-5. }\end{array}$ & $\begin{array}{l}\text { Intervention for } \\
\text { developmental } \\
\text { disorders } \\
\text { (autism, autism } \\
\text { spectrum } \\
\text { disorder), } \\
\text { physical } \\
\text { disabilities, } \\
\text { medical } \\
\text { conditions or } \\
\text { distress about } \\
\text { facing medical } \\
\text { interventions }\end{array}$ \\
\hline $\begin{array}{l}738 . \quad \text { Huang, J., Sherraden, M., \& Purnell, J. Q. (2014). Impacts of } \\
\text { Child Development Accounts on maternal depressive symptoms: } \\
\text { Evidence from a randomized statewide policy experiment. Social } \\
\text { Science \& Medicine, 112, 30-38. }\end{array}$ & $\begin{array}{l}\text { Not a parenting } \\
\text { intervention }\end{array}$ \\
\hline $\begin{array}{l}\text { 739. Hudson JL, Rapee RM, Deveney C, Schniering CA, Lyneham HJ } \\
\text { and Bovopoulos N. 2009. Cognitive-behavioral treatment versus an } \\
\text { active control for children and adolescents with anxiety disorders: a } \\
\text { randomized trial. J Am Acad Child Adolesc Psychiatry. 48(5):533-44. }\end{array}$ & $\begin{array}{l}\text { Not a parenting } \\
\text { intervention }\end{array}$ \\
\hline $\begin{array}{l}740 . \quad \text { Hudson, J. L., Newall, C., Rapee, R. M., Lyneham, H. J., } \\
\text { Schniering, C. C., Wuthrich, V. M., Schneider, S., Seeley-Wait, E., } \\
\text { Edwards, S., Gar, N. S. (2014). The Impact of Brief Parental Anxiety } \\
\text { Management on Child Anxiety Treatment Outcomes: A Controlled } \\
\text { Trial. Journal of clinical child and adolescent psychology, } 43 \text { (3), 370- } \\
\text { 80. }\end{array}$ & $\begin{array}{l}\text { Comparison } \\
\text { condition not a } \\
\text { minimal control; } \\
\text { Parent } \\
\text { component too } \\
\text { small }\end{array}$ \\
\hline $\begin{array}{l}\text { 741. Huesmann, L. R., Maxwell, C. D., Eron, L., Dahlberg, L. L., } \\
\text { Guerra, N. G., Tolan, P. H., et al. (1996). Evaluating a } \\
\text { cognitive/ecological program for the prevention of aggression among } \\
\text { urban children. American journal of preventive medicine, (5 Suppl), } \\
120-128 .\end{array}$ & $\begin{array}{l}\text { Parent } \\
\text { component too } \\
\text { small }\end{array}$ \\
\hline $\begin{array}{l}\text { 742. Huey S, Jr. and Pan D. 2006. Culture-responsive one-session } \\
\text { treatment for phobic Asian Americans: A pilot study. Psychotherapy: } \\
\text { Theory, Research, Practice, Training. Special Issue: Culture, Race, and } \\
\text { Ethnicity in Psychotherapy. 43(4):549-54. }\end{array}$ & $\begin{array}{l}\text { Not a parenting } \\
\text { intervention }\end{array}$ \\
\hline $\begin{array}{l}\text { 743. Huey, S. J., Jr., \& Polo, A. J. (2008). Evidence-based } \\
\text { psychosocial treatments for ethnic minority youth. Journal Of Clinical } \\
\text { Child And Adolescent Psychology, 37, 262-301. }\end{array}$ & Review \\
\hline $\begin{array}{l}\text { 744. Huggenberger, H., Roth, B., Gaab, J., Woessmer, B., Rochlitz, } \\
\text { C., \& Alder, J. (2014). Web-based counseling for families with parental } \\
\text { cancer: First data on effectiveness for children's psychosocial well- } \\
\text { being. Psycho-oncology, } 23 \text { 104-5. }\end{array}$ & $\begin{array}{l}\text { Data not } \\
\text { available/report } \\
\text { ed }\end{array}$ \\
\hline $\begin{array}{l}\text { 745. Huggenberger, H., Roth, B., Gaab, J., Wossmer, B., Rochlitz, C., } \\
\text { \& Alder, J. (2013). Famoca: Family online counseling for families with } \\
\text { parental cancer. [Conference Abstract]. Psycho-Oncology, 22, 256-257. } \\
\text { doi: http://dx.doi.org/10.1111/j.1099-1611.2013.3394 }\end{array}$ & $\begin{array}{l}\text { Not a parenting } \\
\text { intervention }\end{array}$ \\
\hline $\begin{array}{l}\text { 746. Hughes, E. K., Le Grange, D., Yeo, M. S., Campbell, S., Allan, E., } \\
\text { Crosby, R. D., ... \& Sawyer, S. M. (2014). Parent-focused treatment for } \\
\text { adolescent anorexia nervosa: a study protocol of a randomised } \\
\text { controlled trial. BMC psychiatry, 14(1), } 105 .\end{array}$ & $\begin{array}{l}\text { Data not } \\
\text { available/report } \\
\text { ed }\end{array}$ \\
\hline
\end{tabular}




\begin{tabular}{|c|c|}
\hline $\begin{array}{l}\text { 747. Huizenga, L. L. (2012). Expressive writing intervention for } \\
\text { teens whose parents have cancer. Dissertation Abstracts International: } \\
\text { Section B: The Sciences and Engineering, } 72 \text { (12-B). }\end{array}$ & $\begin{array}{l}\text { Not a parenting } \\
\text { intervention }\end{array}$ \\
\hline $\begin{array}{l}\text { 748. Hundert, J., Boyle, M. H., Cunningham, C. E., Duku, E., Heale, } \\
\text { J., McDonald, J., Offord, D. R., \& Racine, Y. (1999). Helping children } \\
\text { adjust-a tri-ministry study: Il. Program effects. Journal of Child } \\
\text { Psychology and Psychiatry and Allied Disciplines, 40, 1061-1073. }\end{array}$ & $\begin{array}{l}\text { Not a parenting } \\
\text { intervention }\end{array}$ \\
\hline $\begin{array}{l}\text { 749. Hunt C. 2007. The effect of an education program on attitudes } \\
\text { and beliefs about bullying and bullying behaviour in junior secondary } \\
\text { school students. Child and Adolescent Mental Health, 12(1), 21-26. }\end{array}$ & $\begin{array}{l}\text { Not a parenting } \\
\text { intervention }\end{array}$ \\
\hline $\begin{array}{l}\text { 750. Hunter SM, Johnson CC, Little-Christian S, Nicklas TA, Harsha } \\
\text { D, Arbeit ML, Webber LS and Berenson GS. 1990. Heart Smart: a } \\
\text { multifaceted cardiovascular risk reduction program for grade school } \\
\text { students. American Journal of Health Promotion, 4(5), 352-360. }\end{array}$ & $\begin{array}{l}\text { No child } \\
\text { internalising } \\
\text { outcomes or not } \\
\text { a major goal }\end{array}$ \\
\hline $\begin{array}{l}\text { 751. Hutchings, J., Bywater, T., Williams, M. E., Lane, E., \& } \\
\text { Whitaker, C. J. (2012). Improvements in maternal depression as a } \\
\text { mediator of child behaviour change. Psychology, 3(9A), 795-801. doi: } \\
\text { 10.4236/psych.2012.329120 }\end{array}$ & $\begin{array}{l}\text { Targets } \\
\text { externalising }\end{array}$ \\
\hline $\begin{array}{l}\text { 752. Hutchings, J., Gardner, F., Bywater, T., Daley, D., Whitaker, C., } \\
\text { Jones, K., et al. (2007). Parenting intervention in Sure Start services for } \\
\text { children at risk of developing conduct disorder: pragmatic randomised } \\
\text { controlled trial. BMJ, 334(7595), 678. doi: bmj.39126.620799.55 }\end{array}$ & $\begin{array}{l}\text { No child } \\
\text { internalising } \\
\text { outcomes or not } \\
\text { a major goal }\end{array}$ \\
\hline $\begin{array}{l}\text { 753. Hutchings, J., Lane, E., \& Kelly, J. (2004). Comparison of two } \\
\text { treatments for children with severely disruptive behaviours: A four- } \\
\text { year follow-up. Behavioural and Cognitive Psychotherapy, 32, 15-30. }\end{array}$ & $\begin{array}{l}\text { Targets } \\
\text { externalising }\end{array}$ \\
\hline $\begin{array}{l}\text { 754. Hwang W-C, Wood JJ and Fujimoto K. 2010. Acculturative } \\
\text { family distancing (AFD) and depression in Chinese American families. } \\
\text { Journal of Consulting and Clinical Psychology. 78(5):655-67. }\end{array}$ & $\begin{array}{l}\text { Not a parenting } \\
\text { intervention }\end{array}$ \\
\hline $\begin{array}{l}755 . \quad \text { Ialongo, N. S., Horn, W. F., Pascoe, J. M., Greenberg, G., } \\
\text { Packard, T., Lopez, M., et al. (1993). The effects of a multimodal } \\
\text { intervention with attention-deficit hyperactivity disorder children: a 9- } \\
\text { month follow-up. Journal of the American Academy of Child and } \\
\text { Adolescent Psychiatry, (1), 182-189. }\end{array}$ & $\begin{array}{l}\text { Parent } \\
\text { component too } \\
\text { small }\end{array}$ \\
\hline $\begin{array}{l}\text { 756. lalongo, N. S., Werthamer, L., Kellam, S. G., Brown, C. H., } \\
\text { Wang, S., \& Lin, Y. (1999). Proximal impact of two first-grade } \\
\text { preventive interventions on the early risk behaviors for later } \\
\text { substance abuse, depression, and antisocial behavior. Am J } \\
\text { Community Psychol, 27(5), 599-641. }\end{array}$ & $\begin{array}{l}\text { No child } \\
\text { internalising } \\
\text { outcomes or not } \\
\text { a major goal }\end{array}$ \\
\hline $\begin{array}{l}\text { 757. IHDP. (1990). Enhancing the outcomes of low-birth-weight, } \\
\text { premature infants: A multisite, randomized trial. JAMA, 263, 3035- } \\
\text { 3042. }\end{array}$ & $\begin{array}{l}\text { Parent } \\
\text { component too } \\
\text { small; No long } \\
\text { term follow-up }\end{array}$ \\
\hline $\begin{array}{l}\text { 758. Ihle W and Jahnke Dr. 2005.The efficacy of family involvement } \\
\text { in the treatment of anxiety disorders and depressive disorders in } \\
\text { childhood and adolescence: Current state of evidence-based } \\
\text { psychotherapy. Kindheit und Entwicklung. 14(1):12-20. }\end{array}$ & Review \\
\hline $\begin{array}{l}\text { 759. Iloabachie, C., Wells, C., Goodwin, B., Baldwin, M., } \\
\text { Vanderplough-Booth, K., Gladstone, T., Murray, M., Fogel, J., \& Van } \\
\text { Voorhees, B. W. (2011). Adolescent and parent experiences with a } \\
\text { primary care/Internet-based depression prevention intervention } \\
\text { (CATCH-IT). Gen Hosp Psychiatry, 33, 543-555. }\end{array}$ & $\begin{array}{l}\text { Not a parenting } \\
\text { intervention }\end{array}$ \\
\hline
\end{tabular}




\begin{tabular}{|c|c|}
\hline $\begin{array}{l}\text { 760. In-Albon T and Schneider S. 2006. Psychotherapy of Childhood } \\
\text { Anxiety Disorders: A Meta-Analysis. Psychotherapy and } \\
\text { Psychosomatics. 76(1):15-24. }\end{array}$ & $\begin{array}{l}\text { Not a parenting } \\
\text { intervention }\end{array}$ \\
\hline $\begin{array}{l}\text { 761. Indrio, F., Di Mauro, A., Riezzo, G., Civardi, E., Intini, C., } \\
\text { Corvaglia, L., ... \& Francavilla, R. (2014). Prophylactic use of a probiotic } \\
\text { in the prevention of colic, regurgitation, and functional constipation: a } \\
\text { randomized clinical trial. JAMA pediatrics, 168(3), 228-233. }\end{array}$ & $\begin{array}{l}\text { Not a parenting } \\
\text { intervention }\end{array}$ \\
\hline $\begin{array}{l}\text { 762. Ingadottir E and Thome M. 2006. Evaluation of a web-based } \\
\text { course for community nurses on postpartum emotional distress. Scand } \\
\text { J Caring Sci. 20(1):86-92. }\end{array}$ & $\begin{array}{l}\text { No child } \\
\text { internalising } \\
\text { outcomes or not } \\
\text { a major goal }\end{array}$ \\
\hline $\begin{array}{l}\text { 763. lobst EA, Alderfer MA, Sahler OJ, Askins MA, Fairclough DL, } \\
\text { Katz ER, Butler RW, Dolgin MJ and Noll RB. 2009. Problem solving and } \\
\text { maternal distress at the time of a child's diagnosis of cancer in two- } \\
\text { parent versus lone-parent households. J Pediatr Psychol. 34(8):817-21. }\end{array}$ & $\begin{array}{l}\text { Not a parenting } \\
\text { intervention }\end{array}$ \\
\hline $\begin{array}{l}\text { 764. Ireys HT, Chernoff R, DeVet KA and Kim Y. 2001. Maternal } \\
\text { outcomes of a randomized controlled trial of a community-based } \\
\text { support program for families of children with chronic illnesses. Arch } \\
\text { Pediatr Adolesc Med. 155(7):771-7. }\end{array}$ & $\begin{array}{l}\text { No child } \\
\text { internalising } \\
\text { outcomes or not } \\
\text { a major goal }\end{array}$ \\
\hline $\begin{array}{l}\text { 765. Ireys HT, Sills EM, Kolodner KB and Walsh BB. 1996. A social } \\
\text { support intervention for parents of children with juvenile rheumatoid } \\
\text { arthritis: results of a randomized trial. Journal of Pediatric Psychology, } \\
\text { 21(5), 633-641. }\end{array}$ & $\begin{array}{l}\text { No child } \\
\text { internalising } \\
\text { outcomes or not } \\
\text { a major goal }\end{array}$ \\
\hline $\begin{array}{l}\text { 766. Irvine, A. B., Biglan, A., Smolkowski, K., Metzler, C. W., \& Ary, } \\
\text { D. V. (1999). The effectiveness of a parenting skills program for } \\
\text { parents of middle school students in small communities. Journal of } \\
\text { consulting and clinical psychology, }(6), 811-825 \text {. }\end{array}$ & $\begin{array}{l}\text { Targets } \\
\text { externalising }\end{array}$ \\
\hline $\begin{array}{l}\text { 767. Ise, E., Kierfeld, F., \& Döpfner, M. (2015). One-Year Follow-Up } \\
\text { of Guided Self-Help for Parents of Preschool Children With } \\
\text { Externalizing Behavior. The journal of primary prevention, 36(1), 33- } \\
40 .\end{array}$ & $\begin{array}{l}\text { Targets } \\
\text { externalising }\end{array}$ \\
\hline $\begin{array}{l}\text { 768. Isler L. Effects of gender on treatment outcome in young } \\
\text { children with ADHD. Dissertation Abstracts International: Section B: } \\
\text { The Sciences and Engineering. [Dissertation Abstract; Dissertation]. } \\
\text { 2008;69(1-B):659. }\end{array}$ & $\begin{array}{l}\text { Comparison } \\
\text { condition not a } \\
\text { minimal control }\end{array}$ \\
\hline $\begin{array}{l}\text { 769. J. Powell;R. Fraser;J. A. Brockway;N. Temkin;K. Bell, (2014). } \\
\text { Improving quality-of-life and emotional well-being for caregivers of } \\
\text { persons with traumatic brain injury: A randomized controlled trial. } \\
\text { Brain injury, } 28(5-6), 798 .\end{array}$ & $\begin{array}{l}\text { Not a parenting } \\
\text { intervention }\end{array}$ \\
\hline $\begin{array}{l}\text { 770. Jackson, B., \& Goodman, E. (2011). Low social status markers: } \\
\text { Do they predict depressive symptoms in adolescence? Race and Social } \\
\text { Problems, 3, 119-128. }\end{array}$ & $\begin{array}{l}\text { Not a parenting } \\
\text { intervention }\end{array}$ \\
\hline $\begin{array}{l}\text { 771. Jackson, C., Cheater, F. M., Harrison, W., Peacock, R., Bekker, } \\
\text { H., West, R., \& Leese, B. (2011). Randomised cluster trial to support } \\
\text { informed parental decision-making for the MMR vaccine. BMC public } \\
\text { health, } 11(1), 475 .\end{array}$ & $\begin{array}{l}\text { No child } \\
\text { internalising } \\
\text { outcomes or not } \\
\text { a major goal }\end{array}$ \\
\hline $\begin{array}{l}\text { 772. Jacobs AK, Roberts MC, Vernberg EM, Nyre JE, Randall CJ and } \\
\text { Puddy RW. 2008. Factors related to outcome in a school-based } \\
\text { intensive mental health program: An examination of nonresponders. } \\
\text { Journal of Child and Family Studies. 17(2):219-31. }\end{array}$ & $\begin{array}{l}\text { Not a parenting } \\
\text { intervention }\end{array}$ \\
\hline
\end{tabular}




\begin{tabular}{|c|c|}
\hline $\begin{array}{l}\text { 773. Jacobs RH, Klein JB, Reinecke MA, Silva SG, Tonev S, Breland- } \\
\text { Noble A, Martinovich Z, Kratochvil CJ, Rezac AJ, Jones J and March JS. } \\
\text { 2008. Ethnic differences in attributions and treatment expectancies } \\
\text { for adolescent depression. International Journal of Cognitive Therapy. } \\
\text { 1(2):163-78. }\end{array}$ & $\begin{array}{l}\text { No child } \\
\text { internalising } \\
\text { outcomes or not } \\
\text { a major goal }\end{array}$ \\
\hline $\begin{array}{l}774 . \quad \text { Jafari Mianaei, S., Alaee Karahroudy, F., Rasouli, M., \& Zayeri, } \\
\text { F. (2011). The effect of creating opportunities for parent } \\
\text { eppowerment (C.O.P.E.) program on maternal stress, anxiety and } \\
\text { participation in NICUs. Pediatric Critical Care Medicine, 12, A101. }\end{array}$ & $\begin{array}{l}\text { No child } \\
\text { internalising } \\
\text { outcomes or not } \\
\text { a major goal }\end{array}$ \\
\hline $\begin{array}{l}\text { 775. Jans T, Philipsen A, Graf E, Schwenck C, Gerlach M and Warnke } \\
\text { A. 2008. The impact of attention deficit/hyperactivity disorder (ADHD) } \\
\text { in mothers on the treatment of their ADHD children - Review and } \\
\text { summary of the study protocol of a randomized controlled multi- } \\
\text { centre trial on parent training. Zeitschrift fur Kinder- und } \\
\text { Jugendpsychiatrie und Psychotherapie. } 36(5): 335-43 \text {. }\end{array}$ & Review \\
\hline $\begin{array}{l}776 . \quad \text { Jansen, M., van Doorn, M. M., Lichtwarck-Aschoff, A., Kuijpers, } \\
\text { R. C., Theunissen, H., Korte, M., van Rossum, J., Wauben, A., \& Granic, } \\
\text { I. (2012). Effectiveness of a cognitive-behavioral therapy (CBT) } \\
\text { manualized program for clinically anxious children: study protocol of a } \\
\text { randomized controlled trial. BMC Psychiatry, } 12,16 .\end{array}$ & $\begin{array}{l}\text { Not a parenting } \\
\text { intervention; } \\
\text { Data not } \\
\text { available/report } \\
\text { ed (protocol } \\
\text { only) }\end{array}$ \\
\hline $\begin{array}{l}\text { 777. Jarrett, M. A., \& Ollendick, T. H. (2012). Treatment of } \\
\text { comorbid attention-deficit/hyperactivity disorder and anxiety in } \\
\text { children: A multiple baseline design analysis. J Consult Clin Psychol, 80, } \\
\text { 239-244. }\end{array}$ & Not an RCT \\
\hline $\begin{array}{l}\text { 778. Jaspers M, De Winter AF, De Meer G, Stewart RE, Verhulst FC, } \\
\text { Ormel J and Reijneveld SA. 2010. Early findings of preventive child } \\
\text { healthcare professionals predict psychosocial problems in } \\
\text { preadolescence: The TRAILS study. The Journal of Pediatrics. } \\
\text { 157(2):316-21. }\end{array}$ & $\begin{array}{l}\text { Not a parenting } \\
\text { intervention }\end{array}$ \\
\hline $\begin{array}{l}\text { 779. Jay SM and Elliott CH. 1990. A stress inoculation program for } \\
\text { parents whose children are undergoing painful medical procedures. J } \\
\text { Consult Clin Psychol. 58(6):799-804. }\end{array}$ & $\begin{array}{l}\text { Intervention for } \\
\text { developmental } \\
\text { disorders } \\
\text { (autism, autism } \\
\text { spectrum } \\
\text { disorder), } \\
\text { physical } \\
\text { disabilities, } \\
\text { medical } \\
\text { conditions or } \\
\text { distress about } \\
\text { facing medical } \\
\text { interventions }\end{array}$ \\
\hline $\begin{array}{l}780 . \quad \text { Jaycox LH, Reivich KJ, Gillham J and Seligman ME. } 1994 . \\
\text { Prevention of depressive symptoms in school children. Behav Res } \\
\text { Ther. 32(8):801-16. }\end{array}$ & $\begin{array}{l}\text { Not a parenting } \\
\text { intervention }\end{array}$ \\
\hline $\begin{array}{l}\text { 781. Jensen PS and Kenny DT. 2004. The effects of yoga on the } \\
\text { attention and behavior of boys with Attention-Deficit/ hyperactivity } \\
\text { Disorder (ADHD). Journal of Attention Disorders, } 7(4), 205-216 \text {. }\end{array}$ & $\begin{array}{l}\text { Not a parenting } \\
\text { intervention }\end{array}$ \\
\hline
\end{tabular}




\begin{tabular}{|c|c|}
\hline $\begin{array}{l}\text { 782. Jensen PS, Youngstrom EA, Steiner H, Findling RL, Meyer RE, } \\
\text { Malone RP, Carlson GA, Coccaro EF, Aman MG, Blair J, Dougherty D, } \\
\text { Ferris C, Flynn L, Green E, Hoagwood K, Hutchinson J, Laughren T, Leve } \\
\text { LD, Novins DK and Vitiello B. 2007. Consensus Report on Impulsive } \\
\text { Aggression as a Symptom Across Diagnostic Categories in Child } \\
\text { Psychiatry: Implications for Medication Studies. Journal of the } \\
\text { American Academy of Child \& Adolescent Psychiatry. 46(3):309-22. }\end{array}$ & $\begin{array}{l}\text { Not a parenting } \\
\text { intervention }\end{array}$ \\
\hline $\begin{array}{l}783 . \quad \text { Jensen, M. R., Wong, J. J., Gonzales, N. A., Dumka, L. E., } \\
\text { Millsap, R., \& Coxe, S. (2014). Long-term effects of a universal family } \\
\text { intervention: Mediation through parent-adolescent conflict. Journal of } \\
\text { Clinical Child \& Adolescent Psychology, 43(3), 415-427. }\end{array}$ & $\begin{array}{l}\text { Parent } \\
\text { component too } \\
\text { small }\end{array}$ \\
\hline $\begin{array}{l}784 . \quad \text { Jensen, P. S., Hinshaw, S. P., Swanson, J. M., Greenhill, L. L., } \\
\text { Conners, C. K., Arnold, L. E., et al. (2001). Findings from the NIMH } \\
\text { Multimodal Treatment Study of ADHD (MTA): implications and } \\
\text { applications for primary care providers. Journal of developmental and } \\
\text { behavioral pediatrics : JDBP, (1), 60-73. }\end{array}$ & $\begin{array}{l}\text { No long term } \\
\text { follow-up }\end{array}$ \\
\hline $\begin{array}{l}\text { 785. Johnson CA, Pentz MA, Weber MD, Dwyer JH, Baer N, } \\
\text { MacKinnon DP, Hansen WB and Flay BR. 1990. Relative effectiveness } \\
\text { of comprehensive community programming for drug abuse prevention } \\
\text { with high-risk and low-risk adolescents. Journal of Consulting and } \\
\text { Clinical Psychology, 58(4), } 447 \text {. }\end{array}$ & $\begin{array}{l}\text { Not a parenting } \\
\text { intervention }\end{array}$ \\
\hline $\begin{array}{l}\text { 786. Johnson JG, Cohen P, Smailes EM, Skodol AE, Brown J and } \\
\text { Oldham JM. 2001. Childhood verbal abuse and risk for personality } \\
\text { disorders during adolescence and early adulthood. Comprehensive } \\
\text { Psychiatry. 42(1):16-23. }\end{array}$ & Not an RCT \\
\hline $\begin{array}{l}\text { 787. Johnson Z, Howell F, Molloy B. Community mothers' } \\
\text { programme: randomised controlled trial of non-professional } \\
\text { intervention in parenting. BMJ 1993;306(6890):1449-52. }\end{array}$ & $\begin{array}{l}\text { No child } \\
\text { internalising } \\
\text { outcomes or not } \\
\text { a major goal }\end{array}$ \\
\hline $\begin{array}{l}\text { 788. Johnson, E. R., Davies, S. L., Aban, I., Mugavero, M. J., } \\
\text { Shrestha, S., \& Kempf, M. C. (2015). Improving Parental Stress Levels } \\
\text { Among Mothers Living with HIV: A Randomized Control Group } \\
\text { Intervention Study. AIDS patient care and STDs, 29(4), 220-228. }\end{array}$ & $\begin{array}{l}\text { No child } \\
\text { internalising } \\
\text { outcomes or not } \\
\text { a major goal }\end{array}$ \\
\hline $\begin{array}{l}\text { 789. Johnston BD, Huebner CE, Tyll LT, Barlow WE and Thompson } \\
\text { RS. 2004. Expanding developmental and behavioral services for } \\
\text { newborns in primary care; Effects on parental well-being, practice, and } \\
\text { satisfaction. American journal of preventive medicine, } 26(4), 356-366 \text {. }\end{array}$ & $\begin{array}{l}\text { Follow-up less } \\
\text { than } 6 \text { months }\end{array}$ \\
\hline $\begin{array}{l}790 . \quad \text { Johnston, B. D., Huebner, C. E., Anderson, M. L., Tyll, L. T., \& } \\
\text { Thompson, R. S. (2006). Healthy steps in an integrated delivery } \\
\text { system: child and parent outcomes at } 30 \text { months. Arch Pediatr Adolesc } \\
\text { Med, } 160(8), 793-800 . \text { doi: } 160 / 8 / 793\end{array}$ & $\begin{array}{l}\text { Not an RCT - } \\
\text { randomisation is } \\
\text { between } 2 \\
\text { active } \\
\text { conditions. } \\
\text { Control } \\
\text { condition not } \\
\text { randomly } \\
\text { assigned. }\end{array}$ \\
\hline $\begin{array}{l}\text { 791. Jomeen J and Martin C. 2004. Is the hospital anxiety and } \\
\text { depression scale (HAPS) a reliable screening tool in early pregnancy? } \\
\text { Psychology \& Health. 19(6):787-800. }\end{array}$ & $\begin{array}{l}\text { Not a parenting } \\
\text { intervention }\end{array}$ \\
\hline
\end{tabular}




\begin{tabular}{|c|c|}
\hline $\begin{array}{l}\text { 792. Jones D, Godwin J, Dodge KA, Bierman KL, Coie JD, Greenberg } \\
\text { MT, Lochman JE, McMahon RJ and Pinderhughes EE. 2010. Impact of } \\
\text { the fast track prevention program on health services use by conduct- } \\
\text { problem youth.Pediatrics, } 125(1), \text { e130-e136. }\end{array}$ & $\begin{array}{l}\text { No child } \\
\text { internalising } \\
\text { outcomes or not } \\
\text { a major goal }\end{array}$ \\
\hline $\begin{array}{l}\text { 793. Jones D, Macias RL, Gold PB, Barreira P and Fisher W. } 2008 . \\
\text { When parents with severe mental illness lose contact with their } \\
\text { children: Are psychiatric symptoms or substance use to blame? } \\
\text { Journal of Loss and Trauma. 13(4):261-87. }\end{array}$ & $\begin{array}{l}\text { Not a parenting } \\
\text { intervention }\end{array}$ \\
\hline $\begin{array}{l}794 . \quad \text { Jones, D. J., Olson, A. L., Forehand, R., Gaffney, C. A., Zens, M. } \\
\text { S., \& Bau, J. J. (2005). A Family-Focused Randomized Controlled Trial } \\
\text { to Prevent Adolescent Alcohol and Tobacco Use: The Moderating } \\
\text { Roles of Positive Parenting and Adolescent Gender. Behavior Therapy, } \\
\text { 36(4), 347-355. doi: 10.1016/s0005-7894(05)80116-9 }\end{array}$ & $\begin{array}{l}\text { No child } \\
\text { internalising } \\
\text { outcomes or not } \\
\text { a major goal } \\
\text { (Targets } \\
\text { prevention of } \\
\text { substance use) }\end{array}$ \\
\hline $\begin{array}{l}795 . \quad \text { Jones, K., Daley, D., Hutchings, J., Bywater, T., \& Eames, C. } \\
\text { (2007). Efficacy of the Incredible Years Basic parent training } \\
\text { programme as an early intervention for children with conduct } \\
\text { problems and ADHD. Child Care Health and Development, 33(6), 749- } \\
756 .\end{array}$ & $\begin{array}{l}\text { No long term } \\
\text { follow-up }\end{array}$ \\
\hline $\begin{array}{l}\text { 796. Jones, K., Daley, D., Hutchings, J., Bywater, T., \& Eames, C. } \\
\text { (2008). Efficacy of the incredible years programme as an early } \\
\text { intervention for children with conduct problems and ADHD: Long-term } \\
\text { follow-up. Child: Care, Health and Development, 34(3), 380-390. }\end{array}$ & $\begin{array}{l}\text { No child } \\
\text { internalising } \\
\text { outcomes or not } \\
\text { a major goal }\end{array}$ \\
\hline $\begin{array}{l}\text { 797. Jones, S., Calam, R., Sanders, M., Diggle, P. J., Dempsey, R., \& } \\
\text { Sadhnani, V. (2014). A pilot web based positive parenting intervention } \\
\text { to help bipolar parents to improve perceived parenting skills and child } \\
\text { outcomes. Behavioural and cognitive psychotherapy, } 42(03), 283-296 .\end{array}$ & $\begin{array}{l}\text { No long term } \\
\text { follow-up }\end{array}$ \\
\hline $\begin{array}{l}\text { 798. Jonsson, U., Goodman, A., von Knorring, A.-L., von Knorring, L., } \\
\text { \& Koupil, I. (2012). School performance and hospital admission due to } \\
\text { unipolar depression: A three-generational study of social causation } \\
\text { and social selection. Social Psychiatry and Psychiatric Epidemiology, } \\
47,1695-1706 .\end{array}$ & $\begin{array}{l}\text { Not a parenting } \\
\text { intervention }\end{array}$ \\
\hline $\begin{array}{l}\text { 799. Jorm AF, Morgan AJ and Wright A. 2008. Interventions that } \\
\text { are helpful for depression and anxiety in young people: A comparison } \\
\text { of clinicians' beliefs with those of youth and their parents. Journal of } \\
\text { Affective Disorders. } 111(2-3): 227-34 \text {. }\end{array}$ & $\begin{array}{l}\text { Not a parenting } \\
\text { intervention }\end{array}$ \\
\hline $\begin{array}{l}\text { 800. Jotzo M and Poets CF. 2005. Helping parents cope with the } \\
\text { trauma of premature birth: an evaluation of a trauma-preventive } \\
\text { psychological intervention. Pediatrics. } 115(4): 915-9 .\end{array}$ & $\begin{array}{l}\text { No child } \\
\text { internalising } \\
\text { outcomes or not } \\
\text { a major goal }\end{array}$ \\
\hline $\begin{array}{l}\text { 801. Jouriles, E. N., McDonald, R., Rosenfield, D., Stephens, N., } \\
\text { Corbitt-Shindler, D., \& Miller, P. C. (2009). Reducing conduct problems } \\
\text { among children exposed to intimate partner violence: a randomized } \\
\text { clinical trial examining effects of Project Support. J Consult Clin } \\
\text { Psychol, 77(4), 705-717. doi: 2009-11168-010 }\end{array}$ & $\begin{array}{l}\text { Targets } \\
\text { externalising }\end{array}$ \\
\hline $\begin{array}{l}\text { 802. Joutsenniemi, K., Laaksonen, M. A., Knekt, P., Haaramo, P., \& } \\
\text { Lindfors, O. (2012). Prediction of the outcome of short- and long-term } \\
\text { psychotherapy based on socio-demographic factors. J Affect Disord, } \\
\text { 141(2-3), 331-342. doi: 10.1016/j.jad.2012.03.027 }\end{array}$ & $\begin{array}{l}\text { Not a parenting } \\
\text { intervention }\end{array}$ \\
\hline
\end{tabular}




\begin{tabular}{|c|c|}
\hline $\begin{array}{l}\text { 803. Joyce Marie, R. (1995). Emotional relief for parents: Is rational- } \\
\text { emotive parent education effective? Journal-of-Rational-Emotive-and- } \\
\text { Cognitive-Behavior-Therapy, (1), 55-75. }\end{array}$ & $\begin{array}{l}\text { No long term } \\
\text { follow-up }\end{array}$ \\
\hline $\begin{array}{l}\text { 804. Judge Santacroce, S., Asmus, K., Kadan-Lottick, N., \& Grey, M. } \\
\text { (2010). Feasibility and preliminary outcomes from a pilot study of } \\
\text { coping skills training for adolescent--young adult survivors of } \\
\text { childhood cancer and their parents. J Pediatr Oncol Nurs, } 27(1), 10-20 . \\
\text { doi: } 1043454209340325\end{array}$ & $\begin{array}{l}\text { No long term } \\
\text { follow-up }\end{array}$ \\
\hline $\begin{array}{l}805 . \quad \text { Juffer, F., Bakermans-Kranenburg, M. J., \& van ljzendoorn, M. } \\
\text { H. (2005). The importance of parenting in the development of } \\
\text { disorganized attachment: Evidence from a preventive intervention } \\
\text { study in adoptive families. Journal of Child Psychology and Psychiatry } \\
\text { and Allied Disciplines, 46(3), 263-274. }\end{array}$ & $\begin{array}{l}\text { No child } \\
\text { internalising } \\
\text { outcomes or not } \\
\text { a major goal }\end{array}$ \\
\hline $\begin{array}{l}\text { 806. Juffer, F., Hoksbergen, R. A. C., Riksen-Walraven, J. M., \& } \\
\text { Kohnstamm, G. A. (1997). Early intervention in adoptive families: } \\
\text { Supporting maternal sensitive responsiveness, infant-mother } \\
\text { attachment, and infant competence. Journal of Child Psychology and } \\
\text { Psychiatry and Allied Disciplines, 38, 1039-1050. }\end{array}$ & $\begin{array}{l}\text { No child } \\
\text { internalising } \\
\text { outcomes or not } \\
\text { a major goal; No } \\
\text { long term } \\
\text { follow-up; Not } \\
\text { an RCT }\end{array}$ \\
\hline $\begin{array}{l}\text { 807. Jungbluth NJ and Shirk SR. 2009. Therapist strategies for } \\
\text { building involvement in cognitive,Äibehavioral therapy for adolescent } \\
\text { depression. Journal of Consulting and Clinical Psychology. 77(6):1179- } \\
84 \text {. }\end{array}$ & $\begin{array}{l}\text { Not a parenting } \\
\text { intervention }\end{array}$ \\
\hline $\begin{array}{l}\text { 808. Kaale, A., Smith, L., \& Sponheim, E. (2012). A randomized } \\
\text { controlled trial of preschool?based joint attention intervention for } \\
\text { children with autism. Journal of Child Psychology and Psychiatry, 53, } \\
\text { 97-105. }\end{array}$ & $\begin{array}{l}\text { Intervention for } \\
\text { developmental } \\
\text { disorders } \\
\text { (autism, autism } \\
\text { spectrum } \\
\text { disorder), } \\
\text { physical } \\
\text { disabilities, } \\
\text { medical } \\
\text { conditions or } \\
\text { distress about } \\
\text { facing medical } \\
\text { interventions }\end{array}$ \\
\hline $\begin{array}{l}\text { 809. Kable, J. A., Coles, C. D., \& Taddeo, E. (2007). Socio-cognitive } \\
\text { habilitation using the math interactive learning experience program } \\
\text { for alcohol-affected children. Alcohol Clin Exp Res, 31(8), 1425-1434. } \\
\text { doi: ACER431 }\end{array}$ & $\begin{array}{l}\text { No long term } \\
\text { follow-up; } \\
\text { Parent } \\
\text { component too } \\
\text { small }\end{array}$ \\
\hline $\begin{array}{l}\text { 810. Kahn James, V. (1978). A Comparison of Manual and Oral } \\
\text { Language Training with Mute Retardates. Final Report. Illinois State } \\
\text { Dept. of Mental Health and Developmental Disabilities. Retrieved from } \\
\text { http://onlinelibrary.wiley.com/o/cochrane/clcentral/articles/680/CN- } \\
\text { 00241680/frame.html }\end{array}$ & $\begin{array}{l}\text { No child } \\
\text { internalising } \\
\text { outcomes or not } \\
\text { a major goal }\end{array}$ \\
\hline
\end{tabular}




\begin{tabular}{|c|c|}
\hline $\begin{array}{l}\text { 811. Kain ZN, Caldwell-Andrews AA, Mayes LC, Weinberg ME, Wang } \\
\text { SM, MacLaren JE and Blount RL. 2007. Family-centered preparation for } \\
\text { surgery improves perioperative outcomes in children: a randomized } \\
\text { controlled trial. Anesthesiology. 106(1):65-74. }\end{array}$ & $\begin{array}{l}\text { Intervention for } \\
\text { developmental } \\
\text { disorders } \\
\text { (autism, autism } \\
\text { spectrum } \\
\text { disorder), } \\
\text { physical } \\
\text { disabilities, } \\
\text { medical } \\
\text { conditions or } \\
\text { distress about } \\
\text { facing medical } \\
\text { interventions }\end{array}$ \\
\hline $\begin{array}{l}\text { 812. Kain ZN, Caramico LA, Mayes LC, Genevro JL, Bornstein MH } \\
\text { and Hofstadter MB. 1998. Preoperative preparation programs in } \\
\text { children: a comparative examination. Anesth Analg. 87(6):1249-55. }\end{array}$ & $\begin{array}{l}\text { Intervention for } \\
\text { developmental } \\
\text { disorders } \\
\text { (autism, autism } \\
\text { spectrum } \\
\text { disorder), } \\
\text { physical } \\
\text { disabilities, } \\
\text { medical } \\
\text { conditions or } \\
\text { distress about } \\
\text { facing medical } \\
\text { interventions }\end{array}$ \\
\hline $\begin{array}{l}\text { 813. Kain ZN, Mayes LC and Caramico LA. 1996. Preoperative } \\
\text { preparation in children: a cross-sectional study. J Clin Anesth. } \\
\text { 8(6):508-14. }\end{array}$ & $\begin{array}{l}\text { Intervention for } \\
\text { developmental } \\
\text { disorders } \\
\text { (autism, autism } \\
\text { spectrum } \\
\text { disorder), } \\
\text { physical } \\
\text { disabilities, } \\
\text { medical } \\
\text { conditions or } \\
\text { distress about } \\
\text { facing medical } \\
\text { interventions }\end{array}$ \\
\hline
\end{tabular}




\begin{tabular}{|c|c|}
\hline $\begin{array}{l}\text { 814. Kain ZN, Mayes LC, Caramico LA, Silver D, Spieker M, Nygren } \\
\text { MM, Anderson G and Rimar S. 1996. Parental presence during } \\
\text { induction of anesthesia. A randomized controlled trial. Anesthesiology, } \\
\text { 84(5), 1060-1067. }\end{array}$ & $\begin{array}{l}\text { Intervention for } \\
\text { developmental } \\
\text { disorders } \\
\text { (autism, autism } \\
\text { spectrum } \\
\text { disorder), } \\
\text { physical } \\
\text { disabilities, } \\
\text { medical } \\
\text { conditions or } \\
\text { distress about } \\
\text { facing medical } \\
\text { interventions }\end{array}$ \\
\hline $\begin{array}{l}\text { 815. Kain ZN, Mayes LC, Wang SM, Caramico LA and Hofstadter } \\
\text { MB. 1998. Parental presence during induction of anesthesia versus } \\
\text { sedative premedication: which intervention is more effective? The } \\
\text { Journal of the American Society of Anesthesiologists, 89(5), 1147- } \\
\text { 1156. }\end{array}$ & $\begin{array}{l}\text { Intervention for } \\
\text { developmental } \\
\text { disorders } \\
\text { (autism, autism } \\
\text { spectrum } \\
\text { disorder), } \\
\text { physical } \\
\text { disabilities, } \\
\text { medical } \\
\text { conditions or } \\
\text { distress about } \\
\text { facing medical } \\
\text { interventions }\end{array}$ \\
\hline $\begin{array}{l}\text { 816. Kain ZN, Mayes LC, Wang SM, Caramico LA, Krivutza DM and } \\
\text { Hofstadter MB. 2000. Parental presence and a sedative premedicant } \\
\text { for children undergoing surgery: a hierarchical study. Anesthesiology, } \\
\text { 92(4), 939-46. }\end{array}$ & $\begin{array}{l}\text { Intervention for } \\
\text { developmental } \\
\text { disorders } \\
\text { (autism, autism } \\
\text { spectrum } \\
\text { disorder), } \\
\text { physical } \\
\text { disabilities, } \\
\text { medical } \\
\text { conditions or } \\
\text { distress about } \\
\text { facing medical } \\
\text { interventions }\end{array}$ \\
\hline
\end{tabular}




\begin{tabular}{|c|c|}
\hline $\begin{array}{l}\text { 817. Kain ZN, Wang SM, Caramico LA, Hofstadter M and Mayes LC. } \\
\text { 1997. Parental desire for perioperative information and informed } \\
\text { consent: a two-phase study.Anesthesia \& Analgesia, 84(2), 299-306. }\end{array}$ & $\begin{array}{l}\text { Intervention for } \\
\text { developmental } \\
\text { disorders } \\
\text { (autism, autism } \\
\text { spectrum } \\
\text { disorder), } \\
\text { physical } \\
\text { disabilities, } \\
\text { medical } \\
\text { conditions or } \\
\text { distress about } \\
\text { facing medical } \\
\text { interventions }\end{array}$ \\
\hline $\begin{array}{l}\text { 818. Kalinauskiene, L., Cekuoliene, D., Van ljzendoorn, M. H., } \\
\text { Bakermans-Kranenburg, M. J., Juffer, F., \& Kusakovskaja, I. (2009). } \\
\text { Supporting insensitive mothers: the Vilnius randomized control trial of } \\
\text { video-feedback intervention to promote maternal sensitivity and } \\
\text { infant attachment security. Child: care, health and development, (5), } \\
\text { 613-623. }\end{array}$ & $\begin{array}{l}\text { No long term } \\
\text { follow-up; No } \\
\text { child } \\
\text { internalising } \\
\text { outcomes } \\
\text { (attachment } \\
\text { only) }\end{array}$ \\
\hline $\begin{array}{l}\text { 819. Kamon J, Budney A and Stanger C. 2005. A contingency } \\
\text { management intervention for adolescent marijuana abuse and } \\
\text { conduct problems. J Am Acad Child Adolesc Psychiatry. 44(6):513-21. }\end{array}$ & Not an RCT \\
\hline $\begin{array}{l}\text { 820. Kang EY, Fields HW, Kiyak A, Beck FM and Firestone AR. } 2009 . \\
\text { Informed consent recall and comprehension in orthodontics: } \\
\text { traditional vs improved readability and processability methods. Am J } \\
\text { Orthod Dentofacial Orthop. 136(4):488 e1-13; discussion-9. }\end{array}$ & $\begin{array}{l}\text { Not a parenting } \\
\text { intervention }\end{array}$ \\
\hline $\begin{array}{l}\text { 821. Kang R, Barnard K, Hammond M, Oshio S, Spencer C, } \\
\text { Thibodeaux B and Williams J. 1995. Preterm infant follow-up project: a } \\
\text { multi-site field experiment of hospital and home intervention } \\
\text { programs for mothers and preterm infants. Public Health Nursing, } \\
\text { 12(3), 171-180. }\end{array}$ & $\begin{array}{l}\text { No long term } \\
\text { follow-up }\end{array}$ \\
\hline $\begin{array}{l}\text { 822. Kapornai, K., \& Vetro, A. (2008). Depression in children. Curr } \\
\text { Opin Psychiatry, 21(1), 1-7. doi:10.1097/YCO.0b013e3282f25b01 }\end{array}$ & Review \\
\hline $\begin{array}{l}\text { 823. Karmaliani, R., Shehzad, S., Hirani, S. S., Asad, N., Akbar Ali } \\
\text { Hirani, S., \& McFarlane, J. (2011). Meeting the } 2015 \text { Millennium } \\
\text { Development Goals with new interventions for abused women. Nurs } \\
\text { Clin North Am, 46, 485-493, vii. }\end{array}$ & $\begin{array}{l}\text { Not a parenting } \\
\text { intervention }\end{array}$ \\
\hline $\begin{array}{l}\text { 824. Karp, E. A., Kuo, A. A. (2015). Maternal mental health after a } \\
\text { child's diagnosis of autism spectrum disorder. JAMA - Journal of the } \\
\text { American Medical Association, } 313 \text { (1), 81-2. }\end{array}$ & $\begin{array}{l}\text { No child } \\
\text { internalising } \\
\text { outcomes or not } \\
\text { a major goal }\end{array}$ \\
\hline $\begin{array}{l}\text { 825. Karver M, Shirk S, Handelsman JB, Fields S, Crisp H, } \\
\text { Gudmundsen G and McMakin D. 2008. Relationship processes in } \\
\text { youth psychotherapy: Measuring alliance, alliance-building behaviors, } \\
\text { and client involvement. Journal of Emotional and Behavioral } \\
\text { Disorders. 16(1):15-28. }\end{array}$ & $\begin{array}{l}\text { Not a parenting } \\
\text { intervention }\end{array}$ \\
\hline
\end{tabular}




\begin{tabular}{|c|c|}
\hline $\begin{array}{l}\text { 826. Kawamura, T., Kawamura, K., Hirose, M., Hashimoto, T., } \\
\text { Higashide, T., Kashihara, Y., . . Harai, H. (2012). Training of } \\
\text { motivational interviewing to parents improved the glycemic control of } \\
\text { the childhood and adolescent type } 1 \text { diabetes: A prospective } \\
\text { randomized control trial. [Conference Abstract]. Pediatric Diabetes, } \\
\text { 13, 26. doi: http://dx.doi.org/10.1111/j.1399-5448.2012.00918.x }\end{array}$ & $\begin{array}{l}\text { Intervention for } \\
\text { developmental } \\
\text { disorders } \\
\text { (autism, autism } \\
\text { spectrum } \\
\text { disorder), } \\
\text { physical } \\
\text { disabilities, } \\
\text { medical } \\
\text { conditions or } \\
\text { distress about } \\
\text { facing medical } \\
\text { interventions }\end{array}$ \\
\hline $\begin{array}{l}\text { 827. Kazak AE, Simms S, Alderfer MA, Rourke MT, Crump T, } \\
\text { McClure K, Jones P, Rodriguez A, Boeving A, Hwang WT and Reilly A. } \\
\text { 2005. Feasibility and preliminary outcomes from a pilot study of a } \\
\text { brief psychological intervention for families of children newly } \\
\text { diagnosed with cancer. J Pediatr Psychol. 30(8):644-55. }\end{array}$ & $\begin{array}{l}\text { Follow-up less } \\
\text { than } 6 \text { months }\end{array}$ \\
\hline $\begin{array}{l}\text { 828. Kazak AE, Simms S, Barakat L, Hobbie W, Foley B, Golomb V } \\
\text { and Best M. 1999. Surviving cancer competently intervention program } \\
\text { (SCCIP): a cognitive-behavioral and family therapy intervention for } \\
\text { adolescent survivors of childhood cancer and their families. Fam } \\
\text { Process. 38(2):175-91. }\end{array}$ & Not an RCT \\
\hline $\begin{array}{l}\text { 829. Kazak, A. E., Alderfer, M. A., Streisand, R., Simms, S., Rourke, } \\
\text { M. T., Barakat, L. P., et al. (2004). Treatment of posttraumatic stress } \\
\text { symptoms in adolescent survivors of childhood cancer and their } \\
\text { families: a randomized clinical trial. J Fam Psychol, 18(3), 493-504. doi: } \\
\text { 10.1037/0893-3200.18.3.493 }\end{array}$ & $\begin{array}{l}\text { Follow-up less } \\
\text { than } 6 \text { months }\end{array}$ \\
\hline $\begin{array}{l}\text { 830. Kazdin AE, Esveldt-Dawson K, French NH and Unis AS. } 1987 . \\
\text { Problem-solving skills training and relationship therapy in the } \\
\text { treatment of antisocial child behavior. Journal of Consulting and } \\
\text { Clinical Psychology. 55(1):76-85. }\end{array}$ & $\begin{array}{l}\text { Not a parenting } \\
\text { intervention }\end{array}$ \\
\hline $\begin{array}{l}\text { 831. Kazdin, A. E. (1997). Parent Management Training: Evidence, } \\
\text { Outcomes, and Issues. Journal of the American Academy of Child \& } \\
\text { Adolescent Psychiatry 36(10): 1349-1356. }\end{array}$ & Review \\
\hline $\begin{array}{l}\text { 832. Kazdin, A. E., Esveldt-Dawson, K., French, N. H., \& Unis, A. S. } \\
\text { (1987). Effects of parent management training and problem-solving } \\
\text { skills training combined in the treatment of antisocial child behavior. } \\
\text { Journal of the American Academy of Child \& Adolescent Psychiatry, } \\
\text { 26(3), 416-424. doi: } 10.1097 / 00004583-198705000-00024\end{array}$ & $\begin{array}{l}\text { Targets } \\
\text { externalising } \\
\text { (antisocial } \\
\text { behaviour) }\end{array}$ \\
\hline $\begin{array}{l}\text { 833. Kazdin, A. E., Mazurick, J. L., \& Bass, D. (1993). Risk for } \\
\text { Attrition in Treatment of Antisocial Children and Families. Journal of } \\
\text { Clinical Child Psychology, 22(1), 2-16. }\end{array}$ & Not an RCT \\
\hline $\begin{array}{l}\text { 834. Kazdin, A. E., Siegel, T. C., \& Bass, D. (1992). Cognitive } \\
\text { problem-solving skills training and parent management training in the } \\
\text { treatment of antisocial behavior in children. J Consult Clin Psychol, } \\
60(5), 733-747 .\end{array}$ & $\begin{array}{l}\text { Targets } \\
\text { externalising }\end{array}$ \\
\hline $\begin{array}{l}\text { 835. Kazdin, A., \& Whitley, M. (2006). Comorbidity, case } \\
\text { complexity, and effects of evidence-based treatment for children } \\
\text { referred for disruptive behavior. Journal of Consulting and Clinical } \\
\text { Psychology, 74, 455-467. }\end{array}$ & Not an RCT \\
\hline
\end{tabular}




\begin{tabular}{|c|c|}
\hline $\begin{array}{l}\text { 836. Kellam 1994. The course and malleability of aggressive } \\
\text { behavior from early first grade into middle school: Results of a } \\
\text { developmental epidemiologically-based preventive trial. J Child } \\
\text { Psychology \& Psychiatry, 35(2), 259-282. }\end{array}$ & $\begin{array}{l}\text { Not a parenting } \\
\text { intervention }\end{array}$ \\
\hline $\begin{array}{l}\text { 837. Kelley ML, Fals-Stewart W. Couples- versus individual-based } \\
\text { therapy for alcholism and drug abuse: Effects on children's } \\
\text { psychosocial functioning. Journal of Consulting and Clinical Psychology } \\
2002 ; 70: 417-427 \text {. }\end{array}$ & $\begin{array}{l}\text { No child } \\
\text { internalising } \\
\text { outcomes or not } \\
\text { a major goal }\end{array}$ \\
\hline $\begin{array}{l}\text { 838. Kelsberg G and St. Anna L. 2006. What are effective } \\
\text { treatments for oppositional defiant behaviors in adolescents? Journal } \\
\text { of Family Practice. 55(10):911-3. }\end{array}$ & Review \\
\hline $\begin{array}{l}\text { 839. Kemp, L., Harris, E., McMahon, C., Matthey, S., Vimpani, G., } \\
\text { Anderson, T., Schmied, V., Aslam, H., \& Zapart, S. (2011). Child and } \\
\text { family outcomes of a long-term nurse home visitation programme: a } \\
\text { randomised controlled trial. Archives of disease in childhood, 96(6), } \\
533 .\end{array}$ & $\begin{array}{l}\text { No child } \\
\text { internalising } \\
\text { outcomes or not } \\
\text { a major goal; No } \\
\text { long term } \\
\text { follow-up }\end{array}$ \\
\hline $\begin{array}{l}\text { 840. Kenardy, J., Thompson, K., Le Brocque, R., \& Olsson, K. (2008). } \\
\text { Information-provision intervention for children and their parents } \\
\text { following pediatric accidental injury. Eur Child Adolesc Psychiatry, } \\
\text { 17(5), 316-325. doi: } 10.1007 / \text { s00787-007-0673-5 }\end{array}$ & $\begin{array}{l}\text { Parent } \\
\text { component too } \\
\text { small }\end{array}$ \\
\hline $\begin{array}{l}\text { 841. Kendall PC and Southam-Gerow MA. 1996. Long-term follow- } \\
\text { up of a cognitive,Äïbehavioral therapy for anxiety-disordered youth. } \\
\text { Journal of Consulting and Clinical Psychology. 64(4):724-30. }\end{array}$ & $\begin{array}{l}\text { Not a parenting } \\
\text { intervention }\end{array}$ \\
\hline $\begin{array}{l}\text { 842. Kendall PC, Brady EU and Verduin TL. 2001. Comorbidity in } \\
\text { childhood anxiety disorders and treatment outcome. J Am Acad Child } \\
\text { Adolesc Psychiatry. 40(7):787-94. }\end{array}$ & $\begin{array}{l}\text { Not a parenting } \\
\text { intervention }\end{array}$ \\
\hline $\begin{array}{l}\text { 843. Kendall PC, Flannery-Schroeder E, Panichelli-Mindel SM, } \\
\text { Southam-Gerow M, Henin A and Warman M. 1997. Therapy for youths } \\
\text { with anxiety disorders: A second randomized clincal trial. Journal of } \\
\text { Consulting and Clinical Psychology.65(3):366-80. }\end{array}$ & $\begin{array}{l}\text { Not a parenting } \\
\text { intervention }\end{array}$ \\
\hline $\begin{array}{l}\text { 844. Kendall PC, Hudson JL, Gosch E, Flannery-Schroeder E, Suveg } \\
\text { C. Cognitive-behavioral therapy for anxiety disordered youth: a } \\
\text { randomized clinical trial evaluating child and family modalities. J } \\
\text { Consult Clin Psychol. 2008 Apr;76(2):282-97. }\end{array}$ & $\begin{array}{l}\text { Parent } \\
\text { component too } \\
\text { small }\end{array}$ \\
\hline $\begin{array}{l}\text { 845. Kendall PC, Khanna MS, Edson A, Cummings C and Harris MS. } \\
\text { 2011. Computers and psychosocial treatment for child anxiety: Recent } \\
\text { advances and ongoing efforts. Depression and Anxiety. 28(1):58-66. }\end{array}$ & Review \\
\hline $\begin{array}{l}\text { 846. Kendall PC, Safford S, Flannery-Schroeder E and Webb A. } \\
\text { 2004. Child Anxiety Treatment: Outcomes in Adolescence and Impact } \\
\text { on Substance Use and Depression at 7.4-Year Follow-Up. Journal of } \\
\text { Consulting and Clinical Psychology. 72(2):276-87. }\end{array}$ & $\begin{array}{l}\text { Not a parenting } \\
\text { intervention }\end{array}$ \\
\hline $\begin{array}{l}\text { 847. Kendall, P. C. (1994). Treating Anxiety Disorders in Children - } \\
\text { Results of a Randomized Clinical-Trial. Journal of Consulting and } \\
\text { Clinical Psychology, 62(1), 100-110. }\end{array}$ & $\begin{array}{l}\text { Not a parenting } \\
\text { intervention }\end{array}$ \\
\hline $\begin{array}{l}\text { 848. Kennard, B., et al. (2008). Maternal Depressive Symptoms in } \\
\text { Pediatric Major Depressive Disorder: Relationship to Acute Treatment } \\
\text { Outcome. Journal of the American Academy of Child \& Adolescent } \\
\text { Psychiatry 47(6): 694-699. }\end{array}$ & $\begin{array}{l}\text { Not a parenting } \\
\text { intervention }\end{array}$ \\
\hline
\end{tabular}




\begin{tabular}{|c|c|}
\hline $\begin{array}{l}\text { 849. Kennedy CM and Riddle, II. 1989. The influence of the timing } \\
\text { of preparation on the anxiety of preschool children experiencing } \\
\text { surgery. Matern Child Nurs J. 18(2):117-32. }\end{array}$ & $\begin{array}{l}\text { Intervention for } \\
\text { developmental } \\
\text { disorders } \\
\text { (autism, autism } \\
\text { spectrum } \\
\text { disorder), } \\
\text { physical } \\
\text { disabilities, } \\
\text { medical } \\
\text { conditions or } \\
\text { distress about } \\
\text { facing medical } \\
\text { interventions }\end{array}$ \\
\hline $\begin{array}{l}\text { 850. Kennedy SJ, Rapee RM, Edwards SL. A selective intervention } \\
\text { program for inhibited preschool-aged children of parents with an } \\
\text { anxiety disorder: effects on current anxiety disorders and } \\
\text { temperament. J Am Acad Child Adolesc Psychiatry. } 2009 \\
\text { Jun;48(6):602-9. }\end{array}$ & $\begin{array}{l}\text { Follow-up less } \\
\text { than } 6 \text { months }\end{array}$ \\
\hline $\begin{array}{l}\text { 851. Kern JK, West EY, Grannemann BD, Greer TL, Snell LM, Cline } \\
\text { LL, VanBeveren TT, Heartwell SF, Kleiber BA and Trivedi MH. } 2004 . \\
\text { Reductions in Stress and Depressive Symptoms in Mothers of } \\
\text { Substance-Exposed Infants, Participating in a Psychosocial Program. } \\
\text { Maternal and Child Health Journal. 8(3):127-36. }\end{array}$ & $\begin{array}{l}\text { No child } \\
\text { internalising } \\
\text { outcomes or not } \\
\text { a major goal }\end{array}$ \\
\hline $\begin{array}{l}852 . \quad \text { Kerr, S. M., Jowett, S. A., \& Smith, L. N. (1996). Preventing } \\
\text { sleep problems in infants: a randomized controlled trial. Journal of } \\
\text { advanced nursing, (5), 938-942. }\end{array}$ & $\begin{array}{l}\text { No child } \\
\text { internalising } \\
\text { outcomes or not } \\
\text { a major goal }\end{array}$ \\
\hline $\begin{array}{l}\text { 853. Kersting, A., Dolemeyer, R., Steinig, J., Walter, F., Kroker, K., } \\
\text { Baust, K., Wagner, B. (2013). Brief Internet-based intervention reduces } \\
\text { posttraumatic stress and prolonged grief in parents after the loss of a } \\
\text { child during pregnancy: A randomized controlled trial. Psychotherapy } \\
\text { and Psychosomatics, } 82 \text { (6), 372-381. }\end{array}$ & $\begin{array}{l}\text { Not a parenting } \\
\text { intervention }\end{array}$ \\
\hline $\begin{array}{l}\text { 854. Kersting, A., Kroker, K., Schlicht, S., Baust, K., \& Wagner, B. } \\
\text { (2011). Efficacy of cognitive behavioral internet-based therapy in } \\
\text { parents after the loss of a child during pregnancy: pilot data from a } \\
\text { randomized controlled trial. Arch Womens Ment Health, } 14,465-477 \text {. }\end{array}$ & $\begin{array}{l}\text { No child } \\
\text { internalising } \\
\text { outcomes or not } \\
\text { a major goal }\end{array}$ \\
\hline $\begin{array}{l}855 . \quad \text { Keshavarzi, Z., Bajoghli, H., Mohamadi, M. R., Salmanian, M., } \\
\text { Kirov, R., Gerber, M., ... \& Brand, S. (2014). In a randomized case- } \\
\text { control trial with 10-years olds suffering from attention } \\
\text { deficit/hyperactivity disorder (ADHD) sleep and psychological } \\
\text { functioning improved during a 12-week sleep-training program. The } \\
\text { World Journal of Biological Psychiatry, 15(8), 609-619. }\end{array}$ & $\begin{array}{l}\text { No long term } \\
\text { follow-up }\end{array}$ \\
\hline $\begin{array}{l}\text { 856. Kettinger LA, Nair P and Schuler ME. 2000. Exposure to } \\
\text { environmental risk factors and parenting attitudes among substance- } \\
\text { abusing women. Am J Drug Alcohol Abuse. } 26(1): 1-11 \text {. }\end{array}$ & $\begin{array}{l}\text { Not a parenting } \\
\text { intervention }\end{array}$ \\
\hline $\begin{array}{l}\text { 857. Keyes, K. M., Vo, T., Wall, M. M., Caetano, R., Suglia, S. F., } \\
\text { Martins, S. S., ... \& Hasin, D. (2015). Racial/ethnic differences in use of } \\
\text { alcohol, tobacco, and marijuana: Is there a cross-over from } \\
\text { adolescence to adulthood?. Social Science \& Medicine, 124, 132-141. }\end{array}$ & $\begin{array}{l}\text { Not a parenting } \\
\text { intervention }\end{array}$ \\
\hline
\end{tabular}




\begin{tabular}{|c|c|}
\hline $\begin{array}{l}\text { 858. Khanna MS and Kendall PC. 2010. Computer-assisted cognitive } \\
\text { behavioral therapy for child anxiety: results of a randomized clinical } \\
\text { trial. J Consult Clin Psychol. 78(5):737-45. }\end{array}$ & $\begin{array}{l}\text { Not a parenting } \\
\text { intervention }\end{array}$ \\
\hline $\begin{array}{l}\text { 859. Khanna, M. S., \& Kendall, P. C. (2009). Exploring the role of } \\
\text { parent training in the treatment of childhood anxiety. J Consult Clin } \\
\text { Psychol, 77(5), 981-986. doi:2009-17643-018 }\end{array}$ & $\begin{array}{l}\text { No long term } \\
\text { follow-up }\end{array}$ \\
\hline $\begin{array}{l}\text { 860. Khodabakhshi Koolaee, A., \& Shahi, A. (2011). The effects of } \\
\text { the triple P-positive parenting program to on schoolage children with } \\
\text { ADHD. European Psychiatry, 26, } 311 .\end{array}$ & $\begin{array}{l}\text { Targets } \\
\text { externalising }\end{array}$ \\
\hline $\begin{array}{l}\text { 861. Kieckhefer, G. M., Trahms, C. M., Churchill, S. S., Kratz, L., } \\
\text { Uding, N., \& Villareale, N. (2014). A randomized clinical trial of the } \\
\text { Building on Family Strengths program: An education program for } \\
\text { parents of children with chronic health conditions. Maternal and Child } \\
\text { Health Journal, 18(3), 563-574. doi: } 10.1007 / \text { s10995-013-1273-2 }\end{array}$ & $\begin{array}{l}\text { No child } \\
\text { internalising } \\
\text { outcomes or not } \\
\text { a major goal }\end{array}$ \\
\hline $\begin{array}{l}\text { 862. Kierfeld, F., \& Döpfner, M. (2006). [Bibliotherapy as a self-help } \\
\text { program for parents of children with externalizing problem behavior]. } \\
\text { Zeitschrift fur Kinder-und Jugendpsychiatrie und Psychotherapie, } \\
\text { 34(5), 377-85. }\end{array}$ & Not an RCT \\
\hline $\begin{array}{l}\text { 863. Kierfeld, F., Ise, E., Hanisch, C., Görtz-Dorten, A., \& Döpfner, } \\
\text { M. (2013). Effectiveness of telephone-assisted parent-administered } \\
\text { behavioural family intervention for preschool children with } \\
\text { externalizing problem behaviour: a randomized controlled trial. } \\
\text { European child \& adolescent psychiatry, 22(9), 553-565. }\end{array}$ & $\begin{array}{l}\text { Targets } \\
\text { externalising; } \\
\text { Follow-up less } \\
\text { than } 6 \text { months }\end{array}$ \\
\hline $\begin{array}{l}\text { 864. Kim, J. (2011). Patterns of parent-child relationship quality, } \\
\text { parent depression and adolescent development outcomes (Order No. } \\
\text { 3485591). Available from ProQuest Central; ProQuest Dissertations \& } \\
\text { Theses Global. (909633794). }\end{array}$ & $\begin{array}{l}\text { Not a parenting } \\
\text { intervention }\end{array}$ \\
\hline $\begin{array}{l}\text { 865. Kim, M., \& Park, I. J. K. (2011). Testing the moderating effect } \\
\text { of parent-adolescent communication on the acculturation gap-distress } \\
\text { relation in Korean American families. Journal of Youth and } \\
\text { Adolescence, 40, 1661-1673. }\end{array}$ & $\begin{array}{l}\text { Not a parenting } \\
\text { intervention }\end{array}$ \\
\hline $\begin{array}{l}\text { 866. Kim, S. E., Le Blanc, A. J., Michalopoulos, C., Azocar, F., } \\
\text { Ludman, E. J., Butler, D. M., \& Simon, G. E. (2011). Does telephone } \\
\text { care management help Medicaid beneficiaries with depression? Am J } \\
\text { Manag Care, 17, e375-382. }\end{array}$ & $\begin{array}{l}\text { Not a parenting } \\
\text { intervention }\end{array}$ \\
\hline $\begin{array}{l}\text { 867. Kincaid, C., Jones, D. J., Cuellar, J., \& Gonzalez, M. (2011). } \\
\text { Psychological control associated with youth adjustment and risky } \\
\text { behavior in African American single mother families. Journal of Child } \\
\text { and Family Studies, 20, 102-110. }\end{array}$ & $\begin{array}{l}\text { Not a parenting } \\
\text { intervention }\end{array}$ \\
\hline $\begin{array}{l}\text { 868. Kindt, K., Kleinjan, M., Janssens, J. M., \& Scholte, R. H. (2014). } \\
\text { Evaluation of a school-based depression prevention program among } \\
\text { adolescents from low-income areas: A randomized controlled } \\
\text { effectiveness trial. International journal of environmental research } \\
\text { and public health, 11(5), 5273-5293. }\end{array}$ & $\begin{array}{l}\text { Not a parenting } \\
\text { intervention }\end{array}$ \\
\hline $\begin{array}{l}\text { 869. King CA, Kramer A, Preuss L, Kerr DC, Weisse L and } \\
\text { Venkataraman S. 2006. Youth-Nominated Support Team for Suicidal } \\
\text { Adolescents (Version 1): a randomized controlled trial. J Consult Clin } \\
\text { Psychol. 74(1):199-206. }\end{array}$ & $\begin{array}{l}\text { Not a parenting } \\
\text { intervention }\end{array}$ \\
\hline
\end{tabular}




\begin{tabular}{|c|c|}
\hline $\begin{array}{l}\text { 870. King NJ, Tonge BJ, Heyne D, Pritchard M, Rollings S, Young D, } \\
\text { Myerson N and Ollendick TH. 1998. Cognitive-behavioral treatment of } \\
\text { school-refusing children: a controlled evaluation. J Am Acad Child } \\
\text { Adolesc Psychiatry. 37(4):395-403. }\end{array}$ & $\begin{array}{l}\text { Follow-up less } \\
\text { than } 6 \text { months }\end{array}$ \\
\hline $\begin{array}{l}\text { 871. Kingree JB and Thompson M. 2000. Mutual help groups, } \\
\text { perceived status benefits, and well-being: a test with adult children of } \\
\text { alcoholics with personal substance abuse problems. Am J Community } \\
\text { Psychol. 28(3):325-42. }\end{array}$ & $\begin{array}{l}\text { Not a parenting } \\
\text { intervention }\end{array}$ \\
\hline $\begin{array}{l}872 . \quad \text { Kingston, D., Austin, M. P., Hegadoren, K., Lasiuk, G., } \\
\text { McDonald, S., Heaman, M., Biringer, A., Sword, W., Giallo, R., Patel, T., } \\
\text { Lane-Smith, M., Zanten, S. V. (2014). Study protocol for a randomized, } \\
\text { controlled, superiority trial comparing the clinical and cost- } \\
\text { effectiveness of integrated online mental health assessment-referral- } \\
\text { care in pregnancy to usual prenatal care on prenatal and postnatal } \\
\text { mental health and infant health and development: The Integrated } \\
\text { Maternal Psychosocial Assessment to Care Trial (IMPACT). Trials, } 15 \\
\text { (1), }\end{array}$ & $\begin{array}{l}\text { Data not } \\
\text { available/report } \\
\text { ed }\end{array}$ \\
\hline $\begin{array}{l}\text { 873. Kirby, J. N., \& Sanders, M. R. (2014). A randomized controlled } \\
\text { trial evaluating a parenting program designed specifically for } \\
\text { grandparents. Behaviour research and therapy, } 52,35-44 .\end{array}$ & $\begin{array}{l}\text { No child } \\
\text { internalising } \\
\text { outcomes or not } \\
\text { a major goal; } \\
\text { Not a parenting } \\
\text { intervention }\end{array}$ \\
\hline $\begin{array}{l}\text { 874. Kitzman, H., Olds, D. L., Henderson, C. R., Hanks, C., Cole, R., } \\
\text { Tatelbaum, R., .. Barnard, K. (1997). Effect of Prenatal and Infancy } \\
\text { Home Visitation by Nurses on Pregnancy Outcomes, Childhood } \\
\text { Injuries, and Repeated Childbearing. JAMA: The Journal of the } \\
\text { American Medical Association, 278(8), 644-652. doi: } \\
\text { 10.1001/jama.1997.03550080054039 }\end{array}$ & $\begin{array}{l}\text { No child } \\
\text { internalising } \\
\text { outcomes or not } \\
\text { a major goal }\end{array}$ \\
\hline $\begin{array}{l}\text { 875. Kjellstrand JM and Eddy JM. 2011. Parental incarceration } \\
\text { during childhood, family context, and youth problem behavior across } \\
\text { adolescence. Journal of Offender Rehabilitation. 50(1):18-36. }\end{array}$ & $\begin{array}{l}\text { Not a parenting } \\
\text { intervention }\end{array}$ \\
\hline $\begin{array}{l}\text { 876. Kjøbli J and Hagen KA. 2009. A mediation model of } \\
\text { interparental collaboration, parenting practices, and child } \\
\text { externalizing behavior in a clinical sample. Family Relations: An } \\
\text { Interdisciplinary Journal of Applied Family Studies. 58(3):275-88. }\end{array}$ & Not an RCT \\
\hline $\begin{array}{l}\text { 877. Kjøbli J and Ogden T. 2009. Gender differences in intake } \\
\text { characteristics and behavior change among children in families } \\
\text { receiving parent management training. Children and Youth Services } \\
\text { Review. 31(8):823-30. }\end{array}$ & $\begin{array}{l}\text { No long term } \\
\text { follow-up }\end{array}$ \\
\hline $\begin{array}{l}\text { 878. Kjøbli, J., Nærde, A., Bjørnebekk, G., \& Askeland, E. (2014). } \\
\text { Maternal mental distress influences child outcomes in brief parent } \\
\text { training. Child and Adolescent Mental Health, 19(3), 171-177. }\end{array}$ & $\begin{array}{l}\text { No long term } \\
\text { follow-up }\end{array}$ \\
\hline $\begin{array}{l}\text { 879. Klaassen MA, Veerkamp JS and Hoogstraten J. 2009. Young } \\
\text { children's Oral Health-Related Quality of Life and dental fear after } \\
\text { treatment under general anaesthesia: a randomized controlled trial. } \\
\text { European journal of oral sciences, } 117(3), 273-278 .\end{array}$ & $\begin{array}{l}\text { Not a parenting } \\
\text { intervention }\end{array}$ \\
\hline
\end{tabular}




\begin{tabular}{|c|c|}
\hline $\begin{array}{l}\text { 880. Kleefman, M., Jansen, D. E., \& Reijneveld, S. A. (2011). The } \\
\text { effectiveness of Stepping Stones Triple P: the design of a randomised } \\
\text { controlled trial on a parenting programme regarding children with } \\
\text { mild intellectual disability and psychosocial problems versus care as } \\
\text { usual. BMC public health, } 11(1), 676 .\end{array}$ & $\begin{array}{l}\text { Intervention for } \\
\text { developmental } \\
\text { disorders } \\
\text { (autism, autism } \\
\text { spectrum } \\
\text { disorder), } \\
\text { physical } \\
\text { disabilities, } \\
\text { medical } \\
\text { conditions or } \\
\text { distress about } \\
\text { facing medical } \\
\text { interventions; } \\
\text { Data not } \\
\text { available/report } \\
\text { ed (protocol) }\end{array}$ \\
\hline $\begin{array}{l}\text { 881. Kleiber C, Craft-Rosenberg M and Harper DC. 2001. Parents as } \\
\text { distraction coaches during IV insertion: A randomized study. Journal of } \\
\text { Pain and Symptom Management. 22(4):851-61. }\end{array}$ & $\begin{array}{l}\text { Intervention for } \\
\text { developmental } \\
\text { disorders } \\
\text { (autism, autism } \\
\text { spectrum } \\
\text { disorder), } \\
\text { physical } \\
\text { disabilities, } \\
\text { medical } \\
\text { conditions or } \\
\text { distress about } \\
\text { facing medical } \\
\text { interventions }\end{array}$ \\
\hline $\begin{array}{l}\text { 882. Klein, M. D., Alcamo, A. M., Beck, A. F., O'Toole, J. K., } \\
\text { McLinden, D., Henize, A., \& Kahn, R. S. (2014). Can a video curriculum } \\
\text { on the social determinants of health affect residents' practice and } \\
\text { families' perceptions of care?. Academic pediatrics, 14(2), 159-166. }\end{array}$ & $\begin{array}{l}\text { Not a parenting } \\
\text { intervention }\end{array}$ \\
\hline $\begin{array}{l}\text { 883. Klemetti S, Kinnunen I, Suominen T, Antila H, Vahlberg T, } \\
\text { Grenman R and Leino-Kilpi } \mathrm{H} \text {. 2010. The effect of preoperative } \\
\text { nutritional face-to-face counseling about child's fasting on parental } \\
\text { knowledge, preoperative need-for-information, and anxiety, in } \\
\text { pediatric ambulatory tonsillectomy. Patient Education and Counseling. } \\
\text { 80(1):64-70. }\end{array}$ & $\begin{array}{l}\text { No child } \\
\text { internalising } \\
\text { outcomes or not } \\
\text { a major goal }\end{array}$ \\
\hline $\begin{array}{l}\text { 884. Klingman, A. (1988). Biblioguidance with kindergartners: } \\
\text { Evaluation of a primary prevention program to reduce fear of the dark. } \\
\text { Journal of Clinical Child Psychology, 17, 237-241. }\end{array}$ & $\begin{array}{l}\text { Not a parenting } \\
\text { intervention; No } \\
\text { long term } \\
\text { follow-up }\end{array}$ \\
\hline
\end{tabular}




\begin{tabular}{|c|c|}
\hline $\begin{array}{l}\text { 885. Klosky JL, Garces-Webb DM, Buscemi J, Schum L, Tyc VL and } \\
\text { Merchant TE. 2007. Examination of an interactive-educational } \\
\text { intervention in improving parent and child distress outcomes } \\
\text { associated with pediatric radiation therapy procedures. Children's } \\
\text { Healthcare, 36(4), 323-334. }\end{array}$ & $\begin{array}{l}\text { Intervention for } \\
\text { developmental } \\
\text { disorders } \\
\text { (autism, autism } \\
\text { spectrum } \\
\text { disorder), } \\
\text { physical } \\
\text { disabilities, } \\
\text { medical } \\
\text { conditions or } \\
\text { distress about } \\
\text { facing medical } \\
\text { interventions }\end{array}$ \\
\hline $\begin{array}{l}\text { 886. Knoche, L. L., Sheridan, S. M., Clarke, B. L., Edwards, C. P., } \\
\text { Marvin, C. A., Cline, K. D., \& Kupzyk, K. A. (2012). Getting ready: } \\
\text { Results of a randomized trial of a relationship-focused intervention on } \\
\text { the parent-infant relationship in rural early head start. Infant mental } \\
\text { health journal, 33(5), 439-458. doi:10.1002/imhj.21320 }\end{array}$ & $\begin{array}{l}\text { No child } \\
\text { internalising } \\
\text { outcomes or not } \\
\text { a major goal }\end{array}$ \\
\hline $\begin{array}{l}\text { 887. Knox, M., Burkhart, K., \& Howe, T. (2011). Effects of the ACT } \\
\text { raising safe kids parenting program on children's externalizing } \\
\text { problems. Family Relations: An Interdisciplinary Journal of Applied } \\
\text { Family Studies, 60, 491-503. }\end{array}$ & $\begin{array}{l}\text { Targets } \\
\text { externalising }\end{array}$ \\
\hline $\begin{array}{l}\text { 888. Koh TH, Butow PN, Coory M, Budge D, Collie LA, Whitehall J } \\
\text { and Tattersall MH. 2007. Provision of taped conversations with } \\
\text { neonatologists to mothers of babies in intensive care: randomised } \\
\text { controlled trial. BMJ. 334(7583):28. }\end{array}$ & $\begin{array}{l}\text { No child } \\
\text { internalising } \\
\text { outcomes or not } \\
\text { a major goal }\end{array}$ \\
\hline $\begin{array}{l}\text { 889. Kohl, P. L., Kagotho, J. N., \& Dixon, D. (2011). Parenting } \\
\text { practices among depressed mothers in the child welfare system. Social } \\
\text { Work Research, } 35,215-225 .\end{array}$ & $\begin{array}{l}\text { Not a parenting } \\
\text { intervention }\end{array}$ \\
\hline $\begin{array}{l}\text { 890. Kolko DJ, Dorn LD, Bukstein OG, Pardini D, Holden EA and Hart } \\
\text { J. 2009. Community vs. clinic-based modular treatment of children } \\
\text { with early-onset ODD or CD: A clinical trial with 3-year follow-up. } \\
\text { Journal of Abnormal Child Psychology: An official publication of the } \\
\text { International Society for Research in Child and Adolescent } \\
\text { Psychopathology. } 37(5): 591-609 .\end{array}$ & $\begin{array}{l}\text { Not a parenting } \\
\text { intervention }\end{array}$ \\
\hline $\begin{array}{l}\text { K91. Kolko, D. J., Campo, J. V., Kilbourne, A. M., \& Kelleher, K. } \\
\text { (2012). Doctor-office collaborative care for pediatric behavioral } \\
\text { problems: a preliminary clinical trial. In Archives of pediatrics \& } \\
\text { adolescent medicine (pp. 224-231). }\end{array}$ & $\begin{array}{l}\text { No long term } \\
\text { follow-up }\end{array}$ \\
\hline $\begin{array}{l}\text { 892. Kolko, D. J., Lindhiem, O., Hart, J., \& Bukstein, O. G. (2014). } \\
\text { Evaluation of a booster intervention three years after acute treatment } \\
\text { for early-onset disruptive behavior disorders. Journal of abnormal } \\
\text { child psychology, } 42(3), 383-398 .\end{array}$ & $\begin{array}{l}\text { Not a parenting } \\
\text { intervention }\end{array}$ \\
\hline $\begin{array}{l}\text { 893. Kollins S, Greenhill L, Swanson J, Wigal S, Abikoff H, } \\
\text { McCracken J, Riddle M, McGough J, Vitiello B, Wigal T, Skrobala A, } \\
\text { Posner K, Ghuman J, Davies M, Cunningham C and Bauzo A. 2006. } \\
\text { Rationale, design, and methods of the Preschool ADHD Treatment } \\
\text { Study (PATS). Journal of the American Academy of Child \& Adolescent } \\
\text { Psychiatry, 45(11), 1275-1283. }\end{array}$ & $\begin{array}{l}\text { Data not } \\
\text { available/report } \\
\text { ed (protocol) }\end{array}$ \\
\hline
\end{tabular}




\begin{tabular}{|c|c|}
\hline $\begin{array}{l}\text { 894. Koniak-Griffin D, Anderson NLR, Verzemnieks I and Brecht ML. } \\
\text { 2000. A public health nursing early intervention program for } \\
\text { adolescent mothers: Outcomes from pregnancy through } 6 \text { weeks } \\
\text { postpartum. Nursing Research. 49(3):130-8. }\end{array}$ & $\begin{array}{l}\text { No child } \\
\text { internalising } \\
\text { outcomes or not } \\
\text { a major goal; No } \\
\text { long term } \\
\text { follow-up }\end{array}$ \\
\hline $\begin{array}{l}\text { 895. Kosterman, R., Hawkins, J. D., Spoth, R., Haggerty, K. P., \& Zhu, } \\
\text { K. (1997). Effects of a preventive parent-training intervention on } \\
\text { observed family interactions: Proximal outcomes from Preparing for } \\
\text { the Drug Free Years. Journal of Community Psychology, 25, 337-352. }\end{array}$ & $\begin{array}{l}\text { No child } \\
\text { internalising } \\
\text { outcomes or not } \\
\text { a major goal }\end{array}$ \\
\hline $\begin{array}{l}\text { 896. Kötter, C., Stemmler, M., Lösel, F., Bühler, A., \& Jaursch, S. } \\
\text { (2011). Mittelfristige Effekte des Präventions-programms EFFEKT-E für } \\
\text { emotional belastete Mütter und ihre Kinder unter besonderer } \\
\text { Berücksichtigung psychosozialer Risikofaktoren. Zeitschrift für } \\
\text { Gesundheitspsychologie, 19, 122-133. }\end{array}$ & $\begin{array}{l}\text { Not in English } \\
\text { (duplicate of } \\
\text { Bühler 2011) }\end{array}$ \\
\hline $\begin{array}{l}897 . \quad \text { Koushede, V., Brixval, C. S., Axelsen, S. F., Lindschou, J., } \\
\text { Winkel, P., Maimburg, R. D., \& Due, P. (2013). Group-based antenatal } \\
\text { birth and parent preparation for improving birth outcomes and } \\
\text { parenting resources: study protocol for a randomised trial. Sex Reprod } \\
\text { Healthc, 4(3), 121-126. doi: 10.1016/j.srhc.2013.08.003 }\end{array}$ & $\begin{array}{l}\text { No child } \\
\text { internalising } \\
\text { outcomes or not } \\
\text { a major goal }\end{array}$ \\
\hline $\begin{array}{l}\text { 898. Krain A, Hudson J, Coles M and Kendall P. 2002. The case of } \\
\text { Molly L.: Use of a family cognitive-behavioral treatment for childhood } \\
\text { anxiety. Clinical Case Studies. 1(4):271-98. }\end{array}$ & Not an RCT \\
\hline $\begin{array}{l}\text { 899. Kramer L, Washo CA. Evaluation of a court-mandated } \\
\text { prevention program for divorcing parents: The Children First program. } \\
\text { Fam Relat. 1993;43:179-186 }\end{array}$ & Not an RCT \\
\hline $\begin{array}{l}\text { 900. Kratochwill TR, Elliott SN, Loitz PA, Sladeczek I and Carlson JS. } \\
\text { 2003. Conjoint Consultation Using Self-Administered Manual and } \\
\text { Videotape Parent-Teacher Training: Effects on Children's Behavioral } \\
\text { Difficulties. School Psychology Quarterly. 18(3):269-302. }\end{array}$ & $\begin{array}{l}\text { No long term } \\
\text { follow-up }\end{array}$ \\
\hline $\begin{array}{l}\text { 901. Krishnakumar A and Black MM. 2003. Family processes within } \\
\text { three-generation households and adolescent mothers' satisfaction } \\
\text { with father involvement. J Fam Psychol. 17(4):488-98. }\end{array}$ & Not an RCT \\
\hline $\begin{array}{l}\text { 902. Kronmüller, K. T., Postelnicu, I., Hartmann, M., Stefini, A., } \\
\text { Geiser-Elze, A., Gerhold, M., ... \& Winkelmann, K. (2005). [Efficacy of } \\
\text { psychodynamic short-term psychotherapy for children and } \\
\text { adolescents with anxiety disorders]. Praxis der Kinderpsychologie und } \\
\text { Kinderpsychiatrie, 54(7), 559-577. }\end{array}$ & $\begin{array}{l}\text { Not a parenting } \\
\text { intervention }\end{array}$ \\
\hline $\begin{array}{l}\text { 903. Kumar, M. M., Cowan, H. R., Kaufman, M., \& Hick, K. M. } \\
\text { (2014). Reading and adolescent parents: A clinical reading } \\
\text { intervention. [Conference Abstract]. Journal of Adolescent Health, 1), } \\
\text { S34-S35. doi: http://dx.doi.org/10.1016/j.jadohealth.2013.10.082 }\end{array}$ & $\begin{array}{l}\text { No child } \\
\text { internalising } \\
\text { outcomes or not } \\
\text { a major goal }\end{array}$ \\
\hline $\begin{array}{l}\text { 904. Kumpfer, K. L., Pinyuchon, M., de Melo, A. T., \& Whiteside, H. } \\
\text { O. (2008). Cultural Adaptation Process for International Dissemination } \\
\text { of the Strengthening Families Program. Evaluation \& the Health } \\
\text { Professions, 31, 226-239. }\end{array}$ & Not an RCT \\
\hline $\begin{array}{l}\text { 905. Kurz H, Neunteufl R, Eichler F, Urschitz M and Tiefenthaler M. } \\
\text { 2002. Does professional counseling improve infant home monitoring? } \\
\text { Evaluation of an intensive instruction program for families using home } \\
\text { monitoring on their babies. Wien Klin Wochenschr. 114(17-18):801-6. }\end{array}$ & $\begin{array}{l}\text { No child } \\
\text { internalising } \\
\text { outcomes or not } \\
\text { a major goal }\end{array}$ \\
\hline
\end{tabular}




\begin{tabular}{|c|c|}
\hline $\begin{array}{l}\text { 906. Kuschel, A., Heinrichs, N., \& Hahlweg, K. (2009). Is a preventive } \\
\text { parenting program effective in reducing a child's externalizing } \\
\text { behavior? European Journal of Developmental Science, 3(3), 299-303. }\end{array}$ & $\begin{array}{l}\text { No child } \\
\text { internalising } \\
\text { outcomes or not } \\
\text { a major goal }\end{array}$ \\
\hline $\begin{array}{l}\text { 907. Kushnir, J., \& Sadeh, A. (2012). Assessment of brief } \\
\text { interventions for nighttime fears in preschool children. European } \\
\text { journal of pediatrics, 171(1), 67-75. doi:10.1007/s00431-011-1488-4 }\end{array}$ & $\begin{array}{l}\text { No child } \\
\text { internalising } \\
\text { outcomes or not } \\
\text { a major goal }\end{array}$ \\
\hline $\begin{array}{l}\text { 908. Kutcher S, Aman M, Brooks SJ, Buitelaar J, van Daalen E, } \\
\text { Fegert Jr, Findling RL, Fisman S, Greenhill LL, Huss M, Kusumakar V, } \\
\text { Pine D, Taylor E and Tyano S. 2004. International consensus statement } \\
\text { on attention-deficit/hyperactivity disorder (ADHD) and disruptive } \\
\text { behaviour disorders (DBDs): Clinical implications and treatment } \\
\text { practice suggestions. European Neuropsychopharmacology. 14(1):11- } \\
28 .\end{array}$ & Not an RCT \\
\hline $\begin{array}{l}\text { 909. Kwon, J. A., Lee, M., Yoo, K. B., \& Park, E. C. (2013). Does the } \\
\text { duration and time of sleep increase the risk of allergic rhinitis? Results } \\
\text { of the 6-year nationwide Korea youth risk behavior web-based survey. } \\
\text { PLoS One, 8(8), e72507. doi: } 10.1371 / \text { journal.pone.0072507 }\end{array}$ & $\begin{array}{l}\text { Not a parenting } \\
\text { intervention }\end{array}$ \\
\hline $\begin{array}{l}\text { 910. Kwon, K., Kim, E., \& Sheridan, S. (2012). Behavioral } \\
\text { competence and academic functioning among early elementary } \\
\text { children with externalizing problems. School Psychology Review, 41, } \\
123-140 .\end{array}$ & Review \\
\hline $\begin{array}{l}\text { 911. Lachenmyer, L. L., Anderson, J. J., Clayton, D. B., Thomas, J. C., } \\
\text { Pope, I. J., Adams, M. C., .. Tanaka, S. T. (2013). Analysis of an } \\
\text { intervention to reduce parental anxiety prior to voiding } \\
\text { cystourethrogram. Journal of pediatric urology, 9(6 part b), 1223- } \\
\text { 1228. doi:10.1016/j.jpurol.2013.05.015 }\end{array}$ & $\begin{array}{l}\text { Intervention for } \\
\text { developmental } \\
\text { disorders } \\
\text { (autism, autism } \\
\text { spectrum } \\
\text { disorder), } \\
\text { physical } \\
\text { disabilities, } \\
\text { medical } \\
\text { conditions or } \\
\text { distress about } \\
\text { facing medical } \\
\text { interventions }\end{array}$ \\
\hline $\begin{array}{l}\text { 912. LaFreniere, P. J., \& Capuano, F. (1997). Preventive intervention } \\
\text { as means of clarifying direction of effects in socialization: Anxious- } \\
\text { withdrawn preschoolers case. Development and Psychopathology, 9, } \\
\text { 551-564. }\end{array}$ & $\begin{array}{l}\text { No long term } \\
\text { follow-up }\end{array}$ \\
\hline $\begin{array}{l}\text { 913. Lam P, Hiscock H, Wake M. Outcomes of infant sleep } \\
\text { problems: a longitudinal study of sleep, behavior, and maternal } \\
\text { wellbeing. Pediatrics. 2003;111(3). }\end{array}$ & $\begin{array}{l}\text { No child } \\
\text { internalising } \\
\text { outcomes or not } \\
\text { a major goal }\end{array}$ \\
\hline $\begin{array}{l}\text { 914. Lambert, K., \& Spinath, B. (2013). [Changes in psychological } \\
\text { stress after interventions in children and adolescents with } \\
\text { mathematical learning disabilities]. Z Kinder Jugendpsychiatr } \\
\text { Psychother, 41(1), 23-34. doi: 10.1024/1422-4917/a000207 }\end{array}$ & $\begin{array}{l}\text { Not a parenting } \\
\text { intervention }\end{array}$ \\
\hline
\end{tabular}




\begin{tabular}{|c|c|}
\hline $\begin{array}{l}915 . \quad \text { Landry, S. H., Smith, K. E., Swank, P. R., \& Guttentag, C. (2008). } \\
\text { A responsive parenting intervention: the optimal timing across early } \\
\text { childhood for impacting maternal behaviors and child outcomes. } \\
\text { Developmental psychology, (5), 1335-1353. }\end{array}$ & $\begin{array}{l}\text { No child } \\
\text { internalising } \\
\text { outcomes or not } \\
\text { a major goal; } \\
\text { Follow-up less } \\
\text { than } 6 \text { months }\end{array}$ \\
\hline $\begin{array}{l}\text { 916. Landsem, I. P., Handegård, B. H., Tunby, J., Ulvund, S. E., \& } \\
\text { Rønning, J. A. (2014). Early intervention program reduces stress in } \\
\text { parents of pre-terms during childhood, a randomized controlled trial. } \\
\text { Trials, 15(1), } 387 .\end{array}$ & $\begin{array}{l}\text { No child } \\
\text { internalising } \\
\text { outcomes or not } \\
\text { a major goal }\end{array}$ \\
\hline $\begin{array}{l}\text { 917. Landsem, I. P., Handegård, B. H., Ulvund, S. E., Kaaresen, P. I., } \\
\text { \& Rønning, J. A. (2015). Early intervention influences positively quality } \\
\text { of life as reported by prematurely born children at age nine and their } \\
\text { parents; a randomized clinical trial. Health and quality of life } \\
\text { outcomes, 13(1), } 25 \text {. }\end{array}$ & $\begin{array}{l}\text { Secondary } \\
\text { analysis }\end{array}$ \\
\hline $\begin{array}{l}\text { 918. Langley JM, Halperin SA and Smith B. 2003. A pilot study to } \\
\text { quantify parental anxiety associated with enrollment of an infant or } \\
\text { toddler in a phase III vaccine trial. Vaccine. 21(25-26):3863-6. }\end{array}$ & $\begin{array}{l}\text { No child } \\
\text { internalising } \\
\text { outcomes or not } \\
\text { a major goal }\end{array}$ \\
\hline $\begin{array}{l}\text { 919. Lanning, B. A., Baier, M. E. M., Ivey-Hatz, J., Krenek, N., \& } \\
\text { Tubbs, J. D. (2014). Effects of Equine Assisted Activities on Autism } \\
\text { Spectrum Disorder. Journal of autism and developmental disorders, } \\
\text { 44(8), 1897-1907. }\end{array}$ & $\begin{array}{l}\text { Intervention for } \\
\text { developmental } \\
\text { disorders } \\
\text { (autism, autism } \\
\text { spectrum } \\
\text { disorder), } \\
\text { physical } \\
\text { disabilities, } \\
\text { medical } \\
\text { conditions or } \\
\text { distress about } \\
\text { facing medical } \\
\text { interventions } \\
\end{array}$ \\
\hline $\begin{array}{l}\text { 920. Lansford JE, Criss MM, Laird RD, Shaw DS, Pettit GS, Bates JE } \\
\text { and Dodge KA. 2011. Reciprocal relations between parents' physical } \\
\text { discipline and children's externalizing behavior during middle } \\
\text { childhood and adolescence. Development and Psychopathology. } \\
\text { 23(1):225-38. }\end{array}$ & $\begin{array}{l}\text { Not a parenting } \\
\text { intervention }\end{array}$ \\
\hline $\begin{array}{l}\text { 921. Lardner DR, Dick BD, Psych R and Crawford S. 2010. The } \\
\text { effects of parental presence in the postanesthetic care unit on } \\
\text { children's postoperative behavior: a prospective, randomized, } \\
\text { controlled study. Anesth Analg. 110(4):1102-8. }\end{array}$ & $\begin{array}{l}\text { Intervention for } \\
\text { developmental } \\
\text { disorders } \\
\text { (autism, autism } \\
\text { spectrum } \\
\text { disorder), } \\
\text { physical } \\
\text { disabilities, } \\
\text { medical } \\
\text { conditions or } \\
\text { distress about } \\
\text { facing medical } \\
\text { interventions }\end{array}$ \\
\hline
\end{tabular}




\begin{tabular}{|c|c|}
\hline $\begin{array}{l}\text { 922. Larsson, B., Fossum, S., Clifford, G., Drugli, M. B., Handegård, } \\
\text { B. H., \& Mørch, W. T. (2009). Treatment of oppositional defiant and } \\
\text { conduct problems in young Norwegian children : results of a } \\
\text { randomized controlled trial. European child \& adolescent psychiatry, } \\
\text { (1), 42-52. }\end{array}$ & $\begin{array}{l}\text { Targets } \\
\text { externalising } \\
\text { (ODD or CD) }\end{array}$ \\
\hline $\begin{array}{l}923 . \quad \text { Last, C. G., Hansen, C., \& Franco, N. (1998). Cognitive- } \\
\text { behavioral treatment of school phobia. Journal of the American } \\
\text { Academy of Child and Adolescent Psychiatry, 37(4), 404-411. }\end{array}$ & $\begin{array}{l}\text { Not a parenting } \\
\text { intervention }\end{array}$ \\
\hline $\begin{array}{l}\text { 924. Lau, A. S., Fung, J. J., \& Yung, V. (2010). Group parent training } \\
\text { with immigrant chinese families: enhancing engagement and } \\
\text { augmenting skills training. Journal Of Clinical Psychology, 66, 880-894. }\end{array}$ & Not an RCT \\
\hline $\begin{array}{l}\text { 925. Lau, A. S., Fung, J. J., Ho, L. Y., Liu, L. L., \& Gudiño, O. G. (2011). } \\
\text { Parent training with high-risk immigrant Chinese families: A pilot } \\
\text { group randomized trial yielding practice-based evidence. Behav Ther, } \\
42,413-426 .\end{array}$ & $\begin{array}{l}\text { Follow-up data } \\
\text { collected only in } \\
\text { active group }\end{array}$ \\
\hline $\begin{array}{l}\text { 926. Lau, J. Y., Pettit, E., \& Creswell, C. (2013). Reducing children's } \\
\text { social anxiety symptoms: exploring a novel parent-administered } \\
\text { cognitive bias modification training intervention. Behaviour research } \\
\text { and therapy, 51(7), 333-337. doi:10.1016/j.brat.2013.03.008 }\end{array}$ & Not an RCT \\
\hline $\begin{array}{l}\text { 927. Lavigne, J. V., Lebailly, S. A., Gouze, K. R., Cicchetti, C., Jessup, } \\
\text { B. W., Arend, R., et al. (2008). Predictor and moderator effects in the } \\
\text { treatment of oppositional defiant disorder in pediatric primary care. J } \\
\text { Pediatr Psychol, 33(5), 462-472. doi: jsm075 }\end{array}$ & $\begin{array}{l}\text { Targets } \\
\text { externalising } \\
\text { (ODD) }\end{array}$ \\
\hline $\begin{array}{l}\text { 928. Lavigne, J. V., Lebailly, S. A., Gouze, K. R., Cicchetti, C., Pochyly, } \\
\text { J., Arend, R., et al. (2008). Treating oppositional defiant disorder in } \\
\text { primary care: a comparison of three models. J Pediatr Psychol, 33(5), } \\
\text { 449-461. doi: jsm074 }\end{array}$ & $\begin{array}{l}\text { Targets } \\
\text { externalising }\end{array}$ \\
\hline $\begin{array}{l}\text { 929. Lavoie M-P, Monfette M-E and Boisvert J-M. } 2005 . \\
\text { Comparative efficacy of in vivo exposure and social skils training in } \\
\text { adolescents with social phobia. Revue Francophone de Clinique } \\
\text { Comportementale et Cognitive. 10(3):7-16. }\end{array}$ & $\begin{array}{l}\text { Not a parenting } \\
\text { intervention }\end{array}$ \\
\hline $\begin{array}{l}\text { 930. Lawes G. Individual parent training implemented by nursery } \\
\text { nurses: evaluation of a programme for mothers of pre-school children. } \\
\text { Behav Psychother 1992;20:239-56. }\end{array}$ & $\begin{array}{l}\text { No long term } \\
\text { follow-up }\end{array}$ \\
\hline $\begin{array}{l}\text { 931. Lay B, Blanz B and Schmidt MH. 2001. Effectiveness of home } \\
\text { treatment in children and adolescents with externalizing psychiatric } \\
\text { disorders. European Child \& Adolescent Psychiatry. 10(5):I80-190. }\end{array}$ & Not an RCT \\
\hline $\begin{array}{l}\text { 932. Layne AE, Bernstein GA, Egan EA and Kushner MG. } 2003 . \\
\text { Predictors of treatment response in anxious-depressed adolescents } \\
\text { with school refusal. Journal of the American Academy of Child \& } \\
\text { Adolescent Psychiatry. 42(3):319-26. }\end{array}$ & $\begin{array}{l}\text { Not a parenting } \\
\text { intervention }\end{array}$ \\
\hline $\begin{array}{l}\text { 933. Lazer, S. (2013). Do callous and unemotional traits in young } \\
\text { children predict change in parenting and child behavior in response to } \\
\text { behavioral parent training? Dissertation Abstracts International: } \\
\text { Section B: The Sciences and Engineering, 73 (8-B E). }\end{array}$ & $\begin{array}{l}\text { No child } \\
\text { internalising } \\
\text { outcomes or not } \\
\text { a major goal }\end{array}$ \\
\hline $\begin{array}{l}\text { 934. Le H-N, Zmuda J, Perry DF and MuV士oz RF. 2010. Transforming } \\
\text { an evidence-based intervention to prevent perinatal depression for } \\
\text { low-income Latina immigrants. American Journal of Orthopsychiatry. } \\
\text { 80(1):34-45. }\end{array}$ & $\begin{array}{l}\text { Not a parenting } \\
\text { intervention }\end{array}$ \\
\hline
\end{tabular}




\begin{tabular}{|c|c|}
\hline $\begin{array}{l}\text { 935. Leathers SJ. 2006. Placement disruption and negative } \\
\text { placement outcomes among adolescents in long-term foster care: The } \\
\text { role of behavior problems. Child Abuse \& Neglect. 30(3):307-24. }\end{array}$ & $\begin{array}{l}\text { Not a parenting } \\
\text { intervention }\end{array}$ \\
\hline $\begin{array}{l}\text { 936. Leathers, S. J., Spielfogel, J. E., Gleeson, J. P., \& Rolock, N. } \\
\text { (2012). Behavior problems, foster home integration, and evidence- } \\
\text { based behavioral interventions: What predicts adoption of foster } \\
\text { children? Children and Youth Services Review, 34, 891-899. }\end{array}$ & $\begin{array}{l}\text { No child } \\
\text { internalising } \\
\text { outcomes or not } \\
\text { a major goal }\end{array}$ \\
\hline $\begin{array}{l}\text { 937. Leathers, S. J., Spielfogel, J. E., McMeel, L. S., \& Atkins, M. S. } \\
\text { (2011). Use of a parent management training intervention with urban } \\
\text { foster parents: A pilot study. Children and Youth Services Review, 33, } \\
\text { 1270-1279. }\end{array}$ & $\begin{array}{l}\text { Not an RCT; } \\
\text { Follow-up less } \\
\text { than } 6 \text { months }\end{array}$ \\
\hline $\begin{array}{l}\text { 938. Lee ML, Cohen SE, Stuber ML and Nade K. 1994. Parent-child } \\
\text { interactions with pediatric bone marrow transplant patients. Journal } \\
\text { of Psychosocial Oncology. 12(4):43-60. }\end{array}$ & Not an RCT \\
\hline $\begin{array}{l}\text { 939. Lee, K. (2003). Maternal Coping Skills as a Moderator Between } \\
\text { Depression and Stressful Life Events: Effects on Children's Behavioral } \\
\text { Problems in an Intervention Program. Journal of Child and Family } \\
\text { Studies, 12(4), 425-437. doi:10.1023/a:1026064007253 }\end{array}$ & $\begin{array}{l}\text { No long term } \\
\text { follow-up } \\
\text { (intervention } \\
\text { lasts for } 36 \\
\text { months) }\end{array}$ \\
\hline $\begin{array}{l}940 . \quad \text { Lee, S. J., Detels, R., Rotheram-Borus, M. J., \& Duan, N. (2007). } \\
\text { The effect of social support on mental and behavioral outcomes } \\
\text { among adolescents with parents with HIV/AIDS. American journal of } \\
\text { public health, (10), 1820-1826. }\end{array}$ & $\begin{array}{l}\text { Secondary } \\
\text { analysis (re- } \\
\text { analysis of data } \\
\text { in Rotheram- } \\
\text { Borus } 2001 \text { using } \\
\text { SEM, no new } \\
\text { data) }\end{array}$ \\
\hline $\begin{array}{l}\text { 941. Legerstee, J. S., Huizink, A. C., van Gastel, W., Liber, J. M., } \\
\text { Treffers, P. D., Verhulst, F. C., et al. (2008). Maternal anxiety predicts } \\
\text { favourable treatment outcomes in anxiety-disordered adolescents. } \\
\text { Acta Psychiatr Scand, 117(4), 289-298. doi: ACP1161 }\end{array}$ & $\begin{array}{l}\text { Parent } \\
\text { component too } \\
\text { small }\end{array}$ \\
\hline $\begin{array}{l}\text { 942. Lehrer JA, Buka S, Gortmaker S and Shrier LA. } 2006 . \\
\text { Depressive symptomatology as a predictor of exposure to intimate } \\
\text { partner violence among US female adolescents and young adults. } \\
\text { Archives of Pediatrics and Adolescent Medicine. } 160(3): 270-6 .\end{array}$ & Not an RCT \\
\hline $\begin{array}{l}\text { 943. Leifer, A. D., et al. (1972). EFFECTS OF MOTHER-INFANT } \\
\text { SEPARATION ON MATERNAL ATTACHMENT BEHAVIOR. Child } \\
\text { Development 43(4): 1203-1218. }\end{array}$ & $\begin{array}{l}\text { Not an RCT; Not } \\
\text { a parenting } \\
\text { intervention }\end{array}$ \\
\hline $\begin{array}{l}944 . \quad \text { Leitch, D. B. (1999). Mother-infant interaction: achieving } \\
\text { synchrony. Nursing research, (1), 55-58. }\end{array}$ & $\begin{array}{l}\text { No long term } \\
\text { follow-up }\end{array}$ \\
\hline $\begin{array}{l}\text { 945. Lemanek, K. L., Ranalli, M., \& Lukens, C. (2009). A randomized } \\
\text { controlled trial of massage therapy in children with sickle cell disease. } \\
\text { J Pediatr Psychol, 34(10), 1091-1096. doi: jsp015 }\end{array}$ & $\begin{array}{l}\text { No long term } \\
\text { follow-up }\end{array}$ \\
\hline $\begin{array}{l}\text { 946. Lemmon CR, Ludwig DA, Howe CA, Ferguson-Smith A and } \\
\text { Barbeau P. 2007. Correlates of adherence to a physical activity } \\
\text { program in young African-American girls. Obesity (Silver Spring). } \\
\text { 15(3):695-703. }\end{array}$ & $\begin{array}{l}\text { Not a parenting } \\
\text { intervention }\end{array}$ \\
\hline $\begin{array}{l}\text { 947. Lenior, M. E., Dingemans, P. M. A. J., Linszen, D. H., de Haan, } \\
\text { L., \& Schene, A. H. (2001). Social functioning and the course of early- } \\
\text { onset schizophrenia: Five-year follow-up of a psychosocial } \\
\text { intervention. British Journal of Psychiatry, 179, 53-58. doi: } \\
\text { 10.1192/bjp.179.1.53 }\end{array}$ & $\begin{array}{l}\text { Not in children } 0 \\
-18\end{array}$ \\
\hline
\end{tabular}




\begin{tabular}{|c|c|}
\hline $\begin{array}{l}\text { 948. Lenze SN, Pautsch J and Luby J. 2011. Parent-child interaction } \\
\text { therapy emotion development: A novel treatment for depression in } \\
\text { preschool children. Depression and Anxiety. 28(2):153-9. }\end{array}$ & Not an RCT \\
\hline $\begin{array}{l}\text { 949. Leonard NR, Gwadz MV, Cleland CM, Vekaria PC and Ferns B. } \\
\text { 2008. Maternal substance use and HIV status: adolescent risk and } \\
\text { resilience. Journal of adolescence, 31(3), 389-405. }\end{array}$ & Not an RCT \\
\hline $\begin{array}{l}\text { 950. Leong J, Cobham VE, de Groot J, McDermott B. Comparing } \\
\text { different modes of delivery: A pilot evaluation of a family-focused, } \\
\text { cognitive-behavioral intervention for anxiety-disordered children. } \\
\text { European Child \& Adolescent Psychiatry. 2009 Apr;18(4):231-9. }\end{array}$ & $\begin{array}{l}\text { Comparison } \\
\text { condition not a } \\
\text { minimal control; } \\
\text { Parent } \\
\text { component too } \\
\text { small }\end{array}$ \\
\hline $\begin{array}{l}\text { 951. Leschied AW, Chiodo D, Whitehead PC and Hurley D. } 2005 . \\
\text { The relationship between maternal depression and child outcomes in } \\
\text { a child welfare sample: Implications for treatment and policy. Child \& } \\
\text { Family Social Work. 10(4):281-91. }\end{array}$ & $\begin{array}{l}\text { Not a parenting } \\
\text { intervention }\end{array}$ \\
\hline $\begin{array}{l}\text { 952. Lesser, J., Koniak-Griffin, D., Huang, R., Takayanagi, S., \& } \\
\text { Cumberland, W. G. (2009). Parental protectiveness and unprotected } \\
\text { sexual activity among Latino adolescent mothers and fathers. AIDS } \\
\text { education and prevention : official publication of the International } \\
\text { Society for AIDS Education, (5 Suppl), 88-102. }\end{array}$ & $\begin{array}{l}\text { No child } \\
\text { internalising } \\
\text { outcomes or not } \\
\text { a major goal }\end{array}$ \\
\hline $\begin{array}{l}\text { 953. Lester P, Rotheram-Borus MJ, Lee S-J, Comulada S, Cantwell S, } \\
\text { Wu N and Lin YY. 2006. Rates and predictors of anxiety and depressive } \\
\text { disorders in adolescents of parents with HIV. Vulnerable Children and } \\
\text { Youth Studies. 1(1):81-101. }\end{array}$ & $\begin{array}{l}\text { Not a parenting } \\
\text { intervention }\end{array}$ \\
\hline $\begin{array}{l}\text { 954. Leung DW and Slep AMS. 2006. Predicting inept discipline: The } \\
\text { role of parental depressive symptoms, anger, and attributions. Journal } \\
\text { of Consulting and Clinical Psychology. 74(3):524-34. }\end{array}$ & $\begin{array}{l}\text { Not a parenting } \\
\text { intervention }\end{array}$ \\
\hline $\begin{array}{l}\text { 955. Leung, C., Sanders, M. R., Leung, S., Mak, R., \& Lau, J. (2003). } \\
\text { An Outcome Evaluation of the Implementation of the Triple P-Positive } \\
\text { Parenting Program in Hong Kong. Family Process, 42(4), 531-544. doi: } \\
\text { 10.1111/j.1545-5300.2003.00531.x }\end{array}$ & $\begin{array}{l}\text { No long term } \\
\text { follow-up }\end{array}$ \\
\hline $\begin{array}{l}\text { 956. Levy, R. L., Langer, S. L., Romano, J. M., Labus, J., Walker, L. S., } \\
\text { Murphy, T. B., ... \& Whitehead, W. E. (2014). Cognitive mediators of } \\
\text { treatment outcomes in pediatric functional abdominal pain. The } \\
\text { Clinical journal of pain, 30(12), 1033-1043. }\end{array}$ & $\begin{array}{l}\text { Intervention for } \\
\text { developmental } \\
\text { disorders } \\
\text { (autism, autism } \\
\text { spectrum } \\
\text { disorder), } \\
\text { physical } \\
\text { disabilities, } \\
\text { medical } \\
\text { conditions or } \\
\text { distress about } \\
\text { facing medical } \\
\text { interventions }\end{array}$ \\
\hline $\begin{array}{l}\text { 957. Levy-Frank, I., Hasson-Ohayon, I., Kravetz, S., \& Roe, D. (2011). } \\
\text { Family psychoeducation and therapeutic alliance focused } \\
\text { interventions for parents of a daughter or son with a severe mental } \\
\text { illness. Psychiatry research, 189(2), 173-179. }\end{array}$ & $\begin{array}{l}\text { No long term } \\
\text { follow-up }\end{array}$ \\
\hline
\end{tabular}




\begin{tabular}{|c|c|}
\hline $\begin{array}{l}\text { 958. Lewin, A. B., Peris, T. S., De Nadai, A. S., McCracken, J. T., \& } \\
\text { Piacentini, J. (2012). Agreement between therapists, parents, patients, } \\
\text { and independent evaluators on clinical improvement in pediatric } \\
\text { obsessive-compulsive disorder. J Consult Clin Psychol, 80(6), 1103- } \\
\text { 1107. doi: } 10.1037 / \text { a0029991 }\end{array}$ & $\begin{array}{l}\text { Not a parenting } \\
\text { intervention }\end{array}$ \\
\hline $\begin{array}{l}\text { 959. Lewinsohn, P. M., Clarke, G. N., Hops, H., \& Andrews, J. (1990). } \\
\text { Cognitive-behavioral treatment for depressed adolescents. Behav. } \\
\text { Ther., (4), 385-401. }\end{array}$ & $\begin{array}{l}\text { Parent } \\
\text { component too } \\
\text { small }\end{array}$ \\
\hline $\begin{array}{l}\text { 960. Lewis CC, Pantell RH and Sharp L. 1991. Increasing patient } \\
\text { knowledge, satisfaction, and involvement: randomized trial of a } \\
\text { communication intervention. Pediatrics. } 88(2): 351-8 .\end{array}$ & $\begin{array}{l}\text { Not a parenting } \\
\text { intervention }\end{array}$ \\
\hline $\begin{array}{l}\text { 961. Lewis FM, Casey SM, Brandt PA, Shands ME and Zahlis EH. } \\
\text { 2006. The enhancing connections program: pilot study of a cognitive- } \\
\text { behavioral intervention for mothers and children affected by breast } \\
\text { cancer. Psychooncology. 15(6):486-97. }\end{array}$ & Not an RCT \\
\hline $\begin{array}{l}\text { 962. Lewis MA, Hatton CL, Salas I, Leake B and Chiofalo N. } 1991 . \\
\text { Impact of the Children's Epilepsy Program on parents. Epilepsia. } \\
\text { 32(3):365-74. }\end{array}$ & $\begin{array}{l}\text { Intervention for } \\
\text { developmental } \\
\text { disorders } \\
\text { (autism, autism } \\
\text { spectrum } \\
\text { disorder), } \\
\text { physical } \\
\text { disabilities, } \\
\text { medical } \\
\text { conditions or } \\
\text { distress about } \\
\text { facing medical } \\
\text { interventions }\end{array}$ \\
\hline $\begin{array}{l}\text { 963. Lewis-Jones S. 2006. Quality of life and childhood atopic } \\
\text { dermatitis: The misery of living with childhood eczema. International } \\
\text { Journal of Clinical Practice. 60(8):984-92. }\end{array}$ & Review \\
\hline $\begin{array}{l}\text { 964. Li G and Dai X-Y. 2009. Control study of cognitive-behavior } \\
\text { therapy in adolescents with Internet addiction disorder. Chinese } \\
\text { Mental Health Journal. 23(7):457-70. }\end{array}$ & $\begin{array}{l}\text { Not a parenting } \\
\text { intervention }\end{array}$ \\
\hline $\begin{array}{l}\text { 965. Li HC and Lopez V. 2008. Effectiveness and appropriateness of } \\
\text { therapeutic play intervention in preparing children for surgery: a } \\
\text { randomized controlled trial study. J Spec Pediatr Nurs. 13(2):63-73. }\end{array}$ & $\begin{array}{l}\text { Intervention for } \\
\text { developmental } \\
\text { disorders } \\
\text { (autism, autism } \\
\text { spectrum } \\
\text { disorder), } \\
\text { physical } \\
\text { disabilities, } \\
\text { medical } \\
\text { conditions or } \\
\text { distress about } \\
\text { facing medical } \\
\text { interventions }\end{array}$ \\
\hline
\end{tabular}




\begin{tabular}{|c|c|}
\hline $\begin{array}{l}\text { 966. Li HC, Lopez V and Lee TL. 2007. Psychoeducational } \\
\text { preparation of children for surgery: the importance of parental } \\
\text { involvement. Patient Educ Couns. 65(1):34-41. }\end{array}$ & $\begin{array}{l}\text { Intervention for } \\
\text { developmental } \\
\text { disorders } \\
\text { (autism, autism } \\
\text { spectrum } \\
\text { disorder), } \\
\text { physical } \\
\text { disabilities, } \\
\text { medical } \\
\text { conditions or } \\
\text { distress about } \\
\text { facing medical } \\
\text { interventions }\end{array}$ \\
\hline $\begin{array}{l}\text { 967. Li Q, Guang Y and Yue G. 2005. [Influence of rehabilitation } \\
\text { education on the psychological status of parents of children with } \\
\text { schizophrenia]. Zhongguo Linchuang Kangfu. 8:98-9. }\end{array}$ & $\begin{array}{l}\text { No child } \\
\text { internalising } \\
\text { outcomes or not } \\
\text { a major goal }\end{array}$ \\
\hline $\begin{array}{l}\text { 968. Liber JM, McLeod BD, Van Widenfelt BM, Goedhart AW, van } \\
\text { der Leeden AJM, Utens EMWJ and Treffers PDA. 2010. Examining the } \\
\text { relation between the therapeutic alliance, treatment adherence, and } \\
\text { outcome of cognitive behavioral therapy for children with anxiety } \\
\text { disorders. Behavior Therapy. } 41(2): 172-86 \text {. }\end{array}$ & $\begin{array}{l}\text { Not a parenting } \\
\text { intervention }\end{array}$ \\
\hline $\begin{array}{l}\text { 969. Liber JM, van Widenfelt BM, Goedhart AW, Utens EMWJ, van } \\
\text { der Leeden AJM, Markus MT and Treffers PDA. 2008. Parenting and } \\
\text { parental anxiety and depression as predictors of treatment outcome } \\
\text { for childhood anxiety disorders: Has the role of fathers been } \\
\text { underestimated? Journal of Clinical Child and Adolescent Psychology. } \\
\text { 37(4):747-58. }\end{array}$ & $\begin{array}{l}\text { Not a parenting } \\
\text { intervention }\end{array}$ \\
\hline $\begin{array}{l}\text { 970. Liber JM, Van Widenfelt BM, Utens EMWJ, Ferdinand RF, Van } \\
\text { der Leeden AJM, Van Gastel W and Treffers PDA. 2008. No differences } \\
\text { between group versus individual treatment of childhood anxiety } \\
\text { disorders in a randomised clinical trial. Journal of Child Psychology and } \\
\text { Psychiatry. 49(8):886-93. }\end{array}$ & $\begin{array}{l}\text { Not a parenting } \\
\text { intervention }\end{array}$ \\
\hline $\begin{array}{l}\text { 971. Lieberman, A. F., Van Horn, P., \& Ippen, C. G. (2005). Toward } \\
\text { evidence-based treatment: child-parent psychotherapy with } \\
\text { preschoolers exposed to marital violence. J Am Acad Child Adolesc } \\
\text { Psychiatry, 44(12), 1241-1248. doi:S0890-8567(09)62235-8 }\end{array}$ & $\begin{array}{l}\text { No long term } \\
\text { follow-up }\end{array}$ \\
\hline $\begin{array}{l}\text { 972. Lieberman, A. F., Weston, D. R., \& Pawl, J. H. (1991). } \\
\text { Preventive intervention and outcome with anxiously attached dyads. } \\
\text { Child Development, 62, 199-209. }\end{array}$ & $\begin{array}{l}\text { No long term } \\
\text { follow-up }\end{array}$ \\
\hline $\begin{array}{l}\text { 973. Lieu TA, Glauber JH, Fuentes-Afflick E and Lo B. 1994. Effects } \\
\text { of vaccine information pamphlets on parents' attitudes. Archives of } \\
\text { pediatrics \& adolescent medicine, 148(9), 921-925. }\end{array}$ & $\begin{array}{l}\text { No child } \\
\text { internalising } \\
\text { outcomes or not } \\
\text { a major goal }\end{array}$ \\
\hline $\begin{array}{l}\text { 974. Liew, J., Johnson, A. Y., Smith, T. R., \& Thoemmes, F. (2011). } \\
\text { Parental expressivity, child physiological and behavioral regulation, } \\
\text { and child adjustment: Testing a three-path mediation model. Early } \\
\text { Education and Development, 22, 549-573. }\end{array}$ & $\begin{array}{l}\text { Not a parenting } \\
\text { intervention }\end{array}$ \\
\hline
\end{tabular}




\begin{tabular}{|c|c|}
\hline $\begin{array}{l}975 . \quad \text { Liles, B. D., Newman, E., LaGasse, L. L., Derauf, C., Shah, R., } \\
\text { Smith, L. M., Arria, A. M., Huestis, M. A., Haning, W., Strauss, A., } \\
\text { DellaGrotta, S., Dansereau, L. M., Neal, C., \& Lester, B. M. (2012). } \\
\text { Perceived child behavior problems, parenting stress, and maternal } \\
\text { depressive symptoms among prenatal methamphetamine users. Child } \\
\text { Psychiatry and Human Development, 43, 943-957. }\end{array}$ & $\begin{array}{l}\text { Not a parenting } \\
\text { intervention }\end{array}$ \\
\hline $\begin{array}{l}\text { 976. Lillehoj CJ, Trudeau L, Spoth R and Madon S. } 2005 . \\
\text { Externalizing behaviors as predictors of substance initiation } \\
\text { trajectories among rural adolescents. Journal of Adolescent Health. } \\
\text { 37(6):493-501. }\end{array}$ & $\begin{array}{l}\text { Not a parenting } \\
\text { intervention }\end{array}$ \\
\hline $\begin{array}{l}\text { 977. Lillehoj CJ, Trudeau L, Spoth R and Wickrama KAS. } 2004 . \\
\text { Internalizing, Social Competence, and Substance Initiation: Influence } \\
\text { of Gender Moderation and a Preventive Intervention. Substance Use \& } \\
\text { Misuse. 39(6):963-91. }\end{array}$ & $\begin{array}{l}\text { Not a parenting } \\
\text { intervention }\end{array}$ \\
\hline $\begin{array}{l}\text { 978. Linares, L. O., Jimenez, J., Nesci, C., Pearson, E., Beller, S., } \\
\text { Edwards, N., \& Levin-Rector, A. (2014). Reducing sibling conflict in } \\
\text { maltreated children placed in foster homes. Prevention Science, 16(2), } \\
\text { 211-221. }\end{array}$ & $\begin{array}{l}\text { Targets } \\
\text { externalising }\end{array}$ \\
\hline $\begin{array}{l}\text { 979. Linares, L. O., Montalto, D., Li, M., \& Oza, V. S. (2006). A } \\
\text { promising parenting intervention in foster care. J Consult Clin Psychol, } \\
\text { 74(1), 32-41. doi: 2006-03253-004 }\end{array}$ & $\begin{array}{l}\text { Follow-up less } \\
\text { than } 6 \text { months }\end{array}$ \\
\hline $\begin{array}{l}\text { 980. Lindwall, J. J., Russell, K., Huang, Q., Zhang, H., Vannatta, K., } \\
\text { Barrera, M., ... \& Phipps, S. (2014). Adjustment in parents of children } \\
\text { undergoing stem cell transplantation. Biology of Blood and Marrow } \\
\text { Transplantation, 20(4), 543-548. }\end{array}$ & $\begin{array}{l}\text { Intervention for } \\
\text { developmental } \\
\text { disorders } \\
\text { (autism, autism } \\
\text { spectrum } \\
\text { disorder), } \\
\text { physical } \\
\text { disabilities, } \\
\text { medical } \\
\text { conditions or } \\
\text { distress about } \\
\text { facing medical } \\
\text { interventions }\end{array}$ \\
\hline $\begin{array}{l}\text { 981. Lipman, E. L., \& Boyle, M. H. (2005). Social support and } \\
\text { education groups for single mothers: a randomized controlled trial of a } \\
\text { community-based program. CMAJ : Canadian Medical Association } \\
\text { journal = journal de l'Association medicale canadienne, (12), 1451- } \\
\text { 1456. }\end{array}$ & $\begin{array}{l}\text { No child } \\
\text { internalising } \\
\text { outcomes or not } \\
\text { a major goal }\end{array}$ \\
\hline $\begin{array}{l}\text { 982. Lipman, E. L., Boyle, M. H., Cunningham, C., Kenny, M., } \\
\text { Sniderman, C., Duku, E., et al. (2006). Testing effectiveness of a } \\
\text { community-based aggression management program for children } 7 \text { to } \\
11 \text { years old and their families. J Am Acad Child Adolesc Psychiatry, } \\
\text { 45(9), 1085-1093. doi:10.1097/01.chi.0000228132.64579.73 }\end{array}$ & $\begin{array}{l}\text { No long term } \\
\text { follow-up; } \\
\text { Parent } \\
\text { component too } \\
\text { small }\end{array}$ \\
\hline $\begin{array}{l}\text { 983. Lipscomb, S. T., Laurent, H., Neiderhiser, J. M., Shaw, D. S., } \\
\text { Natsuaki, M. N., Reiss, D., \& Leve, L. D. (2014). Genetic vulnerability } \\
\text { interacts with parenting and early care and education to predict } \\
\text { increasing externalizing behavior. International Journal of Behavioral } \\
\text { Development, 38(1), 70-80. doi: } \\
\text { http://dx.doi.org/10.1177/0165025413508708 }\end{array}$ & $\begin{array}{l}\text { Not a parenting } \\
\text { intervention }\end{array}$ \\
\hline
\end{tabular}




\begin{tabular}{|c|c|}
\hline $\begin{array}{l}\text { 984. Lipsitz, J. D., Gur, M., Albano, A. M., \& Sherman, B. (2011). A } \\
\text { psychological intervention for pediatric chest pain: development and } \\
\text { open trial. J Dev Behav Pediatr, 32, 153-157. }\end{array}$ & $\begin{array}{l}\text { Intervention for } \\
\text { developmental } \\
\text { disorders } \\
\text { (autism, autism } \\
\text { spectrum } \\
\text { disorder), } \\
\text { physical } \\
\text { disabilities, } \\
\text { medical } \\
\text { conditions or } \\
\text { distress about } \\
\text { facing medical } \\
\text { interventions }\end{array}$ \\
\hline $\begin{array}{l}\text { 985. Little M, Sandler IN, Wolchik SA, Tein J-Y and Ayers TS. } 2009 . \\
\text { Comparing cognitive, relational and stress mechanisms underlying } \\
\text { gender differences in recovery from bereavement-related internalizing } \\
\text { problems. Journal of Clinical Child and Adolescent Psychology. } \\
\text { 38(4):486-500. }\end{array}$ & Not an RCT \\
\hline $\begin{array}{l}\text { 986. Liu C, Wang Y, Xuan X, Xu T, Wang XJ, Tang GZ, Zhao YM and } \\
\text { Wang YF. 2009. [A multi-center randomized controlled trial of social } \\
\text { skills training among children with behavior problems]. Zhonghua yi } \\
\text { xue za zhi, 89(35), 2468-2471. }\end{array}$ & $\begin{array}{l}\text { Not a parenting } \\
\text { intervention }\end{array}$ \\
\hline $\begin{array}{l}\text { 987. Liu J, Raine A, Venables PH and Mednick SA. } 2004 \text {. } \\
\text { Malnutrition at age } 3 \text { years and externalizing behavior problems at } \\
\text { ages } 8,11 \text {, and } 17 \text { years. The American Journal of Psychiatry. } \\
\text { 161(11):2005-13. }\end{array}$ & Not an RCT \\
\hline $\begin{array}{l}\text { 988. Liu, C., \& Feekery, C. (2001). Can asthma education improve } \\
\text { clinical outcomes? An evaluation of a pediatric asthma education } \\
\text { program. J Asthma, 38(3), 269-278. }\end{array}$ & $\begin{array}{l}\text { No child } \\
\text { internalising } \\
\text { outcomes or not } \\
\text { a major goal }\end{array}$ \\
\hline $\begin{array}{l}\text { 989. Llewellyn G, McConnell D, Honey A, Mayes R and Russo D. } \\
\text { 2003. Promoting health and home safety for children of parents with } \\
\text { intellectual disability: a randomized controlled trial. Research in } \\
\text { Developmental Disabilities, } 24(6), 405-431 .\end{array}$ & $\begin{array}{l}\text { No child } \\
\text { internalising } \\
\text { outcomes or not } \\
\text { a major goal }\end{array}$ \\
\hline $\begin{array}{l}\text { 990. Loader S, Shields C, Levenkron JC, Fishel R and Rowley PT. } \\
\text { 2002. Patient vs. physician as the target of educational outreach about } \\
\text { screening for an inherited susceptibility to colorectal cancer. Genet } \\
\text { Test. 6(4):281-90. }\end{array}$ & $\begin{array}{l}\text { Not a parenting } \\
\text { intervention }\end{array}$ \\
\hline $\begin{array}{l}\text { 991. Lochman JE, Boxmeyer C, Powell N, Qu L, Wells K and Windle } \\
\text { M. 2009. Dissemination of the Coping Power program: Importance of } \\
\text { intensity of counselor training. Journal of Consulting and Clinical } \\
\text { Psychology. 77(3):397-409. }\end{array}$ & $\begin{array}{l}\text { Not a parenting } \\
\text { intervention }\end{array}$ \\
\hline $\begin{array}{l}\text { 992. Lochman JE. Parent and family skills training in targeted } \\
\text { prevention programs for at-risk youth. J Prim Prev. 2000;21:253-265 }\end{array}$ & Review \\
\hline $\begin{array}{l}\text { 993. Lochman, J. E., Baden, R. E., Boxmeyer, C. L., Powell, N. P., Qu, } \\
\text { L., Salekin, K. L., \& Windle, M. (2014). Does a booster intervention } \\
\text { augment the preventive effects of an abbreviated version of the } \\
\text { Coping Power Program for aggressive children?. Journal of abnormal } \\
\text { child psychology, 42(3), 367-381. }\end{array}$ & $\begin{array}{l}\text { Targets } \\
\text { externalising }\end{array}$ \\
\hline
\end{tabular}




\begin{tabular}{|c|c|}
\hline $\begin{array}{l}\text { 994. Lock, S., \& Barrett, P. M. (2003). A Longitudinal Study of } \\
\text { Developmental Differences in Universal Preventive Intervention for } \\
\text { Child Anxiety. Behaviour Change, 20, 183-199. }\end{array}$ & $\begin{array}{l}\text { Not a parenting } \\
\text { intervention }\end{array}$ \\
\hline $\begin{array}{l}\text { 995. Loeber R and Farrington DP. 1994. Problems and solutions in } \\
\text { longitudinal and experimental treatment studies of child } \\
\text { psychopathology and delinquency. Journal of Consulting and Clinical } \\
\text { Psychology. 62(5):887-900. }\end{array}$ & Review \\
\hline $\begin{array}{l}\text { 996. Logsdon MC, Birkimer JC, Simpson T and Looney S. } 2005 . \\
\text { Postpartum depression and social support in adolescents. J Obstet } \\
\text { Gynecol Neonatal Nurs. 34(1):46-54. }\end{array}$ & $\begin{array}{l}\text { No child } \\
\text { internalising } \\
\text { outcomes or not } \\
\text { a major goal }\end{array}$ \\
\hline $\begin{array}{l}\text { 997. Logsdon MC, Foltz MP, Stein B, Usui W and Josephson A. } 2010 . \\
\text { Adapting and testing telephone-based depression care management } \\
\text { intervention for adolescent mothers. Arch Womens Ment Health. } \\
\text { 13(4):307-17. }\end{array}$ & $\begin{array}{l}\text { No child } \\
\text { internalising } \\
\text { outcomes or not } \\
\text { a major goal }\end{array}$ \\
\hline $\begin{array}{l}\text { 998. Loitz PA. 2000. Conjoint behavioral consultation using self- } \\
\text { administered manual and videotape parent-teacher training: Effects } \\
\text { on children's behavioral difficulties. Dissertation Abstracts } \\
\text { International: Section B: The Sciences and Engineering. 61(2-B). }\end{array}$ & $\begin{array}{l}\text { No long term } \\
\text { follow-up }\end{array}$ \\
\hline $\begin{array}{l}\text { 999. Long, P., Forehand, R., Wierson, M., \& Morgan, A. (1994). } \\
\text { Does parent training with young noncompliant children have long- } \\
\text { term effects?. Behaviour Research and Therapy, 32(1), 101-107. doi: } \\
\text { 10.1016/0005-7967(94)90088-4 }\end{array}$ & Not an RCT \\
\hline $\begin{array}{l}1000 . \quad \text { Loper, A. B., \& Tuerk, E. H. (2011). Improving the emotional } \\
\text { adjustment and communication patterns of incarcerated mothers: } \\
\text { Effectiveness of a prison parenting intervention. Journal of Child and } \\
\text { Family Studies, 20(1), 89-101. }\end{array}$ & $\begin{array}{l}\text { No child } \\
\text { internalising } \\
\text { outcomes or not } \\
\text { a major goal }\end{array}$ \\
\hline $\begin{array}{l}\text { 1001. Lopez MA, Toprac MG, Crismon ML, Boemer C and } \\
\text { Baumgartner J. 2005. A Psychoeducational Program for Children with } \\
\text { ADHD or Depression and their Families: Results from the CMAP } \\
\text { Feasibility Study. Community Mental Health Journal. 41(1):51-66. }\end{array}$ & Not an RCT \\
\hline $\begin{array}{l}1002 . \quad \text { Losel, F., Stemmler, M., Bender, D. (2013). Long-term } \\
\text { evaluation of a bimodal universal prevention program: Effects on } \\
\text { antisocial development from kindergarten to adolescence. Journal of } \\
\text { Experimental Criminology, } 9 \text { (4), 429-449. }\end{array}$ & $\begin{array}{l}\text { Targets } \\
\text { externalising }\end{array}$ \\
\hline $\begin{array}{l}\text { 1003. Loue S and Sajatovic M. 2008. Research with severely mentally } \\
\text { ill Latinas: Successful recruitment and retention strategies. Journal of } \\
\text { Immigrant and Minority Health. 10(2):145-53. }\end{array}$ & $\begin{array}{l}\text { No child } \\
\text { internalising } \\
\text { outcomes or not } \\
\text { a major goal }\end{array}$ \\
\hline $\begin{array}{l}\text { 1004. Love, J. M., Kisker, E. E., Ross, C., Raikes, H., Constantine, J., } \\
\text { Boller, K., et al. (2005). The effectiveness of early head start for 3-year- } \\
\text { old children and their parents: lessons for policy and programs. } \\
\text { Developmental psychology, (6), 885-901. }\end{array}$ & $\begin{array}{l}\text { No child } \\
\text { internalising } \\
\text { outcomes or not } \\
\text { a major goal }\end{array}$ \\
\hline $\begin{array}{l}\text { 1005. Lown A, Dvorak C, Acree M, Dunn E, Abrahms D, Horn B, } \\
\text { Degelman M, Cowan M and Mehling W. 2011. Massage provides relief } \\
\text { for symptoms in pediatric hematopoietic transplant patients. Psycho- } \\
\text { Oncology. 20(1):70-1. }\end{array}$ & $\begin{array}{l}\text { No long term } \\
\text { follow-up }\end{array}$ \\
\hline $\begin{array}{l}\text { 1006. Lowry-Webster HM, Barrett PM and Dadds MR. 2001. A } \\
\text { universal prevention trial of anxiety and depressive symptomatology } \\
\text { in childhood: Preliminary data from an Australian study. Behaviour } \\
\text { Change. 18(1):36-50. }\end{array}$ & $\begin{array}{l}\text { No long term } \\
\text { follow-up }\end{array}$ \\
\hline
\end{tabular}




\begin{tabular}{|c|c|}
\hline $\begin{array}{l}1007 . \quad \text { Lowry-Webster, H. M., Barrett, P. M., \& Lock, S. (2003). A } \\
\text { universal prevention trial of anxiety symptomology during childhood: } \\
\text { Results at 1-year follow-up. Behaviour Change, 20(1), 25-43. doi: } \\
\text { 10.1375/bech.20.1.25.24843 }\end{array}$ & $\begin{array}{l}\text { Parent } \\
\text { component too } \\
\text { small }\end{array}$ \\
\hline $\begin{array}{l}\text { 1008. Lu Q, Tsao JCl, Myers CD, Kim SC and Zeltzer LK. 2007. Coping } \\
\text { predictors of children's laboratory-induced pain tolerance, intensity, } \\
\text { and unpleasantness. The Journal of Pain. 8(9):708-17. }\end{array}$ & $\begin{array}{l}\text { Not a parenting } \\
\text { intervention }\end{array}$ \\
\hline $\begin{array}{l}\text { 1009. Luby, J., Lenze, S., \& Tillman, R. (2012). A novel early } \\
\text { intervention for preschool depression: findings from a pilot } \\
\text { randomized controlled trial. J Child Psychol Psychiatry, 53, 313-322. }\end{array}$ & $\begin{array}{l}\text { No long term } \\
\text { follow-up }\end{array}$ \\
\hline $\begin{array}{l}1010 . \quad \text { Luecken, L. J., Hagan, M. J., Sandler, I. N., Tein, J. Y., Ayers, T. } \\
\text { S., \& Wolchik, S. A. (2014). Longitudinal mediators of a randomized } \\
\text { prevention program effect on cortisol for youth from parentally } \\
\text { bereaved families. Prevention science, } 15(2), 224-232 .\end{array}$ & $\begin{array}{l}\text { No child } \\
\text { internalising } \\
\text { outcomes or not } \\
\text { a major goal }\end{array}$ \\
\hline $\begin{array}{l}\text { 1011. Luecken, L. J., Hagan, M. J., Sandler, I. N., Tein, J.-Y., Ayers, T. } \\
\text { S., \& Wolchik, S. A. (2010). Cortisol levels six-years after participation } \\
\text { in the family bereavement program. Psychoneuroendocrinology, } \\
\text { 35(5), 785-789. doi: } 10.1016 / \text { j.psyneuen.2009.11.002 }\end{array}$ & $\begin{array}{l}\text { No child } \\
\text { internalising } \\
\text { outcomes or not } \\
\text { a major goal }\end{array}$ \\
\hline $\begin{array}{l}\text { 1012. Luepker RV, Perry CL, Murray DM and Mullis R. } 1988 . \\
\text { Hypertension prevention through nutrition education in youth: a } \\
\text { school-based program involving parents. Health Psychology, } 7(\mathrm{~S}), 233 .\end{array}$ & $\begin{array}{l}\text { Not a parenting } \\
\text { intervention }\end{array}$ \\
\hline $\begin{array}{l}\text { 1013. Lukin, K. (2011). Predictors and moderators of treatment } \\
\text { adherence in depressed youths (Order No. 3476152). Available from } \\
\text { ProQuest Dissertations \& Theses Global. (894118916) }\end{array}$ & $\begin{array}{l}\text { Not a parenting } \\
\text { intervention }\end{array}$ \\
\hline $\begin{array}{l}\text { 1014. Lumpkin PW, Silverman WK, Weems CF, Markham MR and } \\
\text { Kurtines WM. 2002. Treating a heterogeneous set of anxiety disorders } \\
\text { in youths with group cognitive behavioral therapy: A partially } \\
\text { nonconcurrent multiple-baseline evaluation. Behavior Therapy. } \\
\text { 33(1):163-77. }\end{array}$ & $\begin{array}{l}\text { Not a parenting } \\
\text { intervention }\end{array}$ \\
\hline $\begin{array}{l}\text { 1015. Lundahl, B., Riser, H. J., \& Lovejoy, M. C. (2006). A meta- } \\
\text { analysis of parent training: Moderators and follow-up effects. Clinical } \\
\text { Psychology Review, 26, 86-104. }\end{array}$ & Review \\
\hline $\begin{array}{l}\text { 1016. Lynch FL, Hornbrook M, Clarke GN, Perrin N, Polen MR, } \\
\text { O'Connor E and Dickerson J. 2005. Cost-effectiveness of an } \\
\text { intervention to prevent depression in at-risk teens. Arch Gen } \\
\text { Psychiatry. 62(11):1241-8. }\end{array}$ & $\begin{array}{l}\text { Not a parenting } \\
\text { intervention }\end{array}$ \\
\hline $\begin{array}{l}\text { 1017. Lyneham, H. J., \& Rapee, R. M. (2006). Evaluation of therapist- } \\
\text { supported parent-implemented CBT for anxiety disorders in rural } \\
\text { children. Behaviour Research and Therapy, 44(9), 1287-1300. doi: } \\
\text { 10.1016/j.brat.2005.09.009 }\end{array}$ & $\begin{array}{l}\text { Follow-up data } \\
\text { collected only in } \\
\text { active group }\end{array}$ \\
\hline $\begin{array}{l}\text { 1018. Lyon AR and Budd KS. 2010. A community mental health } \\
\text { implementation of Parent-Child Interaction Therapy (PCIT). Journal of } \\
\text { Child and Family Studies. 19(5):654-68. }\end{array}$ & Not an RCT \\
\hline $\begin{array}{l}\text { 1019. Lytle LA, Kubik MY, Perry C, Story M, Birnbaum AS and Murray } \\
\text { DM. 2006. Influencing healthful food choices in school and home } \\
\text { environments: Results from the TEENS study. Preventive Medicine: An } \\
\text { International Journal Devoted to Practice and Theory. 43(1):8-13. }\end{array}$ & $\begin{array}{l}\text { Not a parenting } \\
\text { intervention }\end{array}$ \\
\hline $\begin{array}{l}\text { 1020. Mabe PA, Turner MK and Josephson AM. 2001. Parent } \\
\text { management training. Child and Adolescent Psychiatric Clinics of } \\
\text { North America. 10(3):451-64. }\end{array}$ & Review \\
\hline
\end{tabular}




\begin{tabular}{|c|c|}
\hline $\begin{array}{l}\text { 1021. MacMillan, H. L., \& Canadian Task Force on Preventive Health } \\
\text { Care. (2000). Preventive health care, } 2000 \text { update: prevention of child } \\
\text { maltreatment. CMAJ: Canadian Medical Association journal= journal } \\
\text { de l'Association medicale canadienne, } 163(11), 1451 .\end{array}$ & Review \\
\hline $\begin{array}{l}\text { 1022. MacNeill, V., Nwokoro, C., Griffiths, C., Grigg, J., \& Seale, C. } \\
\text { (2013). Recruiting ethnic minority participants to a clinical trial: A } \\
\text { qualitative study. BMJ Open, 3(4). doi: } \\
\text { http://dx.doi.org/10.1136/bmjopen-2013-002750 }\end{array}$ & Not an RCT \\
\hline $\begin{array}{l}\text { 1023. Madigan, S., Vaillancourt, K., McKibbon, A., \& Benoit, D. } \\
\text { (2015). Trauma and traumatic loss in pregnant adolescents: the impact } \\
\text { of Trauma-Focused Cognitive Behavior Therapy on maternal } \\
\text { unresolved states of mind and Posttraumatic Stress Disorder. } \\
\text { Attachment \& Human Development, } 17(2), 175-198 .\end{array}$ & $\begin{array}{l}\text { No child } \\
\text { internalising } \\
\text { outcomes or not } \\
\text { a major goal }\end{array}$ \\
\hline $\begin{array}{l}\text { 1024. Mager W, Milich R, Harris MJ and Howard A. } 2005 . \\
\text { Intervention groups for adolescents with conduct problems: is } \\
\text { aggregation harmful or helpful? J Abnorm Child Psychol. 33(3):349-62. }\end{array}$ & $\begin{array}{l}\text { Not a parenting } \\
\text { intervention }\end{array}$ \\
\hline $\begin{array}{l}\text { 1025. Mahajan L, Wyllie R, Steffen R, Kay M, Kitaoka G, Dettorre J, } \\
\text { Sarigol S and McCue K. 1998. The effects of a psychological } \\
\text { preparation program on anxiety in children and adolescents } \\
\text { undergoing gastrointestinal endoscopy. J Pediatr Gastroenterol Nutr. } \\
\text { 27(2):161-5. }\end{array}$ & $\begin{array}{l}\text { Intervention for } \\
\text { developmental } \\
\text { disorders } \\
\text { (autism, autism } \\
\text { spectrum } \\
\text { disorder), } \\
\text { physical } \\
\text { disabilities, } \\
\text { medical } \\
\text { conditions or } \\
\text { distress about } \\
\text { facing medical } \\
\text { interventions }\end{array}$ \\
\hline $\begin{array}{l}\text { 1026. Maid R, Smokowski P and Bacallao M. 2008. Family treatment } \\
\text { of childhood anxiety. Child \& Family Social Work. 13(4):433-42. }\end{array}$ & Review \\
\hline $\begin{array}{l}\text { 1027. Mallery, C. J. (2011). The effects of neighborhood context and } \\
\text { parenting factors on adolescent mental health: A multilevel } \\
\text { investigation (Order No. 3434888). Available from ProQuest } \\
\text { Dissertations \& Theses Global. (847800149). }\end{array}$ & $\begin{array}{l}\text { Not a parenting } \\
\text { intervention }\end{array}$ \\
\hline $\begin{array}{l}\text { 1028. Malti, T., Ribeaud, D., \& Eisner, M. P. (2011). The effectiveness } \\
\text { of two universal preventive interventions in reducing children's } \\
\text { externalizing behavior: A cluster randomized controlled trial. Journal } \\
\text { of Clinical Child and Adolescent Psychology, 40,677-692. }\end{array}$ & $\begin{array}{l}\text { Targets } \\
\text { externalising }\end{array}$ \\
\hline $\begin{array}{l}\text { 1029. Manassis K, Wilansky-Traynor P, Farzan N, Kleiman V, Parker K } \\
\text { and Sanford M. 2010. The feelings club: Randomized controlled } \\
\text { evaluation of school-based CBT for anxious or depressive symptoms. } \\
\text { Depression and Anxiety. 27(10):945-52. }\end{array}$ & $\begin{array}{l}\text { Not a parenting } \\
\text { intervention }\end{array}$ \\
\hline $\begin{array}{l}\text { 1030. Manassis, K., Mendlowitz, S. L., Scapillato, D., Avery, D., } \\
\text { Fiksenbaum, L., Freire, M., et al. (2002). Group and individual } \\
\text { cognitive-behavioral therapy for childhood anxiety disorders: a } \\
\text { randomized trial. J Am Acad Child Adolesc Psychiatry, 41(12), 1423- } \\
\text { 1430. doi: S0890-8567(09)60736-X }\end{array}$ & $\begin{array}{l}\text { Parent } \\
\text { component too } \\
\text { small }\end{array}$ \\
\hline $\begin{array}{l}\text { 1031. Manber R, Blasey C and Allen JJB. 2008. Depression symptoms } \\
\text { during pregnancy. Archives of Women's Mental Health. 11(1):43-8. }\end{array}$ & $\begin{array}{l}\text { Not a parenting } \\
\text { intervention }\end{array}$ \\
\hline
\end{tabular}




\begin{tabular}{|c|c|}
\hline $\begin{array}{l}\text { 1032. Manber R, Schnyer RN, Allen JJB, Rush AJ and Blasey CM. } \\
\text { 2004. Acupuncture: A promising treatment for depression during } \\
\text { pregnancy. Journal of Affective Disorders. 83(1):89-95. }\end{array}$ & $\begin{array}{l}\text { Not a parenting } \\
\text { intervention }\end{array}$ \\
\hline $\begin{array}{l}\text { 1033. Mansson ME, Fredrikzon B and Rosberg B. 1992. Comparison } \\
\text { of preparation and narcotic-sedative premedication in children } \\
\text { undergoing surgery. Pediatr Nurs. 18(4):337-42. }\end{array}$ & $\begin{array}{l}\text { Intervention for } \\
\text { developmental } \\
\text { disorders } \\
\text { (autism, autism } \\
\text { spectrum } \\
\text { disorder), } \\
\text { physical } \\
\text { disabilities, } \\
\text { medical } \\
\text { conditions or } \\
\text { distress about } \\
\text { facing medical } \\
\text { interventions }\end{array}$ \\
\hline $\begin{array}{l}\text { 1034. Marcenko MO, Spence M. Home visitation services for at-risk } \\
\text { pregnant and postpartum women: a randomized trial. } \\
\text { AmJOrthopsychiatry.1994;64:468-478. }\end{array}$ & $\begin{array}{l}\text { No long term } \\
\text { follow-up; No } \\
\text { child } \\
\text { internalising } \\
\text { outcomes or not } \\
\text { a major goal }\end{array}$ \\
\hline $\begin{array}{l}\text { 1035. March, S., Spence, S. H., \& Donovan, C. L. (2009). The efficacy } \\
\text { of an internet-based cognitive-behavioral therapy intervention for } \\
\text { child anxiety disorders. J Pediatr Psychol, 34(5), 474-487. doi: jsn099 }\end{array}$ & $\begin{array}{l}\text { Parent } \\
\text { component too } \\
\text { small }\end{array}$ \\
\hline $\begin{array}{l}\text { 1036. Marco, C. A., Wolfson, A. R., Sparling, M., \& Azuaje, A. (2011). } \\
\text { Family socioeconomic status and sleep patterns of young adolescents. } \\
\text { Behavioral Sleep Medicine, 10, 70-80. }\end{array}$ & $\begin{array}{l}\text { Not a parenting } \\
\text { intervention }\end{array}$ \\
\hline $\begin{array}{l}\text { 1037. Margolis JO, Ginsberg B, Dear GL, Ross AK, Goral JE and Bailey } \\
\text { AG. 1998. Paediatric preoperative teaching: effects at induction and } \\
\text { postoperatively. Paediatr Anaesth. 8(1):17-23. }\end{array}$ & $\begin{array}{l}\text { Intervention for } \\
\text { developmental } \\
\text { disorders } \\
\text { (autism, autism } \\
\text { spectrum } \\
\text { disorder), } \\
\text { physical } \\
\text { disabilities, } \\
\text { medical } \\
\text { conditions or } \\
\text { distress about } \\
\text { facing medical } \\
\text { interventions }\end{array}$ \\
\hline $\begin{array}{l}\text { 1038. Margolis PA, Stevens R, Bordley WC, Stuart J, Harlan C, Keyes- } \\
\text { Elstein L and Wisseh S. 2001. From concept to application: the impact } \\
\text { of a community-wide intervention to improve the delivery of } \\
\text { preventive services to children. Pediatrics, 108(3), e42-e42. }\end{array}$ & Not an RCT \\
\hline $\begin{array}{l}\text { 1039. Margolis, K. L. (2014). A pilot feasibility and effectiveness trial } \\
\text { of the family check-up parenting intervention with Spanish } \\
\text { preadolescents and their families: A cultural adaptation and feasibility } \\
\text { study to enhance evidence-based intervention research in spain. } \\
\text { Dissertation Abstracts International: Section B: The Sciences and } \\
\text { Engineering, } 74 \text { (11-B E). }\end{array}$ & $\begin{array}{l}\text { Follow-up less } \\
\text { than } 6 \text { months; } \\
\text { Targets } \\
\text { externalising }\end{array}$ \\
\hline
\end{tabular}




\begin{tabular}{|c|c|}
\hline $\begin{array}{l}\text { 1040. Markie-Dadds, C., \& Sanders, M. R. (2006). Self-directed Triple } \\
\text { P (Positive Parenting Program) for mothers with children at-risk of } \\
\text { developing conduct problems. Behavioural and Cognitive } \\
\text { Psychotherapy, 34(03), 259-275. }\end{array}$ & $\begin{array}{l}\text { No child } \\
\text { internalising } \\
\text { outcomes or not } \\
\text { a major goal; } \\
\text { Follow-up data } \\
\text { collected only in } \\
\text { active group }\end{array}$ \\
\hline $\begin{array}{l}\text { 1041. Markle-Ried, M., Browne, G., Roberts, J., Gafni, A., \& Byrne, C. } \\
\text { (2002). The 2-year costs and effects of a public health nursing case } \\
\text { management intervention on mood-disordered single parents on } \\
\text { social assistance. Journal of Evaluation in Clinical Practice, } 8(1), 45-59 . \\
\text { doi: } 10.1046 / j .1365-2753.2002 .00316 . x\end{array}$ & $\begin{array}{l}\text { No child } \\
\text { internalising } \\
\text { outcomes or not } \\
\text { a major goal }\end{array}$ \\
\hline $\begin{array}{l}\text { 1042. Markowitz JC, Patel SR, Balan IC, Bell MA, Blanco C, Heart } \\
\text { MYHB, Sosa SB and Fernández RL. 2009. Toward an adaptation of } \\
\text { interpersonal psychotherapy for Hispanic patients with DSM-IV major } \\
\text { depressive disorder. Journal of Clinical Psychiatry. 70(2):214-22. }\end{array}$ & $\begin{array}{l}\text { Not a parenting } \\
\text { intervention }\end{array}$ \\
\hline $\begin{array}{l}\text { 1043. Marquis, R. (2014). The gender effects of a foster parent- } \\
\text { delivered tutoring program on foster children's academic skills and } \\
\text { mental health: A randomized field trial. Dissertation Abstracts } \\
\text { International Section A: Humanities and Social Sciences Dissertation } \\
\text { Abstracts International, } 75 \text { (1-a(e). }\end{array}$ & $\begin{array}{l}\text { No child } \\
\text { internalising } \\
\text { outcomes or not } \\
\text { a major goal; No } \\
\text { long term } \\
\text { follow-up; Not a } \\
\text { parenting } \\
\text { program }\end{array}$ \\
\hline $\begin{array}{l}1044 . \quad \text { Marsac, M. L., Hildenbrand, A. K., Kohser, K. L., Winston, F. K., } \\
\text { Li, Y., \& Kassam-Adams, N. (2013). Preventing posttraumatic stress } \\
\text { following pediatric injury: A randomized controlled trial of a web- } \\
\text { based psycho-educational intervention for parents. Journal of } \\
\text { Pediatric Psychology, 38(10), 1101-1111. }\end{array}$ & $\begin{array}{l}\text { Follow-up less } \\
\text { than } 6 \text { months }\end{array}$ \\
\hline $\begin{array}{l}\text { 1045. Martí, A. C., \& Barrachina, M. T. M. 2009. The effects of } \\
\text { mindfulness-based cognitive therapy: A qualitative approach. } \\
\text { Psychology in Spain. 13(1):9-16. }\end{array}$ & $\begin{array}{l}\text { Not a parenting } \\
\text { intervention }\end{array}$ \\
\hline $\begin{array}{l}\text { 1046. Martin, S. R., Chorney, J. M., Tan, E. T., Fortier, M. A., Blount, } \\
\text { R. L., Wald, S. H., Shapiro, N. L., Strom, S. L., Patel, S., \& Kain, Z. N. } \\
\text { (2011). Changing healthcare providers' behavior during pediatric } \\
\text { inductions with an empirically based intervention. Anesthesiology, } \\
\text { 115, 18-27. }\end{array}$ & $\begin{array}{l}\text { Not a parenting } \\
\text { intervention }\end{array}$ \\
\hline $\begin{array}{l}\text { 1047. Martinez, C. R., \& Forgatch, M. S. (2001). Preventing problems } \\
\text { with boys' noncompliance: Effects of a parent training intervention for } \\
\text { divorcing mothers. Journal of Consulting and Clinical Psychology, } \\
69(3), 416-428 .\end{array}$ & $\begin{array}{l}\text { No child } \\
\text { internalising } \\
\text { outcomes or not } \\
\text { a major goal }\end{array}$ \\
\hline $\begin{array}{l}\text { 1048. Martinez, C. R., Jr., \& Eddy, J. M. (2005). Effects of culturally } \\
\text { adapted parent management training on Latino youth behavioral } \\
\text { health outcomes. J Consult Clin Psychol, 73(5), 841-851. doi: 2005- } \\
\text { 13740-007 }\end{array}$ & $\begin{array}{l}\text { No long term } \\
\text { follow-up }\end{array}$ \\
\hline $\begin{array}{l}\text { 1049. Martyn KK, Loveland-Cherry CJ, Villarruel AM, Cabriales EG, } \\
\text { Zhou Y, Ronis DL and Eakin B. 2009. Mexican adolescents' alcohol use, } \\
\text { family intimacy, and parent-adolescent communication. Journal of } \\
\text { Family Nursing. 15(2):152-70. }\end{array}$ & $\begin{array}{l}\text { Not a parenting } \\
\text { intervention }\end{array}$ \\
\hline
\end{tabular}




\begin{tabular}{|c|c|}
\hline $\begin{array}{l}\text { 1050. Masia CL, Klein RG, Storch EA and Corda B. 2001. School-based } \\
\text { behavioral treatment for social anxiety disorder in adolescents: } \\
\text { Results of a pilot study. Journal of the American Academy of Child \& } \\
\text { Adolescent Psychiatry. 40(7):780-6. }\end{array}$ & $\begin{array}{l}\text { Not a parenting } \\
\text { intervention }\end{array}$ \\
\hline $\begin{array}{l}\text { 1051. Masia Warner C, Fisher PH, Shrout PE, Rathor S and Klein RG. } \\
\text { 2007. Treating adolescents with social anxiety disorder in school: An } \\
\text { attention control trial. Journal of Child Psychology and Psychiatry. } \\
\text { 48(7):676-86. }\end{array}$ & $\begin{array}{l}\text { Not a parenting } \\
\text { intervention }\end{array}$ \\
\hline $\begin{array}{l}\text { 1052. Masia-Warner C, Klein RG, Dent HC, Fisher PH, Alvir J, Albano } \\
\text { AM and Guardino M. 2005. School-based intervention for adolescents } \\
\text { with social anxiety disorder: Results of a controlled study. Journal of } \\
\text { Abnormal Child Psychology: An official publication of the International } \\
\text { Society for Research in Child and Adolescent Psychopathology. } \\
\text { 33(6):707-22. }\end{array}$ & $\begin{array}{l}\text { Not a parenting } \\
\text { intervention }\end{array}$ \\
\hline $\begin{array}{l}\text { 1053. Mason MJ, Cheung I and Walker L. 2004. The Social Ecology of } \\
\text { Urban Adolescent Substance Use: A Case Study Utilizing Geographic } \\
\text { Information Systems. The Journal of Primary Prevention. 25(2):271-82. }\end{array}$ & $\begin{array}{l}\text { Not a parenting } \\
\text { intervention }\end{array}$ \\
\hline $\begin{array}{l}\text { 1054. Mason WA, Kosterman R, Haggerty KP, Hawkins JD, Redmond } \\
\text { C, Spoth RL and Shin C. 2008. Dimensions of adolescent alcohol } \\
\text { involvement as predictors of young-adult major depression. Journal of } \\
\text { Studies on Alcohol and Drugs. 69(2):275-85. }\end{array}$ & $\begin{array}{l}\text { Not a parenting } \\
\text { intervention }\end{array}$ \\
\hline $\begin{array}{l}1055 . \quad \text { Mason, W. A., Haggerty, K. P., Fleming, A. P., \& Casey- } \\
\text { Goldstein, M. (2012). Family intervention to prevent depression and } \\
\text { substance use among adolescents of depressed parents. Journal of } \\
\text { Child and Family Studies, 21(6), 891-905. doi: 10.1007/s10826-011- } \\
\text { 9549-x }\end{array}$ & $\begin{array}{l}\text { Follow-up less } \\
\text { than } 6 \text { months }\end{array}$ \\
\hline $\begin{array}{l}\text { 1056. Mason, W. A., Kosterman, R., Hawkins, J. D., Haggerty, K. P., \& } \\
\text { Spoth, R. (2003). Reducing adolescents' growth in substance use and } \\
\text { delinquency: Randomized trial effects of a preventive parent-training } \\
\text { intervention. Prevention Science, 4, 203-213. }\end{array}$ & $\begin{array}{l}\text { No child } \\
\text { internalising } \\
\text { outcomes or not } \\
\text { a major goal }\end{array}$ \\
\hline $\begin{array}{l}\text { 1057. Masuko, I., Futamura, M., Hahashi, K., Ito, K., \& Ohya, Y. } \\
\text { (2009). A randomized evaluator-blinded trial of behavioral } \\
\text { modification program for mothers of children with atopic dermatitis. } \\
\text { Journal of Allergy and Clinical Immunology, } 123(2), \text { S47. }\end{array}$ & $\begin{array}{l}\text { No child } \\
\text { internalising } \\
\text { outcomes or not } \\
\text { a major goal }\end{array}$ \\
\hline $\begin{array}{l}\text { 1058. Matos M, Torres R, Santiago R, Jurado M and Rodríguez I. } \\
\text { 2006. Adaptation of Parent-Child Interaction Therapy for Puerto Rican } \\
\text { Families: A Preliminary Study. Family Process. 45(2):205-22. }\end{array}$ & Not an RCT \\
\hline $\begin{array}{l}\text { 1059. Matos, M., Bauermeister, J. J., \& Bernal, G. (2009). Parent- } \\
\text { Child Interaction Therapy for Puerto Rican Preschool Children with } \\
\text { ADHD and Behavior Problems: A Pilot Efficacy Study. Family Process, } \\
\text { 48, 232-252. }\end{array}$ & $\begin{array}{l}\text { No long term } \\
\text { follow-up }\end{array}$ \\
\hline $\begin{array}{l}\text { 1060. Matson ML, Mahan S and Matson JL. 2009. Parent training: A } \\
\text { review of methods for children with autism spectrum disorders. } \\
\text { Research in Autism Spectrum Disorders. 3(4):868-75. }\end{array}$ & Review \\
\hline $\begin{array}{l}\text { 1061. Matson, J. L., \& Ollendick, T. H. (1977). Issues in toilet training } \\
\text { normal children. Behavior Therapy, 8(4), 549-553. }\end{array}$ & $\begin{array}{l}\text { No child } \\
\text { internalising } \\
\text { outcomes or not } \\
\text { a major goal }\end{array}$ \\
\hline
\end{tabular}




\begin{tabular}{|c|c|}
\hline $\begin{array}{l}\text { 1062. Matsumoto, Y., Sofronoff, K., \& Sanders, M. R. (2007). The } \\
\text { Efficacy and Acceptability of the Triple P-Positive Parenting Program } \\
\text { With Japanese Parents. [Article]. Behaviour Change, 24(4), 205-218. } \\
\text { doi: } 10.1375 / \text { bech.24.4.205 }\end{array}$ & $\begin{array}{l}\text { No long term } \\
\text { follow-up }\end{array}$ \\
\hline $\begin{array}{l}\text { 1063. Matthey S, Kavanagh DJ, Howie P, Barnett B and Charles M. } \\
\text { 2004. Prevention of postnatal distress or depression: an evaluation of } \\
\text { an intervention at preparation for parenthood classes. J Affect Disord. } \\
\text { 79(1-3):113-26. }\end{array}$ & $\begin{array}{l}\text { No child } \\
\text { internalising } \\
\text { outcomes or not } \\
\text { a major goal }\end{array}$ \\
\hline $\begin{array}{l}\text { 1064. Mc Conachie H. 2009. Making sense of autism: What works for } \\
\text { children. Developmental Medicine and Child Neurology. 51:2-3. }\end{array}$ & Review \\
\hline $\begin{array}{l}\text { 1065. McCabe KM, Lucchini SE, Hough RL, Yeh M and Hazen A. } 2005 . \\
\text { The Relation between Violence Exposure and Conduct Problems } \\
\text { among Adolescents: A Prospective Study. American Journal of } \\
\text { Orthopsychiatry. 75(4):575-84. }\end{array}$ & Not an RCT \\
\hline $\begin{array}{l}\text { 1066. McCabe, K. M., Yeh, M., Garland, A. F., Lau, A. S., \& Chavez, G. } \\
\text { (2005). The GANA Program: A Tailoring Approach to Adapting Parent } \\
\text { Child Interaction Therapy for Mexican Americans. Education \& } \\
\text { Treatment of Children, 28(2), 111-129. }\end{array}$ & Not an RCT \\
\hline $\begin{array}{l}\text { 1067. McCabe, K., \& Yeh, M. (2009). Parent-child interaction therapy } \\
\text { for Mexican Americans: a randomized clinical trial. Journal Of Clinical } \\
\text { Child And Adolescent Psychology, 38, 753-759. }\end{array}$ & $\begin{array}{l}\text { No long term } \\
\text { follow-up }\end{array}$ \\
\hline $\begin{array}{l}\text { 1068. McCart MR. 2007. Reducing violence/victimization among } \\
\text { assaulted urban youth. Dissertation Abstracts International: Section B: } \\
\text { The Sciences and Engineering. 67(9-B). }\end{array}$ & $\begin{array}{l}\text { Follow-up less } \\
\text { than } 6 \text { months }\end{array}$ \\
\hline $\begin{array}{l}\text { 1069. McCarthy AM, Cool VA and Hanrahan K. 1998. Cognitive } \\
\text { behavioral interventions for children during painful procedures: } \\
\text { research challenges and program development. J Pediatr Nurs. } \\
\text { 13(1):55-63. }\end{array}$ & $\begin{array}{l}\text { Intervention for } \\
\text { developmental } \\
\text { disorders } \\
\text { (autism, autism } \\
\text { spectrum } \\
\text { disorder), } \\
\text { physical } \\
\text { disabilities, } \\
\text { medical } \\
\text { conditions or } \\
\text { distress about } \\
\text { facing medical } \\
\text { interventions }\end{array}$ \\
\hline $\begin{array}{l}\text { 1070. McCarthy AM, Kleiber C, Hanrahan K, Zimmerman MB, } \\
\text { Westhus N and Allen S. 2010. Impact of parent-provided distraction on } \\
\text { child responses to an IV insertion. Children's Health Care. 39(2):125- } \\
41 .\end{array}$ & $\begin{array}{l}\text { Intervention for } \\
\text { developmental } \\
\text { disorders } \\
\text { (autism, autism } \\
\text { spectrum } \\
\text { disorder), } \\
\text { physical } \\
\text { disabilities, } \\
\text { medical } \\
\text { conditions or } \\
\text { distress about } \\
\text { facing medical } \\
\text { interventions }\end{array}$ \\
\hline
\end{tabular}




\begin{tabular}{|c|c|}
\hline $\begin{array}{l}\text { 1071. McCarthy, A. M., Kleiber, C., Hanrahan, K., Zimmerman, M. B., } \\
\text { Ersig, A., Westhus, N., \& Allen, S. (2014). Matching Doses of } \\
\text { Distraction With Child Risk for Distress During a Medical Procedure: A } \\
\text { Randomized Clinical Trial. Nursing research, 63(6), 397-407. }\end{array}$ & $\begin{array}{l}\text { Intervention for } \\
\text { developmental } \\
\text { disorders } \\
\text { (autism, autism } \\
\text { spectrum } \\
\text { disorder), } \\
\text { physical } \\
\text { disabilities, } \\
\text { medical } \\
\text { conditions or } \\
\text { distress about } \\
\text { facing medical } \\
\text { interventions }\end{array}$ \\
\hline $\begin{array}{l}\text { 1072. McCarton CM, Wallace IF, Bennett FC. Preventive } \\
\text { interventions with low birth weight premature infants: an evaluation } \\
\text { of their success. Semin Perinatol 1995;19:330-40 }\end{array}$ & Review \\
\hline $\begin{array}{l}\text { 1073. McCarton, C. M., Brooks-Gunn, J., Wallace, I. F., Bauer, C. R., } \\
\text { Bennett, F. C., Bernbaum, J. C., Broyles, R. S., Casey, P. H., McCormick, } \\
\text { M. C., Scott, D. T., Tyson, J., Tonascia, J., \& Meinert, C. L. (1997). } \\
\text { Results at age } 8 \text { years of early intervention for low-birth-weight } \\
\text { premature infants. The Infant Health and Development Program. } \\
\text { JAMA, 277, 126-132. }\end{array}$ & $\begin{array}{l}\text { Parent } \\
\text { component too } \\
\text { small }\end{array}$ \\
\hline $\begin{array}{l}\text { 1074. McCarty, C. A., Violette, H. D., Duong, M. T., Cruz, R. A., \& } \\
\text { McCauley, E. (2013). A randomized trial of the Positive Thoughts and } \\
\text { Action program for depression among early adolescents. J Clin Child } \\
\text { Adolesc Psychol, 42(4), 554-563. doi:10.1080/15374416.2013.782817 }\end{array}$ & $\begin{array}{l}\text { Not a parenting } \\
\text { intervention }\end{array}$ \\
\hline $\begin{array}{l}1075 . \quad \text { McConaughy, S. H., Kay, P. J., \& Fitzgerald, M. (1999). The } \\
\text { achieving, Behaving, Caring project for preventing ED: Two-year } \\
\text { outcomes. Journal of Emotional and Behavioral Disorders, 7(4), 224- } \\
\text { 239. doi: } 10.1177 / 106342669900700405\end{array}$ & $\begin{array}{l}\text { No long term } \\
\text { follow-up } \\
\text { (intervention } \\
\text { lasts for } 2 \text { years) }\end{array}$ \\
\hline $\begin{array}{l}1076 . \quad \text { McCormick, E., Kerns, S. E., McPhillips, H., Wright, J., } \\
\text { Christakis, D. A., \& Rivara, F. P. (2014). Training Pediatric Residents to } \\
\text { Provide Parent Education: A Randomized Controlled Trial. Academic } \\
\text { pediatrics, 14(4), 353-360. }\end{array}$ & $\begin{array}{l}\text { No child } \\
\text { internalising } \\
\text { outcomes or not } \\
\text { a major goal }\end{array}$ \\
\hline $\begin{array}{l}\text { 1077. McCusker, C. G., Doherty, N. N., Molloy, B., Rooney, N., } \\
\text { Mulholland, C., Sands, A., et al. (2010). A controlled trial of early } \\
\text { interventions to promote maternal adjustment and development in } \\
\text { infants born with severe congenital heart disease. Child: Care, Health } \\
\text { and Development, 36(1), 110-117. }\end{array}$ & $\begin{array}{l}\text { No child } \\
\text { internalising } \\
\text { outcomes or not } \\
\text { a major goal }\end{array}$ \\
\hline $\begin{array}{l}\text { 1078. McDonald, L., \& Sayger, T. V. (1998). Impact of a Family and } \\
\text { School Based Prevention Program on Protective Factors for High Risk } \\
\text { Youth. Drugs \& Society, 12,61-85. }\end{array}$ & Not an RCT \\
\hline $\begin{array}{l}\text { 1079. McDonald, R., Dodson, M. C., Rosenfield, D., \& Jouriles, E. N. } \\
\text { (2011). Effects of a parenting intervention on features of psychopathy } \\
\text { in children. Journal of Abnormal Child Psychology, } 39,1013-1023 \text {. }\end{array}$ & $\begin{array}{l}\text { Targets } \\
\text { externalising }\end{array}$ \\
\hline
\end{tabular}




\begin{tabular}{|c|c|}
\hline $\begin{array}{l}\text { 1080. McEwen A, Moorthy C, Quantock C, Rose H and Kavanagh R. } \\
\text { 2007. The effect of videotaped preoperative information on parental } \\
\text { anxiety during anesthesia induction for elective pediatric procedures. } \\
\text { Paediatr Anaesth. 17(6):534-9. }\end{array}$ & $\begin{array}{l}\text { Intervention for } \\
\text { developmental } \\
\text { disorders } \\
\text { (autism, autism } \\
\text { spectrum } \\
\text { disorder), } \\
\text { physical } \\
\text { disabilities, } \\
\text { medical } \\
\text { conditions or } \\
\text { distress about } \\
\text { facing medical } \\
\text { interventions }\end{array}$ \\
\hline $\begin{array}{l}\text { 1081. McGilloway, S., Ni Mhaille, G., Bywater, T., Furlong, M., } \\
\text { Leckey, Y., Kelly, P., . . Donnelly, M. (2012). A parenting intervention } \\
\text { for childhood behavioral problems: a randomized controlled trial in } \\
\text { disadvantaged community-based settings. Journal of consulting and } \\
\text { clinical psychology, 80(1), 116-127. doi:10.1037/a0026304 }\end{array}$ & $\begin{array}{l}\text { Targets } \\
\text { externalising }\end{array}$ \\
\hline $\begin{array}{l}\text { 1082. McGilloway, S., NiMhaille, G., Bywater, T., Leckey, Y., Kelly, P., } \\
\text { Furlong, M., ... \& Donnelly, M. (2014). Reducing child conduct } \\
\text { disordered behaviour and improving parent mental health in } \\
\text { disadvantaged families: a 12-month follow-up and cost analysis of a } \\
\text { parenting intervention. European child \& adolescent psychiatry, 23(9), } \\
783-794 .\end{array}$ & $\begin{array}{l}\text { No child } \\
\text { internalising } \\
\text { outcomes or not } \\
\text { a major goal }\end{array}$ \\
\hline $\begin{array}{l}\text { 1083. McIntosh E, Barlow J, Davis H and Stewart-Brown S. } 2009 . \\
\text { Economic evaluation of an intensive home visiting programme for } \\
\text { vulnerable families: a cost-effectiveness analysis of a public health } \\
\text { intervention. Journal of public health, } 31(3), 423-433 .\end{array}$ & $\begin{array}{l}\text { No child } \\
\text { internalising } \\
\text { outcomes or not } \\
\text { a major goal }\end{array}$ \\
\hline $\begin{array}{l}1084 . \quad \text { McKee, L. G., Parent, J., Forehand, R., Rakow, A., Watson, K. } \\
\text { H., Dunbar, J. P., ... \& Compas, B. E. (2014). Reducing youth } \\
\text { internalizing symptoms: Effects of a family-based preventive } \\
\text { intervention on parental guilt induction and youth cognitive style. } \\
\text { Development and psychopathology, 26(02), 319-332. }\end{array}$ & $\begin{array}{l}\text { Parent } \\
\text { component too } \\
\text { small }\end{array}$ \\
\hline $\begin{array}{l}\text { 1085. McLaughlin, A. E., Campbell, F. A., Pungello, E. P., \& Skinner, } \\
\text { M. (2007). Depressive Symptoms in Young Adults: The Influences of } \\
\text { the Early Home Environment and Early Educational Child Care. Child } \\
\text { Development, 78(3), 746-756. doi:10.1111/j.1467-8624.2007.01030.x }\end{array}$ & $\begin{array}{l}\text { Not a parenting } \\
\text { intervention }\end{array}$ \\
\hline $\begin{array}{l}\text { 1086. McLaughlin, K. A., Zeanah, C. H., Fox, N. A., \& Nelson, C. A. } \\
\text { (2012). Attachment security as a mechanism linking foster care } \\
\text { placement to improved mental health outcomes in previously } \\
\text { institutionalized children. In J Child Psychol Psychiatry (pp. 46-55). }\end{array}$ & $\begin{array}{l}\text { No long term } \\
\text { follow-up }\end{array}$ \\
\hline $\begin{array}{l}\text { 1087. McLeod BD and Weisz JR. 2005. The Therapy Process } \\
\text { Observational Coding System-Alliance Scale: Measure Characteristics } \\
\text { and Prediction of Outcome in Usual Clinical Practice. Journal of } \\
\text { Consulting and Clinical Psychology. } 73(2): 323-33 .\end{array}$ & Not an RCT \\
\hline $\begin{array}{l}\text { 1088. McLoyd, V. C. (2011). How money matters for children's } \\
\text { socioemotional adjustment: Family processes and parental } \\
\text { investment. In Health disparities in youth and families (pp. 33-72). } \\
\text { Springer New York. }\end{array}$ & $\begin{array}{l}\text { Not a parenting } \\
\text { intervention }\end{array}$ \\
\hline
\end{tabular}




\begin{tabular}{|c|c|}
\hline $\begin{array}{l}\text { 1089. McMahon B, Holly L, Harrington R, Roberts C and Green J. } \\
\text { 2008. Do larger studies find smaller effects? The example of studies } \\
\text { for the prevention of conduct disorder. European Child \& Adolescent } \\
\text { Psychiatry. 17(7):432-7. }\end{array}$ & Review \\
\hline $\begin{array}{l}\text { 1090. McMahon RJ. 1994. Diagnosis, assessment, and treatment of } \\
\text { externalizing problems in children: The role of longitudinal data. } \\
\text { Journal of Consulting and Clinical Psychology. 62(5):901-17. }\end{array}$ & Review \\
\hline $\begin{array}{l}\text { 1091. McNeil, C. B., Capage, L. C., Bahl, A., \& Blanc, H. (1999). } \\
\text { Importance of early intervention for disruptive behavior problems: } \\
\text { Comparison of treatment and waitlist-control groups. Early Education } \\
\text { and Development, 10, 445-454. }\end{array}$ & $\begin{array}{l}\text { No long term } \\
\text { follow-up }\end{array}$ \\
\hline $\begin{array}{l}\text { 1092. McNeil, C. B., Herschell, A. D., Gurwitch, R. H., \& Clemens- } \\
\text { Mowrer, L. (2005). Training Foster Parents in Parent-Child Interaction } \\
\text { Therapy. [Article]. Education \& Treatment of Children, 28(2), 182-196. }\end{array}$ & Not an RCT \\
\hline $\begin{array}{l}\text { 1093. McRury JM and Zolotor AJ. 2010. A randomized, controlled } \\
\text { trial of a behavioral intervention to reduce crying among infants. J Am } \\
\text { Board Fam Med. 23(3):315-22. }\end{array}$ & $\begin{array}{l}\text { No child } \\
\text { internalising } \\
\text { outcomes or not } \\
\text { a major goal; } \\
\text { Follow-up less } \\
\text { than } 6 \text { months }\end{array}$ \\
\hline $\begin{array}{l}\text { 1094. Meeske, K. A., Sherman-Bien, S., Hamilton, A. S., Olson, A. R., } \\
\text { Slaughter, R., Kuperberg, A., \& Milam, J. (2013). Mental health } \\
\text { disparities between Hispanic and non-Hispanic parents of childhood } \\
\text { cancer survivors. Pediatr Blood Cancer, 60(9), 1470-1477. doi: } \\
\text { 10.1002/pbc.24527 }\end{array}$ & Not an RCT \\
\hline $\begin{array}{l}1095 . \quad \text { Mehling, W. E., Lown, E. A., Dvorak, C. C., Cowan, M. J., Horn, } \\
\text { B. N., Dunn, E. A., . . Hecht, F. M. (2012). Hematopoietic cell } \\
\text { transplant and use of massage for improved symptom management: } \\
\text { Results from a pilot randomized control trial. Evidence-based } \\
\text { Complementary and Alternative Medicine. doi:10.1155/2012/450150 }\end{array}$ & $\begin{array}{l}\text { Intervention for } \\
\text { developmental } \\
\text { disorders } \\
\text { (autism, autism } \\
\text { spectrum } \\
\text { disorder), } \\
\text { physical } \\
\text { disabilities, } \\
\text { medical } \\
\text { conditions or } \\
\text { distress about } \\
\text { facing medical } \\
\text { interventions }\end{array}$ \\
\hline $\begin{array}{l}\text { 1096. Meiser-Stedman R, Smith P, Glucksman E, Yule W and } \\
\text { Dalgleish T. 2007. Parent and child agreement for acute stress } \\
\text { disorder, post-traumatic stress disorder and other psychopathology in } \\
\text { a prospective study of children and adolescents exposed to single- } \\
\text { event trauma. Journal of Abnormal Child Psychology: An official } \\
\text { publication of the International Society for Research in Child and } \\
\text { Adolescent Psychopathology. 35(2):191-201. }\end{array}$ & $\begin{array}{l}\text { Not a parenting } \\
\text { intervention }\end{array}$ \\
\hline $\begin{array}{l}1097 . \quad \text { Mellin LM, Slinkard LA and Irwin CE, Jr. 1987. Adolescent } \\
\text { obesity intervention: validation of the SHAPEDOWN program. J Am } \\
\text { Diet Assoc. 87(3):333-8. }\end{array}$ & $\begin{array}{l}\text { Not a parenting } \\
\text { intervention }\end{array}$ \\
\hline $\begin{array}{l}\text { 1098. Melman, K. N. (1984). Post-divorce interventions: Facilitating } \\
\text { childrens' adjustment. Dissertation Abstracts International, (7-b), } \\
\text { 2252-2253. }\end{array}$ & $\begin{array}{l}\text { No long term } \\
\text { follow-up }\end{array}$ \\
\hline
\end{tabular}




\begin{tabular}{|c|c|}
\hline $\begin{array}{l}\text { 1099. Melnyk BM, Alpert-Gillis L, Feinstein NF, Fairbanks E, Schultz- } \\
\text { Czarniak J, Hust D, Sherman L, LeMoine C, Moldenhauer Z, Small L, } \\
\text { Bender N and Sinkin RA. 2001. Improving cognitive development of } \\
\text { low-birth-weight premature infants with the COPE program: a pilot } \\
\text { study of the benefit of early NICU intervention with mothers. Research } \\
\text { in nursing \& health, 24(5), 373-389. }\end{array}$ & $\begin{array}{l}\text { No child } \\
\text { internalising } \\
\text { outcomes or not } \\
\text { a major goal }\end{array}$ \\
\hline $\begin{array}{l}\text { 1100. Melnyk BM, Alpert-Gillis } L \text {, Hensel PB, Cable-Beiling RC and } \\
\text { Rubenstein JS. 1997. Helping mothers cope with a critically ill child: a } \\
\text { pilot test of the COPE intervention. Res Nurs Health. 20(1):3-14. }\end{array}$ & $\begin{array}{l}\text { Follow-up less } \\
\text { than } 6 \text { months }\end{array}$ \\
\hline $\begin{array}{l}\text { 1101. Melnyk BM, Crean HF, Feinstein NF and Fairbanks E. } 2008 . \\
\text { Maternal anxiety and depression after a premature infant's discharge } \\
\text { from the neonatal intensive care unit: explanatory effects of the } \\
\text { creating opportunities for parent empowerment program. Nurs Res. } \\
\text { 57(6):383-94. }\end{array}$ & $\begin{array}{l}\text { No child } \\
\text { internalising } \\
\text { outcomes or not } \\
\text { a major goal }\end{array}$ \\
\hline $\begin{array}{l}\text { 1102. Melnyk BM, Crean HF, Feinstein NF, Fairbanks E and Alpert- } \\
\text { Gillis LJ. 2007. Testing the theoretical framework of the COPE program } \\
\text { for mothers of critically ill children: an integrative model of young } \\
\text { children's post-hospital adjustment behaviors. J Pediatr Psychol. } \\
\text { 32(4):463-74. }\end{array}$ & $\begin{array}{l}\text { Follow-up less } \\
\text { than } 6 \text { months }\end{array}$ \\
\hline $\begin{array}{l}\text { 1103. Melnyk BM, Feinstein NF, Alpert-Gillis L, Fairbanks E, Crean } \\
\text { HF, Sinkin RA, Stone PW, Small L, Tu X and Gross SJ. 2006. Reducing } \\
\text { premature infants' length of stay and improving parents' mental } \\
\text { health outcomes with the Creating Opportunities for Parent } \\
\text { Empowerment (COPE) neonatal intensive care unit program: a } \\
\text { randomized, controlled trial. Pediatrics. 118(5):e1414-27. }\end{array}$ & $\begin{array}{l}\text { Follow-up less } \\
\text { than } 6 \text { months }\end{array}$ \\
\hline $\begin{array}{l}\text { 1104. Melnyk BM. 1994. Coping with unplanned childhood } \\
\text { hospitalization: effects of informational interventions on mothers and } \\
\text { children. Nurs Res. 43(1):50-5. }\end{array}$ & $\begin{array}{l}\text { Not a parenting } \\
\text { intervention }\end{array}$ \\
\hline $\begin{array}{l}\text { 1105. Melnyk, B. M., \& Feinstein, N. F. (2001). Mediating functions } \\
\text { of maternal anxiety and participation in care on young children's } \\
\text { posthospital adjustment. Res Nurs Health, 24(1), 18-26. doi: } \\
\text { 10.1002/1098-240X(200102)24:1<18::AID-NUR1003>3.0.CO;2-5 }\end{array}$ & $\begin{array}{l}\text { No child } \\
\text { internalising } \\
\text { outcomes or not } \\
\text { a major goal }\end{array}$ \\
\hline $\begin{array}{l}\text { 1106. Mendenhall, A. N., Fristad, M. A., \& Early, T. J. (2009). Factors } \\
\text { influencing service utilization and mood symptom severity in children } \\
\text { with mood disorders: effects of multifamily psychoeducation groups } \\
\text { (MFPGs). Journal of consulting and clinical psychology, (3), 463-473. }\end{array}$ & $\begin{array}{l}\text { Secondary } \\
\text { analysis of study } \\
\text { already excluded } \\
\text { because Parent } \\
\text { component too } \\
\text { small (Fristad et } \\
\text { al 2009) }\end{array}$ \\
\hline $\begin{array}{l}\text { 1107. Mendez, X., Orgiles, M., \& Espada, J. P. (2003). Tratamiento } \\
\text { psicológico de la fobia a la oscuridad en un contexto lúdico: un ensayo } \\
\text { controlado. / Psychological treatment of the phobia of the dark in a } \\
\text { game situation: A controlled essay. Revista de psicopatología y } \\
\text { psicología clínica, 8(3), 199-210. }\end{array}$ & Not in English \\
\hline $\begin{array}{l}\text { 1108. Mendlowitz, S. L. (1997). Coping strategies in children with } \\
\text { anxiety disorders: A cognitive-behavioral therapy outcome study. } \\
\text { [Dissertation Abstract; Dissertation]. Dissertation Abstracts } \\
\text { International: Section B: The Sciences and Engineering, 57(8-B). }\end{array}$ & $\begin{array}{l}\text { No long term } \\
\text { follow-up }\end{array}$ \\
\hline
\end{tabular}




\begin{tabular}{|c|c|}
\hline $\begin{array}{l}\text { 1109. Mendlowitz, S. L., Manassis, K., Bradley, S., Scapillato, D., } \\
\text { Miezitis, S., \& Shaw, B. F. (1999). Cognitive-behavioral group } \\
\text { treatments in childhood anxiety disorders: The role of parental } \\
\text { involvement. Journal of the American Academy of Child \& Adolescent } \\
\text { Psychiatry, 38(10), 1223-1229. doi:10.1097/00004583-199910000- } \\
\text { 00010 }\end{array}$ & $\begin{array}{l}\text { No long term } \\
\text { follow-up }\end{array}$ \\
\hline $\begin{array}{l}\text { 1110. Merry, S. N., McDowell, H. H., Hetrick, S. E., Bir, J. J., \& Muller, } \\
\text { N. (2004). Psychological and/or educational interventions for the } \\
\text { prevention of depression in children and adolescents. Cochrane } \\
\text { Database of Systematic Reviews, (2). } \\
\text { doi:10.1002/14651858.CD003380.pub2 }\end{array}$ & Review \\
\hline $\begin{array}{l}\text { 1111. Metternich, T. W., Plück, J., Wieczorrek, E., Freund-Braier, I., } \\
\text { Hautmann, C., Brix, G., \& Döpfner, M. (2002).PEP: A prevention } \\
\text { program for 3- to 6-year-old children with externalizing problem } \\
\text { behavior. Kindheit und Entwicklung. 11(2):98-106. }\end{array}$ & Review \\
\hline $\begin{array}{l}\text { 1112. Meyer EC, Coll CT, Lester BM, Boukydis CF, McDonough SM } \\
\text { and Oh W. 1994. Family-based intervention improves maternal } \\
\text { psychological well-being and feeding interaction of preterm infants. } \\
\text { Pediatrics. 93(2):241-6. }\end{array}$ & $\begin{array}{l}\text { No child } \\
\text { internalising } \\
\text { outcomes or not } \\
\text { a major goal; No } \\
\text { long term } \\
\text { follow-up }\end{array}$ \\
\hline $\begin{array}{l}\text { 1113. Mian, N. D., Eisenhower, A. S., \& Carter, A. S. (2015). Targeted } \\
\text { Prevention of Childhood Anxiety: Engaging Parents in an Underserved } \\
\text { Community. American journal of community psychology, 55(1-2), 58- } \\
69 .\end{array}$ & Not an RCT \\
\hline $\begin{array}{l}\text { 1114. Mianaei, S. J., Karahroudy, F. A., Rassouli, M., \& Tafreshi, M. Z. } \\
\text { (2014). The effect of Creating Opportunities for Parent Empowerment } \\
\text { program on maternal stress, anxiety, and participation in NICU wards } \\
\text { in Iran. Iran J Nurs Midwifery Res, 19(1), } 94-100 .\end{array}$ & $\begin{array}{l}\text { No child } \\
\text { internalising } \\
\text { outcomes or not } \\
\text { a major goal }\end{array}$ \\
\hline $\begin{array}{l}\text { 1115. Midmer D, Wilson L and Cummings S. 1995. A randomized, } \\
\text { controlled trial of the influence of prenatal parenting education on } \\
\text { postpartum anxiety and marital adjustment. Fam Med. 27(3):200-5. }\end{array}$ & $\begin{array}{l}\text { No child } \\
\text { internalising } \\
\text { outcomes or not } \\
\text { a major goal }\end{array}$ \\
\hline $\begin{array}{l}\text { 1116. Mifsud, C., \& Rapee, R. M. (2005). Early intervention for } \\
\text { childhood anxiety in a school setting: outcomes for an economically } \\
\text { disadvantaged population. Journal of the American Academy of Child } \\
\text { \& Adolescent Psychiatry, (10), 996-1004. }\end{array}$ & $\begin{array}{l}\text { Follow-up less } \\
\text { than } 6 \text { months }\end{array}$ \\
\hline $\begin{array}{l}\text { 1117. Mikirtichan GL and Dzharman OA. 2005. [Sociomedical } \\
\text { characteristics of the family of a child with tuberculosis]. Problemy } \\
\text { tuberkuleza i boleznei legkikh, (7), 28-32. }\end{array}$ & $\begin{array}{l}\text { Not a parenting } \\
\text { intervention }\end{array}$ \\
\hline $\begin{array}{l}\text { 1118. Miklowitz DJ, Axelson DA, George EL, Taylor DO, Schneck CD, } \\
\text { Sullivan AE, et al. Expressed emotion moderates the effects of family- } \\
\text { focused treatment for bipolar adolescents. J Am Acad Child Adolesc } \\
\text { Psychiatry. 2009 Jun;48(6):643-51. }\end{array}$ & $\begin{array}{l}\text { Parent } \\
\text { component too } \\
\text { small }\end{array}$ \\
\hline $\begin{array}{l}1119 . \quad \text { Miklowitz, D. J., Chang, K. D., Taylor, D. O., George, E. L., Singh, } \\
\text { M. K., Schneck, C. D., Dickinson, L. M., Howe, M. E., \& Garber, J. } \\
\text { (2011). Early psychosocial intervention for youth at risk for bipolar lor } \\
\text { II disorder: a one-year treatment development trial. Bipolar Disord, } \\
\text { 13, 67-75. }\end{array}$ & Not an RCT \\
\hline
\end{tabular}




\begin{tabular}{|c|c|}
\hline $\begin{array}{l}\text { 1120. Miklowitz, D. J., Schneck, C. D., Singh, M. K., Taylor, D. O., } \\
\text { George, E. L., Cosgrove, V. E., ... \& Chang, K. D. (2013). Early } \\
\text { intervention for symptomatic youth at risk for bipolar disorder: a } \\
\text { randomized trial of family-focused therapy. Journal of the American } \\
\text { Academy of Child \& Adolescent Psychiatry, 52(2), 121-131. }\end{array}$ & $\begin{array}{l}\text { Parent } \\
\text { component too } \\
\text { small; } \\
\text { Comparison } \\
\text { condition not a } \\
\text { minimal control }\end{array}$ \\
\hline $\begin{array}{l}\text { 1121. Milan S, Kershaw TS, Lewis J, Westdahl C, Rising SS, Patrikios } \\
\text { M and Ickovics JR. 2007. Cargiving history and prenatal depressive } \\
\text { symptoms in low-income adolescent and young adult women: } \\
\text { Moderating and mediating effects. Psychology of Women Quarterly. } \\
\text { 31(3):241-51. }\end{array}$ & Not an RCT \\
\hline $\begin{array}{l}\text { 1122. Milgrom J, Ericksen J, McCarthy R and Gemmill AW. } 2006 . \\
\text { Stressful impact of depression on early mother-infant relations. Stress } \\
\text { and Health: Journal of the International Society for the Investigation of } \\
\text { Stress. 22(4):229-38. }\end{array}$ & Not an RCT \\
\hline $\begin{array}{l}\text { 1123. Milgrom J, Schembri C, Ericksen J, Ross J and Gemmill AW. } \\
\text { 2010. Towards parenthood: An antenatal intervention to reduce } \\
\text { depression, anxiety and parenting difficulties. Journal of affective } \\
\text { disorders, 130(3), 385-394. }\end{array}$ & $\begin{array}{l}\text { No child } \\
\text { internalising } \\
\text { outcomes or not } \\
\text { a major goal }\end{array}$ \\
\hline $\begin{array}{l}\text { 1124. Millar Y, Glaser D, Reilly-Johnson NL, Hurst S-L, Harris K, Skerry } \\
\text { C and Charman T. 2010. Delivering child community psychology } \\
\text { services in the community: Experiences from the NIPPERS project. } \\
\text { Child and Adolescent Mental Health. 15(3):134-41. }\end{array}$ & Not an RCT \\
\hline $\begin{array}{l}\text { 1125. Miller GE and Prinz RJ. 2003. Engagement of Families in } \\
\text { Treatment for Childhood Conduct Problems. Behavior Therapy. Special } \\
\text { Issue: Behaviorally Oriented Interventions for Children With } \\
\text { Aggressive Behavior and/or Conduct Problems. 34(4):517-34. }\end{array}$ & $\begin{array}{l}\text { No child } \\
\text { internalising } \\
\text { outcomes or not } \\
\text { a major goal }\end{array}$ \\
\hline $\begin{array}{l}\text { 1126. Miller L, Gur M, Shanok A and Weissman M. } 2008 \text {. } \\
\text { Interpersonal psychotherapy with pregnant adolescents: Two pilot } \\
\text { studies. Journal of Child Psychology and Psychiatry. 49(7):733-42. }\end{array}$ & $\begin{array}{l}\text { Not a parenting } \\
\text { intervention }\end{array}$ \\
\hline $\begin{array}{l}\text { 1127. Miller VA and Feeny NC. 2003. Modification of Cognitive- } \\
\text { Behavioral Techniques in the Treatment of a Five Year-Old Girl with } \\
\text { Social Phobia. Journal of Contemporary Psychotherapy. 33(4):303-19. }\end{array}$ & Not an RCT \\
\hline $\begin{array}{l}\text { 1128. Miller, L. D., Laye-Gindhu, A., Liu, Y., March, J. S., Thordarson, } \\
\text { D. S., \& Garland, E. J. (2011). Evaluation of a preventive intervention } \\
\text { for child anxiety in two randomized attention-control school trials. } \\
\text { Behav Res Ther, 49, 315-323. }\end{array}$ & $\begin{array}{l}\text { Not a parenting } \\
\text { intervention }\end{array}$ \\
\hline $\begin{array}{l}\text { 1129. Miller, L. S. (1994). Primary prevention of conduct disorder. } \\
\text { Psychiatric Quarterly, 65, 273-285. }\end{array}$ & Review \\
\hline $\begin{array}{l}\text { 1130. Milot T, Éthier LS, St-Laurent D and Provost MA. 2010. The role } \\
\text { of trauma symptoms in the development of behavioral problems in } \\
\text { maltreated preschoolers. Child Abuse \& Neglect. 34(4):225-34. }\end{array}$ & $\begin{array}{l}\text { Not a parenting } \\
\text { intervention }\end{array}$ \\
\hline $\begin{array}{l}\text { 1131. Minde, K., et al. (1980). Self-help groups in a premature } \\
\text { nursery-a controlled evaluation. J Pediatr 96(5): 933-940. }\end{array}$ & $\begin{array}{l}\text { No child } \\
\text { internalising } \\
\text { outcomes or not } \\
\text { a major goal }\end{array}$ \\
\hline $\begin{array}{l}\text { 1132. Minkovitz, C. S., Hughart, N., Strobino, D., Scharfstein, D., } \\
\text { Grason, H., Hou, W., et al. (2003). A practice-based intervention to } \\
\text { enhance quality of care in the first } 3 \text { years of life: the Healthy Steps for } \\
\text { Young Children Program. JAMA, 290(23), 3081-3091. doi: } \\
\text { 10.1001/jama.290.23.3081 }\end{array}$ & $\begin{array}{l}\text { No long term } \\
\text { follow-up } \\
\text { (intervention } \\
\text { provided over } 3 \\
\text { years) }\end{array}$ \\
\hline
\end{tabular}




\begin{tabular}{|c|c|}
\hline $\begin{array}{l}\text { 1133. Minor KI and Elrod P. 1994. The effects of a probation } \\
\text { intervention on juvenile offenders' self-concepts, loci of control, and } \\
\text { perceptions of juvenile justice. Youth \& Society, 25(4), 490-511. }\end{array}$ & $\begin{array}{l}\text { Not a parenting } \\
\text { intervention }\end{array}$ \\
\hline $\begin{array}{l}\text { 1134. Miranda A, Presentacion MJ and Soriano M. } 2002 . \\
\text { Effectiveness of a school-based multicomponent program for the } \\
\text { treatment of children with ADHD. J Learn Disabil. 35(6):546-62. }\end{array}$ & $\begin{array}{l}\text { Not a parenting } \\
\text { intervention }\end{array}$ \\
\hline $\begin{array}{l}\text { 1135. Mirowsky J and Ross CE. 2002. Depression, parenthood, and } \\
\text { age at first birth. Social Science \& Medicine. 54(8):1281-98. }\end{array}$ & $\begin{array}{l}\text { Not a parenting } \\
\text { intervention }\end{array}$ \\
\hline $\begin{array}{l}\text { 1136. Misri S, Kostaras X, Fox D and Kostaras D. 2000. The impact of } \\
\text { partner support in the treatment of postpartum depression. Can J } \\
\text { Psychiatry. 45(6):554-8. }\end{array}$ & $\begin{array}{l}\text { No child } \\
\text { internalising } \\
\text { outcomes or not } \\
\text { a major goal }\end{array}$ \\
\hline $\begin{array}{l}\text { 1137. Misri S, Reebye } P, \text { Milis L and Shah S. 2006. The impact of } \\
\text { treatment intervention on parenting stress in postpartum depressed } \\
\text { mothers: a prospective study. American journal of orthopsychiatry, } \\
76(1), 115-119 .\end{array}$ & $\begin{array}{l}\text { No child } \\
\text { internalising } \\
\text { outcomes or not } \\
\text { a major goal }\end{array}$ \\
\hline $\begin{array}{l}\text { 1138. Mitchell, J. H., Newall, C., Broeren, S., \& Hudson, J. L. (2013). } \\
\text { The role of perfectionism in cognitive behaviour therapy outcomes for } \\
\text { clinically anxious children. Behav Res Ther, 51(9), 547-554. doi: } \\
\text { 10.1016/j.brat.2013.05.015 }\end{array}$ & $\begin{array}{l}\text { Not a parenting } \\
\text { intervention }\end{array}$ \\
\hline $\begin{array}{l}\text { 1139. Mitchell, J. L. (2012). Yoga effects on prenatal depression } \\
\text { (Doctoral dissertation, Fielding Graduate University). }\end{array}$ & $\begin{array}{l}\text { No child } \\
\text { internalising } \\
\text { outcomes or not } \\
\text { a major goal }\end{array}$ \\
\hline $\begin{array}{l}\text { 1140. Mitrani VB, McCabe BE, Robinson C, Weiss-Laxer NS, Feaster } \\
\text { DJ. Structural Ecosystems Therapy for recovering HIV-positive women: } \\
\text { Child, mother, and parenting outcomes. Journal of Family Psychology. } \\
2010 \text { Dec;24(6):746-55. }\end{array}$ & $\begin{array}{l}\text { Secondary } \\
\text { analysis of main } \\
\text { study that aimed } \\
\text { to improve HIV } \\
\text { medication } \\
\text { adherence and } \\
\text { prevent relapse; } \\
\text { No child } \\
\text { internalising } \\
\text { outcomes or not } \\
\text { a major goal }\end{array}$ \\
\hline $\begin{array}{l}\text { 1141. Moeller B, Krattenmacher T and Romer G. 2010. Child mental } \\
\text { health prevention in the context of adult medicine: A randomized } \\
\text { controlled trial of a manualized preventive counseling program for } \\
\text { children of parents with cancer. Psycho-Oncology. 19:S173. }\end{array}$ & $\begin{array}{l}\text { Data not } \\
\text { available/report } \\
\text { ed }\end{array}$ \\
\hline $\begin{array}{l}\text { 1142. Mohebi, S., Sharifirad, G. H., Shahsiah, M., Botlani, S., Matlabi, } \\
\text { M., \& Rezaeian, M. (2012). The effect of assertiveness training on } \\
\text { student's academic anxiety. J Pak Med Assoc, 62, S37-41. }\end{array}$ & $\begin{array}{l}\text { Not a parenting } \\
\text { intervention }\end{array}$ \\
\hline $\begin{array}{l}\text { 1143. Moilanen KL, Shaw DS and Maxwell KL. 2010. Developmental } \\
\text { cascades: Externalizing, internalizing, and academic competence from } \\
\text { middle childhood to early adolescence. Development and } \\
\text { Psychopathology. Special Issue: Developmental cascades: Part } 1 . \\
\text { 22(3):635-53. }\end{array}$ & $\begin{array}{l}\text { Not a parenting } \\
\text { intervention }\end{array}$ \\
\hline
\end{tabular}




\begin{tabular}{|c|c|}
\hline $\begin{array}{l}\text { 1144. Moldenhauer, Z. (2004). Adolescent depression: A primary } \\
\text { care pilot intervention study. [Dissertation Abstract; Dissertation]. } \\
\text { Dissertation Abstracts International: Section B: The Sciences and } \\
\text { Engineering, } 65(2-B), 656 \text {. }\end{array}$ & $\begin{array}{l}\text { follow-up less } \\
\text { than } 6 \text { months; } \\
\text { Parent } \\
\text { component too } \\
\text { small }\end{array}$ \\
\hline $\begin{array}{l}\text { 1145. Monaghan M, Hilliard ME, Cogen FR and Streisand R. } 2011 . \\
\text { Supporting parents of very young children with type } 1 \text { diabetes: } \\
\text { Results from a pilot study. Patient Education and Counseling. } \\
\text { 82(2):271-4. }\end{array}$ & Not an RCT \\
\hline $\begin{array}{l}\text { 1146. Monga, S., Young, A., \& Owens, M. (2009). Evaluating a } \\
\text { cognitive behavioral therapy group program for anxious five to seven } \\
\text { year old children: A pilot study. Depression and Anxiety, 26(3), 243- } \\
\text { 250. doi: } 10.1002 / \text { da.20551 }\end{array}$ & $\begin{array}{l}\text { No long term } \\
\text { follow-up }\end{array}$ \\
\hline $\begin{array}{l}\text { 1147. Moore L, Moore GF, Tapper K, Lynch R, Desousa C, Hale J, } \\
\text { Roberts C and Murphy S. 2007. Free breakfasts in schools: design and } \\
\text { conduct of a cluster randomised controlled trial of the Primary School } \\
\text { Free Breakfast Initiative in Wales [ISRCTN18336527]. BMC Public } \\
\text { Health, } 7(1), 258 .\end{array}$ & $\begin{array}{l}\text { Not a parenting } \\
\text { intervention }\end{array}$ \\
\hline $\begin{array}{l}\text { 1148. Moran G and Diamond G. 2008. Generating nonnegative } \\
\text { attitudes among parents of depressed adolescents: The power of } \\
\text { empathy, concern, and positive regard. Psychother Res. 18(1):97-107. }\end{array}$ & Not an RCT \\
\hline $\begin{array}{l}\text { 1149. Morawska A, Sanders MR. Self-administered behavioral family } \\
\text { intervention for parents of toddlers. Part 1: efficacy. J Consult Clin } \\
\text { Psychol 2006;74:10-9. }\end{array}$ & $\begin{array}{l}\text { No child } \\
\text { internalising } \\
\text { outcomes or not } \\
\text { a major goal; } \\
\text { Follow-up data } \\
\text { collected only in } \\
\text { active group }\end{array}$ \\
\hline $\begin{array}{l}\text { 1150. Morawska, A., Nitschke, F., \& Burrows, S. (2011). Do } \\
\text { testimonials improve parental perceptions and participation in } \\
\text { parenting programmes? Results of two studies. Journal of Child Health } \\
\text { Care, } 1367493510397625 .\end{array}$ & $\begin{array}{l}\text { No child } \\
\text { internalising } \\
\text { outcomes or not } \\
\text { a major goal }\end{array}$ \\
\hline $\begin{array}{l}\text { 1151. Moretti MM, Obsuth I. Effectiveness of an attachment- } \\
\text { focused manualized intervention for parents of teens at risk for } \\
\text { aggressive behaviour: The Connect Program. Journal of Adolescence. } \\
\text { 2009 Dec;32(6):1347-57. }\end{array}$ & Not an RCT \\
\hline $\begin{array}{l}\text { 1152. Moretti, M. M., Obsuth, I., Craig, S. G., \& Bartolo, T. (2015). An } \\
\text { attachment-based intervention for parents of adolescents at risk: } \\
\text { mechanisms of change. Attachment \& human development, 17(2), } \\
\text { 119-135. }\end{array}$ & Not an RCT \\
\hline $\begin{array}{l}\text { 1153. Morgan JF. 2009. Mothers were less likely to be depressed } \\
\text { after a structured behavioural intervention for infant sleep problems. } \\
\text { Evidence-Based Medicine. 14(2):45. }\end{array}$ & Not an RCT \\
\hline $\begin{array}{l}\text { 1154. Morgan, C., Novak, I., Dale, R. C., Guzzetta, A., \& Badawi, N. } \\
\text { (2014). GAME (Goals-Activity-Motor Enrichment): protocol of a single } \\
\text { blind randomised controlled trial of motor training, parent education } \\
\text { and environmental enrichment for infants at high risk of cerebral } \\
\text { palsy. BMC neurology, 14(1), } 203 .\end{array}$ & $\begin{array}{l}\text { Data not } \\
\text { available/report } \\
\text { ed }\end{array}$ \\
\hline
\end{tabular}




\begin{tabular}{|c|c|}
\hline $\begin{array}{l}\text { 1155. Morrell CJ, Slade P, Warner R, Paley G, Dixon S, Walters SJ, } \\
\text { Brugha T, Barkham M, Parry GJ and Nicholl J. 2009. Clinical } \\
\text { effectiveness of health visitor training in psychologically informed } \\
\text { approaches for depression in postnatal women: pragmatic cluster } \\
\text { randomised trial in primary care. BMJ. 338:a3045. }\end{array}$ & $\begin{array}{l}\text { No child } \\
\text { internalising } \\
\text { outcomes or not } \\
\text { a major goal }\end{array}$ \\
\hline $\begin{array}{l}\text { 1156. Morris PA and Hendra R. 2009. Losing the safety net: how a } \\
\text { time-limited welfare policy affects families at risk of reaching time } \\
\text { limits. Dev Psychol. 45(2):383-400. }\end{array}$ & Not an RCT \\
\hline $\begin{array}{l}1157 . \quad \text { Morris PA. 2008. Welfare program implementation and } \\
\text { parents,Äô depression. Social Service Review. 82(4):579-614. }\end{array}$ & $\begin{array}{l}\text { Not a parenting } \\
\text { intervention }\end{array}$ \\
\hline $\begin{array}{l}\text { 1158. Morris, P., \& Michalopoulos, C. (2000). The Self-Sufficiency } \\
\text { Project at } 36 \text { Months: Effects on Children of a Program That Increased } \\
\text { Parental Employment and Income. Ontario: Social Research and } \\
\text { Demonstration Corporation, 3-29. Retrieved from } \\
\text { http://www.mrw.interscience.wiley.com/cochrane/clcentral/articles/ } \\
\text { 343/CN-00635343/frame.html }\end{array}$ & $\begin{array}{l}\text { No long term } \\
\text { follow-up }\end{array}$ \\
\hline $\begin{array}{l}\text { 1159. Morrison-Beedy D, Carey MP, Côté-Arsenault D, Seibold- } \\
\text { Simpson S and Robinson KA. 2008. Understanding sexual abstinence in } \\
\text { urban adolescent girls. Journal of Obstetric, Gynecologic, \& Neonatal } \\
\text { Nursing: Clinical Scholarship for the Care of Women, Childbearing } \\
\text { Families, \& Newborns. 37(2):185-95. }\end{array}$ & $\begin{array}{l}\text { Not a parenting } \\
\text { intervention }\end{array}$ \\
\hline $\begin{array}{l}\text { 1160. Mosack KE, Gore-Felton C, Chartier M and McGarvey E. } 2007 . \\
\text { Individual, peer, and family variables associated with risky sexual } \\
\text { behavior among male and female incarcerated adolescents. Journal of } \\
\text { HIV/AIDS Prevention in Children \& Youth. 8(1):115-33. }\end{array}$ & $\begin{array}{l}\text { Not a parenting } \\
\text { intervention }\end{array}$ \\
\hline $\begin{array}{l}\text { 1161. Moser DK, Dracup K and Doering LV. 1999. Effect of } \\
\text { cardiopulmonary resuscitation training for parents of high-risk } \\
\text { neonates on perceived anxiety, control, and burden. Heart Lung. } \\
\text { 28(5):326-33. }\end{array}$ & $\begin{array}{l}\text { No child } \\
\text { internalising } \\
\text { outcomes or not } \\
\text { a major goal }\end{array}$ \\
\hline $\begin{array}{l}\text { 1162. Moser DK, Dracup K and Doering LV. 2000. Factors } \\
\text { differentiating dropouts from completers in a longitudinal, multicenter } \\
\text { clinical trial. Nurs Res. 49(2):109-16. }\end{array}$ & $\begin{array}{l}\text { No child } \\
\text { internalising } \\
\text { outcomes or not } \\
\text { a major goal }\end{array}$ \\
\hline $\begin{array}{l}\text { 1163. Moss E, Dubois-Comtois K, Cyr C, Tarabulsy GM, St-Laurent D } \\
\text { and Bernier A. 2011. Efficacy of a home-visiting intervention aimed at } \\
\text { improving maternal sensitivity, child attachment, and behavioral } \\
\text { outcomes for maltreated children: A randomized control trial. } \\
\text { Development and Psychopathology. 23(1):195-210. }\end{array}$ & $\begin{array}{l}\text { No long term } \\
\text { follow-up }\end{array}$ \\
\hline $\begin{array}{l}\text { 1164. Moss E, Smolla N, Guerra I, Mazzarello T, Chayer D and } \\
\text { Berthiaume C. 2006. Attachment and self-reported internalizing and } \\
\text { externalizing behavior problems in a school period. Canadian Journal } \\
\text { of Behavioural Science/Revue canadienne des sciences du } \\
\text { comportement. 38(2):142-57. }\end{array}$ & $\begin{array}{l}\text { Not a parenting } \\
\text { intervention }\end{array}$ \\
\hline $\begin{array}{l}\text { 1165. Motlagh MZ, Jazayeri AR, Khoshabi K, Mazaheri MA and } \\
\text { Karimlou M. 2009. Effectiveness of attachment based therapy on } \\
\text { reduction of symptoms in separation anxiety disorder. Iranian Journal } \\
\text { of Psychiatry and Clinical Psychology. 14(4):380-8. }\end{array}$ & Not an RCT \\
\hline $\begin{array}{l}\text { 1166. Mueller, J., Alie, C., Jonas, B., Brown, E., \& Sherr, L. (2011). A } \\
\text { quasi-experimental evaluation of a community-based art therapy } \\
\text { intervention exploring the psychosocial health of children affected by } \\
\text { HIV in South Africa. Trop Med Int Health, 16, 57-66. }\end{array}$ & $\begin{array}{l}\text { Not a parenting } \\
\text { intervention }\end{array}$ \\
\hline
\end{tabular}




\begin{tabular}{|c|c|}
\hline $\begin{array}{l}\text { 1167. Mufson L, Weissman MM, Moreau D and Garfinkel R. } 1999 . \\
\text { Efficacy of interpersonal psychotherapy for depressed adolescents. } \\
\text { Archives of General Psychiatry. 56(6):573-9. }\end{array}$ & $\begin{array}{l}\text { Not a parenting } \\
\text { intervention }\end{array}$ \\
\hline $\begin{array}{l}\text { 1168. Mulkens, S., Fleuren, D., Nederkoorn, C., \& Meijers, J. (2007). } \\
\text { RealFit: Een multidisciplinaire groepsbehandeling voor jongeren met } \\
\text { overgewicht. / RealFit: A multidisciplinary (CBT) group treatment for } \\
\text { obese youngsters. Gedragstherapie, } 40(1), 27-48 .\end{array}$ & $\begin{array}{l}\text { No long term } \\
\text { follow-up }\end{array}$ \\
\hline $\begin{array}{l}\text { 1169. Mullany, B., Barlow, A., Neault, N., Billy, T., Jones, T., Tortice, } \\
\text { I., . . Walkup, J. (2012). The Family Spirit trial for American Indian } \\
\text { teen mothers and their children: CBPR rationale, design, methods and } \\
\text { baseline characteristics. Prevention science, 13(5), 504-518. } \\
\text { doi:10.1007/s11121-012-0277-2 }\end{array}$ & $\begin{array}{l}\text { Data not } \\
\text { available/report } \\
\text { ed (protocol) }\end{array}$ \\
\hline $\begin{array}{l}\text { 1170. Mullin E, Quigley K, Glanville BA. Controlled evaluation of the } \\
\text { impact of a parent training program on child behavior and mothers' } \\
\text { general well-being. Couns Psychol Quart 1994;7:167-79. }\end{array}$ & $\begin{array}{l}\text { Follow-up data } \\
\text { collected only in } \\
\text { active group }\end{array}$ \\
\hline $\begin{array}{l}\text { 1171. Munsch S, Roth B, Michael T, Meyer AH, Biedert E, Roth S, et } \\
\text { al. Randomized controlled comparison of two cognitive behavioral } \\
\text { therapies for obese children: mother versus mother-child cognitive } \\
\text { behavioral therapy. Psychother Psychosom. } 2008 ; 77(4): 235-46 \text {. }\end{array}$ & $\begin{array}{l}\text { Comparison } \\
\text { condition not a } \\
\text { minimal control }\end{array}$ \\
\hline $\begin{array}{l}\text { 1172. Muntz, R., Hutchings, J., Edwards, R. T., Hounsome, B., \& } \\
\text { O'Ceilleachair, A. (2004). Economic evaluation of treatments for } \\
\text { children with severe behavioural problems. J Ment Health Policy Econ, } \\
\text { 7(4), 177-189. }\end{array}$ & $\begin{array}{l}\text { No child } \\
\text { internalising } \\
\text { outcomes or not } \\
\text { a major goal }\end{array}$ \\
\hline $\begin{array}{l}\text { 1173. Muris P, Meesters C and van Melick M. 2002. Treatment of } \\
\text { childhood anxiety disorders; A preliminary comparison between } \\
\text { cognitive-behavioral group therapy and a psychological placebo } \\
\text { intervention. Journal of Behavior Therapy and Experimental } \\
\text { Psychiatry. 33(3-4):143-58. }\end{array}$ & $\begin{array}{l}\text { Not a parenting } \\
\text { intervention }\end{array}$ \\
\hline $\begin{array}{l}\text { 1174. Murphy SA, Johnson C, Cain KC, Gupta AD, Dimond M, Lohan J } \\
\text { and Baugher R. 1998. Broad-spectrum group treatment for parents } \\
\text { bereaved by the violent deaths of their 12- to } 28 \text {-yr-old children: a } \\
\text { randomized controlled trial. Death Studies, 22(3), 209-235. }\end{array}$ & $\begin{array}{l}\text { No child } \\
\text { internalising } \\
\text { outcomes or not } \\
\text { a major goal }\end{array}$ \\
\hline $\begin{array}{l}\text { 1175. Murphy SA. 1997. A bereavement intervention for parents } \\
\text { following the sudden, violent deaths of their 12-28-year-old children: } \\
\text { description and applications to clinical practice. Can J Nurs Res. } \\
\text { 29(4):51-72. }\end{array}$ & $\begin{array}{l}\text { No child } \\
\text { internalising } \\
\text { outcomes or not } \\
\text { a major goal }\end{array}$ \\
\hline $\begin{array}{l}\text { 1176. Murray DW. 2010. Treatment of preschoolers with attention- } \\
\text { deficit/hyperactivity disorder. Current Psychiatry Reports. 12(5):374- } \\
81 .\end{array}$ & Review \\
\hline $\begin{array}{l}\text { 1177. Murray, L., Cooper, P., Wilson, A., \& Romaniuk, H. (2003). } \\
\text { Controlled trial of the short-and long-term effect of psychological } \\
\text { treatment of postpartum depression. 2. Impact on the mother-child } \\
\text { interaction. British Journal of Psychiatry, } 182,420-427 \text {. }\end{array}$ & $\begin{array}{l}\text { No child } \\
\text { internalising } \\
\text { outcomes or not } \\
\text { a major goal }\end{array}$ \\
\hline $\begin{array}{l}\text { 1178. Muzik, M., Rosenblum, K. L., Alfafara, E. A., Schuster, M. M., } \\
\text { Miller, N. M., Waddell, R. M., \& Kohler, E. S. (2015). Mom Power: } \\
\text { preliminary outcomes of a group intervention to improve mental } \\
\text { health and parenting among high-risk mothers. Archives of women's } \\
\text { mental health, } 18(3), 507-521 .\end{array}$ & Not an RCT \\
\hline
\end{tabular}




\begin{tabular}{|c|c|}
\hline $\begin{array}{l}\text { 1179. Muzik, M., Rosenblum, K., Schuster, M., Stanton, E., Alfafara, } \\
\text { E., Ribaudo, J., .. Gorin-Meyer, R. (2013). Mom power: An } \\
\text { attachment-based parenting intervention for trauma-exposed } \\
\text { mothers of young children. [Conference Abstract]. Archives of } \\
\text { Women's Mental Health, 16, S21-S22. doi: } \\
\text { http://dx.doi.org/10.1007/s00737-013-0355-x }\end{array}$ & $\begin{array}{l}\text { Follow-up less } \\
\text { than } 6 \text { months }\end{array}$ \\
\hline $\begin{array}{l}\text { 1180. Myrén KJ, Thernlund G, NylÈn A, Schacht A and Svanborg P. } \\
\text { 2010. Atomoxetine's effect on societal costs in Sweden. Journal of } \\
\text { attention disorders, 13(6), 618-28. }\end{array}$ & $\begin{array}{l}\text { Not a parenting } \\
\text { intervention }\end{array}$ \\
\hline $\begin{array}{l}\text { 1181. Naar-King S, Podolski C-L, Ellis DA, Frey MA and Templin T. } \\
\text { 2006. Social ecological model of illness management in high-risk } \\
\text { youths with type } 1 \text { diabetes. Journal of Consulting and Clinical } \\
\text { Psychology. 74(4):785-9. }\end{array}$ & $\begin{array}{l}\text { Not a parenting } \\
\text { intervention }\end{array}$ \\
\hline $\begin{array}{l}\text { 1182. Nair, P., Schuler, M. E., Black, M. M., Kettinger, L., \& } \\
\text { Harrington, D. (2003). Cumulative environmental risk in substance } \\
\text { abusing women: Early intervention, parenting stress, child abuse } \\
\text { potential and child development. Child Abuse \& Neglect, 27(9), 997- } \\
\text { 1017. doi: } 10.1016 / \text { s0145-2134(03)00169-8 }\end{array}$ & $\begin{array}{l}\text { No child } \\
\text { internalising } \\
\text { outcomes or not } \\
\text { a major goal }\end{array}$ \\
\hline $\begin{array}{l}\text { 1183. Nash, K., Stevens, S., Greenbaum, R., Weiner, J., Koren, G., \& } \\
\text { Rovet, J. (2015). Improving executive functioning in children with fetal } \\
\text { alcohol spectrum disorders. Child Neuropsychology, 21(2), 191-209. }\end{array}$ & $\begin{array}{l}\text { Not a parenting } \\
\text { intervention }\end{array}$ \\
\hline $\begin{array}{l}\text { 1184. Nauta MH, Scholing A, Emmelkamp PM and Minderaa RB. } \\
\text { 2003. Cognitive-behavioral therapy for children with anxiety disorders } \\
\text { in a clinical setting: no additional effect of a cognitive parent training. J } \\
\text { Am Acad Child Adolesc Psychiatry. 42(11):1270-8. }\end{array}$ & $\begin{array}{l}\text { Follow-up less } \\
\text { than } 6 \text { months }\end{array}$ \\
\hline $\begin{array}{l}1185 . \quad \text { Nauta, M. H., Festen, H., Reichart, C. G., Nolen, W. A., Stant, A. } \\
\text { D., Bockting, C. L., van der Wee, N. J., Beekman, A., Doreleijers, T. A., } \\
\text { Hartman, C. A., de Jong, P. J., \& de Vries, S. O. (2012). Preventing } \\
\text { mood and anxiety disorders in youth: a multi-centre RCT in the high } \\
\text { risk offspring of depressed and anxious patients. BMC Psychiatry, 12, } \\
\text { 31. }\end{array}$ & $\begin{array}{l}\text { Not a parenting } \\
\text { intervention }\end{array}$ \\
\hline $\begin{array}{l}\text { 1186. Nauta, M. H., Scholing, A., Emmelkamp, P. M., \& Minderaa, R. } \\
\text { B. (2001). Cognitive-behavioural therapy for anxiety disordered } \\
\text { children in a clinical setting: does additional cognitive parent training } \\
\text { enhance treatment effectiveness? Clinical Psychology and } \\
\text { Psychotherapy, (5), 330-340. }\end{array}$ & $\begin{array}{l}\text { Parent } \\
\text { component too } \\
\text { small }\end{array}$ \\
\hline $\begin{array}{l}\text { 1187. Naylor D, Coates TJ and Kan J. 1984. Reducing distress in } \\
\text { pediatric cardiac catheterization. Am J Dis Child. 138(8):726-9. }\end{array}$ & $\begin{array}{l}\text { Intervention for } \\
\text { developmental } \\
\text { disorders } \\
\text { (autism, autism } \\
\text { spectrum } \\
\text { disorder), } \\
\text { physical } \\
\text { disabilities, } \\
\text { medical } \\
\text { conditions or } \\
\text { distress about } \\
\text { facing medical } \\
\text { interventions }\end{array}$ \\
\hline
\end{tabular}




\begin{tabular}{|c|c|}
\hline $\begin{array}{l}\text { 1188. Neece, C. L. (2014). Mindfulness-based stress reduction for } \\
\text { parents of young children with developmental delays: Implications for } \\
\text { parental mental health and child behavior problems. Journal of } \\
\text { Applied Research in Intellectual Disabilities, 27(2), 174-186. }\end{array}$ & $\begin{array}{l}\text { No long term } \\
\text { follow-up; } \\
\text { Targets } \\
\text { externalising }\end{array}$ \\
\hline $\begin{array}{l}\text { 1189. Nelson, E., Barnard, M., \& Cain, S. (2003). Treating childhood } \\
\text { depression over videoconferencing. Telemedicine Journal and e- } \\
\text { Health, 9, 49-55. }\end{array}$ & $\begin{array}{l}\text { Not a parenting } \\
\text { intervention; No } \\
\text { long term } \\
\text { follow-up }\end{array}$ \\
\hline $\begin{array}{l}\text { 1190. Nelson, G., Westhues, A., \& MacLeod, J. (2003). A meta- } \\
\text { analysis of longitudinal research on preschool prevention programs for } \\
\text { children. Prevention \& Treatment, 6(1), 31a. }\end{array}$ & Review \\
\hline $\begin{array}{l}\text { 1191. Nelson-Gray RO, Keane SP, Hurst RM, Mitchell JT, Warburton } \\
\text { JB, Chok JT and Cobb AR. 2006. A modified DBT skills training program } \\
\text { for oppositional defiant adolescents: Promising preliminary findings. } \\
\text { Behaviour Research and Therapy. 44(12):1811-20. }\end{array}$ & $\begin{array}{l}\text { Not a parenting } \\
\text { intervention }\end{array}$ \\
\hline $\begin{array}{l}\text { 1192. Neto D, Xavier M, Aguiar P, David M, Sardinha L and De } \\
\text { Almeida C. 1997. Sequential combined treatment of heroin addicted } \\
\text { patients in Portugal with naltrexone and family therapy. European } \\
\text { Addiction Research. 3(3):138-45. }\end{array}$ & $\begin{array}{l}\text { No child } \\
\text { internalising } \\
\text { outcomes or not } \\
\text { a major goal }\end{array}$ \\
\hline $\begin{array}{l}\text { 1193. Neuhoff, C. C. (2006). Prescriptive treatment for separation } \\
\text { anxiety disorder: Child therapy versus parent training. Dissertation } \\
\text { Abstracts International, (12-b), } 6931 \text {. }\end{array}$ & $\begin{array}{l}\text { No long term } \\
\text { follow-up }\end{array}$ \\
\hline $\begin{array}{l}\text { 1194. Neumark-Sztainer D, Story M, Hannan PJ, Tharp T and Rex J. } \\
\text { 2003. Factors associated with changes in physical activity: a cohort } \\
\text { study of inactive adolescent girls. Arch Pediatr Adolesc Med. } \\
\text { 157(8):803-10. }\end{array}$ & $\begin{array}{l}\text { Not a parenting } \\
\text { intervention }\end{array}$ \\
\hline $\begin{array}{l}1195 . \quad \text { Newcombe, P. A., Dunn, T. L., Casey, L. M., Sheffield, J. K., } \\
\text { Petsky, H., Anderson-James, S., \& Chang, A. B. (2012). Breathe Easier } \\
\text { Online: evaluation of a randomized controlled pilot trial of an Internet- } \\
\text { based intervention to improve well-being in children and adolescents } \\
\text { with a chronic respiratory condition. J Med Internet Res, 14, e23. }\end{array}$ & $\begin{array}{l}\text { Not a parenting } \\
\text { intervention }\end{array}$ \\
\hline $\begin{array}{l}\text { 1196. Ng SM, Li AM, Lou VW, Tso IF, Wan PY and Chan DF. } 2008 . \\
\text { Incorporating family therapy into asthma group intervention: a } \\
\text { randomized waitlist-controlled trial. Fam Process. 47(1):115-30. }\end{array}$ & $\begin{array}{l}\text { No child } \\
\text { internalising } \\
\text { outcomes or not } \\
\text { a major goal }\end{array}$ \\
\hline $\begin{array}{l}1197 . \quad \text { Nguyen, Q. C., Schmidt, N., Glymour, M. M., Rehkopf, D., \& } \\
\text { Osypuk, T. L. (2012). Did adolescents in higher ses families } \\
\text { differentially benefit from a housing mobility intervention? American } \\
\text { Journal of Epidemiology, } 175, \text { S48. }\end{array}$ & $\begin{array}{l}\text { Not a parenting } \\
\text { intervention }\end{array}$ \\
\hline $\begin{array}{l}\text { 1198. Niccols A. 2009. Immediate and short-term outcomes of the } \\
\text { 'COPEing with Toddler Behaviour' parent group. J Child Psychol } \\
\text { Psychiatry. 50(5):617-26. }\end{array}$ & $\begin{array}{l}\text { Follow-up less } \\
\text { than } 6 \text { months }\end{array}$ \\
\hline $\begin{array}{l}\text { 1199. Niccols, A. (2008). 'Right from the Start': randomized trial } \\
\text { comparing an attachment group intervention to supportive home } \\
\text { visiting. Journal of child psychology and psychiatry, and allied } \\
\text { disciplines, (7), 754-764. }\end{array}$ & $\begin{array}{l}\text { No child } \\
\text { internalising } \\
\text { outcomes or not } \\
\text { a major goal }\end{array}$ \\
\hline $\begin{array}{l}\text { 1200. Nicholson J, Hinden BR, Biebel K, Henry AD and Katz-Leavy J. } \\
\text { 2007. A qualitative study of programs for parents with serious mental } \\
\text { illness and their children: Building practice-based evidence. The } \\
\text { Journal of Behavioral Health Services \& Research. 34(4):395-413. }\end{array}$ & Review \\
\hline
\end{tabular}




\begin{tabular}{|c|c|}
\hline $\begin{array}{l}\text { 1201. Niebel G, Kallweit C, Lange I and Folster-Holst R. 2000. Direct } \\
\text { versus video-based parental education in the treatment of atopic } \\
\text { eczema in children. A controlled pilot study. Hautarzt. 51(6):401-11. }\end{array}$ & $\begin{array}{l}\text { No long term } \\
\text { follow-up; No } \\
\text { child } \\
\text { internalising } \\
\text { outcomes or not } \\
\text { a major goal }\end{array}$ \\
\hline $\begin{array}{l}\text { 1202. Niemeijer AS, Smits-Engelsman BC and Schoemaker MM. } \\
\text { 2007. Neuromotor task training for children with developmental } \\
\text { coordination disorder: a controlled trial. Dev Med Child Neurol. } \\
\text { 49(6):406-11. }\end{array}$ & $\begin{array}{l}\text { Not a parenting } \\
\text { intervention }\end{array}$ \\
\hline $\begin{array}{l}\text { 1203. Niemelä, M., Repo, J., Wahlberg, K.-E., Hakko, H., \& Räsänen, } \\
\text { S. (2012). Pilot evaluation of the impact of structured child-centered } \\
\text { interventions on psychiatric symptom profile of parents with serious } \\
\text { somatic illness: Struggle for Life trial. Journal of Psychosocial } \\
\text { Oncology, 30, 316-330. }\end{array}$ & $\begin{array}{l}\text { No child } \\
\text { internalising } \\
\text { outcomes or not } \\
\text { a major goal }\end{array}$ \\
\hline $\begin{array}{l}\text { 1204. Nievar, M. A., Jacobson, A., Chen, Q., Johnson, U., \& Dier, S. } \\
\text { (2011). Impact of HIPPY on home learning environments of Latino } \\
\text { families. Early Childhood Research Quarterly, 26, 268-277. }\end{array}$ & Not an RCT \\
\hline $\begin{array}{l}\text { 1205. Nilsen, W. (2007). Fostering futures: A preventive intervention } \\
\text { program for school-age children in foster care. Clinical Child } \\
\text { Psychology and Psychiatry, 12(1), 45-63. doi: } \\
\text { 10.1177/1359104507071055 }\end{array}$ & Not an RCT \\
\hline $\begin{array}{l}\text { 1206. Nilsson D and Wadsby M. 2010. Symboldrama, a } \\
\text { psychotherapeutic method for adolescents with dissociative and PTSD } \\
\text { symptoms: A pilot study. Journal of Trauma \& Dissociation. 11(3):308- } \\
21 .\end{array}$ & $\begin{array}{l}\text { Not a parenting } \\
\text { intervention }\end{array}$ \\
\hline $\begin{array}{l}\text { 1207. Nixon RDV, Ellis AA, Nehmy TJ and Ball S-A. 2010. Screening } \\
\text { and predicting posttraumatic stress and depression in children } \\
\text { following single-incident trauma. Journal of Clinical Child and } \\
\text { Adolescent Psychology. 39(4):588-96. }\end{array}$ & $\begin{array}{l}\text { Not a parenting } \\
\text { intervention }\end{array}$ \\
\hline $\begin{array}{l}\text { 1208. Nixon, R. D. V., Sweeney, L., Erickson, D. B., \& Touyz, S. W. } \\
\text { (2003). Parent-child interaction therapy: A comparison of standard } \\
\text { and abbreviated treatments for oppositional defiant preschoolers. } \\
\text { Journal of Consulting and Clinical Psychology, 71(2), 251-260. doi: } \\
\text { 10.1037/0022-006x.71.2.251 }\end{array}$ & $\begin{array}{l}\text { Targets } \\
\text { externalising }\end{array}$ \\
\hline $\begin{array}{l}\text { 1209. Nixon, R.D., Sweney, L., Erickson, D.B., \& Touyz, S.W. (2004). } \\
\text { Parent-child interaction therapy: One and two-year follow-up of } \\
\text { standard and abbreviated treatments for oppositional preschoolers. } \\
\text { Journal of Abnormal Child Psychology, 32, 263-271. }\end{array}$ & $\begin{array}{l}\text { Follow-up data } \\
\text { collected only in } \\
\text { active group; } \\
\text { Targets } \\
\text { externalising }\end{array}$ \\
\hline $\begin{array}{l}\text { 1210. Nock MK and Photos V. 2006. Parent Motivation to Participate } \\
\text { in Treatment: Assessment and Prediction of Subsequent Participation. } \\
\text { Journal of Child and Family Studies. 15(3):345-58. }\end{array}$ & $\begin{array}{l}\text { No child } \\
\text { internalising } \\
\text { outcomes or not } \\
\text { a major goal }\end{array}$ \\
\hline $\begin{array}{l}\text { 1211. Noether CD, Brown V, Finkelstein N, Russell LA, VanDeMark } \\
\text { NR, Morris LS and Graeber C. 2007. Promoting resiliency in children of } \\
\text { mothers with co-occurring disorders and histories of trauma: Impact } \\
\text { of a skills-based intervention program on child outcomes. Journal of } \\
\text { Community Psychology. 35(7):823-43. }\end{array}$ & $\begin{array}{l}\text { Not a parenting } \\
\text { intervention }\end{array}$ \\
\hline
\end{tabular}




\begin{tabular}{|c|c|}
\hline $\begin{array}{l}\text { 1212. Norman E, Sherburn M, Osborne RH and Galea MP. 2010. An } \\
\text { exercise and education program improves well-being of new mothers: } \\
\text { a randomized controlled trial. Phys Ther. } 90(3): 348-55 \text {. }\end{array}$ & $\begin{array}{l}\text { No child } \\
\text { internalising } \\
\text { outcomes or not } \\
\text { a major goal }\end{array}$ \\
\hline $\begin{array}{l}\text { 1213. Nugent K, Brazelton TB. Preventive intervention with infants } \\
\text { and families: the NBAS model. Infant Ment Health J 1989;10:84-99. }\end{array}$ & Not an RCT \\
\hline $\begin{array}{l}\text { 1214. Nurcombe, B., et al. (1984). An intervention program for } \\
\text { mothers of low-birthweight infants: preliminary results. J Am Acad } \\
\text { Child Psychiatry 23(3): 319-325. }\end{array}$ & $\begin{array}{l}\text { No child } \\
\text { internalising } \\
\text { outcomes or not } \\
\text { a major goal }\end{array}$ \\
\hline $\begin{array}{l}\text { 1215. Nyamathi, A., Salem, B. E., Meyer, V., Ganguly, K. K., Sinha, S., } \\
\text { \& Ramakrishnan, P. (2012). Impact of an Asha intervention on } \\
\text { depressive symptoms among rural women living with AIDS in India: } \\
\text { comparison of the Asha-Life and Usual Care program. AIDS Educ Prev, } \\
\text { 24, 280-293. }\end{array}$ & $\begin{array}{l}\text { Not a parenting } \\
\text { intervention }\end{array}$ \\
\hline $\begin{array}{l}\text { 1216. O'Donnell J, Hawkins JD, Catalano RF, Abbott RD and Day LE. } \\
\text { 1995. PREVENTING SCHOOL FAILURE, DRUG USE, AND DELINQUENCY } \\
\text { AMONG LOW-INCOME CHILDREN: Long-Term Intervention in } \\
\text { Elementary Schools. American Journal of Orthopsychiatry. 65(1):87- } \\
\text { 100. }\end{array}$ & $\begin{array}{l}\text { No long term } \\
\text { follow-up; } \\
\text { Parent } \\
\text { component too } \\
\text { small; No child } \\
\text { internalising } \\
\text { outcomes or not } \\
\text { a major goal } \\
\end{array}$ \\
\hline $\begin{array}{l}\text { 1217. Ogden, T., \& Hagen, K. A. (2008). Treatment effectiveness of } \\
\text { Parent Management Training in Norway: a randomized controlled trial } \\
\text { of children with conduct problems. J Consult Clin Psychol, 76(4), 607- } \\
\text { 621. doi: 2008-09736-007 }\end{array}$ & $\begin{array}{l}\text { No long term } \\
\text { follow-up }\end{array}$ \\
\hline $\begin{array}{l}\text { 1218. Ohgi S, Akiyama T, Arisawa K and Shigemori K. } 2004 . \\
\text { Randomised controlled trial of swaddling versus massage in the } \\
\text { management of excessive crying in infants with cerebral injuries. } \\
\text { Archives of disease in childhood, } 89(3), 212-216 .\end{array}$ & $\begin{array}{l}\text { Intervention for } \\
\text { developmental } \\
\text { disorders } \\
\text { (autism, autism } \\
\text { spectrum } \\
\text { disorder), } \\
\text { physical } \\
\text { disabilities, } \\
\text { medical } \\
\text { conditions or } \\
\text { distress about } \\
\text { facing medical } \\
\text { interventions }\end{array}$ \\
\hline $\begin{array}{l}\text { 1219. O'Kearney R, Kang K, Christensen H and Griffiths K. 2009. A } \\
\text { controlled trial of a school-based Internet program for reducing } \\
\text { depressive symptoms in adolescent girls. Depression and Anxiety. } \\
\text { 26(1):65-72. }\end{array}$ & $\begin{array}{l}\text { Not a parenting } \\
\text { intervention }\end{array}$ \\
\hline
\end{tabular}




\begin{tabular}{|c|c|}
\hline $\begin{array}{l}\text { 1220. Olds D, Henderson CR, Cole R, Eckenrode J, Kitzman H, Luckey } \\
\text { D, Pettitt L, Sidora K, Morris P and Powers J. 1998. Long-term effects } \\
\text { of nurse home visitation on children's criminal and antisocial behavior } \\
\text { - 15-year follow-up of a randomized controlled trial. Jama-Journal of } \\
\text { the American Medical Association. } 280(14): 1238-44 .\end{array}$ & $\begin{array}{l}\text { Targets } \\
\text { externalising } \\
\text { (antisocial } \\
\text { behaviour: } \\
\text { running away, } \\
\text { arrests, sex, } \\
\text { substance use, } \\
\text { school } \\
\text { suspension etc) }\end{array}$ \\
\hline $\begin{array}{l}\text { 1221. Olds D, Henderson CR, Kitzman H and Cole R. 1995. Effects of } \\
\text { Prenatal and Infancy Nurse Home Visitation on Surveillance of Child } \\
\text { Maltreatment. Pediatrics. 95(3):365-72. }\end{array}$ & $\begin{array}{l}\text { No child } \\
\text { internalising } \\
\text { outcomes or not } \\
\text { a major goal }\end{array}$ \\
\hline $\begin{array}{l}\text { 1222. Olds DL, Henderson CR, Kitzman HJ, Eckenrode JJ, Cole RE and } \\
\text { Tatelbaum RC. 1999. Prenatal and infancy home visitation by nurses: } \\
\text { Recent findings. Future of Children. 9(1):44-65. }\end{array}$ & $\begin{array}{l}\text { Review of Olds } \\
1995,1998, \text { no } \\
\text { child } \\
\text { internalising } \\
\text { outcomes or not } \\
\text { a major goal }\end{array}$ \\
\hline $\begin{array}{l}\text { 1223. Olds DL, Henderson Jr CR, Chamberli } n \text { R, Tatelbaum R. } \\
\text { Preventing child abuse and neglect: a randomized trial of nurse home } \\
\text { visitation. Pediatrics 1986;78(1):65-78. }\end{array}$ & $\begin{array}{l}\text { No child } \\
\text { internalising } \\
\text { outcomes or not } \\
\text { a major goal }\end{array}$ \\
\hline $\begin{array}{l}\text { 1224. } \text { Olds, D. L. (2006). The Nurse-Family Partnership: An evidence- } \\
\text { based preventive intervention. Infant Mental Health Journal, 27, 5-25. }\end{array}$ & Review \\
\hline $\begin{array}{l}\text { 1225. Olds, D. L., Robinson, J., Pettitt, L., Luckey, D. W., Holmberg, J., } \\
\text { Ng, R. K., et al. (2004). Effects of home visits by paraprofessionals and } \\
\text { by nurses: age } 4 \text { follow-up results of a randomized trial. Pediatrics, } \\
\text { 114(6), 1560-1568. doi: } 114 / 6 / 1560\end{array}$ & $\begin{array}{l}\text { Targets } \\
\text { externalising }\end{array}$ \\
\hline $\begin{array}{l}\text { 1226. Olds, D.L.,Henderson, C.R.,\& Kitzman, H.J.(1994).Does } \\
\text { prenatal and infancy nurse home visitation have enduring effects on } \\
\text { qualities of parental caregiving and child health at } 25 \text { to } 50 \text { months of } \\
\text { life? Pediatrics, } 93,89-98 \text {. }\end{array}$ & $\begin{array}{l}\text { No child } \\
\text { internalising } \\
\text { outcomes or not } \\
\text { a major goal }\end{array}$ \\
\hline $\begin{array}{l}\text { 1227. Olivares, J., Rosa, A. I., \& Piqueras, J. A. (2005). Early detection } \\
\text { and treatment of adolescents with generalized social phobia. } \\
\text { Psicothema, 17(1), 1-8. }\end{array}$ & $\begin{array}{l}\text { Not a parenting } \\
\text { intervention }\end{array}$ \\
\hline $\begin{array}{l}\text { 1228. Olivares-Olivares PJ, Rosa-Alcázar Al and Olivares-Rodríguez J. } \\
\text { 2007. Social validity of adolescent intervention in social phobia: } \\
\text { Parents vs. teachers. Terapia psicológica. 25(1):63-71. }\end{array}$ & $\begin{array}{l}\text { Not a parenting } \\
\text { intervention }\end{array}$ \\
\hline $\begin{array}{l}\text { 1229. Olivares-Olivares PJ, Rosa-Alcázar Al and Olivares-Rodríguez J. } \\
\text { 2008. Does individual attention improve the effect of group treatment } \\
\text { of adolescents with social phobia? International Journal of Clinical and } \\
\text { Health Psychology. } 8(2): 465-81 \text {. }\end{array}$ & $\begin{array}{l}\text { Not a parenting } \\
\text { intervention }\end{array}$ \\
\hline $\begin{array}{l}\text { 1230. Oliver, B. R., Barker, E. D., Mandy, W. P. L., Skuse, D. H., \& } \\
\text { Maughan, B. (2011). Social cognition and conduct problems: A } \\
\text { developmental approach. Journal of the American Academy of Child \& } \\
\text { Adolescent Psychiatry, 50, 385-394. }\end{array}$ & $\begin{array}{l}\text { Not a parenting } \\
\text { intervention }\end{array}$ \\
\hline $\begin{array}{l}\text { 1231. Ollendick TH, Ost LG, Reuterskiold L, Costa N, Cederlund R, } \\
\text { Sirbu C, Davis TE, 3rd and Jarrett MA. 2009. One-session treatment of } \\
\text { specific phobias in youth: a randomized clinical trial in the United } \\
\text { States and Sweden. J Consult Clin Psychol. } 77(3): 504-16 .\end{array}$ & $\begin{array}{l}\text { Not a parenting } \\
\text { intervention }\end{array}$ \\
\hline
\end{tabular}




\begin{tabular}{|c|c|}
\hline $\begin{array}{l}1232 . \quad \text { O'Neill-Murphy K, Liebman M and Barnsteiner JH. 2001. Fever } \\
\text { education: does it reduce parent fever anxiety? Pediatr Emerg Care. } \\
\text { 17(1):47-51. }\end{array}$ & $\begin{array}{l}\text { No child } \\
\text { internalising } \\
\text { outcomes or not } \\
\text { a major goal }\end{array}$ \\
\hline $\begin{array}{l}\text { 1233. Oosterman M and Schuengel C. 2008. Attachment in foster } \\
\text { children associated with caregivers' sensitivity and behavioral } \\
\text { problems. Infant Mental Health Journal. 29(6):609-23. }\end{array}$ & $\begin{array}{l}\text { Not a parenting } \\
\text { intervention }\end{array}$ \\
\hline $\begin{array}{l}1234 . \quad \text { Orte, C., Touza, C., Ballester, L., \& March, M. (2008). Children } \\
\text { of drug-dependent parents: Prevention programme outcomes. } \\
\text { Educational Research, 50(3), 249-260. doi: } \\
10.1080 / 00131880802309390\end{array}$ & $\begin{array}{l}\text { Not an RCT; No } \\
\text { long term } \\
\text { follow-up }\end{array}$ \\
\hline $\begin{array}{l}\text { 1235. Öst, L. G., Cederlund, R., \& Reuterskiöld, L. (2015). Behavioral } \\
\text { treatment of social phobia in youth: Does parent education training } \\
\text { improve the outcome?. Behaviour research and therapy, 67, 19-29. }\end{array}$ & $\begin{array}{l}\text { Follow-up data } \\
\text { collected only in } \\
\text { active group; } \\
\text { Parent } \\
\text { component is } \\
\text { too small }\end{array}$ \\
\hline $\begin{array}{l}\text { 1236. Ostberg, M., \& Rydell, A. M. (2012). An efficacy study of a } \\
\text { combined parent and teacher management training programme for } \\
\text { children with ADHD. Nordic journal of psychiatry, 66(2), 123-130. } \\
\text { doi:10.3109/08039488.2011.641587 }\end{array}$ & $\begin{array}{l}\text { Targets } \\
\text { externalising }\end{array}$ \\
\hline $\begin{array}{l}1237 . \quad \text { Østbye, T., Krause, K. M., Stroo, M., Lovelady, C. A., Evenson, } \\
\text { K. R., Peterson, B. L., . . Zucker, N. L. (2012). Parent-focused change to } \\
\text { prevent obesity in preschoolers: results from the KAN-DO study. } \\
\text { Preventive medicine, 55(3), 188-195. } \\
\text { doi:10.1016/j.ypmed.2012.06.005 }\end{array}$ & $\begin{array}{l}\text { No child } \\
\text { internalising } \\
\text { outcomes or not } \\
\text { a major goal }\end{array}$ \\
\hline $\begin{array}{l}\text { 1238. Ostbye, T., Zucker, N. L., Krause, K. M., Lovelady, C. A., } \\
\text { Evenson, K. R., Peterson, B. L., Bastian, L. A., Swamy, G. K., West, D. G., } \\
\text { \& Brouwer, R. J. (2011). Kids and adults now! Defeat Obesity (KAN- } \\
\text { DO): rationale, design and baseline characteristics. In Contemporary } \\
\text { clinical trials (pp. 461-469). }\end{array}$ & $\begin{array}{l}\text { Data not } \\
\text { available/report } \\
\text { ed (protocol) }\end{array}$ \\
\hline $\begin{array}{l}\text { 1239. Oswald, S. H., Fegert, J. M., \& Goldbeck, L. (2013). [Evaluation } \\
\text { of a training program for child welfare case workers on trauma } \\
\text { sequelae in foster children]. Prax Kinderpsychol Kinderpsychiatr, 62(2), } \\
\text { 128-141. doi: } 10.13109 / \text { prkk.2013.62.2.128 }\end{array}$ & $\begin{array}{l}\text { Not a parenting } \\
\text { intervention }\end{array}$ \\
\hline $\begin{array}{l}1240 . \quad \text { Oswalt KL, Biasini FJ, Wilson LL and Mrug S. 2009. Outcomes of } \\
\text { a massage intervention on teen mothers: a pilot study. Pediatr Nurs. } \\
35(5): 284-9,317 \text {. }\end{array}$ & $\begin{array}{l}\text { No long term } \\
\text { follow-up }\end{array}$ \\
\hline $\begin{array}{l}\text { 1241. Oswalt, K., \& Biasini, F. (2011). Effects of infant massage on } \\
\text { HIV-infected mothers and their infants. J Spec Pediatr Nurs, 16, 169- } \\
178 .\end{array}$ & $\begin{array}{l}\text { No child } \\
\text { internalising } \\
\text { outcomes or not } \\
\text { a major goal }\end{array}$ \\
\hline $\begin{array}{l}\text { 1242. Othman A and Blunden S. 2009. Psychological interventions } \\
\text { for parents of children who have cancer: A meta-analytic review. } \\
\text { Current Pediatric Reviews. 5(2):118-27. }\end{array}$ & $\begin{array}{l}\text { No child } \\
\text { internalising } \\
\text { outcomes or not } \\
\text { a major goal }\end{array}$ \\
\hline $\begin{array}{l}\text { 1243. Ou S-R. 2008. Do GED recipients differ from graduates and } \\
\text { school dropouts?: Findings from an inner-city cohort. Urban } \\
\text { Education. 43(1):83-117. }\end{array}$ & $\begin{array}{l}\text { Not a parenting } \\
\text { intervention }\end{array}$ \\
\hline
\end{tabular}




\begin{tabular}{|c|c|}
\hline $\begin{array}{l}\text { 1244. Overbeek, M. M., de Schipper, J. C., Lamers-Winkelman, F., \& } \\
\text { Schuengel, C. (2012). The effectiveness of a trauma-focused psycho- } \\
\text { educational secondary prevention program for children exposed to } \\
\text { interparental violence: Study protocol for a randomized controlled } \\
\text { trial. Trials, } 13 .\end{array}$ & $\begin{array}{l}\text { Data not } \\
\text { available/report } \\
\text { ed (protocol) }\end{array}$ \\
\hline $\begin{array}{l}\text { 1245. Overbeek, M. M., de Schipper, J. C., Lamers-Winkelman, F., \& } \\
\text { Schuengel, C. (2013). Effectiveness of specific factors in community- } \\
\text { based intervention for child-witnesses of interparental violence: A } \\
\text { randomized trial. Child abuse \& neglect, 37(12), 1202-1214. }\end{array}$ & $\begin{array}{l}\text { Parent } \\
\text { component too } \\
\text { small }\end{array}$ \\
\hline $\begin{array}{l}\text { 1246. Owen, C., Ziebell, L., Lessard, C., Churcher, E., Bourget, V., \& } \\
\text { Villenueve, H. (2012). Interprofessional group intervention for parents } \\
\text { of children age } 3 \text { and younger with feeding difficulties: pilot program } \\
\text { evaluation. Nutr Clin Pract, 27, 129-135. }\end{array}$ & $\begin{array}{l}\text { No child } \\
\text { internalising } \\
\text { outcomes or not } \\
\text { a major goal }\end{array}$ \\
\hline $\begin{array}{l}\text { 1247. Oxford, M. L., Fleming, C. B., Nelson, E. M., Kelly, J. K., Spieker, } \\
\text { S. J. (2013). Randomized trial of Promoting First Relationships: Effects } \\
\text { on maltreated toddlers' separation distress and sleep regulation after } \\
\text { reunification. Children and Youth Services Review, } 35 \text { (12), 1988-1992. }\end{array}$ & $\begin{array}{l}\text { No child } \\
\text { internalising } \\
\text { outcomes or not } \\
\text { a major goal }\end{array}$ \\
\hline $\begin{array}{l}\text { 1248. Ozcan O, Kilic BG, and Aysev A. 2006. Psychopathology in } \\
\text { Parents of Children with School Phobia. Türk psikiyatri dergisi= Turkish } \\
\text { journal of psychiatry. 17(3):173-80. }\end{array}$ & $\begin{array}{l}\text { Not a parenting } \\
\text { intervention }\end{array}$ \\
\hline $\begin{array}{l}\text { 1249. Ozyurt, G., Gencer, O., Ozturk, Y., Ozbek, A. (2014). Long term } \\
\text { effectiveness of Triple P Positive Parenting Program on childhood } \\
\text { anxiety disorders: A randomised controlled trial. European } \\
\text { Neuropsychopharmacology, } 24 \text { S613. }\end{array}$ & $\begin{array}{l}\text { Follow-up less } \\
\text { than } 6 \text { months }\end{array}$ \\
\hline $\begin{array}{l}\text { 1250. Padilla-Walker, L. M., Christensen, K. J., \& Day, R. D. (2011). } \\
\text { Proactive parenting practices during early adolescence: A cluster } \\
\text { approach. Journal of Adolescence, 34, 203-214. }\end{array}$ & Not an RCT \\
\hline $\begin{array}{l}\text { 1251. Padovani FvHP, Carvalho AEIV, Duarte G, Martinez FEg and } \\
\text { Linhares MBM. 2009. Anxiety, dysphoria, and depression symptoms in } \\
\text { mothers of preterm infants. Psychological Reports. 104(2):667-79. }\end{array}$ & $\begin{array}{l}\text { No child } \\
\text { internalising } \\
\text { outcomes or not } \\
\text { a major goal }\end{array}$ \\
\hline $\begin{array}{l}\text { 1252. Paine S, Gradisar M, Dohnt H, Starkey K, Gardner G, } \\
\text { Trenowden S, et al. A randomized controlled trial of cognitive- } \\
\text { behaviour therapy for behavioural insomnia of childhood in school- } \\
\text { aged children. Journal of Sleep Research. 2010;19:30. }\end{array}$ & $\begin{array}{l}\text { Follow-up data } \\
\text { collected only in } \\
\text { active group; } \\
\text { Not a parenting } \\
\text { intervention }\end{array}$ \\
\hline $\begin{array}{l}\text { 1253. Palermo MT, Di Luigi M, Dal Forno G, Dominici C, Vicomandi D, } \\
\text { Sambucioni A, Proietti L and Pasqualetti P. 2006. Externalizing and } \\
\text { Oppositional Behaviors and Karate- }<i>d o</ i>\text { : The }<i>\text { Way }</ i>\text { of } \\
\text { Crime Prevention: A Pilot Study. International Journal of Offender } \\
\text { Therapy and Comparative Criminology. 50(6):654-60. }\end{array}$ & $\begin{array}{l}\text { Not a parenting } \\
\text { intervention }\end{array}$ \\
\hline $\begin{array}{l}\text { 1254. Palermo T and Long A. 2009. Randomized controlled trial of a } \\
\text { web-based psychological treatment for pediatric chronic pain. Journal } \\
\text { of Pain. 10(4):S72. }\end{array}$ & $\begin{array}{l}\text { No long term } \\
\text { follow-up }\end{array}$ \\
\hline
\end{tabular}




\begin{tabular}{|c|c|}
\hline $\begin{array}{l}1255 . \quad \text { Palermo TM and Drotar DD. 1999. Coping with pediatric } \\
\text { ambulatory surgery: Effectiveness of parent- implemented behavioral } \\
\text { distraction strategies. Behavior therapy, 30(4),657-671. }\end{array}$ & $\begin{array}{l}\text { Intervention for } \\
\text { developmental } \\
\text { disorders } \\
\text { (autism, autism } \\
\text { spectrum } \\
\text { disorder), } \\
\text { physical } \\
\text { disabilities, } \\
\text { medical } \\
\text { conditions or } \\
\text { distress about } \\
\text { facing medical } \\
\text { interventions }\end{array}$ \\
\hline $\begin{array}{l}\text { 1256. Palermo TM, Wilson AC, Peters M, Lewandowski A and } \\
\text { Somhegyi H. 2009. Randomized controlled trial of an Internet- } \\
\text { delivered family cognitive-behavioral therapy intervention for children } \\
\text { and adolescents with chronic pain. Pain. 146(1-2):205-13. }\end{array}$ & $\begin{array}{l}\text { Not a parenting } \\
\text { intervention }\end{array}$ \\
\hline $\begin{array}{l}\text { 1257. Palinkas LA, Aarons GA, Chorpita BF, Hoagwood K, Landsverk J } \\
\text { and Weisz JR. 2009. Cultural exchange and the implementation of } \\
\text { evidence-based practices: Two case studies. Research on Social Work } \\
\text { Practice. 19(5):602-12. }\end{array}$ & $\begin{array}{l}\text { No child } \\
\text { internalising } \\
\text { outcomes or not } \\
\text { a major goal }\end{array}$ \\
\hline $\begin{array}{l}\text { 1258. Pan D, Huey SJ, Jr. and Hernandez D. 2011. Culturally adapted } \\
\text { versus standard exposure treatment for phobic Asian Americans: } \\
\text { Treatment efficacy, moderators, and predictors. Cultural Diversity and } \\
\text { Ethnic Minority Psychology. 17(1):11-22. }\end{array}$ & $\begin{array}{l}\text { Not a parenting } \\
\text { intervention }\end{array}$ \\
\hline $\begin{array}{l}\text { 1259. Pantin, H., Coatsworth, J. D., Feaster, D. J., Newman, F. L., } \\
\text { Briones, E., Prado, G., et al. (2003). Familias Unidas: the efficacy of an } \\
\text { intervention to promote parental investment in Hispanic immigrant } \\
\text { families. Prevention science : the official journal of the Society for } \\
\text { Prevention Research, (3), 189-201. }\end{array}$ & $\begin{array}{l}\text { Follow-up less } \\
\text { than } 6 \text { months }\end{array}$ \\
\hline $\begin{array}{l}\text { 1260. Pantin, H., Prado, G., Lopez, B., Huang, S., Tapia, M. I., } \\
\text { Schwartz, S. J., et al. (2009). A randomized controlled trial of Familias } \\
\text { Unidas for Hispanic adolescents with behavior problems. } \\
\text { Psychosomatic Medicine, 71(9), 987-995. doi: } \\
\text { 10.1097/PSY.0b013e3181bb2913 }\end{array}$ & $\begin{array}{l}\text { No child } \\
\text { internalising } \\
\text { outcomes or not } \\
\text { a major goal }\end{array}$ \\
\hline $\begin{array}{l}\text { 1261. Papneja T and Manassis K. 2006. Characterization and } \\
\text { treatment response of anxious children with asthma. Can J Psychiatry. } \\
51(6): 393-6 .\end{array}$ & $\begin{array}{l}\text { Not a parenting } \\
\text { intervention }\end{array}$ \\
\hline $\begin{array}{l}\text { 1262. Pappas DE, Hayden GF and Hendley JO. 2008. Cough and cold } \\
\text { treatments for children: Does anything work? Consultant. 48(3):233-8. }\end{array}$ & $\begin{array}{l}\text { Not a parenting } \\
\text { intervention }\end{array}$ \\
\hline $\begin{array}{l}\text { 1263. Paradis, H. A., Sandler, M., Manly, J. T., \& Valentine, L. (2013). } \\
\text { Building healthy children: Evidence-based home visitation integrated } \\
\text { with pediatric medical homes. Pediatrics, 132(SUPPL.2), S174-S179. } \\
\text { doi: http://dx.doi.org/10.1542/peds.2013-1021R }\end{array}$ & $\begin{array}{l}\text { No child } \\
\text { internalising } \\
\text { outcomes or not } \\
\text { a major goal }\end{array}$ \\
\hline $\begin{array}{l}\text { 1264. Parent, J., Forehand, R. L., Merchant, M. J., Long, N., \& Jones, } \\
\text { D. J. (2011). Predictors of outcome of a parenting group curriculum: a } \\
\text { pilot study. Behav Modif, 35, 370-388. }\end{array}$ & $\begin{array}{l}\text { Targets } \\
\text { externalising }\end{array}$ \\
\hline $\begin{array}{l}\text { 1265. Park, A. (2011). The impact of exposure to domestic violence } \\
\text { on developmental trajectories of depressive symptoms and antisocial } \\
\text { behavior across the transition to adulthood (Order No. 3466780). } \\
\text { Available from ProQuest Dissertations \& Theses Global. (885428286). }\end{array}$ & $\begin{array}{l}\text { Not a parenting } \\
\text { intervention }\end{array}$ \\
\hline
\end{tabular}




\begin{tabular}{|c|c|}
\hline $\begin{array}{l}\text { 1266. Park, H. Y., Heo, J., Subramanian, S. V., Kawachi, I., \& Oh, J. } \\
\text { (2012). Socioeconomic inequalities in adolescent depression in South } \\
\text { Korea: A multilevel analysis. PLoS ONE, 7(10). doi: } \\
\text { 10.1371/journal.pone.0047025 }\end{array}$ & Not an RCT \\
\hline $\begin{array}{l}\text { 1267. Parker H, Swift PG, Botha JL and Raymond NT. 1994. Early } \\
\text { onset diabetes: parents' views. Diabet Med. 11(6):593-6. }\end{array}$ & Not an RCT \\
\hline $\begin{array}{l}\text { 1268. Patterson GR and Fleischman MJ. 1979. Maintenance of } \\
\text { Treatment Effects - Some Considerations Concerning Family Systems } \\
\text { and Follow-up Data. Behavior Therapy. 10(2):168-85. }\end{array}$ & Review \\
\hline $\begin{array}{l}\text { 1269. Patterson GR, DeGarmo DS, Forgatch MS. Systematic changes } \\
\text { in families following prevention trials. Journal of Abnormal Child } \\
\text { Psychology 2004;32:621-633. }\end{array}$ & $\begin{array}{l}\text { No child } \\
\text { internalising } \\
\text { outcomes or not } \\
\text { a major goal }\end{array}$ \\
\hline $\begin{array}{l}\text { 1270. Patterson J, Mockford C and Stewart-Brown S. 2005. Parents' } \\
\text { perceptions of the value of the Webster-Stratton Parenting } \\
\text { Programme: a qualitative study of a general practice based initiative. } \\
\text { Child: care, health and development, } 31(1), 53-64 .\end{array}$ & Not an RCT \\
\hline $\begin{array}{l}\text { 1271. Patterson, G. R., Chamberlain, P. \& Reid, J. B. (1982). A } \\
\text { comparative evaluation of a parent-training program. Behavior } \\
\text { Therapy, } 13,638-650\end{array}$ & $\begin{array}{l}\text { No long term } \\
\text { follow-up }\end{array}$ \\
\hline $\begin{array}{l}\text { 1272. Patterson, J., Barlow, J., Mockford, C., Klimes, I., Pyper, C., \& } \\
\text { Stewart-Brown, S. (2002). Improving mental health through parenting } \\
\text { programmes: block randomised controlled trial. Archives of Disease in } \\
\text { Childhood, } 87(6), 472-477 .\end{array}$ & $\begin{array}{l}\text { Targets } \\
\text { externalising }\end{array}$ \\
\hline $\begin{array}{l}\text { 1273. Pattison, C., \& Lynd-Stevenson, R. M. (2001). The Prevention } \\
\text { of Depressive Symptoms in Children: The Immediate and Long-term } \\
\text { Outcomes of a School-based Program. Behaviour Change, 18, 92-102. }\end{array}$ & $\begin{array}{l}\text { Not a parenting } \\
\text { intervention }\end{array}$ \\
\hline $\begin{array}{l}\text { 1274. Patton GC, Coffey C, Posterino M, Carlin JB and Bowes G. } \\
\text { 2003. Life events and early onset depression: Cause or consequence? } \\
\text { Psychological Medicine: A Journal of Research in Psychiatry and the } \\
\text { Allied Sciences. 33(7):1203-10. }\end{array}$ & $\begin{array}{l}\text { Not a parenting } \\
\text { intervention }\end{array}$ \\
\hline $\begin{array}{l}\text { 1275. Paul F, Jones MC, Hendry C and Adair PM. 2007. The quality of } \\
\text { written information for parents regarding the management of a febrile } \\
\text { convulsion: a randomized controlled trial. J Clin Nurs. 16(12):2308-22. }\end{array}$ & $\begin{array}{l}\text { Intervention for } \\
\text { developmental } \\
\text { disorders } \\
\text { (autism, autism } \\
\text { spectrum } \\
\text { disorder), } \\
\text { physical } \\
\text { disabilities, } \\
\text { medical } \\
\text { conditions or } \\
\text { distress about } \\
\text { facing medical } \\
\text { interventions }\end{array}$ \\
\hline $\begin{array}{l}\text { 1276. Paul, I. M., Williams, J. S., Anzman-Frasca, S., Beiler, J. S., } \\
\text { Makova, K. D., Marini, M. E., ... \& Birch, L. L. (2014). The intervention } \\
\text { nurses start infants growing on healthy trajectories (INSIGHT) study. } \\
\text { BMC pediatrics, } 14(1), 184 .\end{array}$ & $\begin{array}{l}\text { No child } \\
\text { internalising } \\
\text { outcomes or not } \\
\text { a major goal }\end{array}$ \\
\hline
\end{tabular}




\begin{tabular}{|c|c|}
\hline $\begin{array}{l}\text { 1277. Payne KA, Coetzee AR, Mattheyse FJ and Heydenrych JJ. } 1992 . \\
\text { Behavioural changes in children following minor surgery--is } \\
\text { premedication beneficial? Acta Anaesthesiol Belg. 43(3):173-9. }\end{array}$ & $\begin{array}{l}\text { Intervention for } \\
\text { developmental } \\
\text { disorders } \\
\text { (autism, autism } \\
\text { spectrum } \\
\text { disorder), } \\
\text { physical } \\
\text { disabilities, } \\
\text { medical } \\
\text { conditions or } \\
\text { distress about } \\
\text { facing medical } \\
\text { interventions }\end{array}$ \\
\hline $\begin{array}{l}\text { 1278. Pearl ES. 2009. Parent management training for reducing } \\
\text { oppositional and aggressive behavior in preschoolers. Aggression and } \\
\text { Violent Behavior. 14(5):295-305. }\end{array}$ & Review \\
\hline $\begin{array}{l}\text { 1279. Pederson C. 1996. Promoting parental use of } \\
\text { nonpharmacologic techniques with children during lumbar punctures. } \\
\text { Journal of Pediatric Oncology Nursing, 13(1), 21-30. }\end{array}$ & $\begin{array}{l}\text { Intervention for } \\
\text { developmental } \\
\text { disorders } \\
\text { (autism, autism } \\
\text { spectrum } \\
\text { disorder), } \\
\text { physical } \\
\text { disabilities, } \\
\text { medical } \\
\text { conditions or } \\
\text { distress about } \\
\text { facing medical } \\
\text { interventions }\end{array}$ \\
\hline $\begin{array}{l}\text { 1280. Pedro-Carroll, J. L., Sutton, S. E., \& Wyman, P. A. (1999). A } \\
\text { two-year follow-up evaluation of a preventive intervention for young } \\
\text { children of divorce. School Psychology Review, 28(3), } 467 .\end{array}$ & $\begin{array}{l}\text { Not a parenting } \\
\text { intervention; } \\
\text { Not an RCT }\end{array}$ \\
\hline $\begin{array}{l}\text { 1281. Pelchat D, Bisson J, Perreault M, Ricard N and Bouchard JM. } \\
\text { 1998. [Systematic family nursing intervention applied to the birth of a } \\
\text { disabled child: effects on the parents' adaptation]. The Canadian } \\
\text { journal of nursing research= Revue canadienne de recherche en } \\
\text { sciences infirmieres, } 30(3), 99-121 \text {. }\end{array}$ & $\begin{array}{l}\text { Intervention for } \\
\text { developmental } \\
\text { disorders } \\
\text { (autism, autism } \\
\text { spectrum } \\
\text { disorder), } \\
\text { physical } \\
\text { disabilities, } \\
\text { medical } \\
\text { conditions or } \\
\text { distress about } \\
\text { facing medical } \\
\text { interventions }\end{array}$ \\
\hline
\end{tabular}




\begin{tabular}{|c|c|}
\hline $\begin{array}{l}\text { 1282. Pelchat D, Bisson J, Ricard N, Perreault M and Bouchard JM. } \\
\text { 1999. Longitudinal effects of an early family intervention programme } \\
\text { on the adaptation of parents of children with a disability. Int J Nurs } \\
\text { Stud. } 36(6): 465-77 \text {. }\end{array}$ & $\begin{array}{l}\text { Intervention for } \\
\text { developmental } \\
\text { disorders } \\
\text { (autism, autism } \\
\text { spectrum } \\
\text { disorder), } \\
\text { physical } \\
\text { disabilities, } \\
\text { medical } \\
\text { conditions or } \\
\text { distress about } \\
\text { facing medical } \\
\text { interventions }\end{array}$ \\
\hline $\begin{array}{l}\text { 1283. Pendergast MM. The effects of brief interventions on children } \\
\text { of divorce. Dissertation Abstracts International [serial on the Internet]. } \\
\text { 1992; (8-b): Available from: } \\
\text { http://www.mrw.interscience.wiley.com/cochrane/clcentral/articles/ } \\
\text { 367/CN-00711367/frame.html. }\end{array}$ & $\begin{array}{l}\text { No long term } \\
\text { follow-up }\end{array}$ \\
\hline $\begin{array}{l}\text { 1284. Penney, S. R., \& Skilling, T. A. (2012). Moderators of informant } \\
\text { agreement in the assessment of adolescent psychopathology: } \\
\text { Extension to a forensic sample. Psychological Assessment, 24, 386- } \\
401 .\end{array}$ & $\begin{array}{l}\text { Not a parenting } \\
\text { intervention }\end{array}$ \\
\hline $\begin{array}{l}1285 . \quad \text { Perez-Blasco, J., Viguer, P., \& Rodrigo, M. F. (2013). Effects of a } \\
\text { mindfulness-based intervention on psychological distress, well-being, } \\
\text { and maternal self-efficacy in breast-feeding mothers: results of a pilot } \\
\text { study. Arch Womens Ment Health, 16(3), 227-236. doi: } \\
\text { 10.1007/s00737-013-0337-z }\end{array}$ & $\begin{array}{l}\text { No child } \\
\text { internalising } \\
\text { outcomes or not } \\
\text { a major goal }\end{array}$ \\
\hline $\begin{array}{l}\text { 1286. Perrino, T., Pantin, H., Prado, G., Huang, S., Brincks, A., Howe, } \\
\text { G., ... \& Brown, C. H. (2014). Preventing internalizing symptoms among } \\
\text { Hispanic adolescents: A synthesis across Familias Unidas Trials. } \\
\text { Prevention Science, 15(6), 917-928. }\end{array}$ & $\begin{array}{l}\text { Targets } \\
\text { externalising }\end{array}$ \\
\hline $\begin{array}{l}\text { 1287. Perry-Jenkins, M., Smith, J. Z., Goldberg, A. E., \& Logan, J. } \\
\text { (2011). Working-class jobs and new parents' mental health. Journal of } \\
\text { Marriage and Family, } 73,1117-1132 .\end{array}$ & $\begin{array}{l}\text { Not a parenting } \\
\text { intervention }\end{array}$ \\
\hline $\begin{array}{l}\text { 1288. Persaud DI, Barnett SE, Weller SC, Baldwin CD, Niebuhr V and } \\
\text { McCormick DP. 1996. An asthma self-management program for } \\
\text { children, including instruction in peak flow monitoring by school } \\
\text { nurses. J Asthma. 33(1):37-43. }\end{array}$ & $\begin{array}{l}\text { Not a parenting } \\
\text { intervention }\end{array}$ \\
\hline $\begin{array}{l}\text { 1289. Persson G and Nordlund CL. 1985. Agoraphobics and social } \\
\text { phobics: differences in background factors, syndrome profiles and } \\
\text { therapeutic response. Acta Psychiatr Scand. 71(2):148-59. }\end{array}$ & $\begin{array}{l}\text { Not a parenting } \\
\text { intervention }\end{array}$ \\
\hline $\begin{array}{l}\text { 1290. Petch J and Halford WK. 2008. Psycho-education to enhance } \\
\text { couples' transition to parenthood. Clinical Psychology Review. } \\
\text { 28(7):1125-37. }\end{array}$ & Review \\
\hline $\begin{array}{l}\text { 1291. Peterson MA, Hamilton EB and Russell AD. 2009. Starting well: } \\
\text { Facilitating the middle school transition. Journal of Applied School } \\
\text { Psychology. 25(3):286-304. }\end{array}$ & $\begin{array}{l}\text { Not a parenting } \\
\text { intervention }\end{array}$ \\
\hline $\begin{array}{l}\text { 1292. Pettigrew TF. 1959. Regional differences in anti-Negro } \\
\text { prejudice. The Journal of Abnormal and Social Psychology. 59(1):28- } \\
\text { 36. }\end{array}$ & $\begin{array}{l}\text { Not a parenting } \\
\text { intervention }\end{array}$ \\
\hline
\end{tabular}




\begin{tabular}{|c|c|}
\hline $\begin{array}{l}\text { 1293. Pfannenstiel, Annette E, Honig and Alice S. 1988. Father } \\
\text { Sensitivity and Empathy with Infants after a Prenatal Information } \\
\text { Support Program. Washington, D.C: US Department of Education. }\end{array}$ & $\begin{array}{l}\text { No child } \\
\text { internalising } \\
\text { outcomes or not } \\
\text { a major goal }\end{array}$ \\
\hline $\begin{array}{l}\text { 1294. Pfeffer, C. R., Jiang, H., Kakuma, T., Hwang, J., \& Metsch, M. } \\
\text { (2002). Group intervention for children bereaved by the suicide of a } \\
\text { relative. Journal of the American Academy of Child and Adolescent } \\
\text { Psychiatry, (5), 505-513. }\end{array}$ & $\begin{array}{l}\text { No long term } \\
\text { follow-up }\end{array}$ \\
\hline $\begin{array}{l}\text { 1295. Pfiffner } \amalg \text {, Jouriles EN, Brown MM, Etscheidt MA and et al. } \\
\text { 1990. Effects of problem-solving therapy on outcomes of parent } \\
\text { training for single-parent families. Child \& Family Behavior Therapy. } \\
\text { 12(1):1-11. }\end{array}$ & $\begin{array}{l}\text { Follow-up less } \\
\text { than } 6 \text { months }\end{array}$ \\
\hline $\begin{array}{l}1296 . \quad \text { Pfiffner, L. J., Hinshaw, S. P., Owens, E., Zalecki, C., Kaiser, N. } \\
\text { M., Villodas, M., \& McBurnett, K. (2014). A two-site randomized } \\
\text { clinical trial of integrated psychosocial treatment for ADHD-inattentive } \\
\text { type. Journal of consulting and clinical psychology, 82(6), } 1115 .\end{array}$ & $\begin{array}{l}\text { Intervention for } \\
\text { developmental } \\
\text { disorders } \\
\text { (autism, autism } \\
\text { spectrum } \\
\text { disorder), } \\
\text { physical } \\
\text { disabilities, } \\
\text { medical } \\
\text { conditions or } \\
\text { distress about } \\
\text { facing medical } \\
\text { interventions } \\
\end{array}$ \\
\hline $\begin{array}{l}1297 . \quad \text { Pflug, V., Seehagen, S., \& Schneider, S. (2012). Nothing new } \\
\text { since <<little Hans >> and <<little Peter >>? Psychotherapy for anxiety } \\
\text { disorders in childhood and adolescence. Z Kinder Jugendpsychiatr } \\
\text { Psychother, 40, 21-28. }\end{array}$ & Review \\
\hline $\begin{array}{l}\text { 1298. Phillips SD, Hargis MB, Kramer TL, Lensing SY, Taylor JL, Burns } \\
\text { BJ and Robbins JM. 2000. Toward a level playing field: Predicting } \\
\text { factors for the outcomes of mental health treatment for adolescents. } \\
\text { Journal of the American Academy of Child \& Adolescent Psychiatry. } \\
\text { 39(12):1485-95. }\end{array}$ & $\begin{array}{l}\text { Not a parenting } \\
\text { intervention }\end{array}$ \\
\hline $\begin{array}{l}\text { 1299. Phipps, S., Peasant, C., Barrera, M., Alderfer, M. A., Huang, Q., } \\
\text { \& Vannatta, K. (2012). Resilience in children undergoing stem cell } \\
\text { transplantation: results of a complementary intervention trial. } \\
\text { Pediatrics, 129(3), e762-770. doi:10.1542/peds.2011-1816 }\end{array}$ & $\begin{array}{l}\text { Intervention for } \\
\text { developmental } \\
\text { disorders } \\
\text { (autism, autism } \\
\text { spectrum } \\
\text { disorder), } \\
\text { physical } \\
\text { disabilities, } \\
\text { medical } \\
\text { conditions or } \\
\text { distress about } \\
\text { facing medical } \\
\text { interventions }\end{array}$ \\
\hline
\end{tabular}




\begin{tabular}{|c|c|}
\hline $\begin{array}{l}\text { 1300. Piacentini, J., Bergman, R. L., Chang, S., Langley, A., Peris, T., } \\
\text { Wood, J. J., \& McCracken, J. (2011). Controlled comparison of family } \\
\text { cognitive behavioral therapy and psychoeducation/relaxation training } \\
\text { for child obsessive-compulsive disorder. J Am Acad Child Adolesc } \\
\text { Psychiatry, 50, 1149-1161. }\end{array}$ & $\begin{array}{l}\text { Parent } \\
\text { component too } \\
\text { small; 6-month } \\
\text { follow-up only in } \\
\text { responders }\end{array}$ \\
\hline $\begin{array}{l}\text { 1301. Piedra, L. M., Byoun, S.-J., Guardini, L., \& Cintrón, V. (2012). } \\
\text { Improving the parental self-agency of depressed Latino immigrant } \\
\text { mothers: Piloted intervention results. Children and Youth Services } \\
\text { Review, 34, 126-135. }\end{array}$ & $\begin{array}{l}\text { Follow-up less } \\
\text { than } 6 \text { months }\end{array}$ \\
\hline $\begin{array}{l}\text { 1302. Pierce M, Ridout D, Harding D, Keen H and Bradley C. } 2000 \text {. } \\
\text { More good than harm: a randomised controlled trial of the effect of } \\
\text { education about familial risk of diabetes on psychological outcomes. } \\
\text { Br J Gen Pract. } 50(460): 867-71 .\end{array}$ & $\begin{array}{l}\text { Not a parenting } \\
\text { intervention }\end{array}$ \\
\hline $\begin{array}{l}\text { 1303. Pihkala } \mathrm{H} \text { and Johansson EE. 2008. Longing and fearing for } \\
\text { dialogue with children-Depressed parents' way into Beardslee's } \\
\text { preventive family intervention. Nordic Journal of Psychiatry. } \\
62(5): 399-404 \text {. }\end{array}$ & $\begin{array}{l}\text { No child } \\
\text { internalising } \\
\text { outcomes or not } \\
\text { a major goal }\end{array}$ \\
\hline $\begin{array}{l}\text { 1304. Pina AA, Silverman WK, Fuentes RM, Kurtines WM and Weems } \\
\text { CF. 2003. Exposure-based cognitive-behavioral treatment for phobic } \\
\text { and anxiety disorders: Treatment effects and maintenance for } \\
\text { Hispanic/Latino relative to European-American youths. Journal of the } \\
\text { American Academy of Child \& Adolescent Psychiatry. 42(10):1179-87. }\end{array}$ & $\begin{array}{l}\text { Not a parenting } \\
\text { intervention }\end{array}$ \\
\hline $\begin{array}{l}1305 . \quad \text { Pina, A. A., Zerr, A. A., Villalta, I. K., \& Gonzales, N. A. (2012). } \\
\text { Indicated prevention and early intervention for childhood anxiety: A } \\
\text { randomized trial with Caucasian and Hispanic/Latino youth. J Consult } \\
\text { Clin Psychol, 80, 940-946. }\end{array}$ & $\begin{array}{l}\text { Comparison } \\
\text { condition not a } \\
\text { minimal control; } \\
\text { Parent } \\
\text { component too } \\
\text { small }\end{array}$ \\
\hline $\begin{array}{l}\text { 1306. Pincus DB, Santucci LC, Ehrenreich JT and Eyberg SM. } 2008 . \\
\text { The implementation of modified parent-child interaction therapy for } \\
\text { youth with Separation Anxiety Disorder. Cognitive and Behavioral } \\
\text { Practice. 15(2):118-25. }\end{array}$ & Not an RCT \\
\hline $\begin{array}{l}\text { 1307. Pincus, D. B., Eyberg, S. M., \& Choate, M. L. (2005). Adapting } \\
\text { parent-child interaction therapy for young children with separation } \\
\text { anxiety disorder. Education \& Treatment of Children, 28(2), 163-181. }\end{array}$ & $\begin{array}{l}\text { Data not } \\
\text { available/report } \\
\text { ed (protocol) }\end{array}$ \\
\hline $\begin{array}{l}\text { 1308. Pinsker M, Geoffrey K. Comparison of parent-effectiveness } \\
\text { training and behavior modification parent training. Fam Rel } \\
\text { 1981;30:61-8. }\end{array}$ & $\begin{array}{l}\text { Not an RCT; No } \\
\text { child } \\
\text { internalising; } \\
\text { Follow-up data } \\
\text { collected only in } \\
\text { active group }\end{array}$ \\
\hline $\begin{array}{l}\text { 1309. Pisterman, S., McGrath, P., Firestone, P., Goodman, J. T., } \\
\text { Webster, I., \& Mallory, R. (1989). Outcome of parent-mediated } \\
\text { treatment of preschoolers with attention deficit disorder with } \\
\text { hyperactivity. Journal of Consulting and Clinical Psychology, 57(5), } \\
\text { 628-635. doi: } 10.1037 / 0022-006 x .57 .5 .628\end{array}$ & $\begin{array}{l}\text { Follow-up less } \\
\text { than } 6 \text { months }\end{array}$ \\
\hline $\begin{array}{l}\text { 1310. Platt, B., Pietsch, K., Krick, K., Oort, F., \& Schulte-Körne, G. } \\
\text { (2014). Study protocol for a randomised controlled trial of a cognitive- } \\
\text { behavioural prevention programme for the children of parents with } \\
\text { depression: the PRODO trial. BMC psychiatry, } 14(1), 263 \text {. }\end{array}$ & $\begin{array}{l}\text { Data not } \\
\text { available/report } \\
\text { ed }\end{array}$ \\
\hline
\end{tabular}




\begin{tabular}{|c|c|}
\hline $\begin{array}{l}\text { 1311. Pless, I. B., Feeley, N., Gottlieb, L., Rowat, K., Dougherty, G., \& } \\
\text { Willard, B. (1994). A randomized trial of a nursing intervention to } \\
\text { promote the adjustment of children with chronic physical disorders. } \\
\text { Pediatrics, 94(1), 70-75. }\end{array}$ & $\begin{array}{l}\text { No long term } \\
\text { follow-up }\end{array}$ \\
\hline $\begin{array}{l}\text { 1312. Plueck J, Freund-Braier I, Hautmann C, Beckers G, Wieczorrek } \\
\text { E and Doepfner M. 2010. Recruitment in an indicated prevention } \\
\text { program for externalizing behavior - parental participation decisions. } \\
\text { Child and Adolescent Psychiatry and Mental Health. 4(28). }\end{array}$ & $\begin{array}{l}\text { No child } \\
\text { internalising } \\
\text { outcomes or not } \\
\text { a major goal }\end{array}$ \\
\hline $\begin{array}{l}\text { 1313. Podorefsky DL, McDonald-Dowdell M and Beardslee WR. } \\
\text { 2001. Adaptation of preventive interventions for a low-income, } \\
\text { culturally diverse community. J Am Acad Child Adolesc Psychiatry. } \\
\text { 40(8):879-86. }\end{array}$ & $\begin{array}{l}\text { Data not } \\
\text { available/report } \\
\text { ed }\end{array}$ \\
\hline $\begin{array}{l}\text { 1314. Polaha J, Larzelere RE, Shapiro SK and Pettit GS. 2004. Physical } \\
\text { Discipline and Child Behavior Problems: A Study of Ethnic Group } \\
\text { Differences. Parenting: Science and Practice. 4(4):339-60. }\end{array}$ & $\begin{array}{l}\text { Not a parenting } \\
\text { intervention }\end{array}$ \\
\hline $\begin{array}{l}\text { 1315. Pollard, S., Ward, E. M., \& Barkley, R. A. (1984). The Effects of } \\
\text { Parent Training and Ritalin on the Parent-Child Interactions of } \\
\text { Hyperactive Boys. [doi: 10.1300/J019v05n04_04]. Child \& Family } \\
\text { Behavior Therapy, 5(4), 51-70. doi:10.1300/J019v05n04_04 }\end{array}$ & Not an RCT \\
\hline $\begin{array}{l}\text { 1316. Pollitt E, Durnin JV, Husaini M and Jahari A. 2000. Effects of an } \\
\text { energy and micronutrient supplement on growth and development in } \\
\text { undernourished children in Indonesia: methods. European journal of } \\
\text { clinical nutrition, 54, S16-20. }\end{array}$ & $\begin{array}{l}\text { Not a parenting } \\
\text { intervention }\end{array}$ \\
\hline $\begin{array}{l}\text { 1317. Poresky RH and Daniels AM. 2001. Two-year comparison of } \\
\text { income, education, and depression among parents participating in } \\
\text { regular Head Start or supplementary Family Service Center Services. } \\
\text { Psychol Rep. } 88(3 \mathrm{Pt} 1): 787-96 .\end{array}$ & Not an RCT \\
\hline $\begin{array}{l}\text { 1318. Poresky RH, Clark K and Daniels AM. 2000. Longitudinal } \\
\text { characteristics of the Center for Epidemiologic Studies--Depression } \\
\text { Scale. Psychol Rep. 86(3 Pt 1):819-26. }\end{array}$ & $\begin{array}{l}\text { No child } \\
\text { internalising } \\
\text { outcomes or not } \\
\text { a major goal }\end{array}$ \\
\hline $\begin{array}{l}\text { 1319. Post RM, Leverich GS, Fergus E, Miller R and Luckenbaugh D. } \\
\text { 2002. Parental attitudes towards early intervention in children at high } \\
\text { risk for affective disorders. Journal of Affective Disorders. 70(2):117- } \\
\text { 24. }\end{array}$ & Not an RCT \\
\hline $\begin{array}{l}\text { 1320. Posthumus, J. A., Raaijmakers, M. A. J., Maassen, G. H., van } \\
\text { Engeland, H., \& Matthys, W. (2012). Sustained effects of Incredible } \\
\text { Years as a preventive intervention in preschool children with conduct } \\
\text { problems. Journal of Abnormal Child Psychology, 40, 487-500. }\end{array}$ & $\begin{array}{l}\text { Targets } \\
\text { externalising }\end{array}$ \\
\hline $\begin{array}{l}\text { 1321. Pott W, Albayrak Ö, Hebebrand J and Pauli-Pott U. } 2009 . \\
\text { Treating childhood obesity: Family background variables and the } \\
\text { child's success in a weight-control intervention. International Journal } \\
\text { of Eating Disorders. } 42(3): 284-9 .\end{array}$ & Not an RCT \\
\hline $\begin{array}{l}\text { 1322. Powell L, Gilchrist M and Stapley J. 2008. A journey of self- } \\
\text { discovery: an intervention involving massage, yoga and relaxation for } \\
\text { children with emotional and behavioural difficulties attending primary } \\
\text { schools. European Journal of Special Needs Education, 23(4), 403-412. }\end{array}$ & $\begin{array}{l}\text { Not a parenting } \\
\text { intervention }\end{array}$ \\
\hline
\end{tabular}




\begin{tabular}{|c|c|}
\hline $\begin{array}{l}\text { 1323. Powers KS and Rubenstein JS. 1999. Family presence during } \\
\text { invasive procedures in the pediatric intensive care unit: a prospective } \\
\text { study. Arch Pediatr Adolesc Med. 153(9):955-8. }\end{array}$ & $\begin{array}{l}\text { Intervention for } \\
\text { developmental } \\
\text { disorders } \\
\text { (autism, autism } \\
\text { spectrum } \\
\text { disorder), } \\
\text { physical } \\
\text { disabilities, } \\
\text { medical } \\
\text { conditions or } \\
\text { distress about } \\
\text { facing medical } \\
\text { interventions }\end{array}$ \\
\hline $\begin{array}{l}\text { 1324. Pratila MG, Fischer ME, Alagesan R, Reinsel RA and Pratilas D. } \\
\text { 1993. Propofol versus midazolam for monitored sedation: a } \\
\text { comparison of intraoperative and recovery parameters. Journal of } \\
\text { clinical anesthesia, } 5(4), 268-274 \text {. }\end{array}$ & $\begin{array}{l}\text { Not a parenting } \\
\text { intervention }\end{array}$ \\
\hline $\begin{array}{l}\text { 1325. Presnall, N., Webster-Stratton, C. H., \& Constantino, J. N. } \\
\text { (2014). Parent Training: Equivalent Improvement in Externalizing } \\
\text { Behavior for Children With and Without Familial Risk. Journal of the } \\
\text { American Academy of Child \& Adolescent Psychiatry, 53(8), 879-887. }\end{array}$ & $\begin{array}{l}\text { Targets } \\
\text { externalising }\end{array}$ \\
\hline $\begin{array}{l}\text { 1326. Preyde M and Ardal F. 2003. Effectiveness of a parent buddy } \\
\text { program for mothers of very preterm infants in a neonatal intensive } \\
\text { care unit. Canadian Medical Association Journal. 168(8):969-73. }\end{array}$ & $\begin{array}{l}\text { No child } \\
\text { internalising } \\
\text { outcomes or not } \\
\text { a major goal }\end{array}$ \\
\hline $\begin{array}{l}\text { 1327. Price JLS, Day RD and Yorgason JB. 2009. A longitudinal } \\
\text { examination of family processes, demographic variables, and } \\
\text { adolescent weight. Marriage \& Family Review. 45(2-3):310-30. }\end{array}$ & $\begin{array}{l}\text { Not a parenting } \\
\text { intervention }\end{array}$ \\
\hline $\begin{array}{l}\text { 1328. Price, A. M. H., Wake, M., Ukoumunne, O. C., \& Hiscock, H. } \\
\text { (2012). Five-year follow-up of harms and benefits of behavioral infant } \\
\text { sleep intervention: Randomized trial. Pediatrics, } 130,643-651 .\end{array}$ & $\begin{array}{l}\text { No child } \\
\text { internalising } \\
\text { outcomes or not } \\
\text { a major goal }\end{array}$ \\
\hline $\begin{array}{l}\text { 1329. Price, A., Wake, M., Ukoumunne, O., \& Hiscock, H. (2011). } \\
\text { Effects of an infant sleep intervention at child age } 6 \text { years: } \\
\text { Randomised controlled trial. Journal of Sleep Research, 20, } 8 .\end{array}$ & $\begin{array}{l}\text { No child } \\
\text { internalising } \\
\text { outcomes or not } \\
\text { a major goal }\end{array}$ \\
\hline $\begin{array}{l}\text { 1330. Pridham K, Brown R, Clark R, Limbo RK, Schroeder M, } \\
\text { Henriques J and Bohne E. 2005. Effect of guided participation on } \\
\text { feeding competencies of mothers and their premature infants. Res } \\
\text { Nurs Health. 28(3):252-67. }\end{array}$ & $\begin{array}{l}\text { No child } \\
\text { internalising } \\
\text { outcomes or not } \\
\text { a major goal }\end{array}$ \\
\hline $\begin{array}{l}\text { 1331. Prinz RJ, Sanders MR, Shapiro CJ, Whitaker DJ, Lutzker JR: } \\
\text { Population-based prevention of child maltreatment: The U.S. Triple P } \\
\text { system population trial. Prevention Science 2009, 10:1-12. }\end{array}$ & $\begin{array}{l}\text { No child } \\
\text { internalising } \\
\text { outcomes or not } \\
\text { a major goal }\end{array}$ \\
\hline $\begin{array}{l}\text { 1332. Prinz, R. J., \& Miller, G. E. (1994). Family-Based Treatment for } \\
\text { Childhood Antisocial-Behavior - Experimental Influences on Dropout } \\
\text { and Engagement. Journal of Consulting and Clinical Psychology, 62(3), } \\
645-650 .\end{array}$ & $\begin{array}{l}\text { No child } \\
\text { internalising } \\
\text { outcomes or not } \\
\text { a major goal }\end{array}$ \\
\hline
\end{tabular}




\begin{tabular}{|c|c|}
\hline $\begin{array}{l}\text { 1333. Puleo CM and Kendall PC. 2011. Anxiety disorders in typically } \\
\text { developing youth: Autism spectrum symptoms as a predictor of } \\
\text { cognitive-behavioral treatment. Journal of Autism and Developmental } \\
\text { Disorders. 41(3):275-86. }\end{array}$ & $\begin{array}{l}\text { Not a parenting } \\
\text { intervention }\end{array}$ \\
\hline $\begin{array}{l}\text { 1334. Quach, J., Hiscock, H., \& Wake, M. (2009). Impact of sleep } \\
\text { intervention on child behaviour, learning and parent mental health: A } \\
\text { randomised trial. Sleep and Biological Rhythms, 7, A32-A33. }\end{array}$ & $\begin{array}{l}\text { Data not } \\
\text { available/report } \\
\text { ed (conference } \\
\text { abstract) and } \\
\text { duplicate }\end{array}$ \\
\hline $\begin{array}{l}\text { 1335. Quach, J., Hiscock, H., \& Wake, M. (2010). Impact of a brief } \\
\text { school-entry sleep intervention on child and parent outcomes: } \\
\text { Randomised controlled trial. Journal of Paediatrics and Child Health, } \\
46,11 .\end{array}$ & $\begin{array}{l}\text { No child } \\
\text { internalising } \\
\text { outcomes or not } \\
\text { a major goal }\end{array}$ \\
\hline $\begin{array}{l}\text { 1336. Querido JG, Eyberg SM and Boggs SR. 2001. Revisiting the } \\
\text { accuracy hypothesis in families of young children with conduct } \\
\text { problems. Journal of Clinical Child Psychology. 30(2):253-61. }\end{array}$ & $\begin{array}{l}\text { Not a parenting } \\
\text { intervention }\end{array}$ \\
\hline $\begin{array}{l}\text { 1337. Quesnel-Vallée, A., \& Taylor, M. (2012). Socioeconomic } \\
\text { pathways to depressive symptoms in adulthood: Evidence from the } \\
\text { National Longitudinal Survey of Youth 1979. Social Science \& } \\
\text { Medicine, 74, 734-743. }\end{array}$ & $\begin{array}{l}\text { Not a parenting } \\
\text { intervention }\end{array}$ \\
\hline $\begin{array}{l}\text { 1338. R. Theise;K. Y. Huang;D. Kamboukos;G. L. Doctoroff;S. } \\
\text { Dawson-McClure;J. J. Palamar;L. M. Brotman, (2014). Moderators of } \\
\text { Intervention Effects on Parenting Practices in a Randomized } \\
\text { Controlled Trial in Early Childhood. Journal of Clinical Child and } \\
\text { Adolescent Psychology, } 43 \text { (3), 501-509. }\end{array}$ & $\begin{array}{l}\text { Targets } \\
\text { externalising }\end{array}$ \\
\hline $\begin{array}{l}\text { 1339. Radesky, J. S., Zuckerman, B., Silverstein, M., Rivara, F. P., Barr, } \\
\text { M., Taylor, J. A., . . Barr, R. G. (2013). Inconsolable infant crying and } \\
\text { maternal postpartum depressive symptoms. Pediatrics, 131(6), e1857- } \\
\text { e1864. doi: http://dx.doi.org/10.1542/peds.2012-3316 }\end{array}$ & $\begin{array}{l}\text { Not a parenting } \\
\text { intervention }\end{array}$ \\
\hline $\begin{array}{l}\text { 1340. Rafferty Y, Griffin KW and Robokos D. 2010. Maternal } \\
\text { depression and parental distress among families in the Early Head } \\
\text { Start Research and Evaluation Project: Risk factors within the family } \\
\text { setting. Infant Mental Health Journal. 31(5):543-69. }\end{array}$ & $\begin{array}{l}\text { Not a parenting } \\
\text { intervention }\end{array}$ \\
\hline $\begin{array}{l}\text { 1341. Rahman A, lqbal Z, Roberts C and Husain N. 2009. Cluster } \\
\text { randomized trial of a parent-based intervention to support early } \\
\text { development of children in a low-income country. Child: care, health } \\
\text { and development, } 35(1), 56-62 \text {. }\end{array}$ & $\begin{array}{l}\text { No child } \\
\text { internalising } \\
\text { outcomes or not } \\
\text { a major goal }\end{array}$ \\
\hline $\begin{array}{l}\text { 1342. Raider MC, Steele W, Delillo-Storey M, Jacobs J and Kuban C. } \\
\text { 2008. Structured sensory therapy (SITCAP-ART) for traumatized } \\
\text { adjudicated adolescents in residential treatment. Residential } \\
\text { Treatment for Children \& Youth. 25(2):167-85. }\end{array}$ & $\begin{array}{l}\text { Not a parenting } \\
\text { intervention }\end{array}$ \\
\hline $\begin{array}{l}\text { 1343. Raine, A., Portnoy, J., Liu, J., Mahoomed, T., \& Hibbeln, J. R. } \\
\text { (2015). Reduction in behavior problems with omega-3 } \\
\text { supplementation in children aged 8-16 years: a randomized, double- } \\
\text { blind, placebo-controlled, stratified, parallel-group trial. Journal of } \\
\text { Child Psychology and Psychiatry, 56(5), 509-520. }\end{array}$ & $\begin{array}{l}\text { Not a parenting } \\
\text { intervention }\end{array}$ \\
\hline $\begin{array}{l}\text { 1344. Rakow A, Forehand R, Haker K, McKee LG, Champion JE, Potts } \\
\text { J, Hardcastle E, Roberts L and Compas BE. 2011. Use of parental guilt } \\
\text { induction among depressed parents. Journal of Family Psychology. } \\
\text { 25(1):147-51. }\end{array}$ & $\begin{array}{l}\text { Not a parenting } \\
\text { intervention }\end{array}$ \\
\hline
\end{tabular}




\begin{tabular}{|c|c|}
\hline $\begin{array}{l}1345 . \quad \text { Rakow, A., Smith, D., Begle, A. M., \& Ayer, L. (2011). The } \\
\text { association of maternal depressive symptoms with child externalizing } \\
\text { problems: The role of maternal support following child sexual abuse. } \\
\text { Journal of Child Sexual Abuse: Research, Treatment, \& Program } \\
\text { Innovations for Victims, Survivors, \& Offenders, 20, 467-480. }\end{array}$ & $\begin{array}{l}\text { Targets } \\
\text { externalising }\end{array}$ \\
\hline $\begin{array}{l}\text { 1346. Ramey, C. T., et al. (1992). Infant Health and Development } \\
\text { Program for Low Birth Weight, Premature Infants: Program Elements, } \\
\text { Family Participation, and Child Intelligence. Pediatrics 89(3): 454-465. }\end{array}$ & $\begin{array}{l}\text { No long term } \\
\text { follow-up }\end{array}$ \\
\hline $\begin{array}{l}\text { 1347. Ramli M, Adlina S, Suthahar A, Edariah AB, Ariff FM, Narimah } \\
\text { AHH, Nuraliza AS, Fauzi I and Karuthan C. 2008. Depression among } \\
\text { secondary school students: A comparison between urban and rural } \\
\text { populations in a Malaysian community. Hong Kong Journal of } \\
\text { Psychiatry. 18(2):55-61. }\end{array}$ & $\begin{array}{l}\text { Not a parenting } \\
\text { intervention }\end{array}$ \\
\hline $\begin{array}{l}\text { 1348. Ramsauer, B., Lotzin, A., Mühlhan, C., Romer, G., Nolte, T., } \\
\text { Fonagy, P., \& Powell, B. (2014). A randomized controlled trial } \\
\text { comparing Circle of Security Intervention and treatment as usual as } \\
\text { interventions to increase attachment security in infants of mentally ill } \\
\text { mothers: Study Protocol. BMC psychiatry, } 14(1), 24 .\end{array}$ & $\begin{array}{l}\text { Data not } \\
\text { available/report } \\
\text { ed }\end{array}$ \\
\hline $\begin{array}{l}\text { 1349. Rapee RM, Abbott MJ, Lyneham HJ. Bibliotherapy for children } \\
\text { with anxiety disorders using written materials for parents: A } \\
\text { randomized controlled trial. J Consult Clin Psychol. } 2006 \\
\text { Jun;74(3):436-44. }\end{array}$ & $\begin{array}{l}\text { Follow-up less } \\
\text { than } 6 \text { months }\end{array}$ \\
\hline $\begin{array}{l}\text { 1350. Rapee RM. 2003. The influence of comorbidity on treatment } \\
\text { outcome for children and adolescents with anxiety disorders. } \\
\text { Behaviour Research and Therapy. 41(1):105-12. }\end{array}$ & $\begin{array}{l}\text { Not a parenting } \\
\text { intervention }\end{array}$ \\
\hline $\begin{array}{l}\text { 1351. Rapee, R. M. (2002). The development and modification of } \\
\text { temperamental risk for anxiety disorders: prevention of a lifetime of } \\
\text { anxiety?. Biological Psychiatry, 52(10), 947-957. doi: 10.1016/S0006- } \\
\text { 3223(02)01572-X }\end{array}$ & $\begin{array}{l}\text { No long term } \\
\text { follow-up }\end{array}$ \\
\hline $\begin{array}{l}\text { 1352. Rau, J., May, T. W., Pfäfflin, M., Heubrock, D., \& Petermann, F. } \\
\text { (2006). [Education of children with epilepsy and their parents by the } \\
\text { modular education program epilepsy for families (FAMOSES)--results } \\
\text { of an evaluation study]. Die Rehabilitation, } 45(1), 27-39 .\end{array}$ & $\begin{array}{l}\text { Intervention for } \\
\text { developmental } \\
\text { disorders } \\
\text { (autism, autism } \\
\text { spectrum } \\
\text { disorder), } \\
\text { physical } \\
\text { disabilities, } \\
\text { medical } \\
\text { conditions or } \\
\text { distress about } \\
\text { facing medical } \\
\text { interventions }\end{array}$ \\
\hline $\begin{array}{l}\text { 1353. Rauh, V. A., Achenbach, T. M., Nurcombe, B., Howell, C. T., \& } \\
\text { Teti, D. M. (1988). Minimizing Adverse Effects of Low Birthweight: } \\
\text { Four-Year Results of an Early Intervention Program. Child } \\
\text { Development, 59, } 544 .\end{array}$ & $\begin{array}{l}\text { No child } \\
\text { internalising } \\
\text { outcomes or not } \\
\text { a major goal }\end{array}$ \\
\hline $\begin{array}{l}\text { 1354. Rauh, V. A., Nurcombe, B., Achenbach, T., \& Howell, C. (1990). } \\
\text { The Mother-Infant Transaction Program. The content and implications } \\
\text { of an intervention for the mothers of low-birthweight infants. Clinics } \\
\text { in perinatology, 17, 31-45. }\end{array}$ & $\begin{array}{l}\text { No child } \\
\text { internalising } \\
\text { outcomes or not } \\
\text { a major goal }\end{array}$ \\
\hline
\end{tabular}




\begin{tabular}{|c|c|}
\hline $\begin{array}{l}\text { 1355. Raver CC, Jones SM, Li-Grining C, Zhai F, Metzger MW and } \\
\text { Solomon B. 2009. Targeting children's behavior problems in preschool } \\
\text { classrooms: A cluster-randomized controlled trial. Journal of } \\
\text { Consulting and Clinical Psychology. } 77(2): 302-16 \text {. }\end{array}$ & $\begin{array}{l}\text { Not a parenting } \\
\text { intervention }\end{array}$ \\
\hline $\begin{array}{l}\text { 1356. Raviv T and Wadsworth ME. 2010. The efficacy of a pilot } \\
\text { prevention program for children and caregivers coping with economic } \\
\text { strain. Cognitive Therapy and Research.34(3):216-28. }\end{array}$ & Not an RCT \\
\hline $\begin{array}{l}\text { 1357. Ravn, I. H., Smith, L., Smeby, N. A., Kynoe, N. M., Sandvik, L., } \\
\text { Bunch, E. H., \& Lindemann, R. (2012). Effects of early mother-infant } \\
\text { intervention on outcomes in mothers and moderately and late } \\
\text { preterm infants at age } 1 \text { year: a randomized controlled trial. Infant } \\
\text { Behav Dev, 35, 36-47. }\end{array}$ & $\begin{array}{l}\text { No child } \\
\text { internalising } \\
\text { outcomes or not } \\
\text { a major goal }\end{array}$ \\
\hline $\begin{array}{l}\text { 1358. Razza RA, Martin A and Brooks-Gunn J. 2010. Associations } \\
\text { among family environment, sustained attention, and school readiness } \\
\text { for low-income children. Developmental Psychology. } 46(6): 1528-42 \text {. }\end{array}$ & $\begin{array}{l}\text { Not a parenting } \\
\text { intervention }\end{array}$ \\
\hline $\begin{array}{l}\text { 1359. Realmuto, G. M., August, G. J., \& Egan, E. A. (2004). Testing } \\
\text { the goodness-of-fit of a multifaceted preventive intervention for } \\
\text { children at risk for conduct disorder. Canadian journal of psychiatry. } \\
\text { Revue canadienne de psychiatrie, (11), 743-752. }\end{array}$ & $\begin{array}{l}\text { No child } \\
\text { internalising } \\
\text { outcomes or not } \\
\text { a major goal }\end{array}$ \\
\hline $\begin{array}{l}1360 . \quad \text { Reck C, Struben K, Backenstrass M, Stefenelli U, Reinig K, } \\
\text { Fuchs T, Sohn C and Mundt C. 2008. Prevalence, onset and } \\
\text { comorbidity of postpartum anxiety and depressive disorders. Acta } \\
\text { Psychiatrica Scandinavica. 118(6):459-68. }\end{array}$ & $\begin{array}{l}\text { Not a parenting } \\
\text { intervention }\end{array}$ \\
\hline $\begin{array}{l}\text { 1361. Records K and Rice M. 2007. Psychosocial correlates of } \\
\text { depression symptoms during the third trimester of pregnancy. Journal } \\
\text { of Obstetric, Gynecologic, \& Neonatal Nursing: Clinical Scholarship for } \\
\text { the Care of Women, Childbearing Families, \& Newborns. 36(3):231-42. }\end{array}$ & $\begin{array}{l}\text { Not a parenting } \\
\text { intervention }\end{array}$ \\
\hline $\begin{array}{l}\text { 1362. Reebye, P. N., \& Tzoumakis, S. (2012). Clinical home visitation } \\
\text { for children with externalizing disorders: A randomized study. Journal } \\
\text { of Social Service Research, 38, 549-560. }\end{array}$ & $\begin{array}{l}\text { Targets } \\
\text { externalising }\end{array}$ \\
\hline $\begin{array}{l}\text { 1363. Reed GL and Enright RD. 2006. The effects of forgiveness } \\
\text { therapy on depression, anxiety, and posttraumatic stress for women } \\
\text { after spousal emotional abuse. Journal of Consulting and Clinical } \\
\text { Psychology. Special Issue: Benefit-Finding. 74(5):920-9. }\end{array}$ & $\begin{array}{l}\text { Not a parenting } \\
\text { intervention }\end{array}$ \\
\hline $\begin{array}{l}\text { 1364. Reedtz, C., Handegård, B. H., \& Mørch, W. T. (2011). } \\
\text { Promoting positive parenting practices in primary pare: outcomes and } \\
\text { mechanisms of change in a randomized controlled risk reduction trial. } \\
\text { In Scandinavian journal of psychology (pp. 131-137). }\end{array}$ & $\begin{array}{l}\text { No child } \\
\text { internalising } \\
\text { outcomes or not } \\
\text { a major goal; } \\
\text { Targets } \\
\text { externalising } \\
\end{array}$ \\
\hline $\begin{array}{l}1365 . \quad \text { Reese, R. J., Slone, N. C., Soares, N., \& Sprang, R. (2012). } \\
\text { Telehealth for underserved families: An evidence-based parenting } \\
\text { program. Psychological Services, 9, 320-322. }\end{array}$ & $\begin{array}{l}\text { Not an RCT; No } \\
\text { long term } \\
\text { follow-up; } \\
\text { Targets } \\
\text { externalising } \\
\end{array}$ \\
\hline $\begin{array}{l}\text { 1366. Reeves G and Anthony B. 2009. Multimodal treatments versus } \\
\text { pharmacotherapy alone in children with psychiatric disorders: } \\
\text { Implications of access, effectiveness, and contextual treatment. } \\
\text { Pediatric Drugs. 11(3):165-9. }\end{array}$ & Review \\
\hline
\end{tabular}




\begin{tabular}{|c|c|}
\hline $\begin{array}{l}\text { 1367. Regev, D., Kedem, D., \& Guttmann, J. (2012). The effects of } \\
\text { mothers' participation in movement therapy on the emotional } \\
\text { functioning of their school-age children in Israel. Arts in } \\
\text { Psychotherapy, 39(5), 479-488. }\end{array}$ & $\begin{array}{l}\text { Comparison } \\
\text { condition not a } \\
\text { minimal control }\end{array}$ \\
\hline $\begin{array}{l}\text { 1368. Reid JB, Eddy JM, Fetrow RA and Stoolmiller M. } 1999 . \\
\text { Description and Immediate Impacts of a Preventive Intervention for } \\
\text { Conduct Problems. American Journal of Community Psychology. } \\
\text { 27(4):483-518. }\end{array}$ & $\begin{array}{l}\text { No child } \\
\text { internalising } \\
\text { outcomes or not } \\
\text { a major goal; } \\
\text { Parent } \\
\text { component too } \\
\text { small }\end{array}$ \\
\hline $\begin{array}{l}\text { 1369. Reid MJ, Webster-Stratton C and Beauchaine TP. 2001. Parent } \\
\text { Training in Head Start: A Comparison of Program Response Among } \\
\text { African American, Asian American, Caucasian, and Hispanic Mothers. } \\
\text { Prevention Science. 2(4):209-27. }\end{array}$ & $\begin{array}{l}\text { Targets } \\
\text { externalising }\end{array}$ \\
\hline $\begin{array}{l}1370 . \quad \text { Reid, G. J., Stewart, M., Vingilis, E., Dozois, D. J., Wetmore, S., } \\
\text { Jordan, J., ... \& Zaric, G. S. (2013). Randomized trial of distance-based } \\
\text { treatment for young children with discipline problems seen in primary } \\
\text { health care. Family practice, 30(1), 14-24. }\end{array}$ & $\begin{array}{l}\text { Targets } \\
\text { externalising }\end{array}$ \\
\hline $\begin{array}{l}\text { 1371. Reid, J., Webster-Stratton, C., \& Hammond, M. (2003). Follow- } \\
\text { up of children who received the Incredible Years Intervention for } \\
\text { oppositional-defiant disorder: Maintenance and prediction of 2-year } \\
\text { outcome. Behavior Therapy, 34, 471-491. }\end{array}$ & $\begin{array}{l}\text { Follow-up data } \\
\text { collected only in } \\
\text { active group; No } \\
\text { internalising } \\
\text { outcomes or not } \\
\text { a major goal }\end{array}$ \\
\hline $\begin{array}{l}\text { 1372. Reid, M. J., Webster-Stratton, C., \& Baydar, N. (2004). Halting } \\
\text { the development of conduct problems in head start children: The } \\
\text { effects of parent training. Journal of Clinical Child and Adolescent } \\
\text { Psychology, 33, 279-291. }\end{array}$ & $\begin{array}{l}\text { No child } \\
\text { internalising } \\
\text { outcomes or not } \\
\text { a major goal }\end{array}$ \\
\hline $\begin{array}{l}\text { 1373. Reid, M. J., Webster-Stratton, C., \& Hammond, M. (2007). } \\
\text { Enhancing a classroom social competence and problem-solving } \\
\text { curriculum by offering parent training to families of moderate- to high- } \\
\text { risk elementary school children. J Clin Child Adolesc Psychol, 36(4), } \\
\text { 605-620. doi: } 10.1080 / 15374410701662741\end{array}$ & $\begin{array}{l}\text { No long term } \\
\text { follow-up }\end{array}$ \\
\hline $\begin{array}{l}1374 . \quad \text { Reinert DF. 1999. Group intervention for children of } \\
\text { recovering alcoholic parents. Alcoholism Treatment Quarterly. } \\
\text { 17(4):15-27. }\end{array}$ & $\begin{array}{l}\text { Not a parenting } \\
\text { intervention }\end{array}$ \\
\hline $\begin{array}{l}\text { 1375. Renaud, M. (1998). Evaluation of a structured programme of a } \\
\text { group intervention with families experiencing parenting difficulties. In } \\
\text { 5th World Congress on Innovations in Psychiatry. London, UK. 19th- } \\
\text { 22nd May (No. 3-4). }\end{array}$ & $\begin{array}{l}\text { No long term } \\
\text { follow-up }\end{array}$ \\
\hline $\begin{array}{l}1376 . \quad \text { Rennick, J. E., Constantin, E., Stremler, R., Horwood, L., } \\
\text { Antonacci, M., Aita, M., \& Majnemer, A. (2014). Acceptability and } \\
\text { feasibility of an intervention to promote child comfort and } \\
\text { psychological well-being during and following PICU hospitalization. } \\
\text { Pediatric Critical Care Medicine, 15(4_suppl), 9. }\end{array}$ & $\begin{array}{l}\text { Follow-up less } \\
\text { than } 6 \text { months }\end{array}$ \\
\hline $\begin{array}{l}\text { 1377. Resnick MB, Armstrong S, Carter RL. Developmental } \\
\text { intervention program for high-risk premature infants: effects on } \\
\text { development and parent-infant interactions. Dev Behav Pediatr } \\
\text { 1988;9:73-8. }\end{array}$ & $\begin{array}{l}\text { No long term } \\
\text { follow-up }\end{array}$ \\
\hline
\end{tabular}




\begin{tabular}{|c|c|}
\hline $\begin{array}{l}\text { 1378. Resnick, M. B., et al. (1987). Developmental Intervention for } \\
\text { Low Birth Weight Infants: Improved Early Developmental Outcome. } \\
\text { Pediatrics 80(1): 68-74. }\end{array}$ & $\begin{array}{l}\text { No long term } \\
\text { follow-up }\end{array}$ \\
\hline $\begin{array}{l}\text { 1379. Reuland, M. M., \& Teachman, B. A. (2014). Interpretation bias } \\
\text { modification for youth and their parents: A novel treatment for early } \\
\text { adolescent social anxiety. Journal of anxiety disorders, 28(8), 851-864. }\end{array}$ & $\begin{array}{l}\text { Comparison } \\
\text { condition not a } \\
\text { minimal control; } \\
\text { Follow-up less } \\
\text { than } 6 \text { months }\end{array}$ \\
\hline $\begin{array}{l}\text { 1380. Reyno SM and McGrath PJ. 2006. Predictors of parent training } \\
\text { efficacy for child externalizing behavior problems - A meta-analytic } \\
\text { review. Journal of Child Psychology and Psychiatry and Allied } \\
\text { Disciplines. 47(1):99-111. }\end{array}$ & Review \\
\hline $\begin{array}{l}\text { 1381. Reyno, S. M. (2011). Contextual risk factors, parent training } \\
\text { outcomes and dimensions of parenting behaviors: Implications for } \\
\text { parent training interventions (Order No. NR69861). Available from } \\
\text { ProQuest Central; ProQuest Dissertations \& Theses Global. } \\
\text { (850545876). }\end{array}$ & $\begin{array}{l}\text { Targets } \\
\text { externalising }\end{array}$ \\
\hline $\begin{array}{l}\text { 1382. Rhodes KV and Iwashyna TJ. 2007. Child injury risks are close } \\
\text { to home: Parent psychosocial factors associated with child safety. } \\
\text { Maternal and Child Health Journal. 11(3):269-75. }\end{array}$ & $\begin{array}{l}\text { Not a parenting } \\
\text { intervention }\end{array}$ \\
\hline $\begin{array}{l}\text { 1383. Rhodes P, Brown J and Madden S. 2009. The Maudsley model } \\
\text { of family-based treatment for anorexia nervosa: a qualitative } \\
\text { evaluation of parent-to-parent consultation. Journal of marital and } \\
\text { family therapy, } 35(2), 181-192 \text {. }\end{array}$ & $\begin{array}{l}\text { No child } \\
\text { internalising } \\
\text { outcomes or not } \\
\text { a major goal }\end{array}$ \\
\hline $\begin{array}{l}\text { 1384. Ricci G, Bendandi B, Aiazzi R, Patrizi A and Masi M. } 2009 . \\
\text { Three years of Italian experience of an educational program for } \\
\text { parents of young children affected by atopic dermatitis: improving } \\
\text { knowledge produces lower anxiety levels in parents of children with } \\
\text { atopic dermatitis. Pediatr Dermatol. 26(1):1-5. }\end{array}$ & $\begin{array}{l}\text { Intervention for } \\
\text { developmental } \\
\text { disorders } \\
\text { (autism, autism } \\
\text { spectrum } \\
\text { disorder), } \\
\text { physical } \\
\text { disabilities, } \\
\text { medical } \\
\text { conditions or } \\
\text { distress about } \\
\text { facing medical } \\
\text { interventions }\end{array}$ \\
\hline $\begin{array}{l}\text { 1385. Rice } \mathrm{M} \text {, Glasper } \mathrm{A} \text {, Keeton } \mathrm{D} \text { and Spargo P. 2008. The effect of } \\
\text { a preoperative education programme on perioperative anxiety in } \\
\text { children: an observational study. Paediatr Anaesth. 18(5):426-30. }\end{array}$ & $\begin{array}{l}\text { Intervention for } \\
\text { developmental } \\
\text { disorders } \\
\text { (autism, autism } \\
\text { spectrum } \\
\text { disorder), } \\
\text { physical } \\
\text { disabilities, } \\
\text { medical } \\
\text { conditions or } \\
\text { distress about } \\
\text { facing medical } \\
\text { interventions }\end{array}$ \\
\hline
\end{tabular}




\begin{tabular}{|c|c|}
\hline $\begin{array}{l}\text { 1386. Richardson CR. 1997. Parents and pediatric procedures. The } \\
\text { Journal of family practice, } 44(2), 131 .\end{array}$ & $\begin{array}{l}\text { No child } \\
\text { internalising } \\
\text { outcomes or not } \\
\text { a major goal; No } \\
\text { long term } \\
\text { follow-up }\end{array}$ \\
\hline $\begin{array}{l}\text { 1387. Richardson L, McCauley E and Katon W. 2009. Collaborative } \\
\text { care for adolescent depression: A pilot study. General Hospital } \\
\text { Psychiatry. 31(1):36-45. }\end{array}$ & $\begin{array}{l}\text { Not a parenting } \\
\text { intervention }\end{array}$ \\
\hline $\begin{array}{l}\text { 1388. Richardson, B. B. (2010). Alleviating chronic sleep debt in early } \\
\text { adolescence: Can a school based intervention make a } \\
\text { difference?(Order No. 3421687). Available from ProQuest Central; } \\
\text { ProQuest Dissertations \& Theses Global. (753511893). }\end{array}$ & $\begin{array}{l}\text { Not a parenting } \\
\text { intervention }\end{array}$ \\
\hline $\begin{array}{l}\text { 1389. Riley AW, Coiro MJ, Broitman M, Colantuoni E, Hurley KM, } \\
\text { Bandeen-Roche K and Miranda J. 2009. Mental health of children of } \\
\text { low-income depressed mothers: Influences of parenting, family } \\
\text { environment, and raters. Psychiatric Services. 60(3):329-36. }\end{array}$ & $\begin{array}{l}\text { Not a parenting } \\
\text { intervention }\end{array}$ \\
\hline $\begin{array}{l}\text { 1390. Robbins H, Hundley V and Osman LM. 2003. Minor illness } \\
\text { education for parents of young children. J Adv Nurs. 44(3):238-47. }\end{array}$ & $\begin{array}{l}\text { No child } \\
\text { internalising } \\
\text { outcomes or not } \\
\text { a major goal }\end{array}$ \\
\hline $\begin{array}{l}\text { 1391. Roberts C, Kane R, Thomson H, Bishop B and Hart B. 2003. The } \\
\text { prevention of depressive symptoms in rural school children: a } \\
\text { randomized controlled trial. J Consult Clin Psychol. 71(3):622-8. }\end{array}$ & $\begin{array}{l}\text { Not a parenting } \\
\text { intervention }\end{array}$ \\
\hline $\begin{array}{l}\text { 1392. Roberts CM, Kane R, Bishop B, Cross D, Fenton J and Hart B. } \\
\text { 2010. The prevention of anxiety and depression in children from } \\
\text { disadvantaged schools. Behav Res Ther. 48(1):68-73. }\end{array}$ & $\begin{array}{l}\text { Not a parenting } \\
\text { intervention }\end{array}$ \\
\hline $\begin{array}{l}\text { 1393. Robin, A. L., Siegel, P. T., Koepke, T., Moye, A. W., \& Tice, S. } \\
\text { (1994). Family therapy versus individual therapy for adolescent } \\
\text { females with anorexia nervosa. J Dev Behav Pediatr, 15(2), 111-116. }\end{array}$ & $\begin{array}{l}\text { No long term } \\
\text { follow-up }\end{array}$ \\
\hline $\begin{array}{l}\text { 1394. Robinson TN, Matheson DM, Kraemer HC, Wilson DM, } \\
\text { Obarzanek E, Thompson NS, Alhassan S, Spencer TR, Haydel KF, } \\
\text { Fujimoto M, Varady A and Killen JD. 2010. A randomized controlled } \\
\text { trial of culturally tailored dance and reducing screen time to prevent } \\
\text { weight gain in low-income African American girls: Stanford GEMS. } \\
\text { Arch Pediatr Adolesc Med. 164(11):995-1004. }\end{array}$ & $\begin{array}{l}\text { Not a parenting } \\
\text { intervention }\end{array}$ \\
\hline $\begin{array}{l}\text { 1395. Robinson, J., \& Emde, R. N. (2004). Mental Health Moderators } \\
\text { of Early Head Start on Parenting and Child Development: Maternal } \\
\text { Depression and Relationship Attitudes. Parenting: Science and } \\
\text { Practice, 4(1), 73-97. doi:10.1207/s15327922par0401_4 }\end{array}$ & $\begin{array}{l}\text { No child } \\
\text { internalising } \\
\text { outcomes or not } \\
\text { a major goal }\end{array}$ \\
\hline $\begin{array}{l}\text { 1396. Rodgers, A., \& Dunsmuir, S. (2013). A controlled evaluation of } \\
\text { the 'FRIENDS for Life'emotional resiliency programme on overall } \\
\text { anxiety levels, anxiety subtype levels and school adjustment. Child and } \\
\text { Adolescent Mental Health. }\end{array}$ & $\begin{array}{l}\text { Not a parenting } \\
\text { intervention }\end{array}$ \\
\hline $\begin{array}{l}\text { 1397. Rodriguez CM and Eden AM. 2008. Disciplinary style and child } \\
\text { abuse potential: Association with indicators of positive functioning in } \\
\text { children with behavior problems. Child Psychiatry and Human } \\
\text { Development. } 39(2): 123-36 \text {. }\end{array}$ & Not an RCT \\
\hline
\end{tabular}




\begin{tabular}{|c|c|}
\hline $\begin{array}{l}\text { 1398. Rodríguez, G. M., Bagner, D. M., \& Graziano, P. A. (2014). } \\
\text { Parent training for children born premature: A pilot study examining } \\
\text { the moderating role of emotion regulation. Child Psychiatry and } \\
\text { Human Development, } 45(2), 143-152 . \text { doi: } 10.1007 / s 10578-013-0385- \\
7\end{array}$ & $\begin{array}{l}\text { Targets } \\
\text { externalising }\end{array}$ \\
\hline $\begin{array}{l}\text { 1399. Rofey DL, Szigethy EM, Noll RB, Dahl RE, lobst E and Arslanian } \\
\text { SA. 2009. Cognitive,Äibehavioral therapy for physical and emotional } \\
\text { disturbances in Adolescents with polycystic ovary syndrome: A pilot } \\
\text { study. Journal of Pediatric Psychology. 34(2):156-63. }\end{array}$ & Not an RCT \\
\hline $\begin{array}{l}\text { 1400. Rogers, S. J., Estes, A., Lord, C., Vismara, L., Winter, J., } \\
\text { Fitzpatrick, A., Guo, M., \& Dawson, G. (2012). Effects of a brief Early } \\
\text { Start Denver Model (ESDM)-based parent intervention on toddlers at } \\
\text { risk for autism spectrum disorders: A randomized controlled trial. } \\
\text { Journal of the American Academy of Child \& Adolescent Psychiatry, } \\
\text { 51, 1052-1065. }\end{array}$ & $\begin{array}{l}\text { Intervention for } \\
\text { developmental } \\
\text { disorders } \\
\text { (autism, autism } \\
\text { spectrum } \\
\text { disorder), } \\
\text { physical } \\
\text { disabilities, } \\
\text { medical } \\
\text { conditions or } \\
\text { distress about } \\
\text { facing medical } \\
\text { interventions }\end{array}$ \\
\hline $\begin{array}{l}\text { 1401. Roman LA, Gardiner JC, Lindsay JK, Moore JS, Luo Z, Baer LJ, } \\
\text { Goddeeris JH, Shoemaker AL, Barton LR, Fitzgerald HE and Paneth N. } \\
\text { 2009. Alleviating perinatal depressive symptoms and stress: A nurse- } \\
\text { community health worker randomized trial. Archives of Women's } \\
\text { Mental Health. 12(6):379-91. }\end{array}$ & $\begin{array}{l}\text { Not a parenting } \\
\text { intervention }\end{array}$ \\
\hline $\begin{array}{l}\text { 1402. Rosen, L. A., Gabardi, L., Miller, C. D., \& Miller, L. (1990). } \\
\text { Home-based treatment of disruptive junior high school students: An } \\
\text { analysis of the differential effects of positive and negative } \\
\text { consequences. Behavioral Disorders, 15(4), 227-232. }\end{array}$ & $\begin{array}{l}\text { No long term } \\
\text { follow-up }\end{array}$ \\
\hline $\begin{array}{l}\text { 1403. Roskam, I., \& Meunier, J. C. (2012). The determinants of } \\
\text { parental childrearing behavior trajectories: The effects of parental and } \\
\text { child time-varying and time-invariant predictors. International Journal } \\
\text { of Behavioral Development, } 36,186-196 .\end{array}$ & $\begin{array}{l}\text { Not a parenting } \\
\text { intervention }\end{array}$ \\
\hline $\begin{array}{l}\text { 1404. Ross CE and Mirowsky J. 1999. Parental divorce, life-course } \\
\text { disruption and adult depression. Journal of Marriage \& the Family. } \\
61(4): 1034-45 .\end{array}$ & $\begin{array}{l}\text { Not a parenting } \\
\text { intervention }\end{array}$ \\
\hline $\begin{array}{l}\text { 1405. Ross, G. S. (1984). HOME INTERVENTION FOR PREMATURE } \\
\text { INFANTS OF LOW-INCOME FAMILIES. American Journal of } \\
\text { Orthopsychiatry 54(2): 263-270. }\end{array}$ & $\begin{array}{l}\text { No child } \\
\text { internalising } \\
\text { outcomes or not } \\
\text { a major goal }\end{array}$ \\
\hline $\begin{array}{l}\text { 1406. Roth I and Reichle B. 2007. [I keep cool: Relationship oriented } \\
\text { training of prosocial behaviour and constructive conflict solving for } \\
\text { elementary school children]. Prax Kinderpsychol Kinderpsychiatr. } \\
\text { 56(5):463-82. }\end{array}$ & $\begin{array}{l}\text { Not a parenting } \\
\text { intervention }\end{array}$ \\
\hline $\begin{array}{l}\text { 1407. Roth, R. M., Pixley, H. S., Kruck, C. L., Garlinghouse, M. A., } \\
\text { Giancola, P. R., \& Flashman, L. A. (2012). Performance on the Cognitive } \\
\text { Estimation Test in schizophrenia. Applied Neuropsychology, 19, 141- } \\
146 .\end{array}$ & $\begin{array}{l}\text { Not a parenting } \\
\text { intervention }\end{array}$ \\
\hline
\end{tabular}




\begin{tabular}{|c|c|}
\hline $\begin{array}{l}\text { 1408. Rotheram-Borus MJ, Stein JA, Jiraphongsa C, Khumtong S, Lee } \\
\text { SJ and Li L. 2010. Benefits of family and social relationships for Thai } \\
\text { parents living with HIV. Prev Sci. 11(3):298-307. }\end{array}$ & $\begin{array}{l}\text { Not a parenting } \\
\text { intervention }\end{array}$ \\
\hline $\begin{array}{l}\text { 1409. Rotheram-Borus, M. J., Lester, P., Song, J., Lin, Y.-Y., Leonard, } \\
\text { N. R., Beckwith, L., et al. (2006). Intergenerational benefits of family- } \\
\text { based HIV interventions. Journal of Consulting and Clinical Psychology, } \\
\text { 74(3), 622-627. doi: 10.1037/0022-006x.74.3.622 }\end{array}$ & $\begin{array}{l}\text { No child } \\
\text { internalising } \\
\text { outcomes or not } \\
\text { a major goal } \\
\text { (grandchildren } \\
\text { instead) }\end{array}$ \\
\hline $\begin{array}{l}1410 . \quad \text { Rotheram-Borus, M. J., Rice, E., Comulada, W. S., Best, K., Elia, } \\
\text { C., Peters, K., Li, L., Green, S., \& Valladares, E. (2012). Intervention } \\
\text { outcomes among HIV-affected families over } 18 \text { months. AIDS Behav, } \\
\text { 16, 1265-1275. }\end{array}$ & $\begin{array}{l}\text { Parent } \\
\text { component too } \\
\text { small }\end{array}$ \\
\hline $\begin{array}{l}\text { 1411. Rotheram-Borus, M. J., Stein, J. A., \& Lin, Y. Y. (2001). Impact } \\
\text { of parent death and an intervention on the adjustment of adolescents } \\
\text { whose parents have HIV/AIDS. Journal of Consulting \& Clinical } \\
\text { Psychology, (5), 763-773. }\end{array}$ & $\begin{array}{l}\text { Secondary } \\
\text { analysis (re- } \\
\text { analysis of data } \\
\text { in Rotheram- } \\
\text { Borus } 2001 \text { using } \\
\text { SEM, data not } \\
\text { useable) }\end{array}$ \\
\hline $\begin{array}{l}\text { 1412. } \quad \text { Routh, C. P., Hill, J. W., Steele, H., Elliott, C. E., \& Dewey, M. E. } \\
\text { (1995). Maternal Attachment Status, Psychosocial Stressors and } \\
\text { Problem Behavior - Follow-up after Parent Training Courses for } \\
\text { Conduct Disorder. Journal of Child Psychology and Psychiatry and } \\
\text { Allied Disciplines, 36(7), 1179-1198. }\end{array}$ & Not an RCT \\
\hline $\begin{array}{l}\text { 1413. Rowe, H., Wynter, K., Lorgelly, P., Amir, L. H., Ranasinha, S., } \\
\text { Proimos, J., ... \& Fisher, J. (2014). A cluster randomised controlled trial } \\
\text { of a brief couple-focused psychoeducational intervention to prevent } \\
\text { common postnatal mental disorders among women: study protocol. } \\
\text { BMJ open, 4(9), e006436. }\end{array}$ & $\begin{array}{l}\text { No child } \\
\text { internalising } \\
\text { outcomes or not } \\
\text { a major goal }\end{array}$ \\
\hline $\begin{array}{l}\text { 1414. Rozenman, M., Weersing, V. R., \& Amir, N. (2011). A case } \\
\text { series of attention modification in clinically anxious youths. Behav Res } \\
\text { Ther, 49, 324-330. }\end{array}$ & Not an RCT \\
\hline $\begin{array}{l}\text { 1415. Rubin-Vaughan, A. (2011). Interactions between aggressive } \\
\text { children and their mothers: Changes through treatment and the role } \\
\text { of gender (Order No. NR75683). Available from ProQuest Central; } \\
\text { ProQuest Dissertations \& Theses Global. (879637789). }\end{array}$ & $\begin{array}{l}\text { Targets } \\
\text { externalising }\end{array}$ \\
\hline $\begin{array}{l}\text { 1416. Ruma, P. R., Burke, R. V., \& Thompson, R. W. (1996). Group } \\
\text { parent training: Is it effective for children of all ages? Behavior } \\
\text { Therapy, 27, 159-169. }\end{array}$ & $\begin{array}{l}\text { Follow-up data } \\
\text { collected only in } \\
\text { active group }\end{array}$ \\
\hline $\begin{array}{l}1417 . \quad \text { Runfola, C. D., Zucker, N. L., Holle, A. V., Mazzeo, S., Hodges, E. } \\
\text { A., Perrin, E. M., . . Bulik, C. M. (2014). NURTURE: Development and } \\
\text { pilot testing of a novel parenting intervention for mothers with } \\
\text { histories of an eating disorder. International Journal of Eating } \\
\text { Disorders, } 47(1), 1-12 . \text { doi:http://dx.doi.org/10.1002/eat.22178 }\end{array}$ & Not an RCT \\
\hline $\begin{array}{l}1418 . \quad \text { Russell, E. (1996). Identification \& Prevention of Behavioural } \\
\text { Disorders in Children with Learning Disability. Xth World Congress of } \\
\text { Psychiatry. Retrieved from } \\
\text { http://www.mrw.interscience.wiley.com/cochrane/clcentral/articles/ } \\
\text { 338/CN-00320338/frame.html }\end{array}$ & $\begin{array}{l}\text { Data not } \\
\text { available/report } \\
\text { ed }\end{array}$ \\
\hline
\end{tabular}




\begin{tabular}{|c|c|}
\hline $\begin{array}{l}\text { 1419. Ryan, C. S., McCall, R. B., Robinson, D. R., Groark, C. J., Mulvey, } \\
\text { L., \& Plemons, B. W. (2002). Benefits of the Comprehensive Child } \\
\text { Development Program as a function of AFDC receipt and SES. Child } \\
\text { development, (1), 315-328. }\end{array}$ & $\begin{array}{l}\text { No child } \\
\text { internalising } \\
\text { outcomes or not } \\
\text { a major goal }\end{array}$ \\
\hline $\begin{array}{l}\text { 1420. Ryan, S. R., Stanger, C., Thostenson, J., Whitmore, J. J., \& } \\
\text { Budney, A. J. (2013). The impact of disruptive behavior disorder on } \\
\text { substance use treatment outcome in adolescents. J Subst Abuse Treat, } \\
\text { 44(5), 506-514. doi: } 10.1016 / \text { j.jsat.2012.11.003 }\end{array}$ & $\begin{array}{l}\text { No child } \\
\text { internalising } \\
\text { outcomes or not } \\
\text { a major goal }\end{array}$ \\
\hline $\begin{array}{l}\text { 1421. Sagredini, R., Mascheroni, C., Diotto, V., Tranquillini, E., } \\
\text { Paracchini, F., \& Mercuri, P. (2013). Treatment of anxiety at induction } \\
\text { of anaesthesia in children: A randomized controlled trial of non- } \\
\text { pharmacological approach versus midazolam or placebo. [Conference } \\
\text { Abstract]. Paediatric Anaesthesia, } 23 \text { (3), 285. doi: } \\
\text { http://dx.doi.org/10.1111/pan.12097 }\end{array}$ & $\begin{array}{l}\text { Not a parenting } \\
\text { intervention }\end{array}$ \\
\hline $\begin{array}{l}\text { 1422. Sahler, O. J., Dolgin, M. J., Phipps, S., Fairclough, D. L., Askins, } \\
\text { M. A., Katz, E. R., . . Butler, R. W. (2013). Specificity of problem- } \\
\text { solving skills training in mothers of children newly diagnosed with } \\
\text { cancer: results of a multisite randomized clinical trial. J Clin Oncol, } \\
\text { 31(10), 1329-1335. doi: 10.1200/jco.2011.39.1870 }\end{array}$ & $\begin{array}{l}\text { Follow-up less } \\
\text { than } 6 \text { months }\end{array}$ \\
\hline $\begin{array}{l}\text { 1423. Saias, T., Greacen, T., Tubach, F., Dugravier, R., Marcault, E., } \\
\text { Tereno, S., . . Guedeney, A. (2013). Supporting families in challenging } \\
\text { contexts: the CAPEDP project. Glob Health Promot, 20(2 Suppl), 66-70. } \\
\text { doi: } 10.1177 / 1757975913483335\end{array}$ & $\begin{array}{l}\text { Data not } \\
\text { available/report } \\
\text { ed }\end{array}$ \\
\hline $\begin{array}{l}\text { 1424. Sajaniemi N, Makela J, Salokorpi T, et al. Cognitive } \\
\text { performance and attachment patterns at four years of age in } \\
\text { extremely low birth weight infants after early intervention. Eur Child } \\
\text { Adolesc Psychiatry 2001;10:122-9 }\end{array}$ & $\begin{array}{l}\text { No child } \\
\text { internalising } \\
\text { outcomes or not } \\
\text { a major goal }\end{array}$ \\
\hline $\begin{array}{l}\text { 1425. Saldana, L., Chamberlain, P., \& Sheidow, A. (2015). Integrated } \\
\text { treatment for mothers involved in child welfare for substance abuse. } \\
\text { Drug and Alcohol Dependence, (146), e87. }\end{array}$ & $\begin{array}{l}\text { No child } \\
\text { internalising } \\
\text { outcomes or not } \\
\text { a major goal }\end{array}$ \\
\hline $\begin{array}{l}\text { 1426. Salloum A and Overstreet S. 2008. Evaluation of individual and } \\
\text { group grief and trauma interventions for children post disaster. } \\
\text { Journal of Clinical Child and Adolescent Psychology.37(3):459-507. }\end{array}$ & $\begin{array}{l}\text { Not a parenting } \\
\text { intervention }\end{array}$ \\
\hline $\begin{array}{l}\text { 1427. Salloum, A., \& Overstreet, S. (2012). Grief and trauma } \\
\text { intervention for children after disaster: Exploring coping skills versus } \\
\text { trauma narration. Behav Res Ther, 50, 169-179. }\end{array}$ & $\begin{array}{l}\text { Not a parenting } \\
\text { intervention } \\
\text { (child-focused) \& } \\
\text { non active group }\end{array}$ \\
\hline $\begin{array}{l}\text { 1428. Salmela-Aro, K., Read, S., Rouhe, H., Halmesmaki, E., Toivanen, } \\
\text { R. M., Tokola, M. I., \& Saisto, T. (2012). Promoting positive } \\
\text { motherhood among nulliparous pregnant women with an intense fear } \\
\text { of childbirth: RCT intervention. J Health Psychol, 17, 520-534. }\end{array}$ & $\begin{array}{l}\text { No child } \\
\text { internalising } \\
\text { outcomes or not } \\
\text { a major goal }\end{array}$ \\
\hline $\begin{array}{l}\text { 1429. Salome G, de Tychey C, Lighezzolo J, Caludon P, Rebourg- } \\
\text { Roesler C and Flach I. 2009. Evaluation of prevention in perinatality: A } \\
\text { clinical and comparative approach of some determinants involved in } \\
\text { therapeutic grip. Bulletin de Psychologie. 62(1):29-50. }\end{array}$ & $\begin{array}{l}\text { Not a parenting } \\
\text { intervention }\end{array}$ \\
\hline
\end{tabular}




\begin{tabular}{|c|c|}
\hline $\begin{array}{l}\text { 1430. Sanders MR, Markie-Dadds C, Tully LA and Bor W. 2000. The } \\
\text { Triple P-positive parenting program: A comparison of enhanced, } \\
\text { standard, and self-directed behavioral family intervention for parents } \\
\text { of children with early onset conduct problems. Journal of Consulting } \\
\text { and Clinical Psychology. 68(4):624-40. }\end{array}$ & $\begin{array}{l}\text { No child } \\
\text { internalising } \\
\text { outcomes or not } \\
\text { a major goal; } \\
\text { Follow-up data } \\
\text { collected only in } \\
\text { active group }\end{array}$ \\
\hline $\begin{array}{l}\text { 1431. Sanders MR, Pidgeon AM, Gravestock F, Connors MD, Brown S } \\
\text { and Young RW. 2004. Does parental attributional retraining and anger } \\
\text { management enhance the effects of the triple P-positive parenting } \\
\text { program with parents at risk of child maltreatment? Behavior Therapy. } \\
\text { 35(3):513-35. }\end{array}$ & $\begin{array}{l}\text { Comparison } \\
\text { condition not a } \\
\text { minimal control }\end{array}$ \\
\hline $\begin{array}{l}\text { 1432. Sanders MR. 2002. Parenting interventions and the prevention } \\
\text { of serious mental health problems in children. Medical Journal of } \\
\text { Australia. 177(7 SUPPL.):S87-S92. }\end{array}$ & Review \\
\hline $\begin{array}{l}\text { 1433. Sanders, M. R. (1999). Triple P-Positive Parenting Program: } \\
\text { Towards an Empirically Validated Multilevel Parenting and Family } \\
\text { Support Strategy for the Prevention of Behavior and Emotional } \\
\text { Problems in Children. Clinical Child and Family Psychology Review, } \\
\text { 2(2), 71-90. doi:10.1023/a:1021843613840 }\end{array}$ & Review \\
\hline $\begin{array}{l}\text { 1434. Sanders, M. R., \& McFarland, M. (2000). Treatment of } \\
\text { depressed mothers with disruptive children: A controlled evaluation of } \\
\text { cognitive behavioral family intervention. Behavior Therapy, 31(1), 89- } \\
\text { 112. doi: } 10.1016 / \mathrm{s} 0005-7894(00) 80006-4\end{array}$ & $\begin{array}{l}\text { Targets } \\
\text { externalising }\end{array}$ \\
\hline $\begin{array}{l}\text { 1435. Sanders, M. R., Bor, W., \& Morawska, A. (2007). Maintenance } \\
\text { of treatment gains: a comparison of enhanced, standard, and self- } \\
\text { directed Triple P-Positive Parenting Program. J Abnorm Child Psychol, } \\
\text { 35(6), 983-998. doi: } 10.1007 / \mathrm{s} 10802-007-9148-x\end{array}$ & $\begin{array}{l}\text { No child } \\
\text { internalising } \\
\text { outcomes or not } \\
\text { a major goal }\end{array}$ \\
\hline $\begin{array}{l}\text { 1436. Sanders, M. R., Montgomery, D. T., \& Brechman-Toussaint, M. } \\
\text { L. (2000). The mass media and the prevention of child behavior } \\
\text { problems: the evaluation of a television series to promote positive } \\
\text { outcomes for parents and their children. Journal of child psychology } \\
\text { and psychiatry, and allied disciplines, (7), 939-948. }\end{array}$ & $\begin{array}{l}\text { No child } \\
\text { internalising } \\
\text { outcomes or not } \\
\text { a major goal }\end{array}$ \\
\hline $\begin{array}{l}\text { 1437. Sandler I, Miles J, Cookston J and Braver S. 2008. Effects of } \\
\text { father and mother parenting on children's mental health in high- and } \\
\text { low-conflict divorces. Family Court Review. 46(2):282-96. }\end{array}$ & $\begin{array}{l}\text { Not a parenting } \\
\text { intervention }\end{array}$ \\
\hline $\begin{array}{l}\text { 1438. Sandler, I. N., Ayers, T. S., Wolchik, S. A., Tein, J. Y., Kwok, O. } \\
\text { M., Haine, R. A., et al. (2003). The family bereavement program: } \\
\text { efficacy evaluation of a theory-based prevention program for } \\
\text { parentally bereaved children and adolescents. J Consult Clin Psychol, } \\
\text { 71(3), 587-600. }\end{array}$ & $\begin{array}{l}\text { Parent } \\
\text { component too } \\
\text { small }\end{array}$ \\
\hline $\begin{array}{l}\text { 1439. Sandler, I. N., Ma, Y., Tein, J. Y., Ayers, T. S., Wolchik, S., } \\
\text { Kennedy, C., et al. (2010). Long-term effects of the family } \\
\text { bereavement program on multiple indicators of grief in parentally } \\
\text { bereaved children and adolescents. J Consult Clin Psychol, 78(2), 131- } \\
\text { 143. doi: 2010-05835-001 }\end{array}$ & $\begin{array}{l}\text { Parent } \\
\text { component too } \\
\text { small }\end{array}$ \\
\hline $\begin{array}{l}\text { 1440. Sandler, I. N., West, S. G., Baca, L., Pillow, D. R., Gersten, J. C., } \\
\text { Rogosch, F., et al. (1992). Linking empirically based theory and } \\
\text { evaluation: the Family Bereavement Program. Am J Community } \\
\text { Psychol, 20(4), 491-521. }\end{array}$ & $\begin{array}{l}\text { No long term } \\
\text { follow-up }\end{array}$ \\
\hline
\end{tabular}




\begin{tabular}{|c|c|}
\hline $\begin{array}{l}\text { 1441. Sandler, I., Ayers, T. S., Tein, J. Y., Wolchik, S., Millsap, R., } \\
\text { Khoo, S. T., et al. (2010). Six-year follow-up of a preventive } \\
\text { intervention for parentally bereaved youths: a randomized controlled } \\
\text { trial. Arch Pediatr Adolesc Med, 164(10), } 907-914 \text {. doi: } 164 / 10 / 907\end{array}$ & $\begin{array}{l}\text { Parent } \\
\text { component too } \\
\text { small }\end{array}$ \\
\hline $\begin{array}{l}\text { 1442. Sanford M, Boyle M, McCleary L, Miller J, Steele M, Duku E } \\
\text { and Offord D. 2006. A pilot study of adjunctive family psychoeducation } \\
\text { in adolescent major depression: feasibility and treatment effect. J Am } \\
\text { Acad Child Adolesc Psychiatry. 45(4):386-495. }\end{array}$ & $\begin{array}{l}\text { Follow-up less } \\
\text { than } 6 \text { months }\end{array}$ \\
\hline $\begin{array}{l}\text { 1443. Sanford, M., Byrne, C., Williams, S., Atley, S., Miller, J., \& Allin, } \\
\text { H. (2003). A pilot study of a parent-education group for families } \\
\text { affected by depression. Can J Psychiatry, } 48(2), 78-86 .\end{array}$ & $\begin{array}{l}\text { Follow-up less } \\
\text { than } 6 \text { months }\end{array}$ \\
\hline $\begin{array}{l}\text { 1444. Santelices, M. P., Guzmán, G., Aracena, M., Farkas, C., Armijo, } \\
\text { I., Pérez-Salas, C. P., \& Borghini, A. (2011). Promoting secure } \\
\text { attachment: evaluation of the effectiveness of an early intervention } \\
\text { pilot programme with mother-infant dyads in Santiago, Chile. Child: } \\
\text { care, health and development, } 37(2), 203-210 .\end{array}$ & $\begin{array}{l}\text { No long term } \\
\text { follow-up; No } \\
\text { child } \\
\text { internalising } \\
\text { outcomes or not } \\
\text { a major goal }\end{array}$ \\
\hline $\begin{array}{l}1445 . \quad \text { Sassmann, H., deHair, M., Danne, T., \& Lange, K. (2012). } \\
\text { Reducing stress and supporting positive relations in families of young } \\
\text { children with type } 1 \text { diabetes: A randomized controlled study for } \\
\text { evaluating the effects of the DELFIN parenting program. BMC } \\
\text { pediatrics, } 12(1), 152 .\end{array}$ & $\begin{array}{l}\text { Follow-up data } \\
\text { collected only in } \\
\text { active group }\end{array}$ \\
\hline $\begin{array}{l}\text { 1446. Satterfield, J. H., Satterfield, B. T., \& Cantwell, D. P. (1981). } \\
\text { Three-year multimodality treatment study of } 100 \text { hyperactive boys. } \\
\text { The Journal of Pediatrics, 98(4), 650-655. doi: } 10.1016 / \text { S0022- } \\
\text { 3476(81)80788-3 }\end{array}$ & $\begin{array}{l}\text { Not a parenting } \\
\text { intervention }\end{array}$ \\
\hline $\begin{array}{l}\text { 1447. Satterfield, J. H., Satterfield, B. T., \& Schell, A. M. (1987). } \\
\text { Therapeutic Interventions to Prevent Delinquency in Hyperactive Boys. } \\
\text { Journal of the American Academy of Child \& Adolescent Psychiatry, } \\
\text { 26(1), 56-64. [doi: } 10.1097 / 00004583-198701000-00012\end{array}$ & $\begin{array}{l}\text { Not a parenting } \\
\text { intervention }\end{array}$ \\
\hline $\begin{array}{l}1448 . \quad \text { Sawyer, A. C., Lynch, J., Bowering, K., Jeffs, D., Clark, J., } \\
\text { Mpundu-Kaambwa, C., \& Sawyer, M. G. (2014). An equivalence } \\
\text { evaluation of a nurse-moderated group-based internet support } \\
\text { program for new mothers versus standard care: a pragmatic } \\
\text { preference randomised controlled trial. BMC pediatrics, 14(1), } 119 .\end{array}$ & $\begin{array}{l}\text { Data not } \\
\text { available/report } \\
\text { ed }\end{array}$ \\
\hline $\begin{array}{l}\text { 1449. Sayal K, Owen V, White K, Merrell C, Tymms P and Taylor E. } \\
\text { 2010. Impact of early school-based screening and intervention } \\
\text { programs for ADHD on children's outcomes and access to services: } \\
\text { follow-up of a school-based trial at age } 10 \text { years. Archives of pediatrics } \\
\text { \& adolescent medicine, } 164(5), 462-469 \text {. }\end{array}$ & $\begin{array}{l}\text { Not a parenting } \\
\text { intervention }\end{array}$ \\
\hline
\end{tabular}




\begin{tabular}{|c|c|}
\hline $\begin{array}{l}\text { 1450. Scahill, L., McDougle, C. J., Aman, M. G., Johnson, C., Handen, } \\
\text { B., Bearss, K., Dziura, J., Butter, E., Swiezy, N. G., Arnold, L. E., Stigler, } \\
\text { K. A., Sukhodolsky, D. D., Lecavalier, L., Pozdol, S. L., Nikolov, R., } \\
\text { Hollway, J. A., Korzekwa, P., Gavaletz, A., Kohn, A. E., Koenig, K., } \\
\text { Grinnon, S., Mulick, J. A., Yu, S., \& Vitiello, B. (2012). Effects of } \\
\text { risperidone and parent training on adaptive functioning in children } \\
\text { with pervasive developmental disorders and serious behavioral } \\
\text { problems. Journal of the American Academy of Child \& Adolescent } \\
\text { Psychiatry, 51, 136-146. }\end{array}$ & $\begin{array}{l}\text { Intervention for } \\
\text { developmental } \\
\text { disorders } \\
\text { (autism, autism } \\
\text { spectrum } \\
\text { disorder), } \\
\text { physical } \\
\text { disabilities, } \\
\text { medical } \\
\text { conditions or } \\
\text { distress about } \\
\text { facing medical } \\
\text { interventions }\end{array}$ \\
\hline $\begin{array}{l}\text { 1451. Scahill, L., Sukhodolsky, D. G., Bearss, K., Findley, D., Hamrin, } \\
\text { V., Carroll, D. H., et al. (2006). Randomized Trial of Parent } \\
\text { Management Training in Children With Tic Disorders and Disruptive } \\
\text { Behavior. Journal of Child Neurology. Special Issue: Tourette } \\
\text { Syndrome, 21(8), 650-656. doi:10.1177/08830738060210080201 }\end{array}$ & $\begin{array}{l}\text { No long term } \\
\text { follow-up }\end{array}$ \\
\hline $\begin{array}{l}\text { 1452. Scarpa, A., \& Reyes, N. M. (2011). Improving emotion } \\
\text { regulation with CBT in young children with high functioning autism } \\
\text { spectrum disorders: a pilot study. Behav Cogn Psychother, 39, 495- } \\
500 .\end{array}$ & $\begin{array}{l}\text { Intervention for } \\
\text { developmental } \\
\text { disorders } \\
\text { (autism, autism } \\
\text { spectrum } \\
\text { disorder), } \\
\text { physical } \\
\text { disabilities, } \\
\text { medical } \\
\text { conditions or } \\
\text { distress about } \\
\text { facing medical } \\
\text { interventions }\end{array}$ \\
\hline $\begin{array}{l}\text { 1453. Schappin, R., Wijnroks, L., Uniken Venema, M., Wijnberg- } \\
\text { Williams, B., Veenstra, R., Koopman-Esseboom, C., .. Jongmans, M. } \\
\text { (2013). Brief parenting intervention for parents of NICU graduates: a } \\
\text { randomized, clinical trial of Primary Care Triple P. BMC pediatrics, 13, } \\
\text { 69. doi:10.1186/1471-2431-13-69 }\end{array}$ & $\begin{array}{l}\text { Follow-up less } \\
\text { than } 6 \text { months }\end{array}$ \\
\hline $\begin{array}{l}\text { 1454. Scharff L, Marcus DA and Masek BJ. 2002. A controlled study } \\
\text { of minimal-contact thermal biofeedback treatment in children with } \\
\text { migraine. J Pediatr Psychol. 27(2):109-19. }\end{array}$ & $\begin{array}{l}\text { Not a parenting } \\
\text { intervention }\end{array}$ \\
\hline
\end{tabular}




\begin{tabular}{|c|c|}
\hline $\begin{array}{l}\text { 1455. Schiff WB, Holtz KD, Peterson N and Rakusan T. 2001. Effect of } \\
\text { an intervention to reduce procedural pain and distress for children } \\
\text { with HIV infection. Journal of Pediatric Psychology. 26(7):417-27. }\end{array}$ & $\begin{array}{l}\text { Intervention for } \\
\text { developmental } \\
\text { disorders } \\
\text { (autism, autism } \\
\text { spectrum } \\
\text { disorder), } \\
\text { physical } \\
\text { disabilities, } \\
\text { medical } \\
\text { conditions or } \\
\text { distress about } \\
\text { facing medical } \\
\text { interventions }\end{array}$ \\
\hline $\begin{array}{l}\text { 1456. Schiff, S. K., \& Kellan, S. G. (1969). Parent participation in a } \\
\text { community mental health program for first graders. American Journal } \\
\text { of Orthopsychiatry, } 39,282-283 \text {. }\end{array}$ & $\begin{array}{l}\text { Data not } \\
\text { available/report } \\
\text { ed }\end{array}$ \\
\hline $\begin{array}{l}\text { 1457. Schinke SP, Cole KCA and Fang L. 2009. Gender-specific } \\
\text { intervention to reduce underage drinking among early adolescent } \\
\text { girls: A test of a computer-mediated, mother-daughter program. } \\
\text { Journal of Studies on Alcohol and Drugs. } 70(1): 70-7 .\end{array}$ & $\begin{array}{l}\text { Follow-up less } \\
\text { than } 6 \text { months }\end{array}$ \\
\hline $\begin{array}{l}\text { 1458. Schlessel JS, Rappa HA, Lesser M, Pogge D, Ennis R and Mandel } \\
\text { L. 1995. CPR knowledge, self-efficacy, and anticipated anxiety as } \\
\text { functions of infant/child CPR training. Ann Emerg Med. 25(5):618-23. }\end{array}$ & $\begin{array}{l}\text { No child } \\
\text { internalising } \\
\text { outcomes or not } \\
\text { a major goal }\end{array}$ \\
\hline $\begin{array}{l}\text { 1459. Schmaling KB and Hernandez DV. 2008. Problem-solving } \\
\text { treatment for depression among Mexican Americans in primary care. } \\
\text { Journal of Health Care for the Poor and Underserved. 19(2):466-77. }\end{array}$ & $\begin{array}{l}\text { Not a parenting } \\
\text { intervention }\end{array}$ \\
\hline $\begin{array}{l}\text { 1460. Schmidt F and Taylor TK. 2002. Putting empirically supported } \\
\text { treatment into practice: Lessons learned in a children's mental health } \\
\text { center. Professional Psychology: Research and Practice. 33(5):483-9. }\end{array}$ & $\begin{array}{l}\text { No child } \\
\text { internalising } \\
\text { outcomes or not } \\
\text { a major goal }\end{array}$ \\
\hline $\begin{array}{l}\text { 1461. Schmiege, S. J., Khoo, S. T., Sandler, I. N., Ayers, T. S., \& } \\
\text { Wolchik, S. A. (2006). Symptoms of internalizing and externalizing } \\
\text { problems: modeling recovery curves after the death of a parent. Am J } \\
\text { Prev Med, 31(6 Suppl 1), S152-160. doi:S0749-3797(06)00245-5 }\end{array}$ & $\begin{array}{l}\text { Parent } \\
\text { component too } \\
\text { small (re- } \\
\text { analysis using } \\
\text { latent growth } \\
\text { modelling of } \\
\text { Sandler et al } \\
2003 \text { which was } \\
\text { excluded) }\end{array}$ \\
\hline $\begin{array}{l}1462 . \quad \text { Schneider, S., Blatter-Meunier, J., Herren, C., Adornetto, C., In- } \\
\text { Albon, T., \& Lavallee, K. (2011). Disorder-specific cognitive-behavioral } \\
\text { therapy for separation anxiety disorder in young children: A } \\
\text { randomized waiting-list-controlled trial. Psychother Psychosom, 80, } \\
\text { 206-215. }\end{array}$ & $\begin{array}{l}\text { Follow-up less } \\
\text { than } 6 \text { months }\end{array}$ \\
\hline $\begin{array}{l}\text { 1463. Schneider, S., Blatter-Meunier, J., Herren, C., In-Albon, T., } \\
\text { Adornetto, C., Meyer, A., \& Lavallee, K. L. (2013). The efficacy ofa } \\
\text { family-based cognitive-behavioral treatment for separation anxiety } \\
\text { disorder in children aged 8-13: A randomized comparison with a } \\
\text { general anxiety program. Journal of Consulting and Clinical } \\
\text { Psychology, 81(5), 932-940. doi: } 10.1037 / \text { a0032678 }\end{array}$ & $\begin{array}{l}\text { Comparison } \\
\text { condition not a } \\
\text { minimal control }\end{array}$ \\
\hline
\end{tabular}




\begin{tabular}{|c|c|}
\hline $\begin{array}{l}\text { 1464. Schoenfelder, E. (2012). Behavioral and subjective participant } \\
\text { responsiveness to a manualized preventive intervention (Order No. } \\
\text { 3454652). Available from ProQuest Dissertations \& Theses Global. } \\
\text { (871064636). }\end{array}$ & $\begin{array}{l}\text { Secondary } \\
\text { analysis of only } \\
\text { the active arm of } \\
\text { a study } \\
\text { previously } \\
\text { excluded } \\
\text { because Parent } \\
\text { component too } \\
\text { small }\end{array}$ \\
\hline $\begin{array}{l}\text { 1465. Schoenfelder, E. N., Tein, J. Y., Wolchik, S., \& Sandler, I. N. } \\
\text { (2015). Effects of the Family Bereavement Program on Academic } \\
\text { Outcomes, Educational Expectations and Job Aspirations } 6 \text { Years Later: } \\
\text { The Mediating Role of Parenting and Youth Mental Health Problems. } \\
\text { Journal of abnormal child psychology, 43(2), 229-241. }\end{array}$ & $\begin{array}{l}\text { No child } \\
\text { internalising } \\
\text { outcomes or not } \\
\text { a major goal }\end{array}$ \\
\hline $\begin{array}{l}\text { 1466. Scholten, L., Willemen, A. M., Last, B. F., Maurice-Stam, H., van } \\
\text { Dijk, E. M., Ensink, E., ... \& Grootenhuis, M. A. (2013). Efficacy of } \\
\text { psychosocial group intervention for children with chronic illness and } \\
\text { their parents. Pediatrics, 131(4), e1196-e1203. }\end{array}$ & $\begin{array}{l}\text { Parent } \\
\text { component too } \\
\text { small }\end{array}$ \\
\hline $\begin{array}{l}1467 . \quad \text { Scholten, L., Willemen, A. M., Napoleone, E., Maurice-Stam, } \\
\text { H., Last, B. F., van Dijk-Lokkart, E. M., ... \& Schuengel, C. (2015). } \\
\text { Moderators of the efficacy of a psychosocial group intervention for } \\
\text { children with chronic illness and their parents: what works for whom?. } \\
\text { Journal of pediatric psychology, } 40(2), 214-227 .\end{array}$ & $\begin{array}{l}\text { Parent } \\
\text { component too } \\
\text { small }\end{array}$ \\
\hline $\begin{array}{l}\text { 1468. Schreier H, Ladakakos C, Morabito D, Chapman L and Knudson } \\
\text { MM. 2005. Posttraumatic stress symptoms in children after mild to } \\
\text { moderate pediatric trauma: a longitudinal examination of symptom } \\
\text { prevalence, correlates, and parent-child symptom reporting. J Trauma. } \\
\text { 58(2):353-63. }\end{array}$ & $\begin{array}{l}\text { Not a parenting } \\
\text { intervention }\end{array}$ \\
\hline $\begin{array}{l}\text { 1469. Schuhmann, E. M., Foote, R. C., Eyberg, S. M., Boggs, S. R., \& } \\
\text { Algina, J. (1998). Efficacy of parent- child interaction therapy: Interim } \\
\text { report of a randomized trial with short-term maintenance. Journal of } \\
\text { Clinical Child Psychology, } 27,34-45 .\end{array}$ & $\begin{array}{l}\text { Follow-up less } \\
\text { than } 6 \text { months }\end{array}$ \\
\hline $\begin{array}{l}\text { 1470. Schultz, C. L., Schultz, N. C., Bruce, E. J., Smyrnios, K. X., \& et al. } \\
\text { (1993). Psychoeducational support for parents of children with } \\
\text { intellectual disability: An outcome study. International Journal of } \\
\text { Disability, Development and Education, 40(3), 205-216. doi: } \\
\text { 10.1080/0156655930400307 }\end{array}$ & $\begin{array}{l}\text { No child } \\
\text { internalising } \\
\text { outcomes or not } \\
\text { a major goal }\end{array}$ \\
\hline $\begin{array}{l}\text { 1471. Schumann BR. 2005. Effects of child-centered play therapy } \\
\text { and curriculum-based small-group guidance on the behaviors of } \\
\text { children referred for aggression in an elementary school setting } \\
\text { (Texas). Dissertation Abstracts International Section A: Humanities and } \\
\text { Social Sciences. } 65(12-A) \text {. }\end{array}$ & $\begin{array}{l}\text { Not a parenting } \\
\text { intervention }\end{array}$ \\
\hline $\begin{array}{l}\text { 1472. Schwartz, S. E., Rhodes, J. E., Spencer, R., \& Grossman, J. B. } \\
\text { (2013). Youth initiated mentoring: investigating a new approach to } \\
\text { working with vulnerable adolescents. American journal of community } \\
\text { psychology, 52(1-2), 155-169. }\end{array}$ & $\begin{array}{l}\text { Not a parenting } \\
\text { intervention }\end{array}$ \\
\hline $\begin{array}{l}\text { 1473. Schweinhart, L. J. and D. P. Weikart (1997). The high/scope } \\
\text { preschool curriculum comparison study through age } 23 \text {. Early } \\
\text { Childhood Research Quarterly 12(2): } 117-143 \text {. }\end{array}$ & $\begin{array}{l}\text { Not a parenting } \\
\text { intervention }\end{array}$ \\
\hline
\end{tabular}




\begin{tabular}{|c|c|}
\hline $\begin{array}{l}\text { 1474. Sciberras, E., Fulton, M., Efron, D., Oberklaid, F., \& Hiscock, H. } \\
\text { (2011). Managing sleep problems in school aged children with ADHD: } \\
\text { A pilot randomised controlled trial. Sleep Med, } 12,932-935 \text {. }\end{array}$ & $\begin{array}{l}\text { Follow-up less } \\
\text { than } 6 \text { months }\end{array}$ \\
\hline $\begin{array}{l}\text { 1475. Scott S (2005) Do parenting programmes for severe child } \\
\text { antisocial behaviour work over the longer term, and for whom? One } \\
\text { year follow-up of a multi-centre controlled trial. Behavioural and } \\
\text { Cognitive Psychotherapy 33:1-19 }\end{array}$ & $\begin{array}{l}\text { Follow-up data } \\
\text { collected only in } \\
\text { active group }\end{array}$ \\
\hline $\begin{array}{l}\text { 1476. Scott S. 2008. An update on interventions for conduct } \\
\text { disorder. Advances in Psychiatric Treatment. 14(1):61-70. }\end{array}$ & Review \\
\hline $\begin{array}{l}\text { 1477. Scott, K. K., Tepas, J. J., Frykberg, E., Taylor, P. M., \& Plotkin, A. } \\
\text { J. (2002). Turning point: rethinking violence--evaluation of program } \\
\text { efficacy in reducing adolescent violent crime recidivism. The Journal of } \\
\text { trauma, (1), 21-27. }\end{array}$ & $\begin{array}{l}\text { No child } \\
\text { internalising } \\
\text { outcomes or not } \\
\text { a major goal }\end{array}$ \\
\hline $\begin{array}{l}\text { 1478. Scott, M.J., Stradling, S.G., 1987. Evaluation of a Group } \\
\text { Programme for Parents of Problem Children. Behavioural } \\
\text { Psychotherapy 15, 224-239. }\end{array}$ & $\begin{array}{l}\text { Not an RCT; No } \\
\text { child- } \\
\text { internalising } \\
\text { outcomes } \\
\text { measured at } \\
\text { follow-up. }\end{array}$ \\
\hline $\begin{array}{l}\text { 1479. Scott, S., \& O'Connor, T. G. (2012). An experimental test of } \\
\text { differential susceptibility to parenting among emotionally- } \\
\text { dysregulated children in a randomized controlled trial for oppositional } \\
\text { behavior. Journal of child psychology and psychiatry, and allied } \\
\text { disciplines, 53(11), 1184-1193. doi:10.1111/j.1469-7610.2012.02586.x }\end{array}$ & $\begin{array}{l}\text { Targets } \\
\text { externalising }\end{array}$ \\
\hline $\begin{array}{l}\text { 1480. Scott, S., Sylva, K., Doolan, M., Price, J., Jacobs, B., Crook, C., et } \\
\text { al. (2010). Randomised controlled trial of parent groups for child } \\
\text { antisocial behaviour targeting multiple risk factors: the SPOKES } \\
\text { project. Journal of child psychology and psychiatry, and allied } \\
\text { disciplines, (1), 48-57. }\end{array}$ & $\begin{array}{l}\text { Follow-up less } \\
\text { than } 6 \text { months }\end{array}$ \\
\hline $\begin{array}{l}\text { 1481. Scott, S., Webster-Stratton, C., Spender, Q., Doolan, M., } \\
\text { Jacobs, B., \& Aspland, H. (2001). Multicentre controlled trial of } \\
\text { parenting groups for childhood antisocial behaviour in clinical } \\
\text { practiceCommentary: nipping conduct problems in the bud. Bmj, } \\
\text { 323(7306), 194-198. }\end{array}$ & $\begin{array}{l}\text { No long term } \\
\text { follow-up }\end{array}$ \\
\hline $\begin{array}{l}\text { 1482. Sege RD, Perry C, Stigol L, Cohen L, Griffith J, Cohn M and } \\
\text { Spivak H. 1997. Short-term effectiveness of anticipatory guidance to } \\
\text { reduce early childhood risks for subsequent violence. Archives of } \\
\text { pediatrics \& adolescent medicine, 151(4), 392-397. }\end{array}$ & $\begin{array}{l}\text { No child } \\
\text { internalising } \\
\text { outcomes or not } \\
\text { a major goal }\end{array}$ \\
\hline $\begin{array}{l}\text { 1483. Semeniuk, Y., Brown, R. L., Riesch, S. K., Zywicki, M., Hopper, } \\
\text { J., \& Henriques, J. B. (2010). The Strengthening Families Program 10- } \\
\text { 14: influence on parent and youth problem-solving skill. Journal of } \\
\text { psychiatric and mental health nursing, (5), 392-402. }\end{array}$ & $\begin{array}{l}\text { No child } \\
\text { internalising } \\
\text { outcomes or not } \\
\text { a major goal }\end{array}$ \\
\hline $\begin{array}{l}\text { 1484. Serketich, W. J., \& Dumas, J. E. (1996). The effectiveness of } \\
\text { behavioral parent training to modify antisocial behavior in children: A } \\
\text { meta-analysis. Behavior Therapy, 27(2), 171-186. doi: 10.1016/S0005- } \\
\text { 7894(96)80013-X }\end{array}$ & $\begin{array}{l}\text { Meta-analysis of } \\
\text { studies with no } \\
\text { long term } \\
\text { follow-up }\end{array}$ \\
\hline $\begin{array}{l}\text { 1485. Settle, C. J. (2010). The level of parental conflict and children's } \\
\text { behavioral reactions to divorce (Order No. 3432392). Available from } \\
\text { ProQuest Dissertations \& Theses Global. (822233754). }\end{array}$ & Not an RCT \\
\hline
\end{tabular}




\begin{tabular}{|c|c|}
\hline $\begin{array}{l}\text { 1486. Sferrazza, N. (2011). Mothers' attributional biases and its } \\
\text { relation to dysfunctional parenting (Order No. 3459714). Available } \\
\text { from ProQuest Dissertations \& Theses Global. (876014325). }\end{array}$ & Not an RCT \\
\hline $\begin{array}{l}\text { 1487. Shanmugham K, Cano MA, Elliott TR and Davis M. 2009. Social } \\
\text { problem-solving abilities, relationship satisfaction and depression } \\
\text { among family caregivers of stroke survivors. Brain Injury. 23(2):92-100. }\end{array}$ & $\begin{array}{l}\text { Not a parenting } \\
\text { intervention }\end{array}$ \\
\hline $\begin{array}{l}\text { 1488. Shanok AF and Miller L. 2007. Depression and treatment with } \\
\text { inner city pregnant and parenting teens. Archives of Women's Mental } \\
\text { Health. 10(5):199-210. }\end{array}$ & $\begin{array}{l}\text { Not a parenting } \\
\text { intervention }\end{array}$ \\
\hline $\begin{array}{l}\text { 1489. Shapiro, C. J., Kilburn, J., \& Hardin, J. W. (2014). Prevention of } \\
\text { behavior problems in a selected population: Stepping Stones Triple P } \\
\text { for parents of young children with disabilities. Research in } \\
\text { developmental disabilities, 35(11), 2958-2975. }\end{array}$ & $\begin{array}{l}\text { Intervention for } \\
\text { developmental } \\
\text { disorders } \\
\text { (autism, autism } \\
\text { spectrum } \\
\text { disorder), } \\
\text { physical } \\
\text { disabilities, } \\
\text { medical } \\
\text { conditions or } \\
\text { distress about } \\
\text { facing medical } \\
\text { interventions }\end{array}$ \\
\hline $\begin{array}{l}\text { 1490. Shaw DS, Schonberg M, Sherrill J, Huffman D, Lukon J, Obrosky } \\
\text { D and Kovacs M. 2006. Responsivity to Offspring's Expression of } \\
\text { Emotion Among Childhood-Onset Depressed Mothers. Journal of } \\
\text { Clinical Child and Adolescent Psychology. 35(4):490-503. }\end{array}$ & $\begin{array}{l}\text { Not a parenting } \\
\text { intervention }\end{array}$ \\
\hline $\begin{array}{l}\text { 1491. Shaw RJ, Palmer L, Hyte H, Yorgin P and Sarwal M. 2001. Case } \\
\text { study: Treatment adherence in a 13-year-old deaf adolescent male. } \\
\text { Clinical Child Psychology and Psychiatry. 6(4):551-62. }\end{array}$ & Not an RCT \\
\hline $\begin{array}{l}\text { 1492. Shaw, D. S., Connell, A., Dishion, T. J., Wilson, M. N., \& } \\
\text { Gardner, F. (2009). Improvements in maternal depression as a } \\
\text { mediator of intervention effects on early childhood problem behavior. } \\
\text { Dev Psychopathol, 21(2), 417-439. doi: S0954579409000236 }\end{array}$ & $\begin{array}{l}\text { Targets } \\
\text { externalising }\end{array}$ \\
\hline $\begin{array}{l}\text { 1493. Shaw, D. S., Dishion, T. J., Supplee, L., Gardner, F., \& Arnds, K. } \\
\text { (2006). Randomized trial of a family-centered approach to the } \\
\text { prevention of early conduct problems: } 2 \text {-year effects of the family } \\
\text { check-up in early childhood. Journal of Consulting and Clinical } \\
\text { Psychology, } 74(1), 1-9 . \text { doi: } 10.1037 / 0022-006 x .74 .1 .1\end{array}$ & $\begin{array}{l}\text { Targets } \\
\text { externalising }\end{array}$ \\
\hline $\begin{array}{l}\text { 1494. Shaw, R. J., John, N., Lilo, E. A., Jo, B., Benitz, W., Stevenson, D. } \\
\text { K., \& Horwitz, S. M. (2013). Prevention of traumatic stress in mothers } \\
\text { with preterm infants: a randomized controlled trial. Pediatrics, 132(4), } \\
\text { e886-894. doi:10.1542/peds.2013-1331 }\end{array}$ & $\begin{array}{l}\text { No child } \\
\text { internalising } \\
\text { outcomes or not } \\
\text { a major goal }\end{array}$ \\
\hline $\begin{array}{l}\text { 1495. Shaw, R. J., St John, N., Lilo, E., Jo, B., Benitz, W., Stevenson, D. } \\
\text { K., \& Horwitz, S. M. (2014). Prevention of traumatic stress in mothers } \\
\text { of preterms: 6-month outcomes. Pediatrics, 134(2), e481-e488. }\end{array}$ & $\begin{array}{l}\text { No child } \\
\text { internalising } \\
\text { outcomes or not } \\
\text { a major goal }\end{array}$ \\
\hline $\begin{array}{l}\text { 1496. Shechner, T., Rimon-Chakir, A., Britton, J. C., Lotan, D., Apter, } \\
\text { A., Bliese, P. D., . . Bar-Haim, Y. (2014). Attention bias modification } \\
\text { treatment augmenting effects on cognitive behavioral therapy in } \\
\text { children with anxiety: randomized controlled trial. J Am Acad Child } \\
\text { Adolesc Psychiatry, 53(1), 61-71. doi: } 10.1016 / \text { j.jaac.2013.09.016 }\end{array}$ & $\begin{array}{l}\text { Not a parenting } \\
\text { intervention }\end{array}$ \\
\hline
\end{tabular}




\begin{tabular}{|c|c|}
\hline $\begin{array}{l}\text { 1497. Sheeber LB, Johnston C, Chen M, Leve C, Hops H and Davis B. } \\
\text { 2009. Mothers' and fathers' attributions for adolescent behavior: An } \\
\text { examination in families of depressed, subdiagnostic, and } \\
\text { nondepressed youth. Journal of Family Psychology. } 23(6): 871-81 .\end{array}$ & $\begin{array}{l}\text { Not a parenting } \\
\text { intervention }\end{array}$ \\
\hline $\begin{array}{l}\text { 1498. Sheeber, L. B., \& Johnson, J. H. (1994). Evaluation of a } \\
\text { Temperament-Focused, Parent-Training Program. Journal of Clinical } \\
\text { Child Psychology, 23(3), 249-259. }\end{array}$ & $\begin{array}{l}\text { Follow-up less } \\
\text { than } 6 \text { months }\end{array}$ \\
\hline $\begin{array}{l}\text { 1499. Sheffield, J. K., Spence, S. H., Rapee, R. M., Kowalenko, N., } \\
\text { Wignall, A., Davis, A., \& McLoone, J. (2006). Evaluation of universal, } \\
\text { indicated, and combined cognitive-behavioral approaches to the } \\
\text { prevention of depression among adolescents. Journal of Consulting } \\
\text { and Clinical Psychology, 74, 66-79. }\end{array}$ & $\begin{array}{l}\text { Not a parenting } \\
\text { intervention }\end{array}$ \\
\hline $\begin{array}{l}\text { 1500. Shelton, T. L., Barkley, R. A., Crosswait, C., Moorehouse, M., } \\
\text { Fletcher, K., Barrett, S., et al. (2000). Multimethod psychoeducational } \\
\text { intervention for preschool children with disruptive behavior: Two-year } \\
\text { post-treatment follow-up. Journal of Abnormal Child Psychology, (3), } \\
\text { 253-266. }\end{array}$ & $\begin{array}{l}\text { Targets } \\
\text { externalising }\end{array}$ \\
\hline $\begin{array}{l}\text { 1501. Sheridan, S. M., Knoche, L. L., Edwards, C. P., Kupzyk, K. A., } \\
\text { Clarke, B. L., \& Kim, E. M. (2014). Efficacy of the Getting Ready } \\
\text { Intervention and the Role of Parental Depression. Early education and } \\
\text { development, 25(5), 746-769. }\end{array}$ & $\begin{array}{l}\text { No child } \\
\text { internalising } \\
\text { outcomes or not } \\
\text { a major goal }\end{array}$ \\
\hline $\begin{array}{l}\text { 1502. Sherman, B. J., Duarte, C. S., \& Verdeli, H. (2011). Internalizing } \\
\text { and externalizing problems in adolescents from Bahia, Brazil: } \\
\text { Sociodemographic correlates and family environment in boys and } \\
\text { girls. International Journal of Mental Health, } 40,55-76 .\end{array}$ & $\begin{array}{l}\text { Not a parenting } \\
\text { intervention }\end{array}$ \\
\hline $\begin{array}{l}\text { 1503. Sherman, M., \& Bowling, U. (2011). Challenges and } \\
\text { opportunities for intervening with couples in the aftermath of the } \\
\text { global war on terrorism. Journal of Contemporary Psychotherapy, 41, } \\
\text { 209-217. }\end{array}$ & Review \\
\hline $\begin{array}{l}\text { 1504. Shernoff ES and Kratochwill TR. 2007. Transporting an } \\
\text { evidence-based classroom management program for preschoolers } \\
\text { with disruptive behavior problems to a school: An analysis of } \\
\text { implementation, outcomes, and contextual variables. School } \\
\text { Psychology Quarterly. 22(3):449-72. }\end{array}$ & $\begin{array}{l}\text { Not a parenting } \\
\text { intervention }\end{array}$ \\
\hline $\begin{array}{l}\text { 1505. Shochet, I. M., Dadds, M. R., Holland, D., Whitefield, K., } \\
\text { Harnett, P. H., \& Osgarby, S. M. (2001). The efficacy of a universal } \\
\text { school-based program to prevent adolescent depression. Journal of } \\
\text { Clinical Child Psychology, 30(3), 303-315. doi: } \\
\text { 10.1207/s15374424jccp3003_3 }\end{array}$ & Not an RCT \\
\hline $\begin{array}{l}\text { 1506. Short, J. L. (1998). Evaluation of a Substance Abuse Prevention } \\
\text { and Mental Health Promotion Program for Children of Divorce. Journal } \\
\text { of Divorce \& Remarriage, 28, 139-155. }\end{array}$ & $\begin{array}{l}\text { Not a parenting } \\
\text { intervention }\end{array}$ \\
\hline $\begin{array}{l}\text { 1507. Shortt, A. L., Barrett, P. M., \& Fox, T. L. (2001). Evaluating the } \\
\text { FRIENDS program: a cognitive-behavioral group treatment for anxious } \\
\text { children and their parents. J Clin Child Psychol, 30(4), 525-535. }\end{array}$ & $\begin{array}{l}\text { Parent } \\
\text { component too } \\
\text { small }\end{array}$ \\
\hline $\begin{array}{l}\text { 1508. Shuman AL and Shapiro JP. 2002. The effects of preparing } \\
\text { parents for child psychotherapy on accuracy of expectations and } \\
\text { treatment attendance. Community Mental Health Journal, 38(1), 3-16. }\end{array}$ & $\begin{array}{l}\text { No child } \\
\text { internalising } \\
\text { outcomes or not } \\
\text { a major goal }\end{array}$ \\
\hline
\end{tabular}




\begin{tabular}{|c|c|}
\hline $\begin{array}{l}\text { 1509. Siegenthaler, E., Munder, T., \& Egger, M. (2012). Effect of } \\
\text { preventive interventions in mentally ill parents on the mental health } \\
\text { of the offspring: Systematic review and meta-analysis. J Am Acad Child } \\
\text { Adolesc Psychiatry, 51, 8-17.e18. }\end{array}$ & Review \\
\hline $\begin{array}{l}\text { 1510. Sigal, A. B., Wolchik, S. A., Tein, J.-Y., \& Sandler, I. N. (2012). } \\
\text { Enhancing youth outcomes following parental divorce: A longitudinal } \\
\text { study of the effects of the new beginnings program on educational } \\
\text { and occupational goals. Journal of Clinical Child and Adolescent } \\
\text { Psychology, 41, 150-165. }\end{array}$ & $\begin{array}{l}\text { Secondary } \\
\text { analysis (re- } \\
\text { analysis of data } \\
\text { in Wolcik et al } \\
\text { 2002) }\end{array}$ \\
\hline $\begin{array}{l}\text { 1511. Sil, S., Arnold, L. M., Lynch-Jordan, A., Ting, T. V., Peugh, J., } \\
\text { Cunningham, N., ... Kashikar-Zuck, S. (2014). Identifying treatment } \\
\text { responders and predictors of improvement after cognitive-behavioral } \\
\text { therapy for juvenile fibromyalgia. Pain, 155(7), 1206-1212. doi: } \\
\text { 10.1016/j.pain.2014.03.005 }\end{array}$ & $\begin{array}{l}\text { Not a parenting } \\
\text { intervention }\end{array}$ \\
\hline $\begin{array}{l}\text { 1512. Silk JS, Vanderbilt-Adriance E, Shaw DS, Forbes EE, Whalen DJ, } \\
\text { Ryan ND and Dahl RE. 2007. Resilience among children and } \\
\text { adolescents at risk for depression: Mediation and moderation across } \\
\text { social and neurobiological context. Development and } \\
\text { Psychopathology. 19(3):841-65. }\end{array}$ & $\begin{array}{l}\text { Not a parenting } \\
\text { intervention }\end{array}$ \\
\hline $\begin{array}{l}\text { 1513. Silovsky JF, Niec L, Bard D and Hecht DB. 2007. Treatment for } \\
\text { preschool children with interpersonal sexual behavior problems: A } \\
\text { pilot study. Journal of Clinical Child and Adolescent Psychology. } \\
\text { 36(3):378-91. }\end{array}$ & $\begin{array}{l}\text { Not a parenting } \\
\text { intervention }\end{array}$ \\
\hline $\begin{array}{l}\text { 1514. Silver RB, Measelle JR, Armstrong JM and Essex MJ. } 2005 . \\
\text { Trajectories of classroom externalizing behavior: Contributions of child } \\
\text { characteristics, family characteristics, and the teacher-child } \\
\text { relationship during the school transition. Journal of School Psychology. } \\
\text { 43(1):39-60. }\end{array}$ & $\begin{array}{l}\text { Not a parenting } \\
\text { intervention }\end{array}$ \\
\hline $\begin{array}{l}1515 . \quad \text { Silverman WK, Saavedra LM and Pina AA. 2001. Test-retest } \\
\text { reliability of anxiety symptoms and diagnoses with anxiety disorders } \\
\text { interview schedule for }<\mathrm{i}>\mathrm{DSM}-\mathrm{IV}</ \mathrm{i}>\text { : Child and parent versions. } \\
\text { Journal of the American Academy of Child \& Adolescent Psychiatry. } \\
\text { 40(8):937-44. }\end{array}$ & $\begin{array}{l}\text { Not a parenting } \\
\text { intervention }\end{array}$ \\
\hline $\begin{array}{l}\text { 1516. Silverman, W. K., Kurtines, W. M., Ginsburg, G. S., Weems, C. } \\
\text { F., Rabian, B., \& Serafini, L. T. (1999). Contingency management, self- } \\
\text { control, and education support in the treatment of childhood phobic } \\
\text { disorders: a randomized clinical trial. J Consult Clin Psychol, 67(5), 675- } \\
687 .\end{array}$ & $\begin{array}{l}\text { Parent } \\
\text { component too } \\
\text { small }\end{array}$ \\
\hline $\begin{array}{l}\text { 1517. Silverman, W. K., Kurtines, W. M., Jaccard, J., \& Pina, A. A. } \\
\text { (2009). Directionality of change in youth anxiety treatment involving } \\
\text { parents: an initial examination. J Consult Clin Psychol, 77(3), 474-485. } \\
\text { doi: } 2009-08093-010\end{array}$ & $\begin{array}{l}\text { Parent } \\
\text { component too } \\
\text { small }\end{array}$ \\
\hline $\begin{array}{l}\text { 1518. Silverstein, M., Feinberg, E., Cabral, H., Sauder, S., Egbert, L., } \\
\text { Schainker, E., Kamholz, K., Hegel, M., \& Beardslee, W. (2011). } \\
\text { Problem-solving education to prevent depression among low-income } \\
\text { mothers of preterm infants: A randomized controlled pilot trial. Arch } \\
\text { Womens Ment Health, 14, 317-324. }\end{array}$ & $\begin{array}{l}\text { No child } \\
\text { internalising } \\
\text { outcomes or not } \\
\text { a major goal }\end{array}$ \\
\hline
\end{tabular}




\begin{tabular}{|c|c|}
\hline $\begin{array}{l}\text { 1519. Silverstein, M., Hironaka, L. K., Walter, H. J., Feinberg, E., } \\
\text { Sandler, J., Pellicer, M., ... \& Cabral, H. (2015). Collaborative care for } \\
\text { children with ADHD symptoms: a randomized comparative } \\
\text { effectiveness trial. Pediatrics, } 135(4) \text {, e858-e867. }\end{array}$ & $\begin{array}{l}\text { Intervention for } \\
\text { developmental } \\
\text { disorders } \\
\text { (autism, autism } \\
\text { spectrum } \\
\text { disorder), } \\
\text { physical } \\
\text { disabilities, } \\
\text { medical } \\
\text { conditions or } \\
\text { distress about } \\
\text { facing medical } \\
\text { interventions }\end{array}$ \\
\hline $\begin{array}{l}\text { 1520. Simon, E., Dirksen, C., Bogels, S., \& Bodden, D. (2012). Cost- } \\
\text { effectiveness of child-focused and parent-focused interventions in a } \\
\text { child anxiety prevention program. J Anxiety Disord, 26, 287-296. }\end{array}$ & $\begin{array}{l}\text { Secondary } \\
\text { analysis (cost- } \\
\text { effectiveness) } \\
\text { outcomes from } \\
\text { Simon } 2011\end{array}$ \\
\hline $\begin{array}{l}\text { 1521. Simons J, Reynolds J and Morison L. 2001. Randomised } \\
\text { controlled trial of training health visitors to identify and help couples } \\
\text { with relationship problems following a birth. Br J Gen Pract. } \\
\text { 51(471):793-9. }\end{array}$ & $\begin{array}{l}\text { No child } \\
\text { internalising } \\
\text { outcomes or not } \\
\text { a major goal }\end{array}$ \\
\hline $\begin{array}{l}\text { 1522. Singer AJ, Mynster CJ and McMahon BJ. 2003. The effect of IM } \\
\text { ketorolac tromethamine on bleeding time: a prospective, } \\
\text { interventional, controlled study. Am J Emerg Med. 21(5):441-3. }\end{array}$ & $\begin{array}{l}\text { Not a parenting } \\
\text { intervention }\end{array}$ \\
\hline $\begin{array}{l}\text { 1523. Singer GHS and et al. 1988. Stress Management Training for } \\
\text { Parents of Children with Severe Handicaps. Mental retardation, 26(5), } \\
\text { 269-277. }\end{array}$ & $\begin{array}{l}\text { Intervention for } \\
\text { developmental } \\
\text { disorders } \\
\text { (autism, autism } \\
\text { spectrum } \\
\text { disorder), } \\
\text { physical } \\
\text { disabilities, } \\
\text { medical } \\
\text { conditions or } \\
\text { distress about } \\
\text { facing medical } \\
\text { interventions }\end{array}$ \\
\hline $\begin{array}{l}\text { 1524. Singer GHS, Ethridge BL and Aldana SI. 2007. Primary and } \\
\text { secondary effects of parenting and stress management interventions } \\
\text { for parents of children with developmental disabilities: A meta- } \\
\text { analysis. Mental Retardation and Developmental Disabilities Research } \\
\text { Reviews. 13(4):357-69. }\end{array}$ & Review \\
\hline
\end{tabular}




\begin{tabular}{|c|c|}
\hline $\begin{array}{l}\text { 1525. Singer GHS, Glang A, Nixon C, Cooley E, Keras KA, Williams D } \\
\text { and Powers LE. 1994. A comparison of two psychosocial interventions } \\
\text { for parents of children with acquired brain injury: An exploratory } \\
\text { study. The Journal of Head Trauma Rehabilitation, } 9(4), 38-49 .\end{array}$ & $\begin{array}{l}\text { Intervention for } \\
\text { developmental } \\
\text { disorders } \\
\text { (autism, autism } \\
\text { spectrum } \\
\text { disorder), } \\
\text { physical } \\
\text { disabilities, } \\
\text { medical } \\
\text { conditions or } \\
\text { distress about } \\
\text { facing medical } \\
\text { interventions }\end{array}$ \\
\hline $\begin{array}{l}\text { 1526. Singer, GHS. 1985. Stress Management Training for Parents of } \\
\text { Severely Handicapped Children. Eugene, Or.: Oregon Research } \\
\text { Institute. }\end{array}$ & $\begin{array}{l}\text { Intervention for } \\
\text { developmental } \\
\text { disorders } \\
\text { (autism, autism } \\
\text { spectrum } \\
\text { disorder), } \\
\text { physical } \\
\text { disabilities, } \\
\text { medical } \\
\text { conditions or } \\
\text { distress about } \\
\text { facing medical } \\
\text { interventions }\end{array}$ \\
\hline $\begin{array}{l}1527 . \quad \text { Sirbu, W., Cotler, S., \& Jason Leonard, A. (1978). Primary } \\
\text { prevention: Teaching parents behavioral child rearing skills. Family- } \\
\text { Therapy, (2), 163-170. }\end{array}$ & $\begin{array}{l}\text { No long term } \\
\text { follow-up; No } \\
\text { child } \\
\text { internalising } \\
\text { outcomes or not } \\
\text { a major goal }\end{array}$ \\
\hline $\begin{array}{l}\text { 1528. Siu AFY. 2009. Theraplay in the Chinese world: An intervention } \\
\text { program for Hong Kong children with internalizing problems. } \\
\text { International Journal of Play Therapy. 18(1):1-12. }\end{array}$ & $\begin{array}{l}\text { Not a parenting } \\
\text { intervention }\end{array}$ \\
\hline $\begin{array}{l}\text { 1529. Skinner, M. L., Haggerty, K. P., Fleming, C. B., \& Catalano, R. F. } \\
\text { (2009). Predicting functional resilience among young-adult children of } \\
\text { opiate-dependent parents. Journal of Adolescent Health, 44(3), 283- } \\
\text { 290. doi: } 10.1016 / \text { j.jadohealth.2008.07.020 }\end{array}$ & $\begin{array}{l}\text { Targets } \\
\text { externalising }\end{array}$ \\
\hline $\begin{array}{l}\text { 1530. Skinner, M. L., Haggerty, K. P., Fleming, C. B., Catalano, R. F., \& } \\
\text { Gainey, R. R. (2011). Opiate-addicted parents in methadone } \\
\text { treatment: long-term recovery, health, and family relationships. J } \\
\text { Addict Dis, } 30,17-26 .\end{array}$ & $\begin{array}{l}\text { No child } \\
\text { internalising } \\
\text { outcomes or not } \\
\text { a major goal }\end{array}$ \\
\hline $\begin{array}{l}\text { 1531. Sleed, M., Baradon, T., \& Fonagy, P. (2013). New Beginnings } \\
\text { for mothers and babies in prison: a cluster randomized controlled trial. } \\
\text { Attach Hum Dev, 15(4), 349-367. doi:10.1080/14616734.2013.782651 }\end{array}$ & $\begin{array}{l}\text { No child } \\
\text { internalising } \\
\text { outcomes or not } \\
\text { a major goal }\end{array}$ \\
\hline
\end{tabular}




\begin{tabular}{|c|c|}
\hline $\begin{array}{l}\text { 1532. Slifer KJ, DeMore M, Vona-Messersmith N, Pulbrook-Vetter V, } \\
\text { Beck M, Dalhquist L, Bellipanni K and Johnson E. 2009. Comparison of } \\
\text { two brief parent-training interventions for child distress during parent- } \\
\text { administered needle procedures. Children's Health Care, 38(1), 23-48. }\end{array}$ & $\begin{array}{l}\text { Intervention for } \\
\text { developmental } \\
\text { disorders } \\
\text { (autism, autism } \\
\text { spectrum } \\
\text { disorder), } \\
\text { physical } \\
\text { disabilities, } \\
\text { medical } \\
\text { conditions or } \\
\text { distress about } \\
\text { facing medical } \\
\text { interventions }\end{array}$ \\
\hline $\begin{array}{l}1533 . \quad \text { Smeerdijk, M., Keet, R., Haan, L., Barrowclough, C., Linszen, D., } \\
\text { \& Schippers, G. (2014). Feasibility of teaching motivational } \\
\text { interviewing to parents of young adults with recent-onset } \\
\text { schizophrenia and co-occurring cannabis use. Journal of substance } \\
\text { abuse treatment, } 46(3), 340-345 . \text { doi:10.1016/j.jsat.2013.09.006 }\end{array}$ & $\begin{array}{l}\text { Follow-up less } \\
\text { than } 6 \text { months }\end{array}$ \\
\hline $\begin{array}{l}\text { 1534. Smith AP and Kelly AB. 2008. An exploratory study of group } \\
\text { therapy for sexually abused adolescents and nonoffending guardians. } \\
\text { Journal of Child Sexual Abuse. 17(2):101-16. }\end{array}$ & Not an RCT \\
\hline $\begin{array}{l}\text { 1535. Smith CE, Curtas S, Kleinbeck SV, Werkowitch M, Mosier M, } \\
\text { Seidner DL and Steiger E. 2003. Clinical trial of interactive and } \\
\text { videotaped educational interventions reduce infection, reactive } \\
\text { depression, and rehospitalizations for sepsis in patients on home } \\
\text { parenteral nutrition. JPEN J Parenter Enteral Nutr. 27(2):137-45. }\end{array}$ & $\begin{array}{l}\text { Not a parenting } \\
\text { intervention }\end{array}$ \\
\hline $\begin{array}{l}\text { 1536. Smith, A. M., Flannery-Schroeder, E. C., Gorman, K. S., \& Cook, } \\
\text { N. (2014). Parent cognitive-behavioral intervention for the treatment } \\
\text { of childhood anxiety disorders: A pilot study. Behaviour research and } \\
\text { therapy, } 61,156-161 .\end{array}$ & $\begin{array}{l}\text { Follow-up less } \\
\text { than } 6 \text { months }\end{array}$ \\
\hline $\begin{array}{l}1537 . \quad \text { Smokowski, P. R., \& Bacallao, M. (2009). Entre Dos } \\
\text { Mundos/Between Two Worlds youth violence prevention: Comparing } \\
\text { psychodramatic and support group delivery formats. Small Group } \\
\text { Research, 40(1), 3-27. doi: } 10.1177 / 1046496408326771\end{array}$ & $\begin{array}{l}\text { Parent } \\
\text { component too } \\
\text { small }\end{array}$ \\
\hline $\begin{array}{l}\text { 1538. Snyder, F. J., Vuchinich, S., Acock, A., Washburn, I. J., \& Flay, B. } \\
\text { R. (2012). Improving elementary school quality through the use of a } \\
\text { social-emotional and character development program: a matched- } \\
\text { pair, cluster-randomized, controlled trial in Hawai'i. Journal of School } \\
\text { Health, 82(1), 11-20. }\end{array}$ & $\begin{array}{l}\text { Not a parenting } \\
\text { intervention }\end{array}$ \\
\hline
\end{tabular}




\begin{tabular}{|c|c|}
\hline $\begin{array}{l}\text { 1539. Sofronoff K, Attwood T and Hinton S. 2005. A randomised } \\
\text { controlled trial of a CBT intervention for anxiety in children with } \\
\text { Asperger syndrome. Journal of child psychology and psychiatry, } \\
46(11), 1152-1160 .\end{array}$ & $\begin{array}{l}\text { Intervention for } \\
\text { developmental } \\
\text { disorders } \\
\text { (autism, autism } \\
\text { spectrum } \\
\text { disorder), } \\
\text { physical } \\
\text { disabilities, } \\
\text { medical } \\
\text { conditions or } \\
\text { distress about } \\
\text { facing medical } \\
\text { interventions }\end{array}$ \\
\hline $\begin{array}{l}\text { 1540. Solantaus, T., Toikka, S., Alasuutari, M., Beardslee, W. R., \& } \\
\text { Paavonen, E. J. (2009). Safety, feasibility and family experiences of } \\
\text { preventive interventions for children and families with parental } \\
\text { depression. The International Journal of Mental Health Promotion, } \\
\text { 11(4), 15-24. }\end{array}$ & $\begin{array}{l}\text { No long term } \\
\text { follow-up }\end{array}$ \\
\hline $\begin{array}{l}\text { 1541. Solomon, R., Van Egeren, L. A., Mahoney, G., Huber, M. S. Q., } \\
\text { \& Zimmerman, P. (2014). PLAY Project Home Consultation } \\
\text { intervention program for young children with autism spectrum } \\
\text { disorders: a randomized controlled trial. Journal of Developmental and } \\
\text { Behavioral Pediatrics, 35(8), } 475 .\end{array}$ & $\begin{array}{l}\text { Intervention for } \\
\text { developmental } \\
\text { disorders } \\
\text { (autism, autism } \\
\text { spectrum } \\
\text { disorder), } \\
\text { physical } \\
\text { disabilities, } \\
\text { medical } \\
\text { conditions or } \\
\text { distress about } \\
\text { facing medical } \\
\text { interventions }\end{array}$ \\
\hline $\begin{array}{l}\text { 1542. Somech, L. Y., \& Elizur, Y. (2012). Promoting self-regulation } \\
\text { and cooperation in pre-kindergarten children with conduct problems: } \\
\text { A randomized controlled trial. J Am Acad Child Adolesc Psychiatry, 51, } \\
\text { 412-422. }\end{array}$ & $\begin{array}{l}\text { Targets } \\
\text { externalising }\end{array}$ \\
\hline $\begin{array}{l}\text { 1543. Song JE and Park BL. 2010. [The changing pattern of physical } \\
\text { and psychological health, and maternal adjustment between } \\
\text { primiparas who used and those who did not use Sanhujori facilities]. J } \\
\text { Korean Acad Nurs. } 40(4): 503-14 \text {. }\end{array}$ & Not an RCT \\
\hline $\begin{array}{l}\text { 1544. Sonuga-Barke EJ, Daley D and Thompson M. 2002. Does } \\
\text { maternal ADHD reduce the effectiveness of parent training for } \\
\text { preschool children's ADHD? Journal of the American Academy of Child } \\
\text { \& Adolescent Psychiatry, } 41(6), 696-702 \text {. }\end{array}$ & $\begin{array}{l}\text { Follow-up less } \\
\text { than } 6 \text { months; } \\
\text { Targets } \\
\text { externalising }\end{array}$ \\
\hline $\begin{array}{l}\text { 1545. Sonuga-Barke, E. J. S., Daley, D., Thompson, M., Laver- } \\
\text { Bradbury, C., \& Weeks, A. (2001). Parent-based therapies for } \\
\text { preschool attention-deficit/hyperactivity disorder: A randomized, } \\
\text { controlled trial with a community sample. Journal of the American } \\
\text { Academy of Child and Adolescent Psychiatry, } 40(4), 402-408 .\end{array}$ & $\begin{array}{l}\text { Follow-up less } \\
\text { than } 6 \text { months }\end{array}$ \\
\hline $\begin{array}{l}\text { 1546. Sourander, A. (2011). Time?trend changes and psychological } \\
\text { risk factors for soiling: Findings from the Finnish 16?year time?trend } \\
\text { study. Acta Paediatrica, 100, 1276-1280. }\end{array}$ & $\begin{array}{l}\text { Not a parenting } \\
\text { intervention }\end{array}$ \\
\hline
\end{tabular}




\begin{tabular}{|c|c|}
\hline $\begin{array}{l}\text { 1547. Southam-Gerow MA, Chorpita BF, Miller LM and Gleacher AA. } \\
\text { 2008. Are children with anxiety disorders privately referred to a } \\
\text { university clinic like those referred from the public mental health } \\
\text { system? Administration and Policy in Mental Health and Mental } \\
\text { Health Services Research. 35(3):168-80. }\end{array}$ & $\begin{array}{l}\text { Not a parenting } \\
\text { intervention }\end{array}$ \\
\hline $\begin{array}{l}\text { 1548. Southam-Gerow MA, Kendall PC and Weersing VR. } 2001 . \\
\text { Examining outcome variability: Correlates of treatment response in a } \\
\text { child and adolescent anxiety clinic. Journal of Clinical Child Psychology. } \\
\text { 30(3):422-36. }\end{array}$ & $\begin{array}{l}\text { Not a parenting } \\
\text { intervention }\end{array}$ \\
\hline $\begin{array}{l}\text { 1549. Southam-Gerow MA, Silverman WK and Kendall PC. } 2006 . \\
\text { Client Similarities and Differences in Two Childhood Anxiety Disorders } \\
\text { Research Clinics. Journal of Clinical Child and Adolescent Psychology. } \\
\text { 35(4):528-38. }\end{array}$ & $\begin{array}{l}\text { Not a parenting } \\
\text { intervention }\end{array}$ \\
\hline $\begin{array}{l}\text { 1550. Southam-Gerow MA, Weisz JR and Kendall PC. 2003. Youth } \\
\text { With Anxiety Disorders in Research and Service Clinics: Examining } \\
\text { Client Differences and Similarities. Journal of Clinical Child and } \\
\text { Adolescent Psychology. 32(3):375-85. }\end{array}$ & $\begin{array}{l}\text { Not a parenting } \\
\text { intervention }\end{array}$ \\
\hline $\begin{array}{l}\text { 1551. Southam-Gerow MA, Weisz JR, Chu BC, McLeod BD, Gordis EB } \\
\text { and Connor-Smith JK. 2010. Does cognitive behavioral therapy for } \\
\text { youth anxiety outperform usual care in community clinics? An initial } \\
\text { effectiveness test. J Am Acad Child Adolesc Psychiatry. 49(10):1043- } \\
52 .\end{array}$ & $\begin{array}{l}\text { Not a parenting } \\
\text { intervention }\end{array}$ \\
\hline $\begin{array}{l}\text { 1552. Spaccarelli S, Cotler S and Penman D. 1992. Problem-Solving } \\
\text { Skills Training as a Supplement to Behavioral Parent Training. } \\
\text { Cognitive Therapy and Research. 16(1):1-17. }\end{array}$ & $\begin{array}{l}\text { No child } \\
\text { internalising } \\
\text { outcomes or not } \\
\text { a major goal; } \\
\text { Follow-up less } \\
\text { than } 6 \text { months; } \\
\text { Follow-up data } \\
\text { collected only in } \\
\text { active group }\end{array}$ \\
\hline $\begin{array}{l}\text { 1553. Spence SH, Sheffield JK and Donovan CL. 2003. Preventing } \\
\text { adolescent depression: An evaluation of the Problem Solving For Life } \\
\text { program. Journal of Consulting and Clinical Psychology. 71(1):3-13. }\end{array}$ & $\begin{array}{l}\text { Not a parenting } \\
\text { intervention }\end{array}$ \\
\hline $\begin{array}{l}\text { 1554. Spence, S. H., Donovan, C., \& Brechman-Toussaint, M. (2000). } \\
\text { The treatment of childhood social phobia: the effectiveness of a social } \\
\text { skills training-based, cognitive-behavioural intervention, with and } \\
\text { without parental involvement. J Child Psychol Psychiatry, 41(6), 713- } \\
726 .\end{array}$ & $\begin{array}{l}\text { Parent } \\
\text { component too } \\
\text { small; Follow-up } \\
\text { data collected } \\
\text { only in active } \\
\text { group }\end{array}$ \\
\hline
\end{tabular}




\begin{tabular}{|c|c|}
\hline $\begin{array}{l}\text { 1555. Spencer C and Franck LS. 2005. Giving parents written } \\
\text { information about children's anesthesia: are setting and timing } \\
\text { important? Paediatr Anaesth. 15(7):547-53. }\end{array}$ & $\begin{array}{l}\text { Intervention for } \\
\text { developmental } \\
\text { disorders } \\
\text { (autism, autism } \\
\text { spectrum } \\
\text { disorder), } \\
\text { physical } \\
\text { disabilities, } \\
\text { medical } \\
\text { conditions or } \\
\text { distress about } \\
\text { facing medical } \\
\text { interventions }\end{array}$ \\
\hline $\begin{array}{l}\text { 1556. Spielmans GI, Pasek LF and McFall JP. 2007. What are the } \\
\text { active ingredients in cognitive and behavioral psychotherapy for } \\
\text { anxious and depressed children? A meta-analytic review. Clinical } \\
\text { Psychology Review. 27(5):642-54. }\end{array}$ & Review \\
\hline $\begin{array}{l}\text { 1557. Spinelli MG and Endicott J. 2003. Controlled clinical trial of } \\
\text { interpersonal psychotherapy versus parenting education program for } \\
\text { depressed pregnant women. Am J Psychiatry. 160(3):555-62. }\end{array}$ & $\begin{array}{l}\text { No child } \\
\text { internalising } \\
\text { outcomes or not } \\
\text { a major goal }\end{array}$ \\
\hline $\begin{array}{l}\text { 1558. Spinelli, M. G., \& Endicott, J. (2011). Controlled clinical trial of } \\
\text { antepartum interpersonal psychotherapy versus parenting education } \\
\text { program at } 3 \text { NYC sites. Arch Womens Ment Health, 14, S49. }\end{array}$ & $\begin{array}{l}\text { Comparison } \\
\text { condition not a } \\
\text { minimal control }\end{array}$ \\
\hline $\begin{array}{l}1559 . \quad \text { Spinelli, M. G., Endicott, J., \& Goetz, R. R. (2013). Increased } \\
\text { breastfeeding rates in black women after a treatment intervention. } \\
\text { Breastfeeding Medicine, 8(6), 479-484. doi: } \\
\text { http://dx.doi.org/10.1089/bfm.2013.0051 }\end{array}$ & $\begin{array}{l}\text { No child } \\
\text { internalising } \\
\text { outcomes or not } \\
\text { a major goal }\end{array}$ \\
\hline $\begin{array}{l}\text { 1560. Spinelli, M. G., Endicott, J., Goetz, R. R. (2015). Disagreement } \\
\text { between therapist raters and independent evaluators in a controlled } \\
\text { clinical trial of interpersonal psychotherapy for depressed pregnant } \\
\text { women. Journal of Psychiatric Practice, } 21 \text { (2), 114-123. }\end{array}$ & $\begin{array}{l}\text { Data not } \\
\text { available/report } \\
\text { ed }\end{array}$ \\
\hline $\begin{array}{l}\text { 1561. Spinelli, M. G., Endicott, J., Leon, A. C., Goetz, R. R., Kalish, R. } \\
\text { B., Brustman, L. E., .. Schulick, J. L. (2013). A controlled clinical } \\
\text { treatment trial of interpersonal psychotherapy for depressed pregnant } \\
\text { women at } 3 \text { New York city sites. Journal of Clinical Psychiatry, 74(4), } \\
\text { 393-399. doi: http://dx.doi.org/10.4088/JCP.12m07909 }\end{array}$ & $\begin{array}{l}\text { No child } \\
\text { internalising } \\
\text { outcomes or not } \\
\text { a major goal }\end{array}$ \\
\hline $\begin{array}{l}\text { 1562. Spirito, A., Wolff, J. C., Seaboyer, L. M., Hunt, J., Esposito- } \\
\text { Smythers, C., Nugent, N., Zlotnick, C., Miller, I. (2015). Concurrent } \\
\text { Treatment for Adolescent and Parent Depressed Mood and Suicidality: } \\
\text { Feasibility, Acceptability, and Preliminary Findings. Journal of Child } \\
\text { and Adolescent Psychopharmacology, } 25 \text { (2), 131-139. }\end{array}$ & $\begin{array}{l}\text { Parent } \\
\text { component too } \\
\text { small; } \\
\text { Comparison } \\
\text { condition not a } \\
\text { minimal control }\end{array}$ \\
\hline $\begin{array}{l}\text { 1563. Spoth R, Goldberg C and Redmond C. 1999. Engaging families } \\
\text { in longitudinal preventive intervention research: discrete-time survival } \\
\text { analysis of socioeconomic and social-emotional risk factors. J Consult } \\
\text { Clin Psychol. 67(1):157-63. }\end{array}$ & $\begin{array}{l}\text { No child } \\
\text { internalising } \\
\text { outcomes or not } \\
\text { a major goal }\end{array}$ \\
\hline
\end{tabular}




\begin{tabular}{|c|c|}
\hline $\begin{array}{l}\text { 1564. Spoth R, Redmond C and Shin C. 1998. Direct and indirect } \\
\text { latent-variable parenting outcomes of two universal family-focused } \\
\text { preventive interventions: Extending a public health-oriented research } \\
\text { base. Journal of Consulting and Clinical Psychology. 66(2):385-99. }\end{array}$ & $\begin{array}{l}\text { No child } \\
\text { internalising } \\
\text { outcomes or not } \\
\text { a major goal; No } \\
\text { long term } \\
\text { follow-up }\end{array}$ \\
\hline $\begin{array}{l}\text { 1565. Spoth, R. L., Redmond, C., \& Shin, C. (2001). Randomized trial } \\
\text { of brief family interventions for general populations: Adolescent } \\
\text { substance use outcomes } 4 \text { years following baseline. Journal of } \\
\text { Consulting and Clinical Psychology, 69, 627-642. }\end{array}$ & $\begin{array}{l}\text { No child } \\
\text { internalising } \\
\text { outcomes or not } \\
\text { a major goal }\end{array}$ \\
\hline $\begin{array}{l}\text { 1566. Spoth, R., Redmond, C., Shin, C., \& Azevedo, K. (2004). Brief } \\
\text { family intervention effects on adolescent substance initiation: School- } \\
\text { level growth curve analyses } 6 \text { years following baseline. Journal of } \\
\text { Consulting and Clinical Psychology, 72,535-542. }\end{array}$ & $\begin{array}{l}\text { No child } \\
\text { internalising } \\
\text { outcomes or not } \\
\text { a major goal }\end{array}$ \\
\hline $\begin{array}{l}\text { 1567. Sprang, G. (2009). The efficacy of a relational treatment for } \\
\text { maltreated children and their families. Child and Adolescent Mental } \\
\text { Health, 14(2), 81-88. doi:10.1111/j.1475-3588.2008.00499.x }\end{array}$ & $\begin{array}{l}\text { No long term } \\
\text { follow-up }\end{array}$ \\
\hline $\begin{array}{l}\text { 1568. Srai, J. P., Petrie, A., Ryan, F. S., \& Cunningham, S. J. (2013). } \\
\text { Assessment of the effect of combined multimedia and verbal } \\
\text { information vs verbal information alone on anxiety levels before bond- } \\
\text { up in adolescent orthodontic patients: a single-center randomized } \\
\text { controlled trial. Am J Orthod Dentofacial Orthop, 144(4), 505-511. doi: } \\
\text { 10.1016/j.ajodo.2013.06.013 }\end{array}$ & $\begin{array}{l}\text { Not a parenting } \\
\text { intervention }\end{array}$ \\
\hline $\begin{array}{l}\text { 1569. Stabler B and et al. 1981. Facilitating Positive Psychosocial } \\
\text { Adaptation in Children with Cystic Fibrosis by Increasing Family } \\
\text { Communication and Problem-Solving Skills. A Research Report to the } \\
\text { Cystic Fibrosis Foundation. Chapel Hill, NC: North Carolina Univ., } \\
\text { Chapel Hill. School of Medicine. }\end{array}$ & $\begin{array}{l}\text { Intervention for } \\
\text { developmental } \\
\text { disorders } \\
\text { (autism, autism } \\
\text { spectrum } \\
\text { disorder), } \\
\text { physical } \\
\text { disabilities, } \\
\text { medical } \\
\text { conditions or } \\
\text { distress about } \\
\text { facing medical } \\
\text { interventions }\end{array}$ \\
\hline $\begin{array}{l}1570 . \quad \text { Stallard, P., Skryabina, E., Taylor, G., Phillips, R., Daniels, H., } \\
\text { Anderson, R., \& Simpson, N. (2014). Classroom-based cognitive } \\
\text { behaviour therapy (FRIENDS): a cluster randomised controlled trial to } \\
\text { Prevent Anxiety in Children through Education in Schools (PACES). The } \\
\text { Lancet Psychiatry, 1(3), 185-192. }\end{array}$ & $\begin{array}{l}\text { Not a parenting } \\
\text { intervention }\end{array}$ \\
\hline $\begin{array}{l}\text { 1571. Stallman HM and Ralph A. 2007. Reducing risk factors for } \\
\text { adolescent behavioural and emotional problems: a pilot randomised } \\
\text { controlled trial of a self-administered parenting intervention. } \\
\text { Australian e-Journal for the Advancement of Mental Health, 6(2), 125- } \\
\text { 137. }\end{array}$ & $\begin{array}{l}\text { Follow-up less } \\
\text { than } 6 \text { months }\end{array}$ \\
\hline
\end{tabular}




\begin{tabular}{|c|c|}
\hline $\begin{array}{l}\text { 1572. Stanger, C., Ryan, S. R., Fu, H., \& Budney, A. J. (2011). Parent } \\
\text { training plus contingency management for substance abusing families: } \\
\text { A Complier Average Causal Effects (CACE) analysis. Drug Alcohol } \\
\text { Depend, } 118,119-126 .\end{array}$ & $\begin{array}{l}\text { Comparison } \\
\text { condition not a } \\
\text { minimal control; } \\
\text { Targets } \\
\text { externalising; No } \\
\text { long term } \\
\text { follow-up }\end{array}$ \\
\hline $\begin{array}{l}\text { 1573. Stanger, C., Ryan, S. R., Scherer, E. A., Norton, G. E., \& Budney, } \\
\text { A. J. (2015). Clinic-and home-based contingency management plus } \\
\text { parent training for adolescent cannabis use disorders. Journal of the } \\
\text { American Academy of Child \& Adolescent Psychiatry. }\end{array}$ & $\begin{array}{l}\text { No child } \\
\text { internalising } \\
\text { outcomes or not } \\
\text { a major goal }\end{array}$ \\
\hline $\begin{array}{l}\text { 1574. Stark, K. D. (2004). CBT vs CBT plus parent training for girls } \\
\text { with depression. ClinicalTrials.gov [www.clinicaltrials.gov]. Retrieved } \\
\text { from } \\
\text { http://www.mrw.interscience.wiley.com/cochrane/clcentral/articles/ } \\
\text { 281/CN-00497281/frame.html }\end{array}$ & $\begin{array}{l}\text { Parent } \\
\text { component too } \\
\text { small }\end{array}$ \\
\hline $\begin{array}{l}1575 . \quad \text { Stefan, C. A., Miclea, M. (2013). Effects of a multifocused } \\
\text { prevention program on preschool children's competencies and } \\
\text { behavior problems. Psychology in the Schools, } 50 \text { (4), 382-402. }\end{array}$ & $\begin{array}{l}\text { No long term } \\
\text { follow-up }\end{array}$ \\
\hline $\begin{array}{l}\text { 1576. Stehl ML, Kazak AE, Alderfer MA, Rodriguez A, Hwang WT, Pai } \\
\text { AL, Boeving A and Reilly A. 2009. Conducting a randomized clinical trial } \\
\text { of an psychological intervention for parents/caregivers of children } \\
\text { with cancer shortly after diagnosis. J Pediatr Psychol. 34(8):803-16. }\end{array}$ & $\begin{array}{l}\text { No child } \\
\text { internalising } \\
\text { outcomes or not } \\
\text { a major goal }\end{array}$ \\
\hline $\begin{array}{l}\text { 1577. Stevens J, Ammerman RT, Putnam FG and Van Ginkel JB. } 2002 . \\
\text { Depression and trauma history in first-time mothers receiving home } \\
\text { visitation. Journal of Community Psychology. 30(5):551-64. }\end{array}$ & Not an RCT \\
\hline $\begin{array}{l}\text { 1578. Stevens, M. L. (2015). Child directed interaction training: The } \\
\text { impact on the kinship caregiver-child relationship and child } \\
\text { externalizing and internalizing symptoms. Dissertation Abstracts } \\
\text { International: Section B: The Sciences and Engineering, } 75 \text { (7-B E), }\end{array}$ & $\begin{array}{l}\text { Follow-up less } \\
\text { than } 6 \text { months }\end{array}$ \\
\hline $\begin{array}{l}\text { 1579. Stevens, N., Drendel, A. L., \& Weisman, S. J. (2012). Video } \\
\text { education intervention in the emergency department. Academic } \\
\text { Emergency Medicine, 19, S76. }\end{array}$ & $\begin{array}{l}\text { Follow-up less } \\
\text { than } 6 \text { months }\end{array}$ \\
\hline $\begin{array}{l}\text { 1580. Stevenson MD, Bivins CM, O'Brien K and Gonzalez del Rey JA. } \\
\text { 2005. Child life intervention during angiocatheter insertion in the } \\
\text { pediatric emergency department. Pediatr Emerg Care. 21(11):712-8. }\end{array}$ & $\begin{array}{l}\text { Intervention for } \\
\text { developmental } \\
\text { disorders } \\
\text { (autism, autism } \\
\text { spectrum } \\
\text { disorder), } \\
\text { physical } \\
\text { disabilities, } \\
\text { medical } \\
\text { conditions or } \\
\text { distress about } \\
\text { facing medical } \\
\text { interventions }\end{array}$ \\
\hline $\begin{array}{l}\text { 1581. Stewart-Brown, S., Patterson, J., Mockford, C., Barlow, J., } \\
\text { Klimes, I., \& Pyper, C. (2004). Impact of a general practice based group } \\
\text { parenting programme: quantitative and qualitative results from a } \\
\text { controlled trial at } 12 \text { months. Archives of Disease in Childhood, } 89(6) \text {, } \\
519-525 .\end{array}$ & $\begin{array}{l}\text { Targets } \\
\text { externalising } \\
\text { (selected for } \\
\text { high on } \\
\text { externalising) }\end{array}$ \\
\hline
\end{tabular}




\begin{tabular}{|c|c|}
\hline $\begin{array}{l}\text { 1582. Stice E, Rohde P, Seeley JR and Gau JM. 2008. Brief cognitive- } \\
\text { behavioral depression prevention program for high-risk adolescents } \\
\text { outperforms two alternative interventions: A randomized efficacy } \\
\text { trial. Journal of Consulting and Clinical Psychology. 76(4):595-606. }\end{array}$ & $\begin{array}{l}\text { Not a parenting } \\
\text { intervention }\end{array}$ \\
\hline $\begin{array}{l}\text { 1583. Stirtzinger, R., McDermid, S., Grusec, J., Bernardini, S., } \\
\text { Quinlan, K., \& Marshall, M. (2002). Interrupting the inter-generational } \\
\text { cycle in high risk adolescent pregnancy. The Journal of Primary } \\
\text { Prevention, 23(1), 7-22. doi: 10.1023/a:1016535131384 }\end{array}$ & $\begin{array}{l}\text { No child } \\
\text { internalising } \\
\text { outcomes or not } \\
\text { a major goal }\end{array}$ \\
\hline $\begin{array}{l}\text { 1584. Stolberg, A. L., \& Garrison, K. M. (1985). Evaluating a primary } \\
\text { prevention program for children of divorce. American Journal of } \\
\text { Community Psychology, 13, 1110-1124. }\end{array}$ & $\begin{array}{l}\text { Follow-up less } \\
\text { than } 6 \text { months }\end{array}$ \\
\hline $\begin{array}{l}\text { 1585. Stolberg, A. L., \& Mahler, J. (1994). Enhancing treatment gains } \\
\text { in a school-based intervention for children of divorce through skill } \\
\text { training, parental involvement, and transfer procedures. J Consult Clin } \\
\text { Psychol, 62(1), 147-156. }\end{array}$ & $\begin{array}{l}\text { Parent } \\
\text { component too } \\
\text { small }\end{array}$ \\
\hline $\begin{array}{l}\text { 1586. Stolk MN, Mesman J, van Zeijl J, Alink LRA, Bakermans- } \\
\text { Kranenburg MJ, van ljzendoorn MH, Juffer F and Koot HM. 2008. Early } \\
\text { parenting intervention aimed at maternal sensitivity and discipline: A } \\
\text { process evaluation. Journal of Community Psychology. 36(6):780-97. }\end{array}$ & Not an RCT \\
\hline $\begin{array}{l}1587 . \quad \text { Stolk, M. N., Mesman, J., van Zeijl, J., Alink, L. R. A., } \\
\text { Bakermans-Kranenburg, M. J., van ljzendoorn, M. H., et al. (2008). } \\
\text { Early parenting intervention: Family risk and first-time parenting } \\
\text { related to intervention effectiveness. Journal of Child and Family } \\
\text { Studies, } 17(1), 55-83 . \text { doi: } 10.1007 / \text { s10826-007-9136-3 }\end{array}$ & $\begin{array}{l}\text { No long term } \\
\text { follow-up }\end{array}$ \\
\hline $\begin{array}{l}\text { 1588. Stoltz, S., Dekovic, M., van Londen, M., Orobio de Castro, B., } \\
\text { Prinzie, P. (2013). What works for whom, how and under what } \\
\text { circumstances? Testing moderated mediation of intervention effects } \\
\text { on externalizing behavior in children. Social Development, } 22 \text { (2), 406- } \\
425 .\end{array}$ & $\begin{array}{l}\text { Targets } \\
\text { externalising }\end{array}$ \\
\hline $\begin{array}{l}\text { 1589. Stomp-van den Berg SG, van Poppel MN, Hendriksen IJ, } \\
\text { Bruinvels DJ, Uegaki K, de Bruijne MC and van Mechelen W. } 2007 . \\
\text { Improving return-to-work after childbirth: design of the Mom@Work } \\
\text { study, a randomised controlled trial and cohort study. BMC Public } \\
\text { Health. 7:43. }\end{array}$ & $\begin{array}{l}\text { No child } \\
\text { internalising } \\
\text { outcomes or not } \\
\text { a major goal }\end{array}$ \\
\hline $\begin{array}{l}\text { 1590. Stone BP. 2000. Videotape modeling interventions for } \\
\text { selective mutism: A comparative research investigation. Dissertation } \\
\text { Abstracts International Section A: Humanities and Social Sciences. } \\
61(5-A) \text {. }\end{array}$ & $\begin{array}{l}\text { Follow-up less } \\
\text { than } 6 \text { months }\end{array}$ \\
\hline $\begin{array}{l}\text { 1591. Stone S, Raman A and Fleming S. 2010. Behavioral } \\
\text { characteristics among obese/overweight inner-city African American } \\
\text { children: A secondary analysis of participants in a community-based } \\
\text { type } 2 \text { diabetes risk reduction program. Children and Youth Services } \\
\text { Review. 32(6):833-9. }\end{array}$ & $\begin{array}{l}\text { Not a parenting } \\
\text { intervention }\end{array}$ \\
\hline $\begin{array}{l}\text { 1592. Storch EA, Lewin AB, Geffken GR, Morgan JR and Murphy TK. } \\
\text { 2010. The role of comorbid disruptive behavior in the clinical } \\
\text { expression of pediatric obsessive-compulsive disorder. Behaviour } \\
\text { Research and Therapy. } 48(12): 1204-10 \text {. }\end{array}$ & $\begin{array}{l}\text { Not a parenting } \\
\text { intervention }\end{array}$ \\
\hline
\end{tabular}




\begin{tabular}{|c|c|}
\hline $\begin{array}{l}\text { 1593. Storch EA, Murphy TK, Geffken GR, Mann G, Adkins J, Merlo } \\
\text { L, Duke D, Munson M, Swaine Z and Goodman WK. 2006. Cognitive- } \\
\text { behavioral therapy for PANDAS-related obsessive-compulsive } \\
\text { disorder: findings from a preliminary waitlist controlled open trial. J } \\
\text { Am Acad Child Adolesc Psychiatry. 45(10):1171-8. }\end{array}$ & $\begin{array}{l}\text { Not a parenting } \\
\text { intervention }\end{array}$ \\
\hline $\begin{array}{l}\text { 1594. Storch, E. A., Merlo, L. J., Larson, M. J., Geffken, G. R., } \\
\text { Lehmkuhl, H. D., Jacob, M. L., et al. (2008). Impact of comorbidity on } \\
\text { cognitive-behavioral therapy response in pediatric obsessive- } \\
\text { compulsive disorder. Journal of the American Academy of Child \& } \\
\text { Adolescent Psychiatry, } 47(5), 583-592 . \text { doi: } \\
\text { 10.1097/CHI.0b013e31816774b1 }\end{array}$ & $\begin{array}{l}\text { No long term } \\
\text { follow-up }\end{array}$ \\
\hline $\begin{array}{l}\text { 1595. Storeb } \varnothing, \text { O. J., Gluud, C., Winkel, P., \& Simonsen, E. (2012). } \\
\text { Social-skills and parental training plus standard treatment versus } \\
\text { standard treatment for children with ADHD--the randomised SOSTRA } \\
\text { trial. PloS one, } 7(6) \text {, e37280. doi:10.1371/journal.pone.0037280 }\end{array}$ & $\begin{array}{l}\text { Targets } \\
\text { externalising }\end{array}$ \\
\hline $\begin{array}{l}\text { 1596. Storeb } \varnothing \text {, O. J., Pedersen, J., Skoog, M., Thomsen, P. H., Winkel, } \\
\text { P., Gluud, C., \& Simonsen, E. (2011). Randomised social-skills training } \\
\text { and parental training plus standard treatment versus standard } \\
\text { treatment of children with attention deficit hyperactivity disorder - } \\
\text { the SOSTRA trial protocol. In Trials (pp. 18). }\end{array}$ & $\begin{array}{l}\text { Data not } \\
\text { available/report } \\
\text { ed (protocol) }\end{array}$ \\
\hline $\begin{array}{l}\text { 1597. Stover CS, Hahn H, Im JJY and Berkowitz S. 2010. Agreement of } \\
\text { parent and child reports of trauma exposure and symptoms in the } \\
\text { early aftermath of a traumatic event. Psychological Trauma: Theory, } \\
\text { Research, Practice, and Policy. 2(3):159-68. }\end{array}$ & $\begin{array}{l}\text { Not a parenting } \\
\text { intervention }\end{array}$ \\
\hline $\begin{array}{l}1598 . \quad \text { Stover, C. S., Connell, C. M., Leve, L. D., Neiderhiser, J. M., } \\
\text { Shaw, D. S., Scaramella, L. V., Conger, R., \& Reiss, D. (2012). Fathering } \\
\text { and mothering in the family system: Linking marital hostility and } \\
\text { aggression in adopted toddlers. Journal of Child Psychology and } \\
\text { Psychiatry, 53, 401-409. }\end{array}$ & $\begin{array}{l}\text { Not a parenting } \\
\text { intervention }\end{array}$ \\
\hline $\begin{array}{l}\text { 1599. Strachan RG. 1993. Emotional responses to paediatric } \\
\text { hospitalisation. Nursing times, } 89(46), 44-49 \text {. }\end{array}$ & $\begin{array}{l}\text { Intervention for } \\
\text { developmental } \\
\text { disorders } \\
\text { (autism, autism } \\
\text { spectrum } \\
\text { disorder), } \\
\text { physical } \\
\text { disabilities, } \\
\text { medical } \\
\text { conditions or } \\
\text { distress about } \\
\text { facing medical } \\
\text { interventions }\end{array}$ \\
\hline $\begin{array}{l}\text { 1600. Strain, P. S., Steele, P., Ellis, T., \& Timm, M. A. (1982). Long- } \\
\text { Term Effects of Oppositional Child Treatment with Mothers as } \\
\text { Therapists and Therapist Trainers. Journal of Applied Behavior } \\
\text { Analysis, 15(1), 163-169. }\end{array}$ & Not an RCT \\
\hline
\end{tabular}




\begin{tabular}{|c|c|}
\hline $\begin{array}{l}\text { 1601. Straker, L. M., Smith, K. L., Fenner, A. A., Kerr, D. A., McManus, } \\
\text { A., Davis, M. C., . . Abbott, R. A. (2012). Rationale, design and } \\
\text { methods for a staggered-entry, waitlist controlled clinical trial of the } \\
\text { impact of a community-based, family-centred, multidisciplinary } \\
\text { program focussed on activity, food and attitude habits (Curtin } \\
\text { University's Activity, Food and Attitudes Program--CAFAP) among } \\
\text { overweight adolescents. BMC public health, 12, 471. } \\
\text { doi:10.1186/1471-2458-12-471 }\end{array}$ & $\begin{array}{l}\text { Data not } \\
\text { available/report } \\
\text { ed (protocol) }\end{array}$ \\
\hline $\begin{array}{l}\text { 1602. Strayhorn, J. M., \& Weidman, C. S. (1989). Reduction of } \\
\text { attention deficit and internalizing symptoms in preschoolers through } \\
\text { parent-child interaction training. J Am Acad Child Adolesc Psychiatry, } \\
\text { 28(6), 888-896. doi: S0890-8567(09)60213-6 }\end{array}$ & $\begin{array}{l}\text { No long term } \\
\text { follow-up }\end{array}$ \\
\hline $\begin{array}{l}\text { 1603. Streisand R, Mackey ER, Elliot BM, Mednick L, Slaughter IM, } \\
\text { Turek J and Austin A. 2008. Parental anxiety and depression associated } \\
\text { with caring for a child newly diagnosed with type } 1 \text { diabetes: } \\
\text { Opportunities for education and counseling. Patient Education and } \\
\text { Counseling. 73(2):333-8. }\end{array}$ & $\begin{array}{l}\text { Not a parenting } \\
\text { intervention }\end{array}$ \\
\hline $\begin{array}{l}\text { 1604. Stremler R, Hodnett E, Lee K, MacMillan S, Mill C, Ongcangco L } \\
\text { and Willan A. 2006. A behavioral-educational intervention to promote } \\
\text { maternal and infant sleep: a pilot randomized, controlled trial. Sleep. } \\
\text { 29(12):1609-15. }\end{array}$ & $\begin{array}{l}\text { No child } \\
\text { internalising } \\
\text { outcomes or not } \\
\text { a major goal }\end{array}$ \\
\hline $\begin{array}{l}\text { 1605. Suchman, N. E., Decoste, C., McMahon, T. J., Rounsaville, B., \& } \\
\text { Mayes, L. (2011). The Mothers and Toddlers program, an attachment- } \\
\text { based parenting intervention for substance-using women: Results at } \\
\text { 6-week follow-up in a randomized clinical pilot. Infant Mental Health } \\
\text { Journal, 32, 427-449. }\end{array}$ & $\begin{array}{l}\text { Follow-up less } \\
\text { than } 6 \text { months }\end{array}$ \\
\hline $\begin{array}{l}\text { 1606. Suchman, N. E., Decoste, C., Rosenberger, P., \& McMahon, T. } \\
\text { J. (2012). Attachment-based intervention for substance-using } \\
\text { mothers: A preliminary test of the proposed mechanisms of change. } \\
\text { Infant mental health journal, 33(4), 360-371. doi:10.1002/imhj.21311 }\end{array}$ & $\begin{array}{l}\text { No child } \\
\text { internalising } \\
\text { outcomes or not } \\
\text { a major goal; No } \\
\text { long term } \\
\text { follow-up }\end{array}$ \\
\hline $\begin{array}{l}\text { 1607. Sukhodolsky DG, Scahill L, Gadow KD, Arnold LE, Aman MG, } \\
\text { McDougle CJ, McCracken JT, Tierney E, White SW, Lecavalier L and } \\
\text { Vitiello B. 2008. Parent-rated anxiety symptoms in children with } \\
\text { pervasive developmental disorders: Frequency and association with } \\
\text { core autism symptoms and cognitive functioning. Journal of Abnormal } \\
\text { Child Psychology: An official publication of the International Society } \\
\text { for Research in Child and Adolescent Psychopathology. 36(1):117-28. }\end{array}$ & $\begin{array}{l}\text { Not a parenting } \\
\text { intervention }\end{array}$ \\
\hline $\begin{array}{l}\text { 1608. Sukhodolsky, D. G., Gorman, B. S., Scahill, L., Findley, D., \& } \\
\text { McGuire, J. (2013). Exposure and response prevention with or without } \\
\text { parent management training for children with obsessive-compulsive } \\
\text { disorder complicated by disruptive behavior: A multiple-baseline } \\
\text { across-responses design study. Journal of anxiety disorders, 27(3), } \\
\text { 298-305. }\end{array}$ & $\begin{array}{l}\text { Comparison } \\
\text { condition not a } \\
\text { minimal control }\end{array}$ \\
\hline $\begin{array}{l}\text { 1609. Sullivan-Bolyai, S., Grey, M., Deatrick, J., Gruppuso, P., Giraitis, } \\
\text { P., \& Tamborlane, W. (2004). Helping other mothers effectively work } \\
\text { at raising young children with type } 1 \text { diabetes. The Diabetes educator, } \\
\text { 30(3), } 476 .\end{array}$ & $\begin{array}{l}\text { No child } \\
\text { internalising } \\
\text { outcomes or not } \\
\text { a major goal }\end{array}$ \\
\hline
\end{tabular}




\begin{tabular}{|c|c|}
\hline $\begin{array}{l}\text { 1610. Summers JA, Houlding CM and Reitzel J-AM. 2004. Behavior } \\
\text { Management Services for Children with Autism/PDD: Program } \\
\text { Description and Patterns of Referral. Focus on Autism and Other } \\
\text { Developmental Disabilities. 19(2):95-101. }\end{array}$ & $\begin{array}{l}\text { Intervention for } \\
\text { developmental } \\
\text { disorders } \\
\text { (autism, autism } \\
\text { spectrum } \\
\text { disorder), } \\
\text { physical } \\
\text { disabilities, } \\
\text { medical } \\
\text { conditions or } \\
\text { distress about } \\
\text { facing medical } \\
\text { interventions }\end{array}$ \\
\hline $\begin{array}{l}\text { 1611. Supplee LH, Unikel EB and Shaw DS. 2007. Physical } \\
\text { environmental adversity and the protective role of maternal } \\
\text { monitoring in relation to early child conduct problems. Journal of } \\
\text { Applied Developmental Psychology. 28(2):166-83. }\end{array}$ & $\begin{array}{l}\text { Not a parenting } \\
\text { intervention }\end{array}$ \\
\hline $\begin{array}{l}\text { 1612. Surkan PJ, Kawachi I, Ryan LM, Berkman LF, Vieira LMC and } \\
\text { Peterson KE. 2008. Maternal depressive symptoms, parenting self- } \\
\text { efficacy, and child growth. American Journal of Public Health. } \\
\text { 98(1):125-32. }\end{array}$ & $\begin{array}{l}\text { Not a parenting } \\
\text { intervention }\end{array}$ \\
\hline $\begin{array}{l}\text { 1613. Sutton, C. (1992). Training parents to manage difficult } \\
\text { children: A comparison of methods. Behavioural Psychotherapy, 20, } \\
\text { 115-139. }\end{array}$ & $\begin{array}{l}\text { No child } \\
\text { internalising } \\
\text { outcomes or not } \\
\text { a major goal; } \\
\text { Not an RCT }\end{array}$ \\
\hline $\begin{array}{l}\text { 1614. Sutton, C. (1995). Parent training by telephone: A partial } \\
\text { replication! Behavioural and Cognitive Psychotherapy, 23(1), 1-24. doi: } \\
10.1017 / \mathrm{s} 1352465800017598\end{array}$ & $\begin{array}{l}\text { Targets } \\
\text { externalising }\end{array}$ \\
\hline $\begin{array}{l}\text { 1615. Suveg C, Hudson JL, Brewer G, Flannery-Schroeder E, Gosch E, } \\
\text { Kendall PC. Cognitive-behavioral therapy for anxiety-disordered youth: } \\
\text { secondary outcomes from a randomized clinical trial evaluating child } \\
\text { and family modalities. J Anxiety Disord. } 2009 \text { Apr;23(3):341-9. }\end{array}$ & $\begin{array}{l}\text { Parent } \\
\text { component too } \\
\text { small }\end{array}$ \\
\hline $\begin{array}{l}\text { 1616. Svanberg, P. O., Mennet, L., \& Spieker, S. (2010). Promoting a } \\
\text { secure attachment: A primary prevention practice model. Clin Child } \\
\text { Psychol Psychiatry, 15(3), 363-378. doi: 15/3/363 }\end{array}$ & Not an RCT \\
\hline $\begin{array}{l}\text { 1617. Svanborg, P., Thernlund, G., Gustafsson, P. A., Hagglof, B., } \\
\text { Poole, L., \& Kadesjo, B. (2009). Efficacy and safety of atomoxetine as } \\
\text { add-on to psychoeducation in the treatment of attention } \\
\text { deficit/hyperactivity disorder : AAA randomized, double-blind, } \\
\text { placebo-controlled study in stimulant-naive Swedish children and } \\
\text { adolescents. European Child and Adolescent Psychiatry, 18(4), 240- } \\
249 .\end{array}$ & $\begin{array}{l}\text { No long term } \\
\text { follow-up; No } \\
\text { child } \\
\text { internalising } \\
\text { outcomes or not } \\
\text { a major goal }\end{array}$ \\
\hline $\begin{array}{l}\text { 1618. Svanborg, P., Thernlund, G., Gustafsson, P. A., Hagglof, B., } \\
\text { Schacht, A., \& Kadesjo, B. (2009). Atomoxetine improves patient and } \\
\text { family coping in attention deficit/hyperactivity disorder: a randomized, } \\
\text { double-blind, placebo-controlled study in Swedish children and } \\
\text { adolescents. Eur Child Adolesc Psychiatry, 18(12), 725-735. doi: } \\
\text { 10.1007/s00787-009-0031-x }\end{array}$ & $\begin{array}{l}\text { No long term } \\
\text { follow-up }\end{array}$ \\
\hline
\end{tabular}




\begin{tabular}{|c|c|}
\hline $\begin{array}{l}\text { 1619. Swain, J., Hancock, K., Dixon, A., Koo, S., \& Bowman, J. (2013). } \\
\text { Acceptance and commitment therapy for anxious children and } \\
\text { adolescents: study protocol for a randomized controlled trial. Trials, } \\
\text { 14, 140. doi: } 10.1186 / 1745-6215-14-140\end{array}$ & $\begin{array}{l}\text { Data not } \\
\text { available/report } \\
\text { ed }\end{array}$ \\
\hline $\begin{array}{l}\text { 1620. Sweet, M. A., \& Applebaum, M. I. (2004). Is home visiting an } \\
\text { effective strategy? A meta-analytic review of home visiting programs } \\
\text { for families with young children. Child Development, 75, 1435-1456. }\end{array}$ & Review \\
\hline $\begin{array}{l}\text { 1621. Szczepanski R, Gebert N, Hummelink R, Konning J, Schmidt S, } \\
\text { Runde B and Wahn U. 1996. [Outcome of structured asthma } \\
\text { education in childhood and adolescence]. Pneumologie. 50(8):544-8. }\end{array}$ & $\begin{array}{l}\text { Intervention for } \\
\text { developmental } \\
\text { disorders } \\
\text { (autism, autism } \\
\text { spectrum } \\
\text { disorder), } \\
\text { physical } \\
\text { disabilities, } \\
\text { medical } \\
\text { conditions or } \\
\text { distress about } \\
\text { facing medical } \\
\text { interventions }\end{array}$ \\
\hline $\begin{array}{l}\text { 1622. Szigethy E, Whitton SW, Levy-Warren A, DeMaso DR, Weisz J } \\
\text { and Beardslee WR. 2004. Cognitive-Behavioral Therapy for Depression } \\
\text { in Adolescents With Inflammatory Bowel Disease: A Pilot Study. } \\
\text { Journal of the American Academy of Child \& Adolescent Psychiatry. } \\
\text { 43(12):1469-77. }\end{array}$ & $\begin{array}{l}\text { Not a parenting } \\
\text { intervention }\end{array}$ \\
\hline $\begin{array}{l}\text { 1623. Szigethy EM, Ruiz P, DeMaso DR, Shapiro F and Beardslee WR. } \\
\text { 2002. Consultation-liaison psychiatry: A longitudinal and integrated } \\
\text { approach. The American Journal of Psychiatry. 159(3):373-8. }\end{array}$ & Not an RCT \\
\hline $\begin{array}{l}\text { 1624. Tak, Y. R., Van Zundert, R. M., Kuijpers, R. C., Van Vlokhoven, } \\
\text { B. S., Rensink, H. F., \& Engels, R. C. (2012). A randomized controlled } \\
\text { trial testing the effectiveness of a universal school-based depression } \\
\text { prevention program 'Op Volle Kracht' in the Netherlands. BMC Public } \\
\text { Health, 12, } 21 .\end{array}$ & $\begin{array}{l}\text { Not a parenting } \\
\text { intervention }\end{array}$ \\
\hline $\begin{array}{l}\text { 1625. Tarini BA, Singer D, Clark SJ and Davis MM. 2008. Parents' } \\
\text { concern about their own and their children's genetic disease risk: } \\
\text { potential effects of family history vs genetic test results. Archives of } \\
\text { pediatrics \& adolescent medicine, 162(11), 1079-1083. }\end{array}$ & $\begin{array}{l}\text { Not a parenting } \\
\text { intervention }\end{array}$ \\
\hline $\begin{array}{l}\text { 1626. Taubner S, Wiswede D, Nolte T and Roth G. } 2010 . \\
\text { Mentalisierung und externalisierende VerhaltensstVdrungen in der } \\
\text { Adoleszenz. / Mentalization and externalizing behavioral disturbances } \\
\text { during adolescence. Psychotherapeut. 55(4):312-20. }\end{array}$ & $\begin{array}{l}\text { Not a parenting } \\
\text { intervention }\end{array}$ \\
\hline $\begin{array}{l}\text { 1627. Taylor, B. J., Heath, A. L., Galland, B. C., Gray, A. R., Lawrence, } \\
\text { J. A., Sayers, R. M., Dale, K., Coppell, K. J., \& Taylor, R. W. (2011). } \\
\text { Prevention of Overweight in Infancy (POI.nz) study: a randomised } \\
\text { controlled trial of sleep, food and activity interventions for preventing } \\
\text { overweight from birth. BMC Public Health, } 11,942 .\end{array}$ & $\begin{array}{l}\text { Data not } \\
\text { available/report } \\
\text { ed (protocol) }\end{array}$ \\
\hline
\end{tabular}




\begin{tabular}{|c|c|}
\hline $\begin{array}{l}\text { 1628. Taylor, H. L. (2010). A school-based parent intervention } \\
\text { program to improve student behavior problems and the school-family } \\
\text { relationship during the transition to kindergarten (Order No. } \\
\text { 3450971). Available from ProQuest Central; ProQuest Dissertations \& } \\
\text { Theses Global; ProQuest Social Sciences Premium Collection. } \\
\text { (862059708). }\end{array}$ & $\begin{array}{l}\text { Follow-up less } \\
\text { than } 6 \text { months }\end{array}$ \\
\hline $\begin{array}{l}\text { 1629. Taylor, T. K., \& Biglan, A. (1998). Behavioral Family } \\
\text { Interventions for Improving Child-rearing: A Review of the Literature } \\
\text { for Clinicians and Policy Makers. Clinical Child and Family Psychology } \\
\text { Review, 1(1), 41-60. doi:10.1023/a:1021848315541 }\end{array}$ & Review \\
\hline $\begin{array}{l}\text { 1630. Taylor, T. K., Schmidt, F., Pepler, D., \& Hodgins, C. (1998). A } \\
\text { comparison of eclectic treatment with Webster-Stratton's Parents and } \\
\text { Children Series in a children's mental health center: A randomized } \\
\text { controlled trial. Behavior Therapy, 29(2), 221-240. }\end{array}$ & $\begin{array}{l}\text { No long term } \\
\text { follow-up }\end{array}$ \\
\hline $\begin{array}{l}\text { 1631. Teerikangas OM, Aronen ET, Martin RP, Huttunen MO. Effects } \\
\text { of infant temperament and early intervention on the psychiatric } \\
\text { symptoms of adolescents. Journal of the American Academy of Child \& } \\
\text { Adolescent Psychiatry, } 37(10), 1070-1076 \text {. }\end{array}$ & Not an RCT \\
\hline $\begin{array}{l}\text { 1632. Tein JY, Sandler IN, MacKinnon DP, Wolchik SA. How did it } \\
\text { work? Who did it work for? Mediation in the context of a moderated } \\
\text { prevention effect for children of divorce. J Consult Clin Psychol. } 2004 \\
\text { Aug;72(4):617-24. }\end{array}$ & $\begin{array}{l}\text { Secondary } \\
\text { analysis } \\
\text { (mediation) so } \\
\text { can't use data }\end{array}$ \\
\hline $\begin{array}{l}\text { 1633. Tellegen, C. L., \& Sanders, M. R. (2014). A randomized } \\
\text { controlled trial evaluating a brief parenting program with children with } \\
\text { autism spectrum disorders.. Journal of consulting and clinical } \\
\text { psychology, } 82(6), 1193-200 .\end{array}$ & $\begin{array}{l}\text { No child } \\
\text { internalising } \\
\text { outcomes or not } \\
\text { a major goal; } \\
\text { Intervention for } \\
\text { developmental } \\
\text { disorders; } \\
\text { Targets } \\
\text { externalising }\end{array}$ \\
\hline $\begin{array}{l}\text { 1634. Thienemann M, Moore P and Tompkins K. 2006. A parent-only } \\
\text { group intervention for children with anxiety disorders: Pilot study. } \\
\text { Journal of the American Academy of Child \& Adolescent Psychiatry. } \\
\text { 45(1):37-46. }\end{array}$ & Not an RCT \\
\hline $\begin{array}{l}\text { 1635. Thomas DV and Looney SW. 2004. Effectiveness of a } \\
\text { comprehensive psychoeducational intervention with pregnant and } \\
\text { parenting adolescents: a pilot study. J Child Adolesc Psychiatr Nurs. } \\
\text { 17(2):66-77. }\end{array}$ & Not an RCT \\
\hline $\begin{array}{l}\text { 1636. Thomas, R., \& Zimmer-Gembeck, M. J. (2007). Behavioral } \\
\text { outcomes of parent-child interaction therapy and Triple P-Positive } \\
\text { Parenting Program: A review and meta-analysis. Journal of abnormal } \\
\text { child psychology, } 35(3), 475-495 .\end{array}$ & Review \\
\hline $\begin{array}{l}\text { 1637. Thomas, R., \& Zimmer-Gembeck, M. J. (2012). Parent-child } \\
\text { interaction therapy: an evidence-based treatment for child } \\
\text { maltreatment. Child maltreatment, 17(3), 253-266. } \\
\text { doi:10.1177/1077559512459555 }\end{array}$ & $\begin{array}{l}\text { Follow-up less } \\
\text { than } 6 \text { months }\end{array}$ \\
\hline
\end{tabular}




\begin{tabular}{|c|c|}
\hline $\begin{array}{l}\text { 1638. Thompson M. 2002. The provision of a preventative mental } \\
\text { health assessment \& training package to parents and teachers. } \\
\text { National Research Register. Retrieved from } \\
\text { http://onlinelibrary.wiley.com/o/cochrane/clcentral/articles/626/CN- } \\
\text { 00477626/frame.html }\end{array}$ & $\begin{array}{l}\text { Not a parenting } \\
\text { intervention }\end{array}$ \\
\hline $\begin{array}{l}\text { 1639. Thompson MJ, Laver-Bradbury C, Ayres M, Le Poidevin E, } \\
\text { Mead S, Dodds C, Psychogiou L, Bitsakou P, Daley D, Weeks A, } \\
\text { Brotman LM, Abikoff H, Thompson P and Sonuga-Barke EJ. 2009. A } \\
\text { small-scale randomized controlled trial of the revised new forest } \\
\text { parenting programme for preschoolers with attention deficit } \\
\text { hyperactivity disorder. European child \& adolescent psychiatry, 18(10), } \\
\text { 605-616. }\end{array}$ & $\begin{array}{l}\text { Follow-up less } \\
\text { than } 6 \text { months }\end{array}$ \\
\hline $\begin{array}{l}\text { 1640. Thompson R. 2007. Mothers' violence victimization and child } \\
\text { behavior problems: Examining the link. American Journal of } \\
\text { Orthopsychiatry. } 77(2): 306-15 \text {. }\end{array}$ & $\begin{array}{l}\text { Not a parenting } \\
\text { intervention }\end{array}$ \\
\hline $\begin{array}{l}\text { 1641. Thomson H, Ross S, Wilson P, McConnachie A and Watson R. } \\
\text { 2002. Mothers' use of and attitudes to BabyCheck. British Journal of } \\
\text { General Practice. 52(477):314-6. }\end{array}$ & Not an RCT \\
\hline $\begin{array}{l}\text { 1642. Thorell, L. B. (2009). The Community Parent Education } \\
\text { Program (COPE): treatment effects in a clinical and a community- } \\
\text { based sample. Clinical child psychology and psychiatry, (3), 373-387. }\end{array}$ & $\begin{array}{l}\text { No child } \\
\text { internalising } \\
\text { outcomes or not } \\
\text { a major goal }\end{array}$ \\
\hline $\begin{array}{l}\text { 1643. Timmer, S. G., Urquiza, A. J., \& Zebell, N. (2006). Challenging } \\
\text { foster caregiver-maltreated child relationships: The effectiveness of } \\
\text { parent-child interaction therapy. Children and Youth Services Review, } \\
\text { 28(1), 1-19. }\end{array}$ & $\begin{array}{l}\text { Not an RCT; No } \\
\text { long term } \\
\text { follow-up }\end{array}$ \\
\hline $\begin{array}{l}\text { 1644. Timmer, S. G., Urquiza, A. J., Herschell, A. D., McGrath, J. M., } \\
\text { Zebell, N. M., Porter, A. L., \& Vargas, E. C. (2006). Parent-child } \\
\text { interaction therapy: Application of an empirically supported treatment } \\
\text { to maltreated children in foster care. Child Welfare, 85(6), 919-939. }\end{array}$ & Not an RCT \\
\hline $\begin{array}{l}\text { 1645. Tirella LG, Chan W, Cermak SA, Litvinova A, Salas KC and Miller } \\
\text { LC. 2008. Time use in Russian baby homes. Child: Care, Health and } \\
\text { Development. 34(1):77-86. }\end{array}$ & $\begin{array}{l}\text { No child } \\
\text { internalising } \\
\text { outcomes or not } \\
\text { a major goal }\end{array}$ \\
\hline $\begin{array}{l}\text { 1646. Tonge B, Brereton A, Kiomall M, Mackinnon A, King N and } \\
\text { Rinehart N. 2006. Effects on parental mental health of an education } \\
\text { and skills training program for parents of young children with autism: } \\
\text { a randomized controlled trial. J Am Acad Child Adolesc Psychiatry. } \\
\text { 45(5):561-9. }\end{array}$ & $\begin{array}{l}\text { Intervention for } \\
\text { developmental } \\
\text { disorders } \\
\text { (autism, autism } \\
\text { spectrum } \\
\text { disorder), } \\
\text { physical } \\
\text { disabilities, } \\
\text { medical } \\
\text { conditions or } \\
\text { distress about } \\
\text { facing medical } \\
\text { interventions }\end{array}$ \\
\hline $\begin{array}{l}\text { 1647. Tonge BJ, Pullen JM, Hughes GC and Beaufoy J. } 2009 . \\
\text { Effectiveness of psychoanalytic psychotherapy for adolescents with } \\
\text { serious mental illness: } 12 \text { month naturalistic follow-up study. } \\
\text { Australian and New Zealand Journal of Psychiatry. 43(5):467-75. }\end{array}$ & $\begin{array}{l}\text { Not a parenting } \\
\text { intervention }\end{array}$ \\
\hline
\end{tabular}




\begin{tabular}{|c|c|}
\hline $\begin{array}{l}\text { 1648. Tooten, A., Hoffenkamp, H. N., Hall, R. A., Winkel, F. W., Eliëns, } \\
\text { M., Vingerhoets, A. J., \& Bakel, H. J. (2012). The effectiveness of video } \\
\text { interaction guidance in parents of premature infants: a multicenter } \\
\text { randomised controlled trial. BMC pediatrics, } 12,76 \text {. doi:10.1186/1471- } \\
\text { 2431-12-76 }\end{array}$ & $\begin{array}{l}\text { Data not } \\
\text { available/report } \\
\text { ed (protocol) }\end{array}$ \\
\hline $\begin{array}{l}\text { 1649. Topitzes J, Godes O, Mersky JP, Ceglarek S and Reynolds AJ. } \\
\text { 2009. Educational success and adult health: Findings from the Chicago } \\
\text { Longitudinal Study. Prevention Science. 10(2):175-95. }\end{array}$ & $\begin{array}{l}\text { Not a parenting } \\
\text { intervention }\end{array}$ \\
\hline $\begin{array}{l}\text { 1650. Torres, N., Maia, J., Veríssimo, M., Fernandes, M., \& Silva, F. } \\
\text { (2012). Attachment security representations in institutionalized } \\
\text { children and children living with their families: Links to problem } \\
\text { behaviour. Clinical Psychology \& Psychotherapy, 19, 25-36. }\end{array}$ & $\begin{array}{l}\text { Not a parenting } \\
\text { intervention }\end{array}$ \\
\hline $\begin{array}{l}\text { 1651. Toth SL, Rogosch FA, Manly JT and Cicchetti D. 2006. The } \\
\text { efficacy of toddler-parent psychotherapy to reorganize attachment in } \\
\text { the young offspring of mothers with major depressive disorder: A } \\
\text { randomized preventive trial. Journal of Consulting and Clinical } \\
\text { Psychology. } 74(6): 1006-16 \text {. }\end{array}$ & $\begin{array}{l}\text { No long term } \\
\text { follow-up }\end{array}$ \\
\hline $\begin{array}{l}\text { 1652. Toth, S. L., Maughan, A., Manly, J. T., Spagnola, M., \& } \\
\text { Cicchetti, D. (2002). The relative efficacy of two interventions in } \\
\text { altering maltreated preschool children's representational models: } \\
\text { Implications for attachment theory. Development and } \\
\text { psychopathology, 14(04), 877-908. }\end{array}$ & $\begin{array}{l}\text { No child } \\
\text { internalising } \\
\text { outcomes or not } \\
\text { a major goal }\end{array}$ \\
\hline $\begin{array}{l}\text { 1653. Tough SC, Johnston DW, Siever JE, Jorgenson G, Slocombe L, } \\
\text { Lane C and Clarke M. 2006. Does supplementary prenatal nursing and } \\
\text { home visitation support improve resource use in a universal health } \\
\text { care system? A randomized controlled trial in Canada. Birth, 33(3), } \\
\text { 183-194. }\end{array}$ & $\begin{array}{l}\text { No child } \\
\text { internalising } \\
\text { outcomes or not } \\
\text { a major goal }\end{array}$ \\
\hline $\begin{array}{l}\text { 1654. Tough SC, Siever JE and Johnston DW. 2007. Retaining women } \\
\text { in a prenatal care randomized controlled trial in Canada: implications } \\
\text { for program planning. BMC Public Health. 7:148. }\end{array}$ & $\begin{array}{l}\text { No child } \\
\text { internalising } \\
\text { outcomes or not } \\
\text { a major goal }\end{array}$ \\
\hline $\begin{array}{l}\text { 1655. Toulabi, T., Khosh Niyat Nikoo, M., Amini, F., Nazari, H., \& } \\
\text { Mardani, M. (2012). The influence of a behavior modification } \\
\text { interventional program on body mass index in obese adolescents. J } \\
\text { Formos Med Assoc, } 111,153-159 .\end{array}$ & $\begin{array}{l}\text { No long term } \\
\text { follow-up }\end{array}$ \\
\hline $\begin{array}{l}\text { 1656. Toumbourou JW and Gregg ME. 2002. Impact of an } \\
\text { empowerment-based parent education program on the reduction of } \\
\text { youth suicide risk factors. Journal of Adolescent Health, 31(3), 277- } \\
285 \text {. }\end{array}$ & $\begin{array}{l}\text { No long term } \\
\text { follow-up }\end{array}$ \\
\hline
\end{tabular}




\begin{tabular}{|c|c|}
\hline $\begin{array}{l}\text { 1657. Tourigny J. 1998. [Effects of a preoperative educational } \\
\text { intervention on the behavior of parents of three to six-year old } \\
\text { children having day surgery]. Can J Nurs Res. 30(2):135-59. }\end{array}$ & $\begin{array}{l}\text { Intervention for } \\
\text { developmental } \\
\text { disorders } \\
\text { (autism, autism } \\
\text { spectrum } \\
\text { disorder), } \\
\text { physical } \\
\text { disabilities, } \\
\text { medical } \\
\text { conditions or } \\
\text { distress about } \\
\text { facing medical } \\
\text { interventions }\end{array}$ \\
\hline $\begin{array}{l}\text { 1658. Tourigny M, Peladeau N, Doyon M and Bouchard C. } 1998 . \\
\text { [Efficacy of a treatment program for sexually abused children]. Child } \\
\text { Abuse Negl. 22(1):25-43. }\end{array}$ & Not an RCT \\
\hline $\begin{array}{l}\text { 1659. Treiber FA. 1986. A comparison of the positive and negative } \\
\text { consequences approaches upon car restraint usage. Journal of } \\
\text { Pediatric Psychology. 11(1):15-24. }\end{array}$ & $\begin{array}{l}\text { No child } \\
\text { internalising } \\
\text { outcomes or not } \\
\text { a major goal }\end{array}$ \\
\hline $\begin{array}{l}\text { 1660. Trejos-Castillo E and Vazsonyi AT. 2009. Risky sexual behaviors } \\
\text { in first and second generation Hispanic immigrant youth. Journal of } \\
\text { Youth and Adolescence. 38(5):719-31. }\end{array}$ & $\begin{array}{l}\text { Not a parenting } \\
\text { intervention }\end{array}$ \\
\hline $\begin{array}{l}\text { 1661. Tremblay RE, Pagani-Kurtz L, MVCsse LC, Vitaro F and Pihl RO. } \\
\text { 1995. A bimodal preventive intervention for disruptive kindergarten } \\
\text { boys: Its impact through mid-adolescence. Journal of Consulting and } \\
\text { Clinical Psychology. 63(4):560-8. }\end{array}$ & $\begin{array}{l}\text { No child } \\
\text { internalising } \\
\text { outcomes or not } \\
\text { a major goal }\end{array}$ \\
\hline $\begin{array}{l}\text { 1662. Trentacosta CJ, Hyde LW, Shaw DS, Dishion TJ, Gardner F and } \\
\text { Wilson M. 2008. The relations among cumulative risk, parenting, and } \\
\text { behavior problems during early childhood. Journal of Child Psychology } \\
\text { and Psychiatry. 49(11):1211-9. }\end{array}$ & Not an RCT \\
\hline $\begin{array}{l}\text { 1663. Treyvaud K, Rogers S, Matthews J and Allen B. } 2009 . \\
\text { Outcomes following an early parenting center residential parenting } \\
\text { program. J Fam Nurs. 15(4):486-501. }\end{array}$ & Not an RCT \\
\hline $\begin{array}{l}\text { 1664. Trocchio, J. S. (2014). Predictors of change in stress, } \\
\text { interaction styles, and depression in parents of toddlers with autism. } \\
\text { Dissertation Abstracts International Section A: Humanities and Social } \\
\text { Sciences, } 74 \text { (11-A E), }\end{array}$ & $\begin{array}{l}\text { Intervention for } \\
\text { developmental } \\
\text { disorders } \\
\text { (autism, autism } \\
\text { spectrum } \\
\text { disorder), } \\
\text { physical } \\
\text { disabilities, } \\
\text { medical } \\
\text { conditions or } \\
\text { distress about } \\
\text { facing medical } \\
\text { interventions }\end{array}$ \\
\hline
\end{tabular}




\begin{tabular}{|c|c|}
\hline $\begin{array}{l}1665 . \quad \text { Trudeau, L., Spoth, R., Randall, G. K., \& Azevedo, K. (2007). } \\
\text { Longitudinal effects of a universal family-focused intervention on } \\
\text { growth patterns of adolescent internalizing symptoms and } \\
\text { polysubstance use: Gender comparisons. Journal of Youth and } \\
\text { Adolescence, 36(6), 725-740. doi:10.1007/s10964-007-9179-1 }\end{array}$ & $\begin{array}{l}\text { No child } \\
\text { internalising } \\
\text { outcomes or not } \\
\text { a major goal }\end{array}$ \\
\hline $\begin{array}{l}\text { 1666. Trudeau, L., Spoth, R., Randall, G. K., Mason, W. A., \& Shin, C. } \\
\text { (2012). Internalizing symptoms: Effects of a preventive intervention on } \\
\text { developmental pathways from early adolescence to young adulthood. } \\
\text { Journal of Youth and Adolescence, } 41(6), 788-801 . \\
\text { doi:10.1007/s10964-011-9735-6 }\end{array}$ & $\begin{array}{l}\text { No child } \\
\text { internalising } \\
\text { outcomes or not } \\
\text { a major goal }\end{array}$ \\
\hline $\begin{array}{l}1667 . \quad \text { Tsai LY. 1999. Psychopharmacology in autism. Psychosomatic } \\
\text { Medicine. 61(5):651-65. }\end{array}$ & Not an RCT \\
\hline $\begin{array}{l}\text { 1668. Tuerk EH. 2008. Parenting from the inside: Assessing a } \\
\text { curriculum for incarcerated mothers. Dissertation Abstracts } \\
\text { International: Section B: The Sciences and Engineering. 68(11-B). }\end{array}$ & $\begin{array}{l}\text { No long term } \\
\text { follow-up }\end{array}$ \\
\hline $\begin{array}{l}\text { 1669. Turnbull JE, George LK, Landerman R, Swartz MS and Blazer } \\
\text { DG. 1990. Social outcomes related to age of onset among psychiatric } \\
\text { disorders. Journal of Consulting and Clinical Psychology. 58(6):832-9. }\end{array}$ & $\begin{array}{l}\text { Not a parenting } \\
\text { intervention }\end{array}$ \\
\hline $\begin{array}{l}\text { 1670. Turner KMT, Richards M and Sanders MR. 2007. Randomised } \\
\text { clinical trial of a group parent education programme for Australian } \\
\text { Indigenous families. Journal of Paediatrics \& Child Health. 43(6):429- } \\
37 .\end{array}$ & $\begin{array}{l}\text { Follow-up data } \\
\text { collected only in } \\
\text { active group }\end{array}$ \\
\hline $\begin{array}{l}\text { 1671. Turner, K. M., \& Sanders, M. R. (2006). Help when it's needed } \\
\text { first: a controlled evaluation of brief, preventive behavioral family } \\
\text { intervention in a primary care setting. Behav Ther, 37(2), 131-142. doi: } \\
\text { S0005-7894(06)00015-3 }\end{array}$ & $\begin{array}{l}\text { No child } \\
\text { internalising } \\
\text { outcomes or not } \\
\text { a major goal }\end{array}$ \\
\hline $\begin{array}{l}\text { 1672. Tyc VL, Mulhern RK, Fairclough D, Ward PM, Relling MV and } \\
\text { Longmire W. 1993. Chemotherapy induced nausea and emesis in } \\
\text { pediatric cancer patients: external validity of child and parent emesis } \\
\text { ratings. Journal of Developmental \& Behavioral Pediatrics, 14(4), 236- } \\
241 .\end{array}$ & $\begin{array}{l}\text { Not a parenting } \\
\text { intervention }\end{array}$ \\
\hline $\begin{array}{l}\text { 1673. Tyson PD and Sobschak KB. 1994. Perceptual responses to } \\
\text { infant crying after EEG biofeedback assisted stress management } \\
\text { training: implications for physical child abuse. Child Abuse Negl. } \\
\text { 18(11):933-43. }\end{array}$ & $\begin{array}{l}\text { No child } \\
\text { internalising } \\
\text { outcomes or not } \\
\text { a major goal }\end{array}$ \\
\hline $\begin{array}{l}\text { 1674. Tyson PD. 1996. Biodesensitization: biofeedback-controlled } \\
\text { systematic desensitization of the stress response to infant crying. } \\
\text { Biofeedback Self Regul. 21(3):273-90. }\end{array}$ & $\begin{array}{l}\text { No child } \\
\text { internalising } \\
\text { outcomes or not } \\
\text { a major goal }\end{array}$ \\
\hline $\begin{array}{l}\text { 1675. Uddin, M., de los Santos, R., Bakshis, E., Cheng, C., \& Aiello, A. } \\
\text { E. (2011). Building conditions, 5-HTTLPR genotype, and depressive } \\
\text { symptoms in adolescent males and females. Journal of Adolescent } \\
\text { Health, 49, 379-385. }\end{array}$ & $\begin{array}{l}\text { Not a parenting } \\
\text { intervention }\end{array}$ \\
\hline $\begin{array}{l}\text { 1676. Unterhitzenberger, J., \& Rosner, R. (2014). Lessons from } \\
\text { writing sessions: a school-based randomized trial with adolescent } \\
\text { orphans in Rwanda. European journal of psychotraumatology, } 5 .\end{array}$ & $\begin{array}{l}\text { Not a parenting } \\
\text { intervention }\end{array}$ \\
\hline $\begin{array}{l}\text { 1677. Upshur C, Wenz-Gross M and Reed G. 2009. A pilot study of } \\
\text { early childhood mental health consultation for children with } \\
\text { behavioral problems in preschool. Early Childhood Research Quarterly. } \\
\text { 24(1):29-45. }\end{array}$ & $\begin{array}{l}\text { Not a parenting } \\
\text { intervention }\end{array}$ \\
\hline
\end{tabular}




\begin{tabular}{|c|c|}
\hline $\begin{array}{l}\text { 1678. Urquiza, A. J., \& McNeil, C. B. (1996). Parent-Child Interaction } \\
\text { Therapy: An Intensive Dyadic Intervention for Physically Abusive } \\
\text { Families. Child Maltreatment, 1(2), 134-144. doi: } \\
\text { 10.1177/1077559596001002005 }\end{array}$ & Not an RCT \\
\hline $\begin{array}{l}\text { 1679. Uslu, R., Kapci, E. G., \& Erden, G. (2006). Psychoeducation and } \\
\text { expressed emotion by parents of children with learning disorders. } \\
\text { Psychological reports, (2), 291-306. }\end{array}$ & $\begin{array}{l}\text { No long term } \\
\text { follow-up }\end{array}$ \\
\hline $\begin{array}{l}\text { 1680. Uzark K, Klos D, Davis W and Rosenthal A. 1982. Use of } \\
\text { videotape in the preparation of children for cardiac catheterization. } \\
\text { Pediatr Cardiol. 3(4):287-91. }\end{array}$ & $\begin{array}{l}\text { Intervention for } \\
\text { developmental } \\
\text { disorders } \\
\text { (autism, autism } \\
\text { spectrum } \\
\text { disorder), } \\
\text { physical } \\
\text { disabilities, } \\
\text { medical } \\
\text { conditions or } \\
\text { distress about } \\
\text { facing medical } \\
\text { interventions }\end{array}$ \\
\hline $\begin{array}{l}\text { 1681. Vagnoli L, Caprilli S, Robiglio A and Messeri A. 2005. Clown } \\
\text { doctors as a treatment for preoperative anxiety in children: a } \\
\text { randomized, prospective study. Pediatrics. 116(4):e563-7. }\end{array}$ & $\begin{array}{l}\text { Intervention for } \\
\text { developmental } \\
\text { disorders } \\
\text { (autism, autism } \\
\text { spectrum } \\
\text { disorder), } \\
\text { physical } \\
\text { disabilities, } \\
\text { medical } \\
\text { conditions or } \\
\text { distress about } \\
\text { facing medical } \\
\text { interventions }\end{array}$ \\
\hline $\begin{array}{l}\text { 1682. Valdez CR, Mills CL, Barrueco S, Leis J and Riley AW. 2011. A } \\
\text { pilot study of a family-focused intervention for children and families } \\
\text { affected by maternal depression. Journal of Family Therapy. 33(1):3- } \\
\text { 19. }\end{array}$ & Not an RCT \\
\hline $\begin{array}{l}\text { 1683. Valentino, K., Comas, M., Nuttall, A. K., \& Thomas, T. (2013). } \\
\text { Training maltreating parents in elaborative and emotion-rich } \\
\text { reminiscing with their preschool-aged children. Child abuse \& neglect, } \\
\text { 37(8), 585-595. doi:10.1016/j.chiabu.2013.02.010 }\end{array}$ & $\begin{array}{l}\text { No child } \\
\text { internalising } \\
\text { outcomes or not } \\
\text { a major goal }\end{array}$ \\
\hline $\begin{array}{l}\text { 1684. van Aar, J. V., Asscher, J. J., Zijlstra, B. J., Deković, M., \& } \\
\text { Hoffenaar, P. J. (2015). Changes in parenting and child behavior after } \\
\text { the home-start family support program: A 10year follow-up. Children } \\
\text { and Youth Services Review, 53, 166-175. }\end{array}$ & Not an RCT \\
\hline $\begin{array}{l}\text { 1685. van den Hoofdakker BJ, van der Veen-Mulders L, Sytema S, } \\
\text { Emmelkamp PM, Minderaa RB, Nauta MH. Effectiveness of behavioral } \\
\text { parent training for children with ADHD in routine clinical practice: a } \\
\text { randomized controlled study. J Am Acad Child Adolesc Psychiatry. } \\
2007 \text { Oct; } 46(10): 1263-71 .\end{array}$ & $\begin{array}{l}\text { Follow-up data } \\
\text { collected only in } \\
\text { active group }\end{array}$ \\
\hline
\end{tabular}




\begin{tabular}{|c|c|}
\hline $\begin{array}{l}1686 . \quad \text { van den Hoofdakker, B. J., Hoekstra, P. J., van der Veen- } \\
\text { Mulders, L., Sytema, S., Emmelkamp, P. M., Minderaa, R. B., \& Nauta, } \\
\text { M. H. (2014). Paternal influences on treatment outcome of behavioral } \\
\text { parent training in children with attention-deficit/hyperactivity } \\
\text { disorder. European child \& adolescent psychiatry, 23(11), 1071-1079. }\end{array}$ & $\begin{array}{l}\text { Targets } \\
\text { externalising; No } \\
\text { child } \\
\text { internalising } \\
\text { outcomes or not } \\
\text { a major goal; No } \\
\text { long term } \\
\text { follow-up }\end{array}$ \\
\hline $\begin{array}{l}1687 . \quad \text { van den Hoofdakker, B. J., Nauta, M. H., van der Veen- } \\
\text { Mulders, L., Sytema, S., Emmelkamp, P. M., Minderaa, R. B., et al. } \\
\text { (2010). Behavioral parent training as an adjunct to routine care in } \\
\text { children with attention-deficit/hyperactivity disorder: moderators of } \\
\text { treatment response. J Pediatr Psychol, 35(3), 317-326. doi: jsp060 }\end{array}$ & $\begin{array}{l}\text { No long term } \\
\text { follow-up }\end{array}$ \\
\hline $\begin{array}{l}\text { 1688. van der Heijden KB, de Sonneville LMJ and Althaus M. } 2010 . \\
\text { Time-of-day effects on cognition in preadolescents: A TRAILS study. } \\
\text { Chronobiology International. 27(9-10):1870-94. }\end{array}$ & $\begin{array}{l}\text { Not a parenting } \\
\text { intervention }\end{array}$ \\
\hline $\begin{array}{l}1689 . \quad \text { van der Leeden, A. J. M., van Widenfelt, B. M., van der Leeden, } \\
\text { R., Liber, J. M., Utens, E. M. W. J., \& Treffers, P. D. A. (2011). Stepped } \\
\text { care cognitive behavioural therapy for children with anxiety disorders: } \\
\text { A new treatment approach. Behavioural and Cognitive Psychotherapy, } \\
\text { 39(1), 55-75. doi: 10.1017/s1352465810000500 }\end{array}$ & $\begin{array}{l}\text { Not an RCT; } \\
\text { Parent } \\
\text { component too } \\
\text { small }\end{array}$ \\
\hline $\begin{array}{l}\text { 1690. van der Oord S, Prins PJM, Oosterlaan J and Emmelkamp PMG. } \\
\text { 2008. Treatment of attention deficit hyperactivity disorder in children: } \\
\text { Predictors of treatment outcome. European Child \& Adolescent } \\
\text { Psychiatry. 17(2):73-81. }\end{array}$ & $\begin{array}{l}\text { Not a parenting } \\
\text { intervention }\end{array}$ \\
\hline $\begin{array}{l}\text { 1691. van der Oord, S., Prins, P. J., Oosterlaan, J., \& Emmelkamp, P. } \\
\text { M. (2007). Does brief, clinically based, intensive multimodal behavior } \\
\text { therapy enhance the effects of methylphenidate in children with } \\
\text { ADHD? Eur Child Adolesc Psychiatry, 16(1), 48-57. doi: } \\
\text { 10.1007/s00787-006-0574-z }\end{array}$ & $\begin{array}{l}\text { No long term } \\
\text { follow-up }\end{array}$ \\
\hline $\begin{array}{l}\text { 1692. Van Dijk, E. M., Huisman, J., Braam, K. I., Van Dulmen-den } \\
\text { Broeder, E., Veening, M. A., Helder, P. J. M., Sinnema, G., Grootenhuis, } \\
\text { M. A., Merks, J. H., Takken, T., \& Kaspers, G. J. L. (2011). Quality of life } \\
\text { in motion: A combined physical exercise and psychosocial intervention } \\
\text { program for childhood cancer patients. Pediatric Blood and Cancer, } \\
56,1156-1157 .\end{array}$ & $\begin{array}{l}\text { Data not } \\
\text { available/report } \\
\text { ed (protocol) }\end{array}$ \\
\hline $\begin{array}{l}\text { 1693. van Dijk-Lokkart, E. M., Braam, K. I., Kaspers, G. J. L., Veening, } \\
\text { M. A., Grootenhuis, M. A., Streng, I., ... \& Huisman, J. (2014). Effects } \\
\text { on quality of life of participation in a combined physical exercise and } \\
\text { psychosocial intervention program for childhood cancer patients. } \\
\text { Pediatric blood \& cancer, } 61 \text { S160. }\end{array}$ & $\begin{array}{l}\text { Not a parenting } \\
\text { intervention }\end{array}$ \\
\hline $\begin{array}{l}\text { 1694. Van Holen, F., Vanderfaeillie, J., De Maeyer, S., \& Gypen, L. } \\
\text { (2015). Does allocation to a control condition in a Randomized } \\
\text { Controlled Trial affect the routine care foster parents receive?. } \\
\text { Children and Youth Services Review, 49, 48-53. }\end{array}$ & $\begin{array}{l}\text { Data not } \\
\text { available/report } \\
\text { ed }\end{array}$ \\
\hline $\begin{array}{l}\text { 1695. van Loon, L. M. A., Granic, I., \& Engels, R. C. M. E. (2011). The } \\
\text { role of maternal depression on treatment outcome for children with } \\
\text { externalizing behavior problems. Journal of Psychopathology and } \\
\text { Behavioral Assessment, 33, 178-186. }\end{array}$ & $\begin{array}{l}\text { Targets } \\
\text { externalising }\end{array}$ \\
\hline
\end{tabular}




\begin{tabular}{|c|c|}
\hline $\begin{array}{l}\text { 1696. Van Nuil, J. I., Mutwa, P., Asiimwe-Kateera, B., Kestelyn, E., } \\
\text { Vyankandondera, J., Pool, R., ... \& Boer, K. R. (2014). (2014). Let's talk } \\
\text { about sex: a qualitative study of Rwandan adolescents' views on sex } \\
\text { and HIV. PLoS One, } 9 \text { (8), e102933. }\end{array}$ & Not an RCT \\
\hline $\begin{array}{l}\text { 1697. van Oort, F. V. A., Greaves?Lord, K., Ormel, J., Verhulst, F. C., \& } \\
\text { Huizink, A. C. (2011). Risk indicators of anxiety throughout } \\
\text { adolescence: The TRAILS study. Depress Anxiety, 28, 485-494. }\end{array}$ & $\begin{array}{l}\text { Not a parenting } \\
\text { intervention }\end{array}$ \\
\hline $\begin{array}{l}\text { 1698. van Santvoort, F., Hosman, C. M., van Doesum, K. T., \& } \\
\text { Janssens, J. M. (2014). Effectiveness of preventive support groups for } \\
\text { children of mentally ill or addicted parents: a randomized controlled } \\
\text { trial. European child \& adolescent psychiatry, 23(6), 473-484. }\end{array}$ & $\begin{array}{l}\text { Not a parenting } \\
\text { intervention }\end{array}$ \\
\hline $\begin{array}{l}\text { 1699. Van Voorhees BW, Paunesku D, Fogel J and Bell CC. } 2009 . \\
\text { Differences in vulnerability factors for depressive episodes in African } \\
\text { American and European American adolescents. Journal of the National } \\
\text { Medical Association. 101(12):1255-67. }\end{array}$ & $\begin{array}{l}\text { Not a parenting } \\
\text { intervention }\end{array}$ \\
\hline $\begin{array}{l}\text { 1700. Van Zeijl, J., Mesman, J., Van, I. M. H., Bakermans-Kranenburg, } \\
\text { M. J., Juffer, F., Stolk, M. N., et al. (2006). Attachment-based } \\
\text { intervention for enhancing sensitive discipline in mothers of 1- to 3- } \\
\text { year-old children at risk for externalizing behavior problems: a } \\
\text { randomized controlled trial. J Consult Clin Psychol, } 74(6), 994-1005 . \\
\text { doi: } 2006-22003-002\end{array}$ & $\begin{array}{l}\text { No long term } \\
\text { follow-up }\end{array}$ \\
\hline $\begin{array}{l}\text { 1701. Vanderbilt D, Bushley T, Young R and Frank DA. 2009. Acute } \\
\text { posttraumatic stress symptoms among urban mothers with newborns } \\
\text { in the Neonatal Intensive Care Unit; A preliminary study. Journal of } \\
\text { Developmental and Behavioral Pediatrics. 30(1):50-6. }\end{array}$ & $\begin{array}{l}\text { Not a parenting } \\
\text { intervention }\end{array}$ \\
\hline $\begin{array}{l}1702 . \quad \text { Varley CK and Smith CJ. 2003. Anxiety disorders in the child } \\
\text { and teen. Pediatric Clinics of North America. 50(5):1107-38. }\end{array}$ & Review \\
\hline $\begin{array}{l}\text { 1703. Varni JW, Katz ER, Colegrove R, Jr. and Dolgin M. 1993. The } \\
\text { impact of social skills training on the adjustment of children with } \\
\text { newly diagnosed cancer. J Pediatr Psychol. 18(6):751-67. }\end{array}$ & $\begin{array}{l}\text { Not a parenting } \\
\text { intervention }\end{array}$ \\
\hline $\begin{array}{l}\text { 1704. Verdurmen, J. E. E., Koning, I. M., Vollebergh, W. A. M., van } \\
\text { den Eijnden, R. J. J. M., \& Engels, R. C. M. E. (2014). Risk moderation of } \\
\text { a parent and student preventive alcohol intervention by adolescent } \\
\text { and family factors: A cluster randomized trial. Preventive Medicine, } \\
\text { 60, 88-94. doi: http://dx.doi.org/10.1016/j.ypmed.2013.12.027 }\end{array}$ & $\begin{array}{l}\text { No child } \\
\text { internalising } \\
\text { outcomes or not } \\
\text { a major goal }\end{array}$ \\
\hline $\begin{array}{l}\text { 1705. Verreault M and Berthiaume C. 2010. Efficacy of a cognitive- } \\
\text { behavioral therapy for children with a comorbid attention-deficit with } \\
\text { hyperactivity disorder and anxiety disorder. Journal de Thérapie } \\
\text { Comportementale et Cognitive. } 20(3): 93-8 \text {. }\end{array}$ & Not an RCT \\
\hline $\begin{array}{l}\text { 1706. Vines SW and Williams Burgess C. 1994. Effects of a } \\
\text { community health nursing parent-baby (ad)venture program on } \\
\text { depression and other selected maternal-child health outcomes. Public } \\
\text { Health Nursing, 11(3), 188-194. }\end{array}$ & $\begin{array}{l}\text { No child } \\
\text { internalising } \\
\text { outcomes or not } \\
\text { a major goal }\end{array}$ \\
\hline $\begin{array}{l}\text { 1707. Vırıt, O., Akbaş, E., Savaş, H. A., Sertbaş, G., \& Kandemir, H. } \\
\text { (2008). Association between the level of depression and anxiety with } \\
\text { social support in pregnancy. Archives of Neuropsychiatry, 45(1), 9-13. }\end{array}$ & $\begin{array}{l}\text { Not a parenting } \\
\text { intervention }\end{array}$ \\
\hline $\begin{array}{l}\text { 1708. Visser, M. M., Telman, M. D., de Schipper, J. C., Lamers- } \\
\text { Winkelman, F., Schuengel, C., \& Finkenauer, C. (2015). The effects of } \\
\text { parental components in a trauma-focused cognitive behavioral based } \\
\text { therapy for children exposed to interparental violence: study protocol } \\
\text { for a randomized controlled trial. BMC psychiatry, 15(1), } 131 .\end{array}$ & $\begin{array}{l}\text { Data not } \\
\text { available/report } \\
\text { ed }\end{array}$ \\
\hline
\end{tabular}




\begin{tabular}{|c|c|}
\hline $\begin{array}{l}\text { 1709. Vitaro F and Tremblay R. 1994. Impact of a prevention } \\
\text { program on aggressive children's friendships and social adjustment. } \\
\text { Journal of Abnormal Child Psychology. 22(4):457-75. }\end{array}$ & $\begin{array}{l}\text { No child } \\
\text { internalising } \\
\text { outcomes or not } \\
\text { a major goal }\end{array}$ \\
\hline $\begin{array}{l}\text { 1710. Vitiello B, Kratochvil CJ, Silva S, Curry J, Reinecke M, Pathak S, } \\
\text { Waslick B, Hughes CW, Prentice ED, May DE and March JS. } 2007 . \\
\text { Research knowledge among the participants in the Treatment for } \\
\text { Adolescents With Depression Study (TADS). J Am Acad Child Adolesc } \\
\text { Psychiatry. 46(12):1642-50. }\end{array}$ & $\begin{array}{l}\text { Not a parenting } \\
\text { intervention }\end{array}$ \\
\hline $\begin{array}{l}\text { 1711. von Wyl A, Watson M, Glanzmann R and von Klitzing K. } 2008 . \\
\text { [The Basel interdisciplinary clinic for parents with infants and toddlers: } \\
\text { concept and empirical results]. Prax Kinderpsychol Kinderpsychiatr. } \\
57(3): 216-36 \text {. }\end{array}$ & Not an RCT \\
\hline $\begin{array}{l}\text { 1712. Vrieze, D. M. (2011). The role of parental reflective functioning } \\
\text { in promoting attachment for children of depressed mothers in a } \\
\text { toddler-parent psychotherapeutic intervention (Order No. 3478511). } \\
\text { Available from ProQuest Dissertations \& Theses Global. (902742474). }\end{array}$ & $\begin{array}{l}\text { No long term } \\
\text { follow-up }\end{array}$ \\
\hline $\begin{array}{l}\text { 1713. Waddell C, Lavis JN, Abelson J, Lomas J, Shepherd CA, Bird- } \\
\text { Gayson T, Giacomini M and Offord DR. 2005. Research use in } \\
\text { children's mental health policy in Canada: Maintaining vigilance amid } \\
\text { ambiguity. Social Science \& Medicine. 61(8):1649-57. }\end{array}$ & Not an RCT \\
\hline $\begin{array}{l}\text { 1714. Wade SL, Carey J and Wolfe CR. 2006. An online family } \\
\text { intervention to reduce parental distress following pediatric brain } \\
\text { injury. Journal of consulting and clinical psychology, 74(3), } 445 .\end{array}$ & $\begin{array}{l}\text { Intervention for } \\
\text { developmental } \\
\text { disorders } \\
\text { (autism, autism } \\
\text { spectrum } \\
\text { disorder), } \\
\text { physical } \\
\text { disabilities, } \\
\text { medical } \\
\text { conditions or } \\
\text { distress about } \\
\text { facing medical } \\
\text { interventions }\end{array}$ \\
\hline $\begin{array}{l}\text { 1715. Wade SL, Michaud L and Brown TM. 2006. Putting the pieces } \\
\text { together: preliminary efficacy of a family problem-solving intervention } \\
\text { for children with traumatic brain injury. J Head Trauma Rehabil. } \\
\text { 21(1):57-67. }\end{array}$ & $\begin{array}{l}\text { Intervention for } \\
\text { developmental } \\
\text { disorders } \\
\text { (autism, autism } \\
\text { spectrum } \\
\text { disorder), } \\
\text { physical } \\
\text { disabilities, } \\
\text { medical } \\
\text { conditions or } \\
\text { distress about } \\
\text { facing medical } \\
\text { interventions }\end{array}$ \\
\hline $\begin{array}{l}\text { 1716. Wade, S. L., Karver, C. L., Taylor, H. G., Cassedy, A., Stancin, T., } \\
\text { Kirkwood, M. W., \& Brown, T. M. (2014). Counselor-assisted problem } \\
\text { solving improves caregiver efficacy following adolescent brain injury. } \\
\text { Rehabil Psychol, 59(1), 1-9. doi: } 10.1037 / \text { a0034911 }\end{array}$ & $\begin{array}{l}\text { No child } \\
\text { internalising } \\
\text { outcomes or not } \\
\text { a major goal }\end{array}$ \\
\hline
\end{tabular}




\begin{tabular}{|c|c|}
\hline $\begin{array}{l}\text { 1717. Wade, S. L., Walz, N. C., Carey, J. C., \& Williams, K. M. (2008). } \\
\text { Preliminary efficacy of a Web-based family problem-solving treatment } \\
\text { program for adolescents with traumatic brain injury. J Head Trauma } \\
\text { Rehabil, 23(6), 369-377. doi:10.1097/01.HTR.0000341432.67251.48 }\end{array}$ & $\begin{array}{l}\text { No long term } \\
\text { follow-up }\end{array}$ \\
\hline $\begin{array}{l}\text { 1718. Wade, S. L., Walz, N. C., Carey, J., McMullen, K. M., Cass, J., } \\
\text { Mark, E., \& Yeates, K. O. (2011). Effect on behavior problems of teen } \\
\text { online problem-solving for adolescent traumatic brain injury. } \\
\text { Pediatrics, } 128 \text {, e947-953. }\end{array}$ & $\begin{array}{l}\text { Not a parenting } \\
\text { intervention }\end{array}$ \\
\hline $\begin{array}{l}\text { 1719. Wadsby, M., \& Arvidsson, E. (2010). Eight years after-follow- } \\
\text { up study of mothers and children at psychosocial risk who received } \\
\text { early treatment: Does early intervention leave its mark? Child \& } \\
\text { Family Social Work, 15(4), 452-460. doi: } 10.1111 / \text { j.1365- } \\
\text { 2206.2010.00696.x }\end{array}$ & Not an RCT \\
\hline $\begin{array}{l}\text { 1720. Wadsworth, M. E., Santiago, C. D., Einhorn, L., Etter, E. M., } \\
\text { Rienks, S., \& Markman, H. (2011). Preliminary efficacy of an } \\
\text { intervention to reduce psychosocial stress and improve coping in low- } \\
\text { income families. Am J Community Psychol, 48, 257-271. }\end{array}$ & $\begin{array}{l}\text { No long term } \\
\text { follow-up }\end{array}$ \\
\hline $\begin{array}{l}\text { 1721. Wagner JL, Chaney JM, Hommel KA, Page MC, Mullins LL, } \\
\text { White MM and Jarvis JN. 2003. The Influence of Parental Distress on } \\
\text { Child Depressive Symptoms in Juvenile Rheumatic Diseases: The } \\
\text { Moderating Effect of Illness Intrusiveness. Journal of Pediatric } \\
\text { Psychology. 28(7):453-62. }\end{array}$ & $\begin{array}{l}\text { Not a parenting } \\
\text { intervention }\end{array}$ \\
\hline $\begin{array}{l}\text { 1722. Wagner KD, Berard R, Stein MB, Wetherhold E, Carpenter DJ, } \\
\text { Perera P, Gee M, Davy K and Machin A. 2004. A Multicenter, } \\
\text { Randomized, Double-blind, Placebo-Controlled Trial of Paroxetine in } \\
\text { Children and Adolescents with Social Anxiety Disorder. Archives of } \\
\text { General Psychiatry. 61(11):1153-62. }\end{array}$ & $\begin{array}{l}\text { Not a parenting } \\
\text { intervention }\end{array}$ \\
\hline $\begin{array}{l}\text { 1723. Wahler, R. G. (1980). The Insular Mother - Her Problems in } \\
\text { Parent-Child Treatment. Journal of Applied Behavior Analysis, 13(2), } \\
\text { 207-219. }\end{array}$ & Not an RCT \\
\hline $\begin{array}{l}\text { 1724. Waisman Y, Siegal N, Siegal G, Amir L, Cohen H and Mimouni } \\
\text { M. 2005. Role of diagnosis-specific information sheets in parents' } \\
\text { understanding of emergency department discharge instructions. Eur J } \\
\text { Emerg Med. 12(4):159-62. }\end{array}$ & $\begin{array}{l}\text { No child } \\
\text { internalising } \\
\text { outcomes or not } \\
\text { a major goal }\end{array}$ \\
\hline $\begin{array}{l}\text { 1725. Wakefield, C. E., Sansom-Daly, U. M., McGill, B. C., McCarthy, } \\
\text { M., Girgis, A., Grootenhuis, M., ... \& Cohn, R. J. (2015). Online parent- } \\
\text { targeted cognitive-behavioural therapy intervention to improve } \\
\text { quality of life in families of young cancer survivors: study protocol for } \\
\text { a randomised controlled trial. Trials, 16(1), } 153 .\end{array}$ & $\begin{array}{l}\text { Data not } \\
\text { available/report } \\
\text { ed }\end{array}$ \\
\hline
\end{tabular}




\begin{tabular}{|c|c|}
\hline $\begin{array}{l}\text { 1726. Wakimizu R, Kamagata S, Kuwabara T and Kamibeppu K. } 2009 . \\
\text { A randomized controlled trial of an at-home preparation programme } \\
\text { for Japanese preschool children: effects on children's and caregivers' } \\
\text { anxiety associated with surgery. J Eval Clin Pract. 15(2):393-401. }\end{array}$ & $\begin{array}{l}\text { Intervention for } \\
\text { developmental } \\
\text { disorders } \\
\text { (autism, autism } \\
\text { spectrum } \\
\text { disorder), } \\
\text { physical } \\
\text { disabilities, } \\
\text { medical } \\
\text { conditions or } \\
\text { distress about } \\
\text { facing medical } \\
\text { interventions }\end{array}$ \\
\hline $\begin{array}{l}\text { 1727. Walker JG. 2008. A marital intervention program for couples } \\
\text { with chronically ill children. Dissertation Abstracts International: } \\
\text { Section B: The Sciences and Engineering. 68(7-B). }\end{array}$ & $\begin{array}{l}\text { No child } \\
\text { internalising } \\
\text { outcomes or not } \\
\text { a major goal }\end{array}$ \\
\hline $\begin{array}{l}\text { 1728. Walker, H. M., Seeley, J. R., Small, J., Severson, H. H., Graham, } \\
\text { B. A., Feil, E. G., et al. (2009). A randomized controlled trial of the first } \\
\text { step to success early intervention: Demonstration of program efficacy } \\
\text { outcomes in a diverse, urban school district. Journal of Emotional and } \\
\text { Behavioral Disorders, } 17(4), 197-212 \text {. doi: } \\
\text { 10.1177/1063426609341645 }\end{array}$ & $\begin{array}{l}\text { Parent } \\
\text { component too } \\
\text { small; No long } \\
\text { term follow-up }\end{array}$ \\
\hline $\begin{array}{l}\text { 1729. Walkup JT, Labellarte MJ, Riddle MA, Pine D, Greenhill L, Klein } \\
\text { R, Davies M, Sweeney M, Fu C, Abikoff H, Hack S, Klee B, McCracken J, } \\
\text { Bergman L, Piacentini J, March J, Compton S, Robinson J, O'Hara T, } \\
\text { Baker S, Vitiello B, Ritz L and Roper M. 2003. Searching for moderators } \\
\text { and mediators of pharmacological treatment effects in children and } \\
\text { adolescents with anxiety disorders. J Am Acad Child Adolesc } \\
\text { Psychiatry. 42(1):13-21. }\end{array}$ & $\begin{array}{l}\text { Not a parenting } \\
\text { intervention }\end{array}$ \\
\hline $\begin{array}{l}\text { 1730. Wallander, J. L., McClure, E., Biasini, F., Goudar, S. S., Pasha, } \\
\text { O., Chomba, E., et al. (2010). Brain research to ameliorate impaired } \\
\text { neurodevelopment--home-based intervention trial (BRAIN-HIT). BMC } \\
\text { pediatrics, } 27 .\end{array}$ & $\begin{array}{l}\text { Data not } \\
\text { available/report } \\
\text { ed (protocol) }\end{array}$ \\
\hline $\begin{array}{l}\text { 1731. Wallen M and Stagnitti K. 2006. There was insufficient } \\
\text { evidence to conclude whether parent-mediated early intervention was } \\
\text { effective for children with autism: Commentary. Australian } \\
\text { Occupational Therapy Journal. 53(2):137-9. }\end{array}$ & $\begin{array}{l}\text { Intervention for } \\
\text { developmental } \\
\text { disorders } \\
\text { (autism, autism } \\
\text { spectrum } \\
\text { disorder), } \\
\text { physical } \\
\text { disabilities, } \\
\text { medical } \\
\text { conditions or } \\
\text { distress about } \\
\text { facing medical } \\
\text { interventions }\end{array}$ \\
\hline
\end{tabular}




\begin{tabular}{|c|c|}
\hline $\begin{array}{l}\text { 1732. Wang SM, Gaal D, Maranets I, Caldwell-Andrews A and Kain } \\
\text { ZN. 2005. Acupressure and preoperative parental anxiety: a pilot } \\
\text { study. Anesthesia \& Analgesia, 101(3), 666-669. }\end{array}$ & $\begin{array}{l}\text { Intervention for } \\
\text { developmental } \\
\text { disorders } \\
\text { (autism, autism } \\
\text { spectrum } \\
\text { disorder), } \\
\text { physical } \\
\text { disabilities, } \\
\text { medical } \\
\text { conditions or } \\
\text { distress about } \\
\text { facing medical } \\
\text { interventions }\end{array}$ \\
\hline $\begin{array}{l}\text { 1733. Wang Y, Liu C and Wang YF. 2007. Effectiveness of social skills } \\
\text { training among children with behavior problems: A randomized } \\
\text { controlled trial. Beijing da xue xue bao. Yi xue ban= Journal of Peking } \\
\text { University. Health sciences, 39(3), 315-318. }\end{array}$ & $\begin{array}{l}\text { Not a parenting } \\
\text { intervention }\end{array}$ \\
\hline $\begin{array}{l}\text { 1734. Warner CM, Reigada LC, Fisher PH, Saborsky AL and Benkov } \\
\text { KJ. 2009. CBT for anxiety and associated somatic complaints in } \\
\text { pediatric medical settings: An open pilot study. Journal of Clinical } \\
\text { Psychology in Medical Settings. 16(2):169-77. }\end{array}$ & $\begin{array}{l}\text { Not a parenting } \\
\text { intervention }\end{array}$ \\
\hline $\begin{array}{l}\text { 1735. Waters AM, Donaldson J and Zimmer-Gembeck MJ. } 2008 . \\
\text { Cognitive-behavioural therapy combined with an interpersonal skills } \\
\text { component in the treatment of generalised anxiety disorder in } \\
\text { adolescent females: A case series. Behaviour Change. 25(1):35-43. }\end{array}$ & $\begin{array}{l}\text { Not a parenting } \\
\text { intervention }\end{array}$ \\
\hline $\begin{array}{l}\text { 1736. Waters AM, Ford LA, Wharton TA, Cobham VE. Cognitive- } \\
\text { behavioural therapy for young children with anxiety disorders: } \\
\text { Comparison of a child + parent condition versus a parent only } \\
\text { condition. Behaviour Research and Therapy. 2009 Aug; } 47(8): 654-62 \text {. }\end{array}$ & $\begin{array}{l}\text { Follow-up data } \\
\text { collected only in } \\
\text { active group }\end{array}$ \\
\hline $\begin{array}{l}\text { 1737. Waters AM, Wharton TA, Zimmer-Gembeck MJ and Craske } \\
\text { MG. 2008. Threat-based cognitive biases in anxious children: } \\
\text { Comparison with non-anxious children before and after cognitive } \\
\text { behavioural treatment. Behaviour Research and Therapy. 46(3):358- } \\
74 .\end{array}$ & $\begin{array}{l}\text { Not a parenting } \\
\text { intervention }\end{array}$ \\
\hline $\begin{array}{l}\text { 1738. Waxmonsky J, Pelham WE, Gnagy E, Cummings MR, O'Connor } \\
\text { B, Majumdar A, Verley J, Hoffman MT, Massetti GA, Burrows-MacLean } \\
\text { L, Fabiano GA, Waschbusch DA, Chacko A, Arnold FW, Walker KS, } \\
\text { Garefino AC and Robb JA. 2008. The efficacy and tolerability of } \\
\text { methylphenidate and behavior modification in children with attention- } \\
\text { deficit/hyperactivity disorder and severe mood dysregulation. Journal } \\
\text { of Child and Adolescent Psychopharmacology. 18(6):573-88. }\end{array}$ & $\begin{array}{l}\text { Not a parenting } \\
\text { intervention }\end{array}$ \\
\hline $\begin{array}{l}\text { 1739. Waxmonsky, J. G., Wymbs, F. A., Pariseau, M. E., Belin, P. J., } \\
\text { Waschbusch, D. A., Babocsai, L., ... \& Pelham, W. E. (2013). A novel } \\
\text { group therapy for children with ADHD and severe mood dysregulation. } \\
\text { Journal of attention disorders, } 17(6), 527-541 .\end{array}$ & $\begin{array}{l}\text { Parent } \\
\text { component too } \\
\text { small; Follow-up } \\
\text { less than } 6 \\
\text { months; Not an } \\
\text { RCT }\end{array}$ \\
\hline $\begin{array}{l}\text { 1740. Weaver, C. M., Shaw, D. S., Crossan, J. L., Dishion, T. J., \& } \\
\text { Wilson, M. N. (2014). Parent-child conflict and early childhood } \\
\text { adjustment in two-parent low-income families: Parallel developmental } \\
\text { processes. Child Psychiatry \& Human Development, 46(1), 94-107. }\end{array}$ & $\begin{array}{l}\text { Targets } \\
\text { externalising }\end{array}$ \\
\hline
\end{tabular}




\begin{tabular}{|c|c|}
\hline $\begin{array}{l}\text { 1741. Webster Stratton, C. (1992). Individually administered } \\
\text { videotape parent training: Who benefits?. Cognitive-Therapy-and- } \\
\text { Research., (1), 31-52. }\end{array}$ & $\begin{array}{l}\text { Targets } \\
\text { externalising }\end{array}$ \\
\hline $\begin{array}{l}\text { 1742. Webster?Stratton, C., Rinaldi, J., \& Reid, J. M. (2011). Long- } \\
\text { term outcomes of Incredible Years parenting program: Predictors of } \\
\text { adolescent adjustment. Child and Adolescent Mental Health, 16, 38- } \\
46 .\end{array}$ & $\begin{array}{l}\text { Targets } \\
\text { externalising }\end{array}$ \\
\hline $\begin{array}{l}\text { 1743. Webster-Stratton C and Hammond M. 1997. Treating children } \\
\text { with early-onset conduct problems: A comparison of child and parent } \\
\text { training interventions. Journal of Consulting and Clinical Psychology. } \\
\text { 65(1):93-109. }\end{array}$ & $\begin{array}{l}\text { Follow-up data } \\
\text { collected only in } \\
\text { active group }\end{array}$ \\
\hline $\begin{array}{l}\text { 1744. Webster-Stratton C and Reid MJ. 2010. The Incredible Years } \\
\text { parents, teachers, and children training series: A multifaceted } \\
\text { treatment approach for young children with conduct disorders. In: } \\
\text { Evidence-based psychotherapies for children and adolescents (2nd } \\
\text { ed.). (pp. 194-210). New York, NY, US: Guilford Press. xx, 602. }\end{array}$ & Review \\
\hline $\begin{array}{l}\text { 1745. Webster-Stratton C, Hollinsworth T and Kolpacoff M. } 1989 . \\
\text { The long-term effectiveness and clinical significance of three cost- } \\
\text { effective training programs for families with conduct-problem } \\
\text { children. Journal of Consulting and Clinical Psychology. 57(4):550-3. }\end{array}$ & $\begin{array}{l}\text { Comparison } \\
\text { condition not a } \\
\text { minimal control }\end{array}$ \\
\hline $\begin{array}{l}\text { 1746. Webster-Stratton C, Kolpacoff M and Hollinsworth T. } 1988 . \\
\text { Self-Administered Videotape Therapy for Families with Conduct- } \\
\text { Problem Children - Comparison with } 2 \text { Cost-Effective Treatments and a } \\
\text { Control-Group. Journal of Consulting and Clinical Psychology. } \\
\text { 56(4):558-66. }\end{array}$ & $\begin{array}{l}\text { No long term } \\
\text { follow-up }\end{array}$ \\
\hline $\begin{array}{l}\text { 1747. Webster-Stratton C. 1984. Randomized trial of two parent- } \\
\text { training programs for families with conduct-disordered children. } \\
\text { Journal of Consulting and Clinical Psychology. 52(4):666-78. }\end{array}$ & $\begin{array}{l}\text { Follow-up data } \\
\text { collected only in } \\
\text { active group }\end{array}$ \\
\hline $\begin{array}{l}\text { 1748. Webster-Stratton C. 1990. Long-term follow-up of families } \\
\text { with young conduct problem children: From preschool to grade } \\
\text { school. Journal of Clinical Child Psychology. 19(2):144-9. }\end{array}$ & $\begin{array}{l}\text { Comparison } \\
\text { condition not a } \\
\text { minimal control }\end{array}$ \\
\hline $\begin{array}{l}\text { 1749. Webster-Stratton C. 1996. Early onset conduct problems: Does } \\
\text { gender make a difference? Journal of Consulting and Clinical } \\
\text { Psychology. 64(3):540-51. }\end{array}$ & $\begin{array}{l}\text { Not a parenting } \\
\text { intervention }\end{array}$ \\
\hline $\begin{array}{l}\text { 1750. Webster-Stratton C. 1998. Preventing conduct problems in } \\
\text { head start children: Strengthening parenting competencies. Journal of } \\
\text { Consulting and Clinical Psychology.66(5):715-30. }\end{array}$ & $\begin{array}{l}\text { Targets } \\
\text { externalising }\end{array}$ \\
\hline $\begin{array}{l}\text { 1751. Webster-Stratton, C. (1982). The long-term effects of a } \\
\text { videotape modeling parent-training program: Comparison of } \\
\text { immediate and 1-year follow-up results. [doi: 10.1016/S0005- } \\
\text { 7894(82)80026-9]. Behavior Therapy, 13(5), 702-714. }\end{array}$ & Not an RCT \\
\hline $\begin{array}{l}\text { 1752. Webster-Stratton, C. (1985). Predictors of Treatment Outcome } \\
\text { in Parent Training for Conduct Disordered Children. Behavior Therapy, } \\
\text { 16(2), 223-243. }\end{array}$ & Not an RCT \\
\hline $\begin{array}{l}\text { 1753. Webster-Stratton, C. (1990). Enhancing the Effectiveness of } \\
\text { Self-Administered Videotape Parent Training for Families with } \\
\text { Conduct-Problem Children. Journal of Abnormal Child Psychology, } \\
\text { 18(5), 479-492. }\end{array}$ & $\begin{array}{l}\text { No long term } \\
\text { follow-up }\end{array}$ \\
\hline $\begin{array}{l}\text { 1754. Webster-Stratton, C. (1994). Advancing Videotape Parent } \\
\text { Training - a Comparison Study. Journal of Consulting and Clinical } \\
\text { Psychology, 62(3), 583-593. }\end{array}$ & $\begin{array}{l}\text { No long term } \\
\text { follow-up }\end{array}$ \\
\hline
\end{tabular}




\begin{tabular}{|c|c|}
\hline $\begin{array}{l}\text { 1755. Webster-Stratton, C. H., Reid, M. J., \& Beauchaine, T. (2011). } \\
\text { Combining parent and child training for young children with ADHD. } \\
\text { Journal of Clinical Child and Adolescent Psychology, 40, 191-203. }\end{array}$ & $\begin{array}{l}\text { Targets } \\
\text { externalising; No } \\
\text { long term } \\
\text { follow-up }\end{array}$ \\
\hline $\begin{array}{l}\text { 1756. Webster-Stratton, C. H., Reid, M. J., \& Marsenich, L. (2014). } \\
\text { Improving therapist fidelity during implementation of evidence-based } \\
\text { practices: Incredible Years Program. Psychiatric Services, 65(6), 789- } \\
795 .\end{array}$ & $\begin{array}{l}\text { Not a parenting } \\
\text { intervention }\end{array}$ \\
\hline $\begin{array}{l}\text { 1757. Webster-Stratton, C., \& Hammond, M. (1990). Predictors of } \\
\text { Treatment Outcome in Parent Training for Families with Conduct } \\
\text { Problem Children. Behavior Therapy, 21(3), 319-337. }\end{array}$ & Not an RCT \\
\hline $\begin{array}{l}\text { 1758. Webster-Stratton, C., \& Herman, K. C. (2008). The impact of } \\
\text { parent behavior-management training on child depressive symptoms. } \\
\text { Journal of Counseling Psychology, 55(4), 473-484. doi: } \\
\text { 10.1037/a0013664 }\end{array}$ & $\begin{array}{l}\text { No long term } \\
\text { follow-up }\end{array}$ \\
\hline $\begin{array}{l}\text { 1759. Webster-Stratton, C., Reid, J., \& Hammond, M. (2001). Social } \\
\text { skills and problem-solving training for children with early-onset } \\
\text { conduct problems: who benefits? J Child Psychol Psychiatry, 42(7), } \\
\text { 943-952. }\end{array}$ & $\begin{array}{l}\text { Not a parenting } \\
\text { intervention } \\
\text { (child training) }\end{array}$ \\
\hline $\begin{array}{l}\text { 1760. Webster-Stratton, C., Reid, M. J., \& Beauchaine, T. P. (2013). } \\
\text { One-year follow-up of combined parent and child intervention for } \\
\text { young children with ADHD. Journal of Clinical Child \& Adolescent } \\
\text { Psychology, 42(2), 251-261. }\end{array}$ & $\begin{array}{l}\text { Parent } \\
\text { component too } \\
\text { small; Targets } \\
\text { externalising }\end{array}$ \\
\hline $\begin{array}{l}\text { 1761. Webster-Stratton, C., Reid, M. J., \& Stoolmiller, M. (2008). } \\
\text { Preventing conduct problems and improving school readiness: } \\
\text { evaluation of the Incredible Years Teacher and Child Training Programs } \\
\text { in high-risk schools. Journal of Child Psychology and Psychiatry, 49(5), } \\
\text { 471-488. }\end{array}$ & $\begin{array}{l}\text { Not a parenting } \\
\text { intervention }\end{array}$ \\
\hline $\begin{array}{l}\text { 1762. Weersing VR and Weisz JR. 2002. Mechanisms of action in } \\
\text { youth psychotherapy. Journal of Child Psychology and Psychiatry and } \\
\text { Allied Disciplines. 43(1):3-29. }\end{array}$ & Review \\
\hline $\begin{array}{l}\text { 1763. Weihrauch, L., Schäfer, R., \& Franz, M. (2014). Long-term } \\
\text { efficacy of an attachment-based parental training program for single } \\
\text { mothers and their children: a randomized controlled trial. Journal of } \\
\text { Public Health, 22(2), 139-153. }\end{array}$ & $\begin{array}{l}\text { No child } \\
\text { internalising } \\
\text { outcomes or not } \\
\text { a major goal }\end{array}$ \\
\hline $\begin{array}{l}\text { 1764. Weiss BD, Francis L, Senf JH, Heist K and Hargraves R. } 2006 . \\
\text { Literacy Education as Treatment for Depression in Patients with } \\
\text { Limited Literacy and Depression: A Randomized Controlled Trial. } \\
\text { Journal of General Internal Medicine. 21(8):823-8. }\end{array}$ & $\begin{array}{l}\text { Not a parenting } \\
\text { intervention }\end{array}$ \\
\hline $\begin{array}{l}\text { 1765. Weiss, B., Harris, V., Catron, T., \& Han, S. S. (2003). Efficacy of } \\
\text { the RECAP intervention program for children with concurrent } \\
\text { internalizing and externalizing problems. Journal of Consulting and } \\
\text { Clinical Psychology, } 71(2), 364-374 \text {. doi: } 10.1037 / 0022-006 x .71 .2 .364\end{array}$ & $\begin{array}{l}\text { Parent } \\
\text { component too } \\
\text { small }\end{array}$ \\
\hline $\begin{array}{l}\text { 1766. Weissman MM. 2007. Recent non-medication trials of } \\
\text { interpersonal psychotherapy for depression. International Journal of } \\
\text { Neuropsychopharmacology. 10(1):117-22. }\end{array}$ & Review \\
\hline $\begin{array}{l}1767 . \quad \text { Weissman, M. M., Pilowsky, D. J., Wickramaratne, P. J., Talati, } \\
\text { A., Wisniewski, S. R., Fava, M., . . Team, f. t. S. D.-C. (2006). } \\
\text { Remissions in Maternal Depression and Child Psychopathology. JAMA: } \\
\text { The Journal of the American Medical Association, 295(12), 1389-1398. } \\
\text { doi: 10.1001/jama.295.12.1389 }\end{array}$ & $\begin{array}{l}\text { Not a parenting } \\
\text { intervention } \\
\text { (drugs only) }\end{array}$ \\
\hline
\end{tabular}




\begin{tabular}{|c|c|}
\hline $\begin{array}{l}\text { 1768. Weisz JR, Southam-Gerow MA, Gordis EB, Connor-Smith JK, } \\
\text { Chu BC, Langer DA, McLeod BD, Jensen-Doss A, Updegraff A and Weiss } \\
\text { B. 2009. Cognitive-behavioral therapy versus usual clinical care for } \\
\text { youth depression: an initial test of transportability to community } \\
\text { clinics and clinicians. J Consult Clin Psychol. 77(3):383-96. }\end{array}$ & $\begin{array}{l}\text { Not a parenting } \\
\text { intervention }\end{array}$ \\
\hline $\begin{array}{l}\text { 1769. Weisz, J. R., Chorpita, B. F., Palinkas, L. A., Schoenwald, S. K., } \\
\text { Miranda, J., Bearman, S. K., Daleiden, E. L., Ugueto, A. M., Ho, A., } \\
\text { Martin, J., Gray, J., Alleyne, A., Langer, D. A., Southam-Gerow, M. A., \& } \\
\text { Gibbons, R. D. (2012). Testing standard and modular designs for } \\
\text { psychotherapy treating depression, anxiety, and conduct problems in } \\
\text { youth: A randomized effectiveness trial. Arch Gen Psychiatry, 69, 274- } \\
\text { 282. }\end{array}$ & $\begin{array}{l}\text { Not a parenting } \\
\text { intervention }\end{array}$ \\
\hline $\begin{array}{l}\text { 1770. Wells KC, Pelham WE, Kotkin RA, Hoza B, Abikoff HB, } \\
\text { Abramowitz A, Arnold LE, Cantwell DP, Conners CK, Del Carmen R, } \\
\text { Elliott G, Greenhill LL, Hechtman L, Hibbs E, Hinshaw SP, Jensen PS, } \\
\text { March JS, Swanson JM and Schiller E. 2000. Psychosocial treatment } \\
\text { strategies in the MTA study: rationale, methods, and critical issues in } \\
\text { design and implementation. Journal of Abnormal Child Psychology, } \\
\text { 28(6), 483-505. }\end{array}$ & $\begin{array}{l}\text { Data not } \\
\text { available/report } \\
\text { ed (protocol) }\end{array}$ \\
\hline $\begin{array}{l}\text { 1771. Welterlin A. 2010. The Home TEACCHing Program: A study of } \\
\text { the efficacy of a parent training early intervention model. Dissertation } \\
\text { Abstracts International: Section B: The Sciences and Engineering. 71(5- } \\
\text { B). }\end{array}$ & $\begin{array}{l}\text { Intervention for } \\
\text { developmental } \\
\text { disorders } \\
\text { (autism, autism } \\
\text { spectrum } \\
\text { disorder), } \\
\text { physical } \\
\text { disabilities, } \\
\text { medical } \\
\text { conditions or } \\
\text { distress about } \\
\text { facing medical } \\
\text { interventions }\end{array}$ \\
\hline $\begin{array}{l}\text { 1772. Werch CE, Moore MJ and DiClemente CC. 2008. Brief image- } \\
\text { based health behavior messages for adolescents and their parents. } \\
\text { Journal of Child \& Adolescent Substance Abuse. 17(4):19-40. }\end{array}$ & $\begin{array}{l}\text { Follow-up less } \\
\text { than } 6 \text { months }\end{array}$ \\
\hline $\begin{array}{l}\text { 1773. Westermann, G. M., Verheij, F., Winkens, B., Verhulst, F. C., \& } \\
\text { Oort, F. V. (2013). Structured shared decision-making using dialogue } \\
\text { and visualization: a randomized controlled trial. Patient education and } \\
\text { counseling, 90(1), 74-81. doi:10.1016/j.pec.2012.09.014 }\end{array}$ & $\begin{array}{l}\text { No child } \\
\text { internalising } \\
\text { outcomes or not } \\
\text { a major goal }\end{array}$ \\
\hline $\begin{array}{l}\text { 1774. Westrup, B., Böhm, B., Lagercrantz, H., \& Stjernqvist, K. } \\
\text { (2004). Preschool outcome in children born very prematurely and } \\
\text { cared for according to the Newborn Individualized Developmental } \\
\text { Care and Assessment Program (NIDCAP). Acta Paediatrica (Oslo, } \\
\text { Norway: 1992), 93, 498-507. }\end{array}$ & $\begin{array}{l}\text { No child } \\
\text { internalising } \\
\text { outcomes or not } \\
\text { a major goal }\end{array}$ \\
\hline
\end{tabular}




\begin{tabular}{|c|c|}
\hline $\begin{array}{l}\text { 1775. Wetherby, A. M., Guthrie, W., Woods, J., Schatschneider, C., } \\
\text { Holland, R. D., Morgan, L., \& Lord, C. (2014). Parent-implemented } \\
\text { social intervention for toddlers with autism: An RCT. Pediatrics, 134(6), } \\
\text { 1084-1093. }\end{array}$ & $\begin{array}{l}\text { Intervention for } \\
\text { developmental } \\
\text { disorders } \\
\text { (autism, autism } \\
\text { spectrum } \\
\text { disorder), } \\
\text { physical } \\
\text { disabilities, } \\
\text { medical } \\
\text { conditions or } \\
\text { distress about } \\
\text { facing medical } \\
\text { interventions }\end{array}$ \\
\hline $\begin{array}{l}\text { 1776. Wheatley SL, Brugha TS and Shapiro DA. 2003. Exploring and } \\
\text { enhancing engagement to the psychosocial intervention 'Preparing for } \\
\text { Parenthood'. Arch Womens Ment Health. 6(4):275-85. }\end{array}$ & $\begin{array}{l}\text { No child } \\
\text { internalising } \\
\text { outcomes or not } \\
\text { a major goal }\end{array}$ \\
\hline $\begin{array}{l}\text { 1777. White MA, Martin PD, Newton RL, Walden HM, York-Crowe } \\
\text { EE, Gordon ST, Ryan DH and Williamson DA. 2004. Mediators of } \\
\text { weight loss in a family-based intervention presented over the internet. } \\
\text { Obesity Research, 12(7), 1050-1059. }\end{array}$ & $\begin{array}{l}\text { No child } \\
\text { internalising } \\
\text { outcomes or not } \\
\text { a major goal }\end{array}$ \\
\hline $\begin{array}{l}\text { 1778. White, S. W., Ollendick, T., Albano, A. M., Oswald, D., Johnson, } \\
\text { C., Southam-Gerow, M. A., . . Scahill, L. (2013). Randomized } \\
\text { controlled trial: Multimodal Anxiety and Social Skill Intervention for } \\
\text { adolescents with autism spectrum disorder. J Autism Dev Disord, } \\
\text { 43(2), 382-394. doi: 10.1007/s10803-012-1577-x }\end{array}$ & $\begin{array}{l}\text { Intervention for } \\
\text { developmental } \\
\text { disorders } \\
\text { (autism, autism } \\
\text { spectrum } \\
\text { disorder), } \\
\text { physical } \\
\text { disabilities, } \\
\text { medical } \\
\text { conditions or } \\
\text { distress about } \\
\text { facing medical } \\
\text { interventions }\end{array}$ \\
\hline $\begin{array}{l}\text { 1779. Whitebird, R. R., Kreitzer, M., Crain, A. L., Lewis, B. A., Hanson, } \\
\text { L. R., \& Enstad, C. J. (2013). Mindfulness-based stress reduction for } \\
\text { family caregivers: a randomized controlled trial. Gerontologist, 53(4), } \\
\text { 676-686. doi: 10.1093/geront/gns126 }\end{array}$ & $\begin{array}{l}\text { Not in children } 0 \\
-18\end{array}$ \\
\hline $\begin{array}{l}\text { 1780. Whiteside SP and Jacobsen AB. 2010. An uncontrolled } \\
\text { examination of a 5-day intensive treatment for pediatric OCD. Behav } \\
\text { Ther. 41(3):414-22. }\end{array}$ & Not an RCT \\
\hline $\begin{array}{l}\text { 1781. White-Traut, R., Norr, K. F., Fabiyi, C., Rankin, K. M., Li, Z., \& } \\
\text { Liu, L. (2013). Mother-infant interaction improves with a } \\
\text { developmental intervention for mother-preterm infant dyads. Infant } \\
\text { behavior \& development, 36(4), 694-706. } \\
\text { doi:10.1016/j.infbeh.2013.07.004 }\end{array}$ & $\begin{array}{l}\text { No child } \\
\text { internalising } \\
\text { outcomes or not } \\
\text { a major goal }\end{array}$ \\
\hline $\begin{array}{l}\text { 1782. Whittingham, K., Sanders, M., McKinlay, L., \& Boyd, R. N. } \\
\text { (2013). Stepping stones triple p and acceptance and commitment } \\
\text { therapy for parents of children with cerebral palsy: Trial protocol. } \\
\text { Brain impairment, } 14(2), 270-280 . \text { doi:10.1017/BrImp.2013.19 }\end{array}$ & $\begin{array}{l}\text { Data not } \\
\text { available/report } \\
\text { ed }\end{array}$ \\
\hline
\end{tabular}




\begin{tabular}{|c|c|}
\hline $\begin{array}{l}\text { 1783. Whittingham, K., Sanders, M., McKinlay, L., Boyd, R. N. (2014). } \\
\text { Child quality of life and parent psychological adjustment can be } \\
\text { improved with Stepping Stones Triple P and ACT: An RCT. } \\
\text { Developmental medicine and child neurology, } 5675 .\end{array}$ & $\begin{array}{l}\text { No child } \\
\text { internalising } \\
\text { outcomes or not } \\
\text { a major goal }\end{array}$ \\
\hline $\begin{array}{l}\text { 1784. Whittle, S., Yap, M. B. H., Sheeber, L., Dudgeon, P., Yücel, M., } \\
\text { Pantelis, C., Simmons, J. G., \& Allen, N. B. (2011). Hippocampal volume } \\
\text { and sensitivity to maternal aggressive behavior: A prospective study of } \\
\text { adolescent depressive symptoms. Development and Psychopathology, } \\
\text { 23, 115-129. }\end{array}$ & $\begin{array}{l}\text { Not a parenting } \\
\text { intervention }\end{array}$ \\
\hline $\begin{array}{l}\text { 1785. Wiggins TL, Sofronoff K and Sanders MR. 2009. Pathways } \\
\text { Triple P-positive parenting program: effects on parent-child } \\
\text { relationships and child behavior problems. Family Process, 48(4), 517- } \\
530 .\end{array}$ & $\begin{array}{l}\text { Follow-up less } \\
\text { than } 6 \text { months }\end{array}$ \\
\hline $\begin{array}{l}\text { 1786. Wiggs, L., \& Stores, G. (2001). Behavioural treatment for sleep } \\
\text { problems in children with severe intellectual disabilities and daytime } \\
\text { challenging behaviour: effect on mothers and fathers. British journal } \\
\text { of health psychology, } 6(\mathrm{Pt} \mathrm{3}), 257-269 \text {. }\end{array}$ & $\begin{array}{l}\text { No child } \\
\text { internalising } \\
\text { outcomes or not } \\
\text { a major goal }\end{array}$ \\
\hline $\begin{array}{l}\text { 1787. Wildman, B. G., \& Langkamp, D. L. (2012). Impact of location } \\
\text { and availability of behavioral health services for children. Journal of } \\
\text { clinical psychology in medical settings, 19(4), 393-400. }\end{array}$ & $\begin{array}{l}\text { Targets } \\
\text { externalising }\end{array}$ \\
\hline $\begin{array}{l}\text { 1788. Wilkinson-Tough M, Bocci L, Thome K and Herlihy J. 2010. Is } \\
\text { mindfulness-based therapy an effective intervention for obsessive- } \\
\text { intrusive thoughts: A case series. Clinical Psychology \& Psychotherapy. } \\
\text { 17(3):250-68. }\end{array}$ & $\begin{array}{l}\text { Not a parenting } \\
\text { intervention }\end{array}$ \\
\hline $\begin{array}{l}\text { 1789. Williams, M. E., \& Hutchings, J. (2015). A pilot effectiveness } \\
\text { study of the Enhancing Parenting Skills (EPaS) } 2014 \text { programme for } \\
\text { parents of children with behaviour problems: study protocol for a } \\
\text { randomised controlled trial. Trials, } 16(1), 221 .\end{array}$ & $\begin{array}{l}\text { Data not } \\
\text { available/report } \\
\text { ed }\end{array}$ \\
\hline $\begin{array}{l}\text { 1790. Williams, P. D., Williams, A. R., Graff, J. C., Hanson, S., Stanton, } \\
\text { A., Hafeman, C., et al. (2003). A community-based intervention for } \\
\text { siblings and parents of children with chronic illness or disability: the } \\
\text { ISEE study. The Journal of pediatrics, (3), 386-393. }\end{array}$ & $\begin{array}{l}\text { Parent } \\
\text { component too } \\
\text { small }\end{array}$ \\
\hline $\begin{array}{l}\text { 1791. Williams, T. M., \& et al. (1979). The Development of Maternal } \\
\text { Attachment: A Longitudinal Study. Biennial Meeting of the Society for } \\
\text { Research in Child Development. Retrieved from: } \\
\text { http://www.mrw.interscience.wiley.com/cochrane/clcentral/articles/ } \\
657 / \text { CN-00241657/frame.html }\end{array}$ & $\begin{array}{l}\text { No long term } \\
\text { follow-up }\end{array}$ \\
\hline $\begin{array}{l}\text { 1792. Williford, A. P., \& Shelton, T. L. (2008). Using mental health } \\
\text { consultation to decrease disruptive behaviors in preschoolers: } \\
\text { adapting an empirically-supported intervention. Journal of child } \\
\text { psychology and psychiatry, and allied disciplines, (2), 191-200. }\end{array}$ & Not an RCT \\
\hline $\begin{array}{l}\text { 1793. Wilmshurst LA. Treatment programs for youth with emotional } \\
\text { and behavioral disorders: An outcome study of two alternate } \\
\text { approaches. Mental Health Services Research. } 2002 \text { Jun;4(2):85-96. }\end{array}$ & $\begin{array}{l}\text { Comparison } \\
\text { condition not a } \\
\text { minimal control }\end{array}$ \\
\hline $\begin{array}{l}\text { 1794. Wilson AJ, Prapavessis H, Jung ME, Cramp AG, Vascotto J, } \\
\text { Lenhardt L, Shoemaker JK, Watson M, Robinson T and Clarson CL. } \\
\text { 2009. Lifestyle modification and metformin as long-term treatment } \\
\text { options for obese adolescents: study protocol. BMC Public Health. } \\
\text { 9:434. }\end{array}$ & $\begin{array}{l}\text { Not a parenting } \\
\text { intervention }\end{array}$ \\
\hline
\end{tabular}




\begin{tabular}{|c|c|}
\hline $\begin{array}{l}\text { 1795. Wilson, K. R., Havighurst, S. S., \& Harley, A. E. (2012). Tuning in } \\
\text { to Kids: an effectiveness trial of a parenting program targeting } \\
\text { emotion socialization of preschoolers. Journal of family psychology, } \\
\text { 26(1), 56-65. doi:10.1037/a0026480 }\end{array}$ & $\begin{array}{l}\text { Follow-up less } \\
\text { than } 6 \text { months }\end{array}$ \\
\hline $\begin{array}{l}\text { 1796. Wissow LS, Gadomski A, Roter D, Larson S, Brown J, Zachary C, } \\
\text { et al. Improving child and parent mental health in primary care: A } \\
\text { cluster-randomized trial of communication skills training. Pediatrics. } \\
2008 \text { Feb;121(2):266-75. }\end{array}$ & $\begin{array}{l}\text { Not a parenting } \\
\text { intervention }\end{array}$ \\
\hline $\begin{array}{l}1797 . \quad \text { Wissow, L., Gadomski, A., Roter, D., Larson, S., Lewis, B., \& } \\
\text { Brown, J. (2011). Aspects of mental health communication skills } \\
\text { training that predict parent and child outcomes in pediatric primary } \\
\text { care. Patient education and counseling, } 82(2), 226-232 .\end{array}$ & $\begin{array}{l}\text { Not a parenting } \\
\text { intervention }\end{array}$ \\
\hline $\begin{array}{l}\text { 1798. Woitecki, K., \& Dopfner, M. (2012). [Changes in comorbid } \\
\text { symptoms and subjective interference in a habit reversal therapy in } \\
\text { children with chronic tic disorder - a pilot study]. Z Kinder } \\
\text { Jugendpsychiatr Psychother, 40, 181-190. }\end{array}$ & $\begin{array}{l}\text { Not a parenting } \\
\text { intervention }\end{array}$ \\
\hline $\begin{array}{l}\text { 1799. Wolchik SA, Tein J-Y, Sandler IN and Ayers TS. 2006. Stressors, } \\
\text { Quality of the Child-Caregiver Relationship, and Children's Mental } \\
\text { Health Problems After Parental Death: The Mediating Role of Self- } \\
\text { System Beliefs. Journal of Abnormal Child Psychology: An official } \\
\text { publication of the International Society for Research in Child and } \\
\text { Adolescent Psychopathology. 34(2):221-38. }\end{array}$ & $\begin{array}{l}\text { Not a parenting } \\
\text { intervention }\end{array}$ \\
\hline $\begin{array}{l}\text { 1800. Wolchik, S. A., Sandler, I. N., \& Millsap, R. E. (2003). Group } \\
\text { based interventions for mothers and mothers plus children reduced } \\
\text { mental health problems in adolescent children of divorced parents. } \\
\text { Evidence Based Nursing, 6(3), 74-74. }\end{array}$ & $\begin{array}{l}\text { Summary } \\
\text { publication of } \\
\text { Wolchik } 2002\end{array}$ \\
\hline $\begin{array}{l}\text { 1801. Wolchik, S. A., West, S. G., Westover, S., Sandler, I. N., Martin, } \\
\text { A., Lustig, J., et al. (1993). The children of divorce parenting } \\
\text { intervention: outcome evaluation of an empirically based program. } \\
\text { American journal of community psychology, (3), 293-231. }\end{array}$ & $\begin{array}{l}\text { No long term } \\
\text { follow-up }\end{array}$ \\
\hline $\begin{array}{l}\text { 1802. Wolchik, S.; Sandler, I.; Weiss, L.; Winslow, E. New beginnings: } \\
\text { An empirically-based program to help divorced mothers promote } \\
\text { resilience in their children. In: Briesmeister, JM.; Schaefer, CE., editors. } \\
\text { Handbook of parent training: Helping parents prevent and solve } \\
\text { problem behaviors. 3. Hoboken, NJ, US: John Wiley \& Sons Inc; } 2007 . \\
\text { p. 25-62. }\end{array}$ & $\begin{array}{l}\text { Duplicate of } \\
\text { Wolchik } 2002\end{array}$ \\
\hline $\begin{array}{l}\text { 1803. Wolfe DA, Edwards B, Manion I and Koverola C. 1988. Early } \\
\text { Intervention for Parents at Risk of Child-Abuse and Neglect - a } \\
\text { Preliminary Investigation. Journal of Consulting and Clinical } \\
\text { Psychology. 56(1):40-7. }\end{array}$ & $\begin{array}{l}\text { No child } \\
\text { internalising } \\
\text { outcomes or not } \\
\text { a major goal; } \\
\text { Follow-up less } \\
\text { than } 6 \text { months }\end{array}$ \\
\hline $\begin{array}{l}\text { 1804. Wolfson A, Lacks P and Futterman A. 1992. Effects of parent } \\
\text { training on infant sleeping patterns, parents' stress, and perceived } \\
\text { parental competence. J Consult Clin Psychol. 60(1):41-8. }\end{array}$ & $\begin{array}{l}\text { No child } \\
\text { internalising } \\
\text { outcomes or not } \\
\text { a major goal }\end{array}$ \\
\hline $\begin{array}{l}\text { 1805. Woltering, S., Granic, I., Lamm, C., \& Lewis, M. D. (2011). } \\
\text { Neural changes associated with treatment outcome in children with } \\
\text { externalizing problems. Biological Psychiatry, } 70,873-879 .\end{array}$ & $\begin{array}{l}\text { Targets } \\
\text { externalising }\end{array}$ \\
\hline
\end{tabular}




\begin{tabular}{|c|c|}
\hline $\begin{array}{l}\text { 1806. Wong, J. J., Gonzales, N. A., Montaño, Z., Dumka, L., \& Millsap, } \\
\text { R. E. (2014). Parenting intervention effects on parental depressive } \\
\text { symptoms: Examining the role of parenting and child behavior. Journal } \\
\text { of Family Psychology, 28(3), } 267 \text {. }\end{array}$ & $\begin{array}{l}\text { No child } \\
\text { internalising } \\
\text { outcomes or not } \\
\text { a major goal }\end{array}$ \\
\hline $\begin{array}{l}\text { 1807. Wood J. 2006. Effect of anxiety reduction on children's school } \\
\text { performance and social adjustment. Developmental Psychology. } \\
\text { 42(2):345-9. }\end{array}$ & $\begin{array}{l}\text { Not a parenting } \\
\text { intervention }\end{array}$ \\
\hline $\begin{array}{l}\text { 1808. Wood JJ, Drahota A, Sze K, Har K, Chiu A and Langer DA. } 2009 . \\
\text { Cognitive behavioral therapy for anxiety in children with autism } \\
\text { spectrum disorders: a randomized, controlled trial. J Child Psychol } \\
\text { Psychiatry. 50(3):224-34. }\end{array}$ & $\begin{array}{l}\text { Intervention for } \\
\text { developmental } \\
\text { disorders } \\
\text { (autism, autism } \\
\text { spectrum } \\
\text { disorder), } \\
\text { physical } \\
\text { disabilities, } \\
\text { medical } \\
\text { conditions or } \\
\text { distress about } \\
\text { facing medical } \\
\text { interventions }\end{array}$ \\
\hline $\begin{array}{l}\text { 1809. Wood JJ, Drahota A, Sze K, Van Dyke M, Decker K, Fujii C, } \\
\text { Bahng C, Renno P, Hwang WC and Spiker M. 2009. Brief report: effects } \\
\text { of cognitive behavioral therapy on parent-reported autism symptoms } \\
\text { in school-age children with high-functioning autism. J Autism Dev } \\
\text { Disord. 39(11):1608-12. }\end{array}$ & $\begin{array}{l}\text { Intervention for } \\
\text { developmental } \\
\text { disorders } \\
\text { (autism, autism } \\
\text { spectrum } \\
\text { disorder), } \\
\text { physical } \\
\text { disabilities, } \\
\text { medical } \\
\text { conditions or } \\
\text { distress about } \\
\text { facing medical } \\
\text { interventions }\end{array}$ \\
\hline $\begin{array}{l}\text { 1810. Wood JJ, McLeod BD, Piacentini JC, Sigman M. One-year } \\
\text { follow-up of family versus child CBT for anxiety disorders: Exploring } \\
\text { the roles of child age and parental intrusiveness. Child Psychiatry and } \\
\text { Human Development. } 2009 \text { Jun; } 40(2): 301-16 .\end{array}$ & $\begin{array}{l}\text { Comparison } \\
\text { condition not a } \\
\text { minimal control }\end{array}$ \\
\hline $\begin{array}{l}\text { 1811. Wood JJ, Piacentini JC, Southam-Gerow M, Chu BC and Sigman } \\
\text { M. 2006. Family cognitive behavioral therapy for child anxiety } \\
\text { disorders. J Am Acad Child Adolesc Psychiatry. 45(3):314-21. }\end{array}$ & $\begin{array}{l}\text { No long term } \\
\text { follow-up }\end{array}$ \\
\hline $\begin{array}{l}\text { 1812. Wood, J. J. (2003). Child anxiety disorders: Parent-child } \\
\text { communication as a change mechanism. [Dissertation Abstract; } \\
\text { Dissertation]. Dissertation Abstracts International: Section B: The } \\
\text { Sciences and Engineering, 64(6-B). }\end{array}$ & $\begin{array}{l}\text { No long term } \\
\text { follow-up }\end{array}$ \\
\hline $\begin{array}{l}\text { 1813. Wood, J. J., Ehrenreich-May, J., Alessandri, M., Fujii, C., Renno, } \\
\text { P., Laugeson, E., ... \& Storch, E. A. (2015). Cognitive behavioral therapy } \\
\text { for early adolescents with autism spectrum disorders and clinical } \\
\text { anxiety: a randomized, controlled trial. Behavior therapy, 46(1), 7-19. }\end{array}$ & $\begin{array}{l}\text { Not a parenting } \\
\text { intervention }\end{array}$ \\
\hline $\begin{array}{l}\text { 1814. Wood, M. (2011). The safety and efficacy of using a } \\
\text { concentrated intranasal midazolam formulation for paediatric dental } \\
\text { sedation. SAAD Dig, 27, 16-23. }\end{array}$ & $\begin{array}{l}\text { Not a parenting } \\
\text { intervention }\end{array}$ \\
\hline
\end{tabular}




\begin{tabular}{|c|c|}
\hline $\begin{array}{l}\text { 1815. Woolderink M, Smit F, van der Zanden R, Beecham J, Knapp } \\
\text { M, Paulus A and Evers S. 2010. Design of an internet-based health } \\
\text { economic evaluation of a preventive group-intervention for children of } \\
\text { parents with mental illness or substance use disorders. BMC Public } \\
\text { Health. 10:470. }\end{array}$ & $\begin{array}{l}\text { Not a parenting } \\
\text { intervention }\end{array}$ \\
\hline $\begin{array}{l}\text { 1816. Woolderink M. 2009. (Economic) Evaluation of E-mental } \\
\text { health interventions for children of parents with mental illness ( } \mathrm{e}^{\wedge} 3 \\
\text { COPMI). Retrieved from } \\
\text { http://onlinelibrary.wiley.com/o/cochrane/clcentral/articles/358/CN- } \\
\text { 00747358/frame.html. }\end{array}$ & $\begin{array}{l}\text { Not a parenting } \\
\text { intervention }\end{array}$ \\
\hline $\begin{array}{l}\text { 1817. Wray J. 1995. Controlled comparison of three interventions } \\
\text { with parents of children with learning disabilities exhibiting } \\
\text { challenging behaviours. Journal of psychiatric and mental health } \\
\text { nursing, 2(1), 48-48. }\end{array}$ & $\begin{array}{l}\text { Data not } \\
\text { available/report } \\
\text { ed }\end{array}$ \\
\hline $\begin{array}{l}\text { 1818. Wu P, Bird HR, Liu X, Duarte CS, Fuller C, Fan B, Shen S and } \\
\text { Canino GJ. 2010. Trauma, posttraumatic stress symptoms, and } \\
\text { alcohol-use initiation in children. Journal of Studies on Alcohol and } \\
\text { Drugs. 71(3):326-34. }\end{array}$ & $\begin{array}{l}\text { Not a parenting } \\
\text { intervention }\end{array}$ \\
\hline $\begin{array}{l}\text { 1819. Wurtele SK, Kast LC, Miller-Perrin CL and Kondrick PA. } 1989 . \\
\text { Comparison of programs for teaching personal safety skills to } \\
\text { preschoolers. Journal of Consulting and Clinical Psychology, 57(4), } 505 .\end{array}$ & $\begin{array}{l}\text { Not a parenting } \\
\text { intervention }\end{array}$ \\
\hline $\begin{array}{l}\text { 1820. Wurtele, S. K., Kast, L. C., \& Melzer, A. M. (1992). Sexual abuse } \\
\text { prevention education for young children: a comparison of teachers } \\
\text { and parents as instructors. Child abuse \& neglect, (6), 865-876. }\end{array}$ & $\begin{array}{l}\text { Follow-up less } \\
\text { than } 6 \text { months; } \\
\text { No child } \\
\text { internalising } \\
\text { outcomes }\end{array}$ \\
\hline $\begin{array}{l}\text { 1821. Wyatt Kaminski J, Valle LA, Filene JH and Boyle CL. 2008. A } \\
\text { meta-analytic review of components associated with parent training } \\
\text { program effectiveness. Journal of Abnormal Child Psychology. } \\
\text { 36(4):567-89. }\end{array}$ & Review \\
\hline $\begin{array}{l}\text { 1822. Wyman PA, Cross W, Hendricks Brown C, Yu Q, Tu X and } \\
\text { Eberly S. 2010. Intervention to strengthen emotional self-regulation in } \\
\text { children with emerging mental health problems: Proximal impact on } \\
\text { school behavior. Journal of Abnormal Child Psychology: An official } \\
\text { publication of the International Society for Research in Child and } \\
\text { Adolescent Psychopathology. 38(5):707-20. }\end{array}$ & $\begin{array}{l}\text { Not a parenting } \\
\text { intervention }\end{array}$ \\
\hline $\begin{array}{l}\text { 1823. Wymbs BT and Pelham WE, Jr. 2010. Child effects on } \\
\text { communication between parents of youth with and without attention- } \\
\text { deficit/hyperactivity disorder. Journal of Abnormal Psychology. } \\
\text { 119(2):366-75. }\end{array}$ & $\begin{array}{l}\text { Not a parenting } \\
\text { intervention }\end{array}$ \\
\hline $\begin{array}{l}\text { 1824. Wysocki T, Nansel TR, Holmbeck GN, Chen R, Laffel L, } \\
\text { Anderson BJ, Weissberg-Benchell J and Steering Committee of the } \\
\text { Family Management of Childhood Diabetes S. 2009. Collaborative } \\
\text { involvement of primary and secondary caregivers: associations with } \\
\text { youths' diabetes outcomes. Journal of Pediatric Psychology, 34(8), } \\
\text { 869-881. }\end{array}$ & Not an RCT \\
\hline $\begin{array}{l}\text { 1825. Yagmur, S., Mesman, J., Malda, M., Bakermans-Kranenburg, } \\
\text { M. J., \& Ekmekci, H. (2014). Video-feedback intervention increases } \\
\text { sensitive parenting in ethnic minority mothers: a randomized control } \\
\text { trial. Attachment \& human development, 16(4), 371-386. }\end{array}$ & $\begin{array}{l}\text { No long term } \\
\text { follow-up }\end{array}$ \\
\hline
\end{tabular}




\begin{tabular}{|c|c|}
\hline $\begin{array}{l}\text { 1826. Yang KM and Kim SL. 2010. [Effects of a Taegyo program on } \\
\text { parent-fetal attachment and parenthood in first pregnancy couples]. J } \\
\text { Korean Acad Nurs. 40(4):571-9. }\end{array}$ & $\begin{array}{l}\text { No child } \\
\text { internalising } \\
\text { outcomes or not } \\
\text { a major goal }\end{array}$ \\
\hline $\begin{array}{l}\text { 1827. Yang YM, Andrews S, Peterson R, Shah A and Cepeda M. } 2000 . \\
\text { Prenatal sickle cell screening education effect on the follow-up rates } \\
\text { of infants with sickle cell trait. Patient Educ Couns. 39(2-3):185-9. }\end{array}$ & $\begin{array}{l}\text { Not a parenting } \\
\text { intervention }\end{array}$ \\
\hline $\begin{array}{l}\text { 1828. Yap MBH, Whittle S, Yücel M, Sheeber L, Pantelis C, Simmons } \\
\text { JG and Allen NB. 2008. Interaction of parenting experiences and brain } \\
\text { structure in the prediction of depressive symptoms in adolescents. } \\
\text { Archives of General Psychiatry. 65(12):1377-85. }\end{array}$ & $\begin{array}{l}\text { Not a parenting } \\
\text { intervention }\end{array}$ \\
\hline $\begin{array}{l}\text { 1829. Yen, C. F., Chen, Y. M., Cheng, J. W., Liu, T. L., Huang, T. Y., } \\
\text { Wang, P. W., ... \& Chou, W. J. (2014). Effects of cognitive-behavioral } \\
\text { therapy on improving anxiety symptoms, behavioral problems and } \\
\text { parenting stress in Taiwanese children with anxiety disorders and their } \\
\text { mothers. Child Psychiatry \& Human Development, 45(3), 338-347. }\end{array}$ & $\begin{array}{l}\text { Not a parenting } \\
\text { intervention }\end{array}$ \\
\hline $\begin{array}{l}\text { 1830. Yoo, H. J., Bahn, G., Cho, I. H., Kim, E. K., Kim, J. H., Min, J. } \\
\text { W., . . Laugeson, E. A. (2014). A randomized controlled trial of the } \\
\text { korean version of the PEERS parent-assisted social skills training } \\
\text { program for teens with ASD. Autism Research, 7(1), 145-161. doi: } \\
\text { http://dx.doi.org/10.1002/aur.1354 }\end{array}$ & $\begin{array}{l}\text { Intervention for } \\
\text { developmental } \\
\text { disorders } \\
\text { (autism, autism } \\
\text { spectrum } \\
\text { disorder), } \\
\text { physical } \\
\text { disabilities, } \\
\text { medical } \\
\text { conditions or } \\
\text { distress about } \\
\text { facing medical } \\
\text { interventions }\end{array}$ \\
\hline $\begin{array}{l}\text { 1831. Yoo, J. B., Kim, M. J., Cho, S. H., Shin, Y. J., \& Kim, N. C. (2012). } \\
\text { The effects of pre-operative visual information and parental presence } \\
\text { intervention on anxiety, delirium, and pain of post-operative pediatric } \\
\text { patients in PACU. Journal of Korean Academy of Nursing, 42(3), 333- } \\
\text { 341. doi: http://dx.doi.org/10.4040/jkan.2012.42.3.333 }\end{array}$ & $\begin{array}{l}\text { Intervention for } \\
\text { developmental } \\
\text { disorders } \\
\text { (autism, autism } \\
\text { spectrum } \\
\text { disorder), } \\
\text { physical } \\
\text { disabilities, } \\
\text { medical } \\
\text { conditions or } \\
\text { distress about } \\
\text { facing medical } \\
\text { interventions }\end{array}$ \\
\hline $\begin{array}{l}\text { 1832. Yorbik, Ö., \& Birmaher, B. (2003). Pharmacological treatment } \\
\text { of anxiety disorders in children and adolescents. Bulletin of Clinical } \\
\text { Psychopharmacology, 13(3), 133-141. }\end{array}$ & Review \\
\hline $\begin{array}{l}\text { 1833. Yoshikawa H, Magnuson KA, Bos JM and Hsueh J. 2003. Effects } \\
\text { of earnings-supplement policies on adult economic and middle- } \\
\text { childhood outcomes differ for the hardest to employ. Child Dev. } \\
74(5): 1500-21 \text {. }\end{array}$ & $\begin{array}{l}\text { Not a parenting } \\
\text { intervention }\end{array}$ \\
\hline
\end{tabular}




\begin{tabular}{|c|c|}
\hline $\begin{array}{l}\text { 1834. Young JF, Mufson L and Davies M. 2006. Efficacy of } \\
\text { Interpersonal Psychotherapy-Adolescent Skills Training: An indicated } \\
\text { preventive intervention for depression. Journal of Child Psychology } \\
\text { and Psychiatry. 47(12):1254-62. }\end{array}$ & $\begin{array}{l}\text { Not a parenting } \\
\text { intervention }\end{array}$ \\
\hline $\begin{array}{l}\text { 1835. Young JF, Mufson L and Gallop R. 2010. Preventing depression: } \\
\text { A randomized trial of interpersonal psychotherapy-adolescent skills } \\
\text { training. Depression and Anxiety. 27(5):426-33. }\end{array}$ & $\begin{array}{l}\text { Not a parenting } \\
\text { intervention }\end{array}$ \\
\hline $\begin{array}{l}\text { 1836. Younus M and Labellarte MJ. 2002. Insomnia in children: } \\
\text { When are hypnotics indicated? Pediatric Drugs. 4(6):391-403. }\end{array}$ & Review \\
\hline $\begin{array}{l}1837 . \quad \text { Yu DS. 2002. Preventing depressive symptoms in Chinese } \\
\text { children. Prevention \& Treatment, 5(1), 9a. }\end{array}$ & $\begin{array}{l}\text { Not a parenting } \\
\text { intervention }\end{array}$ \\
\hline $\begin{array}{l}\text { 1838. Yu S, Clemens R, Yang H, Li X, Stanton B, Deveaux L, Lunn S, } \\
\text { Cottrell L and Harris C. 2006. Youth and parental perceptions of } \\
\text { parental monitoring and parent-adolescent communication, youth } \\
\text { depression, and youth risk behaviors. Social Behavior and Personality. } \\
\text { 34(10):1297-310. }\end{array}$ & $\begin{array}{l}\text { Not a parenting } \\
\text { intervention }\end{array}$ \\
\hline $\begin{array}{l}\text { 1839. Yuen, E., \& Toumbourou, J. W. (2011). Does family } \\
\text { intervention for adolescent substance use impact parental wellbeing? } \\
\text { A longitudinal evaluation. ANZJFT Australian and New Zealand Journal } \\
\text { of Family Therapy, 32, 249-263. }\end{array}$ & $\begin{array}{l}\text { No child } \\
\text { internalising } \\
\text { outcomes or not } \\
\text { a major goal }\end{array}$ \\
\hline $\begin{array}{l}\text { 1840. Zastowny TR, Kirschenbaum DS and Meng AL. 1986. Coping } \\
\text { skills training for children: effects on distress before, during, and after } \\
\text { hospitalization for surgery. Health Psychol.5(3):231-47. }\end{array}$ & $\begin{array}{l}\text { Intervention for } \\
\text { developmental } \\
\text { disorders } \\
\text { (autism, autism } \\
\text { spectrum } \\
\text { disorder), } \\
\text { physical } \\
\text { disabilities, } \\
\text { medical } \\
\text { conditions or } \\
\text { distress about } \\
\text { facing medical } \\
\text { interventions }\end{array}$ \\
\hline $\begin{array}{l}\text { 1841. Zauszniewski, J. A., Au, T. Y., \& Musil, C. M. (2012). } \\
\text { Resourcefulness training for grandmothers raising grandchildren: is } \\
\text { there a need? Issues in mental health nursing, 33(10), 680-686. } \\
\text { doi:10.3109/01612840.2012.684424 }\end{array}$ & $\begin{array}{l}\text { No child } \\
\text { internalising } \\
\text { outcomes or not } \\
\text { a major goal }\end{array}$ \\
\hline $\begin{array}{l}\text { 1842. Zehnder D, Meuli M and Landolt MA. 2010. Effectiveness of a } \\
\text { single-session early psychological intervention for children after road } \\
\text { traffic accidents: A randomised controlled trial. Child and Adolescent } \\
\text { Psychiatry and Mental Health. 4(7), 1-10. }\end{array}$ & $\begin{array}{l}\text { Not a parenting } \\
\text { intervention }\end{array}$ \\
\hline
\end{tabular}




\begin{tabular}{|c|c|}
\hline $\begin{array}{l}\text { 1843. Zelikovsky N, Rodrigue JR and Gidycz CA. 2001. Reducing } \\
\text { parent distress and increasing parent coping-promoting behavior } \\
\text { during children's medical procedure. Journal of Clinical Psychology in } \\
\text { Medical Settings, } 8(4), 273-281 \text {. }\end{array}$ & $\begin{array}{l}\text { Intervention for } \\
\text { developmental } \\
\text { disorders } \\
\text { (autism, autism } \\
\text { spectrum } \\
\text { disorder), } \\
\text { physical } \\
\text { disabilities, } \\
\text { medical } \\
\text { conditions or } \\
\text { distress about } \\
\text { facing medical } \\
\text { interventions }\end{array}$ \\
\hline $\begin{array}{l}\text { 1844. Zelikovsky N, Rodrigue JR, Gidycz CA and Davis MA. } 2000 . \\
\text { Cognitive behavioral and behavioral interventions help young children } \\
\text { cope during a voiding cystourethrogram. J Pediatr Psychol. 25(8):535- } \\
43 .\end{array}$ & $\begin{array}{l}\text { Intervention for } \\
\text { developmental } \\
\text { disorders } \\
\text { (autism, autism } \\
\text { spectrum } \\
\text { disorder), } \\
\text { physical } \\
\text { disabilities, } \\
\text { medical } \\
\text { conditions or } \\
\text { distress about } \\
\text { facing medical } \\
\text { interventions }\end{array}$ \\
\hline $\begin{array}{l}\text { 1845. Zelkowitz, P., Feeley, N., Shrier, l., Stremler, R., Westreich, R., } \\
\text { Dunkley, D., Steele, R., Rosberger, Z., Lefebvre, F., \& Papageorgiou, A. } \\
\text { (2011). The cues and care randomized controlled trial of a neonatal } \\
\text { intensive care unit intervention: effects on maternal psychological } \\
\text { distress and mother-infant interaction. J Dev Behav Pediatr, 32, 591- } \\
599 .\end{array}$ & $\begin{array}{l}\text { No child } \\
\text { internalising } \\
\text { outcomes or not } \\
\text { a major goal }\end{array}$ \\
\hline $\begin{array}{l}\text { 1846. Zeller MH and Modi AC. 2006. Predictors of Health-Related } \\
\text { Quality of Life in Obese Youth. Obesity Research. 14(1):122-30. }\end{array}$ & $\begin{array}{l}\text { Not a parenting } \\
\text { intervention }\end{array}$ \\
\hline $\begin{array}{l}\text { 1847. Zhai, F., Brooks-Gunn, J., \& Waldfogel, J. (2011). Head Start } \\
\text { and urban children's school readiness: A birth cohort study in } 18 \text { cities. } \\
\text { Developmental Psychology, 47(1), 134-152. doi: 10.1037/a0020784 }\end{array}$ & $\begin{array}{l}\text { Parent } \\
\text { component too } \\
\text { small; Not an } \\
\text { RCT }\end{array}$ \\
\hline $\begin{array}{l}\text { 1848. Zhang XQ, Li RQ and Wang L. 2007. Effect of comprehensive } \\
\text { intervention on parental rearing behavior of key middle school } \\
\text { students. Chinese Mental Health Journal, 21(4), } 280 \text {. }\end{array}$ & $\begin{array}{l}\text { No child } \\
\text { internalising } \\
\text { outcomes or not } \\
\text { a major goal }\end{array}$ \\
\hline $\begin{array}{l}\text { 1849. Zhou, Q., Sandler, I. N., Millsap, R. E., Wolchik, S. A., \& } \\
\text { Dawson-McClure, S. R. (2008). Mother-child relationship quality and } \\
\text { effective discipline as mediators of the 6-year effects of the New } \\
\text { Beginnings Program for children from divorced families. Journal of } \\
\text { Consulting and Clinical Psychology, 76(4), } 579 .\end{array}$ & $\begin{array}{l}\text { Secondary } \\
\text { analysis }\end{array}$ \\
\hline $\begin{array}{l}\text { 1850. Zhu, M. Z., Zhu, X. J., DU, J., \& Zhang, X. L. (2014). [Efficacy of } \\
\text { short-term educational intervention for parents of preschool children } \\
\text { with anxiety.]. Zhongguo dang dai er ke za zhi= Chinese journal of } \\
\text { contemporary pediatrics, 16(9), 901-904. }\end{array}$ & Not in English \\
\hline
\end{tabular}


Appendix B: List of excluded papers and reason(s) for exclusion

\begin{tabular}{|c|c|}
\hline $\begin{array}{l}\text { 1851. Zubrick SR, Ward KA, Silburn SR, Lawrence D, Williams AA, } \\
\text { Blair EM, et al. Prevention of child behaviour problems through } \\
\text { universal implementation of a group behavioural family intervention. } \\
\text { Prev Sci 2005;6:287-304. }\end{array}$ & $\begin{array}{l}\text { Not an RCT; No } \\
\text { child } \\
\text { internalising } \\
\text { outcomes or not } \\
\text { a major goal }\end{array}$ \\
\hline $\begin{array}{l}\text { 1852. Zuwala R and Barber KR. 2001. Reducing anxiety in parents } \\
\text { before and during pediatric anesthesia induction. AANA J. 69(1):21-5. }\end{array}$ & $\begin{array}{l}\text { Intervention for } \\
\text { developmental } \\
\text { disorders } \\
\text { (autism, autism } \\
\text { spectrum } \\
\text { disorder), } \\
\text { physical } \\
\text { disabilities, } \\
\text { medical } \\
\text { conditions or } \\
\text { distress about } \\
\text { facing medical } \\
\text { interventions }\end{array}$ \\
\hline
\end{tabular}


Appendix Table C1. Study participant and sample size c haracteristics

\begin{tabular}{|c|c|c|c|c|c|c|c|c|c|c|}
\hline $\begin{array}{l}\text { Study } \\
\text { ID }\end{array}$ & $\begin{array}{l}\text { Target } \\
\text { Populatio } \\
\text { n }\end{array}$ & $\begin{array}{l}\text { Inclusion } \\
\text { Criteria }\end{array}$ & $\begin{array}{l}\text { Exclusio } \\
\text { n Criteria }\end{array}$ & $\begin{array}{l}\text { Intervent } \\
\text { i on / } \\
\text { rec ruitme } \\
\text { nt setting }\end{array}$ & $\begin{array}{l}\text { Count } \\
\text { ry }\end{array}$ & $\begin{array}{l}\text { Child } \\
\text { age at } \\
\text { recruitm } \\
\text { ent } M \\
\text { (SD), } \\
\text { range }\end{array}$ & $\begin{array}{l}\text { Child } \\
\text { gender } \\
\text { ratio at } \\
\text { recruit } \\
\text { m ent } \\
\text { M/F n } \\
(\text { M/F \%) }\end{array}$ & $\begin{array}{l}\text { Randomi } \\
\text { sed } \\
\text { T (I/C) }\end{array}$ & $\begin{array}{l}\text { Dropouts } \\
\text { T (I/C) n; } \\
\text { T (I/C) \% }\end{array}$ & $\begin{array}{l}\text { Number } \\
\text { analysed } \\
\text { (follow- } \\
\text { up) } \\
\mathbf{T}(\mathrm{I} / \mathrm{C})\end{array}$ \\
\hline $\begin{array}{l}\text { Achenba } \\
\text { ch } \\
\text { (1993) }\end{array}$ & $\begin{array}{l}\text { Selective: } \\
\text { Low-birth } \\
\text { Infants }\end{array}$ & $\begin{array}{l}\text { Infantsweighted } \\
<2.25 \mathrm{~kg} . \\
\text { Gestational age } \\
<37 \text { weeks. } \\
\text { Hospitalised for } \\
\text { at least } 10 \text { days } \\
\text { in the intensive } \\
\text { care nursery. }\end{array}$ & $\begin{array}{l}\text { Infants from } \\
\text { multiple births, } \\
\text { had congenital } \\
\text { anomalies or } \\
\text { severe } \\
\text { neurological } \\
\text { defects, or from } \\
\text { single mothers }\end{array}$ & $\begin{array}{l}\text { Recruitmen } \\
\text { t- Hospital } \\
\text { in Vermont }\end{array}$ & USA & $\mathrm{n} / \mathrm{a}$ & $\begin{array}{l}29 / 26 \\
(53 \% / 47 \% \\
)^{b}\end{array}$ & $78(38 / 40)$ & $\begin{array}{l}23(14 / 9) \\
29.5 \% \\
(36.8 \% / 22 . \\
5 \%)\end{array}$ & $\begin{array}{l}55 \\
(24 / 31)\end{array}$ \\
\hline $\begin{array}{l}\text { Beardsl } \\
\text { ee } \\
(1997)\end{array}$ & $\begin{array}{l}\text { Selective: } \\
\text { Children } \\
\text { of } \\
\text { depressed } \\
\text { parents }\end{array}$ & $\begin{array}{l}\text { Dual/single } \\
\text { parent(s) with } 1 \\
\text { child(8-15) } \\
\text { years old who } \\
\text { had never been } \\
\text { treated for } \\
\text { affective } \\
\text { disorder; at least } \\
1 \text { parent who }\end{array}$ & $\begin{array}{l}\text { Parental } \\
\text { substance } \\
\text { abuse; history } \\
\text { of parental } \\
\text { schizophrenia; } \\
\text { parents with } \\
\text { any crises and } \\
\text { loses focus on } \\
\text { children; }\end{array}$ & $\begin{array}{l}\text { Recruited } \\
\text { froma } \\
\text { large } \\
\text { prepaid } \\
\text { health } \\
\text { maintenanc } \\
\text { e } \\
\text { organisatio } \\
\text { n (HMO) }\end{array}$ & USA & $\begin{array}{l}11.5 \\
(2.03), 8- \\
15 y^{b}\end{array}$ & $\begin{array}{l}32 / 20 \\
(61.5 \% / 38 \\
.5 \%)^{b}\end{array}$ & $\begin{array}{l}\text { Parents/Fa } \\
\text { milies : } 37 \\
\text { (19/18) } \\
\text { single } \\
\text { parent } \\
\\
\text { Children: } \\
\text { 53(29/24) }\end{array}$ & $\begin{array}{l}1(1 / 0) ; \\
1.9 \%(3.4 \% / \\
0)\end{array}$ & $\begin{array}{l}52 \\
(28 / 24)\end{array}$ \\
\hline $\begin{array}{l}\text { Beardsle } \\
\text { e (2003) }\end{array}$ & & $\begin{array}{l}\text { had an episode } \\
\text { of affective } \\
\text { disorder within } \\
18 \text { months } \\
\text { before contact. }\end{array}$ & $\begin{array}{l}\text { familiesin } \\
\text { therapy for } \\
\text { more than } \\
\text { 1x/month; } \\
\text { youngsters in } \\
\text { weekly } \\
\text { psychotherapy } \\
\text { or mood } \\
\text { disorder }\end{array}$ & $\begin{array}{l}\text { Recruited } \\
\text { from a } \\
\text { large } \\
\text { prepaid } \\
\text { HMO in the } \\
\text { Boston } \\
\text { area; } \\
\text { referral } \\
\text { from } \\
\text { mental } \\
\text { health } \\
\text { practitioner } \\
\text { s treating } \\
\text { adult } \\
\text { depression. }\end{array}$ & & $\begin{array}{l}11.6(1.9), \\
8-15 y^{b}\end{array}$ & $\begin{array}{l}69 / 52 \\
(57 \% / 43 \% \\
)^{b}\end{array}$ & $\begin{array}{l}\text { Parents/Fa } \\
\text { milies: } \\
105 \\
(59 / 46) \\
\text { families } \\
\\
\text { Children: } \\
138 \\
(78 / 60)\end{array}$ & $\begin{array}{l}\text { Parents/Fa } \\
\text { milies: } 12 \\
(6 / 6) ; 11 \% \\
(10 \% / 13 \%) \\
\\
\text { Children: } \\
17(9 / 8)\end{array}$ & $\begin{array}{l}\text { Parents/Fa } \\
\text { milies: } 93 \\
(53 / 40) \\
\\
\text { Children: } \\
121 \\
(69 / 52)\end{array}$ \\
\hline
\end{tabular}




\begin{tabular}{|c|c|c|c|c|c|c|c|c|c|c|}
\hline $\begin{array}{l}\text { Study } \\
\text { ID }\end{array}$ & $\begin{array}{l}\text { Target } \\
\text { Populatio } \\
\text { n }\end{array}$ & $\begin{array}{l}\text { Inclusion } \\
\text { Criteria }\end{array}$ & $\begin{array}{l}\text { Exclusio } \\
\text { n Criteria }\end{array}$ & $\begin{array}{l}\text { Intervent } \\
\text { i on / } \\
\text { rec ruitme } \\
\text { nt setting }\end{array}$ & $\begin{array}{l}\text { Count } \\
\text { ry }\end{array}$ & \begin{tabular}{|l} 
Child \\
age at \\
recruitm \\
ent M \\
(SD), \\
range
\end{tabular} & $\begin{array}{l}\text { Child } \\
\text { gender } \\
\text { ratio at } \\
\text { recruit } \\
\text { m ent } \\
\text { M/F n } \\
(M / F \%)\end{array}$ & $\begin{array}{l}\text { Randomi } \\
\text { sed } \\
\text { T (I/C) }\end{array}$ & $\begin{array}{l}\text { Dropouts } \\
\text { T (I/C) n; } \\
\text { T (I/C) \% }\end{array}$ & $\begin{array}{l}\text { Number } \\
\text { analysed } \\
\text { (follow- } \\
\text { up) } \\
T(I / C)\end{array}$ \\
\hline $\begin{array}{l}\text { Beardsle } \\
\text { e (2007) }\end{array}$ & & & & $\begin{array}{l}\text { Recruited } \\
\text { from a } \\
\text { large, } \\
\text { prepaid } \\
\text { HMO, } \\
\text { referral } \\
\text { from } \\
\text { mental } \\
\text { health } \\
\text { practitioner } \\
\text { s treating } \\
\text { adult } \\
\text { depression, } \\
\text { support } \\
\text { groups, } \\
\text { newspaper } \\
\text { advertisem } \\
\text { ents, etc. }\end{array}$ & & $\begin{array}{l}12 \text { (SD } \\
n / a), 8-15 \\
y^{b}\end{array}$ & $n / r$ & $\begin{array}{l}\text { Parents: } \\
200 \\
(106 / 84) \\
\text { Children: } \\
105 \\
(59 / 46)\end{array}$ & $\begin{array}{l}31(15 / 16) \\
15.5 \% \\
(14 \% / 19 \%)\end{array}$ & $\begin{array}{l}\text { Parents: } \\
159 \\
(91 / 68 \\
)\end{array}$ \\
\hline $\begin{array}{l}\text { Black } \\
\text { (2007) }\end{array}$ & $\begin{array}{l}\text { Selective: } \\
\text { I nfants } \\
\text { with } \\
\text { failure to } \\
\text { thrive }\end{array}$ & $\begin{array}{l}<25 \text { months, } \\
\text { gestational age } \\
>36 \text { weeks, birth } \\
\text { weight at least } \\
2500 \text { g; } \\
\text { sustained weight } \\
\text { for age <5th } \\
\text { percentile or } \\
\text { weight for length } \\
<10 \text { th } \\
\text { percentile. }\end{array}$ & $\begin{array}{l}\text { congenital } \\
\text { problems, } \\
\text { disabilities, or } \\
\text { chronic } \\
\text { illnesses }\end{array}$ & $\begin{array}{l}\text { Recruited } \\
\text { from } \\
\text { pediatric } \\
\text { primarycare } \\
\text { clinicsthat } \\
\text { servelow- } \\
\text { income, } \\
\text { urban } \\
\text { communitie } \\
\text { s }\end{array}$ & USA & $\begin{array}{l}(13.1 / 6.5)^{b} \\
c\end{array}$ & $\begin{array}{l}57 / 39 \\
(59 \% / 41 \% \\
)^{b}\end{array}$ & $\begin{array}{l}\text { Children: } \\
130 \\
(66 / 64)\end{array}$ & $\begin{array}{l}34(19 / 15) \\
26 \% \\
(29 \% / 23 \%)\end{array}$ & $\begin{array}{l}96 \\
(47 / 49 \\
)\end{array}$ \\
\hline $\begin{array}{l}\text { Butz } \\
\text { (2001) }\end{array}$ & $\begin{array}{l}\text { Selective: } \\
\text { in-utero } \\
\text { drug- } \\
\text { exposed } \\
\text { (cocaine/ }\end{array}$ & $\begin{array}{l}\text { Maternal } \\
\text { delivery of a } \\
\text { neonate at an } \\
\text { urban hospital, } \\
\text { maternal age 19- }\end{array}$ & $\begin{array}{l}\text { Infants } \\
\text { excluded if <35 } \\
\text { weeks' } \\
\text { gestational } \\
\text { age, required }\end{array}$ & $\begin{array}{l}\text { Recruited } \\
\text { fromurban } \\
\text { hospital } \\
\text { newborn } \\
\text { nurseries } \\
\end{array}$ & USA & $n / a$ & $\begin{array}{l}58 / 59 \\
(50 \% / 50 \% \\
)^{b}\end{array}$ & $\begin{array}{l}204 \\
(96 / 108)\end{array}$ & $\begin{array}{l}87(38 / 49) \\
43 \% \\
(40 \% / 45 \%)\end{array}$ & $\begin{array}{l}100 \\
(49 / 51)\end{array}$ \\
\hline
\end{tabular}




\begin{tabular}{|c|c|c|c|c|c|c|c|c|c|c|}
\hline $\begin{array}{l}\text { Study } \\
\text { ID }\end{array}$ & $\begin{array}{l}\text { Target } \\
\text { Populatio } \\
\text { n }\end{array}$ & $\begin{array}{l}\text { Inclusion } \\
\text { Criteria }\end{array}$ & $\begin{array}{l}\text { Exclusio } \\
\text { n Criteria }\end{array}$ & $\begin{array}{l}\text { Intervent } \\
\text { i on / } \\
\text { rec ruitme } \\
\text { nt setting }\end{array}$ & $\begin{array}{l}\text { Count } \\
\text { ry }\end{array}$ & $\begin{array}{l}\text { Child } \\
\text { age at } \\
\text { recruitm } \\
\text { ent M } \\
\text { (SD), } \\
\text { range }\end{array}$ & $\begin{array}{l}\text { Child } \\
\text { gender } \\
\text { ratio at } \\
\text { recruit } \\
\text { m ent } \\
\text { M/F n } \\
(M / F \%)\end{array}$ & $\begin{array}{l}\text { Randomi } \\
\text { sed } \\
\text { T (I/C) }\end{array}$ & $\begin{array}{l}\text { Dropouts } \\
\text { T (I/C) n; } \\
\text { T (I/C) \% }\end{array}$ & 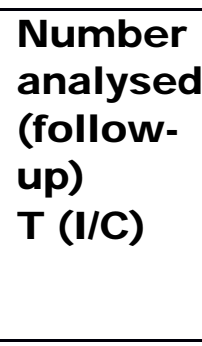 \\
\hline & $\begin{array}{l}\text { opiates) } \\
\text { children }\end{array}$ & $\begin{array}{l}40 \text { years; } \\
\text { maternal use of } \\
\text { cocaine and/or } \\
\text { opiates during } \\
\text { pregnancy. }\end{array}$ & $\begin{array}{l}\text { admission to } \\
\text { the neonatal } \\
\text { ICU for }>24 \\
\text { hours, were } \\
\text { discharged } \\
\text { directly into } \\
\text { nonkinship } \\
\text { foster care or } \\
\text { mothers had } \\
\text { major } \\
\text { psychiatric } \\
\text { diagnosis, } \\
\text { including } \\
\text { schizophrenia } \\
\text { and other } \\
\text { psychotic } \\
\text { disorders. }\end{array}$ & & & & & & & \\
\hline $\begin{array}{l}\text { Carta } \\
\text { (2013) }\end{array}$ & $\begin{array}{l}\text { Selective: } \\
\text { children } \\
\text { fromlow- } \\
\text { income } \\
\text { families; } \\
\text { mother } \\
\text { demonstra } \\
\text { tes at } \\
\text { least one } \\
\text { of } \\
\text { selected } \\
\text { risk } \\
\text { factors for } \\
\text { child } \\
\text { maltreatm } \\
\text { ent }\end{array}$ & $\begin{array}{l}\text { Women between } \\
15-18 \text { years at } \\
\text { first child's birth } \\
\text { OR between } 22- \\
35 \text { years at first } \\
\text { child's birth, with } \\
\text { no formal } \\
\text { education } \\
\text { beyond high } \\
\text { school or GED; } \\
\text { Mothers' oldest } \\
\text { biological child is } \\
\text { between } 4-6 \\
\text { years; Families } \\
\text { anticipate living } \\
\text { in the } 50 \text { mile } \\
\text { vicinity of }\end{array}$ & $\begin{array}{l}\text { Mothers with } \\
\text { major medical } \\
\text { illness that } \\
\text { could interfere } \\
\text { with } \\
\text { participation in } \\
\text { a 2-year } \\
\text { project; Mother } \\
\text { currently lives } \\
\text { in a treatment } \\
\text { program or } \\
\text { rehab center; } \\
\text { Mother has a } \\
\text { serious mental } \\
\text { illness; Mother } \\
\text { lives in a group } \\
\text { home or }\end{array}$ & $\begin{array}{l}\text { Recruitmen } \\
\text { t: Recruited } \\
\text { from } \\
\text { community } \\
\text { health, } \\
\text { early } \\
\text { education, } \\
\text { and social } \\
\text { service } \\
\text { agencies } \\
\text { serving low- } \\
\text { income } \\
\text { families in } \\
\text { metropolita } \\
\text { n South } \\
\text { Bend, } \\
\text { Indiana and }\end{array}$ & USA & $\begin{array}{l}4.56 \\
(0.57), \\
3.5-4.5 y\end{array}$ & $\begin{array}{l}128 / 101 \\
\text { (56\%/44\% } \\
\text { ) }\end{array}$ & $\begin{array}{l}229 \\
(113 / 116)\end{array}$ & $\begin{array}{l}39(24 / 15) ; \\
17 \% \\
(21.2 \% / 12 . \\
9 \%)^{\mathrm{a}}\end{array}$ & $\begin{array}{l}229 \\
(113 / 116)\end{array}$ \\
\hline
\end{tabular}




\begin{tabular}{|c|c|c|c|c|c|c|c|c|c|c|}
\hline $\begin{array}{l}\text { Study } \\
\text { ID }\end{array}$ & $\begin{array}{l}\text { Target } \\
\text { Populatio } \\
\text { n }\end{array}$ & $\begin{array}{l}\text { Inclusion } \\
\text { Criteria }\end{array}$ & $\begin{array}{l}\text { Exclusio } \\
\text { n Criteria }\end{array}$ & $\begin{array}{l}\text { Intervent } \\
\text { i on / } \\
\text { recruitme } \\
\text { nt setting }\end{array}$ & $\begin{array}{l}\text { Count } \\
\text { ry }\end{array}$ & $\begin{array}{l}\text { Child } \\
\text { age at } \\
\text { recruitm } \\
\text { ent M } \\
\text { (SD), } \\
\text { range }\end{array}$ & $\begin{array}{l}\text { Child } \\
\text { gender } \\
\text { ratio at } \\
\text { recruit } \\
\text { m ent } \\
\text { M/F n } \\
(M / F \%)\end{array}$ & $\begin{array}{l}\text { Randomi } \\
\text { sed } \\
\text { T (I/C) }\end{array}$ & $\begin{array}{l}\text { Dropouts } \\
\text { T (I/C) n; } \\
\text { T (I/C) \% }\end{array}$ & $\begin{array}{l}\text { Number } \\
\text { analysed } \\
\text { (follow- } \\
\text { up) } \\
T \text { (I/C) }\end{array}$ \\
\hline & & $\begin{array}{l}\text { Kansas City or } \\
\text { South Bend for } \\
\text { the next } 18 \\
\text { months. Primary } \\
\text { caregiver should } \\
\text { be functionally } \\
\text { literate. Must } \\
\text { speak English or } \\
\text { Spanish fluently. }\end{array}$ & $\begin{array}{l}\text { correctional } \\
\text { program. }\end{array}$ & $\begin{array}{l}\text { metropolita } \\
\text { n Kansas } \\
\text { City. } \\
\text { Interventio } \\
\text { n: Home }\end{array}$ & & & & & & \\
\hline $\begin{array}{l}\text { Cartwrig } \\
\text { ht- } \\
\text { Hatton } \\
(2011)\end{array}$ & $\begin{array}{l}\text { Treatment: } \\
\text { anxious } \\
\text { children }\end{array}$ & $\begin{array}{l}\text { Child age } 9 \text { or } \\
\text { younger; scored } \\
\text { at or above } \\
\text { clinical cut-off on } \\
\text { CBCL } \\
\text { Internalizing } \\
\text { Scale or } \\
\text { Preschool } \\
\text { Behavior } \\
\text { Checklist } \\
\text { Internalising } \\
\text { Scale; or } \\
\text { appeared likely } \\
\text { to have an } \\
\text { anxiety disorder } \\
\text { upon clinical } \\
\text { interview. }\end{array}$ & $\begin{array}{l}\text { The } \\
\text { parent/child } \\
\text { had moderate- } \\
\text { to-severe } \\
\text { learning } \\
\text { difficulties; } \\
\text { child had } \\
\text { moderate-to- } \\
\text { severe autistic } \\
\text { spectrum } \\
\text { disorder. }\end{array}$ & $\begin{array}{l}\text { Recruitmen } \\
\text { t: Mental } \\
\text { Health } \\
\text { Services } \\
\text { referrals } \\
\text { and media } \\
\text { releases. } \\
\text { Interventio } \\
\text { n: donein } \\
\text { research } \\
\text { hospital. }\end{array}$ & UK & $\begin{array}{l}6.6(2.0) \\
2.7-9 y\end{array}$ & $\begin{array}{l}32 / 42 \\
(43.2 \% / 56 \\
.8 \%)\end{array}$ & $74(38 / 36)$ & $\begin{array}{l}16(8 / 8) ; \\
(21 \% / 22 \%)\end{array}$ & $\begin{array}{l}73 \\
(37 / 36)\end{array}$ \\
\hline
\end{tabular}




\begin{tabular}{|c|c|c|c|c|c|c|c|c|c|c|}
\hline $\begin{array}{l}\text { Study } \\
\text { ID }\end{array}$ & $\begin{array}{l}\text { Target } \\
\text { Populatio } \\
\text { n }\end{array}$ & $\begin{array}{l}\text { Inclusion } \\
\text { Criteria }\end{array}$ & $\begin{array}{l}\text { Exclusio } \\
\text { n Criteria }\end{array}$ & $\begin{array}{l}\text { Intervent } \\
\text { i on / } \\
\text { recruitme } \\
\text { nt setting }\end{array}$ & $\begin{array}{l}\text { Count } \\
\text { ry }\end{array}$ & $\begin{array}{l}\text { Child } \\
\text { age at } \\
\text { recruitm } \\
\text { ent } M \\
\text { (SD), } \\
\text { range }\end{array}$ & $\begin{array}{l}\text { Child } \\
\text { gender } \\
\text { ratio at } \\
\text { recruit } \\
\text { m ent } \\
\text { M/F n } \\
(M / F \%)\end{array}$ & $\begin{array}{l}\text { Randomi } \\
\text { sed } \\
\text { T (I/C) }\end{array}$ & $\begin{array}{l}\text { Dropouts } \\
\text { T (I/C) n; } \\
\text { T (I/C) \% }\end{array}$ & $\begin{array}{l}\text { Number } \\
\text { analysed } \\
\text { (follow- } \\
\text { up) } \\
T \text { (I/C) }\end{array}$ \\
\hline $\begin{array}{l}\text { Cheng } \\
\text { (2007) }\end{array}$ & $\begin{array}{l}\text { Universal: } \\
\text { new } \\
\text { mothers }\end{array}$ & $\begin{array}{l}\text { Live births } \\
\text { during J une- } \\
\text { September } \\
\text { 2000, April-J uly } \\
2001 \text {, and } \\
\text { February- } \\
\text { May } 2002 \text {. }\end{array}$ & $\begin{array}{l}\text { Mothers of } \\
\text { nationalities } \\
\text { other than } \\
\text { Japanese, those } \\
\text { who planned to } \\
\text { move out of the } \\
\text { region, or those } \\
\text { who could not } \\
\text { be contacted; } \\
\text { infants with low } \\
\text { birth weight, } \\
\text { premature } \\
\text { delivery, or } \\
\text { congenital } \\
\text { abnormalities. }\end{array}$ & $\begin{array}{l}\text { Recruited } \\
\text { froma } \\
\text { health care } \\
\text { centre }\end{array}$ & JPN & $\begin{array}{l}\text { M (SD) } \\
\text { n/a, Birth }\end{array}$ & $\begin{array}{l}40 / 45 \\
(47 \% / 53 \% \\
)^{b}\end{array}$ & $95(48 / 47)$ & $\begin{array}{l}10(2 / 8) ; \\
10.5 \% \\
(4 \% / 17 \%)\end{array}$ & $\begin{array}{l}85 \\
(46 / 39)\end{array}$ \\
\hline $\begin{array}{l}\text { Cohen } \\
\text { (1997) }\end{array}$ & $\begin{array}{l}\text { Treatment: } \\
\text { sexually } \\
\text { abused } \\
\text { preschool } \\
\text { ers }\end{array}$ & $\begin{array}{l}\text { Substantiated } \\
\text { sexual abuse } \\
\text { within past } 6 \\
\text { months and } \\
\text { child sexually } \\
\text { inappropriate } \\
\text { behaviour or } \\
\text { other behaviour } \\
\text { problems. }\end{array}$ & $\begin{array}{l}\text { Mental } \\
\text { retardation or } \\
\text { pervasive } \\
\text { developmental } \\
\text { disorder, } \\
\text { psychotic } \\
\text { symptoms, a } \\
\text { serious medical } \\
\text { illness, } \\
\text { psychotic } \\
\text { disorder or } \\
\text { active } \\
\text { substance } \\
\text { abuse in the } \\
\text { parent } \\
\text { participating in } \\
\text { treatment, or } \\
\text { the lack of a } \\
\text { long-term }\end{array}$ & $\begin{array}{l}\text { Referred } \\
\text { from } \\
\text { regional } \\
\text { rape crisis } \\
\text { centres, } \\
\text { Child } \\
\text { Protective } \\
\text { Services, } \\
\text { paediatricia } \\
\text { ns, } \\
\text { psychologis } \\
\text { ts, } \\
\text { community } \\
\text { mental } \\
\text { health } \\
\text { agencies, } \\
\text { police } \\
\text { department } \\
\text { s, and the }\end{array}$ & USA & $\begin{array}{l}4.68(S D \\
n / r), 2.11- \\
7.1 y\end{array}$ & $\begin{array}{l}28 / 30 \\
(42 \% / 58 \% \\
)\end{array}$ & $\begin{array}{l}\text { Parents/Fa } \\
\text { milies: } 86 \\
(43 / 43)^{a} \\
\text { Children: } \\
86(\mathrm{I} / \mathrm{C} \mathrm{n/r)}\end{array}$ & $\begin{array}{l}43(15 / 28) ; \\
50 \% \\
(34.9 \% / 65 . \\
1 \%)^{a}\end{array}$ & $\begin{array}{l}43 \\
(28 / 15)\end{array}$ \\
\hline
\end{tabular}




\begin{tabular}{|c|c|c|c|c|c|c|c|c|c|c|}
\hline $\begin{array}{l}\text { Study } \\
\text { ID }\end{array}$ & $\begin{array}{l}\text { Target } \\
\text { Populatio } \\
\text { n }\end{array}$ & $\begin{array}{l}\text { Inclusion } \\
\text { Criteria }\end{array}$ & $\begin{array}{l}\text { Exclusio } \\
\text { n Criteria }\end{array}$ & $\begin{array}{l}\text { Intervent } \\
\text { i on / } \\
\text { recruitme } \\
\text { nt setting }\end{array}$ & $\begin{array}{l}\text { Count } \\
\text { ry }\end{array}$ & $\begin{array}{l}\text { Child } \\
\text { age at } \\
\text { recruitm } \\
\text { ent M } \\
\text { (SD), } \\
\text { range }\end{array}$ & $\begin{array}{l}\text { Child } \\
\text { gender } \\
\text { ratio at } \\
\text { recruit } \\
\text { m ent } \\
\text { M/F n } \\
(M / F \%)\end{array}$ & $\begin{array}{l}\text { Randomi } \\
\text { sed } \\
T(I / C)\end{array}$ & $\begin{array}{l}\text { Dropouts } \\
\text { T (I/C) n; } \\
\text { T (I/C) \% }\end{array}$ & $\begin{array}{l}\text { Number } \\
\text { analysed } \\
\text { (follow- } \\
\text { up) } \\
T \text { (I/C) }\end{array}$ \\
\hline & & & caretaker. & $\begin{array}{l}\text { judicial } \\
\text { system }\end{array}$ & & & & & & \\
\hline $\begin{array}{l}\text { Connell } \\
\text { (2008) }\end{array}$ & $\begin{array}{l}\text { Selective: } \\
\text { Children } \\
\text { at risk of } \\
\text { emotional } \\
\text { and } \\
\text { behaviour } \\
\text { al } \\
\text { problems }\end{array}$ & $\begin{array}{l}\text { Families with a } \\
\text { child between } 2 \text { - } \\
3 \text { years who } \\
\text { were at risk of } \\
\text { future } \\
\text { behavioural } \\
\text { problems in at } \\
\text { least } 2 \text { of } 3 \text { risk } \\
\text { domains: (1) } \\
\text { socioeconomic } \\
\text { (low education } \\
\text { achievementand } \\
\text { low family } \\
\text { income), (2) } \\
\text { family problems } \\
\text { (maternal } \\
\text { depression, daily } \\
\text { parenting } \\
\text { challenges, } \\
\text { substance use, } \\
\text { teen parent } \\
\text { status), (3) child } \\
\text { behaviour } \\
\text { (conduct } \\
\text { problems, high- } \\
\text { conflict } \\
\text { relationships } \\
\text { with adults). }\end{array}$ & $n / r$ & $\begin{array}{l}\text { Recruited } \\
\text { from } \\
\text { familieswith } \\
\text { young } \\
\text { children } \\
\text { who were } \\
\text { engaged in } \\
\text { the Women, } \\
\text { Infants and } \\
\text { Children } \\
\text { Nutrition } \\
\text { Program in } \\
3 \text { cities }\end{array}$ & USA & $\begin{array}{l}28.2 \mathrm{~m} \\
(3.28) \\
2 \mathrm{yOm} \\
2 \mathrm{y} 11 \mathrm{~m}\end{array}$ & $\begin{array}{l}369 / 362 \\
(50.5 \% / 49 \\
.5 \%)\end{array}$ & $\begin{array}{l}731 \\
(367 / 364)\end{array}$ & $\begin{array}{l}112 \\
(54 / 58) ; \\
15.3 \% \\
(14.7 \% / 15 . \\
9 \%)\end{array}$ & $\begin{array}{l}731 \\
(367 / 364)\end{array}$ \\
\hline $\begin{array}{l}\text { Constant } \\
\text { ino } \\
(2001)\end{array}$ & $\begin{array}{l}\text { Selective: } \\
\text { children at } \\
\text { risk of } \\
\text { child } \\
\end{array}$ & $\begin{array}{l}\text { Women with3- } \\
\text { to } 18-\text { month old } \\
\text { infants, residing } \\
\text { in disadvantaged }\end{array}$ & $\mathrm{n} / \mathrm{r}$ & $\begin{array}{l}\text { Recruited } \\
\text { via health } \\
\text { clinics, } \\
\text { community }\end{array}$ & USA & $\begin{array}{l}\text { 8.3(5.7); } \\
3-18 \mathrm{~m}\end{array}$ & $\begin{array}{l}57 / 28 \\
(67 \% / 33 \% \\
)\end{array}$ & $85(51 / 34)$ & $\begin{array}{l}40(27 / 13) ; \\
47.1 \% \\
(47.1 \% / 38 . \\
2 \%)\end{array}$ & $\begin{array}{l}45 \\
(24 / 21)\end{array}$ \\
\hline
\end{tabular}




\begin{tabular}{|c|c|c|c|c|c|c|c|c|c|c|}
\hline $\begin{array}{l}\text { Study } \\
\text { ID }\end{array}$ & $\begin{array}{l}\text { Target } \\
\text { Populatio } \\
\text { n }\end{array}$ & $\begin{array}{l}\text { Inclusion } \\
\text { Criteria }\end{array}$ & $\begin{array}{l}\text { Exclusio } \\
\text { n Criteria }\end{array}$ & $\begin{array}{l}\text { Intervent } \\
\text { i on / } \\
\text { rec ruitme } \\
\text { nt setting }\end{array}$ & $\begin{array}{l}\text { Count } \\
\text { ry }\end{array}$ & $\begin{array}{l}\text { Child } \\
\text { age at } \\
\text { recruitm } \\
\text { ent M } \\
\text { (SD), } \\
\text { range }\end{array}$ & \begin{tabular}{|l|} 
Child \\
gender \\
ratio at \\
recruit \\
m ent \\
M/F n \\
(M/F \%)
\end{tabular} & $\begin{array}{l}\text { Randomi } \\
\text { sed } \\
\text { T (I/C) }\end{array}$ & $\begin{array}{l}\text { Dropouts } \\
\text { T (I/C) n; } \\
\text { T (I/C) \% }\end{array}$ & $\begin{array}{l}\text { Number } \\
\text { analysed } \\
\text { (follow- } \\
\text { up) } \\
T(I / C)\end{array}$ \\
\hline & $\begin{array}{l}\text { abuse or } \\
\text { neglect }\end{array}$ & $\begin{array}{l}\text { urban regions, } \\
\text { who were } \\
\text { already enrolled } \\
\text { in an existing } \\
\text { home visitation } \\
\text { program. }\end{array}$ & & $\begin{array}{l}\text { service } \\
\text { clinics, or } \\
\text { door-to- } \\
\text { door in two } \\
\text { disadvanta } \\
\text { ged urban } \\
\text { regions of } \\
\text { St. Louis. }\end{array}$ & & & & & & \\
\hline $\begin{array}{l}\text { Cowan } \\
\text { (2005) }\end{array}$ & \multirow{2}{*}{$\begin{array}{l}\text { Universal: } \\
\text { Parents of } \\
\text { first child } \\
\text { entering } \\
\text { kindergart } \\
\text { en }\end{array}$} & \multirow{2}{*}{$\begin{array}{l}2 \text { parents living } \\
\text { together with a } \\
\text { first child } \\
\text { entering } \\
\text { kindergarten in } \\
\text { the next fall; and } \\
\text { who completed } \\
\text { an initial 4-page } \\
\text { questionnaire } \\
\text { booklet }\end{array}$} & \multirow[t]{2}{*}{$n / r$} & \multirow{2}{*}{$\begin{array}{l}\text { Recruited } \\
\text { through } \\
\text { daycare } \\
\text { centres, } \\
\text { preschools, } \\
\text { pediatrician } \\
\text { 's offices, } \\
\text { and public } \\
\text { service } \\
\text { announcem } \\
\text { ents in the } \\
\text { media }\end{array}$} & \multirow[t]{2}{*}{ USA } & \multirow[t]{2}{*}{$\begin{array}{l}4.99 \\
(0.56) ; \\
4.5-5.5 \mathrm{y}^{\mathrm{ac}}\end{array}$} & \multirow[t]{2}{*}{$\begin{array}{l}59 / 41 \\
(59 \% / 41 \% \\
)\end{array}$} & $\begin{array}{l}112 \\
(73 / 39)\end{array}$ & $\begin{array}{l}68(51 / 17) ; \\
60.7 \% \\
(69.9 \% / \\
43.6 \%) \\
\end{array}$ & $\begin{array}{l}44 \\
(22 / 22 \\
)\end{array}$ \\
\hline $\begin{array}{l}\text { Cowan } \\
\text { (2011) }\end{array}$ & & & & & & & & & $n / r$ & $n / r$ \\
\hline $\begin{array}{l}\text { Dadds } \\
\text { (2008) }\end{array}$ & $\begin{array}{l}\text { Universal: } \\
\text { preschool } \\
\text { children }\end{array}$ & $\begin{array}{l}\text { Parents of } \\
\text { children aged } 3 \\
\text { to } 7 \text { years from } \\
25 \text { preschools. }\end{array}$ & $\begin{array}{l}\text { Children with } \\
\text { developmental } \\
\text { delay or English } \\
\text { as a second } \\
\text { language were } \\
\text { deleted from } \\
\text { data analyses }\end{array}$ & $\begin{array}{l}\text { Recruited } \\
\text { from } \\
\text { preschools } \\
\text { in Brisbane, } \\
\text { Australia }\end{array}$ & AUS & $\begin{array}{l}4.77(0.47) \\
; 2-6 y\end{array}$ & $\begin{array}{l}389 / 345 \\
(53 \% / 47 \% \\
)\end{array}$ & $\begin{array}{l}734 \\
(355 / 379)\end{array}$ & $\begin{array}{l}\text { Teacher } \\
\text { report: } \\
254 \\
(127 / 127) ; \\
34.6 \% \\
(35.8 \% / 33 . \\
5 \%)\end{array}$ & $\begin{array}{l}\text { Teacher } \\
\text { report: } \\
480 \\
(228 / 252)\end{array}$ \\
\hline $\begin{array}{l}\text { Etter } \\
\text { (2013) }\end{array}$ & $\begin{array}{l}\text { Selective: } \\
\text { Child of } \\
\text { low- } \\
\text { income } \\
\text { couple }\end{array}$ & $\begin{array}{l}\text { In committed } \\
\text { relationships } \\
\text { (married or } \\
\text { cohabiting for at } \\
\text { least } 6 \text { months) } \\
\text { and caring for at } \\
\text { least one child }\end{array}$ & $\begin{array}{l}\text { Prior } \\
\text { experiences } \\
\text { with the } \\
\text { intervention } \\
\text { material, } \\
\text { clearly } \\
\text { intoxicated, not }\end{array}$ & $\begin{array}{l}\text { Recruitmen } \\
\text { t: Flyers, } \\
\text { newspaper } \\
\text { and online } \\
\text { advertisem } \\
\text { ents, media } \\
\text { interviews, }\end{array}$ & USA & $n / r$ & $n / r$ & $\begin{array}{l}301 \\
(229 / 72)\end{array}$ & $\begin{array}{l}\text { FU1: 45 } \\
\text { (38/7); 15\% } \\
(16.6 \% / 9.7 \\
\%) ; \\
\text { FU2: } 78 \\
(67 / 11) ; \\
25.9 \%\end{array}$ & $\begin{array}{l}\text { FU1: } 256 \\
\text { (191/65); } \\
\text { FU2: } 223 \\
(162 / 61)\end{array}$ \\
\hline
\end{tabular}




\begin{tabular}{|c|c|c|c|c|c|c|c|c|c|c|}
\hline $\begin{array}{l}\text { Study } \\
\text { ID }\end{array}$ & $\begin{array}{l}\text { Target } \\
\text { Populatio } \\
\text { n }\end{array}$ & $\begin{array}{l}\text { Inclusion } \\
\text { Criteria }\end{array}$ & $\begin{array}{l}\text { Exclusio } \\
\text { n Criteria }\end{array}$ & $\begin{array}{l}\text { Intervent } \\
\text { i on / } \\
\text { recruitme } \\
\text { nt setting }\end{array}$ & $\begin{array}{l}\text { Count } \\
\text { ry }\end{array}$ & $\begin{array}{l}\text { Child } \\
\text { age at } \\
\text { recruitm } \\
\text { ent M } \\
\text { (SD), } \\
\text { range }\end{array}$ & $\begin{array}{l}\text { Child } \\
\text { gender } \\
\text { ratio at } \\
\text { recruit } \\
\text { m ent } \\
\text { M/F n } \\
\text { (M/F \%) }\end{array}$ & $\begin{array}{l}\text { Randomi } \\
\text { sed } \\
T \text { (I/C) }\end{array}$ & $\begin{array}{l}\text { Dropouts } \\
\text { T (I/C) n; } \\
\text { T (I/C) \% }\end{array}$ & $\begin{array}{l}\text { Number } \\
\text { analysed } \\
\text { (follow- } \\
\text { up) } \\
T \text { (I/C) }\end{array}$ \\
\hline & & together & $\begin{array}{l}\text { fluent in } \\
\text { English. }\end{array}$ & $\begin{array}{l}\text { and } \\
\text { collaboratio } \\
\mathrm{n} \text { with } \\
\text { community } \\
\text { leaders and } \\
\text { organizatio } \\
\text { ns. } \\
\text { Interventio } \\
\mathrm{n}: \\
\text { Workshops } \\
\text { were } \\
\text { conducted } \\
\text { both at the } \\
\text { university } \\
\text { and at two } \\
\text { centrally } \\
\text { located } \\
\text { community } \\
\text { agencies. }\end{array}$ & & & & & $\begin{array}{l}(29.3 \% / 15 . \\
3 \%)\end{array}$ & \\
\hline $\begin{array}{l}\text { Feinber } \\
\text { g } \\
(2010)\end{array}$ & \multirow[t]{2}{*}{$\begin{array}{l}\text { Universal: } \\
\text { couples } \\
\text { expecting } \\
\text { first child }\end{array}$} & \multirow{2}{*}{$\begin{array}{l}\text { First-time } \\
\text { parents must } \\
\text { have been at } \\
\text { least } 18 \text { years of } \\
\text { age, living } \\
\text { together } \\
\text { (regardless of } \\
\text { marital status) } \\
\text { and expecting a } \\
\text { first child. }\end{array}$} & \multirow[t]{2}{*}{$n / r$} & \multirow{2}{*}{$\begin{array}{l}\text { Recruited } \\
\text { from } \\
\text { childbirth } \\
\text { education } \\
\text { programs } \\
\text { at } \\
\text { hospitals, } \\
\text { health } \\
\text { centres, } \\
\text { media } \\
\text { advertising } \\
\text { and word of } \\
\text { mouth. } \\
\text { Delivered } \\
\text { via }\end{array}$} & \multirow[t]{2}{*}{ USA } & \multirow[t]{2}{*}{$n / a$} & \multirow[t]{2}{*}{$n / r$} & \multirow[t]{2}{*}{$\begin{array}{l}169 \\
(89 / 80)\end{array}$} & $\begin{array}{l}27(14 / 13) ; \\
16.0 \% \\
(15.7 \% / 16 . \\
3 \%)^{a}\end{array}$ & $\begin{array}{l}142 \\
(75 / 67)^{\mathrm{a}}\end{array}$ \\
\hline $\begin{array}{l}\text { Feinberg } \\
\text { (2014) }\end{array}$ & & & & & & & & & $\begin{array}{l}71(39 / 32) ; \\
42 \% \\
(43.8 \% / 40 \\
\%)\end{array}$ & $\begin{array}{l}98 \\
(50 / 48)\end{array}$ \\
\hline
\end{tabular}




\begin{tabular}{|c|c|c|c|c|c|c|c|c|c|c|}
\hline $\begin{array}{l}\text { Study } \\
\text { ID }\end{array}$ & $\begin{array}{l}\text { Target } \\
\text { Populatio } \\
\text { n }\end{array}$ & $\begin{array}{l}\text { Inclusion } \\
\text { Criteria }\end{array}$ & $\begin{array}{l}\text { Exclusio } \\
\text { n Criteria }\end{array}$ & $\begin{array}{l}\text { Intervent } \\
\text { i on / } \\
\text { recruitme } \\
\text { nt setting }\end{array}$ & $\begin{array}{l}\text { Count } \\
\text { ry }\end{array}$ & \begin{tabular}{|l} 
Child \\
age at \\
recruitm \\
ent M \\
(SD), \\
range
\end{tabular} & $\begin{array}{l}\text { Child } \\
\text { gender } \\
\text { ratio at } \\
\text { recruit } \\
\text { m ent } \\
\text { M/F n } \\
(M / F \%)\end{array}$ & $\begin{array}{l}\text { Randomi } \\
\text { sed } \\
T(I / C)\end{array}$ & $\begin{array}{l}\text { Dropouts } \\
\text { T (I/C) n; } \\
\text { T (I/C) \% }\end{array}$ & $\begin{array}{l}\text { Number } \\
\text { analysed } \\
\text { (follow- } \\
\text { up) } \\
\text { T (I/C) }\end{array}$ \\
\hline & & & & $\begin{array}{l}\text { childbirth } \\
\text { education } \\
\text { department } \\
\text { s at local } \\
\text { hospitals. }\end{array}$ & & & & & & \\
\hline $\begin{array}{l}\text { Fergusso } \\
\text { n(2013) }\end{array}$ & $\begin{array}{l}\text { Selective: } \\
\text { Families } \\
\text { facing } \\
\text { “multiple } \\
\text { challenges } \\
\text { " }\end{array}$ & $\begin{array}{l}\text { 11-point } \\
\text { screening } \\
\text { measure based } \\
\text { on the measure } \\
\text { used in the } \\
\text { Hawaii Healthy } \\
\text { Start Program. } \\
\text { Screening } \\
\text { covered parent } \\
\text { and family } \\
\text { functioning, } \\
\text { including age of } \\
\text { parents, social } \\
\text { support, } \\
\text { planning of } \\
\text { pregnancy, } \\
\text { parental } \\
\text { substance use, } \\
\text { family financial } \\
\text { situation, and } \\
\text { family violence. } \\
\text { Plunket nurses } \\
\text { were asked to } \\
\text { refer any family } \\
\text { in which } 2 \text { or } \\
\text { more risk factors } \\
\text { were present, or } \\
\text { where there } \\
\text { were serious }\end{array}$ & $n / r$ & $\begin{array}{l}\text { Recruitmen } \\
\text { t: Plunket } \\
\text { nurses } \\
\text { (community } \\
\text { nurses) } \\
\text { visit } \\
\text { families } \\
\text { within } 3 \\
\text { months of } \\
\text { the child's } \\
\text { birth } \\
\text { Interventio } \\
\text { n: Home } \\
\text { visits by } \\
\text { Family } \\
\text { Support } \\
\text { Workers }\end{array}$ & $\mathrm{NZ}$ & $\begin{array}{l}2.2(1.4) ; \\
0.5-14.5 y^{\mathrm{a}}\end{array}$ & $\begin{array}{l}231 / 212 \\
(52 \% / 48 \% \\
)^{a}\end{array}$ & $\begin{array}{l}443 \\
(220 / 223)\end{array}$ & $\begin{array}{l}73(49 / 24) \\
16.5 \% \\
(22.2 \% / 10 . \\
8 \%)\end{array}$ & $\begin{array}{l}370 \\
(171 / 199)\end{array}$ \\
\hline
\end{tabular}




\begin{tabular}{|c|c|c|c|c|c|c|c|c|c|c|}
\hline $\begin{array}{l}\text { Study } \\
\text { ID }\end{array}$ & $\begin{array}{l}\text { Target } \\
\text { Populatio } \\
\text { n }\end{array}$ & $\begin{array}{l}\text { Inclusion } \\
\text { Criteria }\end{array}$ & $\begin{array}{l}\text { Exclusio } \\
\text { n Criteria }\end{array}$ & $\begin{array}{l}\text { Intervent } \\
\text { i on / } \\
\text { rec ruitme } \\
\text { nt setting }\end{array}$ & $\begin{array}{l}\text { Count } \\
\text { ry }\end{array}$ & $\begin{array}{l}\text { Child } \\
\text { age at } \\
\text { recruitm } \\
\text { ent } M \\
\text { (SD), } \\
\text { range }\end{array}$ & $\begin{array}{l}\text { Child } \\
\text { gender } \\
\text { ratio at } \\
\text { recruit } \\
\text { m ent } \\
\text { M/F n } \\
(M / F \%) \\
\end{array}$ & $\begin{array}{l}\text { Randomi } \\
\text { sed } \\
\text { T (I/C) }\end{array}$ & $\begin{array}{l}\text { Dropouts } \\
\text { T (I/C) n; } \\
\text { T (I/C) \% }\end{array}$ & $\begin{array}{l}\text { Number } \\
\text { analysed } \\
\text { (follow- } \\
\text { up) } \\
T \text { (I/C) }\end{array}$ \\
\hline & & $\begin{array}{l}\text { concerns about } \\
\text { the family's } \\
\text { capacity to care } \\
\text { for the child. }\end{array}$ & & & & & & & & \\
\hline $\begin{array}{l}\text { Forgatch } \\
\text { (1999) }\end{array}$ & $\begin{array}{l}\text { Selective: } \\
\text { Divorcing } \\
\text { mothers } \\
\text { with sons } \\
\text { in Grades } \\
1-3\end{array}$ & $\begin{array}{l}\text { Mothers who had } \\
\text { separated from } \\
\text { their partner } \\
\text { within the prior } 3 \\
\text { to } 24 \text { months } \\
\text { and resided with } \\
\text { a biological son } \\
\text { in Grades } 1 \text { to } 3 \text {. }\end{array}$ & $\begin{array}{l}\text { Mothers that } \\
\text { were } \\
\text { cohabiting with } \\
\text { a new partner. }\end{array}$ & $\begin{array}{l}\text { Recruited } \\
\text { via media } \\
\text { advertisem } \\
\text { ents, flyers } \\
\text { and divorce } \\
\text { court } \\
\text { records. }\end{array}$ & USA & $\begin{array}{l}7.8(0.93) \\
6.1-10.4 y\end{array}$ & $\begin{array}{l}238 / 0 \\
(100 \% / 0 \% \\
)\end{array}$ & $\begin{array}{l}238 \\
(153 / 85)\end{array}$ & $\begin{array}{l}81(53 / 28) ; \\
34 \% \\
(34.6 \% / 32 . \\
9 \%)\end{array}$ & $\begin{array}{l}157 \\
(100 / 57)\end{array}$ \\
\hline $\begin{array}{l}\text { Ginsburg } \\
\text { (2009) }\end{array}$ & $\begin{array}{l}\text { Selective: } \\
\text { Children } \\
\text { of parents } \\
\text { with } \\
\text { anxiety } \\
\text { disorders }\end{array}$ & $\begin{array}{l}\text { Children aged 7- } \\
12 \text { years without } \\
\text { an anxiety } \\
\text { disorder and not } \\
\text { receiving } \\
\text { treatment for } \\
\text { anxiety } \\
\text { reduction. } \\
\text { Parents with a } \\
\text { current or } \\
\text { lifetime } \\
\text { diagnosis of an } \\
\text { anxiety disorder } \\
\text { except for PTSD } \\
\text { and ASD. }\end{array}$ & $\begin{array}{l}\text { Child or parent } \\
\text { with a medical } \\
\text { or psychiatric } \\
\text { condition } \\
\text { contraindicatin } \\
\text { g study } \\
\text { intervention. }\end{array}$ & $\begin{array}{l}\text { Recruited } \\
\text { via } \\
\text { newspaper } \\
\text { advertisem } \\
\text { ents, } \\
\text { mailings to } \\
\text { local } \\
\text { doctors and } \\
\text { psychiatrist } \\
\text { s, and } \\
\text { community } \\
\text { flyers. } \\
\text { Interventio } \\
\text { n setting } \\
\text { not } \\
\text { reported. }\end{array}$ & USA & $\begin{array}{l}8.9(1.9) ; \\
\text { Range } \mathrm{n} / \mathrm{r}\end{array}$ & $\begin{array}{l}22 / 18 \\
(55 \% / 45 \% \\
)\end{array}$ & $40(20 / 20)$ & $\begin{array}{l}\text { Follow-up 1: } \\
5(3 / 2) ; \\
12.5 \% \\
(15 \% / 10 \%) \\
\text { Follow-up 2: } \\
7(4 / 3) ; \\
17.5 \% \\
(20 \% / 15 \%)\end{array}$ & $\begin{array}{l}40 \\
(20 / 20)\end{array}$ \\
\hline $\begin{array}{l}\text { Hahlweg } \\
\text { (2010) }\end{array}$ & $\begin{array}{l}\text { Universal: } \\
\text { Parents } \\
\text { with pre- } \\
\text { schoolers }\end{array}$ & $\begin{array}{l}\text { Families with } \\
\text { children aged } 3 \\
\text { to } 6\end{array}$ & $\begin{array}{l}\text { Inadequate } \\
\text { German } \\
\text { language } \\
\text { ability }\end{array}$ & $\begin{array}{l}\text { Recruited } \\
\text { from } \\
\text { preschools } \\
\text { in } \\
\text { Braunschw } \\
\text { eig, }\end{array}$ & GER & $\begin{array}{l}4.5(1.0) \\
2.6-6.0 y\end{array}$ & $\begin{array}{l}144 / 136 \\
(51 \% / 49 \% \\
)\end{array}$ & $\begin{array}{l}280 \\
(186 / 94)\end{array}$ & $\begin{array}{l}48(44 / 4) ; \\
17 \% \\
(24 \% / 4 \%)\end{array}$ & $\begin{array}{l}280 \\
(186 / 94)\end{array}$ \\
\hline
\end{tabular}




\begin{tabular}{|c|c|c|c|c|c|c|c|c|c|c|}
\hline $\begin{array}{l}\text { Study } \\
\text { ID }\end{array}$ & $\begin{array}{l}\text { Target } \\
\text { Populatio } \\
\text { n }\end{array}$ & $\begin{array}{l}\text { Inclusion } \\
\text { Criteria }\end{array}$ & $\begin{array}{l}\text { Exclusio } \\
\text { n Criteria }\end{array}$ & $\begin{array}{l}\text { Intervent } \\
\text { i on / } \\
\text { rec ruitme } \\
\text { nt setting }\end{array}$ & $\begin{array}{l}\text { Count } \\
\text { ry }\end{array}$ & $\begin{array}{l}\text { Child } \\
\text { age at } \\
\text { recruitm } \\
\text { ent M } \\
\text { (SD), } \\
\text { range }\end{array}$ & $\begin{array}{l}\text { Child } \\
\text { gender } \\
\text { ratio at } \\
\text { recruit } \\
\text { m ent } \\
\text { M/F n } \\
(M / F \%)\end{array}$ & $\begin{array}{l}\text { Randomi } \\
\text { sed } \\
\text { T (I/C) }\end{array}$ & $\begin{array}{l}\text { Dropouts } \\
\text { T (I/C) n; } \\
\text { T (I/C) \% }\end{array}$ & $\begin{array}{l}\text { Number } \\
\text { analysed } \\
\text { (follow- } \\
\text { up) } \\
T \text { (I/C) }\end{array}$ \\
\hline & & & & Germany & & & & & & \\
\hline $\begin{array}{l}\text { Hoff } \\
\text { (2004, } \\
2005)\end{array}$ & $\begin{array}{l}\text { Selective: } \\
\text { Parents of } \\
\text { children } \\
\text { newly } \\
\text { diagnosed } \\
\text { with Type } \\
1 \text { diabetes }\end{array}$ & $\begin{array}{l}\text { Children } \\
\text { diagnosed with } \\
\text { Type } 1 \text { diabetes } \\
\text { in the last } 6 \\
\text { months and } \\
\text { aged under } 18 \\
\text { years }\end{array}$ & $\begin{array}{l}\text { Children } \\
\text { diagnosed with } \\
\text { another chronic } \\
\text { illness or who } \\
\text { had a } \\
\text { developmental } \\
\text { disability }\end{array}$ & $\begin{array}{l}\text { Recruitmen } \\
\text { t and } \\
\text { interventio } \\
\mathrm{n} \text { took } \\
\text { place in two } \\
\text { paediatric } \\
\text { endocrinolo } \\
\text { gyclinics }\end{array}$ & USA & $\begin{array}{l}9.36 \\
(4.08) ; 2- \\
18 y^{b}\end{array}$ & $\begin{array}{l}18 / 16 \\
(53 \% / 47 \% \\
)^{b}\end{array}$ & $46(25 / 21)$ & $\begin{array}{l}12(8 / 4) ; \\
26.1 \% \\
(32 \% / 19.0 \\
\%)\end{array}$ & $\begin{array}{l}34(17 / 17 \\
\text { mothers, } \\
14 / 11 \\
\text { fathers) }\end{array}$ \\
\hline $\begin{array}{l}\text { J ohnson } \\
\text { (2005) }\end{array}$ & $\begin{array}{l}\text { Selective: } \\
\text { parents of } \\
\text { preterm } \\
\text { babies }\end{array}$ & $\begin{array}{l}\text { Babies born < } \\
33 \text { weeks } \\
\text { gestational age } \\
\text { to mothers } \\
\text { resident in the } \\
\text { greater Bristol } \\
\text { area, England }\end{array}$ & $\begin{array}{l}\text { English not first } \\
\text { language used } \\
\text { at home }\end{array}$ & $\begin{array}{l}\text { Recruited } \\
\text { from } \\
\text { intensive } \\
\text { care } \\
\text { nurseries. }\end{array}$ & UK & $\begin{array}{l}\text { M (SD) } \\
\text { n/a; birth }\end{array}$ & $\begin{array}{l}127 / 83 \\
(60.5 \% / 39 \\
.5 \%)\end{array}$ & $\begin{array}{l}222 \\
(116 / 106)\end{array}$ & $\begin{array}{l}83(46 / 37) ; \\
37.4 \% \\
(39.7 \% / 34 . \\
9 \%)\end{array}$ & $\begin{array}{l}139 \\
(70 / 69)\end{array}$ \\
\hline $\begin{array}{l}\text { Kaarese } \\
\text { n } \\
(2008)\end{array}$ & \multirow[t]{2}{*}{$\begin{array}{l}\text { Selective: } \\
\text { I nfants } \\
\text { with low } \\
\text { birth } \\
\text { weight }\end{array}$} & \multirow{2}{*}{$\begin{array}{l}\text { Infants witha } \\
\text { birth weight } \\
<2000 g \text { and } \\
\text { where the } \\
\text { mother's native } \\
\text { language was } \\
\text { Norwegian }\end{array}$} & \multirow[t]{2}{*}{$\begin{array}{l}\text { Triplets, infants } \\
\text { with congenital } \\
\text { anomalies }\end{array}$} & \multirow{2}{*}{$\begin{array}{l}\text { Recruitmen } \\
\text { t: NICU } \\
\text { I nterventio } \\
\text { n: } \\
\text { Hospital/Ho } \\
\text { me }\end{array}$} & \multirow[t]{2}{*}{ NOR } & $\begin{array}{l}\text { M (SD) } \\
\text { n/a; birth }\end{array}$ & \multirow[t]{2}{*}{$\begin{array}{l}77 / 69 \\
(52.7 \% / 47 \\
.3 \%)\end{array}$} & \multirow[t]{2}{*}{$\begin{array}{l}146 \\
(72 / 74)\end{array}$} & $\begin{array}{l}16(5 / 11) ; \\
11.0 \% \\
(6.9 \% / 14.9 \\
\%) \text { mothers }\end{array}$ & $\begin{array}{l}136 \\
(67 / 63 \\
\text { mothers, } \\
61 / 52 \\
\text { fathers) }\end{array}$ \\
\hline $\begin{array}{l}\text { Nordhov } \\
\text { (2012) }\end{array}$ & & & & & & $\begin{array}{l}30.0(3.3) \\
\text { at } \\
\text { discharge } \\
\text { c; birth, } \\
\text { gestationa } \\
\text { I weeks }\end{array}$ & & & $\begin{array}{l}19(7 / 12) \\
\text { mothers; } \\
13 \% \\
(9.7 \% / 16.2 \\
\%) \\
13(5 / 8) \\
\text { children; } \\
9.0 \% \\
(6.9 \% / 10.8 \\
\%)\end{array}$ & $\begin{array}{l}133 \\
(67 / 66)\end{array}$ \\
\hline $\begin{array}{l}\text { Kehoe } \\
(2014)\end{array}$ & $\begin{array}{l}\text { Universal: } \\
\text { grade } 6\end{array}$ & $\begin{array}{l}\text { Primary } \\
\text { caregiver of a }\end{array}$ & $\begin{array}{l}\text { Parents without } \\
\text { sufficient }\end{array}$ & $\begin{array}{l}\text { School- } \\
\text { based }\end{array}$ & AUS & $\begin{array}{l}12.1 \\
(0.42) ; 10-\end{array}$ & $\begin{array}{l}110 / 115 \\
(49 \% / 51 \%\end{array}$ & $\begin{array}{l}229 \\
(125 / 104)\end{array}$ & $\begin{array}{l}15(10 / 5) ; \\
6.6 \%\end{array}$ & $\begin{array}{l}\text { Parents: } \\
213\end{array}$ \\
\hline
\end{tabular}




\begin{tabular}{|c|c|c|c|c|c|c|c|c|c|c|}
\hline $\begin{array}{l}\text { Study } \\
\text { ID }\end{array}$ & $\begin{array}{l}\text { Target } \\
\text { Populatio } \\
\text { n }\end{array}$ & \begin{tabular}{|l|} 
Inclusion \\
Criteria
\end{tabular} & $\begin{array}{l}\text { Exclusio } \\
\text { n Criteria }\end{array}$ & $\begin{array}{l}\text { Intervent } \\
\text { i on / } \\
\text { recruitme } \\
\text { nt setting }\end{array}$ & $\begin{array}{l}\text { Count } \\
\text { ry }\end{array}$ & \begin{tabular}{|l} 
Child \\
age at \\
recruitm \\
ent M \\
(SD), \\
range
\end{tabular} & $\begin{array}{l}\text { Child } \\
\text { gender } \\
\text { ratio at } \\
\text { recruit } \\
\text { m ent } \\
\text { M/F n } \\
\text { (M/F \%) }\end{array}$ & $\begin{array}{l}\text { Randomi } \\
\text { sed } \\
\text { T (I/C) }\end{array}$ & $\begin{array}{l}\text { Dropouts } \\
\text { T (I/C) n; } \\
\text { T (I/C) \% }\end{array}$ & $\begin{array}{l}\text { Number } \\
\text { analysed } \\
\text { (follow- } \\
\text { up) } \\
\text { T (I/C) }\end{array}$ \\
\hline & $\begin{array}{l}\text { students } \\
\text { and their } \\
\text { primary } \\
\text { caregiver }\end{array}$ & $\begin{array}{l}\text { grade } 6 \text { student } \\
\text { in a participating } \\
\text { school, who } \\
\text { provided } \\
\text { consent for their } \\
\text { child to } \\
\text { complete } \\
\text { assessments }\end{array}$ & $\begin{array}{l}\text { English } \\
\text { language skills } \\
\text { to answer } \\
\text { questionnaires } \\
\text { or understand } \\
\text { the program } \\
\text { content; } \\
\text { children with a } \\
\text { primary } \\
\text { diagnosis of a } \\
\text { communication } \\
\text { or pervasive } \\
\text { developmental } \\
\text { disorder }\end{array}$ & $\begin{array}{l}\text { recruitment } \\
\text { interventio } \\
\text { n delivered } \\
\text { in local } \\
\text { community } \\
\text { centers }\end{array}$ & & $13 y$ & )$^{\mathrm{b}}$ & & $(8 \% / 4.8 \%)$ & $\begin{array}{l}(114 / 99) \\
\text { Youth: } \\
212 \\
(113 / 99)\end{array}$ \\
\hline $\begin{array}{l}\text { Koldewi } \\
\text { jn } \\
(2010)\end{array}$ & \multirow[t]{2}{*}{$\begin{array}{l}\text { Selective: } \\
\text { Very } \\
\text { Preterm } \\
\text { infants }\end{array}$} & \multirow{2}{*}{$\begin{array}{l}\text { Infants with } \\
\text { gestational ages } \\
\text { (GA) of <32 } \\
\text { weeksand/or } \\
\text { birth weights of } \\
<1500 \text { g, with } \\
\text { parents living in } \\
\text { Amsterdam }\end{array}$} & \multirow[b]{2}{*}{$\begin{array}{l}\text { Infants with } \\
\text { severe } \\
\text { congenital } \\
\text { abnormalities, } \\
\text { infants whose } \\
\text { mothers had a } \\
\text { documented } \\
\text { history of illicit } \\
\text { drug use or } \\
\text { severe physical } \\
\text { or mental } \\
\text { illness, infants } \\
\text { from non- } \\
\text { Dutchspeaking } \\
\text { families for } \\
\text { whom an } \\
\text { interpreter } \\
\text { could not be } \\
\text { arranged, and } \\
\text { infants who }\end{array}$} & \multirow{2}{*}{$\begin{array}{l}\text { Recruitmen } \\
\text { t: NICU } \\
\text { Interventio } \\
\text { n: } \\
\text { Hospital/Ho } \\
\text { me }\end{array}$} & \multirow[t]{2}{*}{ NL } & $\begin{array}{l}\text { I30.2 (2); } \\
\text { Range } \mathrm{n} / \mathrm{r} \text {, } \\
\text { gestationa } \\
\text { I weeks }^{\mathrm{c}}\end{array}$ & $\begin{array}{l}81 / 80 \\
(50.3 \% / 49 \\
.7 \%)\end{array}$ & \multirow[t]{2}{*}{$\begin{array}{l}176 \\
(86 / 90)\end{array}$} & $\begin{array}{l}15(3 / 12) ; \\
8.5 \% \\
(3.5 \% / 13.3 \\
\%)\end{array}$ & $\begin{array}{l}161 \\
(83 / 78)\end{array}$ \\
\hline $\begin{array}{l}\text { Verkerk } \\
\text { (2012) }\end{array}$ & & & & & & $\begin{array}{l}29.8(2.2) \\
\text { Range } \mathrm{n} / \mathrm{r} \\
\text { gestationa } \\
\text { I weeks }^{\mathrm{c}}\end{array}$ & $\begin{array}{l}82 / 81 \\
(50.3 \% / 49 \\
.7 \%)\end{array}$ & & $\begin{array}{l}25(10 / 15) \\
14.2 \% \\
(11.6 \% / 16 \\
6 \%)\end{array}$ & $\begin{array}{l}151 \\
(76 / 75)\end{array}$ \\
\hline
\end{tabular}




\begin{tabular}{|c|c|c|c|c|c|c|c|c|c|c|}
\hline $\begin{array}{l}\text { Study } \\
\text { ID }\end{array}$ & $\begin{array}{l}\text { Target } \\
\text { Populatio } \\
\text { n }\end{array}$ & $\begin{array}{l}\text { Inclusion } \\
\text { Criteria }\end{array}$ & $\begin{array}{l}\text { Exclusio } \\
\text { n Criteria }\end{array}$ & $\begin{array}{l}\text { Intervent } \\
\text { i on / } \\
\text { rec ruitme } \\
\text { nt setting }\end{array}$ & $\begin{array}{l}\text { Count } \\
\text { ry }\end{array}$ & \begin{tabular}{|l} 
Child \\
age at \\
recruitm \\
ent M \\
(SD), \\
range
\end{tabular} & $\begin{array}{l}\text { Child } \\
\text { gender } \\
\text { ratio at } \\
\text { recruit } \\
\text { m ent } \\
\text { M/F n } \\
(M / F \%)\end{array}$ & $\begin{array}{l}\text { Randomi } \\
\text { sed } \\
T(I / C)\end{array}$ & $\begin{array}{l}\text { Dropouts } \\
\text { T (I/C) n; } \\
\text { T (I/C) \% }\end{array}$ & $\begin{array}{l}\text { Number } \\
\text { analysed } \\
\text { (follow- } \\
\text { up) } \\
\text { T (I/C) }\end{array}$ \\
\hline & & & $\begin{array}{l}\text { participated in } \\
\text { other trials on } \\
\text { post- } \\
\text { discharge }\end{array}$ & & & & & & & \\
\hline $\begin{array}{l}\text { Kratoch } \\
\text { will } \\
\text { (2009) }\end{array}$ & $\begin{array}{l}\text { Universal, } \\
\text { parents of } \\
\text { children in } \\
\text { kindergart } \\
\text { en } \\
\text { through to } \\
\text { grade } 2\end{array}$ & $\begin{array}{l}\text { Students in } \\
\text { kindergarten } \\
\text {, grade } 1 \text {, or } \\
\text { grade } 2 \text { in } \\
\text { schools serving } \\
\text { low-income } \\
\text { communities. }\end{array}$ & $\mathrm{n} / \mathrm{r}$ & $\begin{array}{l}\text { Recruited } \\
\text { from } \\
\text { schools } \\
\text { serving low- } \\
\text { income } \\
\text { communitie } \\
\text { s that } \\
\text { showed } \\
\text { increased } \\
\text { rates of } \\
\text { children } \\
\text { with } \\
\text { 'serious } \\
\text { emotional } \\
\text { disturbance } \\
\text { s' }\end{array}$ & USA & $\begin{array}{l}\text { Est. 6(SD } \\
\mathrm{n} / \mathrm{r}) ; 5-7 \mathrm{y}\end{array}$ & \begin{tabular}{|l|}
$57 / 77$ \\
$(42.5 \% / 57$ \\
$.5 \%)$
\end{tabular} & $\begin{array}{l}134 \\
(67 / 67)\end{array}$ & $\begin{array}{l}56(28 / 28) ; \\
41.8 \% \\
(41.8 \% / 41 . \\
8 \%)\end{array}$ & $\begin{array}{l}78 \\
(39 / 39 \\
)\end{array}$ \\
\hline $\begin{array}{l}\text { Lam } \\
\text { (2008) }\end{array}$ & $\begin{array}{l}\text { Selective: } \\
\text { children } \\
\text { living with } \\
\text { an alcohol } \\
\text { abusing } \\
\text { parent }\end{array}$ & $\begin{array}{l}\text { Men aged 18+ } \\
\text { diagnosed with } \\
\text { alcohol abuse or } \\
\text { dependence; } \\
\text { married or } \\
\text { cohabiting with a } \\
\text { female without a } \\
\text { substance use } \\
\text { problem; and } \\
\text { parent of a child } \\
\text { 8-12 years old } \\
\text { living in the } \\
\text { home }\end{array}$ & $n / r$ & $\begin{array}{l}\text { Recruitmen } \\
\mathrm{t} \text { and } \\
\text { interventio } \\
\mathrm{n} \text { took } \\
\text { place in an } \\
\text { outpatient } \\
\text { alcohol } \\
\text { treatment } \\
\text { service. }\end{array}$ & USA & $\begin{array}{l}8.9(2.1)^{c} \\
\text { Range } \mathrm{n} / \mathrm{r}\end{array}$ & $\begin{array}{l}11 / 9 \\
(55 \% / 45 \% \\
)\end{array}$ & $20(10 / 10)$ & $n / r$ & $\begin{array}{l}20 \\
(10 / 10)\end{array}$ \\
\hline Lewis & Selective: & Diagnosed with & Non-English & Recruitmen & USA & $\mathrm{n} / \mathrm{r} ; 8-12$ & $n / r$ & 213 & 90 (47/43); & 123 \\
\hline
\end{tabular}




\begin{tabular}{|c|c|c|c|c|c|c|c|c|c|c|}
\hline $\begin{array}{l}\text { Study } \\
\text { ID }\end{array}$ & $\begin{array}{l}\text { Target } \\
\text { Populatio } \\
\text { n }\end{array}$ & $\begin{array}{l}\text { Inclusion } \\
\text { Criteria }\end{array}$ & $\begin{array}{l}\text { Exclusio } \\
\text { n Criteria }\end{array}$ & $\begin{array}{l}\text { Intervent } \\
\text { i on / } \\
\text { recruitme } \\
\text { nt setting }\end{array}$ & $\begin{array}{l}\text { Count } \\
\text { ry }\end{array}$ & \begin{tabular}{|l} 
Child \\
age at \\
recruitm \\
ent M \\
(SD), \\
range
\end{tabular} & $\begin{array}{l}\text { Child } \\
\text { gender } \\
\text { ratio at } \\
\text { recruit } \\
\text { m ent } \\
\text { M/F n } \\
\text { (M/F \%) }\end{array}$ & $\begin{array}{l}\text { Randomi } \\
\text { sed } \\
T(I / C)\end{array}$ & $\begin{array}{l}\text { Dropouts } \\
\text { T (I/C) n; } \\
\text { T (I/C) \% }\end{array}$ & $\begin{array}{l}\text { Number } \\
\text { analysed } \\
\text { (follow- } \\
\text { up) } \\
T(I / C)\end{array}$ \\
\hline (2015) & $\begin{array}{l}\text { Children } \\
\text { of } \\
\text { mothers } \\
\text { with } \\
\text { breast } \\
\text { cancer }\end{array}$ & $\begin{array}{l}\text { breast cancer } \\
\text { (Stage } 0,1, \text { IIA, } \\
\text { IIB, or III) within } \\
\text { the past } 6 \\
\text { months, married } \\
\text { or in an intimate } \\
\text { heterosexual } \\
\text { relationship, read } \\
\text { and wroteEnglish } \\
\text { among their } \\
\text { languages of } \\
\text { choice, and had a } \\
\text { school-agechild } \\
\text { between } 8 \text { and } \\
12 \text { years of age. } \\
\text { Mothers with } \\
\text { more than one } \\
\text { age-eligible child } \\
\text { were asked to } \\
\text { choose one } \\
\text { referent child for } \\
\text { the study. }\end{array}$ & $\begin{array}{l}\text { speaking } \\
\text { Advanced } \\
\text { stage breast } \\
\text { cancer } \\
\text { Not recently } \\
\text { diagnosed } \\
\text { Single mother } \\
\text { (from study } \\
\text { protocol) }\end{array}$ & $\begin{array}{l}\text { t: } \\
\text { community } \\
\text { based } \\
\text { comprehen } \\
\text { sive cancer } \\
\text { centres, } \\
\text { community } \\
\text { cancer } \\
\text { centres, } \\
\text { private } \\
\text { medical } \\
\text { practices, } \\
\text { and self- } \\
\text { referral. }\end{array}$ & & years & & $(109 / 104)$ & $\begin{array}{l}42 \% \\
(43 \% / 41 \%)\end{array}$ & $(62 / 61)$ \\
\hline $\begin{array}{l}\text { Lieberm } \\
\text { an } \\
\text { (2006) }\end{array}$ & $\begin{array}{l}\text { Selective: } \\
\text { Children } \\
\text { were } \\
\text { exposed } \\
\text { to marital } \\
\text { violence. }\end{array}$ & $\begin{array}{l}\text { Child aged } 3 \text { to } 5 \\
\text { years who had } \\
\text { been exposed to } \\
\text { marital violence } \\
\text { and were } \\
\text { referred due to } \\
\text { clinical concern } \\
\text { about the child's } \\
\text { behaviour or } \\
\text { mother's } \\
\text { parenting. }\end{array}$ & $\begin{array}{l}\text { Perpetrator of } \\
\text { violence was } \\
\text { living in the } \\
\text { home. Mothers } \\
\text { with current } \\
\text { substance } \\
\text { abuse, } \\
\text { homelessness, } \\
\text { mental } \\
\text { retardation or } \\
\text { psychosis. } \\
\text { Children with }\end{array}$ & $\begin{array}{l}\text { Referrals } \\
\text { were } \\
\text { received } \\
\text { from family } \\
\text { court, } \\
\text { domestic } \\
\text { violence } \\
\text { service } \\
\text { providers, } \\
\text { medical } \\
\text { providers, } \\
\text { preschools, }\end{array}$ & USA & $\begin{array}{l}4.06 \\
(0.82) ; 3-5 \\
y\end{array}$ & $\begin{array}{l}36 / 39 \\
(48 \% / 52 \% \\
)\end{array}$ & $75(42 / 33)$ & $\begin{array}{l}25(15 / 10) \\
33.3 \% \\
(35.7 \% / 30 . \\
3 \%)\end{array}$ & $\begin{array}{l}50 \\
(37 / 23)\end{array}$ \\
\hline
\end{tabular}




\begin{tabular}{|c|c|c|c|c|c|c|c|c|c|c|}
\hline $\begin{array}{l}\text { Study } \\
\text { ID }\end{array}$ & $\begin{array}{l}\text { Target } \\
\text { Populatio } \\
\text { n }\end{array}$ & $\begin{array}{l}\text { Inclusion } \\
\text { Criteria }\end{array}$ & $\begin{array}{l}\text { Exclusio } \\
\text { n Criteria }\end{array}$ & $\begin{array}{l}\text { Intervent } \\
\text { i on / } \\
\text { rec ruitme } \\
\text { nt setting }\end{array}$ & $\begin{array}{l}\text { Count } \\
\text { ry }\end{array}$ & $\begin{array}{l}\text { Child } \\
\text { age at } \\
\text { recruitm } \\
\text { ent M } \\
\text { (SD), } \\
\text { range }\end{array}$ & $\begin{array}{l}\text { Child } \\
\text { gender } \\
\text { ratio at } \\
\text { recruit } \\
\text { m ent } \\
\text { M/F n } \\
(M / F \%)\end{array}$ & $\begin{array}{l}\text { Randomi } \\
\text { sed } \\
\text { T (I/C) }\end{array}$ & $\begin{array}{l}\text { Dropouts } \\
\text { T (I/C) n; } \\
\text { T (I/C) \% }\end{array}$ & $\begin{array}{l}\text { Number } \\
\text { analysed } \\
\text { (follow- } \\
\text { up) } \\
T \text { (I/C) }\end{array}$ \\
\hline & & & $\begin{array}{l}\text { mental } \\
\text { retardation, } \\
\text { autism } \\
\text { spectrum } \\
\text { disorder, or } \\
\text { documented } \\
\text { physical or } \\
\text { sexual abuse. }\end{array}$ & $\begin{array}{l}\text { other } \\
\text { agencies, } \\
\text { child } \\
\text { protective } \\
\text { services, } \\
\text { self- } \\
\text { referrals. }\end{array}$ & & & & & & \\
\hline $\begin{array}{l}\text { Lowell } \\
\text { (2011) }\end{array}$ & $\begin{array}{l}\text { Selective } \\
\text { and } \\
\text { Indicated: } \\
\text { Children } \\
\text { who } \\
\text { screened } \\
\text { positivefor } \\
\text { social- } \\
\text { emotional/ } \\
\text { behaviour } \\
\text { al } \\
\text { problems } \\
\text { or parents } \\
\text { screened } \\
\text { high for } \\
\text { psychosoc } \\
\text { ial risk }\end{array}$ & $\begin{array}{l}\text { Child aged } 6 \text { to } \\
36 \text { months, } \\
\text { screened } \\
\text { positive for } \\
\text { social- } \\
\text { emotional/behav } \\
\text { ioural problems } \\
\text { or the parent } \\
\text { screened high } \\
\text { for psychosocial } \\
\text { risk, living in } \\
\text { Bridgeport, } \\
\text { Connecticut, in a } \\
\text { permanent } \\
\text { caregiving } \\
\text { environment }\end{array}$ & $\begin{array}{l}\text { Families with } \\
\text { prior } \\
\text { involvement } \\
\text { with Child } \\
\text { FIRST }\end{array}$ & $\begin{array}{l}\text { Referrals } \\
\text { from } \\
\text { community } \\
\text { providers in } \\
\text { an inner- } \\
\text { citylocation } \\
\text { serving } \\
\text { predominan } \\
\text { tly families } \\
\text { living in } \\
\text { poverty }\end{array}$ & USA & $\begin{array}{l}18.5(9.0) ; \\
5.4-35.9 \\
m\end{array}$ & $\begin{array}{l}69 / 88 \\
(43.9 \% / 56 \\
.1 \%)\end{array}$ & $\begin{array}{l}157(78 / 79 \\
)\end{array}$ & $\begin{array}{l}40(20 / 20) \\
25.5 \% \\
(25.6 \% / 25 . \\
3 \%)\end{array}$ & $\begin{array}{l}117 \\
(58 / 59)\end{array}$ \\
\hline $\begin{array}{l}\text { Luthar } \\
\text { (2000) }\end{array}$ & $\begin{array}{l}\text { Selective: } \\
\text { Children } \\
\text { of heroin- } \\
\text { addicted } \\
\text { mothers }\end{array}$ & $\begin{array}{l}\text { Heroin-addicted } \\
\text { mothers with } \\
\text { children less } \\
\text { than } 16 \text { years of } \\
\text { age and who } \\
\text { reported } \\
\text { problems with } \\
\text { parenting. }\end{array}$ & $\begin{array}{l}\text { Mothers with } \\
\text { cognitive } \\
\text { deficits, } \\
\text { psychotic } \\
\text { thought } \\
\text { processes, and } \\
\text { suicidality. }\end{array}$ & $\begin{array}{l}\text { Recruited } \\
\text { from three } \\
\text { methadone } \\
\text { clinics. }\end{array}$ & USA & $\begin{array}{l}9.7(4.4) ; \\
\text { Range } n / r\end{array}$ & $n / r$ & $61(37 / 24)$ & $\begin{array}{l}14(9 / 5) ; \\
23 \% \\
(24.3 \% / 20 . \\
8 \%)\end{array}$ & $\begin{array}{l}47 \\
(28 / 19)\end{array}$ \\
\hline Luthar & Selective: & Heroin-addicted & Mothers with & Recruited & USA & $9.7(4.4) ;$ & $61 / 66$ & Parents/fa & $19(10 / 9) ;$ & 108 \\
\hline
\end{tabular}




\begin{tabular}{|c|c|c|c|c|c|c|c|c|c|c|}
\hline $\begin{array}{l}\text { Study } \\
\text { ID }\end{array}$ & $\begin{array}{l}\text { Target } \\
\text { Populatio } \\
\text { n }\end{array}$ & $\begin{array}{l}\text { Inclusion } \\
\text { Criteria }\end{array}$ & $\begin{array}{l}\text { Exclusio } \\
\text { n Criteria }\end{array}$ & $\begin{array}{l}\text { Intervent } \\
\text { i on / } \\
\text { recruitme } \\
\text { nt setting }\end{array}$ & $\begin{array}{l}\text { Count } \\
\text { ry }\end{array}$ & $\begin{array}{l}\text { Child } \\
\text { age at } \\
\text { recruitm } \\
\text { ent M } \\
\text { (SD), } \\
\text { range }\end{array}$ & $\begin{array}{l}\text { Child } \\
\text { gender } \\
\text { ratio at } \\
\text { recruit } \\
\text { m ent } \\
\text { M/F n } \\
\text { (M/F \%) }\end{array}$ & $\begin{array}{l}\text { Randomi } \\
\text { sed } \\
T(I / C)\end{array}$ & $\begin{array}{l}\text { Dropouts } \\
\text { T (I/C) n; } \\
\text { T (I/C) \% }\end{array}$ & $\begin{array}{l}\text { Number } \\
\text { analysed } \\
\text { (follow- } \\
\text { up) } \\
\text { T (I/C) }\end{array}$ \\
\hline (2007) & $\begin{array}{l}\text { Children } \\
\text { of heroin- } \\
\text { addicted } \\
\text { mothers }\end{array}$ & $\begin{array}{l}\text { mothers with } \\
\text { children less } \\
\text { than } 16 \text { years of } \\
\text { age and who } \\
\text { reported } \\
\text { problems with } \\
\text { parenting. }\end{array}$ & $\begin{array}{l}\text { cognitive } \\
\text { deficits, } \\
\text { psychotic } \\
\text { thought } \\
\text { processes, } \\
\text { suicidality and } \\
\text { homicidality. }\end{array}$ & $\begin{array}{l}\text { from three } \\
\text { methadone } \\
\text { clinics. }\end{array}$ & & Range $n / r$ & $(48 \% / 52 \%$ & $\begin{array}{l}\text { milies: } \\
127 \\
(60 / 61) \\
\text { Children: } \\
71(\mathrm{I} / \mathrm{Cn} / \mathrm{r})\end{array}$ & $\begin{array}{l}15.0 \% \\
(16.7 \% / 13 . \\
4 \%)\end{array}$ & $(50 / 58)$ \\
\hline $\begin{array}{l}\text { Mason } \\
\text { (2007) }\end{array}$ & $\begin{array}{l}\text { Universal: } \\
\text { parents of } \\
\text { sixth- } \\
\text { graders }\end{array}$ & $\begin{array}{l}\text { Rural schools } \\
\text { that were } \\
\text { eligible for the } \\
\text { school lunch } \\
\text { program and } \\
\text { were in } \\
\text { communities } \\
\text { with populations } \\
\text { less than } 8500 .\end{array}$ & $\mathrm{n} / \mathrm{r}$ & $\begin{array}{l}\text { Recruited } \\
\text { from grade } \\
\text { six classes } \\
\text { in } 22 \\
\text { schools }\end{array}$ & USA & $\begin{array}{l}11.4 \text { (SD } \\
n / r) ; \\
\text { Range n/r }\end{array}$ & $48 \% / 52 \%$ & $\begin{array}{l}\text { Parents/ } \\
\text { families: } \\
\mathrm{n} / \mathrm{r} \\
\text { Children: } \\
429 \\
(221 / 208)\end{array}$ & $\begin{array}{l}\text { Follow up1: } \\
118 \\
(66 / 52) ; \\
27.5 \% \\
(29.9 \% / 25 \\
\%) \\
\text { Follow up2: } \\
143 \\
(76 / 67) ; \\
33.3 \% \\
(34.4 \% / 32 . \\
2 \%) \\
\text { Follow up3: } \\
134 \\
(77 / 57) ; \\
31.2 \% \\
(34.8 \% / 27 . \\
4 \%) \\
\text { Follow up4: } \\
124 \\
(72 / 52) ; \\
28.9 \% \\
\text { ( } 32.6 \% / 25 \\
\%) \\
\end{array}$ & $\begin{array}{l}429 \\
(221 / 208)\end{array}$ \\
\hline
\end{tabular}




\begin{tabular}{|c|c|c|c|c|c|c|c|c|c|c|}
\hline $\begin{array}{l}\text { Study } \\
\text { ID }\end{array}$ & $\begin{array}{l}\text { Target } \\
\text { Populatio } \\
\text { n }\end{array}$ & $\begin{array}{l}\text { Inclusion } \\
\text { Criteria }\end{array}$ & $\begin{array}{l}\text { Exclusio } \\
\text { n Criteria }\end{array}$ & $\begin{array}{l}\text { Intervent } \\
\text { i on / } \\
\text { recruitme } \\
\text { nt setting }\end{array}$ & $\begin{array}{l}\text { Count } \\
\text { ry }\end{array}$ & \begin{tabular}{|l} 
Child \\
age at \\
recruitm \\
ent M \\
(SD), \\
range
\end{tabular} & $\begin{array}{l}\text { Child } \\
\text { gender } \\
\text { ratio at } \\
\text { recruit } \\
\text { m ent } \\
\text { M/F n } \\
\text { (M/F \%) }\end{array}$ & $\begin{array}{l}\text { Randomi } \\
\text { sed } \\
\text { T (I/C) }\end{array}$ & $\begin{array}{l}\text { Dropouts } \\
\text { T (I/C) n; } \\
\text { T (I/C) \% }\end{array}$ & $\begin{array}{l}\text { Number } \\
\text { analysed } \\
\text { (follow- } \\
\text { up) } \\
T(I / C)\end{array}$ \\
\hline $\begin{array}{l}\text { McDonal } \\
d(2006)\end{array}$ & $\begin{array}{l}\text { Universal: } \\
\text { Latino } \\
\text { families } \\
\text { with } \\
\text { primary- } \\
\text { school } \\
\text { aged } \\
\text { children }\end{array}$ & $\begin{array}{l}\text { self-identified } \\
\text { Latino families }\end{array}$ & $n / r$ & $\begin{array}{l}\text { Recruited } \\
\text { from } \\
\text { elementary } \\
\text { schools }\end{array}$ & USA & $\begin{array}{l}7(\mathrm{SDn} / \mathrm{r}) ; \\
\text { Range } \mathrm{n} / \mathrm{r}\end{array}$ & $\begin{array}{l}57 / 73 \\
(43.8 \% / 56 \\
.2 \%)^{b}\end{array}$ & $\begin{array}{l}180(\mathrm{I} / \mathrm{C} \\
\mathrm{n} / \mathrm{r})\end{array}$ & $\begin{array}{l}50(\mathrm{I} / \mathrm{C} \mathrm{n} / \mathrm{r}) \\
27.8 \%(\mathrm{I} / \mathrm{C} \\
\mathrm{n} / \mathrm{r})\end{array}$ & $\begin{array}{l}130 \\
(80 / 50)\end{array}$ \\
\hline $\begin{array}{l}\text { Melynk } \\
\text { (2004) }\end{array}$ & $\begin{array}{l}\text { Selective: } \\
\text { Young } \\
\text { children } \\
\text { unexpecte } \\
\text { dly } \\
\text { hospitalise } \\
\text { d in } \\
\text { paediatric } \\
\text { intensive } \\
\text { care units }\end{array}$ & $\begin{array}{l}\text { Mothers that } \\
\text { could read and } \\
\text { speak English } \\
\text { and their child } \\
\text { had an } \\
\text { unplanned } \\
\text { medical or } \\
\text { surgical } \\
\text { admission to the } \\
\text { PICU, child aged } \\
2-7 \text { years, } \\
\text { expected to } \\
\text { survive, had no } \\
\text { prior ICU } \\
\text { admissions, no } \\
\text { cancer, and no } \\
\text { suspected or } \\
\text { diagnosed } \\
\text { physical or } \\
\text { sexual abuse. }\end{array}$ & $\begin{array}{l}\text { Children who } \\
\text { were } \\
\text { readmitted to } \\
\text { the PICU after } \\
\text { transfer from } \\
\text { the PICU to the } \\
\text { general } \\
\text { paediatric unit } \\
\text { or were } \\
\text { hospitalized in } \\
\text { the PICU for } 21 \\
\text { days. }\end{array}$ & $\begin{array}{l}\text { Children's } \\
\text { hospital }\end{array}$ & USA & $\begin{array}{l}50.3 \\
(19.3) ; 22- \\
88 \mathrm{~m}^{\mathrm{b}}\end{array}$ & $\begin{array}{l}99 / 64 \\
(60.7 \% / 39 \\
.3 \%)^{b}\end{array}$ & \begin{tabular}{|l}
174 \\
$(90 / 84)$
\end{tabular} & $\begin{array}{l}\text { T8: } \\
85(35 / 50) ; \\
48.9 \% \\
(38.9 \% / 59 . \\
5 \%) \\
\\
\text { T9: } \\
106 \\
(47 / 59) ; \\
60.9 \% \\
(52.2 / 70.2 \\
\%)\end{array}$ & $\begin{array}{l}\text { T8: } \\
89 \\
(55 / 34 \\
) \\
\text { T9: } \\
67 \\
(42 / 25)\end{array}$ \\
\hline $\begin{array}{l}\text { Ordway } \\
\text { (2014) }\end{array}$ & $\begin{array}{l}\text { Selective: } \\
\text { Children } \\
\text { of first- } \\
\text { time } \\
\text { parents at } \\
\text { risk due to }\end{array}$ & $\begin{array}{l}\text { Eligibility at } \\
\text { study entry: (a) } \\
\text { Having a first } \\
\text { child, (b) Speak } \\
\text { English, (c) } \\
\text { Obtains primary }\end{array}$ & $\begin{array}{l}\text { No psychoses } \\
\text { or terminal } \\
\text { illnesses (from } \\
\text { study protocol) }\end{array}$ & $\begin{array}{l}\text { Recruitmen } \\
\text { t: local } \\
\text { community } \\
\text { health } \\
\text { centre } \\
\text { Interventio }\end{array}$ & USA & \begin{tabular}{|l|}
$\mathrm{M}(\mathrm{SD})$ \\
$\mathrm{n} / \mathrm{a}$, \\
prenatal
\end{tabular} & $n / r$ & $71(36 / 35)$ & $\begin{array}{l}21(12 / 9) ; \\
29.6 \% \\
(33.3 \% / 25 . \\
7 \%)\end{array}$ & $\begin{array}{l}50 \\
(24 / 26)\end{array}$ \\
\hline
\end{tabular}




\begin{tabular}{|c|c|c|c|c|c|c|c|c|c|c|}
\hline $\begin{array}{l}\text { Study } \\
\text { ID }\end{array}$ & $\begin{array}{l}\text { Target } \\
\text { Populatio } \\
\text { n }\end{array}$ & $\begin{array}{l}\text { Inclusion } \\
\text { Criteria }\end{array}$ & $\begin{array}{l}\text { Exclusio } \\
\text { n Criteria }\end{array}$ & $\begin{array}{l}\text { Intervent } \\
\text { i on / } \\
\text { recruitme } \\
\text { nt setting }\end{array}$ & $\begin{array}{l}\text { Count } \\
\text { ry }\end{array}$ & $\begin{array}{l}\text { Child } \\
\text { age at } \\
\text { recruitm } \\
\text { ent M } \\
\text { (SD), } \\
\text { range }\end{array}$ & $\begin{array}{l}\text { Child } \\
\text { gender } \\
\text { ratio at } \\
\text { recruit } \\
\text { m ent } \\
\text { M/F n } \\
\text { (M/F \%) }\end{array}$ & $\begin{array}{l}\text { Randomi } \\
\text { sed } \\
T(I / C)\end{array}$ & $\begin{array}{l}\text { Dropouts } \\
\text { T (I/C) n; } \\
\text { T (I/C) \% }\end{array}$ & $\begin{array}{l}\text { Number } \\
\text { analysed } \\
\text { (follow- } \\
\text { up) } \\
\text { T (I/C) }\end{array}$ \\
\hline & $\begin{array}{l}\text { multiple } \\
\text { complex } \\
\text { issues } \\
\text { (age, } \\
\text { poverty, } \\
\text { trauma } \\
\text { history } \\
\text { etc.) }\end{array}$ & $\begin{array}{l}\text { care from } \\
\text { community } \\
\text { health centres } \\
\text { (fromprotocol) } \\
\text { Eligibility for } \\
\text { follow-upstudy: } \\
\text { (a) thetargeted } \\
\text { child was } \\
\text { between the } \\
\text { ages of } 3 \text { to } 5 \\
\text { years at the time } \\
\text { of data collection } \\
\text { between March } \\
2010 \text { and March } \\
2011, \text { (b) the } \\
\text { mother had } \\
\text { primary custody } \\
\text { or regular } \\
\text { visitation with } \\
\text { the child, (c) the } \\
\text { dyad lived in } \\
\text { state and/orwas } \\
\text { able to meet in } \\
\text { state for the } \\
\text { data collection, } \\
\text { (d) themother } \\
\text { participated in } \\
\text { the MTB } \\
\text { program or the } \\
\text { control condition } \\
\text { beyond the } \\
\text { initial consent } \\
\text { period. }\end{array}$ & & n: Home & & & & & & \\
\hline
\end{tabular}




\begin{tabular}{|c|c|c|c|c|c|c|c|c|c|c|}
\hline $\begin{array}{l}\text { Study } \\
\text { ID }\end{array}$ & $\begin{array}{l}\text { Target } \\
\text { Populatio } \\
\text { n }\end{array}$ & $\begin{array}{l}\text { Inclusion } \\
\text { Criteria }\end{array}$ & $\begin{array}{l}\text { Exclusio } \\
\text { n Criteria }\end{array}$ & $\begin{array}{l}\text { Intervent } \\
\text { i on / } \\
\text { recruitme } \\
\text { nt setting }\end{array}$ & $\begin{array}{l}\text { Count } \\
\text { ry }\end{array}$ & $\begin{array}{l}\text { Child } \\
\text { age at } \\
\text { recruitm } \\
\text { ent } M \\
\text { (SD), } \\
\text { range }\end{array}$ & $\begin{array}{l}\text { Child } \\
\text { gender } \\
\text { ratio at } \\
\text { recruit } \\
\text { m ent } \\
\text { M/F n } \\
(M / F \%)\end{array}$ & $\begin{array}{l}\text { Randomi } \\
\text { sed } \\
\text { T (I/C) }\end{array}$ & $\begin{array}{l}\text { Dropouts } \\
\text { T (I/C) n; } \\
\text { T (I/C) \% }\end{array}$ & $\begin{array}{l}\text { Number } \\
\text { analysed } \\
\text { (follow- } \\
\text { up) } \\
\text { T (I/C) }\end{array}$ \\
\hline $\begin{array}{l}\text { Oswalt } \\
\text { (2013) }\end{array}$ & $\begin{array}{l}\text { Selective: } \\
\text { Parents of } \\
\text { infants } \\
\text { born pre- } \\
\text { term }\end{array}$ & $\begin{array}{l}\text { Mothers and } \\
\text { fathers, <17 } \\
\text { years who are } \\
\text { literate in } \\
\text { English, who } \\
\text { never had } \\
\text { another infant } \\
\text { admitted to } \\
\text { NICU, and whose } \\
\text { infants had a } \\
\text { gestational age } \\
\text { of 26-34 weeks; } \\
\text { birth weight } \\
\text { >2500 g; } \\
\text { anticipated } \\
\text { survival; } \\
\text { singleton birth; } \\
\text { no severe } \\
\text { handicapping } \\
\text { conditions; not } \\
\text { small for } \\
\text { gestational age; } \\
\text { no grade III or IV } \\
\text { intraventricular } \\
\text { hemorrhage; and } \\
\text { born at thestudy } \\
\text { sites. }\end{array}$ & $\begin{array}{l}\text { Parents were } \\
\text { excluded from } \\
\text { the study if } \\
\text { they made a } \\
\text { personal } \\
\text { decision to } \\
\text { withdraw from } \\
\text { the study or if } \\
\text { their infant had } \\
\text { positive drug } \\
\text { testing. }\end{array}$ & $\begin{array}{l}\text { Recruitmen } \\
\text { t: NICUs in } \\
\text { Upstate } \\
\text { New York. } \\
\text { Interventio } \\
\text { n: Home }\end{array}$ & USA & $\begin{array}{l}\text { 31.3 (SD } \\
\text { n/r); birth; } \\
\text { GA 26.0- } \\
35.0 \\
\text { weeks }\end{array}$ & $\begin{array}{l}126 / 134 \\
(42.5 \% / 51 \\
.5 \%)\end{array}$ & $\begin{array}{l}260 \\
(147 / 113)\end{array}$ & $\begin{array}{l}10(3 / 7) \\
3.8 \% \\
(1.2 \% / 2.7 \% \\
)\end{array}$ & $\begin{array}{l}253(I / C \\
n / r)\end{array}$ \\
\hline $\begin{array}{l}\text { Quach } \\
\text { (2011) }\end{array}$ & $\begin{array}{l}\text { Selective: } \\
\text { Young } \\
\text { children } \\
\text { with sleep } \\
\text { problems }\end{array}$ & $\begin{array}{l}\text { Children starting } \\
\text { primary school } \\
\text { with a moderate } \\
\text { to severe sleep } \\
\text { problem }\end{array}$ & $\begin{array}{l}\text { Insufficient } \\
\text { English and } \\
\text { children with } \\
\text { likely } \\
\text { obstructive } \\
\text { sleep apnea }\end{array}$ & $\begin{array}{l}\text { Recruited } \\
\text { from } 22 \\
\text { primary } \\
\text { schools }\end{array}$ & AUS & $\begin{array}{l}5.7(0.4) \\
\text { Range } n / r\end{array}$ & $\begin{array}{l}27 / 27 \\
(50 \% / 50 \% \\
)\end{array}$ & $\begin{array}{l}108 \\
(54 / 54)\end{array}$ & $\begin{array}{l}2(2 / 0) ; \\
1.9 \% \\
(3.7 \%)\end{array}$ & $\begin{array}{l}106 \\
(52 / 54)\end{array}$ \\
\hline Rapee & Selective: & Score $>30$ on & $\mathrm{n} / \mathrm{r}$ & Interventio & AUS & $46.5(4.8) ;$ & $67 / 79$ & 146 & $30(10 / 20)$ & 116 \\
\hline
\end{tabular}




\begin{tabular}{|c|c|c|c|c|c|c|c|c|c|c|}
\hline $\begin{array}{l}\text { Study } \\
\text { ID }\end{array}$ & $\begin{array}{l}\text { Target } \\
\text { Populatio } \\
\text { n }\end{array}$ & $\begin{array}{l}\text { Inclusion } \\
\text { Criteria }\end{array}$ & $\begin{array}{l}\text { Exclusio } \\
\text { n Criteria }\end{array}$ & $\begin{array}{l}\text { Intervent } \\
\text { i on / } \\
\text { rec ruitme } \\
\text { nt setting }\end{array}$ & $\begin{array}{l}\text { Count } \\
\text { ry }\end{array}$ & $\begin{array}{l}\text { Child } \\
\text { age at } \\
\text { recruitm } \\
\text { ent M } \\
\text { (SD), } \\
\text { range }\end{array}$ & $\begin{array}{l}\text { Child } \\
\text { gender } \\
\text { ratio at } \\
\text { recruit } \\
\text { m ent } \\
\text { M/F n } \\
\text { (M/F \%) }\end{array}$ & $\begin{array}{l}\text { Randomi } \\
\text { sed } \\
\text { T (I/C) }\end{array}$ & $\begin{array}{l}\text { Dropouts } \\
\text { T (I/C) n; } \\
\text { T (I/C) \% }\end{array}$ & $\begin{array}{l}\text { Number } \\
\text { analysed } \\
\text { (follow- } \\
\text { up) } \\
\mathbf{T}(\mathrm{I} / \mathrm{C})\end{array}$ \\
\hline (2005) & \multirow{3}{*}{$\begin{array}{l}\text { Parents of } \\
\text { children } \\
\text { high in } \\
\text { behaviour } \\
\text { al } \\
\text { inhibition }\end{array}$} & \multirow{3}{*}{$\begin{array}{l}\text { Short } \\
\text { temperament } \\
\text { scale for children } \\
\text { Approach } \\
\text { subscale and } \\
\text { who met criteria } \\
\text { for behavioural } \\
\text { inhibition on } \\
\text { laboratory } \\
\text { assessment }\end{array}$} & & \multirow{3}{*}{$\begin{array}{l}\text { n delivered } \\
\text { in } \\
\text { university } \\
\text { clinic, } \\
\text { recruited } \\
\text { from } \\
\text { Sydney } \\
\text { preschools }\end{array}$} & & \multirow[t]{3}{*}{$\begin{array}{l}\text { 36-59 m } \\
\text { (Rapee, } \\
\text { 2010) }\end{array}$} & \multirow[t]{3}{*}{ ) } & \multirow[t]{3}{*}{$(73 / 73)$} & $\begin{array}{l}20.5 \% \\
(13.7 \% / 27 \\
4 \%)\end{array}$ & $(63 / 53)$ \\
\hline $\begin{array}{l}\text { Rapee } \\
\text { (2010) }\end{array}$ & & & & & & & & & $\begin{array}{l}\text { Follow-up 1: } \\
25(8 / 19) ; \\
18.5 \% \\
(11 \% / 26 \%) \\
\text { Follow-up 2: } \\
63(28 / 35) ; \\
43.2 \% \\
(38.4 \% / 47 . \\
9 \%) \\
\text { Follow-up 3: } \\
70(33 / 37) ; \\
47.9 \% \\
(45.2 \% / 50 . \\
7 \%)\end{array}$ & $\begin{array}{l}\text { Follow-up } \\
1: \\
119 \\
(83 / 76) \\
\text { Follow-up } \\
2: \\
83 \\
(45 / 38) \\
\text { Follow-up } \\
3: \\
76 \\
(40 / 36)\end{array}$ \\
\hline $\begin{array}{l}\text { Rapee } \\
\text { (2013) }\end{array}$ & & & & & & & & & $\begin{array}{l}43(23 / 20) ; \\
29 \% \\
(32 \% / 26 \%)\end{array}$ & $\begin{array}{l}103 \\
(49 / 54)\end{array}$ \\
\hline $\begin{array}{l}\text { Rothera } \\
\text { m- } \\
\text { Borus } \\
(2001)\end{array}$ & \multirow[t]{2}{*}{$\begin{array}{l}\text { Selective: } \\
\text { adolescen } \\
\text { ts of } \\
\text { parents } \\
\text { with } \\
\text { HIV/AIDS }\end{array}$} & \multirow[t]{2}{*}{$\begin{array}{l}\text { Financially } \\
\text { needy persons } \\
\text { with AIDS who } \\
\text { requested } \\
\text { services and } \\
\text { were alive } \\
\text { during the } \\
\text { recruitment } \\
\text { period, aged } 25 \\
\text { to } 70 \text { years. had } \\
\text { at least } 1 \\
\text { adolescent child }\end{array}$} & \multirow[t]{2}{*}{$\begin{array}{l}\text { Institutionalizat } \\
\text { ion, } \\
\text { incarceration, } \\
\text { and extreme } \\
\text { illness }\end{array}$} & \multirow[t]{2}{*}{$\begin{array}{l}\text { A } \\
\text { consecutive } \\
\text { series of } \\
\text { parents } \\
\text { living with } \\
\text { HIV was } \\
\text { recruited } \\
\text { from a } \\
\text { comprehen } \\
\text { sive log at } \\
\text { the New } \\
\text { York City }\end{array}$} & \multirow[t]{2}{*}{ USA } & $\begin{array}{l}14.8 \\
(2.10) ; 11- \\
18 y^{b}\end{array}$ & $\begin{array}{l}193 / 219 \\
(47 \% / 53 \% \\
)^{b}\end{array}$ & \multirow[t]{2}{*}{$\begin{array}{l}\text { Parents/ } \\
\text { families: } \\
307 \\
(153 / 154) \\
\text { Children: } \\
423 \\
(212 / 211)\end{array}$} & $\begin{array}{l}\text { Year 1: } \\
68(29 / 39) ; \\
16.1 \% \\
(13.7 \% / 18 . \\
5 \%) \\
\text { Year 2: } \\
24(11 / 13) ; \\
5.7 \% \\
(5.3 \% / 6.2 \% \\
)\end{array}$ & $\begin{array}{l}\text { Year 1: } \\
355 \\
(183 / 172) \\
\text { Year 2: } \\
399 \\
(201 / 198)\end{array}$ \\
\hline Rothera & & & & & & 14.7(2.0); & $198 / 215$ & & $63(22 / 20)$ & 360 \\
\hline
\end{tabular}




\begin{tabular}{|c|c|c|c|c|c|c|c|c|c|c|}
\hline $\begin{array}{l}\text { Study } \\
\text { ID }\end{array}$ & $\begin{array}{l}\text { Target } \\
\text { Populatio } \\
\text { n }\end{array}$ & $\begin{array}{l}\text { Inclusion } \\
\text { Criteria }\end{array}$ & $\begin{array}{l}\text { Exclusio } \\
\text { n Criteria }\end{array}$ & $\begin{array}{l}\text { Intervent } \\
\text { i on / } \\
\text { recruitme } \\
\text { nt setting }\end{array}$ & $\begin{array}{l}\text { Count } \\
\text { ry }\end{array}$ & $\begin{array}{l}\text { Child } \\
\text { age at } \\
\text { recruitm } \\
\text { ent M } \\
\text { (SD), } \\
\text { range }\end{array}$ & $\begin{array}{l}\text { Child } \\
\text { gender } \\
\text { ratio at } \\
\text { recruit } \\
\text { m ent } \\
\text { M/F n } \\
\text { (M/F \%) }\end{array}$ & $\begin{array}{l}\text { Randomi } \\
\text { sed } \\
\text { T (I/C) }\end{array}$ & $\begin{array}{l}\text { Dropouts } \\
\text { T (I/C) n; } \\
\text { T (I/C) \% }\end{array}$ & $\begin{array}{l}\text { Number } \\
\text { analysed } \\
\text { (follow- } \\
\text { up) } \\
T \text { (I/C) }\end{array}$ \\
\hline $\begin{array}{l}\text { m-Borus } \\
(2003)\end{array}$ & & $\begin{array}{l}\text { aged 11-18 } \\
\text { years, and had } \\
\text { the assent of }\end{array}$ & & $\begin{array}{l}\text { Division of } \\
\text { AIDS } \\
\text { Services }\end{array}$ & & $11-18 y^{b}$ & $\begin{array}{l}(48 \% / 52 \% \\
)^{b}\end{array}$ & & $\begin{array}{l}14.9 \% \\
(10.2 \% / 9.3 \\
\%)\end{array}$ & $(169 / 191)$ \\
\hline $\begin{array}{l}\text { Rothera } \\
\text { m-Borus } \\
(2004)\end{array}$ & & $\begin{array}{l}\text { their clinical } \\
\text { social worker } \\
\text { that study } \\
\text { participation was }\end{array}$ & & & & $\begin{array}{l}14.83 \\
(2.02) ; 11- \\
21 y^{b}\end{array}$ & $\begin{array}{l}142.175 \\
(45 \% / 55 \% \\
)^{b}\end{array}$ & & $\begin{array}{l}68(33 / 35) \\
16.1 \% \\
(15.4 \% / 16 . \\
6 \%)\end{array}$ & $\begin{array}{l}317 \\
(156 / 161)\end{array}$ \\
\hline $\begin{array}{l}\text { Rothera } \\
\text { m-Borus } \\
(2006)\end{array}$ & & appropriate & & & & $\begin{array}{l}14.7(2.0) \\
11-19 y^{b}\end{array}$ & $\begin{array}{l}\text { 130/158; } \\
(45 \% / 55 \% \\
)^{b}\end{array}$ & & $\begin{array}{l}19(13 / 6) ; \\
6.2 \% \\
(8.5 \% / 3.9 \%\end{array}$ & $\begin{array}{l}288 \\
(140 / 148)\end{array}$ \\
\hline $\begin{array}{l}\text { Ruffolo } \\
\text { (2005) }\end{array}$ & $\begin{array}{l}\text { Indicated: } \\
\text { children } \\
\text { with } \\
\text { serious }\end{array}$ & $\begin{array}{l}\text { Parents of youth } \\
\text { with serious } \\
\text { emotional } \\
\text { disturbance } \\
\text { enrolled in the } \\
\text { Intensive case } \\
\text { management } \\
\text { programs during } \\
\text { the intake phase } \\
\text { and active for at } \\
\text { least } 6 \text { months }\end{array}$ & $n / r$ & $\begin{array}{l}\text { Recruited } \\
\text { from } \\
\text { community } \\
\text { based } \\
\text { I ntensive } \\
\text { case } \\
\text { manageme } \\
\text { nt } \\
\text { programs }\end{array}$ & USA & $\begin{array}{l}11.47 \text { (SD } \\
n / r) ; \\
\text { Range } n / r\end{array}$ & $n / r$ & $94(58 / 36)$ & $\begin{array}{l}32(32 / 19) ; \\
54.3 \% \\
(55.2 \% / 52 . \\
8 \%)\end{array}$ & $\begin{array}{l}43 \\
(26 / 17)\end{array}$ \\
\hline $\begin{array}{l}\text { Santacru } \\
\text { z (2006) }\end{array}$ & $\begin{array}{l}\text { Treatment: } \\
\text { children } \\
\text { with } \\
\text { darkness } \\
\text { phobia }\end{array}$ & $\begin{array}{l}\text { a) aged 4-8 } \\
\text { years old; b) } \\
\text { Diagnosis of } \\
\text { Darkness } \\
\text { phobia; c) } \\
\text { Maximum scores } \\
\text { for the item } \\
\text { "Fear of the } \\
\text { dark" in the } \\
\text { Children's Fear } \\
\text { Survey } \\
\text { Schedule- }\end{array}$ & $\begin{array}{l}\text { Other anxiety } \\
\text { disorders, } \\
\text { including } \\
\text { separation } \\
\text { anxiety, mental } \\
\text { deficiency, } \\
\text { autism, being } \\
\text { involved in a } \\
\text { therapy, or } \\
\text { psychological } \\
\text { problems which } \\
\text { need treatment }\end{array}$ & $\begin{array}{l}\text { Recruited } \\
\text { from pre } \\
\text { schools }\end{array}$ & SPN & $\begin{array}{l}6.49 \\
(1.46) ; 4-8 \\
y\end{array}$ & $\begin{array}{l}41 / 37 \\
(32 \% / 29 \% \\
)\end{array}$ & $20(27 / 23)$ & $\begin{array}{l}7(7 / 0) ; \\
14.0 \% \\
(25.9 \% / 0)\end{array}$ & $\begin{array}{l}43 \\
(20 / 23)\end{array}$ \\
\hline
\end{tabular}




\begin{tabular}{|c|c|c|c|c|c|c|c|c|c|c|}
\hline $\begin{array}{l}\text { Study } \\
\text { ID }\end{array}$ & $\begin{array}{l}\text { Target } \\
\text { Populatio } \\
\text { n }\end{array}$ & $\begin{array}{l}\text { Inclusion } \\
\text { Criteria }\end{array}$ & $\begin{array}{l}\text { Exclusio } \\
\text { n Criteria }\end{array}$ & $\begin{array}{l}\text { Intervent } \\
\text { i on / } \\
\text { recruitme } \\
\text { nt setting }\end{array}$ & $\begin{array}{l}\text { Count } \\
\text { ry }\end{array}$ & $\begin{array}{l}\text { Child } \\
\text { age at } \\
\text { recruitm } \\
\text { ent M } \\
\text { (SD), } \\
\text { range }\end{array}$ & $\begin{array}{l}\text { Child } \\
\text { gender } \\
\text { ratio at } \\
\text { recruit } \\
\text { m ent } \\
\text { M/F n } \\
(M / F \%)\end{array}$ & $\begin{array}{l}\text { Randomi } \\
\text { sed } \\
\text { T (I/C) }\end{array}$ & $\begin{array}{l}\text { Dropouts } \\
\text { T (I/C) n; } \\
\text { T (I/C) \% }\end{array}$ & $\begin{array}{l}\text { Number } \\
\text { analysed } \\
\text { (follow- } \\
\text { up) } \\
\text { T (I/C) }\end{array}$ \\
\hline & & $\begin{array}{l}\text { Revised; d) }>50 \\
\text { points on the } \\
\text { Dark Fear Scale } \\
\text { (range: } 0-100 \text { ); } \\
\text { and e) Minimum } \\
\text { of six months' } \\
\text { persistence }\end{array}$ & & & & & & & & \\
\hline $\begin{array}{l}\text { Simon } \\
\text { (2011) }\end{array}$ & $\begin{array}{l}\text { Indicated: } \\
\text { children } \\
\text { with high } \\
\text { levels of } \\
\text { anxiety } \\
\text { symptoms }\end{array}$ & $\begin{array}{l}\text { 1. SCARED score } \\
\text { in the highest } \\
15 \% \text {; 2. IQ above } \\
\text { 80; 3. age 8-12; } \\
\text { 4. Normal } \\
\text { elementary } \\
\text { school }\end{array}$ & $\begin{array}{l}\text { 1. Do not } \\
\text { sufficiently } \\
\text { master the } \\
\text { Dutch } \\
\text { language; } 2 \text {. } \\
\text { Have } \\
\text { substantial } \\
\text { learning } \\
\text { problems or a } \\
\text { developmental } \\
\text { delay. }\end{array}$ & $\begin{array}{l}\text { Recruited } \\
\text { from } \\
\text { primary } \\
\text { schools }\end{array}$ & $\mathrm{NL}$ & $\begin{array}{l}9.89 \\
(1.19) ; 8- \\
13 \mathrm{y}^{\mathrm{b}}\end{array}$ & $\begin{array}{l}58 / 67 \\
(73 \% / 84 \% \\
)^{\mathrm{b}}\end{array}$ & $\begin{array}{l}125 \\
(69 / 56)\end{array}$ & $\begin{array}{l}\text { Follow up1: } \\
14(10 / 4) ; \\
11.1 \% \\
\text { ( } 14.5 \% / 7.1 \\
\%) \\
\text { Follow up2: } \\
35(21 / 14) ; \\
28 \% \\
(30.4 \% / 25 \\
\%)\end{array}$ & $\begin{array}{l}90 \\
(48 / 42)\end{array}$ \\
\hline $\begin{array}{l}\text { Solantau } \\
\text { s (2010) }\end{array}$ & $\begin{array}{l}\text { Selective: } \\
\text { children of } \\
\text { parents } \\
\text { with mood } \\
\text { disorder }\end{array}$ & $\begin{array}{l}\text { Patients } \\
\text { diagnosed and } \\
\text { currently treated } \\
\text { for any mood } \\
\text { disorder and had } \\
\text { at least one child } \\
\text { aged 8-16 years } \\
\text { not being } \\
\text { treated for } \\
\text { psychiatric } \\
\text { disorder }\end{array}$ & $\begin{array}{l}\text { Schizophrenia } \\
\text { and life } \\
\text { threatening } \\
\text { stage of a } \\
\text { somatic } \\
\text { disease of the } \\
\text { parent or child, } \\
\text { families with } \\
\text { ongoing family } \\
\text { therapy, } \\
\text { custody dispute } \\
\text { and immediate } \\
\text { need for } \\
\text { involvement of } \\
\text { child protection } \\
\text { services }\end{array}$ & $\begin{array}{l}\text { Recruited } \\
\text { from } 16 \\
\text { health care } \\
\text { units in } 8 \\
\text { regional } \\
\text { national } \\
\text { health } \\
\text { organisatio } \\
\text { ns }\end{array}$ & FIN & $\begin{array}{l}M(S D) n / r ; \\
8-16 y\end{array}$ & $n / r$ & $\begin{array}{l}\text { Parents/fa } \\
\text { milies: } \\
119 \\
(59 / 60) \\
\text { Children: } \\
\mathrm{n} / \mathrm{r}\end{array}$ & $\begin{array}{l}\text { T3: } 40 \\
(20 / 20) ; \\
33.6 \% \\
(33.9 \% / 33 . \\
3 \%) \\
\text { T4: } 35 \\
(19 / 16) ; \\
29.4 \% \\
(322 \% / 26.7 \\
\%)\end{array}$ & $\begin{array}{l}\text { T3: } 79 \\
(39 / 40) \\
\\
\text { T4: } 84 \\
\text { (40/44) }\end{array}$ \\
\hline
\end{tabular}




\begin{tabular}{|c|c|c|c|c|c|c|c|c|c|c|}
\hline $\begin{array}{l}\text { Study } \\
\text { ID }\end{array}$ & $\begin{array}{l}\text { Target } \\
\text { Populatio } \\
\text { n }\end{array}$ & $\begin{array}{l}\text { Inclusion } \\
\text { Criteria }\end{array}$ & $\begin{array}{l}\text { Exclusio } \\
\text { n Criteria }\end{array}$ & $\begin{array}{l}\text { Intervent } \\
\text { i on / } \\
\text { recruitme } \\
\text { nt setting }\end{array}$ & $\begin{array}{l}\text { Count } \\
\text { ry }\end{array}$ & $\begin{array}{l}\text { Child } \\
\text { age at } \\
\text { recruitm } \\
\text { ent } M \\
\text { (SD), } \\
\text { range }\end{array}$ & $\begin{array}{l}\text { Child } \\
\text { gender } \\
\text { ratio at } \\
\text { recruit } \\
\text { m ent } \\
\text { M/F n } \\
\text { (M/F \%) } \\
\end{array}$ & $\begin{array}{l}\text { Randomi } \\
\text { sed } \\
\text { T (I/C) }\end{array}$ & $\begin{array}{l}\text { Dropouts } \\
\text { T (I/C) n; } \\
\text { T (I/C) \% }\end{array}$ & $\begin{array}{l}\text { Number } \\
\text { analysed } \\
\text { (follow- } \\
\text { up) } \\
\text { T (I/C) }\end{array}$ \\
\hline $\begin{array}{l}\text { Spittle } \\
(2010)\end{array}$ & \multirow[t]{2}{*}{$\begin{array}{l}\text { Selective: } \\
\text { Very } \\
\text { Preterm } \\
\text { infants }\end{array}$} & \multirow{2}{*}{$\begin{array}{l}\text { Gestational age } \\
<30 \text { weeks, } \\
\text { English } \\
\text { speaking; I nfants } \\
\text { born at } 30 \\
\text { weeks' } \\
\text { gestational age } \\
\text { and with no } \\
\text { major congenital } \\
\text { abnormalities }\end{array}$} & \multirow{2}{*}{$\begin{array}{l}\text { Congenital } \\
\text { brain } \\
\text { anomalies } \\
\text { associated with } \\
\text { poor } \\
\text { neurodevelopm } \\
\text { entaloutcomes, } \\
\text { still in hospital } \\
\text { at 4weeks } \\
\text { corrected age; } \\
\text { Infants were } \\
\text { excluded if } \\
\text { their family did } \\
\text { not live within a } \\
\text { 100-km radius } \\
\text { of the Royal } \\
\text { Women's } \\
\text { Hospital or } \\
\text { spoke no } \\
\text { English. }\end{array}$} & \multirow[t]{2}{*}{$\begin{array}{l}\text { Recruitmen } \\
\text { t: Royal } \\
\text { Women's/C } \\
\text { hildren's } \\
\text { hospital. } \\
\text { I nterventio } \\
\text { n: Home }\end{array}$} & \multirow[t]{2}{*}{ AUS } & \multirow[t]{2}{*}{$\begin{array}{l}27.3(1.6) ; \\
\text { Range n/r, } \\
\text { gestationa } \\
\text { I weeks }\end{array}$} & \multirow{2}{*}{$\begin{array}{l}\text { Interventi } \\
\text { on: } \\
34 / 27 \\
(56 \% / 44 \% \\
) \\
\text { Control: } \\
27 / 32 \\
(44 \% / 54 \% \\
)\end{array}$} & \multirow[t]{2}{*}{$\begin{array}{l}120 \\
(61 / 59) \\
\\
\text { Parents/fa } \\
\text { milies: } \\
106 \\
(54 / 52)\end{array}$} & $\begin{array}{l}5(3 / 2) \\
4.2 \% \\
(4.9 \% / 3.4 \% \\
)\end{array}$ & $\begin{array}{l}115 \\
(58 / 57 \\
)\end{array}$ \\
\hline $\begin{array}{l}\text { Spencer- } \\
\text { smith } \\
(2012)\end{array}$ & & & & & & & & & $\begin{array}{l}32(15 / 17) ; \\
26.7 \%(24.6 \\
\% / 28.8 \%)\end{array}$ & $\begin{array}{l}88 \\
(46 / 42)\end{array}$ \\
\hline $\begin{array}{l}\text { Strayhor } \\
\text { n (1991) }\end{array}$ & $\begin{array}{l}\text { Selective: } \\
\text { preschool } \\
\text { children }\end{array}$ & $\begin{array}{l}\text { 2-5 year old } \\
\text { children with } \\
\text { adverse } \\
\text { socioeconomic } \\
\text { circumstances } \\
\text { and whose } \\
\text { parents reported } \\
\text { at least one } \\
\text { undesirable } \\
\text { behaviour at } \\
\text { screening. }\end{array}$ & $\begin{array}{l}\text { ESL, child } \\
\text { verbal ability } \\
\text { standard score } \\
<50 \text {. }\end{array}$ & $\begin{array}{l}\text { Recruited } \\
\text { from Head } \\
\text { Start } \\
\text { centers, } \\
\text { advertisem } \\
\text { ents, } \\
\text { referrals } \\
\text { from } \\
\text { pediatrician } \\
\text { s \& mental } \\
\text { health, } \\
\text { word out } \\
\text { mouth. }\end{array}$ & USA & $\begin{array}{l}3 y 9 m(S D \\
n / r) ; \\
2 y 5 m- \\
5 y 7 m\end{array}$ & $\begin{array}{l}36 / 48 \\
(42.9 \% / 57 \\
.1 \%)\end{array}$ & $\begin{array}{l}\text { Parents/fa } \\
\text { milies: } \\
\text { 98(40/48) } \\
\text { Children: } \\
\text { n/r }\end{array}$ & $\begin{array}{l}21 \text { (I/C } \\
\text { unclear) } \\
(20 \%) \mathrm{I} / \mathrm{C} \% \\
\text { unclear }\end{array}$ & $\begin{array}{l}84 \\
(45 / 39)\end{array}$ \\
\hline
\end{tabular}




\begin{tabular}{|c|c|c|c|c|c|c|c|c|c|c|}
\hline $\begin{array}{l}\text { Study } \\
\text { ID }\end{array}$ & $\begin{array}{l}\text { Target } \\
\text { Populatio } \\
\text { n }\end{array}$ & $\begin{array}{l}\text { Inclusion } \\
\text { Criteria }\end{array}$ & $\begin{array}{l}\text { Exclusio } \\
\text { n Criteria }\end{array}$ & $\begin{array}{l}\text { Intervent } \\
\text { i on / } \\
\text { recruitme } \\
\text { nt setting }\end{array}$ & $\begin{array}{l}\text { Count } \\
\text { ry }\end{array}$ & $\begin{array}{l}\text { Child } \\
\text { age at } \\
\text { recruitm } \\
\text { ent } M \\
\text { (SD), } \\
\text { range }\end{array}$ & $\begin{array}{l}\text { Child } \\
\text { gender } \\
\text { ratio at } \\
\text { recruit } \\
\text { m ent } \\
\text { M/F n } \\
(M / F \%)\end{array}$ & $\begin{array}{l}\text { Randomi } \\
\text { sed } \\
\text { T (I/C) }\end{array}$ & $\begin{array}{l}\text { Dropouts } \\
\text { T (I/C) n; } \\
\text { T (I/C) \% }\end{array}$ & $\begin{array}{l}\text { Number } \\
\text { analysed } \\
\text { (follow- } \\
\text { up) } \\
T \text { (I/C) }\end{array}$ \\
\hline & & & & $\begin{array}{l}\text { Interventio } \\
\mathrm{n} \text { setting } \\
\mathrm{n} / \mathrm{r} \text {. }\end{array}$ & & & & & & \\
\hline $\begin{array}{l}\text { Van } \\
\text { Doesum } \\
(2008)\end{array}$ & $\begin{array}{l}\text { Selective: } \\
\text { I nfants of } \\
\text { depressed } \\
\text { mothers }\end{array}$ & $\begin{array}{l}\text { Infant } \leq 12 \\
\text { months, mothers } \\
\text { with DSM-IV } \\
\text { diagnosis of } \\
\text { major } \\
\text { depression/ } \\
\text { dysthymia or } \\
\text { elevated BDI } \\
\text { score, fluent in } \\
\text { Dutch, receiving } \\
\text { outpatient } \\
\text { treatment for } \\
\text { depression }\end{array}$ & $\begin{array}{l}\text { Comorbid } \\
\text { psychotic } \\
\text { disorder, } \\
\text { bipolar, } \\
\text { substance } \\
\text { dependence }\end{array}$ & $\begin{array}{l}\text { Recruitmen } \\
\text { t: referral \& } \\
\text { advertisem } \\
\text { ents. } \\
\text { Interventio } \\
\text { n: Home }\end{array}$ & $\mathrm{NL}$ & $\begin{array}{l}5.7(3.8) \\
1-12 \mathrm{~m}^{\mathrm{c}}\end{array}$ & $\begin{array}{l}51 / 34 \\
(60 \% / 40 \% \\
)\end{array}$ & $85(42 / 43)$ & $\begin{array}{l}14(7 / 7) ; \\
16 \% \\
(16.7 \% / 16 . \\
7 \%)\end{array}$ & $\begin{array}{l}71 \\
(35 / 36)\end{array}$ \\
\hline $\begin{array}{l}\text { Velderm } \\
\text { an } \\
(2006)\end{array}$ & $\begin{array}{l}\text { Selective: } \\
\text { infants of } \\
\text { mothers } \\
\text { with } \\
\text { tentative } \\
\text { (insecure) } \\
\text { attachmen } \\
\text { t } \\
\text { representa } \\
\text { tions }\end{array}$ & $\begin{array}{l}\text { Mothers of } 4 \\
\text { month old first } \\
\text { born infants, } \\
\text { tentative } \\
\text { attachment } \\
\text { representation } \\
\text { classification, } \\
\text { from a particular } \\
\text { city and } \\
\text { neighbouring } \\
\text { villages, pretest } \\
\text { home visit } \\
\text { attended. }\end{array}$ & $\begin{array}{l}<8 \text { years } \\
\text { education, 14+ } \\
\text { yearseducation }\end{array}$ & $\begin{array}{l}\text { Recruitmen } \\
\text { t: city } \\
\text { records, } \\
\text { health } \\
\text { center } \\
\text { records. } \\
\text { Interventio } \\
\text { n: Home }\end{array}$ & $\mathrm{NL}$ & $\begin{array}{l}4(\mathrm{SD} n / \mathrm{r}) ; \\
\text { Range } \mathrm{n} / \mathrm{r} \\
6.8(1.03) \\
\text { months at } \\
\text { pre-test. }\end{array}$ & $\begin{array}{l}40 / 41 \\
(49.4 \% / 50 \\
.6 \%)\end{array}$ & $55(28 / 27)$ & $\begin{array}{l}2(1 / 1) ; \\
3.6 \% \\
(3.6 \% / 3.7 \% \\
)\end{array}$ & $\begin{array}{l}53 \\
(27 / 26)\end{array}$ \\
\hline $\begin{array}{l}\text { Wake } \\
\text { (2011) }\end{array}$ & $\begin{array}{l}\text { Selective: } \\
\text { Children } \\
\text { with low } \\
\text { expressive } \\
\text { vocabular }\end{array}$ & $\begin{array}{l}\text { Children at or } \\
\text { below 20th } \\
\text { percentile on } \\
\text { expressive } \\
\text { vocabulary test }\end{array}$ & $\begin{array}{l}\text { Referral for } \\
\text { cognitive delay, } \\
\text { major medical } \\
\text { conditions, } \\
\text { suspected }\end{array}$ & $\begin{array}{l}\text { Recruitmen } \\
\text { t: Maternal } \\
\text { and child } \\
\text { health } \\
\text { centres. }\end{array}$ & AUS & $\begin{array}{l}18.1 \\
(0.70) ; \\
\text { range } n / r \\
m\end{array}$ & $\begin{array}{l}152 / 149 \\
(50.6 \% / 49 \\
.5 \%)\end{array}$ & $\begin{array}{l}301 \\
(158 / 143)\end{array}$ & $\begin{array}{l}34(18 / 16) ; \\
11.3 \% \\
(11.4 \% / 11 . \\
2 \%)\end{array}$ & $\begin{array}{l}267 \\
(140 / 127)\end{array}$ \\
\hline
\end{tabular}




\begin{tabular}{|c|c|c|c|c|c|c|c|c|c|c|}
\hline $\begin{array}{l}\text { Study } \\
\text { ID }\end{array}$ & $\begin{array}{l}\text { Target } \\
\text { Populatio } \\
\text { n }\end{array}$ & $\begin{array}{l}\text { Inclusion } \\
\text { Criteria }\end{array}$ & $\begin{array}{l}\text { Exclusio } \\
\text { n Criteria }\end{array}$ & $\begin{array}{l}\text { Intervent } \\
\text { i on / } \\
\text { rec ruitme } \\
\text { nt setting }\end{array}$ & $\begin{array}{l}\text { Count } \\
\text { ry }\end{array}$ & $\begin{array}{l}\text { Child } \\
\text { age at } \\
\text { recruitm } \\
\text { ent M } \\
\text { (SD), } \\
\text { range }\end{array}$ & $\begin{array}{l}\text { Child } \\
\text { gender } \\
\text { ratio at } \\
\text { recruit } \\
\text { m ent } \\
\text { M/F n } \\
(M / F \%)\end{array}$ & $\begin{array}{l}\text { Randomi } \\
\text { sed } \\
\text { T (I/C) }\end{array}$ & $\begin{array}{l}\text { Dropouts } \\
\text { T (I/C) n; } \\
\text { T (I/C) \% }\end{array}$ & $\begin{array}{l}\text { Number } \\
\text { analysed } \\
\text { (follow- } \\
\text { up) } \\
T \text { (I/C) }\end{array}$ \\
\hline & $\mathrm{y}$ & & $\begin{array}{l}\text { autism, parents } \\
\text { with } \\
\text { inadequate } \\
\text { English }\end{array}$ & $\begin{array}{l}\text { I nterventio } \\
\mathrm{n}: \\
\text { Community } \\
\text { Centres }\end{array}$ & & & & & & \\
\hline $\begin{array}{l}\text { Walkup } \\
\text { (2009) }\end{array}$ & $\begin{array}{l}\text { Selective: } \\
\text { Children } \\
\text { with } \\
\text { Native } \\
\text { American } \\
\text { mothers }\end{array}$ & $\begin{array}{l}\text { Expectant } \\
\text { American I ndian } \\
\text { mothers aged } \\
12-22 \text { years, } \leq \\
28 \text { weeks } \\
\text { gestation }\end{array}$ & $\begin{array}{l}\text { Mothers with } \\
\text { extreme } \\
\text { medical, social, } \\
\text { or legal } \\
\text { problems that } \\
\text { precluded } \\
\text { participation. } \\
\text { Mother's at } \\
\text { acute risk for } \\
\text { self or others at } \\
\text { recruitment. }\end{array}$ & $\begin{array}{l}\text { Recruitmen } \\
\text { t: prenatal } \\
\text { and school- } \\
\text { based } \\
\text { clinics. } \\
\text { Interventio } \\
\text { n: Home } \\
\text { visits }\end{array}$ & USA & $\begin{array}{l}\text { M and SD } \\
\text { unclear; 3- } \\
35 \text { weeks }\end{array}$ & $n / r$ & $\begin{array}{l}167 \\
(81 / 86)\end{array}$ & $\begin{array}{l}85(44 / 41) \\
50.9 \% \\
(54.3 \% / 47 \\
7 \%)\end{array}$ & $\begin{array}{l}82 \\
(37 / 45)\end{array}$ \\
\hline $\begin{array}{l}\text { Williams } \\
\text { on } \\
(2014)\end{array}$ & $\begin{array}{l}\text { Selective: } \\
\text { Recent } \\
\text { immigrant } \\
\text { Latino } \\
\text { parents }\end{array}$ & $\begin{array}{l}\text { Parent of female } \\
\text { gender with a } \\
\text { child between } \\
\text { the ages of } 7 \\
\text { and } 12 \text {, and } \\
\text { could } \\
\text { not be receiving } \\
\text { concurrent } \\
\text { mental health } \\
\text { treatment. }\end{array}$ & $\mathrm{n} / \mathrm{r}$ & $\begin{array}{l}\text { Recruitmen } \\
\text { t: } \\
\text { Home/com } \\
\text { munity } \\
\text { settings } \\
\text { I nterventio } \\
\text { n setting: } \\
\text { Home }\end{array}$ & USA & $\begin{array}{l}9.47 \\
(1.53), 7- \\
12 \text { y at } \\
\text { pre-test. }\end{array}$ & $\begin{array}{l}87 / 107 \\
(45 \% / 55 \% \\
)^{a}\end{array}$ & $\begin{array}{l}194 \\
(113 / 81)\end{array}$ & $\begin{array}{l}32(13 / 19 ; \\
16.5 \% \\
(11.5 \% / 23 . \\
5 \%)\end{array}$ & $\begin{array}{l}194 \\
(113 / 81)\end{array}$ \\
\hline
\end{tabular}




\begin{tabular}{|c|c|c|c|c|c|c|c|c|c|c|}
\hline $\begin{array}{l}\text { Study } \\
\text { ID }\end{array}$ & $\begin{array}{l}\text { Target } \\
\text { Populatio } \\
\text { n }\end{array}$ & $\begin{array}{l}\text { Inclusion } \\
\text { Criteria }\end{array}$ & $\begin{array}{l}\text { Exclusio } \\
\text { n Criteria }\end{array}$ & $\begin{array}{l}\text { Intervent } \\
\text { i on / } \\
\text { recruitme } \\
\text { nt setting }\end{array}$ & $\begin{array}{l}\text { Count } \\
\text { ry }\end{array}$ & \begin{tabular}{|l} 
Child \\
age at \\
recruitm \\
ent M \\
(SD), \\
range
\end{tabular} & $\begin{array}{l}\text { Child } \\
\text { gender } \\
\text { ratio at } \\
\text { recruit } \\
\text { m ent } \\
\text { M/F n } \\
\text { (M/F \%) }\end{array}$ & $\begin{array}{l}\text { Randomi } \\
\text { sed } \\
\text { T (I/C) }\end{array}$ & $\begin{array}{l}\text { Dropouts } \\
\text { T (I/C) n; } \\
\text { T (I/C) \% }\end{array}$ & $\begin{array}{l}\text { Number } \\
\text { analysed } \\
\text { (follow- } \\
\text { up) } \\
\text { T (I/C) }\end{array}$ \\
\hline $\begin{array}{l}\text { Wolchik } \\
\text { (2000) }\end{array}$ & \multirow[t]{3}{*}{$\begin{array}{l}\text { Selective: } \\
\text { Children } \\
\text { of } \\
\text { divorced } \\
\text { parents }\end{array}$} & \multirow[t]{3}{*}{$\begin{array}{l}\text { Divorcee decree } \\
\text { within last } 2 \\
\text { years, Female } \\
\text { primary } \\
\text { residential } \\
\text { parent with at } \\
\text { least } 1 \text { child 9-12 } \\
\text { years, mother } \\
\text { and child had } \\
\text { adequate } \\
\text { English }\end{array}$} & \multirow{3}{*}{$\begin{array}{l}\text { Mother } \\
\text { remarried or } \\
\text { had a live-in } \\
\text { boyfriend, or } \\
\text { planned to } \\
\text { remarry during } \\
\text { the trial; } \\
\text { expected stable } \\
\text { custody } \\
\text { arrangement; } \\
>1 \text { hour drive } \\
\text { from } \\
\text { intervention } \\
\text { site; } \\
\text { mother/child in } \\
\text { treatment for } \\
\text { psychological } \\
\text { problems; child } \\
\text { in special } \\
\text { education for } \\
\text { mental or } \\
\text { learning } \\
\text { disability/ on } \\
\text { medication for } \\
\text { ADD; child } \\
\text { scored }>17 \text { on } \\
\text { CDI, endorsed } \\
\text { an item on } \\
\text { suicidal } \\
\text { ideation, or } \\
\text { scored >97th } \\
\text { percentile on } \\
\text { CBCL } \\
\text { Externalizing }\end{array}$} & \multirow[t]{3}{*}{$\begin{array}{l}\text { Recruitmen } \\
\text { t: } \\
\text { mail/phone } \\
\text { from Court } \\
\text { records. } \\
\text { Interventio } \\
\text { n: Home }\end{array}$} & \multirow[t]{3}{*}{ USA } & \multirow[t]{3}{*}{$\begin{array}{l}10.4(1.0) \\
9-12 y\end{array}$} & \multirow[t]{3}{*}{$\begin{array}{l}\text { Number of } \\
M \text { and } \mathrm{F} \\
\mathrm{n} / \mathrm{r} ; \\
51 \% / 49 \%\end{array}$} & \multirow[t]{3}{*}{$\begin{array}{l}157 \\
(81 / 76)\end{array}$} & $\begin{array}{l}4(2 / 2) ; \\
2.5 \% \\
(2.5 \% / 2.6 \% \\
)\end{array}$ & $\begin{array}{l}153 \\
(79 / 74)\end{array}$ \\
\hline $\begin{array}{l}\text { Wolchik } \\
\text { (2002) }\end{array}$ & & & & & & & & & $\begin{array}{l}12(4 / 8) ; \\
7.6 \% \\
(4.9 \% / 10.5 \\
\%)\end{array}$ & $\begin{array}{l}145 \\
(77 / 68)\end{array}$ \\
\hline $\begin{array}{l}\text { Wolchik } \\
\text { (2013) }\end{array}$ & & & & & & & & & $\begin{array}{l}17(8 / 9) ; \\
10.8 \% \\
(9.9 \% / 11.8 \\
\%)\end{array}$ & $\begin{array}{l}140 \\
(73 / 67)^{\mathrm{a}}\end{array}$ \\
\hline
\end{tabular}




\begin{tabular}{|c|c|c|c|c|c|c|c|c|c|c|}
\hline $\begin{array}{l}\text { Study } \\
\text { ID }\end{array}$ & $\begin{array}{l}\text { Target } \\
\text { Populatio } \\
\text { n }\end{array}$ & $\begin{array}{l}\text { Inclusion } \\
\text { Criteria }\end{array}$ & $\begin{array}{l}\text { Exclusio } \\
\text { n Criteria }\end{array}$ & $\begin{array}{l}\text { Intervent } \\
\text { i on / } \\
\text { recruitme } \\
\text { nt setting }\end{array}$ & $\begin{array}{l}\text { Count } \\
\text { ry }\end{array}$ & $\begin{array}{l}\text { Child } \\
\text { age at } \\
\text { recruitm } \\
\text { ent } M \\
\text { (SD), } \\
\text { range }\end{array}$ & $\begin{array}{l}\text { Child } \\
\text { gender } \\
\text { ratio at } \\
\text { recruit } \\
\text { m ent } \\
\text { M/F n } \\
(M / F \%)\end{array}$ & $\begin{array}{l}\text { Randomi } \\
\text { sed } \\
\text { T (I/C) }\end{array}$ & $\begin{array}{l}\text { Dropouts } \\
\text { T (I/C) n; } \\
\text { T (I/C) \% }\end{array}$ & $\begin{array}{l}\text { Number } \\
\text { analysed } \\
\text { (follow- } \\
\text { up) } \\
T \text { (I/C) }\end{array}$ \\
\hline & & & subscale & & & & & & & \\
\hline $\begin{array}{l}\text { Wu } \\
\text { (2014) }\end{array}$ & $\begin{array}{l}\text { Selective: } \\
\text { Very } \\
\text { Preterm } \\
\text { infants }\end{array}$ & $\begin{array}{l}\text { Birth weight } \\
<1500 \text { g, } \\
\text { gestational age } \\
<37 \text { weeks, } \\
\text { admission to the } \\
\text { study hospital } \\
\text { within } 7 \text { days of } \\
\text { birth, singleton } \\
\text { birth or the first } \\
\text { child of twins or } \\
\text { multiples }\end{array}$ & $\begin{array}{l}\text { Severe } \\
\text { neonatal } \\
\text { diseases } \\
\text { included major } \\
\text { neurologic } \\
\text { abnormalities }\end{array}$ & $\begin{array}{l}\text { Recruitmen } \\
\text { t: NICU } \\
\text { I nterventio } \\
\text { n: hospital } \\
\text { and } \\
\text { neonatal } \\
\text { clinic }\end{array}$ & Taiwan & $\begin{array}{l}29.6(2.7) \text {, } \\
\text { Range } n / r \text {, } \\
\text { gestationa } \\
\text { I weeks }^{\text {bc }}\end{array}$ & $\begin{array}{l}53 / 52 \\
(50.4 \% / 50 \\
.6 \%)^{\mathrm{b}}\end{array}$ & $\begin{array}{l}115 \\
(57 / 58)\end{array}$ & $\begin{array}{l}20(7 / 13) ; \\
17.4 \% \\
(12.3 \% / 22 . \\
4 \%)\end{array}$ & $\begin{array}{l}95 \\
(50 / 45)\end{array}$ \\
\hline
\end{tabular}

\section{Notes:}

${ }^{a}$ I nformation gathered directly from authors, ${ }^{b}$ I nformation from follow-up sample, rather than sample as randomised, ${ }^{\mathrm{C}}$ I nformation calculated by pooling intervention and control px

$\mathrm{ADD}=$ attention deficit disorder, $\mathrm{ASD}=$ acute stress disorder, $\mathrm{AUS}=$ Australia, $\mathrm{BDI}=\mathrm{Beck}$ Depression Inventory, $\mathrm{C}=\mathrm{control}, \mathrm{CBCL}=\mathrm{Child}$ Behavior Checklist, $\mathrm{CDI}=$ Children's Depression I nventory, ESL = English as a second language, FI N = Finland, FU = Follow-up, GA = gestational age, GER = Germany, I = Intervention, JPN = J apan, $\mathrm{m}=$ month, $\mathrm{n} / \mathrm{a}=$ not applicable, $\mathrm{n} / \mathrm{r}=$ not reported, NICU = neonatal intensive care unit, NL $=$ Netherlands, NOR = Norway, NZ = New Zealand, PICU = pediatric intensive care unit, PTSD = posttraumatic stress disorder, SCARED = Screen for Child Anxiety Related Emotional Disorders, SPN = Spain, T = total, y = years 
Appendix Table C2. Study intervention and comparison c haracteristics

\begin{tabular}{|c|c|c|c|c|c|c|c|c|c|}
\hline $\begin{array}{l}\text { Study } \\
\text { ID }\end{array}$ & $\begin{array}{l}\text { Program } \\
\text { Name }\end{array}$ & $\begin{array}{l}\text { Foc us Of } \\
\text { Interven } \\
t \text { ion }\end{array}$ & Outline of Content & $\begin{array}{l}\text { Delivery } \\
\text { Format }\end{array}$ & $\begin{array}{l}\text { Number / } \\
\text { length of } \\
\text { sessions } \\
\text { schedule } \\
\text { d }\end{array}$ & $\begin{array}{l}\text { Number / } \\
\text { length of } \\
\text { sessions } \\
\text { delivered }\end{array}$ & $\begin{array}{l}\text { Length of } \\
\text { intervent } \\
\text { ion }\end{array}$ & $\begin{array}{l}\text { Direct } \\
\text { intervent } \\
\text { ion with } \\
\text { the } \\
\text { child? }\end{array}$ & Comparison \\
\hline $\begin{array}{l}\text { Achenba } \\
\text { ch } \\
\text { (1993) }\end{array}$ & $\begin{array}{l}\text { Mother- } \\
\text { I nfant } \\
\text { Transactio } \\
\text { n Program }\end{array}$ & $\begin{array}{l}\text { Parent- } \\
\text { child } \\
\text { relationshi } \\
\mathrm{p}, \\
\text { parenting } \\
\text { skills }\end{array}$ & $\begin{array}{l}\text { Mothers were taught } \\
\text { to be more sensitive } \\
\text { and responsive to } \\
\text { their babies' } \\
\text { physiological and } \\
\text { social cues, as well } \\
\text { as techniques of } \\
\text { interactive play } \\
\text { (modeling, } \\
\text { demonstration, } \\
\text { verbal instruction) } \\
\text { and practical } \\
\text { experience handling } \\
\text { the baby. }\end{array}$ & $\begin{array}{l}\text { Individual } \\
\text { sessions } \\
\text { with } \\
\text { mother } \\
\text { and baby, } \\
\text { delivered } \\
\text { by trained } \\
\text { paediatric } \\
\text { nurse. }\end{array}$ & $\begin{array}{l}7 \text { daily in- } \\
\text { hospital } \\
\text { sessions } \\
\text { and } 4 \\
\text { post- } \\
\text { discharge } \\
\text { home } \\
\text { sessions }\end{array}$ & $n / r$ & $\begin{array}{l}\text { About } 14 \\
\text { weeks }\end{array}$ & No & $\begin{array}{l}\text { Usual Care: } \\
\text { Routine medical } \\
\text { and social } \\
\text { services }\end{array}$ \\
\hline $\begin{array}{l}\text { Beardsl } \\
\text { ee } \\
(1997)\end{array}$ & $\mathrm{n} / \mathrm{r}$ & $\begin{array}{l}\text { Parent- } \\
\text { child } \\
\text { relationshi } \\
\text { p, } \\
\text { parental } \\
\text { mental } \\
\text { health }\end{array}$ & $\begin{array}{l}\text { 1) presenting } \\
\text { psychoeducational } \\
\text { material about mood } \\
\text { disorders and about } \\
\text { risks and resilience } \\
\text { in children; 2) linking } \\
\text { the } \\
\text { psychoeducational } \\
\text { material to the } \\
\text { family's life } \\
\text { experience; 3) } \\
\text { decreasing feelings } \\
\text { of guilt and blame in } \\
\text { children; and 4) } \\
\text { helping the children } \\
\text { to develop }\end{array}$ & $\begin{array}{l}\text { Individual } \\
\text { family } \\
\text { sessions } \\
\text { facilitated } \\
\text { by a } \\
\text { clinician: } \\
\text { some } \\
\text { parents- } \\
\text { only, } \\
\text { some } \\
\text { child-only, } \\
\text { and one } \\
\text { family } \\
\text { meeting }\end{array}$ & $\begin{array}{l}6-10 \\
\text { sessions; } \\
\text { length not } \\
\text { specified. } \\
1 \\
\text { refresher } \\
\text { meeting } \\
\text { with } \\
\text { parents } \\
\text { (and child } \\
\text { if } \\
\text { requested } \\
\text { ) } 6 \text { months } \\
\text { after final } \\
\text { interventi } \\
\text { on session }\end{array}$ & $\begin{array}{l}M=7.1 \\
S D=1.3\end{array}$ & $\begin{array}{l}\mathrm{n} / \mathrm{r}, \\
\text { inferred } \\
\sim 6 \\
\text { months } \\
\mathrm{n} / \mathrm{r} \text {, } \\
\text { inferred } \\
\sim 6 \\
\text { months }\end{array}$ & $\begin{array}{l}\text { Yes; some } \\
\text { child-only } \\
\text { sessions } \\
\text { and child } \\
\text { also } \\
\text { participate } \\
\text { d in some } \\
\text { family } \\
\text { meetings }\end{array}$ & $\begin{array}{l}\text { Minimal } \\
\text { Intervention: Two } \\
\text { 1-hour parent- } \\
\text { only standardised } \\
\text { lectures given by } \\
\text { Beardslee, } \\
\text { covering similar } \\
\text { cognitive material } \\
\text { as the } \\
\text { intervention but } \\
\text { not directly linked } \\
\text { to the family's } \\
\text { illness experience. } \\
\text { Parents who } \\
\text { missed lectures } \\
\text { saw videotapes }\end{array}$ \\
\hline
\end{tabular}




\begin{tabular}{|c|c|c|c|c|c|c|c|c|c|}
\hline $\begin{array}{l}\text { Study } \\
\text { ID }\end{array}$ & $\begin{array}{l}\text { Program } \\
\text { Name }\end{array}$ & $\begin{array}{l}\text { Focus of } \\
\text { Interven } \\
t \text { ion }\end{array}$ & Outline of Content & $\begin{array}{l}\text { Delivery } \\
\text { Format }\end{array}$ & $\begin{array}{l}\text { Number / } \\
\text { length of } \\
\text { sessions } \\
\text { schedule } \\
\text { d }\end{array}$ & $\begin{array}{l}\text { Number / } \\
\text { length of } \\
\text { sessions } \\
\text { delivered }\end{array}$ & $\begin{array}{l}\text { Length of } \\
\text { intervent } \\
\text { ion }\end{array}$ & $\begin{array}{l}\text { Direct } \\
\text { intervent } \\
\text { ion with } \\
\text { the } \\
\text { child? }\end{array}$ & Comparison \\
\hline $\begin{array}{l}\text { Beardsle } \\
\text { e (2003) }\end{array}$ & & & $\begin{array}{l}\text { relationships both } \\
\text { within and outside of } \\
\text { the family }\end{array}$ & & $\begin{array}{l}6-11 \\
\text { sessions } \\
\text { including } \\
\text { separate } \\
\text { meetings }\end{array}$ & $\begin{array}{l}M=6.7 \\
S D=1.3 \\
\text { range }= \\
2-9\end{array}$ & & & $\begin{array}{l}\text { with consultation } \\
\text { available. }\end{array}$ \\
\hline $\begin{array}{l}\text { Beardsle } \\
\text { e (2007) }\end{array}$ & & & & & $\begin{array}{l}\text { with } \\
\text { parents } \\
\text { and } \\
\text { children, } \\
\text { family } \\
\text { meetings, } \\
\text { and } \\
\text { telephone } \\
\text { contacts } \\
\text { or } \\
\text { refresher } \\
\text { meetings } \\
\text { at } \\
6-\text { to 9- } \\
\text { month } \\
\text { intervals. }\end{array}$ & & & & \\
\hline $\begin{array}{l}\text { Black } \\
\text { (2007) }\end{array}$ & $n / r$ & $\begin{array}{l}\text { Parenting } \\
\text { skills, } \\
\text { parent- } \\
\text { child } \\
\text { relationshi } \\
\mathrm{p}\end{array}$ & $\begin{array}{l}\text { Support to the } \\
\text { caregiver's personal, } \\
\text { family, and } \\
\text { environmental } \\
\text { needs; opportunities } \\
\text { to model and } \\
\text { promote responsive } \\
\text { parent-infant } \\
\text { interaction; and } \\
\text { problem-solving } \\
\text { strategies regarding } \\
\text { personal, parenting, } \\
\text { and children's } \\
\text { issues. }\end{array}$ & $\begin{array}{l}\text { Home } \\
\text { visitation } \\
\text { by trained } \\
\text { laypersons }\end{array}$ & $\begin{array}{l}\text { One-hour } \\
\text { visits were } \\
\text { scheduled } \\
\text { weekly }\end{array}$ & $\begin{array}{l}M=19.2, \\
S D=11.5, \\
\text { median = } \\
25, \\
\text { interquarti } \\
\text { le range = } \\
18-30, \\
\text { range }=0- \\
47\end{array}$ & 1 year & No & $\begin{array}{l}\text { Usual Care: } \\
\text { Clinical } \\
\text { intervention only. } \\
\text { Multidisciplinary } \\
\text { growth and } \\
\text { nutrition clinic, } \\
\text { clinical services } \\
\text { included referrals } \\
\text { to food programs, } \\
\text { growth } \\
\text { monitoring, } \\
\text { recommended } \\
\text { menus, } \\
\text { videotaped } \\
\text { replays of }\end{array}$ \\
\hline
\end{tabular}




\begin{tabular}{|c|c|c|c|c|c|c|c|c|c|}
\hline $\begin{array}{l}\text { Study } \\
\text { ID }\end{array}$ & $\begin{array}{l}\text { Program } \\
\text { Name }\end{array}$ & $\begin{array}{l}\text { Foc us Of } \\
\text { Interven } \\
\text { t ion }\end{array}$ & Outline of Content & $\begin{array}{l}\text { Delivery } \\
\text { Format }\end{array}$ & $\begin{array}{l}\text { Number / } \\
\text { length of } \\
\text { sessions } \\
\text { schedule } \\
\text { d }\end{array}$ & $\begin{array}{l}\text { Number / } \\
\text { length of } \\
\text { sessions } \\
\text { delivered }\end{array}$ & $\begin{array}{l}\text { Length of } \\
\text { intervent } \\
\text { ion }\end{array}$ & $\begin{array}{l}\text { Direct } \\
\text { intervent } \\
\text { ion with } \\
\text { the } \\
\text { child? }\end{array}$ & Comparison \\
\hline & & & & & & & & & $\begin{array}{l}\text { mothers with their } \\
\text { children during } \\
\text { feeding, and } \\
\text { counselling } \\
\text { regarding feeding } \\
\text { schedules and } \\
\text { behaviour. }\end{array}$ \\
\hline $\begin{array}{l}\text { Butz } \\
(2001)\end{array}$ & $\mathrm{n} / \mathrm{r}$ & $\begin{array}{l}\text { Parenting } \\
\text { skills, } \\
\text { parent- } \\
\text { child } \\
\text { relationshi } \\
\mathrm{p}\end{array}$ & $\begin{array}{l}\text { The nurses } \\
\text { established a caring } \\
\text { relationship with the } \\
\text { caregiver, provided } \\
\text { emotional support, } \\
\text { modelled positive } \\
\text { parent-child } \\
\text { interactions, } \\
\text { monitored infant } \\
\text { health, and taught } \\
\text { skills to enhance } \\
\text { maternal-infant } \\
\text { interaction. }\end{array}$ & $\begin{array}{l}\text { Home } \\
\text { visits by } \\
\text { paediatric } \\
\text { nurse } \\
\text { specialists }\end{array}$ & $\begin{array}{l}16 \\
\text { sessions } \\
\text { (length } \\
\text { not } \\
\text { stated) }\end{array}$ & $\begin{array}{l}M=12.8 \\
S D=3.2 \\
\text { range }=1- \\
20\end{array}$ & $\begin{array}{l}18 \text { months } \\
\text { with more } \\
\text { frequent } \\
\text { visits } \\
\text { during the } \\
\text { first } 6 \\
\text { months }^{\mathrm{a}}\end{array}$ & No & $\begin{array}{l}\text { Usual Care: not } \\
\text { described }\end{array}$ \\
\hline $\begin{array}{l}\text { Carta } \\
(2013)\end{array}$ & $\begin{array}{l}\text { Planned } \\
\text { Activities } \\
\text { Training } \\
\text { (PAT) } \\
\text { suppleme } \\
\text { nted with } \\
\text { cellular } \\
\text { phone } \\
\text { calls and } \\
\text { text } \\
\text { messaging } \\
\text { (CPAT) }\end{array}$ & $\begin{array}{l}\text { Parenting } \\
\text { skills }\end{array}$ & $\begin{array}{l}\text { Part of the SafeCare } \\
\text { parent training } \\
\text { model, aims to } \\
\text { prevent challenging } \\
\text { child behaviour and } \\
\text { improve parent-child } \\
\text { interactions by } \\
\text { focusing on teaching } \\
\text { specific responsive } \\
\text { parenting strategies. } \\
\text { Phone contacts } \\
\text { promoted } \\
\text { engagement and } \\
\text { provided reminders } \\
\text { of newly learned } \\
\text { parenting strategies. }\end{array}$ & $\begin{array}{l}\text { Individual } \\
\text { sessions } \\
\text { with a } \\
\text { family } \\
\text { coach } \\
\text { (research } \\
\text { staff with } \\
\text { a BA } \\
\text { degree). } \\
\text { In addition } \\
\text { to the } \\
\text { home } \\
\text { visits, } \\
\text { they } \\
\text { received } \\
\text { text }\end{array}$ & $\begin{array}{l}5 \text { sessions } \\
+ \\
\text { individuali } \\
\text { sed text } \\
\text { messages } \\
\text { twice/day } \\
+ \text { phone } \\
\text { call } \\
\text { once/week }\end{array}$ & $\mathrm{n} / \mathrm{r}$ & 5 weeks & No & Extended waitlist \\
\hline
\end{tabular}




\begin{tabular}{|c|c|c|c|c|c|c|c|c|c|}
\hline $\begin{array}{l}\text { Study } \\
\text { ID }\end{array}$ & $\begin{array}{l}\text { Program } \\
\text { Name }\end{array}$ & $\begin{array}{l}\text { Focus Of } \\
\text { Interven } \\
\text { t ion }\end{array}$ & Outline of Content & $\begin{array}{l}\text { Delivery } \\
\text { Format }\end{array}$ & $\begin{array}{l}\text { Number / } \\
\text { length of } \\
\text { sessions } \\
\text { schedule } \\
\text { d }\end{array}$ & $\begin{array}{l}\text { Number / } \\
\text { length of } \\
\text { sessions } \\
\text { delivered }\end{array}$ & $\begin{array}{l}\text { Length of } \\
\text { intervent } \\
\text { ion }\end{array}$ & $\begin{array}{l}\text { Direct } \\
\text { intervent } \\
\text { ion with } \\
\text { the } \\
\text { child? }\end{array}$ & Comparison \\
\hline & & & & $\begin{array}{l}\text { messages } \\
\text { and calls }\end{array}$ & & & & & \\
\hline $\begin{array}{l}\text { Cartwrig } \\
\text { ht- } \\
\text { Hatto } \\
\text { n } \\
(2011)\end{array}$ & $\begin{array}{l}\text { Timid to } \\
\text { Tiger }\end{array}$ & $\begin{array}{l}\text { Parenting } \\
\text { skills, } \\
\text { parents as } \\
\text { coach of } \\
\text { child's } \\
\text { problem }\end{array}$ & $\begin{array}{l}\text { Parents were taught } \\
\text { to provide their } \\
\text { children with a } \\
\text { warm, calm, } \\
\text { predictable home } \\
\text { environment, in } \\
\text { which gentle, } \\
\text { positive, discipline } \\
\text { was used to manage } \\
\text { difficult behaviour } \\
\text { and to encourage } \\
\text { confident behaviour; } \\
\text { and to manage } \\
\text { children's anxiety } \\
\text { using cognitive-- } \\
\text { behavioral skills. }\end{array}$ & $\begin{array}{l}\text { Parent } \\
\text { group } \\
\text { sessions } \\
\text { conducted } \\
\text { by } 2 \\
\text { clinical } \\
\text { psychologi } \\
\text { sts }\end{array}$ & $\begin{array}{l}10 \times 2- \\
\text { hour } \\
\text { sessions }\end{array}$ & $\begin{array}{l}5 \text { families } \\
\text { attended } \\
\text { no } \\
\text { sessions; } \\
\text { the rest } \\
\text { attended } \\
\text { a mean of } \\
88 \% \text { of } \\
\text { sessions. }\end{array}$ & 10 weeks & No & $\begin{array}{l}\text { Usual Care: After } \\
10 \text { weeks, control } \\
\text { participants } \\
\text { completed the } \\
\text { post assessment } \\
\text { and were then } \\
\text { seen by referrer } \\
\text { or were given } \\
\text { help finding } \\
\text { appropriate } \\
\text { support. }\end{array}$ \\
\hline $\begin{array}{l}\text { Cheng } \\
\text { (2007) }\end{array}$ & $n / r$ & $\begin{array}{l}\text { Parenting } \\
\text { skills, } \\
\text { parent- } \\
\text { child } \\
\text { relationshi } \\
\text { p }\end{array}$ & $\begin{array}{l}\text { Promotion of } \\
\text { maternal feelings of } \\
\text { competence in } \\
\text { infant caretaking as } \\
\text { well as improvement } \\
\text { of maternal } \\
\text { sensitivity and the } \\
\text { quality of mother- } \\
\text { infant interactions } \\
\text { through modeling or } \\
\text { positive feedback. }\end{array}$ & $\begin{array}{l}\text { Home } \\
\text { visitations } \\
\text { by nurse }\end{array}$ & $\begin{array}{l}\text { Five home } \\
\text { visits, } \\
\text { once a } \\
\text { month, } 1 \\
\text { hour long }\end{array}$ & $n / r$ & 5 months & No & $\begin{array}{l}\text { Usual Care: } \\
\text { Standard centre- } \\
\text { based service } \\
\text { Including } \\
\text { education } \\
\text { regarding } \\
\text { parenting, infant } \\
\text { nutrition, } \\
\text { development, } \\
\text { physical health, } \\
\text { and other services } \\
\text { in conjunction } \\
\text { with infant } \\
\text { medical check- } \\
\text { ups. Plus } \\
\text { psychological } \\
\text { counselling was } \\
\text { made available in }\end{array}$ \\
\hline
\end{tabular}




\begin{tabular}{|c|c|c|c|c|c|c|c|c|c|}
\hline $\begin{array}{l}\text { Study } \\
\text { ID }\end{array}$ & $\begin{array}{l}\text { Program } \\
\text { Name }\end{array}$ & $\begin{array}{l}\text { Foc us Of } \\
\text { Interven } \\
\text { t ion }\end{array}$ & Outline of Content & $\begin{array}{l}\text { Delivery } \\
\text { Format }\end{array}$ & $\begin{array}{l}\text { Number / } \\
\text { length of } \\
\text { sessions } \\
\text { schedule } \\
\text { d }\end{array}$ & $\begin{array}{l}\text { Number / } \\
\text { length of } \\
\text { sessions } \\
\text { delivered }\end{array}$ & $\begin{array}{l}\text { Length of } \\
\text { intervent } \\
\text { ion }\end{array}$ & $\begin{array}{l}\text { Direct } \\
\text { intervent } \\
\text { ion with } \\
\text { the } \\
\text { child? }\end{array}$ & Comparison \\
\hline & & & & & & & & & the town. \\
\hline $\begin{array}{l}\text { Cohen } \\
\text { (1997) }\end{array}$ & $\begin{array}{l}\text { Cognitive- } \\
\text { Behavioral } \\
\text { Therapy } \\
\text { for } \\
\text { Sexually } \\
\text { Abused } \\
\text { Preschoole } \\
\text { rs (CBT- } \\
\text { SAP) }\end{array}$ & $\begin{array}{l}\text { Parenting } \\
\text { skills, } \\
\text { parent- } \\
\text { child } \\
\text { relationshi } \\
\text { p, } \\
\text { parental } \\
\text { mental } \\
\text { health (in } \\
\text { those with } \\
\text { child } \\
\text { abuse } \\
\text { histories), } \\
\text { parents as } \\
\text { coach of } \\
\text { child's } \\
\text { problem }\end{array}$ & $\begin{array}{l}\text { Child: Use of } \\
\text { structured play to } \\
\text { target sexually } \\
\text { inappropriate or } \\
\text { regressive } \\
\text { behaviours, } \\
\text { aggression and } \\
\text { sadness. Parents: } \\
\text { Addresses common } \\
\text { misperceptions } \\
\text { parents hold, } \\
\text { improves parental } \\
\text { skills to provide } \\
\text { emotional support to } \\
\text { child and to manage } \\
\text { regressive and } \\
\text { inappropriate } \\
\text { behaviours, as well } \\
\text { as anxiety } \\
\text { symptoms, maternal } \\
\text { issues related to } \\
\text { own history of } \\
\text { abuse, and legal } \\
\text { issues. }\end{array}$ & $\begin{array}{l}\text { Individual } \\
\text { sessions } \\
\text { with } \\
\text { parent } \\
\text { and } \\
\text { separately } \\
\text { with the } \\
\text { child, } \\
\text { delivered } \\
\text { by } \\
\text { clinicians }\end{array}$ & $\begin{array}{l}12 \text { weekly } \\
\text { sessions; } \\
50 \\
\text { minutes } \\
\text { for } \\
\text { parents } \\
\text { and } 30-40 \\
\text { minutes } \\
\text { for child }\end{array}$ & $n / r$ & 12 weeks & $\begin{array}{l}\text { Yes: child } \\
\text { attends } \\
\text { individual } \\
\text { sessions } \\
\text { with the } \\
\text { clinician. }\end{array}$ & $\begin{array}{l}\text { Attention control: } \\
\text { Non-directive } \\
\text { Supportive } \\
\text { Therapy designed } \\
\text { to control fornon- } \\
\text { specific aspects of } \\
\text { therapy and did } \\
\text { not make } \\
\text { interpretations or } \\
\text { offer advice or } \\
\text { suggestions. }\end{array}$ \\
\hline $\begin{array}{l}\text { Connell } \\
\text { (2008) }\end{array}$ & $\begin{array}{l}\text { Family } \\
\text { Check-Up }\end{array}$ & $\begin{array}{l}\text { Parenting } \\
\text { skills }\end{array}$ & $\begin{array}{l}\text { Sessions focused on } \\
\text { managing child } \\
\text { problem behaviour, } \\
\text { supporting existing } \\
\text { parenting strengths, } \\
\text { identifying services } \\
\text { appropriate to the } \\
\text { family needs, } \\
\text { parenting practices, } \\
\text { other family } \\
\text { management }\end{array}$ & $\begin{array}{l}\text { Individual } \\
\text { sessions } \\
\text { with } \\
\text { clinicians }\end{array}$ & $\begin{array}{l}3 \text { sessions } \\
\text { at age } 2-3 \\
\text { and } 3 \\
\text { sessions } \\
\text { at age } 3- \\
4 \text {. Length } \\
\mathrm{n} / \mathrm{r}\end{array}$ & $\begin{array}{l}\text { Age } 2-3: \\
77.9 \% \\
\text { attended } \\
(M=3.3 \\
S D=2.8) \\
\text { Age 3-4: } \\
65.4 \% \\
\text { attended } \\
(M=2.8 \\
S D=2.70)\end{array}$ & $\begin{array}{l}\mathrm{n} / \mathrm{r} \\
\text { inferred } \\
\sim 1 \text { year }\end{array}$ & No & No Treatment \\
\hline
\end{tabular}




\begin{tabular}{|c|c|c|c|c|c|c|c|c|c|}
\hline $\begin{array}{l}\text { Study } \\
\text { ID }\end{array}$ & $\begin{array}{l}\text { Program } \\
\text { Name }\end{array}$ & $\begin{array}{l}\text { Focus Of } \\
\text { Interven } \\
t \text { ion }\end{array}$ & Outline of Content & $\begin{array}{l}\text { Delivery } \\
\text { Format }\end{array}$ & $\begin{array}{l}\text { Number / } \\
\text { length of } \\
\text { sessions } \\
\text { schedule } \\
\text { d }\end{array}$ & $\begin{array}{l}\text { Number / } \\
\text { length of } \\
\text { sessions } \\
\text { delivered }\end{array}$ & $\begin{array}{l}\text { Length of } \\
\text { intervent } \\
\text { ion }\end{array}$ & $\begin{array}{l}\text { Direct } \\
\text { intervent } \\
\text { ion with } \\
\text { the } \\
\text { child? }\end{array}$ & Comparison \\
\hline & & & $\begin{array}{l}\text { concerns (e.g., co- } \\
\text { parenting), and } \\
\text { contextual issues } \\
\text { (e.g., parental well- } \\
\text { being, marital } \\
\text { adjustment, } \\
\text { housing). }\end{array}$ & & & & & & \\
\hline $\begin{array}{l}\text { Constant } \\
\text { ino } \\
(2001)\end{array}$ & $n / r$ & $\begin{array}{l}\text { Parent- } \\
\text { child } \\
\text { relationshi } \\
\mathrm{p}\end{array}$ & $\begin{array}{l}\text { Parental education } \\
\text { about infant social } \\
\text { and emotional } \\
\text { development; } \\
\text { experience with } \\
\text { interactive play; and } \\
\text { support for young } \\
\text { adults in their role } \\
\text { as parents. }\end{array}$ & $\begin{array}{l}\text { Groups } \\
\text { sessions } \\
\text { with 8-10 } \\
\text { parent- } \\
\text { child } \\
\text { dyads; } \\
\text { each } \\
\text { session } \\
\text { was } \\
\text { delivered } \\
\text { by } 2 \text { early } \\
\text { childhood } \\
\text { educators }\end{array}$ & $\begin{array}{l}10 \\
\text { sessions, } \\
\text { once a } \\
\text { week, } \\
\text { approxima } \\
\text { tely } 1.5 \mathrm{~h}\end{array}$ & $n / r$ & 10 weeks & No & $\begin{array}{l}\text { Usual Care: Home } \\
\text { visitation }\end{array}$ \\
\hline $\begin{array}{l}\text { Cowan } \\
\text { (2005) }\end{array}$ & $n / r$ & $\begin{array}{l}\text { Parent- } \\
\text { child } \\
\text { relationshi } \\
\mathrm{p}\end{array}$ & $\begin{array}{l}\text { Intervention focused } \\
\text { on parents' } \\
\text { individual } \\
\text { adaptation, couple } \\
\text { relationship quality, } \\
\text { relationship quality } \\
\text { in parents' families }\end{array}$ & $\begin{array}{l}\text { Group } \\
\text { sessions } \\
\text { with 4-5 } \\
\text { participant } \\
\text { couples } \\
\text { and a staff } \\
\text { couple }\end{array}$ & $\begin{array}{l}16 \times 2- \\
\text { hour } \\
\text { weekly } \\
\text { sessions }\end{array}$ & $n / r$ & 4 months & No & $\begin{array}{l}\text { Minimal Control: } \\
\text { Opportunity to } \\
\text { consult with the } \\
\text { interviewer team } \\
\text { once a year for } 3 \\
\text { years }\end{array}$ \\
\hline $\begin{array}{l}\text { Cowan } \\
\text { (2011) }\end{array}$ & & & $\begin{array}{l}\text { of origin, quality of } \\
\text { parents' relationship } \\
\text { with the child, } \\
\text { balance between life } \\
\text { stressors and social } \\
\text { supports. Emphasis } \\
\text { was placed on how } \\
\text { issues affect the } \\
\text { relationship between }\end{array}$ & & & & & & \\
\hline
\end{tabular}




\begin{tabular}{|c|c|c|c|c|c|c|c|c|c|}
\hline $\begin{array}{l}\text { Study } \\
\text { ID }\end{array}$ & $\begin{array}{l}\text { Program } \\
\text { Name }\end{array}$ & $\begin{array}{l}\text { Focus Of } \\
\text { Interven } \\
t \text { ion }\end{array}$ & Outline of Content & $\begin{array}{l}\text { Delivery } \\
\text { Format }\end{array}$ & $\begin{array}{l}\text { Number / } \\
\text { length of } \\
\text { sessions } \\
\text { schedule } \\
\text { d }\end{array}$ & $\begin{array}{l}\text { Number / } \\
\text { length of } \\
\text { sessions } \\
\text { delivered }\end{array}$ & $\begin{array}{l}\text { Length of } \\
\text { intervent } \\
\text { ion }\end{array}$ & $\begin{array}{l}\text { Direct } \\
\text { intervent } \\
\text { ion with } \\
\text { the } \\
\text { child? }\end{array}$ & Comparison \\
\hline & & & $\begin{array}{l}\text { parents and their } \\
\text { child }\end{array}$ & & & & & & \\
\hline $\begin{array}{l}\text { Dadds \& } \\
\text { Roth } \\
(2008)\end{array}$ & $\begin{array}{l}\text { REACH for } \\
\text { RESI LIENC } \\
\text { E }\end{array}$ & $\begin{array}{l}\text { Parenting } \\
\text { skills, } \\
\text { parent- } \\
\text { child } \\
\text { relationshi } \\
\text { p }\end{array}$ & $\begin{array}{l}\text { To improve parent- } \\
\text { child interactions, } \\
\text { parents are taught } \\
\text { how to build positive } \\
\text { expectations in their } \\
\text { children through } \\
\text { self-talk, behavioral } \\
\text { change, and } \\
\text { problem-solving } \\
\text { strategies. }\end{array}$ & $\begin{array}{l}\text { Group } \\
\text { sessions } \\
\text { with } \\
\text { parents } \\
\text { conducted } \\
\text { by a } \\
\text { clinician. }\end{array}$ & $\begin{array}{l}6 \\
\text { fortnightly } \\
\text { sessions, } \\
\text { length } \mathrm{n} / \mathrm{r}\end{array}$ & $\begin{array}{l}27.3 \% \\
\text { attended } \\
0 \\
\text { sessions, } \\
12.7 \% \\
\text { attended } \\
1-3 \\
\text { sessions, } \\
21.4 \% \\
\text { attended } \\
4-6 \\
\text { sessions. } \\
\text { The rest } \\
\text { were } \\
\text { 'invitedbut } \\
\text { not } \\
\text { interested' }\end{array}$ & 3 months & No & No Treatment \\
\hline $\begin{array}{l}\text { Etter } \\
(2013)\end{array}$ & $\begin{array}{l}\text { The } \\
\text { Fatherhoo } \\
\text { d, } \\
\text { Relationsh } \\
\text { ip, and } \\
\text { Marriage } \\
\text { Education } \\
\text { program } \\
\text { (FRAME) }\end{array}$ & $\begin{array}{l}\text { Parenting } \\
\text { skills, } \\
\text { Parent- } \\
\text { child } \\
\text { relationshi } \\
\mathrm{p}\end{array}$ & $\begin{array}{l}\text { I ntervention based } \\
\text { on the Family Stress } \\
\text { Model and focused } \\
\text { on relationship } \\
\text { education; parent } \\
\text { stress and coping } \\
\text { skill training; and } \\
\text { child-centred parent } \\
\text { training to create } \\
\text { safer, more stable } \\
\text { couple relationships } \\
\text { and better } \\
\text { environments for } \\
\text { their children. }\end{array}$ & $\begin{array}{l}\text { Group } \\
\text { workshops } \\
\text { led by a } \\
\text { team of } \\
\text { two highly } \\
\text { trained } \\
\text { leaders. } \\
\text { FRAME } \\
\text { includes } \\
\text { extensive } \\
\text { use of } \\
\text { activities, } \\
\text { discussion } \\
\text { s, and } \\
\text { practice/h } \\
\text { omework }\end{array}$ & $\begin{array}{l}\text { Workshop } \\
\text { s involved } \\
14 \text { hours } \\
\text { of content } \\
\text { and were } \\
\text { conducted } \\
\text { on } 3 \\
\text { Saturdays } \\
\text { ( } 6 \text { hours } \\
\text { each) or } 5 \\
\text { weeknight } \\
\text { s ( } 3.5 \\
\text { hours } \\
\text { each). } \\
\text { All } \\
\text { interventi }\end{array}$ & $n / r$ & $n / r$ & No & No Treatment \\
\hline
\end{tabular}




\begin{tabular}{|c|c|c|c|c|c|c|c|c|c|}
\hline $\begin{array}{l}\text { Study } \\
\text { ID }\end{array}$ & $\begin{array}{l}\text { Program } \\
\text { Name }\end{array}$ & $\begin{array}{l}\text { Focus of } \\
\text { Interven } \\
t \text { ion }\end{array}$ & Outline of Content & $\begin{array}{l}\text { Delivery } \\
\text { Format }\end{array}$ & $\begin{array}{l}\text { Number / } \\
\text { length of } \\
\text { sessions } \\
\text { schedule } \\
\text { d }\end{array}$ & $\begin{array}{l}\text { Number / } \\
\text { length of } \\
\text { sessions } \\
\text { delivered }\end{array}$ & $\begin{array}{l}\text { Length of } \\
\text { intervent } \\
\text { ion }\end{array}$ & $\begin{array}{l}\text { Direct } \\
\text { intervent } \\
\text { ion with } \\
\text { the } \\
\text { child? }\end{array}$ & Comparison \\
\hline & & & & $\begin{array}{l}\text { designed } \\
\text { to teach } \\
\text { skills and } \\
\text { principles. }\end{array}$ & $\begin{array}{l}\text { on couples } \\
\text { and } \\
\text { individuals } \\
\text { were also } \\
\text { invited to } \\
\text { attend } \\
\text { booster } \\
\text { sessions. }\end{array}$ & & & & \\
\hline $\begin{array}{l}\text { Feinber } \\
\text { g } \\
(2010)\end{array}$ & $\begin{array}{l}\text { Family } \\
\text { Foundatio } \\
\text { ns }\end{array}$ & $\begin{array}{l}\text { Other (co- } \\
\text { parenting) }\end{array}$ & $\begin{array}{l}\text { Focus on helping } \\
\text { couples become } \\
\text { aware of areas of } \\
\text { coparental } \\
\text { disagreement before } \\
\text { parenthood, and } \\
\text { managing } \\
\text { disagreements } \\
\text { through productive } \\
\text { communication, } \\
\text { problem solving, and } \\
\text { conflictmanagement } \\
\text { techniques. }\end{array}$ & $\begin{array}{l}\text { Group } \\
\text { sessions } \\
\text { of } 6-10 \\
\text { couples } \\
\text { led by a } \\
\text { trained } \\
\text { male and } \\
\text { female } \\
\text { layperson } \\
\text { team }\end{array}$ & $\begin{array}{l}8 \\
\text { sessions: } \\
4 \text { prenatal } \\
\text { and } 4 \\
\text { postnatal }\end{array}$ & $\begin{array}{l}M \text { prenatal } \\
\text { sessions = } \\
3.2 ; M \\
\text { postnatal } \\
=2.3 \\
(80 \% \\
\text { attended } \\
\text { at least } 3 \\
\text { prenatal; } \\
60 \% \text { at } \\
\text { least } 3 \\
\text { postnatal } \\
\text { ) }\end{array}$ & $\mathrm{n} / \mathrm{r}$ & No & No Treatment \\
\hline $\begin{array}{l}\text { Fergusso } \\
\text { n(2013) }\end{array}$ & $\begin{array}{l}\text { Early Start } \\
\text { interventi } \\
\text { on }\end{array}$ & $\begin{array}{l}\text { Parenting } \\
\text { skills }\end{array}$ & $\begin{array}{l}\text { Home visitation } \\
\text { intervention } \\
\text { involving ( } 1 \text { ) } \\
\text { assessment of } \\
\text { family needs, issues, } \\
\text { challenges, } \\
\text { strengths, and } \\
\text { resources; ( } 2 \text { ) } \\
\text { development of a } \\
\text { positive partnership } \\
\text { between the family } \\
\text { support worker and } \\
\text { client; (3) } \\
\text { collaborative }\end{array}$ & $\begin{array}{l}\text { Individual } \\
\text { sessions } \\
\text { provided } \\
\text { by Family } \\
\text { Support } \\
\text { Workers }\end{array}$ & $n / r$ & $n / r$ & $\begin{array}{l}M=37.8 \\
\text { months, } \\
S D=23.6 \\
\text { months, } \\
\text { Median = } \\
48 \\
\text { months, } \\
\text { range 1- } \\
65^{\mathrm{a}}\end{array}$ & No & No Treatment \\
\hline
\end{tabular}




\begin{tabular}{|c|c|c|c|c|c|c|c|c|c|}
\hline $\begin{array}{l}\text { Study } \\
\text { ID }\end{array}$ & $\begin{array}{l}\text { Program } \\
\text { Name }\end{array}$ & $\begin{array}{l}\text { Focus of } \\
\text { Interven } \\
t \text { ion }\end{array}$ & Outline of Content & $\begin{array}{l}\text { Delivery } \\
\text { Format }\end{array}$ & $\begin{array}{l}\text { Number / } \\
\text { length of } \\
\text { sessions } \\
\text { schedule } \\
\text { d }\end{array}$ & $\begin{array}{l}\text { Number / } \\
\text { length of } \\
\text { sessions } \\
\text { delivered }\end{array}$ & $\begin{array}{l}\text { Length of } \\
\text { intervent } \\
\text { ion }\end{array}$ & $\begin{array}{l}\text { Direct } \\
\text { intervent } \\
\text { ion with } \\
\text { the } \\
\text { child? }\end{array}$ & Comparison \\
\hline & & & $\begin{array}{l}\text { problem solving to } \\
\text { devise solutions to } \\
\text { family challenges; } \\
\text { (4) the provisionof } \\
\text { support, mentoring, } \\
\text { and advice to assist } \\
\text { client families to } \\
\text { mobilize their } \\
\text { strengths and } \\
\text { resources; and (5) } \\
\text { involvement with } \\
\text { the family } \\
\text { throughout the } \\
\text { child's preschool } \\
\text { vears }\end{array}$ & & & & & & \\
\hline $\begin{array}{l}\text { Forgatch } \\
\text { (1999) }\end{array}$ & $\begin{array}{l}\text { Parenting } \\
\text { Through } \\
\text { Change }\end{array}$ & $\begin{array}{l}\text { Parenting } \\
\text { Skills }\end{array}$ & $\begin{array}{l}\text { Training in parenting } \\
\text { practices: non- } \\
\text { coercive discipline, } \\
\text { contingent } \\
\text { encouragement, } \\
\text { monitoring and } \\
\text { problem solving; as } \\
\text { well as issues } \\
\text { relevant to divorcing } \\
\text { women: regulating } \\
\text { negative emotions } \\
\text { and managing } \\
\text { interpersonal } \\
\text { conflict. }\end{array}$ & $\begin{array}{l}\text { Group } \\
\text { sessions } \\
\text { with } 6 \text { to } \\
16 \\
\text { mothers, } \\
\text { led by } 2 \\
\text { trained } \\
\text { interventi } \\
\text { onists with } \\
\text { a mixture } \\
\text { of } \\
\text { education } \\
\text { and } \\
\text { experienc } \\
\text { e. Plus a } \\
\text { weekly } \\
\text { phone call } \\
\text { to } \\
\text { encourage } \\
\text { use of the } \\
\text { procedure }\end{array}$ & \begin{tabular}{|l|}
$14-16$ \\
sessions
\end{tabular} & $\begin{array}{l}M=8.5 \\
S D=5.7 \\
\text { range }=0- \\
15\end{array}$ & $\begin{array}{l}16.4 \\
\text { weeks }\end{array}$ & No & No Treatment \\
\hline
\end{tabular}




\begin{tabular}{|c|c|c|c|c|c|c|c|c|c|}
\hline $\begin{array}{l}\text { Study } \\
\text { ID }\end{array}$ & $\begin{array}{l}\text { Program } \\
\text { Name }\end{array}$ & $\begin{array}{l}\text { Foc us Of } \\
\text { Interven } \\
\text { t ion }\end{array}$ & Outline of Content & $\begin{array}{l}\text { Delivery } \\
\text { Format }\end{array}$ & $\begin{array}{l}\text { Number / } \\
\text { length of } \\
\text { sessions } \\
\text { schedule } \\
\text { d }\end{array}$ & $\begin{array}{l}\text { Number / } \\
\text { length of } \\
\text { sessions } \\
\text { delivered }\end{array}$ & $\begin{array}{l}\text { Length of } \\
\text { intervent } \\
\text { ion }\end{array}$ & $\begin{array}{l}\text { Direct } \\
\text { intervent } \\
\text { ion with } \\
\text { the } \\
\text { child? }\end{array}$ & Comparison \\
\hline & & & & $\begin{array}{l}s \text { and to } \\
\text { troublesho } \\
\text { ot } \\
\text { problems } \\
\text { with } \\
\text { homework }\end{array}$ & & & & & \\
\hline $\begin{array}{l}\text { Ginsburg } \\
\text { (2009) }\end{array}$ & $\begin{array}{l}\text { Coping } \\
\text { and } \\
\text { Promoting } \\
\text { Strength } \\
\text { (CAPS) }\end{array}$ & $\begin{array}{l}\text { Parenting } \\
\text { skills, } \\
\text { parent- } \\
\text { child } \\
\text { relationshi } \\
\mathrm{p}\end{array}$ & $\begin{array}{l}\text { Parents taught about } \\
\text { modeling of anxiety } \\
\text { symptoms, } \\
\text { decreasing anxiety- } \\
\text { enhancing parenting } \\
\text { (e.g. } \\
\text { overprotection), and } \\
\text { reducing criticism } \\
\text { and family conflict. } \\
\text { Targets in children } \\
\text { were reducing } \\
\text { anxiety symptoms } \\
\text { and social } \\
\text { avoidance, } \\
\text { maladaptive } \\
\text { cognitions, and poor } \\
\text { problem-solving } \\
\text { skills. }\end{array}$ & $\begin{array}{l}\text { Individual } \\
\text { sessions } \\
\text { with } \\
\text { parents or } \\
\text { parents } \\
\text { with } \\
\text { children } \\
\text { delivered } \\
\text { by } \\
\text { clinicians }\end{array}$ & $\begin{array}{l}6-8 \\
\text { weekly } \\
\text { sessions } \\
\text { plus a 3- } \\
\text { month } \\
\text { booster } \\
\text { session, } \\
60 \\
\text { minutes } \\
\text { each }\end{array}$ & $\begin{array}{l}M=7.47 \\
\text { range }=5- \\
8\end{array}$ & 8 weeks & $\begin{array}{l}\text { Yes: } \\
\text { children } \\
\text { were } \\
\text { invited to } \\
\text { up to } 6\end{array}$ & Extended waitlist \\
\hline $\begin{array}{l}\text { Hahlweg } \\
\text { (2010) }\end{array}$ & $\begin{array}{l}\text { Triple-P } \\
\text { Positive } \\
\text { Parenting } \\
\text { Program } \\
\text { (group) }\end{array}$ & $\begin{array}{l}\text { Parenting } \\
\text { skills }\end{array}$ & $\begin{array}{l}\text { Parents are taught } \\
\text { child management } \\
\text { strategies to } \\
\text { promote children's } \\
\text { competence and } \\
\text { development and to } \\
\text { help parents } \\
\text { manage } \\
\text { misbehaviour. }\end{array}$ & $\begin{array}{l}\text { Group } \\
\text { sessions } \\
\text { with } 6 \text { to } \\
10 \\
\text { families } \\
\text { delivered } \\
\text { by } \\
\text { clinicians }\end{array}$ & $\begin{array}{l}4 \text { weekly } \\
\text { group } \\
\text { sessions, } \\
2 \text { hours } \\
\text { each; plus } \\
\text { four } \\
\text { optional } \\
\text { weekly } \\
15-\text { minute } \\
\text { phone } \\
\text { contacts }\end{array}$ & $\begin{array}{l}23 \% \text { of } \\
\text { whole } \\
\text { sample } \\
\text { did not } \\
\text { attend } \\
\text { any } \\
\text { sessions. } \\
\text { Mothers: } \\
\text { 3-4 } \\
\text { sessions } \\
88.4 \% ;\end{array}$ & 4 weeks & No & No Treatment \\
\hline
\end{tabular}




\begin{tabular}{|c|c|c|c|c|c|c|c|c|c|}
\hline $\begin{array}{l}\text { Study } \\
\text { ID }\end{array}$ & $\begin{array}{l}\text { Program } \\
\text { Name }\end{array}$ & $\begin{array}{l}\text { Foc us Of } \\
\text { Interven } \\
\text { t ion }\end{array}$ & Outline of Content & $\begin{array}{l}\text { Delivery } \\
\text { Format }\end{array}$ & $\begin{array}{l}\text { Number / } \\
\text { length of } \\
\text { sessions } \\
\text { schedule } \\
\text { d }\end{array}$ & $\begin{array}{l}\text { Number / } \\
\text { length of } \\
\text { sessions } \\
\text { delivered }\end{array}$ & $\begin{array}{l}\text { Length of } \\
\text { intervent } \\
\text { ion }\end{array}$ & $\begin{array}{l}\text { Direct } \\
\text { intervent } \\
\text { ion with } \\
\text { the } \\
\text { child? }\end{array}$ & Comparison \\
\hline & & & & & & $\begin{array}{l}\text { Fathers: } \\
69 \% \\
\text { attended } \\
\text { none, and } \\
\text { only } 6.3 \% \\
\text { attended } \\
\text { at least } 3 \\
\text { sessions. }\end{array}$ & & & \\
\hline $\begin{array}{l}\text { Hoff } \\
(2005)\end{array}$ & $n / r$ & Other & $\begin{array}{l}\text { Parents taught skills } \\
\text { to manage } \\
\text { uncertainty around } \\
\text { the meaning and } \\
\text { outcome of their } \\
\text { child's Type } 1 \\
\text { diabetes, including } \\
\text { problem-solving } \\
\text { skills, } \\
\text { communication } \\
\text { skills, and role } \\
\text { clarification }\end{array}$ & $\begin{array}{l}\text { Group } \\
\text { sessions } \\
\text { led by } \\
\text { clinicians } \\
\text { (advanced } \\
\text { clinical } \\
\text { psycholog } \\
\text { ygraduate } \\
\text { students) }\end{array}$ & $\begin{array}{l}\text { Two 2.5- } \\
\text { hour long } \\
\text { sessions }\end{array}$ & $\begin{array}{l}5 \text { families } \\
(20 \%) \\
\text { discontinu } \\
\text { ed or did } \\
\text { not } \\
\text { receive } \\
\text { the } \\
\text { interventi } \\
\text { on. }\end{array}$ & $\begin{array}{l}1 \text { week } \\
\text { (two } \\
\text { weekends) }\end{array}$ & No & $\begin{array}{l}\text { Usual Care: } \\
\text { Routine medical } \\
\text { care and illness } \\
\text { education }\end{array}$ \\
\hline $\begin{array}{l}\text { J ohnson } \\
\text { (2005) }\end{array}$ & $\begin{array}{l}\text { Avon } \\
\text { Premature } \\
\text { Infant } \\
\text { Project } \\
\text { (APIP) }\end{array}$ & $\begin{array}{l}\text { Parents as } \\
\text { coach of } \\
\text { child's } \\
\text { problem }\end{array}$ & $\begin{array}{l}\text { Parents offered } \\
\text { support, help and } \\
\text { advice about the } \\
\text { care and } \\
\text { development of their } \\
\text { child with } \\
\text { developmental } \\
\text { delay, including how } \\
\text { to stimulate their } \\
\text { development } \\
\text { through activities. }\end{array}$ & $\begin{array}{l}\text { Home } \\
\text { visits by } \\
\text { nurses }\end{array}$ & $\begin{array}{l}\text { Visits were } \\
\text { scheduled } \\
\text { weekly for } \\
\text { the first } \\
\text { few } \\
\text { months, } \\
\text { reduced to } \\
2-4 \\
\text { weekly for } \\
\text { the next } \\
\text { year, and } \\
\text { then to } \\
\text { monthly } \\
\text { by } 2 \\
\text { years. } \\
\text { Visits were }\end{array}$ & $\mathrm{n} / \mathrm{r}$ & 2 years & No & $\begin{array}{l}\text { Usual Care: } \\
\text { Regular } \\
\text { outpatient visits } \\
\text { occurred as part } \\
\text { of the normal } \\
\text { neonatal service }\end{array}$ \\
\hline
\end{tabular}




\begin{tabular}{|c|c|c|c|c|c|c|c|c|c|}
\hline $\begin{array}{l}\text { Study } \\
\text { ID }\end{array}$ & $\begin{array}{l}\text { Program } \\
\text { Name }\end{array}$ & $\begin{array}{l}\text { Foc us Of } \\
\text { Interven } \\
\text { t ion }\end{array}$ & Outline of Content & $\begin{array}{l}\text { Delivery } \\
\text { Format }\end{array}$ & $\begin{array}{l}\text { Number / } \\
\text { length of } \\
\text { sessions } \\
\text { schedule } \\
\text { d }\end{array}$ & $\begin{array}{l}\text { Number / } \\
\text { length of } \\
\text { sessions } \\
\text { delivered }\end{array}$ & $\begin{array}{l}\text { Length of } \\
\text { intervent } \\
\text { ion }\end{array}$ & $\begin{array}{l}\text { Direct } \\
\text { intervent } \\
\text { ion with } \\
\text { the } \\
\text { child? }\end{array}$ & Comparison \\
\hline & & & & & $\begin{array}{l}44 \\
\text { minutes } \\
\text { long on } \\
\text { average. }\end{array}$ & & & & \\
\hline $\begin{array}{l}\text { Kaarese } \\
\text { n(2008) }\end{array}$ & \multirow[t]{2}{*}{$\begin{array}{l}\text { Mother- } \\
\text { Infant } \\
\text { Transactio } \\
\text { n Program } \\
\text { (MITP) }\end{array}$} & \multirow[t]{2}{*}{$\begin{array}{l}\text { Parenting } \\
\text { skills, } \\
\text { parent- } \\
\text { child } \\
\text { relationshi } \\
\mathrm{p}\end{array}$} & \multirow[t]{2}{*}{$\begin{array}{l}\text { Teaches parents to } \\
\text { be sensitive to infant } \\
\text { cues and to respond } \\
\text { appropriately to } \\
\text { them in order to } \\
\text { facilitate mutually } \\
\text { satisfying } \\
\text { interactions. }\end{array}$} & \multirow{2}{*}{$\begin{array}{l}8 \text { sessions } \\
\text { ( } 7 \\
\text { individual) } \\
\text { in the } \\
\text { hospital } \\
\text { plus } 4 \\
\text { home } \\
\text { visits, } \\
\text { delivered } \\
\text { by } \\
\text { neonatal } \\
\text { nurses. }\end{array}$} & \multirow{2}{*}{$\begin{array}{l}1 \text { initial } \\
\text { debriefing } \\
\text { session, } \\
\text { followed } \\
\text { by } 7 \text { daily } \\
\text { 1-hour } \\
\text { sessionsin } \\
\text { theweek } \\
\text { before } \\
\text { planned } \\
\text { hospital } \\
\text { discharge, } \\
\text { followed } \\
\text { by } 4 \text { home } \\
\text { visits in } \\
\text { the } 90 \\
\text { days post- } \\
\text { discharge. }\end{array}$} & \multirow{2}{*}{$\begin{array}{l}\text { Allmothers } \\
\text { participate } \\
d \text { in every } \\
\text { session. } \\
\text { Fathers } \\
\text { participate } \\
d \text { in a } \\
\text { median of } \\
6 \text { out of } \\
12 \\
\text { sessions. }\end{array}$} & \multirow[t]{2}{*}{$\begin{array}{l}97 \text { days } \\
\text { (approxim } \\
\text { ately } 3 \\
\text { months) }\end{array}$} & \multirow[t]{2}{*}{ No } & \multirow{2}{*}{$\begin{array}{l}\text { Usual Care: Usua } \\
\text { protocol for } \\
\text { discharge of low } \\
\text { birth weight } \\
\text { infants: offer of } \\
\text { training in baby } \\
\text { massage, visual } \\
\text { and hearing } \\
\text { screening, } \\
\text { consultation with } \\
\text { neonatologist }\end{array}$} \\
\hline $\begin{array}{l}\text { Nordhov } \\
\text { (2012) }\end{array}$ & & & & & & & & & \\
\hline $\begin{array}{l}\text { Kehoe } \\
\text { (2014) }\end{array}$ & $\begin{array}{l}\text { Parent } \\
\text { Education } \\
\text { Program } \\
\text { (Tuning in } \\
\text { toTeens; } \\
\text { TINT) }\end{array}$ & $\begin{array}{l}\text { Parenting } \\
\text { skills, } \\
\text { parent- } \\
\text { child } \\
\text { relationshi } \\
\mathrm{p}\end{array}$ & $\begin{array}{l}\text { Teaches parents } \\
\text { skills in responding } \\
\text { to emotions in ways } \\
\text { that foster closer } \\
\text { parent-adolescent } \\
\text { connection and } \\
\text { enhance emotional } \\
\text { competence in both } \\
\text { parents and youth. } \\
\text { The central focus is } \\
\text { on teaching parents } \\
\text { an adaptive emotion } \\
\text { socialization style }\end{array}$ & $\begin{array}{l}\text { Group } \\
\text { sessions } \\
\text { with 6-13 } \\
\text { parents. } \\
\text { Led by } \\
\text { two } \\
\text { facilitators } \\
\text { (one of } \\
\text { which was } \\
\text { the first or } \\
\text { third } \\
\text { author). } \\
\text { Co-leaders }\end{array}$ & $\begin{array}{l}6 \\
\text { sessions, } \\
\text { each } 120 \\
\text { minutes } \\
\text { long }\end{array}$ & $\begin{array}{l}84 \% \\
\text { completed } \\
4 \text { or more } \\
\text { sessions. } \\
\text { Four } \\
\text { parents } \\
\text { only } \\
\text { attended } \\
\text { one } \\
\text { session. }\end{array}$ & 6 weeks & No & No Treatment \\
\hline
\end{tabular}




\begin{tabular}{|c|c|c|c|c|c|c|c|c|c|}
\hline $\begin{array}{l}\text { Study } \\
\text { ID }\end{array}$ & $\begin{array}{l}\text { Program } \\
\text { Name }\end{array}$ & $\begin{array}{l}\text { Focus of } \\
\text { Interven } \\
t \text { ion }\end{array}$ & Outline of Content & $\begin{array}{l}\text { Delivery } \\
\text { Format }\end{array}$ & $\begin{array}{l}\text { Number / } \\
\text { length of } \\
\text { sessions } \\
\text { schedule } \\
\text { d }\end{array}$ & $\begin{array}{l}\text { Number / } \\
\text { length of } \\
\text { sessions } \\
\text { delivered }\end{array}$ & $\begin{array}{l}\text { Length of } \\
\text { intervent } \\
\text { ion }\end{array}$ & $\begin{array}{l}\text { Direct } \\
\text { intervent } \\
\text { ion with } \\
\text { the } \\
\text { child? }\end{array}$ & Comparison \\
\hline & & & $\begin{array}{l}\text { called 'emotion } \\
\text { coaching'. }\end{array}$ & $\begin{array}{l}\text { were } \\
\text { mental } \\
\text { health } \\
\text { profession } \\
\text { als or } \\
\text { psycholog } \\
\text { y } \\
\text { graduates. }\end{array}$ & & & & & \\
\hline $\begin{array}{l}\text { Koldewi } \\
\text { jn } \\
(2010)\end{array}$ & \multirow[t]{2}{*}{$\begin{array}{l}\text { Infant } \\
\text { Behavioral } \\
\text { Assessme } \\
\text { nt and } \\
\text { I nterventi } \\
\text { on } \\
\text { Program } \\
\text { (IBAI P) }\end{array}$} & \multirow[t]{2}{*}{$\begin{array}{l}\text { Parenting } \\
\text { skills, } \\
\text { parent- } \\
\text { child } \\
\text { relationshi } \\
\text { p }\end{array}$} & \multirow{2}{*}{$\begin{array}{l}\text { Provided } \\
\text { suggestions to } \\
\text { encourage parents } \\
\text { to support their } \\
\text { infant's self- } \\
\text { regulatory efforts } \\
\text { and/or competence; } \\
\text { to adjust the } \\
\text { environment to their } \\
\text { infant's needs; to } \\
\text { support positive } \\
\text { parent-infant } \\
\text { interactions; and to } \\
\text { enhance postural } \\
\text { control and } \\
\text { successful infant } \\
\text { explorations without } \\
\text { distress. The } \\
\text { intervention method } \\
\text { does not only } \\
\text { support the child, } \\
\text { but the parents as } \\
\text { well, by offering } \\
\text { them emotional, } \\
\text { practical and } \\
\text { individual support. }\end{array}$} & \multirow{2}{*}{$\begin{array}{l}\text { 1IBAIP } \\
\text { session } \\
\text { shortly } \\
\text { before } \\
\text { discharge } \\
\text { and } 6 \text { to } 8 \\
\text { home } \\
\text { visits. } \\
\text { Interventi } \\
\text { ons were } \\
\text { carriedout } \\
\text { by } \\
\text { experienc } \\
\text { ed } \\
\text { pediatric } \\
\text { physical } \\
\text { therapists. }\end{array}$} & \multirow{2}{*}{$\begin{array}{l}1 \text { session } \\
\text { pre- } \\
\text { discharge, } \\
\text { and 6-8 } \\
\text { home } \\
\text { visits until } \\
\text { the infant } \\
\text { was } 6 \\
\text { months } \\
\text { corrected } \\
\text { age. Each } \\
\text { session } \\
\text { was 1 } \\
\text { hour's } \\
\text { duration. }{ }^{a}\end{array}$} & \multirow{2}{*}{$\begin{array}{l}81 \text { infants } \\
\text { received } 8 \\
\text { session, } 4 \\
\text { infants } \\
\text { received } \\
6-7 \\
\text { sessions, } \\
1 \text { infant } \\
\text { received } \\
\text { no } \\
\text { interventi } \\
\text { on } \\
\text { sessions. } \\
\text { Length of } \\
\text { session } \\
\text { not } \\
\text { recorded. }{ }^{a}\end{array}$} & \multirow[t]{2}{*}{6 months } & \multirow[t]{2}{*}{ Unclear } & \multirow{2}{*}{$\begin{array}{l}\text { Usual Care: } \\
\text { Control infants } \\
\text { received standard } \\
\text { care (incl. regular } \\
\text { outpatient visits } \\
\text { to the } \\
\text { pediatrician) and, } \\
\text { if required (non- } \\
\text { IBAIP trained), } \\
\text { pediatric physical } \\
\text { therapy. Regular } \\
\text { outpatient visits } \\
\text { to the pediatrician } \\
\text { were standard in } \\
\text { both intervention } \\
\text { and control } \\
\text { groups. }\end{array}$} \\
\hline $\begin{array}{l}\text { Verkerk } \\
\text { (2012) }\end{array}$ & & & & & & & & & \\
\hline $\begin{array}{l}\text { Kratoch } \\
\text { will }\end{array}$ & $\begin{array}{l}\text { Families } \\
\text { and }\end{array}$ & $\begin{array}{l}\text { Parent- } \\
\text { child }\end{array}$ & $\begin{array}{l}\text { Sessions include a } \\
\text { meal at the family }\end{array}$ & $\begin{array}{l}\text { Group } \\
\text { sessions }\end{array}$ & $\begin{array}{l}8 \text { weekly } \\
\text { sessions, }\end{array}$ & $\begin{array}{l}\text { All } \\
\text { families }\end{array}$ & 8 weeks & $\begin{array}{l}\text { Yes. } \\
\text { Children }\end{array}$ & No Treatment \\
\hline
\end{tabular}




\begin{tabular}{|c|c|c|c|c|c|c|c|c|c|}
\hline $\begin{array}{l}\text { Study } \\
\text { ID }\end{array}$ & $\begin{array}{l}\text { Program } \\
\text { Name }\end{array}$ & $\begin{array}{l}\text { Foc us Of } \\
\text { Interven } \\
\text { t ion }\end{array}$ & Outline of Content & $\begin{array}{l}\text { Delivery } \\
\text { Format }\end{array}$ & $\begin{array}{l}\text { Number / } \\
\text { length of } \\
\text { sessions } \\
\text { schedule } \\
\text { d }\end{array}$ & $\begin{array}{l}\text { Number / } \\
\text { length of } \\
\text { sessions } \\
\text { delivered }\end{array}$ & $\begin{array}{l}\text { Length of } \\
\text { intervent } \\
\text { ion }\end{array}$ & $\begin{array}{l}\text { Direct } \\
\text { intervent } \\
\text { ion with } \\
\text { the } \\
\text { child? }\end{array}$ & Comparison \\
\hline (2009) & $\begin{array}{l}\text { Schools } \\
\text { Togethe } \\
\text { r (FAST) }\end{array}$ & $\begin{array}{l}\text { relationshi } \\
\mathrm{p}\end{array}$ & $\begin{array}{l}\text { table, parent-led } \\
\text { family activities, } \\
\text { coaching of parents } \\
\text { to do responsive } \\
\text { play, and parent } \\
\text { support groups. }\end{array}$ & $\begin{array}{l}\text { with } \\
\text { parents } \\
\text { and } \\
\text { children, } \\
\text { delivered } \\
\text { by trained } \\
\text { laypersons }\end{array}$ & $\begin{array}{l}2.5 \text { hours } \\
\text { long }\end{array}$ & $\begin{array}{l}\text { attended } \\
\text { at least } \\
\text { one } \\
\text { session. } \\
90 \% \\
\text { attended } \\
\text { at least } 6 \\
\text { sessions. }\end{array}$ & & $\begin{array}{l}\text { participate } \\
\mathrm{d} \text { in the } \\
\text { sessions. }\end{array}$ & \\
\hline $\begin{array}{l}\text { Lam } \\
\text { (2008) }\end{array}$ & $\begin{array}{l}\text { Parent } \\
\text { Skills with } \\
\text { Behavioral } \\
\text { Couples } \\
\text { Therapy }\end{array}$ & $\begin{array}{l}\text { Parenting } \\
\text { skills, } \\
\text { parental } \\
\text { mental } \\
\text { health }\end{array}$ & $\begin{array}{l}\text { Combined } 8 \text { sessions } \\
\text { of behavioural } \\
\text { couples therapy that } \\
\text { aimed to improve } \\
\text { communication and } \\
\text { problem-solving } \\
\text { skills and to } \\
\text { reinforce sobriety, } \\
\text { with } 4 \text { sessions of } \\
\text { training in parent } \\
\text { skills to improve } \\
\text { parent-child } \\
\text { functioning. In } \\
\text { addition to usual } \\
\text { care ( } 12 \text { sessions of } \\
\text { individual CBT for } \\
\text { substance abuse for } \\
\text { male patients). }\end{array}$ & $\begin{array}{l}\text { Individual } \\
\text { sessions } \\
\text { with both } \\
\text { parents, } \\
\text { led by } \\
\text { clinician }\end{array}$ & $\begin{array}{l}12 \\
\text { sessions } \\
60- \\
\text { minutes } \\
\text { long }\end{array}$ & $\begin{array}{l}84 \% \\
\text { attended } \\
\text { all } \\
\text { sessions }\end{array}$ & 12 weeks & No & $\begin{array}{l}\text { Attention Control: } \\
\text { I ndividual-based } \\
\text { therapy: } 12 \\
\text { sessions with } \\
\text { fathers on coping } \\
\text { skills for } \\
\text { alcoholism, plus } \\
\text { usual care. }\end{array}$ \\
\hline $\begin{array}{l}\text { Lewis } \\
\text { (2015) }\end{array}$ & $\begin{array}{l}\text { The } \\
\text { Enhancing } \\
\text { Connectio } \\
\text { ns } \\
\text { Program }\end{array}$ & $\begin{array}{l}\text { Parent- } \\
\text { child } \\
\text { relationshi } \\
\text { p, } \\
\text { parental } \\
\text { mental } \\
\text { health, } \\
\text { parenting } \\
\text { skills }\end{array}$ & $\begin{array}{l}\text { Teaches diagnosed } \\
\text { mothers cognitive- } \\
\text { behavioral/self-care } \\
\text { methods to manage } \\
\text { their own cancer- } \\
\text { related } \\
\text { emotions as well as } \\
\text { teaching them what } \\
\text { they could do to } \\
\text { support their child's }\end{array}$ & $\begin{array}{l}\text { Fully } \\
\text { scripted } \\
\text { patient } \\
\text { education } \\
\text { counsellin } \\
\text { g sessions } \\
\text { (delivered } \\
\text { by } \\
\text { specially } \\
\text { trained } \\
\end{array}$ & $\begin{array}{l}5 \\
\text { sessions, } \\
60 \\
\text { minutes } \\
\text { long }\end{array}$ & $n / r$ & 8 weeks & No & $\begin{array}{l}\text { Minimal Control: } \\
\text { The control group } \\
\text { were mailed a } \\
\text { printed booklet } \\
\text { that focused on } \\
\text { ways the mother } \\
\text { could be } \\
\text { supportive to her } \\
\text { child about the } \\
\text { breast cancer. }\end{array}$ \\
\hline
\end{tabular}




\begin{tabular}{|c|c|c|c|c|c|c|c|c|c|}
\hline $\begin{array}{l}\text { Study } \\
\text { ID }\end{array}$ & $\begin{array}{l}\text { Program } \\
\text { Name }\end{array}$ & $\begin{array}{l}\text { Focus Of } \\
\text { Interven } \\
t \text { ion }\end{array}$ & Outline of Content & $\begin{array}{l}\text { Delivery } \\
\text { Format }\end{array}$ & $\begin{array}{l}\text { Number / } \\
\text { length of } \\
\text { sessions } \\
\text { schedule } \\
\text { d }\end{array}$ & $\begin{array}{l}\text { Number / } \\
\text { length of } \\
\text { sessions } \\
\text { delivered }\end{array}$ & $\begin{array}{l}\text { Length of } \\
\text { intervent } \\
\text { ion }\end{array}$ & $\begin{array}{l}\text { Direct } \\
\text { intervent } \\
\text { ion with } \\
\text { the } \\
\text { child? }\end{array}$ & Comparison \\
\hline & & & coping. & $\begin{array}{l}\text { nurse); } \\
\text { interactive } \\
\text { booklet } \\
\text { about } \\
\text { breast } \\
\text { cancer to } \\
\text { be read by } \\
\text { the } \\
\text { mother to } \\
\text { the child; } \\
\text { a mother's } \\
\text { workbook; } \\
\text { a child- } \\
\text { completed } \\
\text { activity } \\
\text { booklet; } \\
\text { access by } \\
\text { phone } \\
\text { pager to } \\
\text { the } \\
\text { patient } \\
\text { educator } \\
\text { for } 12 \\
\text { hours } \\
\text { each day } \\
\text { between } \\
\text { the } \\
\text { scheduled } \\
\text { interventi } \\
\text { on } \\
\text { sessions. }\end{array}$ & & & & & $\begin{array}{l}\text { After receiving the } \\
\text { booklet, mothers } \\
\text { were contacted by } \\
\text { a specially trained } \\
\text { master's prepared } \\
\text { patient educator } \\
\text { who used a script } \\
\text { to review key } \\
\text { points in the } \\
\text { booklet and ways } \\
\text { to get the most } \\
\text { from the booklet. } \\
\text { Each call lasted } \\
10 \text { or fewer } \\
\text { minutes and was } \\
\text { digitally recorded. }\end{array}$ \\
\hline $\begin{array}{l}\text { Lieberm } \\
\text { an } \\
\text { (2006) }\end{array}$ & $\begin{array}{l}\text { Child- } \\
\text { Parent } \\
\text { Psychothe } \\
\text { rapy (CPP) }\end{array}$ & $\begin{array}{l}\text { Parent- } \\
\text { child } \\
\text { relationshi } \\
\mathrm{p}\end{array}$ & $\begin{array}{l}\text { Targeted affect } \\
\text { regulation in child } \\
\text { and parent; } \\
\text { changing } \\
\text { maladaptive } \\
\end{array}$ & $\begin{array}{l}\text { Individual } \\
\text { sessions } \\
\text { with } \\
\text { mother } \\
\text { and child, }\end{array}$ & $\begin{array}{l}50 \\
\text { sessions } \\
60- \\
\text { minutes } \\
\text { long once }\end{array}$ & $\begin{array}{l}M=32.1 \\
S D=15.2\end{array}$ & 50 weeks & $\begin{array}{l}\text { Yes - } \\
\text { children } \\
\text { participate } \\
\text { d in the } \\
\text { sessions }\end{array}$ & $\begin{array}{l}\text { Usual Care: Case } \\
\text { management plus } \\
\text { referral for } \\
\text { individual } \\
\text { psychotherapy }\end{array}$ \\
\hline
\end{tabular}




\begin{tabular}{|c|c|c|c|c|c|c|c|c|c|}
\hline $\begin{array}{l}\text { Study } \\
\text { ID }\end{array}$ & $\begin{array}{l}\text { Program } \\
\text { Name }\end{array}$ & $\begin{array}{l}\text { Foc us Of } \\
\text { Interven } \\
\text { t ion }\end{array}$ & Outline of Content & $\begin{array}{l}\text { Delivery } \\
\text { Format }\end{array}$ & $\begin{array}{l}\text { Number / } \\
\text { length of } \\
\text { sessions } \\
\text { schedule } \\
\text { d }\end{array}$ & $\begin{array}{l}\text { Number / } \\
\text { length of } \\
\text { sessions } \\
\text { delivered }\end{array}$ & $\begin{array}{l}\text { Length of } \\
\text { intervent } \\
\text { ion }\end{array}$ & $\begin{array}{l}\text { Direct } \\
\text { intervent } \\
\text { ion with } \\
\text { the } \\
\text { child? }\end{array}$ & Comparison \\
\hline & & & $\begin{array}{l}\text { behaviours in the } \\
\text { child, mother, and } \\
\text { their interaction; } \\
\text { supporting } \\
\text { developmentally } \\
\text { appropriate } \\
\text { interactions and } \\
\text { activities; finding } \\
\text { avenues for conflict } \\
\text { resolution and } \\
\text { restoration of hope } \\
\text { and trust in their } \\
\text { relationship }\end{array}$ & $\begin{array}{l}\text { led by } \\
\text { clinician, } \\
\text { plus } \\
\text { individual } \\
\text { sessions } \\
\text { with the } \\
\text { mother- } \\
\text { only when } \\
\text { indicated. }\end{array}$ & a week & & & $\begin{array}{l}\text { with their } \\
\text { mother }\end{array}$ & \\
\hline $\begin{array}{l}\text { Lowell } \\
\text { (2011) }\end{array}$ & $\begin{array}{l}\text { Child } \\
\text { FIRST } \\
\text { (Child and } \\
\text { Family } \\
\text { Interagenc } \\
\text { y, } \\
\text { Resource, } \\
\text { Support, } \\
\text { and } \\
\text { Training) }\end{array}$ & $\begin{array}{l}\text { Parent- } \\
\text { child } \\
\text { relationshi } \\
\mathrm{p}\end{array}$ & $\begin{array}{l}\text { Parents taught about } \\
\text { understanding and } \\
\text { responding more } \\
\text { effectively to child } \\
\text { behaviour, reciprocal } \\
\text { parent-child play } \\
\text { and positive } \\
\text { interactions. This } \\
\text { involved exploring } \\
\text { connections } \\
\text { between the } \\
\text { parent's past and } \\
\text { current relationships } \\
\text { and feelings toward } \\
\text { the child. }\end{array}$ & $\begin{array}{l}\text { Home } \\
\text { visits by a } \\
\text { clinician } \\
\text { and care- } \\
\text { coordinato } \\
r\end{array}$ & $\begin{array}{l}\text { Weekly } \\
\text { visits of } \\
45-90 \\
\text { minutes } \\
\text { duration, } \\
\text { number } \\
\text { not } \\
\text { reported }\end{array}$ & $\begin{array}{l}M=12.2, \\
S D=6.9 \\
\text { over a } \\
\text { mean of } \\
22 \text { weeks }\end{array}$ & $n / r$ & No & $\begin{array}{l}\text { Usual Care: not } \\
\text { described }\end{array}$ \\
\hline $\begin{array}{l}\text { Luthar } \\
\text { (2000) }\end{array}$ & $\begin{array}{l}\text { Relational } \\
\text { Psychothe } \\
\text { rapy } \\
\text { Mothers' } \\
\text { Group } \\
\text { (RPMG) }\end{array}$ & $\begin{array}{l}\text { Parenting } \\
\text { skills, } \\
\text { parental } \\
\text { mental } \\
\text { health }\end{array}$ & $\begin{array}{l}\text { Maternal mental } \\
\text { health and } \\
\text { functioning, and } \\
\text { parenting skills } \\
\text { (avoiding violent } \\
\text { conflict, alternatives } \\
\text { to physical } \\
\text { punishment, age- }\end{array}$ & $\begin{array}{l}\text { Group } \\
\text { sessions } \\
\text { of } 4 \text { to } 8 \\
\text { mothers } \\
\text { led by a } \\
\text { clinician } \\
\text { and a } \\
\text { drug }\end{array}$ & $\begin{array}{l}24 \\
\text { sessions } \\
\text { once a } \\
\text { week, } \\
\text { duration } \\
\text { not } \\
\text { reported }\end{array}$ & $\begin{array}{l}\text { 86\% of } \\
\text { mothers } \\
\text { completed } \\
\text { treatment } \\
\text { (missed } \\
\text { no more } \\
\text { than } 2 \\
\text { consecutiv }\end{array}$ & 24 weeks & No & $\begin{array}{l}\text { Usual Care: } \\
\text { Standard } \\
\text { methadone } \\
\text { treatment } \\
\text { consisting of 1-hr } \\
\text { addiction } \\
\text { counselling group } \\
\text { each week, plus }\end{array}$ \\
\hline
\end{tabular}




\begin{tabular}{|c|c|c|c|c|c|c|c|c|c|}
\hline $\begin{array}{l}\text { Study } \\
\text { ID }\end{array}$ & $\begin{array}{l}\text { Program } \\
\text { Name }\end{array}$ & $\begin{array}{l}\text { Focus Of } \\
\text { Interven } \\
t \text { ion }\end{array}$ & Outline of Content & $\begin{array}{l}\text { Delivery } \\
\text { Format }\end{array}$ & $\begin{array}{l}\text { Number / } \\
\text { length of } \\
\text { sessions } \\
\text { schedule } \\
\text { d }\end{array}$ & $\begin{array}{l}\text { Number / } \\
\text { length of } \\
\text { sessions } \\
\text { delivered }\end{array}$ & $\begin{array}{l}\text { Length of } \\
\text { intervent } \\
\text { ion }\end{array}$ & $\begin{array}{l}\text { Direct } \\
\text { intervent } \\
\text { ion with } \\
\text { the } \\
\text { child? }\end{array}$ & Comparison \\
\hline & & & $\begin{array}{l}\text { appropriate limits in } \\
\text { disciplining children, } \\
\text { warmparenting). }\end{array}$ & $\begin{array}{l}\text { counselor, } \\
\text { in addition } \\
\text { to } \\
\text { standard } \\
\text { methadon } \\
\text { e } \\
\text { treatment. }\end{array}$ & & $\begin{array}{l}\text { e sessions } \\
\text { and } \\
\text { attended } \\
\text { at least } \\
\text { half of all } \\
\text { group } \\
\text { meetings) }\end{array}$ & & & $\begin{array}{l}\text { methadone and } \\
\text { periodic meetings } \\
\text { with case } \\
\text { managers to } \\
\text { secure basic } \\
\text { needs. }\end{array}$ \\
\hline $\begin{array}{l}\text { Luthar } \\
\text { (2007) }\end{array}$ & $\begin{array}{l}\text { Relational } \\
\text { Psychothe } \\
\text { rapy } \\
\text { Mothers' } \\
\text { Group } \\
\text { (RPMG) }\end{array}$ & $\begin{array}{l}\text { Parenting } \\
\text { skills, } \\
\text { parental } \\
\text { mental } \\
\text { health }\end{array}$ & $\begin{array}{l}\text { Maternal mental } \\
\text { health and } \\
\text { functioning, and } \\
\text { parenting skills } \\
\text { (avoiding violent } \\
\text { conflict, alternatives } \\
\text { to physical } \\
\text { punishment, age- } \\
\text { appropriate limits in } \\
\text { disciplining children, } \\
\text { warmparenting). }\end{array}$ & $\begin{array}{l}\text { Group } \\
\text { sessionsof } \\
3 \text { to } 8 \\
\text { mothers } \\
\text { led by a } \\
\text { clinician, in } \\
\text { addition } \\
\text { to } \\
\text { standard } \\
\text { methadon } \\
\text { e } \\
\text { treatment. }\end{array}$ & $\begin{array}{l}24 \\
\text { sessions } \\
\text { once a } \\
\text { week, } \\
\text { duration } \\
\text { not } \\
\text { reported }\end{array}$ & $\begin{array}{l}58 \% \text { of } \\
\text { mothers } \\
\text { completed } \\
\text { treatment } \\
\text { (missed } \\
\text { no more } \\
\text { than } 2 \\
\text { consecutiv } \\
\text { e sessions } \\
\text { and } \\
\text { attended } \\
\text { at least } \\
\text { half of all } \\
\text { group } \\
\text { meetings) }\end{array}$ & 24 weeks & No & $\begin{array}{l}\text { Attention Control: } \\
\text { Recovery Training } \\
\text { (an equivalent } \\
\text { dose of group } \\
\text { therapy focused } \\
\text { on the processes } \\
\text { of addiction, } \\
\text { recovery, and } \\
\text { relapse } \\
\text { prevention). }\end{array}$ \\
\hline $\begin{array}{l}\text { Mason } \\
\text { (2007) }\end{array}$ & $\begin{array}{l}\text { Preparing } \\
\text { for the } \\
\text { Drug Free } \\
\text { Years } \\
\text { (PDFY) }\end{array}$ & $\begin{array}{l}\text { Parenting } \\
\text { skills, } \\
\text { parent- } \\
\text { child } \\
\text { relationshi } \\
\text { p }\end{array}$ & $\begin{array}{l}\text { Families taught } \\
\text { about involvement } \\
\text { and interaction } \\
\text { within thefamily; } \\
\text { family rules about } \\
\text { substance use, } \\
\text { monitoring and } \\
\text { disciplining children; } \\
\text { resisting peer } \\
\text { influence to use } \\
\text { drugs; anger and } \\
\text { family conflict; and } \\
\text { developing bonding. }\end{array}$ & $\begin{array}{l}\text { Group } \\
\text { sessions } \\
\text { with on } \\
\text { average } \\
10 \\
\text { families in } \\
\text { each } \\
\text { group, led } \\
\text { by } 2 \text { group } \\
\text { leaders }\end{array}$ & $\begin{array}{l}\text { 52-hour } \\
\text { weekly } \\
\text { sessions }\end{array}$ & $\begin{array}{l}56 \% \\
\text { agreed to } \\
\text { participate } \\
\text { in } \\
\text { program. } \\
\text { Of these, } \\
94 \% \\
\text { attended } \\
3 \text { or more } \\
\text { sessions, } \\
93 \% \\
\text { attended } \\
\text { at least } 4 \\
\text { sessions, }\end{array}$ & 5 weeks & $\begin{array}{l}\text { Yes - } \\
\text { children } \\
\text { participate } \\
d \text { in one of } \\
\text { the five } \\
\text { sessions } \\
\text { with their } \\
\text { parents. }\end{array}$ & $\begin{array}{l}\text { Minimal } \\
\text { Intervention: } \\
\text { Minimal contact, } \\
\text { parents were } \\
\text { provided with } 4 \\
\text { informational } \\
\text { facts sheets on } \\
\text { adolescent } \\
\text { development. }\end{array}$ \\
\hline
\end{tabular}




\begin{tabular}{|c|c|c|c|c|c|c|c|c|c|}
\hline $\begin{array}{l}\text { Study } \\
\text { ID }\end{array}$ & $\begin{array}{l}\text { Program } \\
\text { Name }\end{array}$ & $\begin{array}{l}\text { Foc us Of } \\
\text { Interven } \\
\text { t ion }\end{array}$ & Outline of Content & $\begin{array}{l}\text { Delivery } \\
\text { Format }\end{array}$ & $\begin{array}{l}\text { Number / } \\
\text { length of } \\
\text { sessions } \\
\text { schedule } \\
\text { d }\end{array}$ & $\begin{array}{l}\text { Number / } \\
\text { length of } \\
\text { sessions } \\
\text { delivered }\end{array}$ & $\begin{array}{l}\text { Length of } \\
\text { intervent } \\
\text { ion }\end{array}$ & $\begin{array}{l}\text { Direct } \\
\text { intervent } \\
\text { ion with } \\
\text { the } \\
\text { child? }\end{array}$ & Comparison \\
\hline & & & & & & $\begin{array}{l}61 \% \\
\text { attended } \\
\text { all } 5 \\
\text { sessions. }\end{array}$ & & & \\
\hline $\begin{array}{l}\text { McDonal } \\
\text { d (2006) }\end{array}$ & $\begin{array}{l}\text { Families } \\
\text { and } \\
\text { Schools } \\
\text { Togethe } \\
\text { r (FAST) }\end{array}$ & $\begin{array}{l}\text { Parent- } \\
\text { child } \\
\text { relationshi } \\
\mathrm{p}\end{array}$ & $\begin{array}{l}\text { Aims to increase } \\
\text { parent involvement } \\
\text { in schools and } \\
\text { improve children's } \\
\text { wellbeing. Activities } \\
\text { support parents to } \\
\text { help their child } \\
\text { connect the cultures } \\
\text { of home and school } \\
\text { and enhance } \\
\text { relationships. } \\
\text { Sessions consist of } \\
\text { sharing a meal, } \\
\text { family } \\
\text { communication, } \\
\text { group songs, playing } \\
\text { games, and parental } \\
\text { peer support. }\end{array}$ & $\begin{array}{l}\text { Group } \\
\text { sessionsof } \\
5 \text { to } 15 \\
\text { familiesled } \\
\text { by a } \\
\text { collaborati } \\
\text { ve team of } \\
\text { profession } \\
\text { als and } \\
\text { parents }\end{array}$ & $\begin{array}{l}8 \text { sessions } \\
\text { of } 2.25 \\
\text { hours long }\end{array}$ & $\begin{array}{l}90 \% \\
\text { attended } \\
\text { at least } \\
\text { one } \\
\text { session, } \\
85 \% \text { of } \\
\text { these } \\
\text { completed } \\
5 \text { sessions } \\
\text { and } \\
\text { 'graduate } \\
\text { d' ( } 76.5 \% \\
\text { of total) }\end{array}$ & 8 weeks & $\begin{array}{l}\text { Yes - } \\
\text { children } \\
\text { participate } \\
\text { in the first } \\
\text { hour of } \\
\text { the } 2.25 \\
\text { hour } \\
\text { sessions. }\end{array}$ & $\begin{array}{l}\text { Minimal } \\
\text { I ntervention: } \\
\text { Family Education } \\
\text { (FAME) condition. } \\
\text { Parents were sent } \\
8 \text { weekly mailings } \\
\text { of parenting skills } \\
\text { booklets and were } \\
\text { invited to a formal } \\
\text { parenting lecture. }\end{array}$ \\
\hline $\begin{array}{l}\text { Melynk } \\
\text { (2004) }\end{array}$ & COPE & $\begin{array}{l}\text { Parents as } \\
\text { coach of } \\
\text { child's } \\
\text { problem }\end{array}$ & $\begin{array}{l}\text { Aims to increase } \\
\text { parents' knowledge } \\
\text { and understanding } \\
\text { of the rangeof } \\
\text { behaviours and } \\
\text { emotions that young } \\
\text { children typically } \\
\text { display during and } \\
\text { after hospitalisation, } \\
\text { and to direct parent } \\
\text { participation in their } \\
\text { children's emotional } \\
\text { and physical care to } \\
\text { enhance coping }\end{array}$ & $\begin{array}{l}\text { Audiotape, } \\
\text { written } \\
\text { informatio } \\
\mathrm{n} \text { and } \\
\text { telephone } \\
\text { call }\end{array}$ & $\begin{array}{l}\text { 3 phases: } \\
\text { (1) audio } \\
\text { tape and } \\
\text { written } \\
\text { material } \\
\text { during } \\
\text { stay in } \\
\text { PICU, (2) } \\
\text { audiotape } \\
\text { and } \\
\text { written } \\
\text { material } \\
\text { plus } \\
\text { workbook }\end{array}$ & $n / r$ & $n / r$ & No & $\begin{array}{l}\text { Attention Control: } \\
\text { Audiotaped and } \\
\text { written } \\
\text { information on the } \\
\text { services and } \\
\text { policies of the } \\
\text { PICU and the } \\
\text { general paediatric } \\
\text { unit and a parent- } \\
\text { child activity } \\
\text { workbook } \\
\text { containing } \\
\text { "control" } \\
\text { activities (eg, }\end{array}$ \\
\hline
\end{tabular}




\begin{tabular}{|c|c|c|c|c|c|c|c|c|c|}
\hline $\begin{array}{l}\text { Study } \\
\text { ID }\end{array}$ & $\begin{array}{l}\text { Program } \\
\text { Name }\end{array}$ & $\begin{array}{l}\text { Foc us Of } \\
\text { Interven } \\
\text { t ion }\end{array}$ & Outline of Content & $\begin{array}{l}\text { Delivery } \\
\text { Format }\end{array}$ & $\begin{array}{l}\text { Number / } \\
\text { length of } \\
\text { sessions } \\
\text { schedule } \\
\text { d }\end{array}$ & $\begin{array}{l}\text { Number / } \\
\text { length of } \\
\text { sessions } \\
\text { delivered }\end{array}$ & $\begin{array}{l}\text { Length of } \\
\text { intervent } \\
\text { ion }\end{array}$ & $\begin{array}{l}\text { Direct } \\
\text { intervent } \\
\text { ion with } \\
\text { the } \\
\text { child? }\end{array}$ & Comparison \\
\hline & & & outcomes. & & $\begin{array}{l}\text { delivered } \\
\text { after } \\
\text { transfer } \\
\text { fromPICU } \\
\text { to general } \\
\text { paediatric } \\
\text { unit, (3) } \\
\text { telephone } \\
\text { call } 2 \text { to } 3 \\
\text { days after } \\
\text { hospital } \\
\text { discharge. }\end{array}$ & & & & $\begin{array}{l}\text { reading a non- } \\
\text { hospital-related } \\
\text { book. Plus a } \\
\text { phone call } \\
\text { informing mothers } \\
\text { to contact doctor } \\
\text { if any unusual } \\
\text { symptoms appear } \\
\text { after discharge. }\end{array}$ \\
\hline $\begin{array}{l}\text { Ordway } \\
\text { (2014) }\end{array}$ & $\begin{array}{l}\text { Minding } \\
\text { the Baby } \\
\text { (MTB) }\end{array}$ & $\begin{array}{l}\text { Parenting } \\
\text { skills, } \\
\text { parent- } \\
\text { child } \\
\text { relationshi } \\
\text { p, parent } \\
\text { mental } \\
\text { health }\end{array}$ & $\begin{array}{l}\text { Deliverers support } \\
\text { reflective parenting, } \\
\text { promote the mother- } \\
\text { infant attachment } \\
\text { relationship, and } \\
\text { model and foster a } \\
\text { range of parenting } \\
\text { skills. }\end{array}$ & $\begin{array}{l}\text { An } \\
\text { interdiscip } \\
\text { linary } \\
\text { team of a } \\
\text { pediatric } \\
\text { nurse } \\
\text { practitione } \\
\text { r and } \\
\text { clinical } \\
\text { social } \\
\text { worker } \\
\text { provide } \\
\text { weekly } \\
\text { individual } \\
\text { home } \\
\text { visits to } \\
\text { first time } \\
\text { mothers } \\
\text { beginning } \\
\text { prenatally } \\
\text { until their } \\
\text { children } \\
\text { are } 2 \\
\text { years old. }\end{array}$ & $\begin{array}{l}\text { Visits, } \\
\text { beginning } \\
\text { prenatally } \\
\text { (late } \\
\text { second or } \\
\text { early third } \\
\text { trimester), } \\
\text { are } \\
\text { conducted } \\
\text { weekly up } \\
\text { until the } \\
\text { child is } 24 \\
\text { months. } \\
\text { Each visit } \\
\text { is } \\
\text { approxima } \\
\text { tely } 1 \mathrm{hr} \text {. }\end{array}$ & $n / r$ & 24 months & No & $\begin{array}{l}\text { Usual Care: } \\
\text { Routine primary } \\
\text { care at } \\
\text { community health } \\
\text { centre }\end{array}$ \\
\hline
\end{tabular}




\begin{tabular}{|c|c|c|c|c|c|c|c|c|c|}
\hline $\begin{array}{l}\text { Study } \\
\text { ID }\end{array}$ & $\begin{array}{l}\text { Program } \\
\text { Name }\end{array}$ & $\begin{array}{l}\text { Focus of } \\
\text { Interven } \\
t \text { ion }\end{array}$ & Outline of Content & $\begin{array}{l}\text { Delivery } \\
\text { Format }\end{array}$ & $\begin{array}{l}\text { Number / } \\
\text { length of } \\
\text { sessions } \\
\text { schedule } \\
\text { d }\end{array}$ & $\begin{array}{l}\text { Number / } \\
\text { length of } \\
\text { sessions } \\
\text { delivered }\end{array}$ & $\begin{array}{l}\text { Length of } \\
\text { intervent } \\
\text { ion }\end{array}$ & $\begin{array}{l}\text { Direct } \\
\text { intervent } \\
\text { ion with } \\
\text { the } \\
\text { child? }\end{array}$ & Comparison \\
\hline $\begin{array}{l}\text { Oswalt } \\
\text { (2013) }\end{array}$ & $\begin{array}{l}\text { Creating } \\
\text { Opportunit } \\
\text { ies for } \\
\text { Parent } \\
\text { Empower } \\
\text { ment } \\
\text { (COPE) }\end{array}$ & $\begin{array}{l}\text { Parenting } \\
\text { skills, } \\
\text { parent- } \\
\text { child } \\
\text { relationshi } \\
\text { p }\end{array}$ & $\begin{array}{l}\text { Intervention } \\
\text { provided information } \\
\text { about their preterm } \\
\text { infant, including } \\
\text { information that } \\
\text { reduces ambiguity } \\
\text { about their infant's } \\
\text { appearance and } \\
\text { behaviours, and how } \\
\text { the parent should } \\
\text { care for them to } \\
\text { enhance their } \\
\text { development. Also } \\
\text { includes practical } \\
\text { advice to facilitate } \\
\text { implementation of } \\
\text { the information. }\end{array}$ & $\begin{array}{l}\text { Audiotape } \\
\text { s and } \\
\text { workbook, } \\
\text { delivered } \\
\text { by 'trained } \\
\text { interventi } \\
\text { onists' in } 7 \\
\text { phases } \\
\text { fromNICU } \\
\text { admission } \\
\text { to } 18 \\
\text { months } \\
\text { corrected } \\
\text { age }\end{array}$ & $\begin{array}{l}7 \text { phases - } \\
\text { length not } \\
\text { specified }\end{array}$ & $n / r$ & 18 months & No & $\begin{array}{l}\text { Attention Control: } \\
\text { Audiotapes and } \\
\text { written } \\
\text { information } \\
\text { delivered at the } \\
\text { same times as the } \\
\text { COPE phases, } \\
\text { including } \\
\text { information about } \\
\text { hospital services, } \\
\text { discharge } \\
\text { information, } \\
\text { information } \\
\text { regarding } \\
\text { immunizations, } \\
\text { and age } \\
\text { appropriate child } \\
\text { safety and } \\
\text { nutrition. }\end{array}$ \\
\hline $\begin{array}{l}\text { Quach } \\
(2011)\end{array}$ & $n / r$ & $\begin{array}{l}\text { Parents as } \\
\text { coach of } \\
\text { child's } \\
\text { problem }\end{array}$ & $\begin{array}{l}\text { Parents taught } \\
\text { normal sleep } \\
\text { requirements for } \\
\text { children and } \\
\text { behavioural sleep } \\
\text { strategies and they } \\
\text { created a sleep } \\
\text { management plan }\end{array}$ & $\begin{array}{l}\text { One } \\
\text { individual } \\
\text { consultati } \\
\text { on, plus a } \\
\text { telephone } \\
\text { consultati } \\
\text { on, plus } \\
\text { an } \\
\text { optional } \\
\text { individual } \\
\text { consultati } \\
\text { on, } \\
\text { delivered } \\
\text { by } \\
\text { clinician or } \\
\text { nurse. }\end{array}$ & $\begin{array}{l}2 \text { to } 3 \\
\text { consultati } \\
\text { ons (first } \\
\text { consultati } \\
\text { on } 45 \\
\text { minutes, } \\
\text { phone call } \\
\text { for } 20 \\
\text { minutes, } \\
\text { 3rd } \\
\text { consultati } \\
\text { on } 30 \\
\text { minutes) }\end{array}$ & $n / r$ & 3 weeks & No & No Treatment \\
\hline
\end{tabular}




\begin{tabular}{|c|c|c|c|c|c|c|c|c|c|}
\hline $\begin{array}{l}\text { Study } \\
\text { ID }\end{array}$ & $\begin{array}{l}\text { Program } \\
\text { Name }\end{array}$ & $\begin{array}{l}\text { Focus of } \\
\text { Interven } \\
\text { t ion }\end{array}$ & Outline of Content & $\begin{array}{l}\text { Delivery } \\
\text { Format }\end{array}$ & $\begin{array}{l}\text { Number / } \\
\text { length of } \\
\text { sessions } \\
\text { schedule } \\
\text { d }\end{array}$ & $\begin{array}{l}\text { Number / } \\
\text { length of } \\
\text { sessions } \\
\text { delivered }\end{array}$ & $\begin{array}{l}\text { Length of } \\
\text { intervent } \\
\text { ion }\end{array}$ & $\begin{array}{l}\text { Direct } \\
\text { intervent } \\
\text { ion with } \\
\text { the } \\
\text { child? }\end{array}$ & Comparison \\
\hline $\begin{array}{l}\text { Rapee } \\
(2005)\end{array}$ & \multirow[t]{3}{*}{$\begin{array}{l}\text { Parent } \\
\text { Education } \\
\text { Program } \\
\text { (Cool Little } \\
\text { Kids) }\end{array}$} & \multirow[t]{3}{*}{$\begin{array}{l}\text { Parenting } \\
\text { skills, } \\
\text { parents as } \\
\text { coach of } \\
\text { child's } \\
\text { problem }\end{array}$} & \multirow{3}{*}{$\begin{array}{l}\text { Parents taught } \\
\text { anxiety } \\
\text { psychoeducation, } \\
\text { basic principles of } \\
\text { parent-management } \\
\text { techniques and the } \\
\text { importance of } \\
\text { overprotection in } \\
\text { maintaining anxiety, } \\
\text { application of } \\
\text { exposure hierarchies } \\
\text { with their child, } \\
\text { cognitive } \\
\text { restructuring of their } \\
\text { own worries, relapse } \\
\text { prevention }\end{array}$} & \multirow{3}{*}{$\begin{array}{l}\text { Group } \\
\text { sessions } \\
\text { with } \\
\text { approxima } \\
\text { tely } 6 \text { sets } \\
\text { of parents, } \\
\text { delivered } \\
\text { byclinician }\end{array}$} & \multirow[t]{3}{*}{$\begin{array}{l}6 \text { sessions } \\
90 \\
\text { minutes } \\
\text { long }\end{array}$} & \multirow{3}{*}{$\begin{array}{l}95 \% \text { of } \\
\text { mothers } \\
\text { and } 45 \% \\
\text { of fathers } \\
\text { attended } \\
\text { at least } \\
\text { one } \\
\text { session. } \\
\text { Most } \\
\text { sessions } \\
\text { ( } 5 \text { or } 6) \\
\text { were } \\
\text { attended } \\
\text { by } 73 \% \text { of } \\
\text { mothers } \\
\text { and } 23 \% \\
\text { of fathers. }\end{array}$} & \multirow[t]{3}{*}{9 weeks } & \multirow[t]{3}{*}{ No } & \multirow[t]{3}{*}{ No Treatment } \\
\hline $\begin{array}{l}\text { Rapee } \\
\text { (2010) }\end{array}$ & & & & & & & & & \\
\hline $\begin{array}{l}\text { Rapee } \\
\text { (2013) }\end{array}$ & & & & & & & & & \\
\hline $\begin{array}{l}\text { Rothera } \\
\text { m- } \\
\text { Borus } \\
(2001)\end{array}$ & \multirow[t]{2}{*}{$n / r$} & \multirow[t]{2}{*}{$\begin{array}{l}\text { Parenting } \\
\text { skills, } \\
\text { parent- } \\
\text { child } \\
\text { relationshi } \\
\text { p, } \\
\text { parental } \\
\text { mental } \\
\text { health }\end{array}$} & \multirow{2}{*}{$\begin{array}{l}\text { Module 1: parents } \\
\text { focused on their } \\
\text { adaptation to } \\
\text { positive HIV } \\
\text { serostatus, learned } \\
\text { to cope with } \\
\text { negative affect } \\
\text { related to their } \\
\text { health status, made } \\
\text { decisions regarding } \\
\text { disclosure, and } \\
\text { formulated a plan } \\
\text { for helping their } \\
\text { children cope with } \\
\text { the diagnosis. }\end{array}$} & \multirow{2}{*}{$\begin{array}{l}\text { Module 1: } \\
4 \text { weekly } \\
\text { parent } \\
\text { group } \\
\text { sessions; } \\
\text { Module 2: } \\
8 \text { weekly } \\
\text { sessions } \\
\text { including } \\
\text { parent- } \\
\text { only } \\
\text { groups, } \\
\text { adolescen } \\
\text { t-only } \\
\text { groups, }\end{array}$} & \multirow[t]{2}{*}{$\begin{array}{l}\text { Parents: } \\
12 \text { weeks } \\
\text { (Saturdays } \\
\text { ), each for } \\
2 \times 2 \text { hourly } \\
\text { sessions; } \\
\text { Adolescen } \\
\text { ts: } 16 \\
\text { sessions }\end{array}$} & \multirow{2}{*}{$\begin{array}{l}\text { Parents: } \\
M=17.2, \\
S D=11.8, \\
\text { median=1 } \\
6 . \text { Adolesc } \\
\text { ents: } \\
M=12.1 \text {, } \\
S D=8, \\
\text { median=1 } \\
1 .\end{array}$} & \multirow[t]{2}{*}{$\begin{array}{l}12 \text { weeks } \\
\text { (Saturdays }\end{array}$} & \multirow[t]{2}{*}{$\begin{array}{l}\text { Yes- } \\
\text { Module } 2 \\
\text { has } 8 \\
\text { parent- } \\
\text { adolescen } \\
\text { t group } \\
\text { sessions }\end{array}$} & \multirow[t]{2}{*}{$\begin{array}{l}\text { Usual Care: } \\
\text { Parents assigned } \\
\text { to social worker } \\
\text { who provided } \\
\text { access to range of } \\
\text { services (e.g., } \\
\text { child care, food } \\
\text { banks, medical } \\
\text { care etc) }\end{array}$} \\
\hline $\begin{array}{l}\text { Rothera } \\
\text { m-Borus } \\
(2003)\end{array}$ & & & & & & & & & \\
\hline
\end{tabular}




\begin{tabular}{|c|c|c|c|c|c|c|c|c|c|}
\hline $\begin{array}{l}\text { Study } \\
\text { ID }\end{array}$ & $\begin{array}{l}\text { Program } \\
\text { Name }\end{array}$ & $\begin{array}{l}\text { Focus of } \\
\text { Interven } \\
t \text { ion }\end{array}$ & Outline of Content & $\begin{array}{l}\text { Delivery } \\
\text { Format }\end{array}$ & $\begin{array}{l}\text { Number / } \\
\text { length of } \\
\text { sessions } \\
\text { schedule } \\
\text { d }\end{array}$ & $\begin{array}{l}\text { Number / } \\
\text { length of } \\
\text { sessions } \\
\text { delivered }\end{array}$ & $\begin{array}{l}\text { Length of } \\
\text { intervent } \\
\text { ion }\end{array}$ & $\begin{array}{l}\text { Direct } \\
\text { intervent } \\
\text { ion with } \\
\text { the } \\
\text { child? } \\
\end{array}$ & Comparison \\
\hline $\begin{array}{l}\text { Rothera } \\
\text { m-Borus } \\
\text { (2006) }\end{array}$ & & & $\begin{array}{l}\text { Module 2: parents } \\
\text { focused on initiating } \\
\text { custody plans, } \\
\text { reducing their } \\
\text { adolescent's risk } \\
\text { behaviours, and } \\
\text { creating and } \\
\text { maintaining positive } \\
\text { family routines while } \\
\text { ill. Module 2 } \\
\text { adolescent sessions } \\
\text { focused on youths' } \\
\text { adaptation to their } \\
\text { parents' illness, } \\
\text { improving parent- } \\
\text { youth relationships, } \\
\text { and reducing youths' } \\
\text { risk acts. }\end{array}$ & $\begin{array}{l}\text { and } \\
\text { parent- } \\
\text { adolescen } \\
\text { t groups. } \\
\text { Delivered } \\
\text { by trained } \\
\text { social } \\
\text { workers } \\
\text { and } \\
\text { clinical } \\
\text { psycholog } \\
\text { ygraduate } \\
\text { students }\end{array}$ & & & & & \\
\hline $\begin{array}{l}\text { Ruffolo } \\
\text { (2005) }\end{array}$ & $\begin{array}{l}\text { Support, } \\
\text { Empower } \\
\text { ment, and } \\
\text { Education } \\
\text { Group } \\
\text { (SEE) }\end{array}$ & $\begin{array}{l}\text { Parenting } \\
\text { skills, } \\
\text { parental } \\
\text { mental } \\
\text { health }\end{array}$ & $\begin{array}{l}\text { Intervention } \\
\text { addresses the key } \\
\text { components of } \\
\text { social support, } \\
\text { parent education, } \\
\text { and parental } \\
\text { empowerment } \\
\text { through group } \\
\text { problem solving and } \\
\text { interactive } \\
\text { knowledge } \\
\text { development } \\
\text { activities. }\end{array}$ & $\begin{array}{l}\text { Group } \\
\text { sessions } \\
\text { by trained } \\
\text { mental } \\
\text { health } \\
\text { profession } \\
\text { als and } \\
\text { parent } \\
\text { leaders }\end{array}$ & $\begin{array}{l}2 \text { sessions } \\
\text { per month } \\
\text { for at least } \\
2 \text { hours } \\
\text { each }\end{array}$ & $\mathrm{n} / \mathrm{r}$ & $n / r$ & No & $\begin{array}{l}\text { Usual Care: } \\
\text { workers provide } \\
\text { home visits, } \\
\text { support, crisis } \\
\text { intervention and } \\
\text { case management } \\
\text { service }\end{array}$ \\
\hline $\begin{array}{l}\text { Santacru } \\
\text { z (2006) }\end{array}$ & $\begin{array}{l}\text { Bibliothera } \\
\text { py and } \\
\text { games }\end{array}$ & $\begin{array}{l}\text { Parents as } \\
\text { coach of } \\
\text { child's } \\
\text { problem }\end{array}$ & $\begin{array}{l}\text { Parents are trained } \\
\text { to carry out } \\
\text { treatment at home, } \\
\text { with } 2 \text { components: } \\
\text { (1) a treatmentbook }\end{array}$ & $\begin{array}{l}\text { Individual } \\
\text { sessions } \\
\text { to train } \\
\text { parents; } \\
\text { no }\end{array}$ & $\begin{array}{l}\text { Five } \\
\text { sessions } \\
\text { of } \sim 45 \\
\text { minutes } \\
\text { each over }\end{array}$ & $n / r$ & 5 weeks & No & No Treatment \\
\hline
\end{tabular}




\begin{tabular}{|c|c|c|c|c|c|c|c|c|c|}
\hline $\begin{array}{l}\text { Study } \\
\text { ID }\end{array}$ & $\begin{array}{l}\text { Program } \\
\text { Name }\end{array}$ & $\begin{array}{l}\text { Focus Of } \\
\text { Interven } \\
t \text { ion }\end{array}$ & Outline of Content & $\begin{array}{l}\text { Delivery } \\
\text { Format }\end{array}$ & $\begin{array}{l}\text { Number / } \\
\text { length of } \\
\text { sessions } \\
\text { schedule } \\
\text { d }\end{array}$ & $\begin{array}{l}\text { Number / } \\
\text { length of } \\
\text { sessions } \\
\text { delivered }\end{array}$ & $\begin{array}{l}\text { Length of } \\
\text { intervent } \\
\text { ion }\end{array}$ & $\begin{array}{l}\text { Direct } \\
\text { intervent } \\
\text { ion with } \\
\text { the } \\
\text { child? }\end{array}$ & Comparison \\
\hline & & & $\begin{array}{l}\text { where the hero isa } \\
\text { coping model } \\
\text { (imaginary } \\
\text { exposure), and ( } 2 \text { ) } \\
\text { nine games to } \\
\text { overcome night-time } \\
\text { fear (in vivo } \\
\text { exposure). }\end{array}$ & $\begin{array}{l}\text { informatio } \\
\text { n on } \\
\text { trainers }\end{array}$ & $\begin{array}{l}\text { a } 1 \text { month } \\
\text { period, } 1 \\
\text { session } \\
\text { each week }\end{array}$ & & & & \\
\hline $\begin{array}{l}\text { Simon } \\
\text { (2011) }\end{array}$ & $n / r$ & $\begin{array}{l}\text { Parenting } \\
\text { skills, } \\
\text { parental } \\
\text { mental } \\
\text { health, } \\
\text { parents as } \\
\text { coach of } \\
\text { child's } \\
\text { problem }\end{array}$ & $\begin{array}{l}\text { Parents were trained } \\
\text { as lay therapists to } \\
\text { help their child } \\
\text { overcome his or her } \\
\text { anxieties by } \\
\text { applying the } \\
\text { principles of } \\
\text { stepwise exposure- } \\
\text { in-vivo, cognitive } \\
\text { restructuring, } \\
\text { modeling, task- } \\
\text { concentration } \\
\text { training and } \\
\text { relaxation, and } \\
\text { reinforcement. } \\
\text { Parents worked on } \\
\text { their own anxieties } \\
\text { and anxiety- } \\
\text { enhancing parenting } \\
\text { and on their } \\
\text { coparenting } \\
\text { relationship. The } \\
\text { father's role was } \\
\text { also highlighted. }\end{array}$ & $\begin{array}{l}\text { Group } \\
\text { sessions + } \\
\text { telephone } \\
\text { sessions, } \\
\text { delivered } \\
\text { by } \\
\text { psycholog } \\
\text { y } \\
\text { graduates }\end{array}$ & $\begin{array}{l}3 \text { group } \\
\text { sessions + } \\
5 \times 15 m i n \\
\text { telephone } \\
\text { sessions }\end{array}$ & $n / r$ & $n / r$ & No & No Treatment \\
\hline $\begin{array}{l}\text { Solantau } \\
\text { s (2010) }\end{array}$ & $\begin{array}{l}\text { Family } \\
\text { Talk } \\
\text { I nterventi } \\
\text { on }\end{array}$ & $\begin{array}{l}\text { Parent- } \\
\text { child } \\
\text { relationshi } \\
\text { p, }\end{array}$ & $\begin{array}{l}\text { (1) presenting } \\
\text { psychoeducational } \\
\text { material about mood } \\
\text { disorders and about }\end{array}$ & $\begin{array}{l}\text { Individual } \\
\text { family } \\
\text { sessions } \\
\text { facilitated }\end{array}$ & $\begin{array}{l}\text { At least } 6 \\
\text { sessions } \\
\text { for } \\
\text { families }\end{array}$ & $\begin{array}{l}94.6 \% \\
\text { attended } \\
\text { all } \\
\text { sessions }\end{array}$ & $n / r$ & Yes & $\begin{array}{l}\text { Minimal } \\
\text { Intervention: Let's } \\
\text { Talk About } \\
\text { Children: child- }\end{array}$ \\
\hline
\end{tabular}




\begin{tabular}{|c|c|c|c|c|c|c|c|c|c|}
\hline $\begin{array}{l}\text { Study } \\
\text { ID }\end{array}$ & $\begin{array}{l}\text { Program } \\
\text { Name }\end{array}$ & $\begin{array}{l}\text { Foc us Of } \\
\text { Interven } \\
t \text { ion }\end{array}$ & Outline of Content & $\begin{array}{l}\text { Delivery } \\
\text { Format }\end{array}$ & $\begin{array}{l}\text { Number / } \\
\text { length of } \\
\text { sessions } \\
\text { schedule } \\
\text { d }\end{array}$ & $\begin{array}{l}\text { Number / } \\
\text { length of } \\
\text { sessions } \\
\text { delivered }\end{array}$ & $\begin{array}{l}\text { Length of } \\
\text { intervent } \\
\text { ion }\end{array}$ & $\begin{array}{l}\text { Direct } \\
\text { intervent } \\
\text { ion with } \\
\text { the } \\
\text { child? }\end{array}$ & Comparison \\
\hline & & $\begin{array}{l}\text { parental } \\
\text { mental } \\
\text { health }\end{array}$ & $\begin{array}{l}\text { risks and resilience } \\
\text { in children; ( } 2 \text { ) } \\
\text { linking the } \\
\text { psychoeducational } \\
\text { material to the } \\
\text { family's life } \\
\text { experience; (3) } \\
\text { decreasing feelings } \\
\text { of guilt and blame in } \\
\text { children; and (4) } \\
\text { helping the children } \\
\text { to develop } \\
\text { relationships both } \\
\text { within and outsideof } \\
\text { the family }\end{array}$ & $\begin{array}{l}\text { by a } \\
\text { clinician: } \\
\text { some } \\
\text { parents- } \\
\text { only, } \\
\text { some } \\
\text { child-only, } \\
\text { and one } \\
\text { family } \\
\text { meeting }\end{array}$ & $\begin{array}{l}\text { with one } \\
\text { child, } \\
\text { more } \\
\text { sessions } \\
\text { with more } \\
\text { children. } \\
\text { Length } \mathrm{n} / \mathrm{r}\end{array}$ & & & & $\begin{array}{l}\text { focussed } \\
\text { discussion with } \\
\text { parent to assess } \\
\text { thechild's } \\
\text { situation and to } \\
\text { provide } \\
\text { information on } \\
\text { how parents can } \\
\text { support their } \\
\text { children (15-45 } \\
\text { minutes, with up } \\
\text { to } 2 \text { sessions). } \\
\text { Both conditions } \\
\text { also received a } \\
\text { self-help guide } \\
\text { called How Can I } \\
\text { Help My Children, } \\
\text { A Guide Bookfor } \\
\text { Parents with } \\
\text { Mental Health } \\
\text { Problems and a } \\
\text { standard } \\
\text { information } \\
\text { booklet about } \\
\text { depression. }\end{array}$ \\
\hline
\end{tabular}




\begin{tabular}{|c|c|c|c|c|c|c|c|c|c|}
\hline $\begin{array}{l}\text { Study } \\
\text { ID }\end{array}$ & $\begin{array}{l}\text { Program } \\
\text { Name }\end{array}$ & $\begin{array}{l}\text { Foc us of } \\
\text { Interven } \\
\text { t ion }\end{array}$ & Outline of Content & $\begin{array}{l}\text { Delivery } \\
\text { Format }\end{array}$ & $\begin{array}{l}\text { Number / } \\
\text { length of } \\
\text { sessions } \\
\text { schedule } \\
\text { d }\end{array}$ & $\begin{array}{l}\text { Number / } \\
\text { length of } \\
\text { sessions } \\
\text { delivered }\end{array}$ & $\begin{array}{l}\text { Length of } \\
\text { intervent } \\
\text { ion }\end{array}$ & $\begin{array}{l}\text { Direct } \\
\text { intervent } \\
\text { ion with } \\
\text { the } \\
\text { child? }\end{array}$ & Comparison \\
\hline $\begin{array}{l}\text { Spencer } \\
\text {-Smith } \\
(2010)\end{array}$ & $\begin{array}{l}\text { VIBeS } \\
\text { (Victorian } \\
\text { Infant } \\
\text { Brain } \\
\text { Studies) } \\
\text { Plus } \\
\text { home- } \\
\text { based } \\
\text { preventive } \\
\text { care } \\
\text { program. }\end{array}$ & $\begin{array}{l}\text { Parenting } \\
\text { skills, } \\
\text { parent- } \\
\text { child } \\
\text { relationshi } \\
\text { p, } \\
\text { parental } \\
\text { mental } \\
\text { health }\end{array}$ & $\begin{array}{l}\text { The program aimsto } \\
\text { educate the primary } \\
\text { caregiver(s) about } \\
\text { evidence-based } \\
\text { interventions for } \\
\text { improving infant } \\
\text { self-regulation, } \\
\text { postural stability, } \\
\text { coordination and } \\
\text { strength, parent } \\
\text { mental health, and } \\
\text { the parent- infant } \\
\text { relationship. }\end{array}$ & $\begin{array}{l}\text { Individual } \\
\text { family } \\
\text { sessions } \\
\text { with team } \\
\text { of } \\
\text { psychologi } \\
\text { st \& } \\
\text { physiother } \\
\text { apist. All } \\
\text { participant } \\
\text { s had } \\
\text { standard } \\
\text { follow-up } \\
\text { care. }\end{array}$ & $\begin{array}{l}9 \text { sessions } \\
\text { (1.5-2hrs) }\end{array}$ & $\begin{array}{l}M=8.9 \\
S D=0.4 \\
\text { range }=6- \\
9\end{array}$ & 11 months & No & $\begin{array}{l}\text { Usual Care: } \\
\text { Families in both } \\
\text { the intervention } \\
\text { and control } \\
\text { groups had access } \\
\text { to a maternal } \\
\text { child health nurse } \\
\text { in the community } \\
\text { who assessed } \\
\text { developmental } \\
\text { progress of the } \\
\text { infant and } \\
\text { performed } \\
\text { surveillance such } \\
\text { as height and } \\
\text { weight checks. } \\
\text { Referral could be } \\
\text { made to early } \\
\text { intervention } \\
\text { services by the } \\
\text { infant's health } \\
\text { care team at any } \\
\text { time. }\end{array}$ \\
\hline $\begin{array}{l}\text { Strayhor } \\
\text { n (1991) }\end{array}$ & $n / r$ & $\begin{array}{l}\text { Parenting } \\
\text { skills, } \\
\text { parent- } \\
\text { child } \\
\text { relationshi } \\
\text { p }\end{array}$ & $\begin{array}{l}\text { Modelling \& role- } \\
\text { play on } \\
\text { conversation, story } \\
\text { reading, and } \\
\text { dramatic play } \\
\text { focusing on prosocial } \\
\text { behaviour and } \\
\text { teaching parents to } \\
\text { have fun with andto } \\
\text { instruct their } \\
\text { children, as } \\
\text { contrasted with a } \\
\text { heavier emphasis on }\end{array}$ & $\begin{array}{l}\text { Group \& } \\
\text { individual } \\
\text { sessions } \\
\text { by } \\
\text { research } \\
\text { assistant/ } \\
\text { paraprofes } \\
\text { sional }\end{array}$ & $\begin{array}{l}\text { Four to } \\
\text { five } 2- \\
\text { hourly } \\
\text { group } \\
\text { meetings } \\
+2-3 \\
\text { sessions } \\
\text { observing } \\
\text { the } \\
\text { modelling } \\
\text { of child } \\
\text { interaction } \\
\text { by the }\end{array}$ & $\begin{array}{l}M=12.5 \\
\text { hours of } \\
\text { training }\end{array}$ & $n / r$ & $\begin{array}{l}\text { Yes- } \\
\text { research } \\
\text { assistants } \\
\text { modelled } \\
\text { parent- } \\
\text { child } \\
\text { interaction } \\
\text { s with the } \\
\text { child }\end{array}$ & $\begin{array}{l}\text { Minimal } \\
\text { Intervention: } \\
\text { Control group } \\
\text { watched } 2 \\
\text { videotapes on a } \\
\text { subset of } \\
\text { information from } \\
\text { intervention } \\
\text { group: time-out\& } \\
\text { positive } \\
\text { reinforcement; } \\
\text { plus a pamphlet } \\
\text { summarizing the }\end{array}$ \\
\hline
\end{tabular}




\begin{tabular}{|c|c|c|c|c|c|c|c|c|c|}
\hline $\begin{array}{l}\text { Study } \\
\text { ID }\end{array}$ & $\begin{array}{l}\text { Program } \\
\text { Name }\end{array}$ & $\begin{array}{l}\text { Foc us Of } \\
\text { Interven } \\
t \text { ion }\end{array}$ & Outline of Content & $\begin{array}{l}\text { Delivery } \\
\text { Format }\end{array}$ & $\begin{array}{l}\text { Number / } \\
\text { length of } \\
\text { sessions } \\
\text { schedule } \\
\text { d }\end{array}$ & $\begin{array}{l}\text { Number / } \\
\text { length of } \\
\text { sessions } \\
\text { delivered }\end{array}$ & $\begin{array}{l}\text { Length of } \\
\text { intervent } \\
\text { ion }\end{array}$ & $\begin{array}{l}\text { Direct } \\
\text { intervent } \\
\text { ion with } \\
\text { the } \\
\text { child? }\end{array}$ & Comparison \\
\hline & & & child compliance. & & $\begin{array}{l}\text { research } \\
\text { assistant } \\
+ \\
\text { research- } \\
\text { assistant } \\
\text { monitored } \\
\text { parent- } \\
\text { child play } \\
\text { sessions }\end{array}$ & & & & $\begin{array}{l}\text { content of the } \\
\text { Intervention } \\
\text { group training }\end{array}$ \\
\hline $\begin{array}{l}\text { Van } \\
\text { Doesum } \\
\text { (2008) }\end{array}$ & $n / r$ & $\begin{array}{l}\text { Parenting } \\
\text { skills, } \\
\text { parent- } \\
\text { child } \\
\text { relationshi } \\
\mathrm{p}\end{array}$ & $\begin{array}{l}\text { Videotapes of } \\
\text { mothers and infants } \\
\text { were analysed and } \\
\text { intervention was } \\
\text { tailored to each } \\
\text { family, using: } \\
\text { modelling, cognitive } \\
\text { restructuring, } \\
\text { practical pedagogical } \\
\text { support, baby } \\
\text { massage. } \\
\text { Focus was on } \\
\text { mother's sensitivity } \\
\text { to infant signals }\end{array}$ & $\begin{array}{l}\text { Home } \\
\text { visits with } \\
\text { mother } \\
\text { and child } \\
\text { (and } \\
\text { father if } \\
\text { present), } \\
\text { delivered } \\
\text { by } \\
\text { prevention } \\
\text { specialists }\end{array}$ & $\begin{array}{l}8-10 \text { home } \\
\text { visits, 1- } \\
1.5 \text { hours } \\
\text { long }\end{array}$ & $n / r$ & $\begin{array}{l}\text { Mean of } 4 \\
\text { months }\end{array}$ & No & $\begin{array}{l}\text { Minimal } \\
\text { Intervention: } 3 \\
\text { phone calls } \\
\text { providing general } \\
\text { parenting skills } \\
\text { advice (not } \\
\text { mother-child } \\
\text { interaction; only } \\
\text { general } \\
\text { information about } \\
\text { child-rearing } \\
\text { skills) }\end{array}$ \\
\hline $\begin{array}{l}\text { Velderm } \\
\text { an } \\
\text { (2006) }\end{array}$ & $\begin{array}{l}\text { Video- } \\
\text { Feedback } \\
\text { I nterventi } \\
\text { on to } \\
\text { Promote } \\
\text { Positive } \\
\text { Parenting } \\
\text { (VIPP) }\end{array}$ & $\begin{array}{l}\text { Parenting } \\
\text { skills, } \\
\text { parent- } \\
\text { child } \\
\text { relationshi } \\
\mathrm{p}\end{array}$ & $\begin{array}{l}\text { Brochures and } \\
\text { feedback on } \\
\text { videotaped parent- } \\
\text { child interactions } \\
\text { focusing on sensitive } \\
\text { parenting. }\end{array}$ & $\begin{array}{l}\text { Home } \\
\text { visits } \\
\text { videotapin } \\
\text { g mother- } \\
\text { infant } \\
\text { interaction } \\
\text {, with } \\
\text { feedback } \\
\& \\
\text { discussion } \\
\text { with the } \\
\text { mother. } \\
\text { Home }\end{array}$ & $\begin{array}{l}4 \text { visits, } \\
1.5 \text { hours }\end{array}$ & $n / r$ & 3 months & No & No Treatment \\
\hline
\end{tabular}




\begin{tabular}{|c|c|c|c|c|c|c|c|c|c|}
\hline $\begin{array}{l}\text { Study } \\
\text { ID }\end{array}$ & $\begin{array}{l}\text { Program } \\
\text { Name }\end{array}$ & $\begin{array}{l}\text { Focus Of } \\
\text { Interven } \\
t \text { ion }\end{array}$ & Outline of Content & $\begin{array}{l}\text { Delivery } \\
\text { Format }\end{array}$ & $\begin{array}{l}\text { Number / } \\
\text { length of } \\
\text { sessions } \\
\text { schedule } \\
\text { d }\end{array}$ & $\begin{array}{l}\text { Number / } \\
\text { length of } \\
\text { sessions } \\
\text { delivered }\end{array}$ & $\begin{array}{l}\text { Length of } \\
\text { intervent } \\
\text { ion }\end{array}$ & $\begin{array}{l}\text { Direct } \\
\text { intervent } \\
\text { ion with } \\
\text { the } \\
\text { child? }\end{array}$ & Comparison \\
\hline & & & & $\begin{array}{l}\text { visits } \\
\text { conducted } \\
\text { by an } \\
\text { interventi } \\
\text { onist with } \\
\text { a } \\
\text { university } \\
\text { degree in } \\
\text { education } \\
\text { and child } \\
\text { studies ( } 2 \\
\text { of the } \\
\text { authors). }\end{array}$ & & & & & \\
\hline $\begin{array}{l}\text { Wake } \\
\text { (2011) }\end{array}$ & $\begin{array}{l}\text { You Make } \\
\text { the } \\
\text { Difference } \\
\text { (modified) }\end{array}$ & $\begin{array}{l}\text { Parenting } \\
\text { skills, } \\
\text { parents as } \\
\text { coach of } \\
\text { child's } \\
\text { problem }\end{array}$ & $\begin{array}{l}\text { Teaches child- } \\
\text { centred, interaction- } \\
\text { promoting and } \\
\text { language-modelling } \\
\text { responsive } \\
\text { interaction } \\
\text { strategies targeting } \\
\text { the development of } \\
\text { vocabulary and } \\
\text { multi-word phrases. } \\
\text { Encourage parents } \\
\text { to follow child's } \\
\text { interests in } \\
\text { interactions, how to } \\
\text { engage and sustain } \\
\text { interactions with } \\
\text { child, and extending } \\
\text { information shared } \\
\text { with child. }\end{array}$ & $\begin{array}{l}\text { Group } \\
\text { sessions } \\
\text { by an } \\
\text { interventi } \\
\text { onist with } \\
\text { either a } \\
\text { speech } \\
\text { pathology } \\
\text { or } \\
\text { psycholog } \\
\text { y } \\
\text { backgroun } \\
\text { d }\end{array}$ & $\begin{array}{l}6 \times 2 \text { hour } \\
\text { sessions }\end{array}$ & $\begin{array}{l}M=4.5 \\
S D=1.6 \\
\text { range }=1- \\
6\end{array}$ & 6 weeks & No & $\begin{array}{l}\text { Usual Care: Usual } \\
\text { care from } \\
\text { maternal and } \\
\text { child health nurse } \\
\text { focusing on } \\
\text { general health, } \\
\text { development, and } \\
\text { advice }\end{array}$ \\
\hline $\begin{array}{l}\text { Walkup } \\
\text { (2009) }\end{array}$ & $\begin{array}{l}\text { Family } \\
\text { Spirit } \\
\text { Interventi } \\
\text { on }\end{array}$ & $\begin{array}{l}\text { Parenting } \\
\text { skills, } \\
\text { parental } \\
\text { mental }\end{array}$ & $\begin{array}{l}\text { Developmentally } \\
\text { timed prenatal and } \\
\text { infant-care parenting } \\
\text { skills, family }\end{array}$ & $\begin{array}{l}\text { Home } \\
\text { visits by } \\
\text { paraprofes } \\
\text { sionals }\end{array}$ & $\begin{array}{l}25 \times 1 \text { hour } \\
\text { visits }\end{array}$ & $\begin{array}{l}\text { Median = } \\
20 \text { out of } \\
25 \text { visits }\end{array}$ & $\begin{array}{l}\text { Approxima } \\
\text { tely } 9 \\
\text { months - } \\
\text { Unclear; }\end{array}$ & No & $\begin{array}{l}\text { Attention Control: } \\
\text { Breastfeeding and } \\
\text { nutrition lessons } \\
\text { via home visits - }\end{array}$ \\
\hline
\end{tabular}




\begin{tabular}{|c|c|c|c|c|c|c|c|c|c|}
\hline $\begin{array}{l}\text { Study } \\
\text { ID }\end{array}$ & $\begin{array}{l}\text { Program } \\
\text { Name }\end{array}$ & $\begin{array}{l}\text { Foc us Of } \\
\text { Interven } \\
\text { t ion }\end{array}$ & Outline of Content & $\begin{array}{l}\text { Delivery } \\
\text { Format }\end{array}$ & $\begin{array}{l}\text { Number / } \\
\text { length of } \\
\text { sessions } \\
\text { schedule } \\
\text { d }\end{array}$ & $\begin{array}{l}\text { Number / } \\
\text { length of } \\
\text { sessions } \\
\text { delivered }\end{array}$ & $\begin{array}{l}\text { Length of } \\
\text { intervent } \\
\text { ion }\end{array}$ & $\begin{array}{l}\text { Direct } \\
\text { intervent } \\
\text { ion with } \\
\text { the } \\
\text { child? }\end{array}$ & Comparison \\
\hline & & health & $\begin{array}{l}\text { planning, substance } \\
\text { abuse prevention, } \\
\text { problem-solving and } \\
\text { coping skills }\end{array}$ & & & & $\begin{array}{l}\text { from } 28 \\
\text { weeks } \\
\text { gestation } \\
\text { to } 6 \\
\text { months } \\
\text { post- } \\
\text { partum }\end{array}$ & & $\begin{array}{l}23 \text { scheduled } \\
\text { sessions }\end{array}$ \\
\hline $\begin{array}{l}\text { Williams } \\
\text { on } \\
(2014)\end{array}$ & $\begin{array}{l}\text { Madres a } \\
\text { Madres } \\
\text { (Mothers } \\
\text { to } \\
\text { Mothers) }\end{array}$ & $\begin{array}{l}\text { Parenting } \\
\text { skills, } \\
\text { Parent- } \\
\text { child } \\
\text { relationshi } \\
\text { p }\end{array}$ & $\begin{array}{l}\text { Each session } \\
\text { consists of } \\
\text { instruction in four } \\
\text { core content areas: } \\
\text { (1) normative child } \\
\text { development and } \\
\text { related social } \\
\text { competencies, (2) } \\
\text { positiveparent-child } \\
\text { interaction } \\
\text { techniques, (3) } \\
\text { positive behavioural } \\
\text { management } \\
\text { strategies, and (4) } \\
\text { service navigation to } \\
\text { support access to } \\
\text { community } \\
\text { resources. }\end{array}$ & $\begin{array}{l}\text { Home } \\
\text { visits to } \\
\text { mothers } \\
\text { by } \\
\text { 'promotor } \\
\text { as'. Visual } \\
\text { materials, } \\
\text { video } \\
\text { segments, } \\
\text { interactive } \\
\text { role-plays, } \\
\text { and } \\
\text { worksheet } \\
\text { s are used } \\
\text { to teach } \\
\text { interventi } \\
\text { on } \\
\text { content. }\end{array}$ & $\begin{array}{l}4 \\
\text { sessions, } \\
2 \text { hours } \\
\text { each, bi- } \\
\text { weekly }\end{array}$ & $\begin{array}{l}M=3.82, \\
S D=0.73, \\
\text { range =0- } \\
4) .106 \\
\text { dyads } \\
(94 \%) \\
\text { attended } \\
\text { all } \\
\text { interventi } \\
\text { on } \\
\text { sessions. }^{a}\end{array}$ & $\begin{array}{l}2-3 \\
\text { months }\end{array}$ & No & Waitlist control \\
\hline $\begin{array}{l}\begin{array}{l}\text { Wolchik } \\
\text { (2002) }\end{array} \\
\text { Wolchik } \\
\text { (2013) }\end{array}$ & $\begin{array}{l}\text { New } \\
\text { Beginning } \\
\text { s Program } \\
\text { (NBP) }\end{array}$ & $\begin{array}{l}\text { Parenting } \\
\text { skills, } \\
\text { parent- } \\
\text { child } \\
\text { relationshi } \\
\mathrm{p}\end{array}$ & $\begin{array}{l}\text { Intervention focused } \\
\text { on improving } \\
\text { mother-child and } \\
\text { father-child } \\
\text { relationships and } \\
\text { taught skills for } \\
\text { effective discipline } \\
\text { and managing } \\
\text { interparental conflict }\end{array}$ & $\begin{array}{l}\text { Group } \\
\text { sessions } \\
\text { by } 2 \\
\text { clinicians; } \\
\text { and } \\
\text { individual } \\
\text { sessions }\end{array}$ & $\begin{array}{l}\text { Mothers: } \\
11 \text { ( } 1.75 \\
\text { hour) } \\
\text { group } \\
\text { sessions + } \\
2 \text { ( } 1 \text { hour) } \\
\text { individual } \\
\text { home } \\
\text { visits }\end{array}$ & $\begin{array}{l}M=10.8 \\
S D=3.6\end{array}$ & $\begin{array}{l}\text { Approx.13 } \\
\text { weeks }\end{array}$ & No & $\begin{array}{l}\text { Minimal } \\
\text { Intervention: Self- } \\
\text { study books about } \\
\text { adjusting to } \\
\text { divorce given to } \\
\text { parents and } \\
\text { children based on } \\
\text { syllabi to guide } \\
\text { reading }\end{array}$ \\
\hline$\overline{\mathrm{Wu}}$ & Clinic- & Parenting & Intervention infants & Individual & 5 in- & $M=$ & 12 months & No & Usual care: \\
\hline
\end{tabular}




\begin{tabular}{|c|c|c|c|c|c|c|c|c|c|}
\hline $\begin{array}{l}\text { Study } \\
\text { ID }\end{array}$ & $\begin{array}{l}\text { Program } \\
\text { Name }\end{array}$ & $\begin{array}{l}\text { Focus of } \\
\text { Interven } \\
t \text { ion }\end{array}$ & Outline of Content & $\begin{array}{l}\text { Delivery } \\
\text { Format }\end{array}$ & $\begin{array}{l}\text { Number / } \\
\text { length of } \\
\text { sessions } \\
\text { schedule } \\
\text { d }\end{array}$ & $\begin{array}{l}\text { Number / } \\
\text { length of } \\
\text { sessions } \\
\text { delivered }\end{array}$ & $\begin{array}{l}\text { Length of } \\
\text { intervent } \\
\text { ion }\end{array}$ & $\begin{array}{l}\text { Direct } \\
\text { intervent } \\
\text { ion with } \\
\text { the } \\
\text { child? }\end{array}$ & Comparison \\
\hline (2014) & $\begin{array}{l}\text { based } \\
\text { interventi } \\
\text { on } \\
\text { program } \\
\text { (CBIP) }\end{array}$ & $\begin{array}{l}\text { skills, } \\
\text { parent- } \\
\text { child } \\
\text { relationshi } \\
\mathrm{p}\end{array}$ & $\begin{array}{l}\text { received in-hospital } \\
\text { and after-discharge } \\
\text { interventions } \\
\text { emphasizing child-, } \\
\text { parent-, and dyad-- } \\
\text { focused services and } \\
\text { neonatal clinic visits } \\
\text { involving ( } 1 \text { ) } \\
\text { Modulation of NICU } \\
\text { and home (2) } \\
\text { Teaching of child } \\
\text { developmental skills } \\
\text { (3) Feeding support } \\
\text { (4) Massage (5) } \\
\text { Parent support and } \\
\text { education (6) } \\
\text { Interaction activities }\end{array}$ & $\begin{array}{l}\text { sessions } \\
\text { plus a } \\
\text { book \&CD } \\
\text { containing } \\
\text { age- } \\
\text { appropriat } \\
\text { e } \\
\text { interventi } \\
\text { on } \\
\text { activities. } \\
\text { Delivered } \\
\text { by nurse, } \\
\text { physical } \\
\text { therapist, } \\
\text { and } \\
\text { neonatolo } \\
\text { gist. }\end{array}$ & $\begin{array}{l}\text { hospital } \\
\text { sessions, } \\
8 \text { after } \\
\text { discharge } \\
\text { sessionsin } \\
\text { the } \\
\text { hospital } \\
\text { for CBIP } \\
\text { and } 8 \\
\text { neonatal } \\
\text { clinic } \\
\text { visits. The } \\
\text { average } \\
\text { duration } \\
\text { for in- } \\
\text { hospital } \\
\text { interventi } \\
\text { on was } \\
\text { around } 40 \\
\text { min and } \\
30 \text { min for } \\
\text { after- } \\
\text { discharge. } \\
\text { a }\end{array}$ & $\begin{array}{l}12.56, S D \\
=1.12^{\mathrm{a}}\end{array}$ & & & $\begin{array}{l}\text { Standard } \\
\text { developmental } \\
\text { care consisting of } \\
\text { child-focused in- } \\
\text { hospital } \\
\text { interventions and } \\
\text { neonatal clinic } \\
\text { visits. }\end{array}$ \\
\hline
\end{tabular}

\section{Notes}

${ }^{\mathrm{a}}=$ Information gathered directly from authors

$\mathrm{M}=$ Mean, $\mathrm{n} / \mathrm{r}=$ Not reported, $\mathrm{PICU}=$ Pediatric intensive care unit, $\mathrm{SD}=$ standard deviation 
Appendix Table C3. Risk of bias

\begin{tabular}{|c|c|c|c|c|c|}
\hline Study ID & Random Sequence Generation & Allocation Concealment & $\begin{array}{l}\text { Blinding of Outcome } \\
\text { Assessment }\end{array}$ & $\begin{array}{l}\text { Incomplete Outcome } \\
\text { Data }\end{array}$ & Selective Reporting \\
\hline Achenbach (1993) & Low Risk: coin toss & $\begin{array}{l}\text { Low Risk: participants were } \\
\text { assigned by toss of a coin to } \\
\text { experimental and control } \\
\text { groups "after recruitment" } \\
\text { (Nurcombe 1984, p.320) }\end{array}$ & $\begin{array}{l}\text { High Risk for CBCL, } \\
\text { Unclear for TRF: Unclear } \\
\text { whether teachers were } \\
\text { blinded to condition } \\
\text { (TRF). CBCL is parent } \\
\text { report. } \\
\text { TRF blindings were not } \\
\text { checked. }\end{array}$ & $\begin{array}{l}\text { High Risk: Dropouts } \\
>15 \% \text { and assessment } \\
\text { completers only used } \\
\text { in analyses. } \\
\text { Imputation method: } \\
\text { assessment } \\
\text { completers only }\end{array}$ & $\begin{array}{l}\text { High Risk for CBCL and } \\
\text { TRF: Outcomes reported } \\
\text { incompletely ("not- } \\
\text { significant", no further } \\
\text { data); No protocol } \\
\text { available }\end{array}$ \\
\hline Beardslee (1997) & $\begin{array}{l}\text { Risk Unclear: "A balanced block } \\
\text { randomisation procedure with } \\
\text { blocks of } 4 \text {, stratified by family } \\
\text { type (single- vs dual-parent } \\
\text { families) was used" (p.198) }\end{array}$ & $\begin{array}{l}\text { Risk Unclear: supporting data } \\
\mathrm{n} / \mathrm{r}\end{array}$ & $\begin{array}{l}\text { High Risk for CDI, CBCL } \\
\text { and YSR. Unclear for K- } \\
\text { SLICE: CDI and YSR are } \\
\text { child-report and CBCL is } \\
\text { maternal-report. Unclear } \\
\text { who conducted K-SLICE } \\
\text { interviews. }\end{array}$ & $\begin{array}{l}\text { Low Risk: Only one } \\
\text { drop out (<15\%) } \\
\text { which was excluded } \\
\text { from analyses; not } \\
\text { likely to have } \\
\text { significant impact on } \\
\text { the observed effect } \\
\text { size } \\
\text { Imputation method: } \\
\text { assessment } \\
\text { completers only }\end{array}$ & $\begin{array}{l}\text { High Risk for CDI, CBCL } \\
\text { and YSR. Unclear for K- } \\
\text { SLICE: Results stated 'no } \\
\text { group differences' on } \\
\text { the CDI, CBCL and YSR, } \\
\text { but no data reported; } \\
\text { No protocol available }\end{array}$ \\
\hline Beardslee (2003) & $\begin{array}{l}\text { Risk Unclear: supporting data } \\
\mathrm{n} / \mathrm{r}\end{array}$ & $\begin{array}{l}\text { Risk Unclear: supporting data } \\
\mathrm{n} / \mathrm{r}\end{array}$ & $\begin{array}{l}\text { High Risk: YSR is child- } \\
\text { report. } \\
\text { K-SLICE: "It was } \\
\text { impossible for individual }\end{array}$ & $\begin{array}{l}\text { Low Risk: Relatively } \\
\text { low missing data } \\
(<15 \%) \text { and balanced } \\
\text { across groups }\end{array}$ & $\begin{array}{l}\text { High Risk: K-SLICE } \\
\text { diagnoses not reported } \\
\text { for each group; no } \\
\text { protocol available }\end{array}$ \\
\hline Beardslee (2007) & & & group status" (p.705) & $\begin{array}{l}\text { Imputation method: } \\
\text { assessment } \\
\text { completers only }\end{array}$ & \\
\hline Black (2007) & $\begin{array}{l}\text { Low Risk: Used a random } \\
\text { numbers table }\end{array}$ & $\begin{array}{l}\text { Risk Unclear: Insufficient } \\
\text { information to permit } \\
\text { judgement of low or high risk }\end{array}$ & $\begin{array}{l}\text { High for } C B C L \text {, low for } \\
\text { TRF: } C B C L \text { is maternal- } \\
\text { report, TRF is teacher- } \\
\text { report and "teachers were } \\
\text { unaware of } \\
\text { children's...intervention }\end{array}$ & $\begin{array}{l}\text { High Risk: Dropouts } \\
>15 \% \text { and included } \\
\text { only assessment } \\
\text { completers in } \\
\text { analyses, which may } \\
\text { have induced bias in }\end{array}$ & $\begin{array}{l}\text { Risk Unclear: Insufficient } \\
\text { information to permit } \\
\text { judgement of low or } \\
\text { high risk; no protocol } \\
\text { available }\end{array}$ \\
\hline
\end{tabular}




\begin{tabular}{|c|c|c|c|c|c|}
\hline Study ID & Random Sequence Generation & Allocation Concealment & $\begin{array}{l}\text { Blinding of Outcome } \\
\text { Assessment }\end{array}$ & $\begin{array}{l}\text { Incomplete Outcome } \\
\text { Data }\end{array}$ & Selective Reporting \\
\hline & & & $\begin{array}{l}\text { history" (p.62); Blinding } \\
\text { not checked for TRF. }\end{array}$ & $\begin{array}{l}\text { observed effect size. } \\
\text { Imputation method: } \\
\text { assessments } \\
\text { completers only }\end{array}$ & \\
\hline Butz (2001) & $\begin{array}{l}\text { Low Risk: "After informed } \\
\text { consent was obtained, mother- } \\
\text { infant dyads were randomized } \\
\text { into the INT or standard care } \\
\text { (SC) groups by selecting an } \\
\text { envelope with a computer- } \\
\text { generated random number. } \\
\text { Subjects with odd numbers } \\
\text { were assigned to the INT group, } \\
\text { and those with even numbers } \\
\text { were assigned to the SC group." } \\
\text { (p.1030) }\end{array}$ & $\begin{array}{l}\text { Unclear: Not specified whether } \\
\text { envelopes were opaque, sealed } \\
\text { and sequentially numbered }\end{array}$ & $\begin{array}{l}\text { High Risk: } \mathrm{CBCL} \text { is } \\
\text { maternal report; blinding } \\
\text { information notavailable }\end{array}$ & $\begin{array}{l}\text { High Risk: Significant } \\
\text { proportion of missing } \\
\text { data (>15\%) and } \\
\text { included only } \\
\text { assessment } \\
\text { completers in } \\
\text { analyses, which may } \\
\text { have induced bias in } \\
\text { observed effectsize } \\
\text { Imputation method: } \\
\text { assessment } \\
\text { completers only }\end{array}$ & $\begin{array}{l}\text { Risk Unclear: Insufficient } \\
\text { information to permit } \\
\text { judgement of low or } \\
\text { high risk; no protocol } \\
\text { available }\end{array}$ \\
\hline Carta (2013) & $\begin{array}{l}\text { Unclear: Method of } \\
\text { randomisation } \mathrm{n} / \mathrm{r} \text { "Upon } \\
\text { consent and enrolment, } \\
\text { mothers were randomly } \\
\text { assigned to } 1 \text { of } 3 \text { conditions" } \\
\text { (p.S168) }\end{array}$ & $\begin{array}{l}\text { Unclear: Insufficientinformation } \\
\text { provided }\end{array}$ & $\begin{array}{l}\text { High Risk: BASC2-PRS is } \\
\text { parent report, not blind to } \\
\text { allocation. }\end{array}$ & $\begin{array}{l}\text { Low Risk: Dropout }> \\
\text { 15\% but MI used } \\
\text { Imputation method: } \\
\text { MI }\end{array}$ & $\begin{array}{l}\text { Risk Unclear: Protocol } \\
\text { available but primary } \\
\text { outcome measure } \\
\text { stated is 'Child } \\
\text { maltreatment' at } 12 \\
\text { months, hence not } \\
\text { relevant in the current } \\
\text { paper. Protocol also } \\
\text { stated multi-method ax } \\
\text { (only mother-report in } \\
\text { this paper) but this may } \\
\text { have referred to the } \\
\text { primary outcome } \\
\text { measure; Protocol } \\
\text { available }\end{array}$ \\
\hline $\begin{array}{l}\text { Cartwright-Hatton } \\
\text { (2011) }\end{array}$ & $\begin{array}{l}\text { Low Risk: "The allocation } \\
\text { method was minimization with } \\
\text { random element using three }\end{array}$ & $\begin{array}{l}\text { Low Risk: "Telephone } \\
\text { randomization (with concealed } \\
\text { allocation) was conducted byan }\end{array}$ & $\begin{array}{l}\text { High Risk for CBCL, } \\
\text { SCARED, Low Risk for ADIS } \\
\text { and MASC. Unclear for }\end{array}$ & $\begin{array}{l}\text { High Risk: Significant } \\
\text { proportion of missing } \\
\text { data }(>15 \%) \text { and used }\end{array}$ & $\begin{array}{l}\text { High Risk: Protocol } \\
\text { reported that TRF and } \\
\text { DSM diagnoses would }\end{array}$ \\
\hline
\end{tabular}




\begin{tabular}{|c|c|c|c|c|c|}
\hline Study ID & Random Sequence Generation & Allocation Concealment & $\begin{array}{l}\text { Blinding of Outcome } \\
\text { Assessment }\end{array}$ & $\begin{array}{l}\text { Incomplete Outcome } \\
\text { Data }\end{array}$ & Selective Reporting \\
\hline & $\begin{array}{l}\text { factors: source of referral; } \\
\text { child's age and gender" (p.244) }\end{array}$ & independent agency." (p.244) & $\begin{array}{l}\text { TRF: CBCL and SCARED } \\
\text { are parent report. MASC } \\
\text { is child self-report. ADIS } \\
\text { conducted by research } \\
\text { assistant who was } \\
\text { "blinded to group } \\
\text { allocation". TRF by } \\
\text { teachers but it was } \mathrm{n} / \mathrm{r} \\
\text { whether they were aware } \\
\text { of the child's condition; } \\
\text { ADIS blinding not } \\
\text { checked. }\end{array}$ & $\begin{array}{l}\text { LOCF, which may have } \\
\text { induced bias in } \\
\text { observed effectsize } \\
\text { Imputation method: } \\
\text { LOCF }\end{array}$ & $\begin{array}{l}\text { be the main outcomes } \\
\text { at } 12 \text {-month follow-up, } \\
\text { but paper reported that } \\
\text { insufficient TRF data was } \\
\text { received hence not } \\
\text { reported; protocol was } \\
\text { available }\end{array}$ \\
\hline Cheng (2007) & $\begin{array}{l}\text { Low Risk: "A random number } \\
\text { table was computer generated } \\
\text { and used to determine the } \\
\text { intervention status" (p.684) }\end{array}$ & $\begin{array}{l}\text { Low Risk: Allocation "was } \\
\text { performed by a clerical officer } \\
\text { who was not involved in } \\
\text { determining eligibility." (p.684) }\end{array}$ & $\begin{array}{l}\text { High Risk: } \mathrm{CBCL} \text { was } \\
\text { mother-reported }\end{array}$ & $\begin{array}{l}\text { Unclear: Low dropout } \\
\text { (<15\%) but higher in } \\
\text { control group: the } \\
\text { number lost to follow- } \\
\text { up was similar across } \\
\text { groups ( } 3 \text { from } \\
\text { control, } 2 \text { from } \\
\text { intervention), but the } \\
\text { control group had } 5 \\
\text { dropouts prior to } \\
\text { baseline assessment. } \\
\text { Unclear whether } \\
\text { these families } \\
\text { dropped out for } \\
\text { reasons that may } \\
\text { have influenced } \\
\text { observed effect size } \\
\text { (e.g. treatment } \\
\text { expectancies) } \\
\text { Imputation method: } \\
\text { assessment } \\
\text { completers only }\end{array}$ & $\begin{array}{l}\text { Risk Unclear: Insufficient } \\
\text { information to permit } \\
\text { judgement of low or } \\
\text { high risk; protocol not } \\
\text { available }\end{array}$ \\
\hline Cohen (1997) & Low Risk: "Treatment & Low Risk: "Allocation was & High Risk: $C B C L$ is parent- & High Risk: Dropouts & High Risk: The Preschool \\
\hline
\end{tabular}




\begin{tabular}{|c|c|c|c|c|c|}
\hline Study ID & Random Sequence Generation & Allocation Concealment & $\begin{array}{l}\text { Blinding of Outcome } \\
\text { Assessment }\end{array}$ & $\begin{array}{l}\text { Incomplete Outcome } \\
\text { Data }\end{array}$ & Selective Reporting \\
\hline & $\begin{array}{l}\text { assignment was randomized } \\
\text { with the use of Efron's biased } \\
\text { coin toss (Efron, 1980)." (Cohen } \\
\text { 1996, p.45) }\end{array}$ & $\begin{array}{l}\text { concealed from researchers and } \\
\text { participants prior to } \\
\text { assignment. I believe the } \\
\text { randomization list had been } \\
\text { generated by an independent } \\
\text { statistical consultant; only one } \\
\text { random assignment at a time } \\
\text { was available to the PI. After } \\
\text { the assessment was completed, } \\
\text { the PI accessed the next } \\
\text { random assignment on the list } \\
\text { and scheduled the family with } \\
\text { the appropriate therapist. The } \\
\text { therapist informed the family of } \\
\text { the treatment they would } \\
\text { receive at the time of the first } \\
\text { appointment." }\end{array}$ & report & $\begin{array}{l}>15 \% \text { and available } \\
\text { cases only. } \\
\text { Imputation method: } \\
\text { assessment } \\
\text { completers only }\end{array}$ & $\begin{array}{l}\text { Symptom Self-Report } \\
\text { was used but not } \\
\text { reported because "it } \\
\text { was not found to be } \\
\text { useful" (Cohen } 1997, \\
\text { p.1230); protocol not } \\
\text { available }\end{array}$ \\
\hline Connell (2008) & $\begin{array}{l}\text { Low Risk: Randomisation } \\
\text { sequence was computer } \\
\text { generated (p.7 Shaw 2009) }\end{array}$ & $\begin{array}{l}\text { Low Risk: Randomisation was } \\
\text { conducted by a staff member } \\
\text { who had not been involved in } \\
\text { recruitment and sealed } \\
\text { envelopes were used. }\end{array}$ & $\begin{array}{l}\text { High Risk: } \mathrm{CBCL} \text { was } \\
\text { mother-report }\end{array}$ & $\begin{array}{l}\text { Low Risk: Dropouts } \\
\text { >15\%; but MI was } \\
\text { used } \\
\text { Imputation method: } \\
\text { assessment } \\
\text { completers only }\end{array}$ & $\begin{array}{l}\text { Risk Unclear: Insufficient } \\
\text { information to permit } \\
\text { judgement of low or } \\
\text { high risk; protocol not } \\
\text { available }\end{array}$ \\
\hline Constantino (2001) & $\begin{array}{l}\text { Risk Unclear: } n / r \text {, just stated } \\
\text { "randomised" after collection of } \\
\text { baseline data }\end{array}$ & Risk Unclear: support $n / r$ & $\begin{array}{l}\text { High Risk: } \mathrm{CBCL} \text { is parent } \\
\text { report }\end{array}$ & $\begin{array}{l}\text { High Risk: High } \\
\text { dropouts ( }>15 \%) \text {. Only } \\
\text { those who completed } \\
\text { at least } 7 / 10 \text { sessions } \\
\text { were analysed. } \\
\text { Imputation method: } \\
\text { assessment } \\
\text { completers only }\end{array}$ & $\begin{array}{l}\text { High Risk: CBCL data not } \\
\text { reported by group; just } \\
\text { stated "there were no } \\
\text { between-group } \\
\text { differences in } \\
\text { internalizing, } \\
\text { externalizing or total } \\
\text { behavior problem scores } \\
\text { on the Child Behavior } \\
\text { Checklist" (p.1578) and } \\
\text { provided M and SDs for } \\
\text { whole sample; no }\end{array}$ \\
\hline
\end{tabular}




\begin{tabular}{|c|c|c|c|c|c|}
\hline Study ID & Random Sequence Generation & Allocation Concealment & $\begin{array}{l}\text { Blinding of Outcome } \\
\text { Assessment }\end{array}$ & $\begin{array}{l}\text { Incomplete Outcome } \\
\text { Data }\end{array}$ & Selective Reporting \\
\hline & & & & & protocol available \\
\hline Cowan (2005) & \multirow[t]{2}{*}{$\begin{array}{l}\text { Risk Unclear: } \mathrm{n} / \mathrm{r} \text {, just stated } \\
\text { 'random assignment' }\end{array}$} & \multirow{2}{*}{$\begin{array}{l}\text { High Risk: Randomisation } \\
\text { (stratified by score on marital } \\
\text { satisfaction measure) was } \\
\text { conducted by a data manager, } \\
\text { but "by the time the staff } \\
\text { arrived to conduct the initial } \\
\text { interview, the couples had } \\
\text { already been randomly } \\
\text { allocated to one of three } \\
\text { conditions" (p.284) }\end{array}$} & \multirow[t]{2}{*}{$\begin{array}{l}\text { Low Risk: Child } \\
\text { internalizing was based on } \\
\text { teachers' responses to the } \\
\text { Child Adaptive Behavior } \\
\text { Inventory (CABI) - } \\
\text { Teachers were blind to } \\
\text { which children were } \\
\text { participants in the study. }\end{array}$} & $\begin{array}{l}\text { High Risk: High } \\
\text { dropouts }(>15 \%) \text { and } \\
\text { not balanced across } \\
\text { groups. } \\
\text { Imputation method: } \\
\text { assessment } \\
\text { completers only }\end{array}$ & $\begin{array}{l}\text { Risk Unclear: Insufficient } \\
\text { information to permit } \\
\text { judgement of low or } \\
\text { high risk }\end{array}$ \\
\hline Cowan (2011) & & & & $\begin{array}{l}\text { Low Risk: Dropout }> \\
\text { 15\%, but maximum } \\
\text { likelihood methods } \\
\text { used. } \\
\text { Imputation method: } \\
\text { Maximum likelihood } \\
\text { via growth curve } \\
\text { methodology }\end{array}$ & $\begin{array}{l}\text { High Risk: Data not } \\
\text { reported, just stated "a } \\
\text { model summarizing the } \\
\text { trajectory of } \\
\text { anxiety/depression fit } \\
\text { the data well, but there } \\
\text { were no significant } \\
\text { intervention effects } \\
\text { detected" (for efficacy } \\
\text { analyses, ITT analyses } \\
\text { not reported at all). }\end{array}$ \\
\hline $\begin{array}{l}\text { Dadds \& Roth } \\
\text { (2008) }\end{array}$ & $\begin{array}{l}\text { High Risk: "Schools were ranked } \\
\text { according to SES status of } \\
\text { parents and alternate schools } \\
\text { were allocated using the list, } \\
\text { with minor adjustments to } \\
\text { balance numbers of } \\
\text { participants." }\end{array}$ & $\begin{array}{l}\text { High Risk: Allocation } \\
\text { concealment does not seem } \\
\text { possible (especially for } \\
\text { investigators) giventhe } \\
\text { alternation of allocation }\end{array}$ & $\begin{array}{l}\text { Low Risk for ADIS, unclear } \\
\text { for SCBE-SF: ADIS } \\
\text { interviewers were "naïve } \\
\text { to the participants' } \\
\text { groups" (p.325).SCBE-SF: } \\
\text { Unclear whether teachers } \\
\text { were aware of child's } \\
\text { intervention condition; } \\
\text { ADIS blinding not } \\
\text { checked. }\end{array}$ & $\begin{array}{l}\text { High Risk: Dropouts } \\
>15 \% \text { and Ax } \\
\text { completers only. } \\
\text { Authors commented } \\
\text { that missing outcome } \\
\text { data likely to be } \\
\text { related to true } \\
\text { outcome: "differential } \\
\text { dropout and self- } \\
\text { selection rates were } \\
\text { evident" (p.327) } \\
\text { Imputation method: } \\
\text { assessment } \\
\text { completers only }\end{array}$ & $\begin{array}{l}\text { Risk Unclear: Insufficient } \\
\text { information to permit } \\
\text { judgement of low or } \\
\text { high risk; protocol not } \\
\text { unavailable }\end{array}$ \\
\hline Etter (2013) & Low Risk: "Randomization was & Low Risk: Random assignments & High Risk: $C B C L$ is parent- & Low Risk: Dropout< & Risk Unclear: Insufficient \\
\hline
\end{tabular}




\begin{tabular}{|c|c|c|c|c|c|}
\hline Study ID & Random Sequence Generation & Allocation Concealment & $\begin{array}{l}\text { Blinding of Outcome } \\
\text { Assessment }\end{array}$ & $\begin{array}{l}\text { Incomplete Outcome } \\
\text { Data }\end{array}$ & Selective Reporting \\
\hline & $\begin{array}{l}\text { accomplished by the assessors } \\
\text { using the online random } \\
\text { number generator: } \\
\text { http://www.randomizer.org/for } \\
\text { m.htm to request unique sets of } \\
\text { block randomization with a } \\
\text { range of 1-4. Assignments were } \\
\text { printed by a member of the } \\
\text { research team and put into a } \\
\text { secure, opaque envelope in the } \\
\text { order they were generated, and } \\
\text { the envelopes were kept in that } \\
\text { order. After a couple's baseline } \\
\text { assessment was completed, a } \\
\text { member of the assessment } \\
\text { team who had no knowledge of } \\
\text { the contents of the envelope } \\
\text { handed the couple the sealed } \\
\text { envelope containing their } \\
\text { assignment." }\end{array}$ & $\begin{array}{l}\text { were put into secure, opaque } \\
\text { envelopes by a different } \\
\text { member of the research team } \\
\text { to the person who enrolled } \\
\text { participants. }\end{array}$ & report & $\begin{array}{l}15 \% \text { for FU1, but }> \\
15 \% \text { for FU2, but HLM } \\
\text { (MI-comparable) } \\
\text { analysis used } \\
\text { Imputation method: } \\
\text { HLM (MI-comparable) }\end{array}$ & $\begin{array}{l}\text { information to permit } \\
\text { judgement of low or } \\
\text { high risk; protocol not } \\
\text { unavailable }\end{array}$ \\
\hline Feinberg (2010) & \multirow[t]{2}{*}{$\begin{array}{l}\text { Low Risk: "In order to evaluate } \\
\text { the efficacy of the intervention, } \\
\text { respondents were randomly } \\
\text { assigned to intervention and } \\
\text { control groups by the study } \\
\text { coordinately, using a random } \\
\text { number table."a }\end{array}$} & \multirow[t]{2}{*}{ Risk Unclear: support $n / r$} & $\begin{array}{l}\text { High Risk: CBCL is mother- } \\
\text { report }\end{array}$ & $\begin{array}{l}\text { High Risk: }>15 \% \\
\text { overall dropout and } \\
\text { Ax completers only. } \\
\text { Imputation method: } \\
\text { assessment } \\
\text { completers only }\end{array}$ & \multirow[t]{2}{*}{$\begin{array}{l}\text { Risk Unclear: Insufficient } \\
\text { information to permit } \\
\text { judgement of low or } \\
\text { high risk; protocol not } \\
\text { unavailable }\end{array}$} \\
\hline Feinberg (2014) & & & $\begin{array}{l}\text { High Risk for SDQ, Low } \\
\text { Risk for CBCL: SDQ is } \\
\text { parent report (not blind } \\
\text { to allocation), } C B C L \text { is } \\
\text { teacher-report. Blinding } \\
\text { of evaluators notchecked. }\end{array}$ & $\begin{array}{l}\text { High Risk: Dropout > } \\
15 \% \text { and Ax } \\
\text { completers only. An } \\
\text { analysis using MI } \\
\text { found the main effect } \\
\text { for CBCL internalising } \\
\text { was nonsignificant } \\
\text { (versus significant } \\
\text { when using complete }\end{array}$ & \\
\hline
\end{tabular}




\begin{tabular}{|c|c|c|c|c|c|}
\hline Study ID & Random Sequence Generation & Allocation Concealment & $\begin{array}{l}\text { Blinding of Outcome } \\
\text { Assessment }\end{array}$ & $\begin{array}{l}\text { Incomplete Outcome } \\
\text { Data }\end{array}$ & Selective Reporting \\
\hline & & & & $\begin{array}{l}\text { cases). } \\
\text { Imputation method: } \\
\text { Ax completers only. } \\
\text { MI analyses } \\
\text { conducted but not } \\
\text { reported }\end{array}$ & \\
\hline Fergusson (2013) & $\begin{array}{l}\text { Low Risk: Computer-generated } \\
\text { series of random numbers } \\
\text { (p.298) }\end{array}$ & $\begin{array}{l}\text { Low Risk: "The investigators did } \\
\text { not have direct contact with the } \\
\text { participants (information was } \\
\text { collected by interviewers who } \\
\text { were not involved in the } \\
\text { randomisation process). } \\
\text { Participants and Early Start staff } \\
\text { who were involved in the trial } \\
\text { had no knowledge of the } \\
\text { random assignment of any } \\
\text { participant until after an } \\
\text { individual had agreed to } \\
\text { participate in the trial." }\end{array}$ & $\begin{array}{l}\text { High Risk: Parent and } \\
\text { teacher completed SDQ, } \\
\text { not blind to allocation }\end{array}$ & $\begin{array}{l}\text { Low Risk: Dropout } \\
>15 \% \text { and not } \\
\text { balanced across } \\
\text { groups. Used } \\
\text { Assessments only. } \\
\text { Results with MI } \\
\text { similar to observed. } \\
\text { Imputation method: } \\
\text { MI (reported in } \\
\text { Supplement) }\end{array}$ & $\begin{array}{l}\text { Risk Unclear: protocol } \\
\text { available but } \\
\text { retrospectively } \\
\text { registered; protocol } \\
\text { available but } \\
\text { retrospectively } \\
\text { registered. }\end{array}$ \\
\hline Forgatch (1999) & $\begin{array}{l}\text { Low Risk: "Randomization was } \\
\text { done by computer-the person } \\
\text { who ran the program had no } \\
\text { contact with the families." }\end{array}$ & $\begin{array}{l}\text { Low Risk: "Mothers were } \\
\text { recruited and given information } \\
\text { about the study. Once they } \\
\text { consented to participate, they } \\
\text { were randomly assigned to } \\
\text { experimental or no-treatment } \\
\text { control. Randomization was } \\
\text { done by computer-the person } \\
\text { who ran the program had no } \\
\text { contact with the families." }\end{array}$ & $\begin{array}{l}\text { High Risk for Mother- } \\
\text { report; low risk for Child- } \\
\text { report: High Risk for } \\
\text { Mother-report Anxiety } \\
\text { and Depressed mood; low } \\
\text { risk for Child-report } \\
\text { Depressed mood }\end{array}$ & $\begin{array}{l}\text { High Risk: } \\
\text { Dropouts }>15 \% \text { and Ax } \\
\text { completers only. } \\
\text { Imputation method: } \\
\text { assessment } \\
\text { completers only }\end{array}$ & $\begin{array}{l}\text { High Risk: Mother- } \\
\text { report of child } \\
\text { depression and anxiety } \\
\text { involved an } \\
\text { inadequately justified } \\
\text { selection of } C B C L \text { items, } \\
\text { instead of validated } \\
\text { (sub)scales; protocol not } \\
\text { available }\end{array}$ \\
\hline Ginsburg (2009) & $\begin{array}{l}\text { Low Risk: "Families were } \\
\text { randomised equally to CAPS or } \\
\text { the control condition; } \\
\text { Participants were randomized } \\
\text { 1:1 to intervention and control } \\
\text { using randomization website }\end{array}$ & $\begin{array}{l}\text { Low Risk: "Families were } \\
\text { randomised equally to CAPS or } \\
\text { the control condition" - } \\
\text { Insufficient information about } \\
\text { when the numbers were } \\
\text { generated by the websiteto }\end{array}$ & $\begin{array}{l}\text { Low Risk for ADIS and } \\
\text { high risk for SCARED: } \\
\text { Independent evaluators } \\
\text { conducted the ADIS } \\
\text { interviews. Both parent } \\
\text { and child reports via }\end{array}$ & $\begin{array}{l}\text { Low Risk: Dropouts } \\
<15 \% \text { for FU1 and } \\
>15 \% \text { for FU2 but MI } \\
\text { was used. } \\
\text { Imputation method: }\end{array}$ & $\begin{array}{l}\text { Low Risk: Protocol } \\
\text { stated that primary } \\
\text { outcome is ADIS } \\
\text { diagnoses, which are } \\
\text { reported clearly in } \\
\text { paper. Paper also }\end{array}$ \\
\hline
\end{tabular}




\begin{tabular}{|c|c|c|c|c|c|}
\hline Study ID & Random Sequence Generation & Allocation Concealment & $\begin{array}{l}\text { Blinding of Outcome } \\
\text { Assessment }\end{array}$ & $\begin{array}{l}\text { Incomplete Outcome } \\
\text { Data }\end{array}$ & Selective Reporting \\
\hline & generated numbers." ${ }^{\prime a}$ & $\begin{array}{l}\text { permit judgement of risk; } \\
\text { assignment was concealed from } \\
\text { researchers/evaluators and } \\
\text { families until they were deemed } \\
\text { eligible then they were } \\
\text { assigned... so they did not know } \\
\text { in advance whether the next } \\
\text { eligible family would be } \\
\text { assigned to the control or } \\
\text { intervention groups. }\end{array}$ & $\begin{array}{l}\text { SCARED are high risk } \\
\text { because they were both } \\
\text { directly involved in the } \\
\text { intervention; ADIS } \\
\text { blinding not checked }\end{array}$ & $\mathrm{Ml}$ & $\begin{array}{l}\text { reports ADIS Child } \\
\text { Anxiety Severity and C } \\
\text { and P-report SCARED as } \\
\text { secondary outcomes; } \\
\text { protocol available. }\end{array}$ \\
\hline Hahlweg (2010) & $\begin{array}{l}\text { Risk Unclear: "preschools were } \\
\text { randomly assigned to either the } \\
\text { intervention or control } \\
\text { condition" (p.3) }\end{array}$ & Risk Unclear: $\mathrm{n} / \mathrm{r}$ & $\begin{array}{l}\text { High for } \mathrm{CBCL} \text {, unclear for } \\
\text { TRF: } \mathrm{CBCL} \text { is parent } \\
\text { report. Unclear whether } \\
\text { teachers were blind to } \\
\text { child's allocation; TRF } \\
\text { blinding not checked }\end{array}$ & $\begin{array}{l}\text { Low Risk: Dropouts } \\
<15 \% \text { and balanced } \\
\text { across groups. } \\
\text { Imputation method: } \\
\text { LOCF }\end{array}$ & $\begin{array}{l}\text { Risk Unclear: Insufficient } \\
\text { information to permit } \\
\text { judgement; protocol not } \\
\text { available. }\end{array}$ \\
\hline Hoff (2005) & $\begin{array}{l}\text { Risk Unclear: Families were } \\
\text { randomised to the intervention } \\
\text { or the control group. }\end{array}$ & $\begin{array}{l}\text { Low Risk: A staff member } \\
\text { unaware of the child's or } \\
\text { parent's identity conducted } \\
\text { randomisation by randomly } \\
\text { selecting sealed slips of paper } \\
\text { on which numbers indicated } \\
\text { group assignment (p.335) }\end{array}$ & $\begin{array}{l}\text { High Risk: BASC-PRS is } \\
\text { rated by parents (not } \\
\text { blind to allocation) }\end{array}$ & $\begin{array}{l}\text { High Risk: As treated } \\
\text { analysis done with } \\
\text { substantial departure } \\
\text { of the intervention } \\
\text { received from that } \\
\text { assigned at } \\
\text { randomisation. } \\
\text { Dropouts above 15\% } \\
\text { Imputation method: } \\
\text { assessment } \\
\text { completers only }\end{array}$ & $\begin{array}{l}\text { Risk Unclear: Insufficient } \\
\text { information to permit } \\
\text { judgement; protocol not } \\
\text { available. }\end{array}$ \\
\hline Johnson (2005) & $\begin{array}{l}\text { Low Risk: Randomisation was } \\
\text { arranged in blocks of six using } \\
\text { random number tables for each } \\
\text { stratum (p.F5, Johnson 1998) }\end{array}$ & $\begin{array}{l}\text { Low Risk: The allocation was by } \\
\text { sealed opaque envelopes } \\
\text { identified by stratificationgroup } \\
\text { and consecutively numbered. } \\
\text { These were prepared by the } \\
\text { lead author and opened by } \\
\text { recruitment nurses. (p.F5, } \\
\text { Johnson 1998) }\end{array}$ & $\begin{array}{l}\text { High Risk: } \mathrm{CBCL} \\
\text { completed by parent (not } \\
\text { blind to allocation) }\end{array}$ & $\begin{array}{l}\text { High Risk: Dropouts } \\
\text { above } 15 \% \text { and } \\
\text { available cases only. } \\
\text { Imputation method: } \\
\text { assessment } \\
\text { completers only }\end{array}$ & $\begin{array}{l}\text { High Risk: Medians only } \\
\text { with no decimal places. } \\
\text { Can't be entered in } \\
\text { meta-analysis; protocol } \\
\text { not available }\end{array}$ \\
\hline
\end{tabular}




\begin{tabular}{|c|c|c|c|c|c|}
\hline Study ID & Random Sequence Generation & Allocation Concealment & $\begin{array}{l}\text { Blinding of Outcome } \\
\text { Assessment }\end{array}$ & $\begin{array}{l}\text { Incomplete Outcome } \\
\text { Data }\end{array}$ & Selective Reporting \\
\hline Kaaresen (2008) & \multirow[t]{2}{*}{$\begin{array}{l}\text { Low Risk: Randomisation was } \\
\text { arranged in blocks of } 4 \text { and } 6 \\
\text { using computer generated } \\
\text { random numbers and stratified } \\
\text { by gestation }\end{array}$} & \multirow[t]{2}{*}{$\begin{array}{l}\text { Low Risk: The allocation was by } \\
\text { sealed opaque envelopes, } \\
\text { identified by stratificationgroup } \\
\text { and consecutively numbered. } \\
\text { The envelopes were prepared } \\
\text { by an independent researcher. } \\
\text { (p.e10 Kaaresen 2006) }\end{array}$} & \multirow[t]{2}{*}{$\begin{array}{l}\text { High Risk: } \mathrm{CBCL} \\
\text { completed by parent (not } \\
\text { blind to allocation) }\end{array}$} & $\begin{array}{l}\text { Low Risk: Dropouts } \\
\text { less than } 15 \% \text { and } \\
\text { fairly even across } \\
\text { groups. } \\
\text { Imputation method: } \\
\text { assessment } \\
\text { completers only }\end{array}$ & \multirow[t]{2}{*}{$\begin{array}{l}\text { Risk Unclear: Insufficient } \\
\text { information to permit } \\
\text { judgement; protocol not } \\
\text { available }\end{array}$} \\
\hline Nordhov (2012) & & & & $\begin{array}{l}\text { Low Risk: Dropouts } \\
\text { less than 15\%, } \\
\text { although CONT>INT } \\
\text { Imputation method: } \\
\text { Ax completers only } \\
\end{array}$ & \\
\hline Kehoe (2014) & $\begin{array}{l}\text { Low Risk: Allocation ratio 1:1, } \\
\text { using a computer randomizer }\end{array}$ & $\begin{array}{l}\text { Risk Unclear: } \\
\text { Insufficient information to } \\
\text { permit judgement of low or } \\
\text { high risk }\end{array}$ & $\begin{array}{l}\text { High Risk for SCAS/CDI } \\
\text { parent report, low risk for } \\
\text { SCAS/CDI child report: } \\
\text { SCAS/CDI rated by parent, } \\
\text { not blind to allocation. } \\
\text { Child not involved in } \\
\text { intervention so SCAS/CDI } \\
\text { child report is low risk; } \\
\text { blinding not checked for } \\
\text { child }\end{array}$ & $\begin{array}{l}\text { Low Risk: Dropout } \\
<15 \% \text {, although more } \\
\text { in intervention group } \\
\text { than control } \\
\text { Imputation method: } \\
\text { assessment } \\
\text { completers only }\end{array}$ & $\begin{array}{l}\text { Risk Unclear: Protocol } \\
\text { was retrospectively } \\
\text { registered; protocol } \\
\text { available; but } \\
\text { retrospectively } \\
\text { registered. }\end{array}$ \\
\hline Koldewijn (2010) & $\begin{array}{l}\text { Low Risk: Randomization into a } \\
\text { control or intervention group } \\
\text { was computer-generated, } \\
\text { stratified for gestational age } \\
\text { ( }<30 \text { and }>30 \text { weeks) and } \\
\text { recruitment site. }\end{array}$ & $\begin{array}{l}\text { Risk Unclear: Insufficient } \\
\text { information to permit } \\
\text { judgment. Only stated "Because } \\
\text { the interventions were } \\
\text { administered by the same } \\
\text { person who performed the } \\
\text { baseline assessment, } \\
\text { randomization was done aftera } \\
\text { baseline video recording of the } \\
\text { infant's neurobehavioral } \\
\text { performance at } 35 \text { to } 38 \text { weeks } \\
\text { postmenstrual age. }\end{array}$ & $\begin{array}{l}\text { High Risk: } \mathrm{CBCL} \\
\text { completed by parent (not } \\
\text { blind to allocation) }\end{array}$ & $\begin{array}{l}\text { High Risk: Dropout } \\
>15 \% \text { AND MI not } \\
\text { used } \\
\text { Imputation method: } \\
\text { Assessment } \\
\text { completers only }\end{array}$ & $\begin{array}{l}\text { Risk Unclear: Insufficient } \\
\text { information to permit } \\
\text { judgement; protocol } \\
\text { was retrospectively } \\
\text { registered, and } \mathrm{CBCL} \\
\text { was not listed as an } \\
\text { outcome measure. In } \\
\text { the paper, only M\&SD } \\
\text { per group was reported, } \\
\text { but non-significant } \\
\text { linear regression } \\
\text { statistics (adjusted for }\end{array}$ \\
\hline
\end{tabular}




\begin{tabular}{|c|c|c|c|c|c|}
\hline Study ID & Random Sequence Generation & Allocation Concealment & $\begin{array}{l}\text { Blinding of Outcome } \\
\text { Assessment }\end{array}$ & $\begin{array}{l}\text { Incomplete Outcome } \\
\text { Data }\end{array}$ & Selective Reporting \\
\hline & & $\begin{array}{l}\text { Randomization into the control } \\
\text { or intervention group was } \\
\text { computer generated, stratified } \\
\text { for GA and recruitment site, } \\
\text { with twins assigned to the same } \\
\text { group." (Koldewijn 2009, p.34) }\end{array}$ & & & $\begin{array}{l}\text { covariates) were not } \\
\text { provided. }\end{array}$ \\
\hline Verkerk (2012) & & & & $\begin{array}{l}\text { Low Risk: Dropouts< } \\
15 \% \\
\text { Imputation method: } \\
\text { Assessment } \\
\text { completers only }\end{array}$ & $\begin{array}{l}\text { Risk Unclear: Protocol } \\
\text { was retrospectively } \\
\text { registered, and } \mathrm{CBCL} \\
\text { was not listed as an } \\
\text { outcome measure. } \\
\text { Paper reported data } \\
\text { transparently and in a } \\
\text { manner that is adequate } \\
\text { for inclusion in a meta- } \\
\text { analysis; but insufficient } \\
\text { information to permit } \\
\text { judgement. }\end{array}$ \\
\hline Kratochwill (2009) & $\begin{array}{l}\text { Risk Unclear: Students were } \\
\text { randomly assigned either to } \\
\text { participate in the FAST program } \\
\text { or to serve as non-FAST } \\
\text { controls. (p.253) }\end{array}$ & $\begin{array}{l}\text { Risk Unclear: Students were } \\
\text { randomly assigned either to } \\
\text { participate in the FAST program } \\
\text { or to serve as non-FAST } \\
\text { controls. }\end{array}$ & $\begin{array}{l}\text { Low Risk for TRF, high risk } \\
\text { for CBCL: Teachers at } \\
\text { follow-up were blind to } \\
\text { the participants' } \\
\text { experimental condition. } \\
\text { Parents rated the CBCL } \\
\text { (not blind to allocation); } \\
\text { blinding not checked. }\end{array}$ & $\begin{array}{l}\text { High Risk: Dropouts } \\
\text { above } 15 \% \text { and } \\
\text { available cases only. } \\
\text { Control and } \\
\text { intervention students } \\
\text { were 'yoked', such } \\
\text { that data were } \\
\text { excluded if half a pair } \\
\text { was missing data. } \\
\text { Imputation method: } \\
\text { assessment } \\
\text { completers only }\end{array}$ & $\begin{array}{l}\text { High Risk: Data not } \\
\text { reported in a way that is } \\
\text { useable in meta- } \\
\text { analysis. }\end{array}$ \\
\hline
\end{tabular}




\begin{tabular}{|c|c|c|c|c|c|}
\hline Study ID & Random Sequence Generation & Allocation Concealment & $\begin{array}{l}\text { Blinding of Outcome } \\
\text { Assessment }\end{array}$ & $\begin{array}{l}\text { Incomplete Outcome } \\
\text { Data }\end{array}$ & Selective Reporting \\
\hline Lam (2008) & $\begin{array}{l}\text { Risk Unclear: Participants were } \\
\text { randomised }\end{array}$ & $\begin{array}{l}\text { Risk Unclear: Insufficient } \\
\text { information to permit } \\
\text { judgement of low or high risk }\end{array}$ & $\begin{array}{l}\text { High Risk for } C B C L \text {, low } \\
\text { risk for } C D I \text { and } R C M A S \text { : } \\
\text { Parents rated } C B C L \text { (not } \\
\text { blind to allocation), } \\
\text { children rated } C D I \text { and } \\
\text { RCMAS and did not } \\
\text { receive intervention }\end{array}$ & $\begin{array}{l}\text { Risk Unclear: } \\
\text { Dropouts not } \\
\text { adequately described. } \\
\text { Imputation method: } \\
\text { MI }\end{array}$ & $\begin{array}{l}\text { Risk Unclear: Insufficient } \\
\text { information to permit } \\
\text { judgement; protocol not } \\
\text { available }\end{array}$ \\
\hline Lewis (2015) & $\begin{array}{l}\text { Low Risk: The randomization } \\
\text { was done in blocks of } 2 \text { for each } \\
\text { of the } 6 \text { sites individually. A } \\
\text { standard randomization table } \\
\text { from a statistics book wasused. }\end{array}$ & $\begin{array}{l}\text { Low Risk: The randomization } \\
\text { group was determined before } \\
\text { baseline data was collected. A } \\
\text { sealed letter was sent with the } \\
\text { evaluation team member when } \\
\text { they went to collect baseline } \\
\text { data. After the evaluation team } \\
\text { member left the house, the } \\
\text { participant was instructed to } \\
\text { open the letter which revealed } \\
\text { their group. }\end{array}$ & $\begin{array}{l}\text { High Risk for CBCL parent, } \\
\text { Low Risk for RCMAS and } \\
\text { CDI child report: } C B C L \\
\text { rated by parent - not blind } \\
\text { to allocation. Child not } \\
\text { involved in intervention } \\
\text { so RCMAS and CDI low } \\
\text { risk }\end{array}$ & $\begin{array}{l}\text { Low Risk: Dropouts > } \\
15 \% \text { but used } \\
\text { maximum likelihood } \\
\text { methods which is } \\
\text { equivalent to MI } \\
\text { Imputation method: } \\
\text { maximum likelihood } \\
\text { methods }\end{array}$ & $\begin{array}{l}\text { Risk Unclear: Protocol } \\
\text { was retrospectively } \\
\text { registered, and changes } \\
\text { made to protocol } \\
\text { outcome measures. }\end{array}$ \\
\hline Lieberman (2006) & $\begin{array}{l}\text { Risk Unclear: "Dyads were } \\
\text { randomly assigned" }\end{array}$ & $\begin{array}{l}\text { Unclear: Insufficient information } \\
\text { to permit judgement of low or } \\
\text { high risk }\end{array}$ & $\begin{array}{l}\text { High Risk for } \mathrm{CBCL} \text { : } \\
\text { Mother rated } \mathrm{CBCL} \text { (not } \\
\text { blind to allocation) }\end{array}$ & $\begin{array}{l}\text { High Risk: Dropouts } \\
\text { above } 15 \% \text { and } \\
\text { available cases only. } 7 \\
\text { in the intervention } \\
\text { group not assessed } \\
\text { because their } \\
\text { treatment ended } \\
\text { before the 6-month } \\
\text { follow-up was added } \\
\text { to the study } \\
\text { Imputation method: } \\
\text { assessment } \\
\text { completers only }\end{array}$ & $\begin{array}{l}\text { High Risk: Not all of the } \\
\text { study's pre-specified } \\
\text { relevant primary } \\
\text { outcomes have been } \\
\text { reported; protocol } \\
\text { available. Protocol lists } \\
\text { Traumatic stress } \\
\text { symptomatology (DC0-3 } \\
\text { Traumatic Stress } \\
\text { Disorder) as a primary } \\
\text { outcome, yet not } \\
\text { included in paper. }\end{array}$ \\
\hline Lowell (2011) & $\begin{array}{l}\text { Low Risk: "Families were } \\
\text { randomised by coin toss" }\end{array}$ & $\begin{array}{l}\text { Risk Unclear: "families were } \\
\text { randomised by coin toss by the } \\
\text { principal investigator... after } \\
\text { completing the baseline }\end{array}$ & $\begin{array}{l}\text { High Risk: Parents rated } \\
\text { ITSEA (not blind to } \\
\text { allocation) }\end{array}$ & $\begin{array}{l}\text { High Risk: Dropouts } \\
\text { above } 15 \% \text { and } \\
\text { available cases only. }\end{array}$ & $\begin{array}{l}\text { Risk Unclear: Insufficient } \\
\text { information to permit } \\
\text { judgement; protocol not } \\
\text { available }\end{array}$ \\
\hline
\end{tabular}




\begin{tabular}{|c|c|c|c|c|c|}
\hline Study ID & Random Sequence Generation & Allocation Concealment & $\begin{array}{l}\text { Blinding of Outcome } \\
\text { Assessment }\end{array}$ & $\begin{array}{l}\text { Incomplete Outcome } \\
\text { Data }\end{array}$ & Selective Reporting \\
\hline & & $\begin{array}{l}\text { assessment" (p.197) - } \\
\text { Insufficient information to } \\
\text { permit judgement of low or } \\
\text { high risk }\end{array}$ & & $\begin{array}{l}\text { Imputation method: } \\
\text { assessment } \\
\text { completers only }\end{array}$ & \\
\hline Luthar (2000) & $\begin{array}{l}\text { Risk Unclear: "Mothers were } \\
\text { randomised to either the RPMG } \\
\text { or comparison condition" }\end{array}$ & $\begin{array}{l}\text { Risk Unclear: "Mothers were } \\
\text { randomised to either the RPMG } \\
\text { or comparison condition" }\end{array}$ & $\begin{array}{l}\text { High Risk for parent } \\
\text { report BASC, low risk for } \\
\text { child BASC: BASC rated by } \\
\text { parent (not blind to } \\
\text { allocation), child reported } \\
\text { BASC not did not receive } \\
\text { intervention. }\end{array}$ & $\begin{array}{l}\text { High Risk: Dropouts } \\
\text { above } 15 \% \text { and } \\
\text { available cases only. } \\
\text { Imputation method: } \\
\text { assessment } \\
\text { completers only }\end{array}$ & $\begin{array}{l}\text { Risk Unclear: Insufficient } \\
\text { information to permit } \\
\text { judgement; protocol not } \\
\text { available }\end{array}$ \\
\hline Luthar (2007) & $\begin{array}{l}\text { Low Risk: Mothers were } \\
\text { randomized using the URN } \\
\text { Randomization Program } \\
\text { procedures }\end{array}$ & $\begin{array}{l}\text { Risk Unclear: Method of } \\
\text { concealment not adequately } \\
\text { described "Mothers were } \\
\text { randomized using the URN } \\
\text { Randomization Program } \\
\text { procedures" }\end{array}$ & $\begin{array}{l}\text { High Risk for BASC parent } \\
\text { rated, low risk for BASC } \\
\text { child and CDI: BASC } \\
\text { (parent rated) not blind to } \\
\text { allocation, BASC (child } \\
\text { rated) and CDI (child } \\
\text { rated) did not receive } \\
\text { intervention }\end{array}$ & $\begin{array}{l}\text { Low Risk: Dropouts } \\
\text { below } 15 \% \text { and } \\
\text { balanced across } \\
\text { groups } \\
\text { Imputation method: } \\
\text { No imputation, used } \\
\text { random effects } \\
\text { regression models }\end{array}$ & $\begin{array}{l}\text { Risk Unclear: Insufficient } \\
\text { information to permit } \\
\text { judgement; protocol not } \\
\text { available }\end{array}$ \\
\hline Mason (2007) & $\begin{array}{l}\text { Low Risk: "Random assignment } \\
\text { was computer generated by a } \\
\text { data analyst" (p.629, Spoth } \\
\text { 2001) }\end{array}$ & $\begin{array}{l}\text { Low Risk: "Random assignment } \\
\text { was computer generated by a } \\
\text { data analyst and assignment } \\
\text { information was then provided } \\
\text { to program implementation } \\
\text { staff who sent letters to schools } \\
\text { informing them of their } \\
\text { assignment" (p.629, Spoth } \\
\text { 2001) }\end{array}$ & $\begin{array}{l}\text { High Risk: Outcome rated } \\
\text { by child, who did receive } \\
\text { intervention }\end{array}$ & $\begin{array}{l}\text { Low Risk: Dropouts } \\
\text { above } 15 \% \text { but } \\
\text { maximum likelihood } \\
\text { estimation used in the } \\
\text { analyses } \\
\text { Imputation method: } \\
\text { Maximum likelihood } \\
\text { estimation used }\end{array}$ & $\begin{array}{l}\text { Risk Unclear: Insufficient } \\
\text { information to permit } \\
\text { judgement; protocol not } \\
\text { available }\end{array}$ \\
\hline McDonald (2006) & $\begin{array}{l}\text { Risk Unclear: "Classrooms...were } \\
\text { randomly assigned" }\end{array}$ & $\begin{array}{l}\text { Risk Unclear: Method of } \\
\text { concealment not described }\end{array}$ & $\begin{array}{l}\text { High Risk: "Teachers were } \\
\text { generally unaware of the } \\
\text { condition of the } \\
\text { participating students." } \\
\text { Blinding not checked. }\end{array}$ & $\begin{array}{l}\text { High Risk: Dropouts } \\
\text { above } 15 \% \text { and } \\
\text { available cases only } \\
\text { Imputation method: } \\
\text { assessment } \\
\text { completers only }\end{array}$ & $\begin{array}{l}\text { Risk Unclear: Insufficient } \\
\text { information to permit } \\
\text { judgement; protocol not } \\
\text { available }\end{array}$ \\
\hline
\end{tabular}




\begin{tabular}{|c|c|c|c|c|c|}
\hline Study ID & Random Sequence Generation & Allocation Concealment & $\begin{array}{l}\text { Blinding of Outcome } \\
\text { Assessment }\end{array}$ & $\begin{array}{l}\text { Incomplete Outcome } \\
\text { Data }\end{array}$ & Selective Reporting \\
\hline Melynk (2004) & $\begin{array}{l}\text { Risk Unclear: subjects were } \\
\text { randomly assigned }\end{array}$ & $\begin{array}{l}\text { Risk Unclear: Method of } \\
\text { concealment not described }\end{array}$ & $\begin{array}{l}\text { High Risk for PHSI, high } \\
\text { risk for BASC: Outcomes } \\
\text { rated by parent, not blind } \\
\text { to allocation }\end{array}$ & $\begin{array}{l}\text { High Risk: Dropouts } \\
\text { above } 15 \% \text { and } \\
\text { available cases only } \\
\text { Imputation method: } \\
\text { assessment } \\
\text { completers only }\end{array}$ & $\begin{array}{l}\text { High Risk: Data not } \\
\text { presented for PHSI } \\
\text { (child); protocol not } \\
\text { available }\end{array}$ \\
\hline Ordway (2014) & $\begin{array}{l}\text { Risk Unclear: Insufficient } \\
\text { evidence to permit assessment } \\
\text { of risk }\end{array}$ & $\begin{array}{l}\text { Low Risk: "random assignment } \\
\text { of prenatal care groups (sealed } \\
\text { envelope method)" p394 Sadler } \\
2013\end{array}$ & $\begin{array}{l}\text { High Risk CBCL, C-TRF Risk } \\
\text { Unclear: CBCL completed } \\
\text { by parent (not blind to } \\
\text { allocation); unclear } \\
\text { whether } \\
\text { teacher/alternate } \\
\text { caregivers would have } \\
\text { known allocation status }\end{array}$ & $\begin{array}{l}\text { High Risk: Dropout } \\
>15 \% \text { and Ax } \\
\text { completers only } \\
\text { Imputation method: } \\
\text { assessment } \\
\text { completers only }\end{array}$ & $\begin{array}{l}\text { Risk Unclear: Protocol is } \\
\text { provided for the larger } \\
\text { RCT (Sadler 2013) and } \\
\text { does not include follow- } \\
\text { up assessments. }\end{array}$ \\
\hline Oswalt (2013) & $\begin{array}{l}\text { Risk Unclear: "Participating } \\
\text { parents were assigned randomly } \\
\text { to receive either the COPE or } \\
\text { placebo control program by 4- } \\
\text { week blocks of time to decrease } \\
\text { staff-to-parent and parent-to- } \\
\text { parent contamination (i.e., the } \\
\text { likelihood that mothers in the } \\
\text { NICU who were in different } \\
\text { study groups would share } \\
\text { content that was provided to } \\
\text { them in their experimental } \\
\text { program)." (Melnyk 2008, } \\
\text { p.287) }\end{array}$ & $\begin{array}{l}\text { Low Risk: "Parents who agreed } \\
\text { to participate completed } \\
\text { baseline measures and were } \\
\text { randomly assigned to study } \\
\text { conditions by using a blinded } \\
\text { system generated by the } \\
\text { investigators. Groupassignment } \\
\text { was concealed by using opaque, } \\
\text { sealed envelopes. The } \\
\text { envelopes were not opened by } \\
\text { the interventionists until after } \\
\text { the subjects were enrolled." } \\
\text { (Melnyk 2006, p. e1416) and } \\
\text { "The random assignment, which } \\
\text { was hidden from the enrolling } \\
\text { research assistants until after } \\
\text { informed consent was } \\
\text { obtained" (Melnyk 2006, p. } \\
\text { e1417) }\end{array}$ & $\begin{array}{l}\text { Low Risk: If parents were } \\
\text { unaware whether their } \\
\text { intervention was in fact } \\
\text { the 'real' one, then the } \\
\text { risk is low even though } \\
\mathrm{CBCL} \text { was mother-report; } \\
\text { blinding was checked: } \\
\text { Manipulation checks } \\
\text { showed that each group } \\
\text { demonstrated more } \\
\text { knowledge of the } \\
\text { domains covered in their } \\
\text { intervention, suggesting } \\
\text { the control group may not } \\
\text { have known that their } \\
\text { intervention was not the } \\
\text { 'real' one. }\end{array}$ & $\begin{array}{l}\text { Low Risk: Drop-out < } \\
\text { 15\%, Used FIML } \\
\text { which is equivalent to } \\
\text { MI "Missing data } \\
\text { were accounted for } \\
\text { using the full } \\
\text { information maximum } \\
\text { likelihood estimator, } \\
\text { which provides more } \\
\text { unbiased parameter } \\
\text { estimates and } \\
\text { standard errors than } \\
\text { listwise deletion" } \\
\text { Imputation method: } \\
\text { MI }\end{array}$ & $\begin{array}{l}\text { Low Risk: Protocol } \\
\text { included child 'negative } \\
\text { behaviors' as one of the } \\
\text { primary outcomes in the } \\
\text { research questions; and } \\
\text { this was reported in the } \\
\text { paper; protocol not } \\
\text { available }\end{array}$ \\
\hline Quach (2011) & $\begin{array}{l}\text { Low Risk: An independent } \\
\text { statistician produceda }\end{array}$ & $\begin{array}{l}\text { Low Risk: Allocation...performed } \\
\text { by a researcher not otherwise }\end{array}$ & $\begin{array}{l}\text { High Risk for SDQ: SDQ } \\
\text { rated by parent, not blind }\end{array}$ & $\begin{array}{l}\text { Low Risk: Dropouts } \\
\text { below } 15 \% \text { and }\end{array}$ & $\begin{array}{l}\text { Low Risk: all relevant } \\
\text { outcomes are reported; }\end{array}$ \\
\hline
\end{tabular}




\begin{tabular}{|c|c|c|c|c|c|}
\hline Study ID & Random Sequence Generation & Allocation Concealment & $\begin{array}{l}\text { Blinding of Outcome } \\
\text { Assessment }\end{array}$ & $\begin{array}{l}\text { Incomplete Outcome } \\
\text { Data }\end{array}$ & Selective Reporting \\
\hline & $\begin{array}{l}\text { computer-generated } \\
\text { randomisation sequence }\end{array}$ & involved in the study & to allocation & $\begin{array}{l}\text { balanced across } \\
\text { groups } \\
\text { Imputation method: } \\
\text { assessment } \\
\text { completers only }\end{array}$ & protocol available \\
\hline Rapee (2005) & \multirow[t]{3}{*}{$\begin{array}{l}\text { Low Risk: randomised by coin } \\
\text { toss }\end{array}$} & \multirow[t]{3}{*}{$\begin{array}{l}\text { Risk Unclear: Insufficient } \\
\text { information to permit } \\
\text { judgement of low or high risk }\end{array}$} & $\begin{array}{l}\text { Low Risk for ADIS: } \\
\text { Interviews were } \\
\text { conducted by } \\
\text { psychologists who were } \\
\text { blind to group } \\
\text { membership; Blinding not } \\
\text { checked }\end{array}$ & \multirow[t]{3}{*}{$\begin{array}{l}\text { High Risk: Dropouts } \\
\text { above } 15 \% \text { and } \\
\text { available cases only } \\
\text { Imputation method: } \\
\text { assessment } \\
\text { completers only }\end{array}$} & $\begin{array}{l}\text { Risk Unclear: Insufficient } \\
\text { information to permit } \\
\text { judgement; protocol } \\
\text { available, but } \\
\text { retrospectively } \\
\text { registered }\end{array}$ \\
\hline Rapee (2010) & & & \multirow{2}{*}{$\begin{array}{l}\text { Low Risk for ADIS, high } \\
\text { risk for SCAS parent } \\
\text { report and SMFQ-P, low } \\
\text { risk for SCAS child report } \\
\text { and SMFQ child report: } \\
\text { SCAS rated by parent, not } \\
\text { blind to allocation. ADIS } \\
\text { interviews were } \\
\text { conducted by } \\
\text { psychologists who were } \\
\text { blind to group } \\
\text { membership. Child not } \\
\text { involved in intervention } \\
\text { so SCAS child report is low } \\
\text { risk; blinding not checked. }\end{array}$} & & $\begin{array}{l}\text { Risk Unclear: All } \\
\text { relevant outcomes are } \\
\text { reported; protocol } \\
\text { available, but } \\
\text { retrospectively } \\
\text { registered. }\end{array}$ \\
\hline Rapee (2013) & & & & & $\begin{array}{l}\text { Risk Unclear: Insufficient } \\
\text { information to permit } \\
\text { judgement; protocol } \\
\text { available, but } \\
\text { retrospectively } \\
\text { registered }\end{array}$ \\
\hline $\begin{array}{l}\text { Rotheram-Borus } \\
\text { (2001) }\end{array}$ & \multirow[t]{2}{*}{$\begin{array}{l}\text { Low Risk: "Families ... were } \\
\text { randomly assigned by a pre- } \\
\text { programmed laptop computer } \\
\text { at the end of the baseline } \\
\text { assessment session" ( } p .1218 \text {, } \\
\text { Rotheram-Borus 2003) }\end{array}$} & \multirow[t]{2}{*}{$\begin{array}{l}\text { Low Risk: Pre-programmed } \\
\text { computer randomisation post } \\
\text { baseline assessment could not } \\
\text { have been foreseen }\end{array}$} & \multirow[t]{2}{*}{$\begin{array}{l}\text { High Risk: Adolescent self- } \\
\text { report on BSI }\end{array}$} & \multirow{2}{*}{$\begin{array}{l}\text { Low Risk: Trend } \\
\text { analysis does not } \\
\text { impute for missing } \\
\text { data and instead uses } \\
\text { all available data at } \\
\text { every wave; but is } \\
\text { considered as good as } \\
\text { Ml in terms of } \\
\text { method of treatment }\end{array}$} & $\begin{array}{l}\text { Risk Unclear: Insufficient } \\
\text { information to permit } \\
\text { judgement of low or } \\
\text { high risk; Protocol not } \\
\text { available }\end{array}$ \\
\hline $\begin{array}{l}\text { Rotheram-Borus } \\
\text { (2003) }\end{array}$ & & & & & $\begin{array}{l}\text { Risk Unclear: Insufficient } \\
\text { information to permit } \\
\text { judgement of low or }\end{array}$ \\
\hline
\end{tabular}




\begin{tabular}{|c|c|c|c|c|c|}
\hline Study ID & Random Sequence Generation & Allocation Concealment & $\begin{array}{l}\text { Blinding of Outcome } \\
\text { Assessment }\end{array}$ & $\begin{array}{l}\text { Incomplete Outcome } \\
\text { Data }\end{array}$ & Selective Reporting \\
\hline & & & & $\begin{array}{l}\text { of missing data. } \\
\text { Imputation method: } \\
\text { Ml-comparable }\end{array}$ & $\begin{array}{l}\text { high risk. However, } \\
\text { whilst the } 2001 \text { paper } \\
\text { reported BSI depression } \\
\text { and anxiety subscale }\end{array}$ \\
\hline $\begin{array}{l}\text { Rotheram-Borus } \\
(2004)\end{array}$ & & & & $\begin{array}{l}\text { High Risk: } \\
\text { Dropouts }>15 \% \text { and } \\
\text { assessment } \\
\text { completers only. } \\
\text { Imputation method: } \\
\text { assessment } \\
\text { completers only }\end{array}$ & $\begin{array}{l}\text { findings, the } 2003 \text { and } \\
2004 \text { papers did not. No } \\
\text { rationale for this } \\
\text { omission was provided; } \\
\text { Protocol not available }\end{array}$ \\
\hline $\begin{array}{l}\text { Rotheram-Borus } \\
(2006)\end{array}$ & & & & $\begin{array}{l}\text { High Risk: Dropouts } \\
<15 \% \text { but not } \\
\text { balanced between } \\
\text { groups; this } \\
\text { imbalance may } \\
\text { influence effects } \\
\text { found } \\
\text { Imputation method: } \\
\text { assessment } \\
\text { completers only }\end{array}$ & \\
\hline Ruffolo (2005) & $\begin{array}{l}\text { Low Risk: Parents were "were } \\
\text { randomly assigned to one of the } \\
\text { two treatment conditions using } \\
\text { Efron's (1971) coin procedure" } \\
\text { (p.202) }\end{array}$ & $\begin{array}{l}\text { Risk Unclear: "Ninety-four } \\
\text { parents who signed a written } \\
\text { consent form that indicated } \\
\text { their willingness to be part of } \\
\text { the study were randomly } \\
\text { assigned to one of the two } \\
\text { treatment conditions using } \\
\text { Efron's (1971) coin procedure, } \\
\text { which permits experimenters to } \\
\text { randomly assign more } \\
\text { participants to the intervention } \\
\text { condition (ICM plus SEE group)." } \\
\text {--but not stated whether the }\end{array}$ & $\begin{array}{l}\text { High Risk: } \mathrm{CBCL} \text { is parent } \\
\text { report }\end{array}$ & $\begin{array}{l}\text { Low Risk: Dropouts > } \\
15 \% \text { but used } \\
\text { maximum likelihood } \\
\text { methods. } \\
\text { Imputation method: } \\
\text { Ml-comparable }\end{array}$ & $\begin{array}{l}\text { Risk Unclear: Insufficient } \\
\text { information to permit } \\
\text { judgement of low or } \\
\text { high risk; protocol not } \\
\text { available }\end{array}$ \\
\hline
\end{tabular}




\begin{tabular}{|c|c|c|c|c|c|}
\hline Study ID & Random Sequence Generation & Allocation Concealment & $\begin{array}{l}\text { Blinding of Outcome } \\
\text { Assessment }\end{array}$ & $\begin{array}{l}\text { Incomplete Outcome } \\
\text { Data }\end{array}$ & Selective Reporting \\
\hline & & $\begin{array}{l}\text { randomisation was, e.g. done } \\
\text { centrally (PI could not } \\
\text { foresee/predict the next group } \\
\text { allocation) }\end{array}$ & & & \\
\hline Santacruz (2006) & $\begin{array}{l}\text { Risk Unclear: Method of random } \\
\text { sequence generation not } \\
\text { adequately described }\end{array}$ & $\begin{array}{l}\text { Risk Unclear: Method of } \\
\text { concealment is not described or } \\
\text { not described in sufficient detail } \\
\text { to allow a definite judgement }\end{array}$ & $\begin{array}{l}\text { High Risk: Both primary } \\
\text { outcomes Bed Time } \\
\text { Recording and Dark } \\
\text { Behavior Recording- } \\
\text { Modified were parent- } \\
\text { report. }\end{array}$ & $\begin{array}{l}\text { High Risk: Dropouts < } \\
15 \% \text { but not balanced } \\
\text { across groups and } \\
\text { analyses included } \\
\text { Assessment } \\
\text { completers only } \\
\text { Imputation method: } \\
\text { assessment } \\
\text { completers only }\end{array}$ & $\begin{array}{l}\text { Risk Unclear: insufficient } \\
\text { information to permit } \\
\text { judgement of low or } \\
\text { high risk; protocol not } \\
\text { available }\end{array}$ \\
\hline Simon (2011) & $\begin{array}{l}\text { Risk Unclear: Method of random } \\
\text { sequence generation not } \\
\text { adequately described: "After } \\
\text { screening, the high-anxious } \\
\text { children and (both) their } \\
\text { parents were randomized to } \\
\text { one of three conditions" (p.206) }\end{array}$ & $\begin{array}{l}\text { Risk Unclear: Method of } \\
\text { concealment is not described or } \\
\text { not described in sufficient detail } \\
\text { to allow a definite judgement }\end{array}$ & $\begin{array}{l}\text { High Risk for SCARED-71, } \\
\text { low risk for ADIS: } \\
\text { Independent evaluators } \\
\text { conducted the ADIS } \\
\text { interviews; SCARED-71 is } \\
\text { child self-report; blinding } \\
\text { was not checked }\end{array}$ & $\begin{array}{l}\text { High Risk: Dropouts } \\
<15 \% \text { at FU1 but not } \\
\text { balanced across } \\
\text { groups, and dropouts } \\
>15 \% \text { by FU2 and } \\
\text { used LOCF } \\
\text { Imputation method: } \\
\text { LOCF }\end{array}$ & $\begin{array}{l}\text { High Risk: Protocol } \\
\text { stated that the primary } \\
\text { outcome is "Number of } \\
\text { children that have not } \\
\text { developed anxiety } \\
\text { disorders" and } \\
\text { secondary outcome is } \\
\text { SCARED symptoms, but } \\
\text { paper reports "ADIS- } \\
\text { improved' presence and } \\
\text { severity) and symptoms; } \\
\text { protocol available }\end{array}$ \\
\hline Solantaus (2010) & $\begin{array}{l}\text { Low Risk: Randomised into two } \\
\text { groups using computerized } \\
\text { block randomisation }\end{array}$ & $\begin{array}{l}\text { Risk Unclear: "The consenting } \\
\text { families were randomized into } \\
\text { two groups using computerized } \\
\text { block randomization with block } \\
\text { sizes 6-8." (p.885) --does not } \\
\text { specify whether allocation was } \\
\text { done centrally or if the block } \\
\text { sizes were randomlyassigned }\end{array}$ & $\begin{array}{l}\text { High Risk: SCARED and } \\
\text { SDQ both rated by } \\
\text { parents, not blind to } \\
\text { allocation }\end{array}$ & $\begin{array}{l}\text { High Risk: Dropouts } \\
\text { above } 15 \% \text { and } \\
\text { available cases only } \\
\text { Imputation method: } \\
\text { assessment } \\
\text { completers only }\end{array}$ & $\begin{array}{l}\text { Risk Unclear: Insufficient } \\
\text { information to permit } \\
\text { judgement; protocol not } \\
\text { available }\end{array}$ \\
\hline Spittle (2010) & $\begin{array}{l}\text { Low Risk: "Infants will be } \\
\text { randomly allocated to the }\end{array}$ & $\begin{array}{l}\text { Low Risk: "Infants will be } \\
\text { randomly allocated to the }\end{array}$ & $\begin{array}{l}\text { High Risk: Caregiver } \\
\text { report ITSEA }\end{array}$ & $\begin{array}{l}\text { Low Risk: dropout } \\
<15 \% \text {, and balanced }\end{array}$ & $\begin{array}{l}\text { Risk Unclear: ITSEA } \\
\text { means SDs and p's }\end{array}$ \\
\hline
\end{tabular}




\begin{tabular}{|c|c|c|c|c|c|}
\hline Study ID & Random Sequence Generation & Allocation Concealment & $\begin{array}{l}\text { Blinding of Outcome } \\
\text { Assessment }\end{array}$ & $\begin{array}{l}\text { Incomplete Outcome } \\
\text { Data }\end{array}$ & Selective Reporting \\
\hline & \multirow[t]{2}{*}{$\begin{array}{l}\text { intervention and control groups } \\
\text { by a computer-generated } \\
\text { random sequence, with the } \\
\text { treatment allocation concealed } \\
\text { in opaque envelopes; only the } \\
\text { trial statistician will have access } \\
\text { to the code." (Spittle 2009, p.7) }\end{array}$} & \multirow[t]{2}{*}{$\begin{array}{l}\text { intervention and controlgroups } \\
\text { by a computer-generated } \\
\text { random sequence, with the } \\
\text { treatment allocation concealed } \\
\text { in opaque envelopes; only the } \\
\text { trial statistician will have access } \\
\text { to the code." (Spittle 2009, p.7) }\end{array}$} & & $\begin{array}{l}\text { across groups } \\
\text { Imputation method: } \\
\text { assessment } \\
\text { completers only }\end{array}$ & $\begin{array}{l}\text { reported in paper, but } \\
\text { protocol was } \\
\text { retrospectively } \\
\text { registered and did not } \\
\text { specify actual measure } \\
\text { of the outcome of } \\
\text { interest "behavioural } \\
\text { and emotional } \\
\text { functioning". Hence } \\
\text { insufficient detail to } \\
\text { permit judgement; } \\
\text { Protocol available, but } \\
\text { retrospectively } \\
\text { registered }\end{array}$ \\
\hline $\begin{array}{l}\text { Spencer-Smith } \\
\text { (2012) }\end{array}$ & & & $\begin{array}{l}\text { High Risk: BASC } \\
\text { completed by primary } \\
\text { caregiver, not blind to } \\
\text { allocation }\end{array}$ & $\begin{array}{l}\text { High Risk: Drop-out< } \\
\text { 15\%, not balanced } \\
\text { across groups, } \\
\text { Assessment } \\
\text { completers only } \\
\text { Imputation method: } \\
\text { assessment } \\
\text { completers only }\end{array}$ & $\begin{array}{l}\text { Risk Unclear: Not } \\
\text { specified in protocol } \\
\text { what measure will be } \\
\text { used to assess child } \\
\text { behavioural outcomes, } \\
\text { but data on BASC were } \\
\text { all presented clearly in } \\
\text { Table 4; Protocol } \\
\text { available; but } \\
\text { retrospectively } \\
\text { registered }\end{array}$ \\
\hline Strayhorn (1991) & $\begin{array}{l}\text { Low Risk: Random assignment } \\
\text { by drawing facedown card, } \\
\text { sequentially, from a tabletop }\end{array}$ & $\begin{array}{l}\text { Risk Unclear: insufficient } \\
\text { information provided }\end{array}$ & $\begin{array}{l}\text { Low Risk: Behar scale is } \\
\text { teacher report, who is } \\
\text { blind to intervention } \\
\text { status; blinding was } \\
\text { checked: } 0 \% \text { of teachers } \\
\text { interviewed for awareness } \\
\text { of study groups (none } \\
\text { was) }\end{array}$ & $\begin{array}{l}\text { High Risk: dropout > } \\
\text { 15\%, no MI } \\
\text { Imputation method: } \\
\text { assessment } \\
\text { completers only }\end{array}$ & $\begin{array}{l}\text { High Risk: Composite } \\
\text { Behar scores reported } \\
\text { for parent ratings, sub } \\
\text { scores + composite for } \\
\text { teacher ratings, CBCL } \\
\text { scores not reported; } \\
\text { protocol not available }\end{array}$ \\
\hline $\begin{array}{l}\text { Van Doesum } \\
\text { (2008) }\end{array}$ & $\begin{array}{l}\text { Low Risk: Participants were } \\
\text { randomly assigned after } \\
\text { baseline assessment, using a }\end{array}$ & $\begin{array}{l}\text { Risk Unclear: "after a baseline } \\
\text { assessment, participants were } \\
\text { randomly assigned to the }\end{array}$ & $\begin{array}{l}\text { High Risk: ITSEA is mother } \\
\text { report }\end{array}$ & $\begin{array}{l}\text { High Risk: dropout > } \\
15 \%, \text { no MI }\end{array}$ & $\begin{array}{l}\text { Low Risk: Outcomes of } \\
\text { interest from protocol } \\
\text { reported; protocol }\end{array}$ \\
\hline
\end{tabular}




\begin{tabular}{|c|c|c|c|c|c|}
\hline Study ID & Random Sequence Generation & Allocation Concealment & $\begin{array}{l}\text { Blinding of Outcome } \\
\text { Assessment }\end{array}$ & $\begin{array}{l}\text { Incomplete Outcome } \\
\text { Data }\end{array}$ & Selective Reporting \\
\hline & $\begin{array}{l}\text { computer-generated } \\
\text { randomization sequence }\end{array}$ & $\begin{array}{l}\text { intervention group or the } \\
\text { control group. The two groups } \\
\text { were balanced in sets of 10, } \\
\text { each with a computer- } \\
\text { generated randomization } \\
\text { sequence." (p.549-550) -- the } \\
\text { size of each set was known to } \\
\text { the researcher, hence there } \\
\text { remains a risk for selection bias; } \\
\text { but would be low risk if done } \\
\text { centrally however that } \\
\text { information is unclear }\end{array}$ & & $\begin{array}{l}\text { Imputation method: } \\
\text { assessment } \\
\text { completers only }\end{array}$ & available \\
\hline Velderman (2006) & $\begin{array}{l}\text { Risk Unclear: randomly assigned } \\
\text { (p.473) }\end{array}$ & Risk Unclear: support $n / r$ & $\begin{array}{l}\text { High Risk: mother report } \\
\mathrm{CBCL}\end{array}$ & $\begin{array}{l}\text { Low Risk: dropout } \\
<15 \%, \text { no } \mathrm{MI} \\
\text { Imputation method: } \\
\text { assessment } \\
\text { completers only }\end{array}$ & $\begin{array}{l}\text { Risk Unclear: insufficient } \\
\text { detail to permit } \\
\text { judgement }\end{array}$ \\
\hline Wake (2011) & $\begin{array}{l}\text { Low Risk: computer-generated } \\
\text { randomisation by blinded } \\
\text { statistician (p.2) }\end{array}$ & $\begin{array}{l}\text { Low Risk: allocation } \\
\text { concealment (of health centres) } \\
\text { by blinded statistician (p.2) }\end{array}$ & $\begin{array}{l}\text { High Risk: Parent } \\
\text { completed CBCL }\end{array}$ & $\begin{array}{l}\text { Low Risk: dropout< } \\
15 \% \text { and balanced } \\
\text { across groups } \\
\text { Imputation method: } \\
\text { assessment } \\
\text { completers only }\end{array}$ & $\begin{array}{l}\text { Low Risk: all outcome } \\
\text { data presented } \\
\text { according to protocol; } \\
\text { protocol available }\end{array}$ \\
\hline Walkup (2009) & $\begin{array}{l}\text { Low Risk:computer-generated } \\
\text { randomization (p.593) }\end{array}$ & $\begin{array}{l}\text { Low Risk: "The randomization } \\
\text { sequence, generated by the } \\
\text { Web site } \\
\text { http://randomization.com was } \\
\text { stored confidentially by the data } \\
\text { manager in Baltimore, MD. } \\
\text { Randomization was revealed to } \\
\text { participants after the baseline } \\
\text { assessment." (p.593)--study was } \\
\text { conducted in New Mexico and } \\
\text { Arizona; hence infer that }\end{array}$ & $\begin{array}{l}\text { High Risk: Parent } \\
\text { completed ITSEA }\end{array}$ & $\begin{array}{l}\text { Low Risk: dropout > } \\
15 \% \text {, but generalized } \\
\text { linear mixed models } \\
\text { were used } \\
\text { Imputation method: } \\
\text { GLMM }\end{array}$ & $\begin{array}{l}\text { Risk Unclear: insufficient } \\
\text { detail to permit } \\
\text { judgement; protocol not } \\
\text { available }\end{array}$ \\
\hline
\end{tabular}




\begin{tabular}{|c|c|c|c|c|c|}
\hline Study ID & Random Sequence Generation & Allocation Concealment & $\begin{array}{l}\text { Blinding of Outcome } \\
\text { Assessment }\end{array}$ & $\begin{array}{l}\text { Incomplete Outcome } \\
\text { Data }\end{array}$ & Selective Reporting \\
\hline & & $\begin{array}{l}\text { allocation was concealed from } \\
\text { both participants and } \\
\text { interventionists prior to } \\
\text { assignment. }\end{array}$ & & & \\
\hline Williamson (2014) & $\begin{array}{l}\text { Low Risk: "For every } 10 \text { families, } \\
\text { a computerized randomization } \\
\text { model assigned } 6 \text { families to the } \\
\text { intervention condition and } 4 \\
\text { families to the wait-list control } \\
\text { condition." }\end{array}$ & $\begin{array}{l}\text { Risk Unclear: Intervention and } \\
\text { wait-list control groups were } \\
\text { allocated using computerized } \\
\text { randomization, which was } \\
\text { completed by the research } \\
\text { team. A member of the } \\
\text { research team then provided } \\
\text { separate lists to the } \\
\text { intervention staff. Lists were } \\
\text { kept electronically in password } \\
\text { protected excel files with } \\
\text { passwords known only to the } \\
\text { research team, and paper } \\
\text { copies of these lists were kept } \\
\text { locked in research team office. } \\
\text { Assessors independent of } \\
\text { intervention staff completed } \\
\text { assessment and were blinded to } \\
\text { intervention condition }\end{array}$ & $\begin{array}{l}\text { High Risk: SCBE-30 is } \\
\text { mother report }\end{array}$ & $\begin{array}{l}\text { Low Risk: Dropouts } \\
>15 \% \text { but FIML used } \\
\text { Imputation method: } \\
\text { FIML }\end{array}$ & $\begin{array}{l}\text { Risk Unclear: insufficient } \\
\text { detail to permit } \\
\text { judgement; protocol not } \\
\text { available }\end{array}$ \\
\hline Wolchik (2000) & \multirow[t]{2}{*}{$\begin{array}{l}\text { Low Risk: Randomisation } \\
\text { software utilised (Wolchik2002 } \\
\text { p.1876) }\end{array}$} & \multirow{2}{*}{$\begin{array}{l}\text { Low Risk: "After completion of } \\
\text { the pretest, families were } \\
\text { randomly assigned to one of } \\
\text { three conditions: mother, dual- } \\
\text { component, or LC. } \\
\text { Randomization was conducted } \\
\text { by project staff other than the } \\
\text { investigators and interviewers. } \\
\text { A computer-generated } \\
\text { algorithm developed by a } \\
\text { researcher not involved in the } \\
\text { trial was used to assign families } \\
\text { to condition." (Wolchik2013 }\end{array}$} & $\begin{array}{l}\text { High Risk for composite of } \\
C B C L, C D I \text {, and RCMAS; } \\
\text { low risk for TCRS: CBCL is } \\
\text { mother report; CDI \& } \\
\text { RCMAS are child report } \\
\text { (not involved in } \\
\text { intervention) and TCRS is } \\
\text { teacher report; blinding } \\
\text { was not checked }\end{array}$ & \multirow[t]{2}{*}{$\begin{array}{l}\text { Low Risk: dropout< } \\
15 \% \text { and balanced } \\
\text { across groups } \\
\text { Imputation method: } \\
\text { assessment } \\
\text { completers only }\end{array}$} & \multirow[t]{2}{*}{$\begin{array}{l}\text { Risk Unclear: insufficient } \\
\text { detail to permit } \\
\text { judgement; protocol not } \\
\text { available }\end{array}$} \\
\hline Wolchik (2002) & & & $\begin{array}{l}\text { High Risk for composite of } \\
\text { CBCL, CDI, and RCMAS: } \\
\text { CBCL is mother report; } \\
\text { CDI \& RCMAS are child }\end{array}$ & & \\
\hline
\end{tabular}




\begin{tabular}{|c|c|c|c|c|c|}
\hline Study ID & Random Sequence Generation & Allocation Concealment & $\begin{array}{l}\text { Blinding of Outcome } \\
\text { Assessment }\end{array}$ & $\begin{array}{l}\text { Incomplete Outcome } \\
\text { Data }\end{array}$ & Selective Reporting \\
\hline & & p.664) & $\begin{array}{l}\text { report (not involved in } \\
\text { intervention) }\end{array}$ & & \\
\hline Wolchik (2013) & & & $\begin{array}{l}\text { Low Risk for the DIS, High } \\
\text { Risk for internalising } \\
\text { composite: Interviewers } \\
\text { blind to group conducted } \\
\text { the DIS, internalising } \\
\text { composite combined } \\
\text { mother report ABCL and } \\
\text { mothers were not blind. }\end{array}$ & $\begin{array}{l}\text { Low Risk: Dropouts } \\
>15 \% \text { and balanced } \\
\text { across groups } \\
\text { Imputation method: } \\
\text { MI for categorical } \\
\text { variables, maximum- } \\
\text { likelihood estimation } \\
\text { for continuous } \\
\text { variables. }\end{array}$ & \\
\hline Wu (2014) & $\begin{array}{l}\text { Low Risk: "computer-generated } \\
\text { random numbers with } \\
\text { stratification by gestationalage } \\
\text { and hospital" (p. 2386). }\end{array}$ & $\begin{array}{l}\text { Risk Unclear: "The random } \\
\text { sequence was concealed from } \\
\text { the parents, the clinical staff, } \\
\text { and the research assistants in } \\
\text { charge of participant } \\
\text { recruitment." (p. 2386) } \\
\text { Insufficient information about } \\
\text { method of concealment. }\end{array}$ & $\begin{array}{l}\text { High Risk: } \mathrm{CBCL} \text { is parent } \\
\text { report (not blind to } \\
\text { allocation). }\end{array}$ & $\begin{array}{l}\text { High Risk: Dropout } \\
>15 \% \text { AND not } \\
\text { balanced across } \\
\text { groups AND MI not } \\
\text { used (Ax completers } \\
\text { only) } \\
\text { Imputation method: } \\
\text { assessment } \\
\text { completers only. }\end{array}$ & $\begin{array}{l}\text { Low Risk: Protocol did } \\
\text { not mention behavioral } \\
\text { outcomes of interest; } \\
\text { however, paper } \\
\text { reported data } \\
\text { transparently and in a } \\
\text { manner that is adequate } \\
\text { for inclusion in a meta- } \\
\text { analysis. }\end{array}$ \\
\hline
\end{tabular}

Notes

${ }^{a}=$ Information gathered directly fromauthors

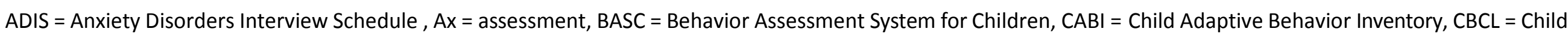
Behavior Checklist, $\mathrm{CDI}=$ Children's Depression Inventory, CONT = control, FIML = full information maximum likelihood, FU1 = follow-up 1, FU2 = follow-up 2, GLMM = generalized linear mixed models, HLM = Hierarchical Linear Modeling, INT = intervention, ITSEA = Infant-Toddler Social and Emotional Assessment, ITT = Intention-to-treat, KSLICE = Kiddie-Streamlined Longitudinal Interval Continuation Evaluation, LOCF = last outcome carried forward, MM = Mean, ASC = Multidimensional Anxiety Scale for Children, $\mathrm{MI}=$ multiple imputation, $\mathrm{n} / \mathrm{r}=$ not reported, $\mathrm{PHSI}=$ Post-Hospital Stress Index for Children, $\mathrm{PI}=$ primary investigator, RCMAS = Revised Children's Manifest Anxiety Scale, SCARED = Screen for Child Anxiety-Related Emotional Disorders, SCAS = Spence Children's Anxiety Scale, SCBE-30 = Social Competence and Behavior-30, SCBE-SF = Social Competence and Behavior Evaluation-Short Form, SD = Standard deviation, SDQ = Strengths and Difficulties Questionnaire, SMFQ = Short Mood and Feelings Questionnaire, TCRS = Teacher-Child Rating Scale, TRF = Teacher Report Form, YSR = Youth Self-Report 


\section{Highlights}

- Preventive parenting interventions can reduce child internalizing problems.

- The effects of parenting interventions can last for up to 11 years.

- Preventive interventions directed primarily at parents can have long-term benefits for children.

- We should increase parental involvement in preventing child internalizing problems. 


\section{University Library}

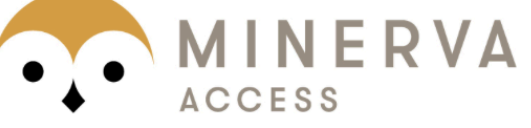

A gateway to Melbourne's research publications

Minerva Access is the Institutional Repository of The University of Melbourne

Author/s:

Yap, MBH;Morgan, AJ;Cairns, K;Jorm, AF;Hetrick, SE;Merry, S

Title:

Parents in prevention: A meta-analysis of randomized controlled trials of parenting interventions to prevent internalizing problems in children from birth to age 18

Date:

2016-12-01

Citation:

Yap, M. B. H., Morgan, A. J., Cairns, K., Jorm, A. F., Hetrick, S. E. \& Merry, S. (2016). Parents in prevention: A meta-analysis of randomized controlled trials of parenting interventions to prevent internalizing problems in children from birth to age 18. CLINICAL PSYCHOLOGY REVIEW, 50, pp.138-158. https://doi.org/10.1016/j.cpr.2016.10.003.

Persistent Link:

http://hdl.handle.net/11343/123521 